\title{
REGRESSÃO COM ERROS DE MEDIDA E PONTOS DE MUDANÇA UTILIZANDO METODOLOGIA BAYESIANA
}

\author{
Daisy Gomes de Souza Tu
}

TESE APRESENTADA

$\mathrm{AO}$

INSTITUTO DE MATEMÁTICA E ESTATÍSTICA

DA

UNIVERSIDADE DE SÃO PAULO

PARA

OBTENÇÃO DO GRAU

$\mathrm{DE}$

DOUTOR EM ESTATÍSTICA

Área de Concentração: Estatística

Orientador: Prof. Dr. Heleno Bolfarine

-- São Paulo. dezembro de 2003 - 


\title{
REGRESSÃO COM ERROS DE MEDIDA E PONTOS DE MUDANÇA UTILIZANDO \\ METODOLOGIA BAYESIANA
}

\begin{abstract}
A tese foi defendida por Daisy Gomes de Souza Tu e aprovada pela Comissão Examinadora em 14 de maio de 2004. Este exemplar corresponde à redação final, devidamente corrigido segundo as recomendações dessa Comissão.
\end{abstract}

\section{Comissão Julgadora:}

- Prof. Dr. Heleno Bolfarine (orientador) - IME-USP

- Prof. Dra. Pilar L.Iglesias - PUC-CHILE

- Prof. Dr. Hélio Migon - IM-LFRJ

- Prof. Dr. Filidor E. V. Labra-IMECC-LNICAMP

- Prof. Dra. Marcia Branco- MME-USP 
Dedico este trabalho ao meu Senhor e Saliador Jesus, que me amou e deu a sua vida por mim. 


\section{Resumo}

O objetivo principal desse estudo foi analisar os modelos de regressão linear estrutural Normal e $t$-Student com erros nas variáveis, com mudança abrupta em um ou mais parâmetros após o k-ésimo ponto desconhecido (ponto de mudança) de uma seqüência finita de observações, utilizando métodos bayesianos de análise de dados.

A análise inferencial envolveu o problema de detectar a existência de mudança nos parâmetros, estimar o ponto de mudança $\mathrm{k}$ e os demais parâmetros, e também fazer a análise preditiva.

O modelo Normal com erros de medida e sem pontos de mudança, foi analisado sob diferentes escolhas de distribuições à priori incluindo também prioris impróprias.

Foram demonstrados teoremas que estabelecem condições para a existência das distribuições posterioris nos modelos Normal e $t$-Student com erros dependentes (com e sem ponto de mudança) sob algumas escolhas específicas de prioris impróprias.

Alguns algoritmos do tipo MCMC ("Markov Chain Monte Carlo") foram utilizados com o objetivo de amostrar das posterioris, como os algoritmos de "Gibbs", "Grouped Gibbs", "Modified and Collapsed Gibbs" e "Metrópolis-Hastings em Gibbs". Foi feita uma análise de sensibilidade a variações nos valores dos seguintes hiperparâmetros: a razão das variâncias residuais (nos modelos identificáveis) e o número de graus de liberdade da distribuição $t$ (quando este é assumido conhecido).

A qualidade do ajuste dos modelos foi avaliada utilizando resíduos bayesianos de validação cruzada. 


\section{Abstract}

The main purpose of this study was to analyze the Normal and Student-t linear regression with measurement error models with an abrupt change in a finite sequence of observations, using bayesian methods for data analysis.

The inferential analysis considered the problem of detecting the existence of parameter changes, estimating the change point $\mathrm{k}$ and the remaining parameters, and also doing predictive analysis.

The Normal measurement error model without change point was analyzed under diferent prior distributions including improper priors.

Conditions for the existence of the posterior distribution under some specific improper prior distributions were demonstrated for the Normal and Student-t with dependent error models (with and without change point).

Some MCMC ("Markov Chain Monte Carlo") were used with the purpose of sampling from the posteriors like for instance: Gibbs, Grouped Gibbs, Modified and Collapsed Gibbs and Metropolis-Hastings in Gibbs.

A sensitivity analysis to diferent values of the following hyperparameters was done: the ratio of the residual variances (on the identifiable models) and the number of degrees of freedom of the Student-t distribution (when it was assumed known).

To asses model adequacy, bayesian cross-validation residuals were used. 


\section{Agradecimentos}

A Deus, por ter me sustentado nos momentos difíceis da doença de meu marido e durante toda a trajetória desse trabalho. A Ele sejam dadas a Honra, a Glória e o Poder para sempre.

Ao meu orientador, o Professor Heleno Bolfarine, realmente pelo privilégio que tive de trabalhar sob sua orientação e pelo respeito, confiança, compreensão e sua enorme dedicação durante todo o desenvolvimento desse trabalho.

Ao Professor Chen Ming-Hui, pela orientação que possibilitou o desenvolvimento dos teoremas sobre a existência da posteriori do modelo estrutural Normal do Capítulo 2, e também por checar meus primeiros programas computacionais durante o semestre em que estive na "Worcester Polytechnic Institute - U. S. A.".

À Professora Pilar Iglesias, eu realmente agradeço pelas sugestões e esclarecimentos nos vários encontros que tivemos.

Ao Professor Alexandre Kawano da Escola Politécnica, pelas dicas na fase de demonstração de um dos teoremas sobre a existência da posteriori no modelo $t$ dependente.

Ao Walter eu agradeço imensamente por seu trabalho notável e eficiente na edição dessa tese, e também por conseguir sempre arranjar um tempo para esse trabalho, e não somente isso, mas por sua enorme paciência.

Aos meus queridos amigos Marcia, Dione, Luiz Gustavo, Arthur, Denise, Oswaldo e Cátia, pela amizade, boa vontade e encorajamento com que sempre me presentearam.

A CAPES e ao $\mathrm{CNPq}$, respectivamente, pela bolsa de doutorado no país e a bolsa sanduíche (PDEE).

Finalmente, em especial, à minha mãe, por suas orações e sua força e também 
por eu poder sempre contar com seu amor incondicional. Também ao meu marido, que sempre desejou e procurou fazer o melhor por mim. 


\section{Sumário}

1 Introdução 1

1.1 Caracterização geral dos problemas de ponto de mudança . . . . . . . 1

1.2 O problema alvo desse estudo . . . . . . . . . . . . . . 3

1.3 Levantamento bibliográfico . . . . . . . . . . . . . . 6

2 Regressão linear Normal com erros nas variáveis 11

Introdução . . . . . . . . . . . . . . . . . . . . . 11

2.1 O modelo estrutural e o modelo funcional com erros Normais na estatística clássica . . . . . . . . . . . . . . . . . . . 12

2.2 O modelo estrutural e o modelo funcional na estatística bayesiana . . 15

2.2 .1 O modelo bayesiano estrutural . . . . . . . . . . . 16

2.2 .2 O modelo bayesiano funcional . . . . . . . . . . . . 23

2.3 O modelo estrutural identificável com particular priori de componentes independentes . . . . . . . . . . . . . 25

2.4 Métodos computacionais descritos na literatura . . . . . . . . 28

2.5 Implementação de algoritmos para o modelo da seção 2.3 . . . . . . 29

2.5.1 Distribuições condicionais completas à posteriori baseadas nos dados observados . . . . . . . . . . . . . . . . . 29

2.5.2 Distribuições condicionais completas à posteriori baseadas nos dados completos $(D) \ldots \ldots \ldots \ldots . \ldots . \ldots 32$

2.5.3 Implementação do algoritmo original de Gibbs . . . . . . . . 32

2.5.4 Implementação do algoritmo de Gibbs em blocos . . . . . . . . 34 


\subsubsection{Implementação do algoritmo "modified collapsed}

Gibbs" . . . . . . . . . . . . . . . . 36

2.5.6 Implementação do algoritmo híbrido de Metropolis-Hastings em Gibbs . . . . . . . . . . . . . . . . . . 38

2.6 O modelo estrutural não identificável com particular priori de componentes independentes . . . . . . . . . . . . . . 44 42

2.7 O modelo estrutural com particular priori própria de componentes condicionalmente independentes . . . . . . . . . . 44 46

2.7.1 O modelo com restrição de identificabilidade . . . . . . . . 46

2.7.2 O modelo sem restrições de identificabilidade . . . . . . . . . . 49

2.8 Estabelecimento das condições para a existência da distribuição à posteriori sob diferentes escolhas de prioris impróprias . . . . . . . . 52

2.8.1 Condições necessárias e suficientes no caso do modelo estrutural identificável . . . . . . . . . . . . . . . . 53

2.8.2 Condições necessárias e suficientes no caso do modelo não identificável . . . . . . . . . . . . . 76

2.9 Estimação do modelo . . . . . . . . . . . . . . . . . 81

2.10 Avaliação do ajuste e análise de sensibilidade . . . . . . . . . . 84

2.10.1 Sensibilidade do modelo a diferentes valores da razão das variâncias residuais . . . . . . . . . . . . . 85

2.10 .2 Avaliação da qualidade do ajuste . . . . . . . . . 85

2.11 Algumas medidas de comparação de modelos . . . . . . . . . . . 98

2.12 EXEMPLOS . . . . . . . . . . . . . . . . . . 103

2.12.1 Análise do modelo estrutural normal com restrição de identificabilidade . . . . . . . . . . . . . . . 103

2.12.2 Análise do modelo estrutural normal sem restrição de identificabilidade . . . . . . . . . . . . . . . . . 118

2.12.3 O modelo estrutural com restrição de identificabilidade e priori imprópria . . . . . . . . . . . . . . . . 126 
2.12.4 O modelo estrutural sem restrição de identificabilidade e priori imprópria . . . . . . . . . . . . . . 136

2.12 .5 Resumo dos resultados: . . . . . . . . . . . . . . . . 143

3 O modelo linear estrutural identificável $t$-Student com erros nas variáveis 146 Introdução . . . . . . . . . . . . . . . . . . . . . 146

3.1 O modelo estrutural $t$-independente com o número de graus de liberdade conhecido . . . . . . . . . . . . . . . . . 149

3.1.1 Definição do modelo . . . . . . . . . . . . . . . . . 149

3.1.2 Implementação de algoritmos MCMC no modelo com particular priori própria de componentes independentes . . . . . 155

3.1 .3 Distribuição preditiva . . . . . . . . . . . . . . . 157

3.1 .4 Avaliação do ajuste . . . . . . . . . . . . . . . 158

$3.2 \mathrm{O}$ modelo estrutural $t$-independente com graus de liberdade descon-

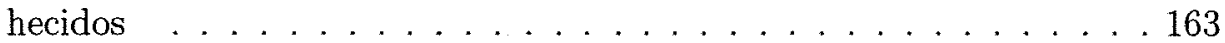

3.2 .1 Definição do modelo . . . . . . . . . . . . . . 164

3.2.2 Implementação de algoritmos MCMC no modelo $t$-independente para duas particulares escolhas de priori própria . . . 165

3.3 O modelo estrutural $t$-dependente com o número de graus de liberdade conhecido . . . . . . . . . . . . . . 166

3.3.1 Definição do modelo . . . . . . . . . . . . . . . . . 166

3.3.2 Implementação de algoritmos MCMC no modelo com particular priori própria de componentes independentes . . . . . . 170

3.3.3 Distribuição preditiva . . . . . . . . . . . . . . . . . . . 171

3.3.4 Avaliação do ajuste . . . . . . . . . . . . . . . . . 172

3.4 O modelo estrutural $t$-dependente com graus de liberdade desconheci-

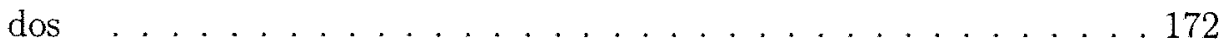


3.5 Condições para a existência da posteriori no modelo estrutural $t$ Student com erros dependentes e número de graus de liberdade co-

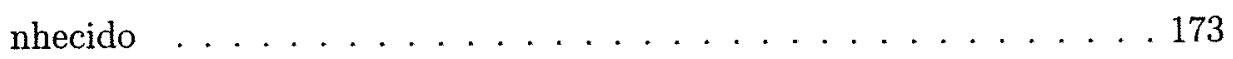

3.6 Sensibilidade do modelo a diferentes valores do número de graus de liberdade . . . . . . . . . . . . . . . . . . . . 182

3.7 Algumas medidas de comparação de modelos . . . . . . . . . . . 183

4 Uma análise do modelo estrutural Normal com erros nas variáveis $\begin{array}{ll}\text { com um ponto de mudança desconhecido } & 187\end{array}$ Introdução . . . . . . . . . . . . . . . . . . . . 187

4.1 O modelo estrutural com um ponto de mudança e priori com componentes independentes . . . . . . . . . . . . . . 189

4.2 A análise do modelo que permite mudança em todos os parâmetros com particular priori de componentes independentes . . . . . . . . 194

4.2 .1 Distribuição à posteriori . . . . . . . . . . . . . . . 195

4.2.2 Distribuições condicionais completas à posteriori baseadas nos dados completos . . . . . . . . . . . . . . . . 195

4.2.3 Estimação do modelo que permite mudança em todos os parâmetros . . . . . . . . . . . . . . 197

4.3 Implementação do algoritmo de Gibbs no modelo que permite mudança em um subconjunto fixado de parâmetros . . . . . . . . . 199

4.4 Detecção de mudança . . . . . . . . . . . . . . . . . 202

4.4.1 Procedimento 1 - Um teste baseado na distribuição à posteriori de $k \ldots \ldots \ldots . \ldots . \ldots . \ldots 203$

4.4.2 Procedimento 2 - Uso da distribuição à posteriori da diferença (ou quociente) entre os parâmetros. . . . . . . . . . . . 208

4.4.3 Procedimeto 3 - Uso de critérios de seleção ou escolha de modelos . . . . . . . . . . . . . . . 208

4.5 Distribuição preditiva e avaliação do ajuste . . . . . . . . . 218

4.5.1 Distribuição preditiva . . . . . . . . . . . . . . . 219 
4.5.2 Densidade condicional preditiva de validação cruzada . . . . 220

4.5.3 Resíduos de validação cruzada . . . . . . . . . . . . . 223

4.6 Estabelecimento das condições para a existência da posteriori sob algumas escolhas de prioris impróprias . . . . . . . . . 2225

4.6.1 Condições necessárias e suficientes no modelo que permite mudança em todos os parâmetros . . . . . . . . . . . 225

4.6.2 Condições suficientes no modelo que permite mudança apenas

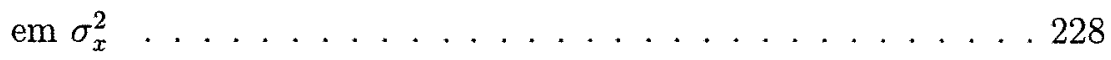

4.7 EXEMPLOS . . . . . . . . . . . . . . . . . . 231

4.7.1 Análise do modelo estrutural normal identificável . . . . . . 231

4.7.2 Análise do modelo estrutural não identificável . . . . . . . . 245

4.7.3 Análise do procedimento 1 para detetar mudança baseada na distribuição à posteriori de $k$. . . . . . . . . . . . . . . 259

4.7.4 Análise do procedimento 2 para detetar mudança baseada na distribuição à posteriori das diferenças (ou quocientes) . . . . 269

4.7.5 Análise do procedimento 3 para detetar mudança baseada nos critérios de seleção de modelos. . . . . . . . . . . . 275

4.7.6 Resumo dos resultados . . . . . . . . . . . . . . . 278

5 Uma análise do modelo linear estrutural $t$-Student com erros nas variáveis com um ponto de mudança desconhecido

5.1 O modelo $t$ com erros independentes, com particular priori de componentes independentes . . . . . . . . . . . . . . . . 281

5.1.1 O modelo que permite mudança em todos os parâmetros após o ponto de mudança $k \ldots \ldots . \ldots . \ldots 281$

5.1.2 Implementação do algoritmo de Gibbs no modelo que permite mudança em todos os parâmetros . . . . . . . . . . . . 284

5.1.3 Implementação do algoritmo de Gibbs no modelo com mudança em um subconjunto qualquer de parâmetros . . . . 286 
5.2 O modelo $t$-dependente com particular priori de componentes independentes . . . . . . . . . . . . . . . 288

5.2.1 O modelo que permite mudança em todos os parâmetros após o ponto $k \ldots \ldots \ldots \ldots \ldots \ldots \ldots$

5.2.2 Implementação do algoritmo de Gibbs no modelo com mudança em um subconjunto qualquer de parâmetros . . . . . 290

5.3 Detecção de mudança e estimação do modelo . . . . . . . . . . . . 291

5.4 Condições para a existência da posteriori no modelo $t$-dependente sob diferentes escolhas de prioris impróprias . . . . . . . . . . . . 291

5.4.1 Condições no caso do modelo que permite mudança em todos os parâmetros . . . . . . . . . . . . . . . . 292

5.4.2 Condições no caso do modelo com mudança apenas em $\sigma_{x}^{2} \quad \ldots 295$

5.5 Distribuição preditiva e avaliação do ajuste . . . . . . . . . . . 298

6 Considerações Finais $\quad 299$

6.0.1 Resultados do estudo sobre ponto de mudança . . . . . . . 299

6.0 .2 Perspectivas para trabalhos futuros . . . . . . . . . . 303

Apêndice

305

Seção 2.4 Métodos Computacionais Descritos na Literatura . . . . . . . . 305

2.4 .1 Classificação Geral . . . . . . . . . . . . . . . . 305

2.4.2 Métodos MCMC: idéias básicas . . . . . . . . . . . . 310

O algoritmo de Metrópolis-Hastings . . . . . . . . . . 312

O algoritmo original de Gibbs . . . . . . . . . 316

2.4 .3 O algoritmo de Gibbs em Blocos . . . . . . . . . . . . 318

2.4.4 O algoritmo "Collapsed Gibbs" . . . . . . . . . . . . . 320

2.4.5 O algoritmo Híbrido de Metropolis-Hastings em Gibbs . . . . . 324

2.4.6 Diagnósticos de Convergência . . . . . . . . . . . . 326

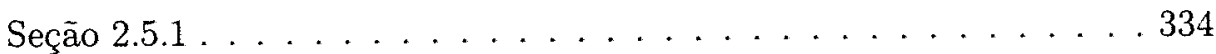

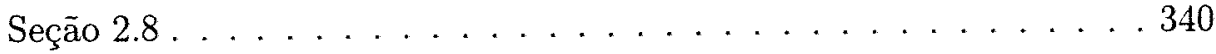




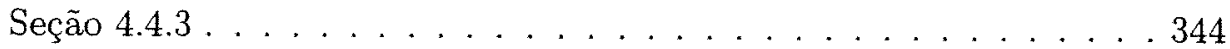

(a) O critério do Fator de Bayes . . . . . . . . . . . . . . 344

(b) O critério do Pseudo Fator de Bayes - formulação geral . . . . . . 347

(c) O critério "BIC" ("Bayesian Information Criterion") . . . . . . 347 


\section{Capítulo 1}

\section{Introdução}

\subsection{Caracterização geral dos problemas de ponto de mudança}

Dizemos que um problema tem um ponto de mudança se dada uma seqüência de observações ordenadas pelo tempo ou espaço ou outra variável qualquer, a distribuição dessas observações muda após um determinado ponto desconhecido dessa seqüência. Podemos definir o problema da seguinte maneira:

Dada a sequêencia de variáveis aleatórias (ou vetores aleatórios) $\underset{\sim}{z}, z_{2}, \ldots$ com distribuições dadas respectivamente por $F_{1}, F_{2}, \ldots$ onde os índices $1,2, \ldots$ são, por exemplo, "instantes de tempo". Dizemos que o índice $k$, ou o ponto $k$, é o ponto de mudança desconhecido dessa seqüência se $F_{1}=F_{2}=\cdots=F_{k} \neq F_{k+1}=F_{k+2}=\cdots$

Vale salientar ainda o fato de que as observações não precisam ser tomadas necessariamente em intervalos "de tempo" igualmente espaçados, e que a definição no caso de múltiplos pontos de mudança é semelhante.

Um exemplo de aplicação é ilustrado em Worsley (1983) que estudou a incidência de um defeito de nascença denominado "Talipes" numa região da Nova Zelândia entre 1960 e 1976 . No ano de 1965 o herbicida $245-\mathrm{T}$ foi utilizado pela primeira vez nessa região e o interesse era saber se isso coincidia com um aumento aparente da incidência de crianças nascidas com essa doença. O modelo proposto considerou o 
número de crianças nascidas com Talipes no ano $i$ como tendo distribuição binomial $B\left(n_{i}, p_{i}\right), i=1,2, \ldots, 17$, onde $n_{i}=$ número total de nascimentos no ano $i$ e $p_{i}=$ probabilidade de uma criança nascer com a doença no ano $i$. O autor propôs um teste para testar $H_{0}: p_{1}=p_{2}=\cdots=p_{17}$ versus $H_{1}: p_{1}=\cdots=p_{k} \neq p_{k+1}=p_{k+2}=$ $\cdots=p_{17}$, onde $k$ é o ponto de mudança desconhecido. O interesse era então saber se o ponto de mudança $k$ coincidia com a data de introdução do pesticida, para que providências necessárias pudessem ser tomadas (como a verificação, por meio de uma análise bioquímica, de uma possível relação causal e, nesse caso, a tomada de medidas governamentais para a comercialização do produto).

Segundo Brodsky e Darkvosky (1993) os problemas de ponto de mudança tem vários aspectos e podem ser classificados por exemplo quanto às seguintes características:

(1) Quanto ao método de obtenção dos dados. Temos dois tipos de problemas, os seqüenciais e os de tamanho de amostra fixados. Nos procedimentos seqüenciais os dados são examinados (quanto à homogeneidade) a cada nova observação. Nesse caso, a seqüência de observações $\left\{\underset{\sim}{z_{i}}\right\}_{i}$ é infinita (já que o tamanho da amostra é determinado por uma regra de parada que depende dos dados). Nos problemas de amostras fixas (problemas retrospectivos), o processo de aquisição de dados é completado antes da hipótese de homogeneidade ser testada. Nesse caso, temos uma seqüência finita $\{\underset{\sim}{z}\}_{i=1}^{n}$.

(2) Quanto à informação à priori sobre $F_{i}$. Nesse caso, podemos ter problemas de ponto de mudança paramétricos, semi-paramétricos e não-paramétricos.

(3) Quanto às características dos dados. Podemos ter modelos com tempo discreto ou tempo contínuo, uni ou multidimensionais, como também os modelos com observações dependentes ou independentes. 
(4) Quanto ao tipo de mudança. Temos os modelos com mudanças abruptas e os modelos com mudanças graduais. Um exemplo de mudança abrupta num modelo de regressão linear simples sem erro de medida é quando $Y_{i}=\alpha_{1}+\beta_{1} X_{i}$, para $i=1, \ldots, k$, e $Y_{i}=\alpha_{2}+\beta_{2} X_{i}$, para $i=k+1, \ldots, n$, e os $X_{i}$ 's não estão necessariamente em ordem crescente no tempo. No caso, por exemplo, em que $X_{1} \leq X_{2} \leq \cdots \leq X_{n}$ e além disso existe $\gamma$ tal que $\alpha_{1}+\beta_{1} \gamma=$ $\alpha_{2}+\beta_{2} \gamma$, onde $X_{k} \leq \gamma<X_{k+1}$, temos um modelo com mudança gradual, ou sem descontinuidade, conhecido como "regressão segmentada" ou "two-phase regression".

(5) Quanto ao número de mudanças. Nessa categoria podemos ter os modelos com um único ponto de mudança e os modelos com múltiplos pontos de mudança.

Para a inferência em modelos com pontos de mudança temos basicamente dois aspectos a serem considerados. O primeiro é o de determinar se existem mudanças e o segundo é o de localizar a posição desses pontos (dado que há mudança), como também estimar as outras quantidades de interesse e também fazer a análise preditiva

\subsection{O problema alvo desse estudo}

Nesse trabalho o objetivo principal é fazer inferência sobre o modelo de regressão linear estrutural com erros nas variáveis e com mudança abrupta nos parâmetros após o $k$-ésimo "instante de tempo" (desconhecido) numa sequiência finita de vetores de observações independentes $\left(\begin{array}{l}Y_{1} \\ X_{1}\end{array}\right), \ldots,\left(\begin{array}{l}Y_{n} \\ X_{n}\end{array}\right)$ indexadas pelo "tempo". Nesse trabalho vamos estudar também modelos que permitem mudanças em um subconjunto fixado qualquer de parâmetros e que a relação entre as variáveis observáveis, $Y_{i}$ e $X_{i}$, permanece linear ao longo do "tempo" (isto é, antes e depois do ponto de mudança 
$k$ ); além disso vamos assumir que tanto a variável aleatória latente como os erros de medida são normalmente distribuídos, com parâmetros que podem mudar ao longo do "tempo". O caso em que os erros têm uma distribuição $t$-Student também é analisado.

A análise inferencial, que envolve o problema de detetar a existência de mudança, localizar a(s) posição(ōes) do(s) ponto(s) de mudança, estimar os demais parâmetros e fazer a análise preditiva é desenvolvida utilizando-se métodos bayesianos.

$\mathrm{Na}$ literatura, até o momento, muito poucos resultados têm sido publicados nessa linha; temos basicamente os artigos de Chang e Huang (1997) e Chong (1999).

Chang e Huang (1997), utilizando metodologia clássica (frequentista) analisaram o modelo que permite mudança apenas na média da covariável e somente no caso em que os erros (e a covariável) são normalmente distribuídos. Nas aplicações o modelo de Chang e Huang (1997) é muito limitado, já que permite mudança apenas na média da covariável, o que não é muito realista para a maioria dos problemas de regressão na prática. Outra limitação é que o procedimento para detectar mudança proposto pelos autores está baseado nas propriedades assintóticas da estatística da razão de verossimilhança e a eficiência desse teste para pequenas (ou médias) amostras ainda não foi estudada. Além disso, os resultados assintóticos sobre a consistência dos estimadores no caso em que há mudança estão baseados em hipóteses extremamente fortes.

Chong (1999) estudou também o modelo linear com erros nas variáveis do ponto de vista da estatística clássica e se limitou ao caso de mudança apenas no coeficiente angular da reta de regressão (mantendo os demais parâmetros sem mudança); além disso, mostrou a consistência do estimador do ponto de mudança e derivou a distribuição assintótica de um teste tipo Wald para detetar mudança.

Nossa proposta é mais geral em vários sentidos: 
(1) Podemos analisar modelos que permitem mudanças em todos os parâmetros (como também em qualquer subconjunto de parâmetros de interesse); conseqüentemente, podemos escolher o "melhor modelo". Nas aplicações isso é vital, já que uma análise exploratória dos dados pode não "revelar" a modelagem mais adequada pelo fato de que o ponto de mudança é desconhecido.

(2) Deixando à parte razões filosóficas, a metodologia bayesiana para tratar de problemas de pontos de mudança é a mais natural e conveniente por diversas razões práticas.

(a) Não se apóia em resultados assintóticos: Do ponto de vista bayesiano, adotada uma função perda, os estimadores que minimizam o risco à posteriori são ótimos. Segundo Zacks (1983), a obtenção, por exemplo, dos estimadores de máxima verossimilhança e a derivação de suas propriedades assintóticas pode ser bem complicada, mesmo nos problemas mais simples de ponto de mudança. Segundo Smith (1975) e Hinkley (1970), além do problema assintótico, os procedimentos baseados somente na verossimilhança apresentam dificuldades nos problemas de ponto de mudança, pelo fato de que a função de verossimilhança é frequentemente multimodal.

(b) A mesma metodologia de análise que é usada no modelo que permite mudança em um subconjunto qualquer de parâmetros é usada também no modelo que permite mudança em todos os parâmetros, ao contrário do procedimento de Chang e Huang (1997) que funciona somente para o caso de mudança na média (e que talvez possa eventualmente ser estendido somente para o caso de mudança no intercepto da reta de regressão, mantendo-se os demais parâmetros sem mudança).

(c) Usando metodologia bayesiana podemos investigar a existência de múl- 
tiplos pontos de mudança de forma bem simples, usando por exemplo o método da segmentação binária descrito no capítulo 4, que consiste simplesmente em aplicar repetidas vezes o mesmo procedimento construído para detetar um único ponto de mudança. $\mathrm{Na}$ estatística clássica temos o problema do nível de significância e portanto nem sempre isso pode ser feito.

(d) Do ponto de vista computacional, a análise bayesiana também é relativamente simples, principalmente utilizando as técnicas computacionais do tipo MCMC disponíveis atualmente na literatura. Além disso, é suficiente implementar basicamente um único programa computacional.

Assim, de modo geral, a estatística bayesiana para problemas de ponto de mudança é (a) mais natural, (b) tecnicamente mais simples, (c) permite incorporar ao modelo informação subjetiva disponível na área da aplicação e (d) permite-nos obter mais resultados.

\subsection{Levantamento bibliográfico}

$\mathrm{Na}$ literatura, os primeiros estudos sobre pontos de mudança apareceram na década de 50 principalmente na área de controle de qualidade e tratavam principalmente do problema de mudança na média de uma sequência de variáveis aleatórias independentes e normalmente distribuídas. Posteriormente estendeu-se o estudo nas áreas de regressão com observações independentes, séries temporais, análise de sobrevivência e outras, de modo que a literatura sobre os problemas de ponto de mudança é atualmente muito vasta.

O modelo de regressão linear simples com mudança abrupta (não contínua) após um ponto de mudança desconhecido, no caso em que a covariável é medida sem 
erros e o tamanho da amostra é fixado (caso não seqüencial, ou restrospectivo) foi descrito por Quandt $(1958,1960)$, que usou o método de máxima verossimilhança para estimar os parâmetros do modelo e derivou um teste baseado no critério da razão de verossimilhança para testar a existência de mudança nos parâmetros da equação de regressão. Nessa linha (Estatística Clássica), muitos trabalhos têm sido publicados desde então para tratar desse problema. Sob o enfoque da Estatística Bayesiana esse modelo foi estudado por Ferreira (1975) para o caso em que o número de pontos de mudança é conhecido. As posterioris dos parâmetros, seus valores esperados como também intervalos de credibilidade e testes foram derivados.

Continuando a focalizar na literatura bayesiana desses modelos de regressão, apresentamos a seguir alguns dos trabalhos relevantes nessa linha:

Broemeling e Choy (1981) desenvolveram um teste bayesiano para detetar mudança nos parâmetros da equação de regressão do modelo com no máximo um ponto de mudança. Esse teste é baseado na distribuição marginal à posteriori do ponto de mudança, onde a probabilidade à posteriori de que não haja mudança, $p(K=n)$, é comparada com a probabilidade de que haja uma mudança $(1-p(K=n))$.

Holbert (1982) investigou o problema de estimação do modelo de regressão linear múltipla onde ocorre mudança abrupta em um único ponto, como também a estimação do modelo de regressão simples segmentado ("two-fase regression") usando priori imprópria e obtendo a distribuição de probabilidade marginal à posteriori do ponto de mudança.

Moen et al. (1985) generalizou o trabalho de Choy e Broemeling (1980) estudando o modelo linear multivariado usando uma priori própria Normal Multivariada - Wishart e obtendo as marginais à posteriori do ponto de mudança, bem como das matrizes de regressão e da matriz de precisão.

Land e Broemeling (1983) estudaram o modelo linear geral que admite um único 
ponto de mudança (mudança abrupta) num período fixado, assumindo que todos os parâmetros são desconhecidos e tomando uma particular priori própria. Eles também derivaram a distribuição preditiva à posteriori de observacões futuras.

Moen e Broemeling (1985) obtiveram a densidade preditiva à posteriori para o modelo linear multivariado com um único ponto de mudança (mudança abrupta) na matriz dos parâmetros da regressão, também sob o enfoque retrospectivo, ou seja, não sequencial.

Kim (1991) apresentou um teste bayesiano para testar se existe mudança na reta de regressão usando a região de credibilidade HPD.

Carlin (1992) fez uma abordagem geral de modelos hierárquicos bayesianos com um único ponto de mudança (abrupta) incluindo uma aplicação em regressão, utilizando o amostrador de Gibbs para estimar as densidades marginais à posteriori de interesse.

Wang e Lee (1993) estudaram o modelo com mudança abrupta apenas no intercepto da reta de regressão, obtendo a distribuição marginal à posteriori tanto do ponto de mudança como da diferença dos interceptos (e também dos parâmetros que não mudam); além disso utilizaram a priori de Jeffreys comparando com a priori "uniforme" encontrada na maioria dos estudos.

Leiva (1994) estendeu o trabalho de Broemeling e Choy (1981) para o caso em que o número de pontos de mudança é desconhecido.

Lyazrhi (1997) estudou o problema de mudança abrupta no modelo de regressão linear normal com no máximo um ponto de mudança propondo um procedimento bayesiano ótimo para testar a hipótese $H_{0}$ de que não há nenhuma mudança versus a hipótese $\bigcup_{i=1}^{n-1} H_{i}$, onde $H_{i}$ é a hipótese que pelo menos um parâmetro muda após a i-ésima observação. O procedimento é baseado na função perda quadrática quando escolhemos a hipótese $H_{i}$ ao invés da verdadeira hipótese $H_{j}$. 
Lyazrhi e Caussinus (1997) propõem um procedimento global baseado na teoria de decisão bayesiana para detetar no máximo $p$ pontos de mudança (onde $p$ é desconhecido) no modelo de regressão linear Normal.

Os trabalhos citados até este ponto tratam dos problemas não sequenciais de regressão linear com erros independentes onde a(s) covariável(eis) são medidas sem erros e a(s) mudança(s) não são contínuas (isto é são abruptas).

No caso dos modelos com erros nas variáveis e mudança nos parâmetros temos muito poucos resultados na literatura e somente sob o enfoque clássico. Fizemos um levantamento bibliográfico pelo CIS (Current Index to Statistics) desde 1975 e apuramos os seguintes trabalhos de Teeter (1979, 1985), Gbur e Dahm (1985), Chang e Huang (1997) e Chong (1999).

Teeter (1979) estudou o modelo linear segmentado com erros nas variáveis formado por duas retas que se interceptam e mostrou que o estimador do ponto de interseç̧ão das duas retas, baseado nos estimadores de mínimos quadrados dos coeficientes das retas, não é assintoticamente não-viesado e derivou uma aproximação assintótica para o erro quadrático médio desse estimador. Teeter (1985), estudando ainda o mesmo modelo, propõe uma técnica geral para obter uma estimativa do ponto de mudança da intersecção dos dois submodelos que se interceptam.

Gbur e Dahm (1985) estudaramm também o modelo segmentado simples com erros nas variáveis, derivando o estimador do ponto de intersecção das duas retas pelo método dos momentos e obtendo sua distribuição assintótica.

Grimshaw (1992) estudou o modelo linear-platô com erros nas variáveis em duas situações; primeiramente tomando-se observações repetidas para $X_{i}$ (para um mesmo $Y_{i}$ ) e também tomando-se observações repetidas para cada elemento do par $\left(X_{i}, Y_{i}\right)$. O autor analisou o estimador de mínimos quadrados dos parâmetros quanto à consistência em ambos os casos. 
Os resultados de Chang e Huang (1997) e Chong (1999), descritos brevemente na seção anterior, são os mais relevantes no contexto de regressão com erros nas variáveis com ponto de mudança. 


\section{Capítulo 2}

\section{Regressão linear Normal com erros nas variáveis}

\section{Introdução}

Inicialmente, sob o enfoque da estatística clássica, comparamos o modelo linear estrutural simples com erros nas variáveis (no caso em que os erros são normalmente distribuídos e aditivos) com o modelo linear funcional sem pontos de mudança. A seguir comparamos esses dois modelos na formulação bayesiana.

No restante do capítulo concentramo-nos na análise bayesiana do modelo de regressão linear estrutural simples com erros (e covariável) normalmente distribuídos para várias escolhas de distribuição à priori. Estudamos tanto o modelo com restrição de identificabilidade como o modelo sem restrições de identificabilidade (aproveitando o fato de que a metodologia bayesiana não está limitada apenas a situações de identificabilidade).

Para amostrar das posterioris implementamos quatro algorítmos do tipo "Markov Chain Monte Carlo": o algoritmo de Gibbs, o "Grouped Gibbs", o "Modified and Collapsed Gibbs" e o algoritmo de "Metropolis-Hastings em Gibbs".

Nesse capítulo também derivamos quatro teoremas que estabelecem as condições necessárias e suficientes para a existência das distribuições posterioris partindo de 
um conjunto específico de prioris impróprias.

Uma análise de sensibilidade com relação a diferentes valores da razão das variâncias residuais $(\lambda)$ também é feita para o modelo identificável.

A qualidade do ajuste é também avaliada usando residuos bayesianos de validação cruzada.

Finalmente descrevemos alguns métodos de seleção ou escolha de modelos encontrados na literatura aplicados ao modelo estrutural Normal.

\subsection{O modelo estrutural e o modelo funcional com erros Normais na estatística clássica}

\section{O Modelo Estrutural}

O modelo linear estrutural aditivo simples com erros de medida e covariável normalmente distribuídos é definido pelas equações

$$
\begin{aligned}
Y_{i} & =y_{i}+e_{i}, \text { com } y_{i}=\alpha+\beta x_{i} \\
\text { e } X_{i} & =x_{i}+u_{i}
\end{aligned}
$$

onde $x_{i}$ é uma variável aleatória latente (não observável), $e_{i}$ e $u_{i}$ são as componentes de erro e $X_{i}$ e $Y_{i}$ são variáveis aleatórias observáveis para $i=1,2, \ldots, n$. Além disso, assumimos que $\left(e_{i}, u_{i}, x_{i}\right)^{T}$ são independentes e identicamente distribuidos (i.i.d.) com

$$
\left(\begin{array}{c}
e_{i} \\
u_{i} \\
x_{i}
\end{array}\right) \stackrel{\text { i.i.d. }}{\sim} N_{3}\left(\left(\begin{array}{c}
0 \\
0 \\
\mu
\end{array}\right) ;\left(\begin{array}{ccc}
\sigma_{e}^{2} & 0 & 0 \\
0 & \sigma_{u}^{2} & 0 \\
0 & 0 & \sigma_{x}^{2}
\end{array}\right)\right) \text {, para } i=1,2, \ldots, n .
$$

O espaço paramétrico do modelo é o conjunto

$$
\Theta=\left\{\underset{\underline{\theta}}{\theta}=\left(\alpha, \beta, \mu, \sigma_{x}^{2}, \sigma_{u}^{2}, \sigma_{e}^{2}\right)^{T}: \alpha, \beta, \mu \in \mathbb{R} \quad \text { e } \quad \sigma_{x}^{2}, \sigma_{u}^{2}, \sigma_{e}^{2} \in \mathbb{R}^{+}\right\}
$$

Como conseqüência de (2.1.1), os dados "completos" $\left(X_{i}, Y_{i}, x_{i}\right)^{T}, i=1, \ldots, n$ 
têm a seguinte distribuição normal:

$$
\left(\begin{array}{c}
Y_{i} \\
X_{i} \\
x_{i}
\end{array}\right) \stackrel{\text { i.i.j. }}{\sim} N_{3}\left(\left(\begin{array}{c}
\alpha+\beta \mu \\
\mu \\
\mu
\end{array}\right) ;\left(\begin{array}{ccc}
\beta^{2} \sigma_{x}^{2}+\sigma_{e}^{2} & \beta \sigma_{x}^{2} & \beta \sigma_{x}^{2} \\
\beta \sigma_{x}^{2} & \sigma_{x}^{2}+\sigma^{2} & \sigma_{x}^{2} \\
\beta \sigma_{x}^{2} & \sigma_{x}^{2} & \sigma_{x}^{2}
\end{array}\right)\right),
$$

para $i=1,2, \ldots, n$.

Os dados observados $\left(Y_{i}, X_{i}\right)^{T}, i=1, \ldots, n$, têm a seguinte distribuição conjunta:

$$
\left(\begin{array}{c}
Y_{i} \\
X_{i}
\end{array}\right) \stackrel{\text { i.i.j. }}{\sim} N_{2}\left(\left(\begin{array}{c}
\alpha+\beta \mu \\
\mu
\end{array}\right) ;\left(\begin{array}{cc}
\beta^{2} \sigma_{x}^{2}+\sigma_{e}^{2} & \beta \sigma_{x}^{2} \\
\beta \sigma_{x}^{2} & \sigma_{x}^{2}+\sigma_{u}^{2}
\end{array}\right)\right) \text {, para } i=1,2, \ldots, n,
$$

Seja $D_{\text {obs }}=\left\{\left(Y_{i}, X_{i}\right)^{T}, i=1, \ldots, n\right\}$ o conjunto dos dados observados e $D=$ $\left\{\left(Y_{i}, X_{i}, x_{i}\right)^{T}, i=1, \ldots, n\right\}$ os "dados completos". Então a função de verossimilhança baseada nos dados observados é dada por

$$
\begin{aligned}
& L\left(\underset{\sim}{\theta} \mid D_{\mathrm{obs}}\right) \propto|\Sigma|^{-\frac{n}{2}} \exp \left\{-\frac{1}{2|\Sigma|}\left[\left(\beta^{2} \sigma_{x}^{2}+\sigma_{e}^{2}\right) \sum_{i=1}^{n}\left(X_{i}-\mu\right)^{2}\right.\right. \\
& \left.\left.-2 \beta \sigma_{x}^{2} \sum_{i=1}^{n}\left(Y_{i}-\alpha-\beta \mu\right)\left(X_{i}-\mu\right)+\left(\sigma_{x}^{2}+\sigma_{u}^{2}\right) \sum_{i=1}^{n}\left(Y_{i}-\alpha-\beta \mu\right)^{2}\right]\right\}
\end{aligned}
$$

onde $|\Sigma|=\beta^{2} \sigma_{u}^{2} \sigma_{x}^{2}+\sigma_{e}^{2} \sigma_{x}^{2}+\sigma_{u}^{2} \sigma_{e}^{2}$, e a função de verossimilhança baseada nos dados completos (D) é dada por

$$
\begin{aligned}
& L(\underset{\theta}{\theta} \mid D) \propto \\
& \left(\sigma_{u}^{2} \sigma_{e}^{2} \sigma_{x}^{2}\right)^{-\frac{n}{2}} \exp \left\{-\frac{1}{2}\left[\frac{\sum_{i=1}^{n}\left(Y_{i}-\alpha-\beta x_{i}\right)^{2}}{\sigma_{e}^{2}}+\frac{\sum_{i=1}^{n}\left(X_{i}-x_{i}\right)^{2}}{\sigma_{u}^{2}}+\frac{\sum_{i=1}^{n}\left(x_{i}-\mu\right)^{2}}{\sigma_{x}^{2}}\right]\right\} .
\end{aligned}
$$

É fato bastante conhecido que este modelo não é idenficável (Fuller, 1987), já que a distribuição conjunta dos dados observados dada em (2.1.2) depende de seis parâmetros: $\alpha, \beta, \mu, \sigma_{x}^{2}, \sigma_{u}^{2}$ e $\sigma_{e}^{2}$ mas somente cinco podem ser estimados (já que a estatística suficiente tem dimensão 5).

Sabemos por definição que um modelo é identificável se e sòmente se para todo $\underline{\sim}_{1}$ e ${\underset{\sim}{2}}_{2}$ pertencentes ao espaço paramétrico, com $\underline{\sim}_{1} \neq \underline{\sim}_{2}$ então $f\left(\theta_{1}\right) \neq f\left(\theta_{2}\right)$. Portanto um modelo é não identificável se e sòmente se $\exists \underset{\sim}{\theta_{1}} \neq{\underset{\sim}{2}}_{2}$ tal que $f\left(\theta_{1}\right)=$ 
$f\left(\underline{\theta}_{2}\right)$. No caso do modelo estrutural com $\underset{\sim}{\theta}=\left(\alpha, \beta, \mu, \sigma_{x}^{2}, \sigma_{e}^{2}, \sigma_{u}^{2}\right)$, podemos exibir dois pontos ${\underset{\sim}{1}}_{1}=(1,1,1,1,1,1)^{T}$ e ${\underset{\sim}{2}}_{2}=(1.5,0.5,1.0,2.0,1.5,0.0)^{T}$ que levam à mesma distribuição.

$$
\left(\begin{array}{c}
Y_{i} \\
X_{i}
\end{array}\right) \sim N\left(\left(\begin{array}{c}
2 \\
1
\end{array}\right) ;\left(\begin{array}{cc}
2 & 1 \\
1 & 2
\end{array}\right)\right) .
$$

Para assegurar identificabilidade deve-se fazer alguma suposição extra sobre o modelo. Algumas das suposições mais comumente encontradas na literatura são as seguintes:
(a) $\sigma_{e}^{2} / \sigma_{u}^{2}=\lambda$, onde $\lambda$ é conhecido;
(b) $\sigma_{e}^{2}$ ou $\sigma_{u}^{2}$ é conhecido;
(c) $\sigma_{x}^{2} /\left(\sigma_{x}^{2}+\sigma_{e}^{2}\right)$ é conhecido.

\section{O modelo funcional}

$\mathrm{Na}$ estatística clássica o modelo funcional aditivo com erros normalmente distribuídos é definido pelas equações

$$
\begin{aligned}
Y_{i} & =\alpha+\beta x_{i}+e_{i} \\
\text { e } X_{i} & =x_{i}+u_{i}
\end{aligned}
$$

onde $Y_{i}$ e $X_{i}$ são variáveis aleatórias observáveis, $e_{i}$ e $u_{i}$ são os erros aleatórios, $x_{i}$ é parâmetro desconhecido, onde

$$
\left(\begin{array}{c}
e_{i} \\
u_{i}
\end{array}\right) \stackrel{\text { i.i.d. }}{\sim} N\left(\left(\begin{array}{l}
0 \\
0
\end{array}\right) ;\left(\begin{array}{cc}
\sigma_{e}^{2} & 0 \\
0 & \sigma_{u}^{2}
\end{array}\right)\right), \text { para } i=1,2, \ldots, n .
$$

O espaço paramétrico é o conjunto

$$
\Theta=\left\{\underline{\theta}=\left(\alpha, \beta, \sigma_{e}^{2}, \sigma_{u}^{2}, x_{1}, \ldots, x_{n}\right)^{T} \in \mathbb{R}^{2} \times \mathbb{R}^{+^{2}} \times \mathbb{R}^{n}\right\} .
$$

A função de verossimilhança nesse caso é dada por

$$
L\left(\underset{\theta}{\theta} \mid D_{\text {obs }}\right) \propto\left(\sigma_{u}^{2} \cdot \sigma_{e}^{2}\right)^{-\frac{n}{2}} \exp \left\{-\frac{1}{2}\left[\frac{\sum_{i=1}^{n}\left(Y_{i}-\alpha-\beta x_{i}\right)^{2}}{\sigma_{e}^{2}}+\frac{\sum_{i=1}^{n}\left(X_{i}-x_{i}\right)^{2}}{\sigma_{u}^{2}}\right]\right\}
$$


que é ilimitada. Entretanto, pode-se eliminar este problema acrescentando-se ao modelo, por exemplo, a suposição que $\sigma_{e}^{2}=\lambda \sigma_{u}^{2}$, onde $\lambda$ é conhecido.

No modelo funcional temos que $x_{1}, x_{2}, \ldots, x_{n}$ são constantes desconhecidas e portanto ele é apropriado quando as únicas unidades experimentais de interesse estão representadas nos dados disponíveis (isto é, os $x_{i}$ 's não são uma amostra aleatória de alguma população mas a própria população) (Carrol e Stefanski, 1995).

Quando as unidades experimentais representadas nos dados constituem uma amostra aleatória de alguma população, então é apropriado tratar $x_{1}, x_{2}, \ldots, x_{n}$ como variáveis aleatórias i.i.d. e nesse caso é razoável utilizar um modelo estrutural (Stefansky, 2000).

\subsection{O modelo estrutural e o modelo funcional na estatística bayesiana}

Como na literatura bayesiana, a diferença entre os modelos funcional e estrutural não está muito clara, nossa intençãoo nessa seção é procurar esclarecer o leitor sobre esse ponto.

Inicialmente apresentamos duas formulações alternativas do modelo estrutural (a formulação 1 e a formulação 2). A formulação 2 é a que aparece mais comumente na literatura no contexto do modelos com erros nas variáveis, além disso é mais fácil comparar os modelos estrutural e funcional usando essa formulação. Entretanto a formulação 1 é a que traz maior vantagem do ponto de vista computacional.

$\mathrm{Na}$ subseção 2.2 .1 a seguir, descrevemos o modelo estrutural aditivo Normal por meio de duas formulações equivalentes. Na subseção 2.2 .2 definimos o modelo funcional aditivo Normal fazendo uma comparação com o modelo estrutural. 


\subsubsection{O modelo bayesiano estrutural}

\section{Formulação 1 do modelo estrutural Normal}

Tomamos o modelo clássico definido pelas equações

$$
\begin{aligned}
& Y_{i}=\alpha+\beta x_{i}+e_{i} \\
& \text { e } \quad X_{i}=x_{i}+u_{i},
\end{aligned}
$$

onde

$\left(Y_{i}, X_{i}\right)^{T}, i=1, \ldots, n$ são os vetores de observações, e $x_{1}, \ldots, x_{n}$ são as v.a. latentes (não observáveis),

$$
\left(\begin{array}{c}
e_{i} \\
u_{i} \\
x_{i}
\end{array}\right) \mid \theta \stackrel{\text { i.i.d. }}{\sim} N\left(\left(\begin{array}{c}
0 \\
0 \\
\mu
\end{array}\right) ;\left(\begin{array}{ccc}
\sigma_{e}^{2} & 0 & 0 \\
0 & \sigma_{u}^{2} & 0 \\
0 & 0 & \sigma_{x}^{2}
\end{array}\right)\right) \text {, para } i=1,2, \ldots, n,
$$

e acrescentamos a suposição de que o vetor de parâmetros $\underset{\sim}{\theta}=\left(\alpha, \beta, \mu, \sigma_{x}^{2}, \sigma_{e}^{2}, \sigma_{u}^{2}\right)^{T}$ tem distribuição à priori $\pi(\underset{\sim}{\theta})$. Nessa formulação, por convenção não incluímos as variáveis latentes $x_{1}, x_{2}, \ldots, x_{n}$ no vetor de parâmetros pelo fato de que esses $x_{i}$ 's, além de desconhecidos (isto é, não observáveis), são realizações de um processo amostral (diferentemente das quantidades $\alpha, \beta, \mu, \sigma_{x}^{2}, \sigma_{e}^{2}, \sigma_{u}^{2}$ ). Assim, o espaço paramétrico nesse caso é o conjunto

$$
\Theta=\left\{\underset{\theta}{\theta}=\left(\alpha, \beta, \mu, \sigma_{x}^{2}, \sigma_{e}^{2}, \sigma_{u}^{2}\right)^{T}: \underset{\theta}{\theta} \in \mathbb{R}^{3} \times \mathbb{R}_{+}^{3}\right\} .
$$

\section{Distribuição à posteriori}

Chamando de $D_{\text {obs }}$ o conjunto de dados observados, $\left\{\left(X_{i}, Y_{i}\right)^{T}, i=1, \ldots, n\right\}$, temos que a distribuição à posteriori de $\underset{\sim}{\theta}=\left(\alpha, \beta, \mu, \sigma_{x}^{2}, \sigma_{e}^{2}, \sigma_{u}^{2}\right)^{T}$ é dada por

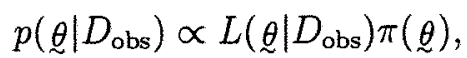

onde $L\left(\underset{\sim}{\theta} \mid D_{\text {obs }}\right)$ é a verossimilhança baseada nos dados observados dada em (2.1.4) e $\pi(\underset{\sim}{\theta})$ a distribuição à priori de $\underset{\sim}{\theta}$. Portanto,

$$
p\left(\underset{\sim}{\theta} \mid D_{\text {obs }}\right) \propto|\Sigma|^{-\frac{n}{2}} \exp \left\{-\frac{1}{2|\Sigma|}\left[\left(\beta^{2} \sigma_{x}^{2}+\sigma_{e}^{2}\right) \sum_{i=1}^{n}\left(X_{i}-\mu\right)^{2}\right.\right.
$$




$$
\left.\left.-2 \beta \sigma_{x}^{2} \sum_{i=1}^{n}\left(Y_{i}-\alpha-\beta \mu\right)\left(X_{i}-\mu\right)+\left(\sigma_{x}^{2}+\sigma^{2}\right) \sum_{i=1}^{n}\left(Y_{i}-\alpha-\beta \mu\right)^{2}\right]\right\} . \pi(\theta)
$$

onde $|\Sigma|=\beta^{2} \sigma_{u}^{2} \sigma_{x}^{2}+\sigma_{e}^{2} \sigma_{x}^{2}+\sigma_{u}^{2} \sigma_{e}^{2}$.

\section{Distribuição à posteriori em função dos "dados completos"}

É importante ressaltar que a distribuição à posteriori $p\left(\underset{\theta}{\theta} \mid D_{\text {obs }}\right)$ também é uma distribuição marginal de $p\left(\underset{\sim}{\theta} \underset{\sim}{x} \mid D_{\text {obs }}\right)$, ou seja, $p\left(\underset{\sim}{\theta} \mid D_{\text {obs }}\right)=\int_{\mathscr{X}=\mathbb{R}^{n}} p\left(\underset{\sim}{\theta} \underset{\sim}{x} \mid D_{\text {obs }}\right) \mathrm{d} \underset{\sim}{x}$. Então, chamando de $D$ o conjunto de "dados completos", $D=\left\{\left(Y_{i}, X_{i}, x_{i}\right)^{T}, i=\right.$ $1, \ldots, n\}$, temos que

$$
p\left(\underset{\sim}{\theta} \mid D_{\mathrm{obs}}\right)=\int_{\mathscr{\Psi}=\mathbb{R}^{n}} p\left(\underset{\sim}{\theta} \underset{\sim}{x} \mid D_{\mathrm{obs}}\right) \mathrm{d} \underset{\sim}{x} \propto \int_{\mathscr{X}=\mathbb{R}^{n}} L(\underset{\sim}{\theta} \mid D) \pi(\underset{\sim}{\theta}) \mathrm{d} \underset{\sim}{x},
$$

onde $L(\underset{\sim}{\theta} \mid D)$ é a verossimilhança baseada nos dados completos dada em (2.1.5) e $\pi(\underset{\theta}{\theta})$ a priori de $\underset{\sim}{\theta}$.

Portanto a distribuição à posteriori $p\left(\underset{\theta}{\theta} \mid D_{\mathrm{obs}}\right)$ também é proporcional a

$$
\begin{aligned}
& \int_{\mathfrak{X}=\mathbb{R}^{n}}\left(\sigma_{u}^{2} \sigma_{e}^{2} \sigma_{x}^{2}\right)^{-\frac{n}{2}} \exp \left\{-\frac{1}{2}\left[\frac{\sum_{i=1}^{n}\left(Y_{i}-\alpha-\beta x_{i}\right)^{2}}{\sigma_{e}^{2}}+\frac{\sum_{i=1}^{n}\left(X_{i}-x_{i}\right)^{2}}{\sigma_{u}^{2}}\right.\right. \\
& \left.\left.+\frac{\sum_{i=1}^{n}\left(x_{i}-\mu\right)^{2}}{\sigma_{x}^{2}}\right]\right\} \pi(\theta) \mathrm{d} \underset{\sim}{x} .
\end{aligned}
$$

Observação 1: É importante ressaltar que a distribuição à posteriori $p\left(\underset{\theta}{\theta} \mid D_{\mathrm{obs}}\right)$ pode então ser explorada computacionalmente de duas maneiras: a primeira usando a fórmula (2.2.2), que não é função de $x_{1}, x_{2}, \ldots, x_{n}$, e a segunda usando o fato de que $p\left(\theta \mid D_{\text {obs }}\right)$ é distribuição marginal de $\left.p\left(\underset{\sim}{x}, \underset{\sim}{x} \mid D_{\text {obs }}\right)\right)$.

Observação 2: É importante observar também que na formulação 1 do modelo, se a distribuição à priori de $\underset{\sim}{\theta}=\left(\alpha, \beta, \mu, \sigma_{x}^{2}, \sigma^{2}\right)^{T}$ for totalmente especificada, isto é, se os hiperparâmetros forem conhecidos, temos um modelo com apenas um nível hierárquico. 


\section{Distribuição preditiva}

Seja $\underset{\sim}{U_{i}}=\left(\begin{array}{c}Y_{i} \\ X_{i}\end{array}\right), i=1, \ldots, n$. A distribuição preditiva à posteriori nesse modelo é dada por

$$
p\left(\underset{\sim}{U_{f}} \mid D_{\mathrm{obs}}\right)=\int_{\Theta} p\left({\underset{\sim}{U}}_{f}, \underset{\sim}{\theta} \mid D_{\mathrm{obs}}\right) \mathrm{d} \underset{\sim}{\theta}=\int_{\Theta} p\left(\underset{\sim}{U_{f}} \mid \theta\right) p\left(\underset{\sim}{\theta} \mid D_{\mathrm{obs}}\right) \mathrm{d} \underset{\sim}{\theta}
$$

onde $\bigcup_{f}$ é uma observação futura de $\left(\begin{array}{l}Y \\ X\end{array}\right)$, isto é, $\underset{\sim}{U_{f}}=\left(\begin{array}{l}Y_{f} \\ X_{f}\end{array}\right)$,

$$
\left.\underset{\sim}{U_{f}}\right|_{\underline{\theta}} \sim N\left[\left(\begin{array}{c}
\alpha+\beta \mu \\
\mu
\end{array}\right) ;\left(\begin{array}{cc}
\beta^{2} \sigma_{x}^{2}+\sigma_{e}^{2} & \beta \sigma_{x}^{2} \\
\beta \sigma_{x}^{2} & \sigma_{x}^{2}+\sigma_{u}^{2}
\end{array}\right)\right],
$$

e $p\left(\underset{\theta}{\theta} \mid D_{\text {obs }}\right)$ é a distribuição à posteriori de $\theta=\left(\alpha, \beta, \mu, \sigma_{x}^{2}, \sigma_{e}^{2}, \sigma_{\mu}^{2}\right)^{T}$. Portanto,

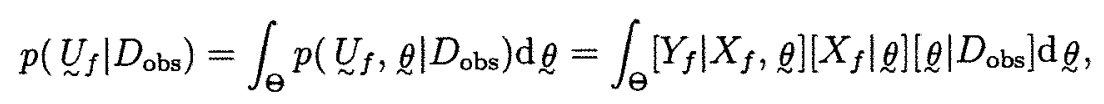

onde $\left[\underset{\sim}{\theta} \mid D_{\mathrm{obs}}\right]$ representa a distribuição à posteriori de $\underset{\sim}{\underset{\sim}{\theta}}$, além disso $\left[X_{f} \mid \underset{\sim}{\theta}\right] \mathrm{e}\left[Y_{f} \mid X_{f}, \underset{\sim}{\theta}\right]$ representam respectivamente as distribuições condicionais de $X_{f} \mid \underset{\sim}{\theta}$ e $Y_{f} \mid X_{f}, \underset{\sim}{\theta}$ onde

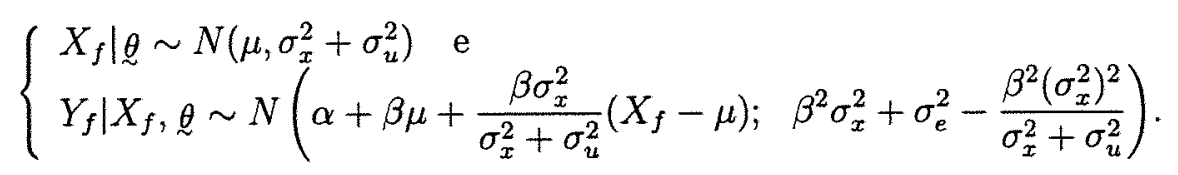

Geração de uma observação futura:

Para gerar uma observação $\underset{\sim}{U_{f}}$, basta gerar inicialmente um $\underset{\sim}{\theta}$ da distribuição à posteriori $\left[\underset{\sim}{\theta} \mid D_{\text {obs }}\right]$; a seguir, para esse $\underset{\sim}{\theta}$ fixado, gerar um valor $X_{f}$ da distribuição $\left[X_{f} \mid \theta\right]$ e finalmente gerar $Y_{f}$ de $\left[Y_{f} \mid X_{f}, \theta\right]$.

Se quisermos gerar um valor $Y_{f}$ da distribuição preditiva $\left[Y_{f} \mid X_{f}=X_{0}, D_{\text {obs }}\right]$ basta gerar $\underset{\sim}{\theta}$ de $\left[\underset{\sim}{\theta} \mid D_{\text {obs }}\right]$ e depois $Y_{f}$ de $\left[Y_{f} \mid X_{0}, \theta\right]$, já que $\left[Y_{f} \mid X_{f}=X_{0}, D_{\text {obs }}\right]$ é dada por $\int_{\Theta}\left[Y_{f} \mid X_{0}, \underset{\sim}{\theta}\right]\left[\underset{\sim}{\theta} \mid D_{\mathrm{obs}}\right] \mathrm{d} \theta$.

Semelhantemente, podemos gerar uma observação futura $X_{f}$ dado que $Y_{f}=Y_{0}$. A distribuição preditiva de $X_{f} \mid Y_{f}=Y_{0}, D_{\text {obs }}$ nesse caso é dada por

$$
\int_{\theta}\left[X_{f} \mid Y_{0}, \theta\right]\left[\underset{\sim}{\theta} \mid D_{\mathrm{obs}}\right] \mathrm{d} \underset{\sim}{\theta},
$$


onde

$$
X_{f} \mid Y_{f}=Y_{0}, \theta \sim N\left(\mu+\frac{\beta \sigma_{x}^{2}}{\beta^{2} \sigma_{x}^{2}+\sigma_{e}^{2}}\left(Y_{0}-\alpha-\beta \mu\right) ; \quad \sigma_{x}^{2}+\sigma_{u}^{2}-\frac{\beta^{2}\left(\sigma_{x}^{2}\right)^{2}}{\beta^{2} \sigma_{x}^{2}+\sigma_{e}^{2}}\right) .
$$

Observação: Uma alternativa para gerar uma observação futura $\underset{\sim}{U_{f}}$ :

A distribuição preditiva $p\left(\underset{\sim}{U} \mid D_{\text {obs }}\right)$ pode ser escrita também como

$$
\begin{aligned}
& p\left(\underset{\sim}{U_{f}} \mid D_{\mathrm{obs}}\right)=\int_{\Re_{f}=\mathbb{R}} \int_{\Theta} p\left(U_{f}, \theta, x_{f} \mid D_{\mathrm{obs}}\right) \mathrm{d} \underset{\underset{\Theta}{ } \mathrm{d} x_{f}}{ } \\
& =\int_{x_{f}=\mathbb{R}} \int_{\Theta}\left[{\underset{\sim}{f}}_{f} \mid x_{f}, \theta\right]\left[x_{f} \mid \underline{\sim}\right]\left[\underset{\sim}{\theta} \mid D_{\mathrm{obs}}\right] \mathrm{d} \underset{\sim}{\theta} \mathrm{d} x_{f},
\end{aligned}
$$

onde

$$
\underset{\sim}{U_{f}} \mid x_{f}, \theta \sim N\left[\left(\begin{array}{c}
\alpha+\beta x_{f} \\
x_{f}
\end{array}\right),\left(\begin{array}{cc}
\sigma_{e}^{2} & 0 \\
0 & \sigma_{u}^{2}
\end{array}\right)\right]
$$

e portanto para gerar uma observação ${\underset{\sim}{f}}_{f}$ basta gerar $\underset{\sim}{\theta}$ da distribuição à posteriori $\left[\underset{\theta}{\theta} \mid D_{\text {obs }}\right]$, depois $x_{f}$ da distribuição $\left[x_{f} \mid \theta\right]$, depois $X_{f}$ de $\left[X_{f} \mid \underset{\sim}{\theta}, x_{f}\right]$ e depois $Y_{f}$ de $\left[Y_{f} \mid X_{f}, x_{f}, \underset{\sim}{\theta}\right]$, onde $x_{f}\left|\underset{\sim}{\theta} \sim N\left(\mu, \sigma_{x}^{2}\right), X_{f}\right| \underset{\sim}{\theta}, x_{f} \sim N\left(x_{f}, \sigma_{u}^{2}\right)$ e $Y_{f} \mid X_{f}, x_{f}, \underset{\sim}{\theta} \sim N(\alpha+$ $\left.\beta x_{f}, \sigma_{e}^{2}\right)$.

\section{Formulação 2 do modelo estrutural Normal}

Seja o modelo definido pelas equações

$$
\begin{array}{ll} 
& Y_{i}=\alpha+\beta x_{i}+e_{i} \\
\text { e } \quad & X_{i}=x_{i}+u_{i},
\end{array}
$$

onde $\left(\begin{array}{c}e_{i} \\ u_{i}\end{array}\right) \stackrel{*}{*} \stackrel{\text { i.i.d. }}{\sim} N_{2}\left(\left(\begin{array}{l}0 \\ 0\end{array}\right) ;\left(\begin{array}{cc}\sigma_{e}^{2} & 0 \\ 0 & \sigma_{u}^{2}\end{array}\right)\right)$, para $i=1, \ldots, n$.

Nessa formulação o vetor de parâmetros agora é $\theta^{*}=\left(\alpha, \beta, \sigma_{e}^{2}, \sigma_{u}^{2}, x_{1}, \ldots, x_{n}\right)^{T}$, onde à priori $x_{i} \mid \alpha, \beta, \sigma_{e}^{2}, \sigma_{u}^{2}, \Phi \stackrel{\text { i.i.d. }}{\sim} N\left(\mu, \sigma_{x}^{2}\right)^{T}, i=1, \ldots, n$, onde $\phi=\left(\mu, \sigma_{x}^{2}\right)$ é o vetor de hiperparâmetros desconhecidos.

\section{Função de verossimilhança}


A função de verossimilhança, $L\left(\theta^{*} \mid D_{\text {obs }}\right)$, nesse caso será igual a:

$$
\left(\sigma_{u}^{2} \cdot \sigma_{e}^{2}\right)^{-\frac{n}{2}} \exp \left\{-\frac{1}{2}\left[\frac{\sum_{i=1}^{n}\left(Y_{i}-\alpha-\beta x_{i}\right)^{2}}{\sigma_{e}^{2}}+\frac{\sum_{i=1}^{n}\left(X_{i}-x_{i}\right)^{2}}{\sigma_{u}^{2}}\right]\right\}
$$

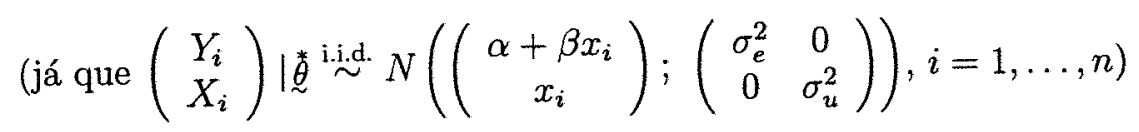

\section{Distribuição à posteriori}

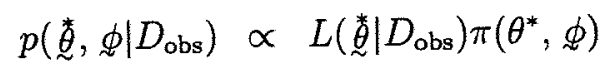

$$
\begin{aligned}
& =L\left(\underset{\sim}{\stackrel{*}{\theta}} \mid D_{\mathrm{obs}}\right) \pi\left(\underset{\sim}{x} \mid \alpha, \beta, \sigma_{e}^{2}, \sigma_{u}^{2} \phi\right) \pi\left(\alpha, \beta, \sigma_{e}^{2} \sigma_{u}^{2} \phi\right),
\end{aligned}
$$

onde $\pi\left(\underset{\sim}{x} \mid \alpha, \beta, \sigma_{e}^{2}, \sigma_{u}^{2}, \phi\right) \propto\left(\sigma_{x}^{2}\right)^{-\frac{n}{2}} \exp \left\{-\frac{1}{2} \frac{\sum_{i=1}^{n}\left(x_{i}-\mu\right)^{2}}{\sigma_{x}^{2}}\right\}$.

Portanto,

$$
\begin{aligned}
\text { Portanto, } & \\
p\left(\stackrel{*}{\otimes}, \phi \mid D_{\text {obs }}\right) \propto & \left(\sigma_{u}^{2} \sigma_{e}^{2} \sigma_{x}^{2}\right)^{-\frac{n}{2}} \exp \left\{-\frac{1}{2}\left[\frac{\sum_{i=1}^{n}\left(Y_{i}-\alpha-\beta x_{i}\right)^{2}}{\sigma_{e}^{2}}+\frac{\sum_{i=1}^{n}\left(X_{i}-x_{i}\right)^{2}}{\sigma_{u}^{2}}\right.\right. \\
& \left.\left.+\frac{\sum_{i=1}^{n}\left(x_{i}-\mu\right)^{2}}{\sigma_{x}^{2}}\right]\right\} \pi\left(\alpha, \beta, \sigma_{e}^{2}, \sigma_{u}^{2}, \phi\right) .
\end{aligned}
$$

onde $\pi\left(\alpha, \beta, \sigma_{e}^{2}, \sigma_{u}^{2}, \phi\right)=\pi\left(\alpha, \beta, \mu, \sigma_{x}^{2}, \sigma_{e}^{2}, \sigma_{u}^{2}\right)$.

Notemos que se $\pi\left(\alpha, \beta, \sigma_{e}^{2}, \sigma_{u}^{2}, \phi\right)$ tiver hiperparâmetros conhecidos temos um modelo com dois níveis hierarquicos. Notemos também que a expressão em (2.2.7) coincide com a expressão dentro do sinal de integração dada em (2.2.3) (assim, a posteriori em função dos dados completos dada pela formulação $1 \mathrm{em}(2.2 .3)$ é a marginal da posteriori na formulação 2 , quando eliminamos as variáveis $x_{1}, x_{2}, \ldots, x_{n}$ por integração).

\section{Distribuição preditiva}

A distribuição preditiva $p\left(\underset{\sim}{U} \mid D_{\text {obs }}\right)$ na formulação 2 é dada por

$$
p\left(\underset{\sim}{U_{f}} \mid D_{\mathrm{obs}}\right)=\int_{\Theta^{*}} \int_{\Phi} p\left(\underset{\sim}{U_{f}}, \stackrel{*}{\stackrel{\theta}{*}}, \phi, \mid D_{\mathrm{obs}}\right) \mathrm{d} \phi \mathrm{d} \stackrel{*}{\underset{\sim}{*}}
$$


onde $\underset{\sim}{U_{f}}=\left(\begin{array}{l}Y_{f} \\ X_{f}\end{array}\right), \Theta^{*}=\left\{\stackrel{*}{\theta}=\left(\alpha, \beta, \sigma_{e}^{2}, \sigma_{u}^{2}, \underset{\sim}{x}\right)^{T}: \underset{\sim}{\stackrel{*}{\theta}} \in \mathbb{R}^{2} \times \mathbb{R}_{+}^{2} \times \mathbb{R}^{n}\right\}$ e $\Phi=$ $\left\{\left(\mu, \sigma_{x}^{2}\right)^{T}: \mu \in \mathbb{R}, \sigma_{x}^{2} \in \mathbb{R}^{+}\right\}$. Portanto,

$$
p\left(\underset{\sim}{U_{f}} \mid D_{\mathrm{obs}}\right)=\int_{\Theta} \int_{\mathscr{X}=\mathbb{R}^{n}} p\left(\underset{\sim}{U_{f}}, \underset{\sim}{\theta} \underset{\sim}{x} \mid D_{\mathrm{obs}}\right) \mathrm{d} \underset{\sim}{\underset{\mathrm{d}}{\mathrm{d}} \underset{\sim}{x}}
$$

onde $\underset{\sim}{\theta}=\left(\alpha, \beta, \mu, \sigma_{x}^{2}, \sigma_{e}^{2}, \sigma_{u}^{2}\right)^{T}, \underset{\sim}{x}=\left(x_{1}, \ldots, x_{n}\right)^{T}, \Theta=\left\{\underset{\sim}{\theta} \mid \alpha, \beta, \mu \in \mathbb{R}\right.$ e $\sigma_{x}^{2}, \sigma_{e}^{2}, \sigma_{u}^{2} \in$ $\left.\mathbb{R}^{+}\right\}$e $x=\left\{\underset{\sim}{x}: x_{i} \in \mathbb{R}\right\}$. Portanto,

$$
\begin{aligned}
p\left(\underset{\sim}{U_{f}} \mid D_{\mathrm{obs}}\right) & =\int_{\Theta} \int_{\mathscr{x}}\left[\underset{\sim}{U_{f}} \mid \underset{\sim}{\theta}\right]\left[\underset{\sim}{\theta}, \underset{\sim}{x} \mid D_{\mathrm{obs}}\right] \mathrm{d} \underset{\sim}{\theta} \mathrm{d} \underset{\sim}{x} \\
& =\int_{\mathscr{X}} \int_{\Theta}\left[Y_{f} \mid X_{f}, \underset{\sim}{\theta}\right]\left[X_{f} \mid \underset{\sim}{\theta}\right]\left[\underset{\sim}{\theta} \underset{\sim}{x} \mid D_{\mathrm{obs}}\right] \mathrm{d} \underset{\underset{\sim}{\theta} \mathrm{d} \underset{\sim}{x}}{ }
\end{aligned}
$$

onde $\left[\underset{\underset{ }{\theta}}{\theta}, x \mid D_{\text {obs }}\right]$ é a distribuição à posteriori dada em (2.2.7), as distribuições $\left[X_{f} \mid \theta\right]$ e $\left[Y_{f} \mid X_{f}, \theta\right]$ são dadas em (2.2.4).

Para gerar uma observação ${\underset{\sim}{f}}_{f}$, basta gerar $\underset{\sim}{\theta} \operatorname{de}\left[\underset{\sim}{\underset{\sim}{x}} \underset{\sim}{x} \mid D_{\text {obs }}\right]$, depois $X_{f}$ de $\left[X_{f} \mid \underline{\sim}\right]$ e depois $Y_{f}$ de $\left[Y_{f} \mid X_{f}, \theta\right]$ (da mesma maneira descrita na formulação 1).

Observação: Outra forma de escrever a posteriori preditiva é

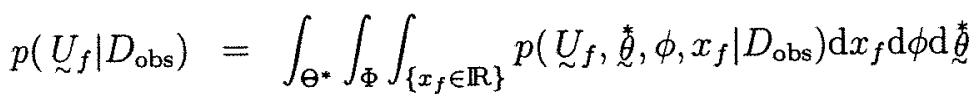

$$
\begin{aligned}
& =\int_{\mathfrak{X}} \int_{\Theta} \int_{\left\{x_{f} \in \mathbb{R}\right\}} p\left(\underset{\sim}{U_{f}}, \underset{\sim}{\theta} \underset{\sim}{x}, x_{f} \mid D_{\text {obs }}\right) \mathrm{d} \underset{\sim}{\underset{\sim}{x} f} \mathrm{~d} \underset{\sim}{\theta} \mathrm{d} \underset{\sim}{x},
\end{aligned}
$$

onde $\underset{\sim}{\theta}=\left(\alpha, \beta, \mu, \sigma_{x}^{2}, \sigma_{e}^{2}, \sigma_{u}^{2}\right)^{T}$ e $\underset{\sim}{x}=\left(x_{1}, \ldots, x_{n}\right)^{T}$ e portanto

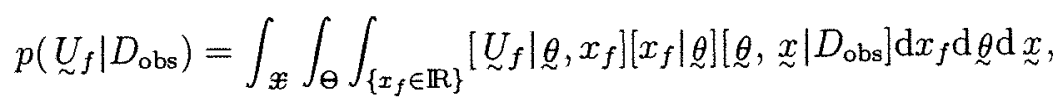

onde $x_{f} \mid \underset{\sim}{\theta} \sim N\left(\mu, \sigma_{x}^{2}\right)$ e $\underset{\sim}{U_{f}} \mid \underset{\sim}{\theta}, x_{f} \sim N\left(\left(\begin{array}{c}\alpha+\beta x_{f} \\ x_{f}\end{array}\right),\left(\begin{array}{cc}\sigma_{e}^{2} & 0 \\ 0 & \sigma_{u}^{2}\end{array}\right)\right)$.

Observamos que podemos gerar $\bigcup_{f}$ da mesma maneira que na Observação da formulação 1 .

Do que foi apresentado, podemos ver que as duas formulações são equivalentes em termos de inferência. Entretanto, no restante desse trabalho estaremos utilizando 
a formulação 1 também por razões didáticas e computacionais. Nessa formulação é mais direto ver que a posteriori de $\left(\alpha, \beta, \mu, \sigma_{x}^{2}, \sigma_{e}^{2}, \sigma_{u}^{2}\right)$ pode ser estudada de duas formas: uma em função dos dados completos, como em (2.2.3), e outra em função somente dos dados observados como em (2.2.2), o que amplia as opções de algoritmos para amostrar da posteriori.

Comentários: $\mathrm{O}$ modelo estrutural bayesiano com erros nas variáveis, como sabemos, não é identificável (já que a função de verossimilhança não é identificável). Em princípio, teoricamente, isso não é problema. Entretanto nas aplicações o algoritmo de Gibbs para amostrar da posteriori de modelos não identificáveis (que será descrito nesse capítulo) pode apresentar problemas de convergência segundo Gelfand e Sahu (1999). Além disso, se a priori não é "informativa", o problema pode piorar bastante e a recomendação dos autores nesse caso é que se escolha uma priori "adequada", que não seja nem "muito pouco informativa", mas também que não seja "extremamente precisa" para não limitar o aprendizado proveniente dos dados. Segundo os autores, o problema é achar esse "meio termo" o que geralmente requer um trabalho considerável de simulação.

Uma outra saída para o problema computacional decorrente da falta de identificabilidade é simplesmente acrescentar uma suposição ao modelo como, por exemplo, adotar uma das restrições de identificabilidade descritas em (2.1.6).

Com relação à existência da posteriori, ressaltamos que sempre que a priori for própria, a posteriori também será própria, entretanto podemos ter posterioris próprias para certas escolhas de prioris impróprias. Na Seção 2.5.1 estudamos as condições para a existência da posteriori do modelo estrutural sob diferentes escolhas de prioris impróprias quando incorporamos ao modelo a restrição de idenficabilidade: $\sigma_{e}^{2}=\lambda \sigma_{u}^{2}$, onde $\lambda$ é conhecido e na Seção 2.5.2 fazemos um estudo semelhante para 
o modelo sem restrições.

\subsubsection{O modelo bayesiano funcional}

Tomamos o modelo aditivo definido pelas equações

$$
\begin{aligned}
& Y_{i}=\alpha+\beta x_{i}+e_{i} \\
& \text { e } \quad X_{i}=x_{i}+u_{i},
\end{aligned}
$$

onde $Y_{i}$ e $X_{i}$ são as variáveis aleatórias observáveis, $x_{i}$ são quantidades fixadas desconhecidas, $e_{i}$ e $u_{i}$ os erros aleatórios, onde

$$
\left(\begin{array}{c}
e_{i} \\
u_{i}
\end{array}\right) \stackrel{\text { i.i.d. }}{\sim} N_{2}\left(\left(\begin{array}{l}
0 \\
0
\end{array}\right) ;\left(\begin{array}{cc}
\sigma_{e}^{2} & 0 \\
0 & \sigma_{u}^{2}
\end{array}\right)\right) \text {, para } i=1,2, \ldots, n,
$$

e onde $\underset{\sim}{\stackrel{*}{*}}=\left(\alpha, \beta, \sigma_{u}^{2}, \sigma_{e}^{2}, \underset{\sim}{x}\right)^{T}$ onde $\left(x_{1}, \ldots, x_{n}\right)^{T}$ é o vetor de parâmetros, e incorpo-

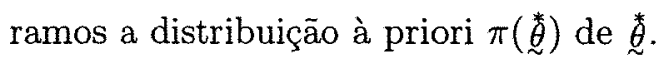

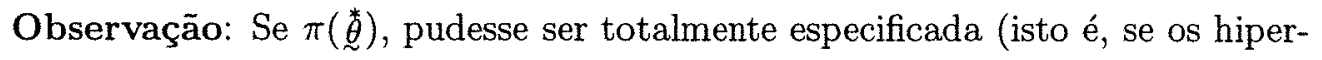
parâmetros de $\pi(\underset{\underset{\sim}{*}}{)}$ fosem conhecidos), então teríamos um modelo não hierárquico. Se, entretanto, a distribuição dos $x_{i}$ 's depende de hiperparâmetros $\phi_{i}$ desconhecidos, para $i=1, \ldots, n$, então temos um modelo hierárquico.

\section{Distribuição à posteriori}

A distribuição à posteriori sob o modelo, onde $\phi_{i}$ é desconhecido, é dada por

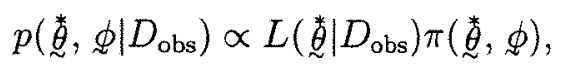

onde $\phi=\left(\Phi_{1}, \phi_{2}, \ldots, \phi_{n}\right)^{T}, \phi_{i}=\left(\mu_{i}, \sigma_{x_{i}}^{2}\right), i=1, \ldots, n, L\left(\underset{v}{\theta} \mid D_{\mathrm{obs}}\right)$ é a verossimilhança e $\pi(\underset{\theta}{*}, \phi)$ é a distribuição à priori de $\underset{\sim}{*}$ e $\phi$, onde

$$
L\left(\underset{\stackrel{*}{*} \mid}{\mid} \mid D_{\mathrm{obs}}\right) \propto\left(\sigma_{u}^{2} \sigma_{e}^{2}\right)^{-\frac{n}{2}} \exp \left\{-\frac{1}{2}\left[\frac{\sum_{i=1}^{n}\left(Y_{i}-\alpha-\beta x_{i}\right)^{2}}{\sigma_{e}^{2}}+\frac{\sum_{i=1}^{n}\left(X_{i}-x_{i}\right)^{2}}{\sigma_{e}^{2}}\right]\right\}
$$

(já que $\left.\left(\begin{array}{c}X_{i} \\ Y_{1}\end{array}\right) \mid \theta^{*} \sim N\left(\left(\begin{array}{c}\alpha+\beta x_{i} \\ x_{i}\end{array}\right) ;\left(\begin{array}{cc}\sigma_{e}^{2} & 0 \\ 0 & \sigma_{u}^{2}\end{array}\right)\right), i=1, \ldots, n\right)$. 
É interessante comparar esta pasteriori com a posteriori sob o modelo estrutural dada em (2.2.6) e (2.2.7) e notar que se pudessemos assumir que $x_{i} \mid \phi_{i}, \alpha, \beta, \sigma_{e}^{2}, \sigma_{u}^{2} \stackrel{\text { i.i.d. }}{\sim} N\left(\mu, \sigma_{x}^{2}\right)$ para $i=1, \ldots, n$, onde $\phi_{i}=\phi=\left(\mu, \sigma_{x}^{2}\right)^{T}$ é desconhecido, então as posterioris coincidiriam. É fácil ver também (usando a formulação 2 do modelo estrutural) que as posterioris dos modelos estrutural e funcional podem coindidir não somente no caso Normal (erros e covariável normais) mas em qualquer outro caso, desde que $\pi\left(\underset{\sim}{x} \mid \alpha, \beta, \sigma_{e}^{2}, \sigma_{u}^{2}, \not\right)$ seja a mesma nos dois modelos ( ou seja, $x_{i} \mid \phi_{i}, \alpha, \beta, \sigma_{e}^{2}, \sigma_{u}^{2}$ sejam independentes e identicamente distribuídos para $i=1, \ldots, n)$.

$\mathrm{Na}$ estatística Bayesiana, portanto, a diferença entre os modelos estrutural e funcional está somente na escolha da distribuição de $\underset{\sim}{x}=\left(x_{1}, x_{2}, \ldots, x_{n}\right)^{T}$, isto é, na especificação do modelo. No caso estrutural assumimos que $x_{i} \mid \alpha, \beta, \sigma_{u}^{2}, \sigma_{e}^{2}, \phi_{i}$ são independentes e indenticamente distribuídos para $i=1, \ldots, n$, isto é, $\underset{\sim}{x}=\left(x_{1}, \ldots, x_{n}\right)^{T}$ constitui uma amostra aleatória (na Seção 2.2 .1 , tomamos $\pi\left(x_{i} \mid \alpha, \beta, \sigma_{u}^{2} \sigma_{e}^{2}, \not{D}\right) \stackrel{\text { i.i.d. }}{\sim}$ $N\left(\mu, \sigma_{x}^{2}\right)$, onde $\phi=\left(\mu, \sigma_{x}^{2}\right)$ para $\left.i=1, \ldots, n\right)$. No caso funcional, conceitualmente, os $x_{i}$ 's são quantidades (desconhecidas) que não estão sujeitas a flutuações amostrais e por isso não nos parece razoável assumir que os $x_{i}$ 's sejam i.i.d. devido à interpretação amostral que isso acarretaria. Em um modelo funcional a distribuição de $\pi\left(x_{i} \mid \alpha, \beta, \sigma_{u}^{2} \sigma_{e}^{2} \phi_{i}\right)$ pode também depender de $i$, para $i=1, \ldots, n$ (Carrol e Stefanski, 1995) como também pode ser imprópria, já que dificilmente teríamos uma interpretação amostral nesse caso (Florens et al., 1974).

Segundo Stephens e Dellaportas (1992), embora na estatística clássica os modelos funcional e estrutural difiram bastante quanto à análise e quanto aos procedimentos de estimação, na formulação Bayesiana a diferença é apenas uma questão de especificação do modelo ou seja uma questão de especificar a distribuição de $\underset{\sim}{x} \mid \alpha, \beta, \sigma_{e}^{2}, \sigma_{u}^{2}, \phi$ onde $\phi=\left(\phi_{1}, \ldots, \phi_{n}\right)$. 


\subsection{O modelo estrutural identificável com parti- cular priori de componentes independentes}

Nesta seção apresentamos o modelo linear estrutural Normal com erros nas variáveis no caso em que a distribuição à priori é própria e tem componentes independentes Normais ou Gama Invertida, assumindo a restrição de identificabilidade $\sigma_{e}^{2}=\lambda \sigma_{u}^{2}$, onde $\lambda$ é conhecido.

\section{O modelo}

No modelo estrutural descrito em (2.2.1) acrescentamos a restrição $\sigma_{e}^{2}=\lambda \sigma_{u}^{2}$, onde $\sigma_{u}^{2}=\sigma^{2}$ e $\lambda$ é conhecido, obtendo o seguinte modelo identificável, definido pelas equações

$$
\begin{aligned}
& Y_{i}=\alpha+\beta x_{i}+e_{i} \\
& \text { e } \quad X_{i}=x_{i}+u_{i},
\end{aligned}
$$

onde

$$
\left(\begin{array}{c}
e_{i} \\
u_{i} \\
x_{i}
\end{array}\right) \stackrel{\text { i.j.d. }}{\sim} N\left(\left(\begin{array}{c}
0 \\
0 \\
\mu
\end{array}\right)\left(\begin{array}{ccc}
\lambda \sigma^{2} & 0 & 0 \\
0 & \sigma^{2} & 0 \\
0 & 0 & \sigma_{x}^{2}
\end{array}\right)\right), i=1,2, \ldots, n,
$$

onde $\underset{\sim}{\theta}=\left(\alpha, \beta, \mu, \sigma_{x}^{2}, \sigma^{2}\right)^{T}$ é o vetor de parâmetros.

Acrescentamos a esse modelo a suposição à priori de que $\alpha, \beta, \mu, \sigma_{x}^{2}$ e $\sigma^{2}$ são variáveis aleatórias independentes, $\operatorname{com} \alpha, \beta, \mu$ tendo distribuição normal e $\sigma_{x}^{2}, \sigma^{2}$ tendo distribuição Gama Invertida, como segue:

$$
\begin{aligned}
& \pi(\alpha) \sim N\left(a, \sigma_{\alpha}^{2}\right), \pi(\beta) \sim N\left(b, \sigma_{\beta}^{2}\right), \pi(\mu) \sim N\left(m, \sigma_{\mu}^{2}\right) \\
& \sigma_{x}^{2} \sim I G(c, d) \text { e } \sigma^{2} \sim I G(f, g)\left(\text { isto é, } \pi\left(\sigma^{2}\right) \propto\left(\sigma^{2}\right)^{-(f+1)} \exp \left\{-\frac{g}{\sigma^{2}}\right\}\right)
\end{aligned}
$$

onde $\lambda, a, \sigma_{\alpha}^{2}, b, \sigma_{\beta}^{2}, m, \sigma_{\mu}^{2}, c, d, f$ e $g$ são os hiperparâmetros conhecidos.

Observação: Embora vamos manter (até o final da tese) o valor de $\lambda$ em todas as equações que envolvem o modelo com restrição de identificabilidade $\sigma_{e}^{2}=\lambda \sigma_{u}^{2}$. É 
importante lembrar que não existe perda de generalidade em trabalhar sempre com $\lambda=1$ (pois sempre podemos dividir a equação $Y_{i}=\alpha+\beta x_{i}+e_{i}$ por $\sqrt{\lambda}$ e fazer $Y_{i}^{*}=\frac{Y_{i}}{\sqrt{\lambda}}, \alpha^{*}=\frac{\alpha}{\sqrt{\lambda}}, \beta^{*}=\frac{\beta}{\sqrt{\lambda}}$ e $e_{i}^{*}=\frac{e_{i}}{\sqrt{\lambda}}$, de tal modo que $\operatorname{Var}\left(e_{i}^{*}\right)=\frac{\sigma_{e}^{2}}{\lambda}=\frac{\lambda \sigma_{u}^{2}}{\lambda}=$ $\sigma_{u}^{2}$ (isto é $\sigma_{e^{*}}^{2}=\sigma_{u}^{2}$ ).

Assim, trabalhando-se com as variáveis observadas $Y_{i}^{*}$ e $X_{i}$ obtemos os estimadores $\hat{\alpha}^{*}$ e $\hat{\beta}^{*}$ e finalmente $\hat{\alpha}=\hat{\alpha}^{*} \sqrt{\lambda}$ e $\hat{\beta}=\hat{\beta}^{*} \sqrt{\lambda}$.

\section{Distribuição à posteriori}

Na seção 2.2.1, usando a formulação 1 , vimos que a posteriori $p\left(\underset{\theta}{\theta} \mid D_{\text {obs }}\right)=p(\alpha, \beta, \mu$, $\left.\sigma_{x}^{2}, \sigma^{2} \mid D_{\text {obs }}\right)$ pode ser escrita de duas maneiras, uma em função da verossimilhança baseada nos dados observados $\left(L\left(\underset{\theta}{\theta} \mid D_{\text {obs }}\right)\right)$, que é dada por

$$
p\left(\underset{\sim}{\theta} \mid D_{\mathrm{obs}}\right) \propto L\left(\underset{\sim}{\theta} \mid D_{\mathrm{obs}}\right) \pi(\underset{\sim}{\theta}),
$$

e que chamamos de "posteriori baseada nos dados observados". Outra maneira, em função da verossimilhança baseada nos dados completos $(L(\underset{\theta}{\theta} \mid D)$ onde $D=$ $\left.\left(D_{\text {obs }}, \underset{\sim}{x}\right)\right)$, que é dada por

$$
p\left(\underset{\sim}{\theta} \mid D_{\mathrm{obs}}\right)=\int_{\mathscr{X}=\mathbb{R}^{n}} p\left(\underset{\sim}{x}, \underset{\sim}{\theta} \mid D_{\mathrm{obs}}\right) \mathrm{d} \underset{\sim}{x} \propto \int_{\mathscr{X}=\mathbb{R}^{n}} L(\underset{\theta}{\theta} \mid D) \pi(\underset{\sim}{\theta}) \mathrm{d} \underset{\sim}{\theta},
$$

e que chamamos de "posteriori baseada nos dados completos".

\section{Posteriori baseada nos dados observados (Dobs)}

No modelo definido por (2.3.1) e (2.3.2), a posteriori é dada por

$$
\begin{aligned}
& p\left(\theta \mid D_{\text {obs }}\right) \propto|\Sigma|^{-\frac{n}{2}}\left(\sigma_{x}^{2}\right)^{-(c+1)}\left(\sigma^{2}\right)^{-(f+1)} \exp \left\{-\frac{1}{2}\left[\left(\left(\beta^{2} \sigma_{x}^{2}+\lambda \sigma^{2}\right) \sum_{i=1}^{n}\left(X_{i}-\mu\right)^{2}\right.\right.\right. \\
& \left.-2 \beta \sigma_{x}^{2} \sum_{i=1}^{n}\left(Y_{i}-\alpha-\beta \mu\right)\left(X_{i}-\mu\right)+\left(\sigma_{x}^{2}+\sigma^{2}\right) \sum_{i=1}^{n}\left(Y_{i}-\alpha-\beta \mu\right)^{2}\right) /|\Sigma| \\
& \left.\left.+\frac{(\alpha-a)^{2}}{\sigma_{\alpha}^{2}}+\frac{(\beta-b)^{2}}{\sigma_{\beta}^{2}}+\frac{(\mu-m)^{2}}{\sigma_{\mu}^{2}}+\frac{2 d}{\sigma_{x}^{2}}+\frac{2 g}{\sigma^{2}}\right]\right\}
\end{aligned}
$$


onde $|\Sigma|=\beta^{2} \sigma^{2} \sigma_{x}^{2}+\lambda \sigma^{2} \sigma_{x}^{2}+\lambda\left(\sigma^{2}\right)^{2}$.

\section{Posteriori baseada nos dados completos}

$$
p\left(\underset{\sim}{\theta} \mid D_{\mathrm{obs}}\right)=\int_{\mathfrak{X}} p\left(\underset{\sim}{\theta} \underset{\sim}{x} \mid D_{\mathrm{obs}}\right) \mathrm{d} \underset{\sim}{x}
$$

onde $p\left(\underset{\sim}{\theta} \underset{\sim}{x} \mid D_{\text {obs }}\right)$ é proporcional a

$$
\begin{aligned}
& \left(\sigma_{x}^{2}\right)^{-\left(\frac{n}{2}+c+1\right)}\left(\sigma^{2}\right)^{-(n+f+1)} \exp \left\{-\frac{1}{2}\left[\frac{\sum_{i=1}^{n}\left(Y_{i}-\alpha-\beta x_{i}\right)^{2}}{\lambda \sigma^{2}}+\frac{\sum_{i=1}^{n}\left(X_{i}-x_{i}\right)^{2}}{\sigma^{2}}\right.\right. \\
& \left.\left.+\frac{\sum_{i=1}^{n}\left(x_{i}-\mu\right)^{2}}{\sigma_{x}^{2}}+\frac{(\alpha-a)^{2}}{\sigma_{\alpha}^{2}}+\frac{(\beta-b)^{2}}{\sigma_{\beta}^{2}}+\frac{(\mu-m)^{2}}{\sigma_{\mu}^{2}}+\frac{2 d}{\sigma_{x}^{2}}+\frac{2 g}{\sigma^{2}}\right]\right\}
\end{aligned}
$$

A distribuição à posteriori de $\underset{\sim}{\theta}=\left(\alpha, \beta, \mu, \sigma_{x}^{2}, \sigma^{2}\right)^{T}$ na forma exata é dada por

$$
\begin{aligned}
& p\left(\underset{\theta}{\theta} \mid D_{\text {obs }}\right)=\frac{L\left(\underset{\theta}{\theta} \mid D_{\text {obs }}\right) \pi(\theta)}{\int_{\theta} L\left(\underset{\sim}{\theta} \mid D_{\text {obs }}\right) \pi(\underset{\theta}{\theta}) \mathrm{d} \theta} \\
& \left(\text { ou } \frac{\int_{\mathscr{X}} L(\underset{\theta}{\theta} \mid D) \pi(\theta) \mathrm{d} \underset{\sim}{x}}{\int_{\Theta} \int_{\mathscr{X}} L(\underset{\sim}{\theta} \mid D) \pi(\theta) \mathrm{d} \underset{\sim}{\underset{\sim}{x} \mathrm{~d}} \underset{\sim}{\theta}} \text {, onde } D=\left(D_{\mathrm{obs}}, \underset{\sim}{x}\right)\right) \text {. }
\end{aligned}
$$

Podemos ver, com base em (2.3.3) e (2.3.4), que a posteriori portanto não tem uma forma fechada pois a constante de normalização (ou proporcionalidade) dada por $\int_{\Theta} L\left(\underset{\sim}{\theta} \mid D_{\text {obs }}\right) \pi(\underset{\sim}{\theta}) \mathrm{d} \underset{\sim}{\theta}\left(\right.$ ou $\int_{\Theta} \int_{\mathscr{X}} L(\underset{\sim}{\theta} \mid D) \pi(\underset{\sim}{\theta}) \mathrm{d} \underset{\sim}{x} \mathrm{~d} \underset{\sim}{\theta}$ ) não pode ser obtida analiticamente, já que essas integrais não têm primitivas na forma explícita; apenas o núcleo da posteriori baseada nos dados observados $\left(L\left(\underset{\sim}{\theta} \mid D_{\mathrm{obs}}\right) \pi(\underset{\sim}{\theta})\right)$ e o núcleo de $p\left(\underset{\sim}{\theta} \underset{\sim}{x} \mid D_{\text {obs }}\right)$ têm forma conhecida, dada por (2.3.3) e (2.3.4), respectivamente. Além disso, as distribuições marginais e condicionais à posteriori, bem como as medidas resumo da posteriori (como, por exemplo, os momentos e os quantis) obviamente não tem fórmulas fechadas pois requerem ainda mais integrais para serem calculadas (agora também em relação ao numerador de (2.3.5)).

$\mathrm{Na}$ literatura existem vários procedimentos numéricos computacionais para tratar desse tipo de problema e na próxima seção descrevemos alguns dos mais conhecidos na estatística, incluindo por exemplo algoritmos de simulação para amostrar de uma distribuição à posteriori. 


\subsection{Métodos computacionais descritos na litera- tura}

A análise do modelo estrutural Normal, bem como de todos os modelos propostos no restante desse trabalho requer a utilização de procedimentos computacionais. Nosso objetivo então é descrever alguns dos métodos mais populares utilizados na estatística e também apresentar algumas técnicas de diagnósticos de convergência. Entretanto para não quebrar a seqüência das seções colocamos esse tópico na seção 2.4 do Apêndice. As subseções dentro do Apêndice 2.4 abordam os seguintes tópicos:

Na subseção 2.4.1 fazemos uma classificação geral dos métodos computacionais, descrevendo brevemente alguns dos algoritmos mais conhecidos.

Na subseção 2.4.2 apresentamos as idéias básicas sobre os métodos de simulação do tipo MCMC ("Markov Chain Monte Carlo") descrevendo o algoritmo de Metropolis-Hastings e o algoritmo original de Gibbs (Geman e Geman, 1984, e Gelfand e Smith, 1990).

Nas subseções 2.4.3 e 2.4.4 descrevemos duas variações do algoritmo de Gibbs, o "Gibbs em Blocos" (ou "Grouped Gibbs") e o "Collapsed Gibbs" (Liu, 1994). Estes métodos tem o objetivo de acelerar a convergência do Gibbs original.

$\mathrm{Na}$ subseção 2.4.5 descrevemos o algoritmo híbrido de Metropolis-Hastings em Gibbs (Müller, 1991), conhecido como "Localized Metropolis-Hastings".

Finalmente, na subseção 2.4.6 do Apêndice apresentamos algumas das técnicas de diagnóstico de convergência e critérios de parada dos algoritmos encontrados na literatura. 


\subsection{Implementação de algoritmos para o modelo da seção 2.3}

Nessa seção apresentamos inicialmente as distribuições condicionais completas à posteriori obtidas a partir do núcleo da "distribuição à posteriori baseada nos dados observados" (que é dada em (2.3.3)). Apresentamos também as condicionais completas à posteriori obtidas a partir do núcleo da distribuição conjunta $p\left(\underset{\sim}{x}, \underset{\sim}{\theta} \mid D_{\text {obs }}\right)$ (dada em (2.3.4)). Essas distribuições condicionais serão úteis para implementar os algoritmos MCMC dessa seção.

\subsubsection{Distribuições condicionais completas à posteriori ba- seadas nos dados observados}

Baseando-se na posteriori em (2.3.3) (sob o modelo estrutural na formulação 1), obtemos a seguir as distribuições condicionais completas univariadas de $\alpha$ e $\mu$ (que são Normais), a distribuição condicional completa conjunta de $\alpha$ e $\mu$ (que também é Normal), e as distribuições condicionais completas univariadas de $\beta, \sigma_{x}^{2}$ e $\sigma^{2}$ que são distribuições conhecidas a menos de constantes de proporcionalidade (ou seja, apenas os seus núcleos são conhecidos). Essas distribuições estão descritas a seguir.

- A distribuição de $\alpha$ dado $\beta, \mu, \sigma_{x}^{2}, \sigma^{2}$ e $D_{\text {obs }}$ é dada por

$$
\begin{gathered}
\alpha \mid \beta, \mu, \sigma_{x}^{2}, \sigma^{2}, D_{\mathrm{obs}} \sim \\
N\left(\frac{n \sigma_{\alpha}^{2}\left[\sigma_{x}^{2}(\bar{Y}-\beta \bar{X})+\sigma^{2}(\bar{Y}-\beta \mu)\right]+a|\Sigma|}{n \sigma_{\alpha}^{2}\left(\sigma_{x}^{2}+\sigma^{2}\right)+|\Sigma|} ; \frac{\sigma_{\alpha}^{2}|\Sigma|}{n \sigma_{\alpha}^{2}\left(\sigma_{x}^{2}+\sigma^{2}\right)+|\Sigma|}\right) \\
|\Sigma|=\beta^{2} \sigma^{2} \sigma_{x}^{2}+\lambda \sigma^{2} \sigma_{x}^{2}+\lambda\left(\sigma^{2}\right)^{2}
\end{gathered}
$$

onde

- A distribuição de $\mu$ dado $\alpha, \beta, \sigma_{x}^{2}, \sigma^{2}$ e $D_{\text {obs }}$ é a seguinte:

$$
\begin{aligned}
& \mu \mid \alpha, \beta, \sigma_{x}^{2}, \sigma^{2}, D_{\text {obs }} \sim \\
& N\left(\frac{n \sigma_{\mu}^{2}\left[\lambda \sigma^{2} \bar{X}+\beta \sigma^{2}(\bar{Y}-\alpha)\right]+m|\Sigma|}{n \sigma_{\mu}^{2}\left[\sigma^{2} \lambda+\beta^{2} \sigma^{2}\right]+|\Sigma|} ; \frac{\sigma_{\mu}^{2}|\Sigma|}{n \sigma_{\mu}^{2}\left[\sigma^{2} \lambda+\beta^{2} \sigma^{2}\right]+|\Sigma|}\right),
\end{aligned}
$$


onde $|\Sigma|$ é dada em (2.5.2).

- A distribuição conjunta de $\alpha$ e $\mu$ dado $\beta, \sigma_{x}^{2}, \sigma^{2}$ e $D_{\text {obs }}$ é dada por

$$
\pi\left(\alpha, \mu \mid \beta \sigma_{x}^{2}, \sigma^{2}, D_{\mathrm{obs}}\right)=\pi\left(\alpha \mid \mu, \beta, \sigma_{x}^{2}, \sigma^{2}, D_{\mathrm{obs}}\right) . \pi\left(\mu \mid \beta, \sigma_{x}^{2}, \sigma^{2}, D_{\mathrm{obs}}\right)
$$

onde $\pi\left(\alpha \mid \mu, \beta, \sigma_{x}^{2}, \sigma^{2}, D_{\text {obs }}\right)$ é dada em (2.5.1) e

$$
\left.\mu \mid \beta, \sigma_{x}^{2}, \sigma^{2}, D_{\mathrm{obs}}\right) \sim N\left(\frac{B}{A}, \frac{1}{A}\right)
$$

onde

$$
\begin{aligned}
& A=\frac{n \sigma^{2}\left(\lambda+\beta^{2}\right)}{|\Sigma|}+\frac{1}{\sigma_{\mu}^{2}}-\frac{n^{2} \sigma_{\alpha}^{2} \beta^{2}\left(\sigma^{2}\right)^{2}}{|\Sigma|\left(n \sigma_{\alpha}^{2}\left(\sigma_{x}^{2}+\sigma^{2}\right)+|\Sigma|\right)} \\
& B=\frac{n \sigma^{2}(\lambda \bar{X}+\beta \bar{Y})}{|\Sigma|}+\frac{m}{\sigma_{\mu}^{2}}-\frac{n \beta \sigma^{2}\left[n \sigma_{\alpha}^{2}\left(\bar{Y}\left(\sigma_{x}^{2}+\sigma^{2}\right)-\beta \sigma_{x}^{2} \bar{X}\right)+a|\Sigma|\right]}{|\Sigma|\left(n \sigma_{\alpha}^{2}\left(\sigma_{x}^{2}+\sigma^{2}\right)+|\Sigma|\right)}
\end{aligned}
$$

e $|\Sigma|$ é dada em (2.5.2). A distribuição (2.5.5) foi obtida integrando-se a posteriori em (2.3.3) em relação a $\alpha$ e depois obtendo-se a condicional completa em relação a $\mu$.

- A distribuição condicional de $\beta$ dado $\alpha, \mu, \sigma_{x}^{2}, \sigma^{2}$ e $D_{\text {obs }}$ é

$$
\pi\left(\beta \mid \alpha, \mu, \sigma_{x}^{2}, \sigma^{2}, D_{\text {obs }}\right) \propto|\Sigma|^{-\frac{n}{2}} \exp \left\{-\frac{1}{2 \sigma_{\beta}^{2}|\Sigma|}\left[\beta^{2} . A-2 \beta . B\right]\right\}
$$

onde

$$
\begin{aligned}
& A=\sigma_{\beta}^{2}\left[\sigma_{x}^{2} \sum_{i=1}^{n}\left(X_{i}-\mu\right)^{2}+2 n \mu \sigma_{x}^{2}(\bar{X}-\mu)+n \mu^{2}\left(\sigma_{x}^{2}+\sigma^{2}\right)\right]+|\Sigma| \\
& B=\sigma_{\beta}^{2}\left[n \mu \sigma^{2}(\bar{Y}-\alpha)+\sigma_{x}^{2}\left(\sum_{i=1}^{n} X_{i} Y_{i}-n \alpha \bar{X}\right)\right]+b|\Sigma|
\end{aligned}
$$

e $|\Sigma|$ é dado por (2.5.2).

De (2.5.3) podemos ver que a distribuição condicional de $\beta$ baseada nos dados observados não é Normal, já que $A, B$ e $|\Sigma|$ são funções de $\beta$. 
- A distribuição condicional de $\sigma_{x}^{2}$ dado $\alpha, \beta, \mu, \sigma^{2}, D_{\text {obs }}$ é dada por

$$
\pi\left(\sigma_{x}^{2} \mid \alpha, \beta, \mu, \sigma^{2}, D_{\text {obs }}\right) \propto|\Sigma|^{-\frac{n}{2}}\left(\sigma_{x}^{2}\right)^{-(c+1)} \exp \left\{-\frac{1}{2}\left[\frac{\sigma_{x}^{2} \sum_{i=1}^{n}\left(Y_{i}-\alpha-\beta X_{i}\right)^{2}}{|\Sigma|}+\frac{2 d}{\sigma_{x}^{2}}\right]\right\}
$$

onde $|\Sigma|$ é dada por (2.5.2).

- A distribuição condicional de $\sigma^{2}$ dado $\alpha, \beta, \mu, \sigma_{x}^{2}, \sigma^{2}, D_{\text {obse }}$ dada por

$$
\begin{aligned}
& \pi\left(\sigma^{2} \mid \alpha, \beta, \mu, \sigma_{x}^{2}, D_{\text {obs }}\right) \\
& \propto|\Sigma|^{-\frac{n}{2}}\left(\sigma^{2}\right)^{-(f+1)} \exp \left\{-\frac{1}{2}\left\{\frac{\sigma^{2}}{|\Sigma|}\left[\sum_{i=1}^{n}\left(Y_{i}-\alpha-\beta \mu\right)^{2}+\lambda \sum_{i=1}^{n}\left(X_{i}-\mu\right)^{2}\right]+\frac{2 g}{\sigma^{2}}\right\}\right\}
\end{aligned}
$$

onde $|\Sigma|$ é dada em (2.5.2).

As fórmulas (2.5.1) a (2.5.8) são desenvolvidas na seção 2.5.1 do Apêndice A.

Observação: Das distribuições condicionais completas à posteriori dadas em (2.5.6) (2.5.7) e (2.5.8) conhecemos apenas o núcleo. Nesse caso, podemos utilizar por exemplo o algoritmo híbrido de Metropolis-Hastings dentro do Gibbs, que utiliza o algoritmo Metropolis-Hastings para amostrar de cada condicional completa univariada desconhecida dada acima (na subseção 2.4.5 descrevemos os passos desse algoritmo com detalhe). Uma outra saída seria utilizar a "posteriori baseada nos dados completos" e amostrar da distribuição conjunta $p\left(\underset{\sim}{x}, \underset{\sim}{\theta} \mid D_{\text {obs }}\right)$ dada em (2.3.4) por meio do algoritmo de Gibbs, que nesse caso é muito simples de ser implementado, já que todas as distribuições condicionais completas necessárias são conhecidas como vemos na subseção a seguir. Note que, uma vez obtida uma amostra de $p\left(\underset{\sim}{x}, \underset{\sim}{\theta} \mid D_{\text {obs }}\right)$, podemos então descartar a parte da amostra referente a $\underset{\sim}{x}$, já que $p\left(\underset{\sim}{\theta} \mid D_{\text {obs }}\right)$ é uma distribuição marginal de $p\left(\underset{\sim}{x}, \underset{\sim}{\theta} \mid D_{\text {obs }}\right)$. 


\subsubsection{Distribuições condicionais completas à posteriori ba- seadas nos dados completos $(D)$}

A seguir apresentamos as distribuições condicionais completas e $\alpha, \beta, \mu, \sigma_{x}^{2}, \sigma^{2}$ e $\underset{\sim}{x}$ obtidas a partir de $p(\underset{\sim}{x}, \underset{\underset{ }{\theta}}{)} \propto L(\underset{\sim}{\theta} \mid D) \pi(\underset{\sim}{\theta})$ dada em (2.3.4).

Nesse caso as distribuições condicionais de $\alpha, \beta$ e $\mu$ são Normais e as de $\sigma_{x}^{2}$ e $\sigma^{2}$ são Gama Invertida, com os respectivos parâmetros dados por:

$$
\begin{aligned}
\alpha \mid \beta, \mu, \sigma_{x}^{2}, \sigma^{2}, D & \sim N\left(\frac{n \sigma_{\alpha}^{2}(\bar{Y}-\beta \bar{x})+\lambda a \sigma^{2}}{n \sigma_{\alpha}^{2}+\lambda \sigma^{2}} ; \frac{\lambda \sigma^{2} \sigma_{\alpha}^{2}}{n \sigma_{\alpha}^{2}+\lambda \sigma^{2}}\right) \\
\beta \mid \alpha, \mu, \sigma_{x}^{2}, \sigma^{2}, D & \sim N\left(\frac{\sigma_{\beta}^{2}\left(\sum_{i=1}^{n} x_{i} Y_{i}-n \alpha \bar{x}\right)+\lambda \sigma^{2} b}{\sigma_{\beta}^{2} \sum_{i=1}^{n} x_{i}^{2}+\lambda \sigma^{2}} ; \frac{\lambda \sigma^{2} \sigma_{\beta}^{2}}{\sigma_{\beta}^{2} \sum_{i=1}^{n} x_{i}^{2}+\lambda \sigma^{2}}\right), \\
\mu \mid \alpha, \beta, \sigma_{x}^{2}, \sigma^{2}, D & \sim N\left(\frac{n \bar{x} \sigma_{\mu}^{2}+\sigma_{x}^{2} m}{n \sigma_{\mu}^{2}+\sigma_{x}^{2}} ; \frac{\sigma_{x}^{2} \sigma_{\mu}^{2}}{n \sigma_{\mu}^{2}+\sigma_{x}^{2}}\right) \\
\sigma_{x}^{2} \mid \alpha, \beta, \mu, \sigma^{2}, D & \sim I G\left(\frac{n}{2}+c ; d+\frac{1}{2} \sum_{i=1}^{n}\left(x_{i}-\mu\right)^{2}\right) \\
\sigma^{2} \mid \alpha, \beta, \mu, \sigma_{x}^{2}, D & \sim I G\left(n+f ; g+\frac{1}{2}\left(\frac{\sum_{i=1}^{n}\left(Y_{i}-\alpha-\beta x_{i}\right)^{2}}{\lambda}+\sum_{i=1}^{n}\left(X_{i}-x_{i}\right)^{2}\right)\right)
\end{aligned}
$$

e cada uma das variáveis $x_{i}, i=1, \ldots, n$, tem a seguinte distribuição condicional completa:

$$
x_{i} \mid \alpha, \beta, \mu, \sigma_{x}^{2}, D_{\mathrm{obs}}, \underset{\sim}{x}(-i) \sim N\left(\frac{\sigma_{x}^{2}\left(\beta Y_{i}-\beta \alpha+\lambda X_{i}\right)+\lambda \sigma^{2} \mu}{\sigma_{x}^{2}\left(\beta^{2}+\lambda\right)+\lambda \sigma^{2}} ; \frac{\lambda \sigma^{2} \sigma_{x}^{2}}{\sigma_{x}^{2}\left(\beta^{2}+\lambda\right)+\lambda \sigma^{2}}\right) .
$$

Observação: A "introdução" das variáveis latentes $x_{1}, \ldots, x_{n}$ leva às condicionais à posteriori serem Normais ou Gamas Invertidas e, portanto, mais simples de serem amostradas computacionalmente.

\subsubsection{Implementação do algoritmo original de Gibbs}

Para amostrar da posteriori $p\left(\underset{\sim}{\theta} \mid D_{\text {obs }}\right)$ sob o modelo em (2.3.1) podemos amostrar da distribuição $p\left(\underset{\sim}{x}, \theta \mid D_{\text {obs }}\right)$ dada em (2.3.4) utilizando o algoritmo de Gibbs. Para isso 
usamos as distribuições condicionais completas unidimensionais dadas por (2.5.9) até (2.5.14). Os passos do algoritmo são os seguintes:

Passo 0: Escolher arbitrariamente um ponto inicial ${\underset{\theta}{(0)}}^{(0)}\left(\alpha^{(0)}, \beta^{(0)}, \mu^{(0)}, \sigma_{x}^{2^{(0)}}, \sigma^{2^{(0)}}\right)^{T}$.

Faça $j=0$.

Passo 1: Gerar ${\underset{\sim}{x}}^{(j+1)},{\underset{\sim}{(j+1)}}^{(j+1}(\underbrace{(j+1)}, \alpha^{(j+1)}, \beta^{(j+1)}, \mu^{(j+1)}, \sigma_{x}^{2^{(j+1)}}, \sigma^{2^{(j+1)}})^{T}$ como segue

- gerar ${\underset{\sim}{x}}^{(j+1)}=\left(x_{1}^{(j+1)}, \ldots, x_{n}^{(j+1)}\right)^{T}$, onde

$$
x_{i}^{(j+1)} \sim \pi\left(x_{i} \mid \alpha^{(j)}, \beta^{(j)}, \mu^{(j)}, \sigma_{x}^{2^{(j)}},{\sigma^{2}}^{(j)}, D_{\mathrm{obs}}\right)
$$

isto é,

$$
\begin{aligned}
x_{i}^{(j+1)} \sim & N\left(\frac{\sigma_{x}^{2^{(j)}}\left(\beta^{(j)} Y_{i}-\beta^{(j)} \alpha^{(j)}+\lambda X_{i}\right)+\lambda{\sigma^{2}}^{(j)} \mu^{(j)}}{\sigma_{x}^{2^{(j)}}\left(\beta^{2^{(j)}}+\lambda\right)+\lambda \sigma^{2^{(j)}}} ; \frac{\lambda{\sigma^{2(j)}}^{2^{(j)}}}{\sigma_{x}^{2^{(j)}}\left(\beta^{2^{(j)}}+\lambda\right)+\lambda \sigma^{2^{(j)}}}\right), \\
& i=1, \ldots, n .
\end{aligned}
$$

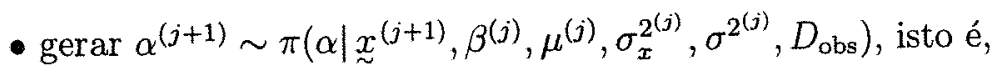

$$
\alpha^{(j+1)} \sim N\left(\frac{n \sigma_{\alpha}^{2}\left(\bar{Y}-\beta^{(j)} \bar{x}^{(j+1)}\right)+\lambda a \sigma^{2^{(j)}}}{n \sigma_{\alpha}^{2}+\lambda \sigma^{2^{(j)}}} ; \frac{\lambda \sigma^{2^{(j)}} \sigma_{\alpha}^{2}}{n \sigma_{\alpha}^{2}+\lambda \sigma^{2^{(j)}}}\right)
$$

- $\operatorname{gerar} \beta^{(j+1)} \sim \pi\left(\beta \mid \underset{\sim}{x}{ }^{(j+1)}, \alpha^{(j+1)}, \mu^{(j)}, \sigma_{x}^{2^{(j)}}, \sigma^{2^{(j)}}, D_{\mathrm{obs}}\right)$, isto é,

$$
\beta^{(j+1)} \sim N\left(\frac{\sigma_{\beta}^{2}\left(\sum_{i=1}^{n} x_{i}^{(j+1)} Y_{i}-n \alpha^{(j+1)} \bar{x}^{(j+1)}\right)+\lambda \sigma^{2^{(j)}} b}{\sigma_{\beta}^{2} \sum_{i=1}^{n}\left(x_{i}^{(j+1)}\right)^{2}+\lambda{\sigma^{2(j)}}^{(j)}} ; \frac{\lambda{\sigma^{2}}^{(j)} \sigma_{\beta}^{2}}{\sigma_{\beta}^{2} \sum_{i=1}^{n}\left(x_{i}^{(j+1)}\right)^{2}+\lambda \sigma^{2^{(j)}}}\right),
$$

- $\operatorname{gerar} \mu^{(j+1)} \sim \pi\left(\mu \mid{\underset{\sim}{x}}^{(j+1)}, \alpha^{(j+1)}, \beta^{(j+1)}, \sigma_{x}^{2^{(j)}}, \sigma^{2^{(j)}}, D_{\text {obs }}\right)$, isto é,

$$
\mu^{(j+1)} \sim N\left(\frac{n \bar{x}^{(j+1)} \sigma_{\mu}^{2}+\sigma_{x}^{2^{(j)}} m}{n \sigma_{\mu}^{2}+\sigma_{x}^{2^{(j)}}} ; \frac{\sigma_{x}^{2^{(j)}} \sigma_{\mu}^{2}}{n \sigma_{\mu}^{2}+\sigma_{x}^{2^{(j)}}}\right),
$$

- gerar $\sigma_{x}^{2^{(j+1)}} \sim \pi\left(\sigma_{x}^{2} \mid{\underset{\sim}{x}}^{(j+1)}, \alpha^{(j+1)}, \beta^{(j+1)}, \mu^{(j+1)}, \sigma^{2^{(j)}}, D_{\mathrm{obs}}\right)$, isto é,

$$
\sigma_{x}^{2^{(j+1)}} \sim I G\left(\frac{n}{2}+c ; d+\frac{1}{2} \sum_{i=1}^{n}\left(x_{i}^{(j+1)}-\mu^{(j+1)}\right)^{2}\right)
$$


- $\operatorname{gerar} \sigma^{2^{(j+1)}} \sim \pi\left(\sigma^{2} \mid \underset{\sim}{x^{(j+1)}}, \alpha^{(j+1)}, \beta^{(j+1)}, \mu^{(j+1)}, \sigma_{x}^{2^{(j+1)}}, D_{\text {obs }}\right)$, isto é,

$$
\sigma^{2^{(j+1)}} \sim I G\left(n+f ; g+\frac{1}{2}\left(\frac{\sum_{i=1}^{n}\left(Y_{i}-\alpha^{(j+1)}-\beta^{(j+1)} x_{i}^{(j+1)}\right)^{2}}{\lambda}+\sum_{i=1}^{n}\left(X_{i}-x_{i}^{(j+1)}\right)^{2}\right)\right) .
$$

Passo 2: Faça $j=j+1$ e vá para o Passo 1 (até a convergência).

\subsubsection{Implementação do algoritmo de Gibbs em blocos}

$\mathrm{Na}$ seção anterior aplicamos o amostrador de Gibbs para amostrar da posteriori $p\left(\underset{\sim}{\theta} \underset{\sim}{x} \mid D_{\text {obs }}\right)$ em (2.3.4), usando as distribuições condicionais completas em (2.5.9) a (2.5.14). É importante notar entretanto que $\underset{\sim}{x}=\left(x_{1}, \ldots, x_{n}\right)$ aparece em cada uma das fórmulas de 2.5.9) a (2.5.14), o que significa que existe uma considerável dependência entre cada uma das componentes de $\underset{\sim}{\theta}$ e $\underset{\sim}{x}$ (e também entre as iterações), o que pode eventualmente prejudicar o desempenho desse algoritmo. Uma alternativa nesse caso seria usar o algoritmo de Gibbs em blocos.

Nessa seção vamos utilizar o algoritmo de Gibbs em blocos para amostrar de $p\left(\underset{\sim}{x} \underset{\sim}{\theta} \mid D_{\text {obs }}\right)$ dada em (2.3.4). Para isso vamos considerar quatro grupos de componentes, $\{\alpha, \mu, \underset{\sim}{x}\},\{\beta\},\left\{\sigma_{x}^{2}\right\},\left\{\sigma^{2}\right\}$, onde a distribuição conjunta $\pi\left(\alpha, \mu, \underset{\sim}{x} \mid \beta, \sigma_{x}^{2}, \sigma^{2}\right.$, $D_{\text {obs }}$ ) é uma Normal de dimensão $n+2$ e portanto muito simples de ser amostrada já que $\pi\left(\alpha, \mu, \underset{\sim}{x} \mid \beta, \sigma_{x}^{2}, \sigma^{2}, D_{\text {obs }}\right)=\pi\left(\underset{\sim}{x} \mid \alpha, \mu, \beta, \sigma_{x}^{2}, \sigma^{2}, D_{\text {obs }}\right) \pi\left(\alpha, \mu \mid \beta, \sigma_{x}^{2}, \sigma^{2}, D_{\text {obs }}\right)$, onde os $x_{i} \mid \alpha, \beta, \mu, \sigma_{x}^{2}, \sigma^{2}, D_{\text {obs }}$, para $i=1, \ldots, n$, são condicionalmente independentes com distribuição dada por (2.5.14), além disso, a distribuição conjunta de $\alpha, \mu \mid \beta, \sigma_{x}^{2}, \sigma^{2}$, $D_{\text {obs }}$ é dada em (2.5.4). As componentes $\beta, \sigma_{x}^{2}$ e $\sigma^{2}$ são amostradas das distribuições em (2.5.10), (2.5.12) e (2.5.13), respectivamente. Com essas informações podemos implementar o algoritmo de Gibbs em blocos e a seguir damos os passos desse algoritmo.

\section{Algoritmo de Gibbs em Blocos}

A seguir damos os passos desse algoritmo para amostrar da distribuição conjunta 
$p\left(\underset{\sim}{\theta} \underset{\sim}{x} \mid D_{\text {obs }}\right)$ dada em $(2.3 .4)$.

Passo 0: escolher ${\underset{\theta}{(0)}}^{(0)}=\left(\beta^{(0)}, \sigma_{x}^{2^{(0)}}, \sigma^{2^{(0)}}\right)^{T}$ e faça $j=0$.

Passo 1: $\operatorname{gerar}\left(\mu^{(j+1)}, \alpha^{(j+1)},{\underset{\sim}{(j+1)}}^{T}\right.$ conjuntamente da seguinte maneira:

- gerar $\mu^{(j+1)} \sim \pi\left(\mu \mid \beta^{(j)}, \sigma_{x}^{2^{(j)}}, \sigma^{2^{(j)}}, D_{\text {obs }}\right)$ que é

$$
\begin{aligned}
& N\left(\frac{B^{(j)}}{A^{(j)}} ; \frac{1}{A^{(j)}}\right), \text { onde } \\
& A^{(j)}=\frac{n \sigma^{2^{(j)}}\left(\lambda+\left(\beta^{(j)}\right)^{2}\right)}{\left|\Sigma^{(j)}\right|}+\frac{1}{\sigma_{\mu}^{2}}-\frac{n^{2} \sigma_{\alpha}^{2}\left(\beta^{(j)}\right)^{2}\left(\sigma^{2^{(j)}}\right)^{2}}{\left|\Sigma^{(j)}\right|\left(n \sigma_{\alpha}^{2}\left(\sigma_{x}^{2^{(j)}}+\sigma^{2^{(j)}}\right)+\left|\Sigma^{(j)}\right|\right)} \\
& B^{(j)}=\frac{n \sigma^{2^{(j)}}\left(\lambda \bar{X}+\beta^{(j)} \bar{Y}\right)}{\left|\Sigma^{(j)}\right|}+\frac{m}{\sigma_{\mu}^{2}} \\
& -\frac{n \beta^{(j)} \sigma^{2^{(j)}}\left[n \sigma_{\alpha}^{2}\left(\bar{Y}\left(\sigma_{x}^{2^{(j)}}+\sigma^{2^{(j)}}\right)-\beta^{(j)} \sigma_{x}^{2^{(j)}} \bar{X}\right)+a\left|\Sigma^{(j)}\right|\right]}{\left|\Sigma^{(j)}\right|\left(n \sigma_{\alpha}^{2}\left(\sigma_{x}^{2^{(j)}}+\sigma^{2^{(j)}}\right)+\left|\Sigma^{(j)}\right|\right)}
\end{aligned}
$$

onde $\quad\left|\Sigma^{(j)}\right|=\left(\beta^{(j)}\right)^{2} \sigma^{2^{(j)}} \sigma_{x}^{2^{(j)}}+\lambda \sigma^{2^{(j)}} \sigma_{x}^{2^{(j)}}+\lambda\left(\sigma^{2^{(j)}}\right)^{2}$

- gerar $\alpha^{(j+1)} \sim \pi\left(\alpha \mid \mu^{(j+1)}, \beta^{(j)}, \sigma_{x}^{2^{(j)}}, \sigma^{2^{(j)}}, D_{\text {obs }}\right)$ que é a seguinte normal

$$
N\left(\frac{n \sigma_{\alpha}^{2}\left[\sigma_{x}^{2^{(j)}}\left(\bar{Y}-\beta^{(j)} \bar{X}\right)+\sigma^{2^{(j)}}\left(\bar{Y}-\beta^{(j)} \mu^{(j+1)}\right)\right]+a\left|\Sigma^{(j)}\right|}{n \sigma_{\alpha}^{2}\left(\sigma_{x}^{2^{(j)}}+\sigma^{2^{(j)}}\right)+\left|\Sigma^{(j)}\right|} ; \frac{\sigma_{\alpha}^{2}\left|\Sigma^{(j)}\right|}{n \sigma_{\alpha}^{2}\left(\sigma_{x}^{2^{(j)}}+\sigma^{2^{(j)}}\right)+\left|\Sigma^{(j)}\right|}\right)
$$

onde $\left|\Sigma^{(j)}\right|$ é dada por (2.5.16),

- gerar as variáveis latentes ${\underset{\sim}{x}}^{(j+1)}=\left(x_{1}^{(j+1)}, \ldots, x_{n}^{(j+1)}\right)^{T}$ do seguinte modo:

$$
\begin{aligned}
& \text { gerar } x_{i}^{(j+1)} \sim \pi\left(x_{i} \mid \mu^{(j+1)}, \alpha^{(j+1)}, \beta^{(j)}, \sigma_{x}^{2^{(j)}}, \sigma^{2^{(j)}}, D_{\text {obs }}\right), \text { para } \\
& i=1,2, \ldots, n, \text { onde } \pi\left(x_{i} \mid \mu, \alpha, \beta, \sigma_{x}^{2}, \sigma^{2}, D_{\text {obs }}\right) \text { é dada } \\
& \text { por (2.5.14). }
\end{aligned}
$$

Passo 2: Gerar $\left(\beta^{(j+1)}, \sigma_{x}^{2^{(j+1)}}, \sigma^{2^{(j+1)}}\right)$ como no algoritmo original de Gibbs da subseção 2.5.2:

- gerar $\beta^{(j+1)} \sim \pi\left(\beta \mid \mu^{(j+1)}, \alpha^{(j+1)}, \sigma_{x}^{2^{(j)}}, \sigma^{2^{(j)}}, x_{\sim}^{(j+1)}, D_{\mathrm{obs}}\right)$,

onde $\pi\left(\beta \mid \mu, \alpha, \sigma_{x}^{2}, \sigma^{2}, \underset{\sim}{x}, D_{\text {obs }}\right)$ é dada em (2.5.10), 
- gerar $\sigma_{x}^{2^{(j+1)}} \sim \pi\left(\sigma_{x}^{2} \mid \mu^{(j+1)}, \alpha^{(j+1)}, \beta^{(j+1)}, \sigma^{2^{(j)}}, \underset{\sim}{x^{(j+1)}}, D_{\mathrm{obs}}\right)$,

onde $\pi\left(\sigma_{x}^{2} \mid \mu, \alpha, \beta, \sigma^{2}, \underset{\sim}{x}, D_{\text {obs }}\right)$ é dada em (2.5.12),

- $\operatorname{gerar} \sigma^{2^{(j+1)}} \sim \pi\left(\sigma^{2} \mid \mu^{(j+1)}, \alpha^{(j+1)}, \beta^{(j+1)}, \sigma_{x}^{2^{(j+1)}}, \underset{\sim}{x^{(j+1)}}, D_{\mathrm{obs}}\right)$,

onde $\pi\left(\sigma^{2} \mid \mu, \alpha, \beta, \sigma_{x}^{2}, \underset{\sim}{x}, D_{\text {obs }}\right)$ é dada em (2.5.13).

Passo 3: Fazer $i=i+1$ e voltar para o passo 1 .

\subsubsection{Implementação do algoritmo "modified collapsed Gibbs"}

Nesta subseção vamos utilizar o algoritmo "modified collapsed Gibbs" para amostrar da distribuição $p\left(\underset{\sim}{\theta} \underset{\sim}{x} \mid D_{\text {obs }}\right)$ dada em (2.3.4).

A idéia é num primeiro passo gerar $(\alpha, \mu)$, não de $\pi\left(\alpha, \mu \mid \beta, \sigma_{x}^{2}, \sigma^{2}, \underset{\sim}{x}, D_{\mathrm{obs}}\right)$, mas de $\pi\left(\alpha, \mu \mid \beta, \sigma_{x}^{2}, \sigma^{2}, D_{\text {obs }}\right.$ ), onde $\underset{\sim}{x}$ foi eliminado por integração (isto é, $\left.\pi\left(\alpha, \mu \mid \beta, \sigma_{x}^{2}, \sigma^{2}, D_{\mathrm{obs}}\right)=\int_{\dddot{x}} \pi\left(\alpha, \mu, \underset{\sim}{x} \mid \beta, \sigma_{x}^{2}, \sigma^{2}, D_{\mathrm{obs}}\right) \mathrm{d} \underset{\sim}{x}\right)$ e num segundo passo gerar $\left(\underset{\sim}{x}, \beta, \sigma_{x}^{2}, \sigma^{2}\right)$ de $\pi\left(\underset{\sim}{x}, \beta, \sigma_{x}^{2}, \sigma^{2} \mid \alpha, \mu, D_{\text {obs }}\right)$, que pode ser feito por meio de um miniGibbs utilizando as condicionais completas $\pi\left(\underset{\sim}{x} \mid \alpha, \beta, \mu, \sigma_{x}^{2}, \sigma^{2}, D_{\mathrm{obs}}\right), \pi\left(\beta \mid \alpha, \mu, \sigma_{x}^{2}, \sigma^{2}, \underset{\sim}{x}\right.$, $\left.D_{\text {obs }}\right), \pi\left(\sigma_{x}^{2} \mid \alpha, \beta, \mu, \sigma^{2}, \underset{\sim}{x}, D_{\text {obs }}\right)$ e $\pi\left(\sigma^{2} \mid \alpha, \beta, \mu, \sigma_{x}^{2}, \underset{\sim}{x}, D_{\text {obs }}\right)$, dadas respectivamente por $(2.5 .14),(2.5 .10),(2.5 .12)$ e (2.5.13)

Os passos do algoritmo "modified collapsed Gibbs" são os seguintes:

Passo 0: faça $i=0$, escolha $\left(\beta^{(0)}, \sigma_{x}^{2^{(0)}}, \sigma^{2^{(0)}}\right)$ e faça $j=1$;

Passo 1: gere $\left(\alpha^{(i+1)}, \mu^{(i+1)}\right)$ de $\pi\left(\alpha, \mu \mid \beta^{(i)}, \sigma_{x}^{2^{(i)}},{\sigma^{2}}^{(i)}\right)$ do seguinte modo:

- gere $\mu^{(i+1)}$ de $\pi\left(\mu \mid \beta^{(i)}, \sigma_{x}^{2^{(i)}}, \sigma^{2^{(i)}}, D_{\text {obs }}\right)$, onde $\pi\left(\mu \mid \beta, \sigma_{x}^{2}, \sigma^{2}, D_{\text {obs }}\right)$ é dada em (2.5.5),

- gere $\alpha^{(i+1)}$ de $\pi\left(\alpha \mid \mu^{(i+1)}, \beta^{(i)}, \sigma_{x}^{2^{(i)}}, \sigma^{2^{(i)}}, D_{\text {obs }}\right)$, onde $\pi\left(\alpha \mid \mu, \beta, \sigma_{x}^{2}, \sigma^{2}, D_{\text {obs }}\right)$ é dada em (2.5.1); 
Passo 2: gere $\left(\underset{\sim}{x_{(j)}^{(i+1)}}, \beta_{(j)}^{(i+1)}, \sigma_{x_{(j)}^{2}}^{2^{(i+1)}}, \sigma_{(j)}^{2^{(i+1)}}\right)$ de $\pi\left(\underset{\sim}{x}, \beta, \sigma_{x}^{2}, \sigma_{j}^{2} \mid \alpha^{(i+1)}, \mu^{(i+1)}, D_{\text {obs }}\right)$ da seguinte maneira:

- gere $\underset{\sim}{x_{(j)}^{(i+1)}}$ de $\pi\left(\underset{\sim}{x} \mid \alpha^{(i+1)}, \mu^{(i+1)}, \beta_{(j)}^{(i)}, \sigma_{x(j)}^{2^{(i)}}, \sigma_{(j)}^{2^{(i)}}, D_{\text {obs }}\right)$ do seguinte modo: gere $x_{k(j)}^{(i+1)}$ de $\pi\left(x_{k} \mid \alpha^{(i+1)}, \mu^{(i+1)}, \beta_{(j)}^{(i)}, \sigma_{x_{(j)}}^{2^{(i)}}, \sigma_{(j)}^{2^{(i)}}, D_{\text {obs }}\right)$ para $k=1,2, \ldots, n$, onde $\pi\left(x_{k} \mid \alpha, \mu, \beta, \sigma_{x}^{2}, \sigma^{2}, D_{\text {obs }}\right)$ é dada em (2.5.14),

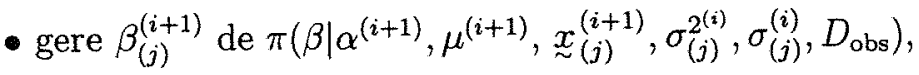
onde $\pi\left(\beta \mid \alpha, \mu, \underset{\sim}{x}, \sigma_{x}^{2}, \sigma^{2}, D_{\text {obs }}\right)$ é dada em (2.5.10),

- gere $\sigma_{x_{(j)}^{2(i+1)}}$ de $\pi\left(\sigma_{x}^{2} \mid \alpha^{(i+1)}, \mu^{(i+1)}, \underset{\sim(j)}{x_{(j+1)}^{(i+1)}}, \beta_{(j)}^{(i+1)}, \sigma_{(j)}^{2^{(i)}}, D_{\mathrm{obs}}\right)$, onde $\pi\left(\sigma_{x}^{2} \mid \alpha, \mu, \beta, \sigma^{2}, \underset{\sim}{x}, D_{\text {obs }}\right)$ é dada em (2.5.12)

- gere $\sigma_{(j)}^{2^{(i+1)}}$ de $\pi\left(\sigma^{2} \mid \alpha^{(i+1)}, \mu^{(i+1)}, \underset{\sim}{x_{(j)}^{(i+1)}}, \beta_{(j)}^{(i+1)}, \sigma_{x_{(j)}}^{2^{(i+1)}}, D_{\text {obs }}\right)$, onde $\pi\left(\sigma^{2} \mid \alpha, \beta, \mu, \sigma_{x}^{2}, \sigma^{2}, \underset{\sim}{x}, D_{\text {obs }}\right)$ é dada em (2.5.13);

Passo 3: se $j<N$, faça $j=j+1$ e volte para o passo 2 (onde $N$ é o número de iterações do mini-Gibbs);

Passo 4: faça $i=i+1$ e volte para o passo 1 .

\section{Observações}

1. A diferença entre o "grouped" e o "modified collapsed Gibbs" para amostrar da distribuição em (2.3.4) (quando apenas o vetor $\underset{\sim}{x}$ é eliminado por integração) é que no último utilizamos um mini-Gibbs nas componentes $\underset{\sim}{x}, \beta, \sigma_{x}^{2}$ e $\sigma^{2}$.

2. Uma outra maneira de implementar o algoritmo "modified collapsed Gibbs" é no passo 1 amostrar apenas o vetor $\underset{\sim}{x}$, e não da distribuição $\pi\left(\underset{\sim}{x} \mid \underset{\sim}{\theta}, D_{\mathrm{obs}}\right)$, mas de $\pi\left(\underset{\sim}{x} \mid D_{\text {obs }}\right)$ (onde $\underset{\sim}{\theta}$ é eliminado integrando-se $p\left(\underset{\sim}{\theta} \underset{\sim}{x} \mid D_{\text {obs }}\right)$ em relação a $\underset{\sim}{\theta}$ ) e no passo 2 amostrar $\underset{\sim}{\theta}$ de $\pi\left(\underset{\sim}{\theta} \underset{\sim}{x}, D_{\text {obs }}\right)$. No primeiro passo aplicamos 
um mini-Gibbs até a convergência, utilizando para isso as distribuições condicionais completas $\pi\left(x_{i} \mid x_{(-i)}, D_{\mathrm{obs}}\right)$, para $i=1, \ldots, n$, e no segundo passo aplicamos outro mini-Gibbs até que convirja, utilizando para isso as condicionais completas $\pi\left(\alpha \mid \beta, \mu, \sigma_{x}^{2}, \sigma^{2}, \underset{\sim}{x}, D_{\mathrm{obs}}\right), \pi\left(\beta \mid \alpha, \mu, \sigma_{x}^{2}, \sigma^{2}, \underset{\sim}{x}, D_{\mathrm{obs}}\right), \pi\left(\mu \mid \alpha, \beta, \sigma_{x}^{2}, \sigma^{2}\right.$, $\left.\underset{\sim}{x}, D_{\text {obs }}\right), \pi\left(\sigma_{x}^{2} \mid \alpha, \beta, \mu, \sigma^{2}, \underset{\sim}{x}, D_{\text {obs }}\right)$ e $\pi\left(\sigma^{2} \mid \alpha, \beta, \mu, \sigma_{x}^{2}, \underset{\sim}{x}, D_{\text {obs }}\right)$.

Para obter a distribuição $\pi\left(\underset{\sim}{x} \mid D_{\text {obs }}\right)$ precisamos integrar a posteriori conjunta $p\left(\underset{\sim}{\theta} \underset{\sim}{x} \mid D_{\text {obs }}\right)$ em relação a $\underset{\sim}{\theta}=\left(\alpha, \beta, \mu, \sigma_{x}^{2}, \sigma_{e}^{2}\right)$, para então obter as condicionais completas $\pi\left(x_{i} \mid x_{(-i)}, D_{\text {obs }}\right)$ para $i=1, \ldots, n$.

\subsubsection{Implementação do algoritmo híbrido de Metropolis- Hastings em Gibbs}

Para amostrar da "posteriori baseada nos dados observados", dada em (2.3.3), utilizando o algoritmo de Metropolis-Hastings em Gibbs, devemos fazer inicialmente as seguintes observações:

1. $\alpha$ e $\mu$ podem ser facilmente gerados da distribuição $\pi\left(\alpha, \mu \mid \beta, \sigma_{x}^{2}, \sigma^{2}, D_{\text {obs }}\right)$ que é Normal bivariada

$$
\pi\left(\alpha, \mu \mid \beta, \sigma_{x}^{2}, \sigma^{2}, D_{\mathrm{obs}}\right)=\pi\left(\alpha \mid \mu, \beta, \sigma_{x}^{2}, \sigma^{2}, D_{\mathrm{obs}}\right) \pi\left(\mu \mid \beta, \sigma_{x}^{2}, \sigma^{2}, D_{\mathrm{obs}}\right)
$$

onde $\pi\left(\alpha \mid \mu, \beta, \sigma_{x}^{2}, \sigma^{2}, D_{\text {obs }}\right)$ é uma Normal dada em $(2.5 .1)$ e $\pi\left(\mu \mid \beta, \sigma_{x}^{2}, \sigma^{2}, D_{\text {obs }}\right)$ é uma Normal dada por (2.5.5).

2. A distribuição condicional completa à posteriori de $\beta$ dada em (2.5.6) é proporcional ao produto

$$
\pi\left(\beta \mid \alpha, \mu, \sigma_{x}^{2}, \sigma^{2}, D_{\text {obs }}\right) \propto \psi_{1}\left(\beta \mid \alpha, \mu, \sigma_{x}^{2}, \sigma^{2}, D_{\text {obs }}\right) \exp \left\{-\frac{1}{2 \sigma_{\beta}^{2}}(\beta-b)^{2}\right\},
$$

onde o segundo fator é o núcleo de uma $N\left(b, \sigma_{\beta}^{2}\right)$, onde $\sigma_{\beta}^{2}$ é conhecido e

$$
\psi_{1}\left(\beta \mid \alpha, \mu, \sigma_{x}^{2}, \sigma^{2}, D_{\text {obs }}\right)=|\Sigma|^{-\frac{n}{2}} \exp \left\{-\frac{1}{2 \sigma_{\beta}^{2}|\Sigma|}\left(\beta^{2} C-2 \beta D\right)\right\},
$$


é uma função uniformemente limitada em $\beta$, onde

$$
\begin{aligned}
C & =\sigma_{\beta}^{2}\left[\sigma_{x}^{2} \sum_{i=1}^{n}\left(X_{i}-\mu\right)^{2}+2 n \mu \sigma_{x}^{2}(\bar{X}-\mu)+n \mu^{2}\left(\sigma_{x}^{2}+\sigma^{2}\right)\right], \\
D & =\sigma_{\beta}^{2}\left[n \mu \sigma^{2}(\bar{Y}-\alpha)+\sigma_{x}^{2}\left(\sum_{i=1}^{n} X_{i} Y_{i}-n \alpha \bar{X}\right)\right] \\
\text { e } \quad|\Sigma| & =\beta^{2} \sigma^{2} \sigma_{x}^{2}+\lambda \sigma^{2}\left(\sigma_{x}^{2}+\sigma^{2}\right) .
\end{aligned}
$$

- A distribuição condicional completa à posteriori de $\sigma_{x}^{2}$, dada em (2.5.7), é proporcional ao produto

$$
\pi\left(\sigma_{x}^{2} \mid \alpha, \beta, \mu, \sigma^{2}, D_{\text {obs }}\right) \propto \psi_{2}\left(\sigma_{x}^{2} \mid \alpha, \beta, \mu, \sigma^{2}, D_{\text {obs }}\right) \cdot\left(\sigma_{x}^{2}\right)^{-(c+1)} \exp \left\{-\frac{d}{\sigma_{x}^{2}}\right\}
$$

onde o segundo fator é o núcleo de uma distribuição $I G(c, d)$ e

$$
\psi_{2}\left(\sigma_{x}^{2} \mid \alpha, \beta, \mu, \sigma^{2}, D_{\mathrm{obs}}\right)=|\Sigma|^{-\frac{n}{2}} \exp \left\{\frac{-\sigma_{x}^{2} \sum_{i=1}^{n}\left(Y_{i}-\alpha-\beta X_{i}\right)^{2}}{2|\Sigma|}\right\},
$$

onde $|\Sigma|=\sigma_{x}^{2}\left(\beta^{2} \sigma^{2}+\lambda \sigma^{2}\right)+\lambda\left(\sigma^{2}\right)^{2}$. Além disso, $\psi_{2}$ é uma função uniformemente limitada $\left(\mathrm{em} \sigma_{x}^{2}\right)$.

- A distribuição condicional completa à posteriori de $\sigma^{2}$, dada em (2.5.8) pode ser escrita como

$$
\pi\left(\sigma^{2} \mid \alpha, \beta, \mu, \sigma_{x}^{2}, D_{\mathrm{obs}}\right) \propto \psi_{3}\left(\alpha, \beta, \mu, \sigma_{x}^{2}, \sigma^{2}, D_{\mathrm{obs}}\right) \cdot\left(\sigma^{2}\right)^{-\left(f+1+\frac{n}{2}\right)} \exp \left\{-\frac{g}{\sigma^{2}}\right\},
$$

onde o segundo fator é o núcleo de uma $I G\left(f+\frac{n}{2} ; g\right)$ e

$$
\psi_{3}\left(\alpha, \beta, \mu, \sigma_{x}^{2}, \sigma^{2}, D_{\text {obs }}\right)=\left(\frac{|\Sigma|}{\sigma^{2}}\right)^{-\frac{n}{2}} \exp \left\{-\frac{\sigma^{2}}{|\Sigma|}\left[\sum_{i=1}^{n}\left(Y_{i}-\alpha-\beta \mu\right)^{2}+\lambda \sum_{i=1}^{n}\left(X_{i}-\mu\right)^{2}\right]\right\}
$$

onde $|\Sigma|=\sigma^{2}\left[\left(\beta^{2} \sigma_{x}^{2}+\lambda \sigma_{x}^{2}\right)+\lambda \sigma^{2}\right]$. Além disso, $\psi_{3}$ é uniformemente limitada $\left(\mathrm{em} \sigma^{2}\right)$

A seguir apresentamos os passos do algoritmo híbrido de Metropolis-Hastings em Gibbs para esse problema.

Passos do Algoritmo de Metropolis-Hastings em Gibbs (para o caso particular em que $T=1$ ) 
Passo 0: Escolher $\dot{\theta}^{(0)}=\left(\beta^{(0)}, \sigma_{x}^{2^{(0)}}, \sigma^{2^{(0)}}\right)^{T}$ e faça $j=0$.

Passo 1: Gerar $\underline{\theta}^{(i+1)}=\left(\alpha^{(i+1)}, \mu^{(i+1)}, \beta^{(i+1)}, \sigma_{x}^{2^{(x+1)}}, \sigma^{2^{(i+1)}}\right)^{T}$ da seguinte maneira:

- gerar $\left(\alpha^{(j+1)}, \mu^{(j+1)}\right)$ conjuntamente do seguinte modo:

- gerar $\mu^{(j+1)} \sim \pi\left(\mu \mid \beta^{(j)}, \sigma_{x}^{2^{(j)}}, \sigma^{2^{(j)}}, D_{\text {obs }}\right)$ que é dada em (2.5.15) e (2.5.16)

- $\operatorname{gerar} \alpha^{(j+1)} \sim \pi\left(\alpha \mid \mu^{(j+1)}, \beta^{(j)}, \sigma_{x}^{2^{(j)}}, \sigma^{2^{(j)}}, D_{\text {obs }}\right)$,

onde $\pi\left(\alpha \mid \mu^{(j+1)}, \beta^{(j)}, \sigma_{x}^{2^{(j)}}, \sigma^{2^{(j)}}, D_{\text {obs }}\right)$ é dada em (2.5.17).

- gerar $\beta^{(j+1)} \sim \pi\left(\beta \mid \alpha^{(j+1)}, \mu^{(j+1)}, \sigma_{x}^{2^{(j)}}, \sigma^{2^{(j)}}, D_{\text {obs }}\right)$ da seguinte maneira:

- gerar o candidato $\beta^{*(j+1)}$ a partir de $N\left(b, \sigma_{\beta}^{2}\right)$ e $U$ da $U(0,1)$,

- fazer

$$
\alpha\left(\beta^{*(j+1)} \mid \beta^{(j)}\right)=\min \left\{\frac{\psi_{1}\left(\beta^{*(j+1)} \mid \alpha^{(j+1)}, \mu^{(j+1)}, \sigma_{x}^{2^{(j)}}, \sigma^{2^{(j)}}, D_{\mathrm{obs}}\right)}{\psi_{1}\left(\beta^{(j)} \mid \alpha^{(j+1)}, \mu^{(j+1)}, \sigma_{x}^{2^{(j)}}, \sigma^{2^{(j)}}, D_{\mathrm{obs}}\right)} ; 1\right\}
$$

onde

$$
\begin{aligned}
& \psi_{1}\left(\beta^{*(j+1)} \mid \alpha^{(j+1)}, \mu^{(j+1)}, \sigma_{x}^{2^{(j)}}, \sigma^{2^{(j)}}, D_{\text {obs }}\right) \\
& =\left|\Sigma^{*}\right|^{-\frac{n}{2}} \exp \left\{-\frac{1}{2 \sigma_{\beta}^{2}\left|\Sigma^{*}\right|}\left(\left(\beta^{*(j+1)}\right)^{2} C-\beta^{*(j+1)}\right) D\right\} \\
& \psi_{1}\left(\beta^{(j)} \mid \alpha^{(j+1)}, \mu^{(j+1)}, \sigma_{x}^{2^{(j)}}, \sigma^{2^{(j)}}\right) \\
& =\left|\Sigma^{(j)}\right|^{-\frac{n}{2}} \exp \left\{-\frac{1}{2 \sigma_{\beta}^{2}\left|\Sigma^{(j)}\right|}\left(\left(\beta^{(j)}\right)^{2} C-\beta^{(j)}\right) D\right\}
\end{aligned}
$$

onde

$$
\begin{aligned}
& C= \sigma_{\beta}^{2}\left[\sigma_{x}^{2^{(j)}} \sum_{i=1}^{n}\left(X_{i}-\mu^{(j+1)}\right)^{2}+2 n \mu^{(j+1)} \sigma_{x}^{2^{(j)}}\left(\bar{X}-\mu^{(j+1)}\right)\right. \\
&\left.+n\left(\mu^{(j+1)}\right)^{2}\left(\sigma_{x}^{2^{(j)}}+\sigma^{2^{(j)}}\right)\right] \\
& D= \sigma_{\beta}^{2}\left[n \mu^{(j+1)} \sigma^{2^{(j)}}\left(\bar{Y}-\alpha^{(j+1)}\right)+\sigma_{x}^{2^{(j)}}\left(\sum_{i=1}^{n} X_{i} Y_{i}-n \alpha^{(j+1)} \bar{X}\right)\right] \\
&\left|\Sigma^{*}\right|=\left(\beta^{*(j+1)}\right)^{2} \sigma^{2^{(j)}} \sigma_{e}^{2^{(j)}}+\lambda \sigma^{2^{(j)}}\left(\sigma_{x}^{2^{(j)}}+\sigma^{2^{(j)}}\right) \\
&\left|\Sigma^{(j)}\right|=\left(\beta^{(j)}\right)^{2} \sigma^{2^{(j)}} \sigma_{e}^{2^{(j)}}+\lambda \sigma^{2^{(j)}}\left(\sigma_{x}^{2^{(j)}}+\sigma^{2^{(j)}}\right) .
\end{aligned}
$$


- Se $U<\alpha\left(\beta^{*(j+1)} \mid \beta^{(j)}\right)$, faça $\beta^{(j+1)}=\beta^{*(j+1)} ;$ caso contrário faça $\beta^{(j+1)}=\beta^{(j)}$.

- Gerar $\sigma_{x}^{2^{(j+1)}} \sim \pi\left(\sigma_{x}^{2} \mid \alpha^{(j+1)}, \mu^{(j+1)}, \beta^{(j+1)}, \sigma^{2^{(j)}}, D_{\text {obs }}\right)$ do seguinte modo:

- gerar um candidato $\sigma_{x}^{2^{*(j+1)}}$ a partir da $I G(c, d)$ e gerar $U \sim U(0,1)$,

- fazer

$\alpha\left(\sigma_{x}^{2^{*(j+1)}} \mid \sigma_{x}^{2^{(j)}}\right)=\min \left\{\frac{\psi_{2}\left(\sigma_{x}^{2^{*(j+1)}} \mid \alpha^{(j+1)}, \mu^{(j+1)}, \beta^{(j+1)}, \sigma^{2^{(j)}}, D_{\mathrm{obs}}\right)}{\psi_{2}\left(\sigma_{x}^{2(j)} \mid \alpha^{(j+1)}, \mu^{(j+1)}, \beta^{(j+1)}, \sigma^{2^{(j)}}, D_{\mathrm{obs}}\right)} ; 1\right\}$

onde

$$
\begin{aligned}
& \psi_{2}\left(\sigma_{x}^{2^{*(j+1)}} \mid \alpha^{(j+1)}, \mu^{(j+1)}, \beta^{(j+1)}, \sigma^{2^{(j)}}, D_{\mathrm{obs}}\right)=\left|\Sigma^{*}\right|^{-\frac{n}{2}} \exp \left\{-\frac{\sigma_{x}^{2^{*(j+1)}}}{2\left|\Sigma^{*}\right|} \cdot A\right\} \\
& \psi_{2}\left(\sigma_{x}^{2^{(j)}} \mid \alpha^{(j+1)}, \mu^{(j+1)}, \beta^{(j+1)}, \sigma^{2^{(j)}}, D_{\mathrm{obs}}\right)=|\Sigma|^{-\frac{n}{2}} \exp \left\{-\frac{\sigma_{x}^{2^{(j)}}}{2|\Sigma|} \cdot A\right\} \\
& \text { onde } \quad \begin{array}{l}
A=\sum_{i=1}^{n}\left(Y_{i}-\alpha^{(i+1)}-\beta^{(i+1)} X_{i}\right)^{2} \\
\left|\Sigma^{*}\right|=\sigma_{x}^{2^{*(j+1)}}\left[\left(\beta^{(j+1)}\right)^{2}{\sigma^{2}}^{(j)}+\lambda \sigma^{2^{(j)}}\right]+\lambda\left(\sigma^{2^{(j)}}\right)^{2} \\
|\Sigma|=\sigma_{x}^{2^{(j)}}\left(\left(\beta^{(j+1)}\right)^{2} \sigma^{2^{(j)}}+\lambda \sigma^{2^{(j)}}\right)+\lambda\left(\sigma^{2^{(j)}}\right)^{2} .
\end{array}
\end{aligned}
$$

- Se $U<\alpha\left(\sigma_{x}^{2^{*(j+1)}} \mid{\sigma_{x}^{2(j)}}^{2}\right.$, faça $\sigma_{x}^{2^{(j+1)}}=\sigma_{x}^{2^{*(j+1)}}$; caso contrário faça $\sigma_{x}^{2^{(j+1)}}=\sigma_{x}^{2^{(j)}}$

- Gerar $\sigma^{2^{(j+1)}} \sim \pi\left(\sigma^{2} \mid \alpha^{(j+1)}, \mu^{(j+1)}, \beta^{(j+1)}, \sigma_{x}^{2^{(j+1)}}, D_{\text {obs }}\right)$ da seguinte forma:

- gerar um candidato $\sigma^{2^{*(j+1)}}$ da $I G\left(f+\frac{n}{2} ; g\right)$ e gerar $U$ da $U(0,10)$,

- fazer

$$
\alpha\left(\sigma^{2^{*(j+1)}} \mid \sigma^{2^{(j)}}\right)=\min \left\{\frac{\psi_{3}\left(\sigma^{2^{*(j+1)}} \mid \alpha^{(j+1)}, \mu^{(j+1)}, \beta^{(j+1)}, \sigma_{x}^{2^{(j+1)}}, D_{\mathrm{obs}}\right)}{\psi_{3}\left(\sigma^{2^{(j)}} \mid \alpha^{(j+1)}, \mu^{(j+1)}, \beta^{(j+1)}, \sigma_{x}^{2^{(j+1)}}, D_{\mathrm{obs}}\right)} ;\right\}
$$

onde

$$
\begin{aligned}
& \psi_{3}\left(\sigma^{2^{*(j+1)}} \mid \alpha^{(j+1)}, \beta^{(j+1)}, \mu^{(j+1)}, \sigma_{x}^{2^{(j+1)}}, D_{\text {obs }}\right) \\
& =\left(A^{*}\right)^{-\frac{n}{2}} \exp \left\{-\frac{1}{A^{*}}\left[\sum_{i=1}^{n}\left(Y_{i}-\alpha^{(i+1)}-\beta^{(i+1)} \mu^{(i+1)}\right)^{2}+\lambda \sum_{i=1}^{n}\left(X_{i}-\mu^{(i+1)}\right)^{2}\right]\right\}
\end{aligned}
$$




$$
\begin{aligned}
& \psi_{3}\left(\sigma^{2^{(j)}} \mid \alpha^{(j+1)}, \beta^{(j+1)}, \mu^{(j+1)}, \sigma_{x}^{2^{(j+1)}}, D_{\mathrm{obs}}\right) \\
& =(A)^{-\frac{n}{2}} \exp \left\{-\frac{1}{A}\left[\sum_{i=1}^{n}\left(Y_{i}-\alpha^{(i+1)}-\beta^{(i+1)} \mu^{(i+1)}\right)^{2}+\lambda \sum_{i=1}^{n}\left(X_{i}-\mu^{(i+1)}\right)^{2}\right]\right\}
\end{aligned}
$$

onde

$$
\begin{gathered}
A^{*}=\left(\beta^{(j+1)}\right)^{2} \sigma_{x}^{2^{(j+1)}}+\lambda \sigma_{x}^{2^{(j+1)}}+\lambda \sigma^{2^{*(j+1)}}, \\
A=\left(\beta^{(j+1)}\right)^{2} \sigma_{x}^{2^{(j+1)}}+\lambda \sigma_{x}^{2^{(j+1)}}+\lambda \sigma^{2^{(j)}}
\end{gathered}
$$

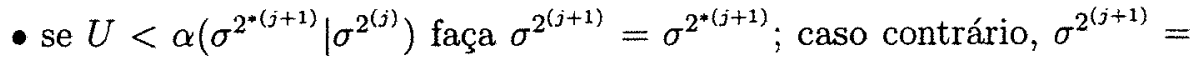
$\sigma^{2^{(j)}}$.

Passo 2: Faça $i=i+1$ e volte ao passo 1 .

Observação: Agrupando-se as componentes $\alpha$ e $\mu$ (isto é, amostrando-se conjuntamente $\alpha$ e $\mu$, ao invés de individualmente) ganha-se em termos de velocidade de convergência do algoritmo.

\subsection{O modelo estrutural não identificável com par- ticular priori de componentes independentes}

No modelo estrutural Normal sem restrição de identificabilidade em (2.2.1), dado pelas equações

$$
\begin{gathered}
Y_{i}=\alpha+\beta x_{i}+e_{i}, \\
\text { e } X_{i}=x_{i}+u_{i}, \quad \text { onde } \\
\left(\begin{array}{c}
e_{i} \\
u_{i} \\
x_{i}
\end{array}\right) \stackrel{\text { i.j.d. }}{\sim} N\left(\left(\begin{array}{l}
0 \\
0 \\
\mu
\end{array}\right) ;\left(\begin{array}{ccc}
\sigma_{e}^{2} & 0 & 0 \\
0 & \sigma_{u}^{2} & 0 \\
0 & 0 & \sigma_{x}^{2}
\end{array}\right)\right), i=1,2, \ldots, n,
\end{gathered}
$$

e onde $\underset{\sim}{\theta}=\left(\alpha, \beta, \mu, \sigma_{x}^{2}, \sigma_{u}^{2}, \sigma_{e}^{2}\right)^{T}$, acrescentamos a suposição à priori que $\alpha, \beta, \mu$, $\sigma_{x}^{2}, \sigma_{e}^{2}$ e $\sigma_{u}^{2}$ são independentes, com $\alpha \sim N\left(a, \sigma_{\alpha}^{2}\right), \beta \sim N\left(b, \sigma_{\beta}^{2}\right), \mu \sim N\left(m, \sigma_{\mu}^{2}\right)$, $\sigma_{x}^{2} \sim I G(c, d), \sigma_{u}^{2} \sim I G(f, g)$ e $\sigma_{e}^{2} \sim I G(p, q)$, onde $a, b, m, \sigma_{\alpha}^{2}, \sigma_{\beta}^{2}, \sigma_{\mu}^{2}, c, d, f, g, p$ e $q$ são hiperparâmetros conhecidos.

Distribuição à posteriori baseada nos dados observados $\left(D_{\text {obs }}\right)$ 
A posteriori baseada nos dados observados sob esse modelo é dada por

$$
p\left(\underset{\sim}{\theta} \mid D_{\text {obs }}\right) \propto L\left(\underset{\sim}{\theta} \mid D_{\text {obs }}\right) \pi(\underset{\theta}{\theta}), \text { onde } L\left(\underset{\sim}{\theta} \mid D_{\text {obs }}\right) \text { é dada por }(2.1 .4)
$$

$\mathrm{e} \pi(\underset{\theta}{\theta})=\pi(\alpha) \cdot \pi(\beta) \cdot \pi(\mu) \cdot \pi\left(\sigma_{x}^{2}\right) \cdot \pi\left(\sigma_{e}^{2}\right) \cdot \pi\left(\sigma_{u}^{2}\right)$, isto é,

$$
\begin{aligned}
& \begin{array}{l}
p\left(\theta \mid D_{\mathrm{obs}}\right) \propto|\Sigma|^{-\frac{n}{2}}\left(\sigma_{x}^{2}\right)^{-(c+1)}\left(\sigma_{u}^{2}\right)^{-(f+1)}\left(\sigma_{e}^{2}\right)^{-(p+1)} \exp \left\{-\frac{1}{2}\left[\left(\left(\beta^{2} \sigma_{x}^{2}+\sigma_{e}^{2}\right) \sum_{i=1}^{n}\left(X_{i}-\mu\right)^{2}\right.\right.\right. \\
\left.-2 \beta \sigma_{x}^{2} \sum\left(Y_{i}-\alpha-\beta \mu\right)\left(X_{i}-\mu\right)+\left(\sigma_{x}^{2}+\sigma_{u}^{2}\right) \sum_{i=1}^{n}\left(Y_{i}-\alpha-\beta \mu\right)^{2}\right) /|\Sigma| \\
\left.\left.+\frac{(\alpha-a)^{2}}{\sigma_{\alpha}^{2}}+\frac{(\beta-b)^{2}}{\sigma_{\beta}^{2}}+\frac{(\mu-m)^{2}}{\sigma_{\mu}^{2}}+\frac{2 d}{\sigma_{x}^{2}}+\frac{2 g}{\sigma_{u}^{2}}+\frac{2 q}{\sigma_{e}^{2}}\right]\right\} \\
\text { onde } \quad|\Sigma|=\beta^{2} \sigma_{u}^{2} \sigma_{x}^{2}+\sigma_{e}^{2} \sigma_{x}^{2}+\sigma_{e}^{2} \sigma_{u}^{2}
\end{array}
\end{aligned}
$$

Posteriori baseada nos dados completos $(D)$

$$
p\left(\underset{\theta}{\theta} \mid D_{\text {obs }}\right)=\int_{\mathscr{X}} p\left(\underset{\sim}{x}, \underset{\theta}{\theta} \mid D_{\text {obs }}\right) \mathrm{d} \underset{\sim}{x}
$$

onde $p\left(\underset{\sim}{x}, \underset{\theta}{\theta} \mid D_{\text {obs }}\right) \propto L(\underset{\sim}{\theta} \mid D) \pi(\underset{\sim}{\theta})$ e $L(\underset{\sim}{\theta} \mid D)$ é dada por $(2.1 .5)$ e $\pi(\theta)=\pi(\alpha) \pi(\beta)$. .$\pi(\mu) \pi\left(\sigma_{x}^{2}\right) \pi\left(\sigma_{u}^{2}\right) \pi\left(\sigma_{e}\right)$, ou seja,

$$
\begin{aligned}
& p\left(\underset{\sim}{x}, \underset{\theta}{\theta} \mid D_{\mathrm{obs}}\right) \propto\left(\sigma_{x}^{2}\right)^{-\left(\frac{n}{2}+c+1\right)}\left(\sigma_{u}^{2}\right)^{-\left(\frac{n}{2}+f+1\right)}\left(\sigma_{e}^{2}\right)^{-\left(\frac{n}{2}+p+1\right)} \exp \left\{-\frac{1}{2}\left[\frac{\sum_{i=1}^{n}\left(Y_{i}-\alpha-\beta x_{i}\right)^{2}}{\sigma_{e}^{2}}\right.\right. \\
& \quad+\frac{\sum_{i=1}^{n}\left(X_{i}-x_{i}\right)^{2}}{\sigma_{u}^{2}}+\frac{\sum_{i=1}^{n}\left(x_{i}-\mu\right)^{2}}{\sigma_{x}^{2}}+\frac{(\alpha-a)^{2}}{\sigma_{\alpha}^{2}}+\frac{(\beta-b)^{2}}{\sigma_{\beta}^{2}}+\frac{(\mu-m)^{2}}{\sigma_{\mu}^{2}}+\frac{2 d}{\sigma_{x}^{2}} \\
& \left.\left.\quad+\frac{2 g}{\sigma_{u}^{2}}+\frac{2 q}{\sigma_{e}^{2}}\right]\right\} .
\end{aligned}
$$

\section{Distribuições condicionais completas "baseadas nos dados observados"}

Usando a posteriori em (2.6.2) obtemos as condicionais completas univariadas de $\alpha$ e de $\mu$ que são Normais, a distribuição condicional completa conjunta de $\alpha$ e $\mu$ (que também é Normal) e as distribuições condicionais completas univariadas de 
$\beta, \sigma_{x}^{2}, \sigma_{u}^{2}, \sigma_{e}^{2}$ que são conhecidas apenas por seus núcleos. Essas distribuições são apresentadas a seguir.

- $\alpha \mid \beta, \mu, \sigma_{x}^{2}, \sigma_{u}^{2}, \sigma_{e}^{2}, D_{\mathrm{obs}}$

$\sim N\left(\frac{n \sigma_{\alpha}^{2}\left[\sigma_{x}^{2}(\bar{Y}-\beta \bar{X})+\sigma_{u}^{2}(\bar{Y}-\beta \mu)\right]+a|\Sigma|}{n \sigma_{\alpha}^{2}\left(\sigma_{x}^{2}+\sigma_{u}^{2}\right)+|\Sigma|} ; \frac{\sigma_{\alpha}^{2}|\Sigma|}{n \sigma_{\alpha}^{2}\left(\sigma_{x}^{2}+\sigma_{u}^{2}\right)+|\Sigma|}\right)$

onde $|\Sigma|$ é dada em (2.6.3),

- $\mu \mid \alpha, \beta, \sigma_{x}^{2}, \sigma_{u}^{2}, \sigma_{e}^{2}, D_{\mathrm{obs}}$

$\sim N\left(\frac{n \sigma_{\mu}^{2}\left[\lambda \sigma^{2} \bar{X}+\beta \sigma_{u}^{2}(\bar{Y}-\alpha)\right]+m|\Sigma|}{n \sigma_{\mu}^{2}\left(\sigma_{e}^{2}+\beta^{2} \sigma_{u}^{2}\right)+|\Sigma|} ; \frac{\sigma_{\mu}^{2}|\Sigma|}{n \sigma_{\mu}^{2}\left(\sigma_{e}^{2}+\beta^{2} \sigma_{u}^{2}\right)+|\Sigma|}\right)$,

onde $|\Sigma|$ é dada em (2.6.3),

- $\pi\left(\alpha, \mu \mid \beta, \sigma_{x}^{2}, \sigma_{e}^{2}, \sigma_{u}^{2}, D_{\text {obs }}\right)=$

$\pi\left(\alpha \mid \beta, \mu, \sigma_{x}^{2}, \sigma_{e}^{2}, \sigma_{u}^{2}, D_{\mathrm{obs}}\right) \pi\left(\mu \mid \beta, \sigma_{x}^{2}, \sigma_{e}^{2}, \sigma_{u}^{2}, D_{\mathrm{obs}}\right)$,

onde $\alpha \mid \beta, \mu, \sigma_{x}^{2}, \sigma_{e}^{2}, \sigma_{u}^{2} D_{\text {obs }}$ tem distr. dada por (2.6.5) e

$\left.\mu \mid \beta, \sigma_{x}^{2}, \sigma_{u}^{2}, \sigma_{e}^{2}, D_{\text {obs }}\right) \sim N\left(\frac{B}{A} ; \frac{1}{A}\right)$, onde

$A=\frac{n\left(\sigma_{e}^{2}+\beta^{2} \sigma_{u}^{2}\right)}{|\Sigma|}+\frac{1}{\sigma_{\mu}^{2}}-\frac{n^{2} \sigma_{\alpha}^{2} \beta^{2}\left(\sigma_{u}^{2}\right)^{2}}{|\Sigma|\left[n \sigma_{\alpha}^{2}\left(\sigma_{x}^{2}+\sigma_{u}^{2}\right)+|\Sigma|\right]}$,

$B=\frac{n\left(\bar{X} \sigma_{e}^{2}+\bar{Y} \beta \sigma_{u}^{2}\right)}{|\Sigma|}+\frac{m}{\sigma_{\mu}^{2}}-\frac{n \beta \sigma_{u}^{2}\left[n \sigma_{\alpha}^{2}\left(\bar{Y}\left(\sigma_{x}^{2}+\sigma_{u}^{2}\right)-\beta \sigma_{x}^{2} \bar{X}\right)+a|\Sigma|\right]}{|\Sigma|\left[n \sigma_{\alpha}^{2}\left(\sigma_{x}^{2}+\sigma_{u}^{2}\right)+|\Sigma|\right]}$

e $|\Sigma|$ é dada por (2.6.3).

- $\pi\left(\beta \mid \alpha, \mu, \sigma_{x}^{2}, \sigma_{u}^{2}, \sigma_{e}^{2}, D_{\text {obs }}\right) \propto|\Sigma|^{-\frac{\pi}{2}} \exp \left\{-\frac{1}{2 \sigma_{\beta}^{2}|\Sigma|}\left[\beta^{2} C-2 \beta D\right]\right\}$,

onde

$C=\sigma_{\beta}^{2}\left[\sigma_{x}^{2} \sum_{i=1}^{n}\left(X_{i}-\mu\right)^{2}+2 n \mu \sigma_{x}^{2}(\bar{X}-\mu)+n \mu^{2}\left(\sigma_{x}^{2}+\sigma_{u}^{2}\right)\right]+|\Sigma|$,

$D=\sigma_{\beta}^{2}\left[n \mu \sigma^{2}(\bar{Y}-\alpha)+\sigma_{x}^{2}\left(\sum_{i=1}^{n} X_{i} Y_{i}-n \alpha \bar{X}\right)\right]+b|\Sigma|$

$|\Sigma|$ é dada por (2.6.3),

- $\pi\left(\sigma_{x}^{2} \mid \alpha, \mu, \sigma_{u}^{2}, \sigma_{x}^{2}, D_{\mathrm{obs}}\right)$

$\propto|\Sigma|^{-\frac{n}{2}}\left(\sigma_{x}^{2}\right)^{-(c+1)} \exp \left\{-\frac{1}{2}\left[\frac{\sigma_{x}^{2} \sum_{i=1}^{n}\left(Y_{i}-\alpha-\beta X_{i}\right)^{2}}{|\Sigma|}+\frac{2 d}{\sigma_{x}^{2}}\right]\right\}$, 
onde $|\Sigma|$ é dada em (2.6.3),

- $\pi\left(\sigma_{u}^{2} \mid \alpha, \mu, \beta, \sigma_{x}^{2}, \sigma_{e}^{2}, D_{\text {obs }}\right)$

$\propto|\Sigma|^{-\frac{n}{2}}\left(\sigma_{u}^{2}\right)^{-(f+1)} \exp \left\{-\frac{1}{2}\left[\frac{\sigma_{u}^{2} \sum_{i=1}^{n}\left(Y_{i}-\alpha-\beta \mu\right)^{2}}{|\Sigma|}+\frac{2 g}{\sigma_{u}^{2}}\right]\right\}$

onde $|\Sigma|$ é dada por (2.6.3),

- $\pi\left(\sigma_{e}^{2} \mid \alpha, \beta, \mu, \sigma_{x}^{2}, \sigma_{u}^{2}, D_{\mathrm{obs}}\right)$

$\propto|\Sigma|^{-\frac{n}{2}}\left(\sigma_{e}^{2}\right)^{-(p+1)} \exp \left\{-\frac{1}{2}\left[\frac{\sigma_{e}^{2} \sum_{i=1}^{n}\left(X_{i}-\mu\right)^{2}}{|\Sigma|}+\frac{2 q}{\sigma_{e}^{2}}\right]\right\}$

onde $|\Sigma|$ é dada por (2.6.3).

Distribuições condicionais completas à posteriori "baseadas nos dados completos"

Usando a posteriori em (2.6.4), obtemos as seguintes distribuições condicionais completas:

- $\pi\left(\alpha \mid \beta, \mu, \sigma_{x}^{2}, \sigma_{u}^{2}, \sigma_{e}^{2}, \underset{\sim}{x}, D_{\mathrm{obs}}\right) \sim N\left(\frac{n \sigma_{\alpha}^{2}(\bar{Y}-\beta \bar{x})+a \sigma_{e}^{2}}{n \sigma_{\alpha}^{2}+\sigma_{e}^{2}} ; \frac{\sigma_{e}^{2} \sigma_{\alpha}^{2}}{n \sigma_{\alpha}^{2}+\sigma_{e}^{2}}\right)$,

- $\pi\left(\beta \mid \alpha, \mu, \sigma_{x}^{2}, \sigma_{u}^{2}, \sigma_{e}^{2}, D_{\mathrm{obs}}\right) \sim N\left(\frac{\sigma_{\beta}^{2}\left(\sum_{i=1}^{n} x_{i} Y_{i}-n \alpha \bar{x}\right)+b \sigma_{e}^{2}}{\sigma_{\beta}^{2} \sum_{i=1}^{n} x_{i}^{2}+\sigma_{e}^{2}} ; \frac{\sigma_{e}^{2} \sigma_{\beta}^{2}}{\sigma_{\beta}^{2} \sum_{i=1}^{n} x_{i}^{2}+\sigma_{e}^{2}}\right)$

- $\pi\left(\mu \mid \alpha, \beta, \sigma_{x}^{2}, \sigma_{u}^{2}, \sigma_{e}^{2}, \underset{\sim}{x}, D_{\text {obs }}\right)$ é Normal dada em (2.5.11),

- $\pi\left(\sigma_{x}^{2} \mid \alpha, \beta, \mu, \sigma_{u}^{2}, \sigma_{e}^{2}, \underset{\sim}{x}, D_{\text {obs }}\right)$ é Gama Invertida dada em (2.5.12),

- $\pi\left(\sigma_{u}^{2} \mid \alpha, \beta, \mu, \sigma_{x}^{2}, \sigma_{e}^{2}, \underset{\sim}{x}, D_{\mathrm{obs}}\right) \sim I G\left(\frac{n}{2}+f ; \frac{1}{2} \sum_{i=1}^{n}\left(X_{i}-x_{i}\right)^{2}+g\right)$,

- $\pi\left(\sigma_{e}^{2} \mid \alpha, \beta, \mu, \sigma_{x}^{2}, \sigma_{u}^{2}, \underset{\sim}{x}, D_{\text {obs }}\right) \sim I G\left(\frac{n}{2}+p ; \frac{1}{2} \sum_{i=1}^{n}\left(Y_{i}-\alpha-\beta x_{i}\right)^{2}+q\right)$,

- $\pi\left(x_{i} \mid \alpha, \beta, \mu, \sigma_{x}^{2}, \sigma_{u}^{2}, \sigma_{e}^{2}, x_{(-i)}, D_{\text {obs }}\right)=\pi\left(x_{i} \mid \alpha, \beta, \mu, \sigma_{x}^{2}, \sigma_{u}^{2}, \sigma_{e}^{2}, D_{\text {obs }}\right)$

$\sim N\left(\frac{\sigma_{x}^{2}\left[\beta \sigma_{u}^{2}\left(Y_{i}-\alpha\right)+X_{i} \sigma_{e}^{2}\right]+\sigma_{e}^{2} \sigma_{u}^{2} \mu}{\sigma_{u}^{2} \sigma_{x}^{2} \beta^{2}+\sigma_{e}^{2} \sigma_{x}^{2}+\sigma_{e}^{2} \sigma_{u}^{2}} ; \frac{\sigma_{e}^{2} \sigma_{u}^{2} \sigma_{x}^{2}}{\sigma_{u}^{2} \sigma_{x}^{2} \beta^{2}+\sigma_{e}^{2} \sigma_{x}^{2}+\sigma_{e}^{2} \sigma_{u}^{2}}\right)$,

para $i=1, \ldots, n$. 


\section{Implementação de Algoritmos}

Podemos utilizar as distribuições condicionais completas acima para implementar o algoritmo original de Gibbs, o Gibbs em blocos, o Metropolis-Hastings em Gibbs e o "Collapsed Gibbs" de forma totalmente semelhante à Seção 2.5 .

\subsection{O modelo estrutural com particular priori própria de componentes condicionalmente independentes}

Nesta seção analisamos tanto o modelo Normal com a restrição de identificabilidade $\sigma_{e}^{2}=\lambda \sigma_{u}^{2}$, onde $\lambda$ é conhecido, como também o modelo Normal sem restrições de identificabilidade, usando em cada caso uma particular priori própria com componentes de locação condicionalmente independentes.

\subsubsection{O modelo com restrição de identificabilidade}

No modelo estrutural dado pelas equações

$$
\begin{gathered}
Y_{i}=\alpha+\beta x_{i}+e_{i} \\
X_{i}=x_{i}+u_{i}, \quad \text { onde } \\
\left(\begin{array}{c}
e_{i} \\
u_{i} \\
x_{i}
\end{array}\right) \sim N\left(\left(\begin{array}{c}
0 \\
0 \\
\mu
\end{array}\right) ;\left(\begin{array}{ccc}
\lambda \sigma^{2} & 0 & 0 \\
0 & \sigma^{2} & 0 \\
0 & 0 & \sigma_{x}^{2}
\end{array}\right)\right), i=1, \ldots, n,
\end{gathered}
$$

onde $\underset{\sim}{\theta}=\left(\alpha, \beta, \mu, \sigma_{x}^{2}, \sigma^{2}\right)^{T}$ e $\sigma_{e}^{2}=\lambda \sigma_{u}^{2}=\lambda \sigma^{2}$ com $\lambda$ conhecido e acrescentamos a suposição à priori que $\pi\left(\underset{\underset{\alpha}{\theta})}{\operatorname{si}}=\pi\left(\alpha \mid \sigma^{2}\right) \pi\left(\beta \mid \sigma^{2}\right) \pi\left(\mu \mid \sigma_{x}^{2}\right) \pi\left(\sigma_{x}^{2}\right) \pi\left(\sigma^{2}\right)\right.$,

onde $\alpha\left|\sigma^{2} \sim N\left(a, \chi_{01}^{2} \sigma^{2}\right), \beta\right| \sigma^{2} \sim N\left(b, \chi_{02}^{2} \sigma^{2}\right), \mu \mid \sigma_{x}^{2} \sim N\left(m, \tau_{0}^{2} \sigma_{x}^{2}\right)$ e $\sigma_{x}^{2} \sim I G\left(\nu_{01}, \lambda_{01}\right)$, $\sigma^{2} \sim I G\left(\nu_{02}, \lambda_{02}\right)$, onde $a, b, m, \chi_{01}^{2}, \chi_{02}^{2}, \tau_{0}^{2}, \nu_{01}, \nu_{02}, \lambda_{01}, \lambda_{02}$ são os hiperparâmetros conhecidos.

A escolha dessa priori baseou-se primeiramente na analogia com o modelo de regressão clássico (sem erros de medida em $X$ ) com priori conjugada, além do fato 
de que em muitas situações na prática é bastante razoável assumir que parâmetros de locação dependem de parâmetros de escala.

Distribuição à posteriori, baseada nos dados observados

$$
p\left(\underset{\sim}{\theta} \mid D_{\mathrm{obs}}\right) \propto L\left(\underset{\sim}{(\theta} \mid D_{\mathrm{obs}}\right) \pi(\underset{\sim}{\theta})
$$

onde $L\left(\underset{\sim}{\theta} \mid D_{\text {obs }}\right)$ é obtida de (2.1.4) quando fazemos $\sigma_{e}^{2}=\lambda \sigma_{u}^{2}$ e $\sigma_{u}^{2}=\sigma^{2}$.

Posteriori baseada nos dados completos $(D)$

$$
p\left(\underset{\sim}{\theta} \mid D_{\mathrm{obs}}\right)=\int_{\mathscr{X}} p\left(\underset{\sim}{x}, \underset{\sim}{\theta} \mid D_{\mathrm{obs}}\right) \mathrm{d} \underset{\sim}{x}
$$

onde $p\left(\underset{\sim}{x}, \underset{\sim}{\theta} \mid D_{\text {obs }}\right) \propto L(\underset{\sim}{\theta} \mid D) \pi(\underset{\sim}{\theta})$ e $L(\underset{\sim}{\theta} \mid D)$ é obtida de (2.1.5) quando fazemos $\sigma_{e}^{2}=\lambda \sigma_{u}^{2}$ e $\sigma_{u}^{2}=\sigma^{2}$

Distribuições condicionais completas à posteriori "baseadas nos dados observados"

Usando a posteriori em (2.7.2) obtemos as seguintes distribuições:

- $\alpha \mid \beta, \mu, \sigma_{x}^{2}, \sigma^{2}, D_{\text {obs }} \sim$ Normal dada em (2.5.1), substituindo $\sigma_{\alpha}^{2}$ por $\chi_{01}^{2} \sigma^{2}$;

- $\mu \mid \alpha, \beta, \sigma_{x}^{2}, \sigma^{2}, D_{\text {obs }} \sim$ Normal dada em (2.5.3) substituindo $\sigma_{\mu}^{2}$ por $\tau_{0}^{2} \sigma_{x}^{2}$

- $\pi\left(\alpha, \mu \mid \beta, \sigma_{x}^{2}, \sigma^{2}, D_{\text {obs }}\right)=\pi\left(\alpha \mid \beta, \mu, \sigma_{x}^{2}, \sigma^{2}, D_{\text {obs }}\right) \pi\left(\beta \mid \mu, \sigma_{x}^{2}, \sigma^{2}, D_{\text {obs }}\right)$, onde $\mu \mid \beta, \sigma_{x}^{2}, \sigma^{2}, D_{\text {obs }}$ tem distribuição dada em (2.5.5) substituindo $\sigma_{\mu}^{2}$ por $\tau_{0}^{2} \sigma_{x}^{2}$ e $\sigma_{\alpha}^{2}$ por $\chi_{01}^{2} \sigma^{2}$;

- $\beta \mid \alpha, \mu, \sigma_{x}^{2} \sigma^{2}, D_{\text {obs }}$ tem distribuição dada em (2.5.6) substituindo $\sigma_{\beta}^{2}$ por $\chi_{02}^{2} \sigma^{2}$,

- $\sigma_{x}^{2} \mid \alpha, \beta, \mu, \sigma^{2}, D_{\text {obs }}$ tem distribuição dada em (2.5.7) substituindo $c$ por $\nu_{01}$ e $d$ por $\lambda_{01}$; 
- $\sigma^{2} \mid \alpha, \beta, \mu, \sigma_{x}^{2}, D_{\text {obs }}$ tem distribuição dada em (2.5.8) substituindo $f$ por $\nu_{02}$ e $g$ por $\lambda_{02}$.

\section{Distribuiçōes condicionais completas à posteriori "baseadas nos dados} completos"

Usando a posteriori em (2.7.3) obtemos as seguintes distribuições condicionais:

- $\alpha \mid \beta, \mu, \sigma_{x}^{2}, \sigma^{2}, \underset{\sim}{x}, D_{\text {obs }} \sim$ Normal dada em (2.5.9) substituindo $\sigma_{\alpha}$ por $\chi_{01}^{2} \sigma^{2}$, ou seja,

$$
\alpha \mid \beta, \mu, \sigma_{x}^{2}, \sigma^{2}, \underset{\sim}{x}, D_{\mathrm{obs}} \sim N\left(\frac{n \chi_{01}^{2}(\bar{Y}-\beta \bar{x})+\lambda a}{n \chi_{01}^{2}+\lambda} ; \frac{\lambda \chi_{01}^{2} \sigma^{2}}{n \chi_{01}^{2}+\lambda}\right)
$$

- $\beta \mid \alpha, \mu, \sigma_{x}^{2}, \sigma^{2}, \underset{\sim}{x}, D_{\text {obs }} \sim$ Normal dada em (2.5.10) substituindo $\sigma_{\beta}^{2}$ por $\chi_{02}^{2} \sigma^{2}$, ou seja,

$$
\beta \mid \alpha, \mu, \sigma_{x}^{2}, \sigma^{2}, \underset{\sim}{x}, D_{\mathrm{obs}} \sim N\left(\frac{\chi_{02}^{2}\left[\sum_{i=1}^{n} x_{i} Y_{i}-n \alpha \bar{x}\right]+\lambda b}{\chi_{02}^{2} \sum_{i=1}^{n} x_{i}^{2}+\lambda} ; \frac{\lambda \chi_{02}^{2} \sigma^{2}}{\chi_{02}^{2} \sum_{i=1}^{n} x_{i}^{2}+\lambda}\right)
$$

- $\mu \mid \alpha, \beta, \sigma_{x}^{2}, \sigma^{2}, \underset{\sim}{x}, D_{\text {obs }} \sim$ Normal dada em (2.5.11) substituindo $\sigma_{\mu}^{2}$ por $\tau_{0}^{2} \sigma_{x}^{2}$, ou seja,

$$
\mu \mid \alpha, \beta, \sigma_{x}^{2}, \sigma^{2}, D_{\mathrm{obs}} \sim N\left(\frac{n \bar{x} \tau_{0}^{2}+m}{n \tau_{0}^{2}+1} ; \frac{\tau_{0}^{2} \sigma_{x}^{2}}{n \tau_{0}^{2}+1}\right)
$$

- $\left.\sigma_{x}^{2} \mid \alpha, \beta, \mu, \sigma^{2}, \underset{\sim}{x}, D_{\mathrm{obs}}\right) \sim$ Gama Invertida dada em (2.5.12) substituindo $c$ por $\nu_{01}$ e $d$ por $\lambda_{01}$

- $\sigma^{2} \mid \alpha, \beta, \mu, \sigma_{x}^{2}, \underset{\sim}{x}, D_{\mathrm{obs}} \sim$ Gama Invertida dada em (2.5.13) substituindo $f$ por $\nu_{02}$ e $q$ por $\lambda_{02}$

- $x_{i} \mid \alpha, \beta, \mu, \sigma_{x}^{2}, \sigma^{2}, x_{(-i)}, D_{\text {obs }} \sim$ Normal dada em (2.5.14).

\section{Implementação de algoritmos}


Utilizando as distribuições condicionais completas à posteriori obtidas acima, podemos implementar os algoritmos de Gibbs, "grouped Gibbs", "collapsed Gibbs" e Metropolis em Gibbs (para amostrar da posteriori), de forma totalmente semelhante à seção 2.5 .

\subsubsection{O modelo sem restrições de identificabilidade}

No modelo estrutural

$$
\begin{aligned}
& Y_{i}=\alpha+\beta x_{i}+e_{i}, \\
& X_{i}=x_{i}+u_{i},
\end{aligned}
$$

onde

$$
\left(\begin{array}{c}
e_{i} \\
u_{i} \\
x_{i}
\end{array}\right) \sim N\left(\left(\begin{array}{c}
0 \\
0 \\
\mu
\end{array}\right) ;\left(\begin{array}{ccc}
\sigma_{e}^{2} & 0 & 0 \\
0 & \sigma_{u}^{2} & 0 \\
0 & 0 & \sigma_{x}^{2}
\end{array}\right)\right), i=1, \ldots, n,
$$

onde $\underset{\sim}{\theta}=\left(\alpha, \beta, \mu, \sigma_{x}^{2}, \sigma_{u}^{2}, \sigma_{e}^{2}\right)^{T}$, acrescentamos a suposição à priori sobre $\underset{\sim}{\theta}$ que

$$
\pi(\underset{\sim}{\theta})=\pi\left(\alpha \mid \sigma_{e}^{2}\right) \pi\left(\beta \mid \sigma_{e}^{2}\right) \pi\left(\mu \mid \sigma_{x}^{2}\right) \pi\left(\sigma_{x}^{2}\right) \pi\left(\sigma_{u}^{2}\right) \pi\left(\sigma_{e}^{2}\right)
$$

onde $\alpha\left|\sigma_{e}^{2} \sim N\left(a, \chi_{01}^{2} \sigma_{e}^{2}\right), \beta\right| \sigma_{e}^{2} \sim N\left(b, \chi_{02}^{2} \sigma_{e}^{2}\right), \mu \mid \sigma_{x}^{2} \sim N\left(m, \tau_{0}^{2} \sigma_{x}^{2}\right), \sigma_{x}^{2} \sim I G\left(\nu_{01}, \lambda_{01}\right)$, $\sigma_{u}^{2} \sim I G\left(\nu_{02}, \lambda_{02}\right), \sigma_{e}^{2} \sim I G\left(\nu_{03}, \lambda_{03}\right)$.

\section{Distribuição à posteriori}

A posteriori baseada nos dados observados, então é dada por

$$
p\left(\underset{\sim}{\theta} \mid D_{\text {obs }}\right) \propto L\left(\underset{\sim}{\theta} \mid D_{\text {obs }}\right) \pi(\underset{\sim}{\theta}),
$$

onde $L\left(\underset{\sim}{\theta} \mid D_{\text {obs }}\right)$ é dado por (2.1.4) e $\pi(\underset{\theta}{\theta})$ é dada acima.

A posteriori "baseada nos dados completos" $(D)$ é dada por

$$
p\left(\underset{\sim}{\theta} \mid D_{\mathrm{obs}}\right)=\int_{\mathfrak{X}} p\left(\underset{\sim}{x}, \underset{\sim}{\theta} \mid D_{\mathrm{obs}}\right) \mathrm{d} \underset{\sim}{x}
$$

onde $L(\underset{\sim}{\theta} \mid D)$ é dada em (2.1.5) e $\pi(\theta)$ é dada acima. 
Distribuições condicionais completas à posteriori baseadas nos dados observados

Usando a posteriori em (2.7.4), obtemos as seguintes distribuições condicionais:

- $\alpha \mid \beta, \mu, \sigma_{x}^{2}, \sigma_{u}^{2}, \sigma_{e}^{2}, D_{\mathrm{obs}} \sim$ Normal dada por (2.6.5), substituindo $\sigma_{\alpha}^{2}$ por $\chi_{01}^{2} \sigma_{e}^{2}$;

- $\mu \mid \alpha, \beta, \sigma_{x}^{2}, \sigma_{u}^{2}, \sigma_{e}^{2}, D_{\mathrm{obs}} \sim$ Normal dada por (2.6.6), substituindo $\sigma_{\mu}^{2}$ por $\tau_{0}^{2} \sigma_{x}^{2}$

- $\pi\left(\alpha, \mu \mid \beta, \sigma_{x}^{2}, \sigma^{2}, D_{\text {obs }}\right)=\pi\left(\alpha \mid \beta, \mu, \sigma_{x}^{2}, \sigma^{2}, D_{\text {obs }}\right) \pi\left(\beta \mid \mu, \sigma_{x}^{2}, \sigma^{2}, D_{\text {obs }}\right)$, onde $\mu \mid \beta, \sigma_{x}^{2}, \sigma_{u}^{2}, \sigma_{e}^{2}, D_{\text {obs }} \sim$ Normal dada por (2.6.7), substituindo $\sigma_{\mu}^{2}$ por $\tau_{0}^{2} \sigma_{x}^{2}$ e $\sigma_{\alpha}$ por $\chi_{01}^{2} \sigma_{x}^{2}$

- $\beta \mid \alpha, \mu, \sigma_{x}^{2}, \sigma_{u}^{2}, \sigma_{e}^{2}, D_{\text {obs }}$ tem distribuição dada por (2.6.8), substituindo $\sigma_{\beta}^{2}$ por $\chi_{02}^{2} \sigma_{e}^{2}$

- $\sigma_{x}^{2} \mid \alpha, \beta, \mu, \sigma_{u}^{2}, \sigma_{e}^{2}, D_{\text {obs }}$ tem distribuição dada por (2.6.9), substituindo $c$ por $\nu_{01}$ e $d$ por $\lambda_{01}$;

- $\sigma_{u}^{2} \mid \alpha, \beta, \mu, \sigma_{x}^{2}, \sigma_{e}^{2}, D_{\text {obs }}$ tem distribuição dada por (2.6.10), substituindo $f$ por $\nu_{02}$ e $g$ por $\lambda_{02}$

- $\sigma_{e}^{2} \mid \alpha, \beta, \mu, \sigma_{x}^{2}, \sigma_{u}^{2}, D_{\text {obs }}$ tem distribuição dada por (2.6.11), substituindo $p$ por $\nu_{03}$ e $q$ por $\lambda_{03}$.

Distribuições condicionais completas à posteriori "baseada nos dados completos", $(D)$

Usando a posteriori em (2.7.5) obtemos as seguintes distribuições condicionais completas: 
- $\alpha \mid \beta, \mu, \sigma_{x}^{2}, \sigma_{u}^{2}, \sigma_{e}^{2}, \underset{\sim}{x}, D_{\mathrm{obs}} \sim$ Normal dada em (2.6.12), substituindo $\sigma_{\alpha}^{2}$ por $\chi_{01}^{2} \sigma_{e}^{2}$, ou seja,

$$
N\left(\frac{n \chi_{01}^{2}(\bar{Y}-\beta \bar{x})+a}{n \chi_{01}^{2}+1} ; \frac{\chi_{01}^{2} \sigma_{e}^{2}}{n \chi_{01}^{2}+1}\right)
$$

- $\beta \mid \alpha, \mu, \sigma_{x}^{2}, \sigma_{u}^{2}, \sigma_{e}^{2}, \underset{\sim}{x}, D_{\text {obs }} \sim$ Normal dada em (2.6.13), substituindo $\sigma_{\beta}^{2}$ por $\chi_{02}^{2} \sigma_{e}^{2}$, isto é,

$$
N\left(\frac{\chi_{02}^{2}\left(\sum_{i=1}^{n} x_{i} Y_{i}-n \alpha \bar{x}\right)+b}{\chi_{02}^{2} \sum_{i=1}^{n} x_{i}^{2}+1} ; \frac{\chi_{02}^{2} \sigma_{e}^{2}}{\chi_{02}^{2} \sum_{i=1}^{n} x_{i}^{2}+1}\right) ;
$$

- $\mu \mid \alpha, \beta, \sigma_{x}^{2}, \sigma_{e}^{2}, \underset{\sim}{x}, D_{\mathrm{obs}} \sim$ Normal dada em (2.6.14), substituindo $\sigma_{\mu}^{2}$ por $\tau_{0}^{2} \sigma_{x}^{2}$, isto é,

$$
N\left(\frac{n \bar{x} \tau_{0}^{2}+m}{n \tau_{0}^{2}+1} ; \frac{\tau_{0}^{2} \sigma_{x}^{2}}{n \tau_{0}^{2}+1}\right)
$$

- $\sigma_{x}^{2} \mid \alpha, \beta, \mu, \sigma_{u}^{2}, \sigma_{x}^{2}, \underset{\sim}{x}, D_{\mathrm{obs}} \sim$ Gama Invertida dada em (2.6.15), substituindo $c$ por $\nu_{01}$ e $d$ por $\lambda_{01}$, isto é,

$$
I G\left(\frac{n}{2}+\nu_{01} ; \lambda_{01}+\frac{1}{2} \sum_{i=1}^{n}(x-\mu)^{2}\right) ;
$$

- $\sigma_{u}^{2} \mid \alpha, \beta, \mu, \sigma_{x}^{2}, \sigma_{e}^{2}, \underset{\sim}{x}, D_{\text {obs }} \sim$ Gama Invertida dada em (2.6.16), substituindo $f$ por $\nu_{02}$ e $g$ por $\lambda_{02}$, isto é,

$$
I G\left(\frac{n}{2}+\nu_{02} ; \frac{1}{2} \sum_{i=1}^{n}\left(X_{i}-x_{i}\right)^{2}+\lambda_{02}\right)
$$

- $\sigma_{e}^{2} \mid \alpha, \beta, \mu, \sigma_{x}^{2}, \sigma_{u}^{2}, \underset{\sim}{x}, D_{\text {obs }} \sim$ Gama Invertida dada em (2.6.17), substituindo $p$ por $\nu_{03}$ e $q$ por $\lambda_{03}$, isto é,

$$
I G\left(\frac{n}{2}+\nu_{03} ; \frac{1}{2} \sum_{i=1}^{n}\left(Y_{i}-\alpha-\beta x_{i}\right)^{2}+\lambda_{03}\right)
$$

- $x_{i} \mid \alpha, \beta, \mu, \sigma_{x}^{2}, \sigma_{u}^{2}, \sigma_{e}^{2}, x_{(-i)}, D_{\mathrm{obs}} \sim$ Normal dada por (2.6.18), para $i=1, \ldots, n$. 


\section{Implementação de algoritmos}

Para amostrar da posteriori, podemos utilizar as distribuições condicionais completas acima para implementar os algoritmos de Gibbs, "grouped Gibbs", "collapsed Gibbs" e Metropolis-Hastings de forma semelhante à Seção 2.5 .

\subsection{Estabelecimento das condições para a existência da distribuição à posteriori sob diferentes es- colhas de prioris impróprias}

Nesta seção investigamos se a distribuição à posteriori sob o modelo estrutural normal é própria quando escolhemos um conjunto específico de prioris impróprias, tanto no caso com restrição como nocaso sem restrição de identificabilidade. O objetivo é estabelecer restrições sobre o tamanho da amostra e sobre os hiperparâmetros dessas prioris para que as correspondentes distribuições à posteriori sejam próprias.

Obviamente, é importante verificar se a posteriori é própria para que se possa fazer inferência. Sabemos que o uso de priori imprópria tem sido alvo de críticas por violar o "Princípio da Coerência" na estatística bayesiana, mas sabemos também que nem sempre uma priori própria é garantia de bons resultados. Por exemplo, é muito comum (quando não temos "muita informação" à priori) escolhermos uma distribuição à priori própria com variância grande (ou seja, uma "priori própria vaga"), como por exemplo a distribuição $\pi\left(\sigma^{2}\right) \propto\left(\sigma^{2}\right)^{-(\alpha+1)} \exp \left\{\frac{-\beta}{\sigma^{2}}\right\}$, com $|\beta|$ bem pequeno, em vez da distribuição imprópria $\pi\left(\sigma^{2}\right) \propto\left(\sigma^{2}\right)^{-(\alpha+1)}$ (que é o limite da distribuição acima quando $\beta \rightarrow 0$ ). Sobre esse tipo de problema, Berger (2000) faz a seguinte afirmação: "... A noção comum de que é mais seguro ou mais garantido (em termos de desempenho) usar uma priori "vaga" do que uma priori imprópria (pelo fato de que teoricamente a posteriori é própria no primeiro caso) é simplesmente 
errada". O autor comenta esse problema apresentando um exemplo em que o uso de uma priori vaga pode levar a resultados absurdos.

\subsubsection{Condições necessárias e suficientes no caso do modelo estrutural identificável}

\section{O modelo}

$$
\begin{aligned}
& Y_{i}=\alpha+\beta x_{i}+e_{i}, \\
& X_{i}=x_{i}+u_{i}
\end{aligned}
$$

onde $\left\{\left(X_{i}, Y_{i}\right)^{T}, i=1,2, \ldots, n\right\}$ é o conjunto dos dados observados $\left(D_{\mathrm{obs}}\right)$,

$$
\begin{aligned}
& \left\{x_{i}, i=1, \ldots, n\right\}: \text { dados latentes, } \\
& \left\{\left(e_{i}, u_{i}\right)^{T}, i=1, \ldots, n\right\}: \text { erros aleatórios. }
\end{aligned}
$$

Com a suposição

$$
\left(\begin{array}{c}
e_{i} \\
u_{i} \\
x_{i}
\end{array}\right) \stackrel{\text { i.i.j. }}{\sim}\left(\left(\begin{array}{c}
0 \\
0 \\
\mu
\end{array}\right) ;\left(\begin{array}{ccc}
\sigma_{e}^{2} & 0 & 0 \\
0 & \sigma_{u}^{2} & 0 \\
0 & 0 & \sigma_{x}^{2}
\end{array}\right)\right), i=1, \ldots, n,
$$

e com a restrição de identificabilidade $\sigma_{e}^{2}=\lambda \sigma_{u}^{2}$ e $\sigma_{u}^{2}=\sigma^{2}$ com $\lambda$ conhecido.

$$
\Theta=\left\{\underset{\theta}{\theta}=\left(\alpha, \beta, \mu, \sigma_{x}^{2}, \sigma^{2}\right)^{T}: \alpha, \beta, \mu \in \mathbb{R} \text { e } \sigma_{x}^{2}, \sigma^{2} \in \mathbb{R}^{+}\right\} \text {, onde } \Theta \text { é o espaço }
$$

paramétrico.

\section{Distribuição à priori para $\theta$}

Nessa subseção apresentamos quatro modelos de distribuição à priori imprópria não informativas e parcialmente informativas. As posteriores obtidas dessas prioris é que serão avaliadas quanto à existência.

Nesses modelos as distribuições à priori $\pi(\underset{\sim}{\theta})$ são da forma

$$
\pi(\underset{\sim}{\theta})=\pi(\alpha) \pi(\beta) \pi(\mu) \pi\left(\sigma_{x}^{2}\right) \pi\left(\sigma^{2}\right)
$$

onde as "marginais" de $\alpha, \beta, \mu, \sigma_{x}^{2}$ e $\sigma^{2}$ são dadas a seguir. 
Modelo 1: $\pi(\alpha) \propto 1 ; \pi(\beta) \propto 1 ; \pi(\mu) \propto 1 ; \pi\left(\sigma_{x}^{2}\right) \propto\left(\sigma_{x}^{2}\right)^{-(c+1)} \exp \left\{-\frac{d}{\sigma_{x}^{2}}\right\}$, onde $c, d>0$ e $\pi\left(\sigma^{2}\right) \propto\left(\sigma^{2}\right)^{-(f+1)} \exp \left\{-\frac{g}{\sigma^{2}}\right\}$, onde $f, g>0$ (isto é, $\sigma_{x}^{2} \sim I G(c, d)$ e $\left.\sigma^{2} \sim I G(f, g)\right)$.

Modelo $2: \pi(\alpha) \propto 1 ; \pi(\beta) \propto 1 ; \pi(\mu) \propto 1 ; \pi\left(\sigma_{x}^{2}\right) \propto\left(\sigma_{x}^{2}\right)^{-r}, r>0$ e $\pi\left(\sigma^{2}\right) \sim I G(f, g)$ (esta priori coincide com a do modelo 1 quando $c=r-1$ e $d=0$ ).

Modelo $3: \pi(\alpha) \propto 1 ; \pi(\beta) \propto 1 ; \pi(\mu) \propto 1 ; \pi\left(\sigma_{x}^{2}\right) \sim I G(c, d) ; \pi\left(\sigma^{2}\right) \propto\left(\sigma^{2}\right)^{-s}$ (coincide com a priori do modelo 1 quando $f=s-1$ e $g=0$ ).

Modelo 4: $\pi(\alpha) \propto 1 ; \pi(\beta) \propto 1 ; \pi(\mu) \propto 1 ; \pi\left(\sigma_{x}^{2}\right) \propto\left(\sigma_{x}^{2}\right)^{-r}, r>0$ e $\pi\left(\sigma^{2}\right) \propto\left(\sigma^{2}\right)^{-s}$, $s>0$ (é idêntica à priori do modelo 1 quando $c=r-1, f=s-1, d=0 \mathrm{e}$ $g=0)$.

A escolha dos modelos acima baseou-se em alguns argumentos que daremos a seguir e principalmente na analogia com o modelo linear sem erros nas variáveis.

Um resultado bastante conhecido demonstrado por Berger (1980) é que se uma variável aleatória $z$ tem densidade da forma $f(z \mid \theta)=f(z-\theta)$ (ou seja, se $\theta$ é um parâmetro de locação), então a priori de $\theta$ deve ser uniforme no domínio de variação de $\theta$ para que ela seja invariante sob transformação de locação (isto é, sob transformação da forma $Y=z+c$, onde $c$ é uma constante); portanto, se $-\infty<\theta<\infty$, a priori "não informativa" para $\theta$ é dado por $\pi(\theta) \propto 1, \theta \in \mathbb{R}$. Segundo Carlin e Louis (2000), embora a distribuição uniforme na reta seja imprópria, inferência bayesiana ainda pode ser feita se a distribuição à posteriori correspondente for própria. Berger (1985) demonstrou também que se $\sigma^{2}$ é um parâmetro de escala então a priori de $\sigma^{2}$ deve ser da forma $\pi\left(\sigma^{2}\right) \propto \frac{1}{\sigma^{2}}$ para ser invariante sob transformação de escala (e portanto a priori "não informativa" para $\sigma^{2}>0$ é dada por $\pi\left(\sigma^{2}\right) \propto \frac{1}{\sigma^{2}}$ (que nesse caso é também imprópria)). 
Segundo Carlin e Louis (2000) e Berger (1980) quando o modelo tem simultaneamente um parâmetro de locação $\theta$ e outro de escala $\sigma^{2}$, o procedimento mais simples comumente usado para obter priori "não informativa" é considerar independência à priori entre esses parâmetros (embora a rigor o conceito de independência está reservado apenas para as distribuições próprias) e tomar a priori $\pi\left(\theta, \sigma^{2}\right)$ como o produto das distribuições de $\theta$ e de $\sigma^{2}$ separadamente. Assim, a priori não informativa nesse caso é dada por $\pi(\theta, \sigma) \propto \frac{1}{\sigma}$ (que é imprópria quando $\theta \in \mathbb{R}$ ou $\sigma^{2} \in \mathbb{R}^{+}$).

No modelo linear Normal sem erros nas covariáveis, da forma $Y_{i}=\beta_{1}+\beta_{2} x_{2 i}+$ $\cdots+\beta_{k} x_{k i}$ com $e_{i} \sim N\left(0, \sigma^{2}\right), i=1,2, \ldots, n$ independentes, temos que $\sigma^{2}$ é um parâmetro de escala e $\beta=\left(\begin{array}{c}\beta_{1} \\ \vdots \\ \beta_{k}\end{array}\right)$ é um "parâmetro de locação num sentido mais amplo" (já que a verossimilhança pode ser escrita como

$$
L\left(\underset{\sim}{\beta}, \sigma^{2} \mid \underset{\sim}{Y}\right) \propto\left(\frac{s^{2}}{\sigma^{2}}\right)^{\frac{n}{2}} \exp \left\{-\frac{s^{2}}{2 \sigma^{2}}\left[(n-k)+\frac{(\underset{\sim}{\beta}-\underset{\sim}{\beta})^{\top} \underset{\sim}{X^{\top}} \underset{\sim}{X}(\underset{\sim}{\beta}-\underset{\sim}{\beta})}{s^{2}}\right]\right\},
$$

onde $s^{2}=\frac{(Y-Y)^{\top}(Y-Y)}{n-k}$ e $\hat{\beta}=\left(\underset{\sim}{X^{\top}} \underset{\sim}{X}\right)^{-1} \underset{\sim}{X} \underset{\sim}{Y}$ (Box e Tiao, 1973) e assim podemos ver que $\underset{\sim}{\beta}$ é um parâmetro de locação da distribuição de $\hat{\beta}$ ). Nesse caso, a distribuição à priori não informativa seria dada por

$$
\pi(\underset{\sim}{\beta}, \sigma)=p(\underset{\sim}{\beta}) p\left(\sigma^{2}\right) \propto \frac{1}{\sigma^{2}}
$$

Nesse modelo, a priori (não informativa) de Jeffreys, que é a raiz quadrada do determinante da matriz de informação de Fisher, é dada por

$$
\pi\left(\underset{\sim}{\beta}, \sigma^{2}\right) \propto \frac{1}{\left(\sigma^{2}\right)^{\frac{k}{2}-1}}, \quad \sigma^{2}>0 .
$$

No caso do modelo Normal com erros nas variáveis a priori de Jeffreys não pode ser obtida pois a matriz de informação de Fisher não pode ser obtida analiticamente (lembrando que para a análise das condições de existência da posteriori é necessário ter a expressão analítica da priori) 
Segundo Carlin e Louis (2000), a obtenção da priori de Jeffreys no caso multidimensional pode ser impraticável especialmente quando a dimensão do problema é alta e um procedimento bastante usado na prática é tomar o produto das distribuições de cada componente. Segundo os autores, esse procedimento é freqüentemente justificado com base no argumento de que a idéia de ignorância é consistente com a idéia de ìndependência (embora numa distribuição imprópria a noção formal de independência não se aplica por não se tratar de uma distribuição de probabilidade).

As prioris para o modelo com erros nas variáveis, dados pelos modelos de 1 a 4 do início da seção, foram construídos levando-se em conta os argumentos acima. Para todos os quatro modelos, a priori conjunta $\pi(\underset{\sim}{\theta})$ é tomada como o produto das distribuições de cada componente; além disso, fizemos analogia com os modelos lineares sem erros nas variáveis considerando $\alpha$ e $\beta$ como "parâmetros de locação" num sentido mais amplo. Como $\mu$ é claramente um parâmetro de locação, tomamos para $\alpha, \beta$ e $\mu$ a priori não informativa uniforme na reta. Para as componentes de escala $\sigma_{x}^{2}$ e $\sigma^{2}$ atribuímos ou a distribuição Gama Invertida com os respectivos parâmetros, ou então as distribuições impróprias não informativas $\pi\left(\sigma_{x}^{2}\right) \propto \frac{1}{\left(\sigma_{x}^{2}\right)^{r}}$ para $\sigma_{x}^{2}$ e $\pi\left(\sigma^{2}\right) \propto \frac{1}{\left(\sigma^{2}\right)^{s}}$ para $\sigma^{2}$, onde os hiperparâmetros $r$ e $s$ são escolhidos de tal modo que a posteriori seja própria, com isso quisemos dar uma certa flexibilidade na escolha. Em cada um dos teoremas nas próximas subseções obtemos os intervalos dos possíveis valores desses hiperparâmetros.

Achamos que vale a pena notar ainda que as distribuições impróprias $\frac{1}{\left(\sigma_{x}^{2}\right)^{r}}$ e $\frac{1}{\left(\sigma^{2}\right)^{s}}$ podem ser pensadas como sendo o limite, respectivamente, das distribuições $I G(c, d)$ e $I G(f, g)$ quando $d$ e $g \rightarrow 0$ e $r=c+1$ e $s=f+1$.

Vale a pena também notar que o "grau de informação" das prioris dos modelos propostos no início, vai diminuindo quando passamos do modelo 1 para os modelos 
2, 3 e 4. No modelo 1 , as componentes $\sigma_{x}^{2}$ e $\sigma^{2}$ tem distribuição própria (e portanto informativas para valores convenientes dos hiperparâmetros), já no modelo 4 essas duas componentes tem distribuição não informativa.

Observação: Usamos o termo "priori não informativa" várias vezes no texto acima. A seguir apresentamos esse conceito como é conhecido na literatura.

Segundo Box e Tiao (1973), uma priori não informativa (sobre $\theta$ ) não é aquela que representa ignorância total (sobre $\theta$ ) mas a que representa uma situação em que o conhecimento à priori (sobre $\theta$ ) é pequeno relativamente ao conhecimento (ou informação) que se espera obter dos dados; em outras palavras, $\pi(\theta)$ é uma priori não informativa se ela tem um impacto mínimo na distribuição à posteriori de $\theta$ (ou ainda, que a verossimilhança domina sobre $\pi(\theta)$ ). Na literatura existem vários métodos com o objetivo de construir priores com essas características. Uma possível maneira de expressar essa idéia segundo Box e Tiao (1973), é dizer que $\pi(\theta)$ não favorece nenhum valor de $\theta$ em especial. Assim, as seguintes distribuições, $\pi(\theta)=\frac{1}{n}$, $\theta \in\left\{\theta_{1}, \ldots, \theta_{n}\right\}, \pi(\theta)=\frac{1}{b-a}, a<\theta<b$, e a distribuição imprópria $\pi(\theta) \propto 1, \theta \in \mathbb{R}$, seriam exemplos de priores não informativas (Box e Tiao expressam este estado de indiferença com prioris localmente uniformes). Essa maneira de definir uma priori não informativa é bastante questionada pelo fato de que priores uniformes não são invariantes sob reparametrizaçōes. Uma saída para remediar o problema é tratar cada problema como um problema diferente e assim procurar a reparametrização que seja mais adequada para o particular problema que se está estudando, e somente então aí aplicar uma priori uniforme nesta escala (para mais detalhes sobre este assunto ver Box e Tiao, 1973, páginas 23 e 24).

Pode-se também argumentar por exemplo que uma "boa priori não informativa" não deveria ser afetada quando se usa diferentes parametrizações do mesmo mod- 
elo. Na literatura temos a conhecida priori de Jeffreys que é invariante sob transformações (ou reparametrizações) bijetoras. Outro exemplo é o caso das famílias de distribuições com parâmetros de locação (ou de escala); nesse caso uma priori "não informativa razoável" deveria ser invariante sob transformações de locação (ou escala) nos dados. Berger (1980) demonstra que a distribuição à priori de um parâmetro de locação com essa característica deve ser do tipo $\pi(\theta) \propto 1$ e a priori de um parâmetro de escala que seja invariante sob transformação de escala nos dados deve ser do tipo $\pi\left(\sigma^{2}\right) \propto \frac{1}{\sigma^{2}}$.

Outro tipo de priori, que está relacionada bem de perto com o conceito de priori não informativa é a chamada "priori de referência" (ver Kass e Wasserman, 1996, e Bernardo, 1979).

\section{Distribuição à posteriori}

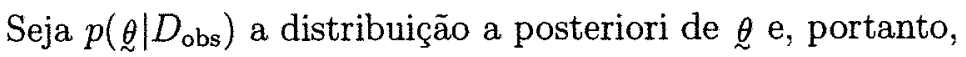

$$
p\left(\underset{\sim}{\theta} \mid D_{\mathrm{obs}}\right) \propto L\left(\underset{\sim}{\left(\theta \mid D_{\mathrm{obs}}\right) \pi(\underset{\sim}{\theta})}\right.
$$

onde $L\left(\underset{\theta}{\theta} \mid D_{\text {obs }}\right)$ é a função de verossimilhança e $\pi(\underset{\sim}{\theta})$ é a distribuição à priori de $\underset{\sim}{\theta}$.

Nosso objetivo é encontrar as condições necessárias e suficientes para que a posteriori seja própria, isto é, $\int_{\Theta} L\left(\theta \mid D_{\text {obs }}\right) \pi(\underset{\theta}{\theta}) \mathrm{d} \theta<\infty$, onde

$$
\begin{aligned}
L\left(\underset{\theta}{\theta} \mid D_{\mathrm{obs}}\right) \propto & \frac{1}{\left[\beta^{2} \sigma_{x}^{2} \sigma^{2}+\lambda \sigma_{x}^{2} \sigma^{2}+\lambda\left(\sigma^{2}\right)^{2}\right]^{n / 2}} \exp \left\{-\frac{1}{2}\left[\left(\beta^{2} \sigma_{x}^{2}+\lambda \sigma^{2}\right) \sum_{i=1}^{n}\left(X_{i}-\mu\right)^{2}\right.\right. \\
& \left.\left.-2 \beta \sigma_{x}^{2} \sum_{i=1}^{n}\left(Y_{i}-\alpha-\beta \mu\right)\left(X_{i}-\mu\right)+\left(\sigma_{x}^{2}+\sigma^{2}\right) \sum_{i=1}^{n}\left(Y_{i}-\alpha-\beta \mu\right)^{2}\right]\right\} .
\end{aligned}
$$

O problema é que, independentemente da escolha de $\pi(\underset{\theta}{\theta})$, o cálculo da integral $\int_{\Theta} L\left(\underset{\sim}{\theta} \mid D_{\mathrm{obs}}\right) \pi(\underset{\sim}{\theta}) \mathrm{d} \underset{\sim}{\theta}$ é muito complicado. Para contornar o problema "reintroduzimos" as variáveis latentes $\underset{\sim}{x}=\left(x_{1}, \ldots, x_{n}\right)^{T}$ escrevendo a posteriori como

$$
p\left(\underset{\sim}{\theta} \mid D_{\mathrm{obs}}\right)=\int_{\mathscr{X}=\left\{\underset{\sim}{x}: \underset{\sim}{\left.x \in \mathbb{R}^{n}\right\}}\right.} p\left(\underset{\sim}{\theta}, \underset{\sim}{x} \mid D_{\mathrm{obs}}\right) \mathrm{d} \underset{\sim}{x} \propto \int_{x} L(\underset{\sim}{\theta} \mid D) \pi(\underset{\sim}{\theta}) \mathrm{d} \underset{\sim}{x},
$$


onde $L(\underset{\sim}{\theta} \mid D)$ é a verossimilhança para os dados completos, dada por

$$
\begin{aligned}
L(\underset{g}{\theta} \mid D) \propto & {\left[\left(\sigma^{2}\right)^{2} \sigma_{x}^{2}\right]^{-n / 2} \exp \left\{-\frac{1}{2}\left[\frac{\sum_{i=1}^{n}\left(Y_{i}-\alpha-\beta x_{i}\right)^{2}}{\lambda \sigma^{2}}+\frac{\sum_{i=1}^{n}\left(X_{i}-x_{i}\right)^{2}}{\sigma^{2}}\right.\right.} \\
& \left.\left.+\frac{\sum_{i=1}^{n}\left(x_{i}-\mu\right)^{2}}{\sigma_{x}^{2}}\right]\right\} .
\end{aligned}
$$

e portanto

$$
\int_{\Theta} p\left(\underset{\sim}{\theta} \mid D_{\mathrm{obs}}\right) \mathrm{d} \underset{\sim}{\theta} \propto \int_{\Theta} \int_{\mathscr{X}} L(\underset{\sim}{\theta} \mid D) \pi(\underset{\sim}{\theta}) \mathrm{d} \underset{\sim}{x} \mathrm{~d} \underset{\sim}{\theta}
$$

Esta última integral é muito mais tratável do que a anterior e, portanto, ela será utilizada para demonstrar a existência da posteriori.

Mais à frente enunciamos e demonstramos os teoremas que estabelecem as condições necessárias e suficientes para a existência das posteriores que correspondem às priores especificadas anteriormente. Entretanto, antes disso iremos demonstrar um lema cujo resultado será muito útil nas demonstrações.

Lema 2.8.1 Se $\underset{\sim}{x} \times$ é um vetor de $p$ componentes, entâo

(a) $\int_{\left\{\underset{\sim}{x} \in \mathbb{R}^{p}:\|\underset{\sim}{x}\|<\varepsilon\right\}} \frac{1}{\|\underset{\sim}{x}\|^{\lambda}} \mathrm{d} \underset{\sim}{x}<\infty$ se e somente se $\lambda<p$;

(b) $\int_{\left\{\underset{\sim}{x} \in \mathbb{R}^{n}:\|\underset{\sim}{x}\|>\varepsilon\right\}} \frac{1}{\|\underset{\sim}{x}\|^{\lambda}} \mathrm{d} \underset{\sim}{x}<\infty \Leftrightarrow \lambda>p$

, onde $\|\underset{\sim}{x}\|$ é a norma Euclideana de $\underset{\sim}{x}$.

Demonstração do caso (a): Se $p=1$, a demonstração é trivial. Se $p \geq 2$, podemos usar a seguinte mudança de coordenadas: $\underset{\sim}{x}=r \underset{\sim}{d}$, onde

$$
\underset{\sim}{d=}\left(\begin{array}{l}
\operatorname{sen}\left(\theta_{1}\right) \operatorname{sen}\left(\theta_{2}\right) \operatorname{sen}\left(\theta_{3}\right) \cdots \operatorname{sen}\left(\theta_{p-3}\right) \operatorname{sen}\left(\theta_{p-2}\right) \operatorname{sen}\left(\theta_{p-1}\right) \\
\operatorname{sen}\left(\theta_{1}\right) \operatorname{sen}\left(\theta_{2}\right) \operatorname{sen}\left(\theta_{3}\right) \cdots \operatorname{sen}\left(\theta_{p-3}\right) \operatorname{sen}\left(\theta_{p-2}\right) \cos \left(\theta_{p-1}\right) \\
\operatorname{sen}\left(\theta_{1}\right) \operatorname{sen}\left(\theta_{2}\right) \operatorname{sen}\left(\theta_{3}\right) \cdots \operatorname{sen}\left(\theta_{p-3}\right) \cos \left(\theta_{p-2}\right) \\
\operatorname{sen}\left(\theta_{1}\right) \operatorname{sen}\left(\theta_{2}\right) \operatorname{sen}\left(\theta_{3}\right) \cdots \cos \left(\theta_{p-3}\right) \\
\vdots \\
\operatorname{sen}\left(\theta_{1}\right) \operatorname{sen}\left(\theta_{2}\right) \cos \left(\theta_{3}\right) \\
\operatorname{sen}\left(\theta_{1}\right) \cos \left(\theta_{2}\right) \\
\cos \left(\theta_{1}\right)
\end{array}\right) \text { e } r \geq 0
$$


Esta transformação é uma generalização da chamada "transformação em "coordenadas esféricas" (quando $p=2$ temos a transformação em coordenadas polares e quando $p=3$ a transformação em coordenadas esféricas). É fácil ver que $\|d\|=1$ e o valor absoluto do jacobiano da transformação é

$$
\begin{aligned}
& |J|=r^{p-1}\left|\operatorname{sen}^{p-2} \theta_{1} \operatorname{sen}^{p-3} \theta_{2} \cdots \operatorname{sen}^{1} \theta_{p-2}\right| \text {. Portanto, } \\
& \int_{\|\underset{\sim}{x}\|<\varepsilon} \frac{1}{\|\underset{\sim}{x}\|^{\lambda}} \mathrm{d} \underset{\sim}{x}
\end{aligned}
$$

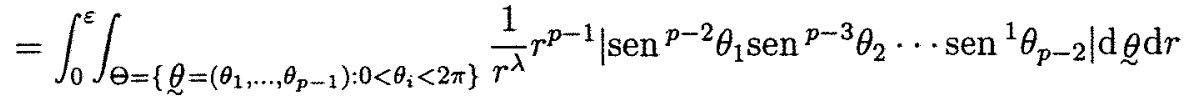

$$
\begin{aligned}
& =\int_{0}^{\varepsilon} \int_{\Theta=\left\{\theta=\left(\theta_{1}, \ldots, \theta_{p-1}\right): 0<\theta_{i}<2 \pi\right\}} \frac{1}{r^{\lambda-p+1}}\left|\operatorname{sen}^{p-2} \theta_{1} \cdots \operatorname{sen}^{1} \theta_{p-2}\right| \mathrm{d} \underset{\theta}{\theta} \mathrm{d} r
\end{aligned}
$$

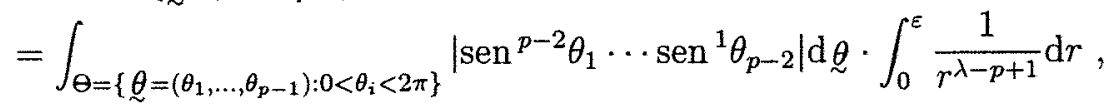

que é finita se se somente se $\lambda-p+1<1$, ou seja, $\lambda<p$. (Note que a integral em $\Theta$ é finita já que a função no integrando é limitada e o domínio de integração $\Theta$ é um conjunto limitado.)

A demonstração do Lema 2.8.1(b) é semelhante.

Teorema 2.8.2 A condição necessária e suficiente para a existência da distribuição $\grave{a}$ posteriori do modelo Normal em (2.8.1) com priori dada por $\pi(\underset{\sim}{\theta}) \propto \pi\left(\sigma_{x}^{2}\right) \pi\left(\sigma^{2}\right)$, onde $\sigma_{x}^{2} \sim I G(c, d)$ e $\sigma^{2} \sim I G(f, g)$, é que $n \geq 3$ (onde $n$ é o tamanho da amostra).

Demonstração: Primeiramente devemos calcular a integral

$$
\int_{\Theta} p\left(\underset{\sim}{\theta} \mid D_{\mathrm{obs}}\right) \mathrm{d} \underset{\sim}{\theta}=\int_{\mathscr{X}} \int_{\Theta} p\left(\underset{\sim}{\theta} \underset{\sim}{x} \mid D_{\mathrm{obs}}\right) \mathrm{d} \underset{\sim}{x} \mathrm{~d} \underset{\sim}{\theta}
$$

onde $p\left(\underset{\sim}{\theta} \underset{\sim}{x} \mid D_{\text {obs }}\right) \propto L(\underset{\sim}{\theta} \mid D) \pi(\underset{\sim}{\theta})$ e depois verificar sob que condições ela é finita.

A distribuição conjunta à posteriori de $\underset{\sim}{\theta}$ e $\underset{\sim}{x}, p\left(\underset{\sim}{\theta} \underset{\sim}{x} \mid D_{\text {obs }}\right)$, sob o modelo do Teorema 2.8 .2 é

$$
\begin{aligned}
p\left(\underline{\theta}, \underset{\sim}{x} \mid D_{\text {obs }}\right) \propto & \left(\sigma_{x}^{2}\right)^{-\left(\frac{n}{2}+c+1\right)}\left(\sigma^{2}\right)^{-(n+f+1)} \exp \left\{-\frac{1}{2}\left[\frac{2 d}{\sigma_{x}^{2}}+\frac{2 g}{\sigma^{2}}\right.\right. \\
& \left.\left.+\frac{\sum_{i=1}^{n}\left(Y_{i}-\alpha-\beta x_{i}\right)^{2}}{\lambda \sigma^{2}}+\frac{\sum_{i=1}^{n}\left(X_{i}-x_{i}\right)^{2}}{\sigma^{2}}+\frac{\sum_{i=1}^{n}\left(x_{i}-\mu\right)^{2}}{\sigma_{x}^{2}}\right]\right\} .
\end{aligned}
$$


Integrando inicialmente esta função em relação a $\alpha$, temos que (ver seção 2.8 do Apêndice A)

$$
\begin{aligned}
& \int_{-\infty}^{\infty} p\left(\underset{\sim}{\theta}, \underset{\sim}{x} \mid D_{\mathrm{obs}}\right) \mathrm{d} \alpha \propto\left(\sigma_{x}^{2}\right)^{-\left(\frac{n}{2}+c+\frac{1}{2}\right)}\left(\sigma^{2}\right)^{-(n+f+1)} \exp \left\{-\frac{1}{2}\left[\frac{2 d}{\sigma_{x}^{2}}+\frac{2 g}{\sigma^{2}}\right.\right. \\
& \left.\left.+\frac{\sum_{i=1}^{n}\left(X_{i}-x_{i}\right)^{2}}{\sigma^{2}}+\frac{\sum_{i=1}^{n}\left(x_{i}-\mu\right)^{2}}{\sigma_{x}^{2}}+\frac{\sum_{i=1}^{n}\left(Y_{i}-\beta x_{i}\right)^{2}-n(\bar{Y}-\beta \bar{x})^{2}}{\lambda \sigma^{2}}\right]\right\} .
\end{aligned}
$$

A seguir, integramos em relação $\mu$ (ver Apêndice A, seção 2.8), obtendo

$$
\begin{aligned}
\int_{-\infty}^{\infty} \int_{-\infty}^{\infty} p\left(\underset{\sim}{\theta}, \underset{\sim}{x} \mid D_{\text {obs }}\right) \mathrm{d} \alpha \mathrm{d} \mu \propto\left(\sigma_{x}^{2}\right)^{-\left(\frac{n}{2}+c+\frac{1}{2}\right)}\left(\sigma^{2}\right)^{-\left(n+f+\frac{1}{2}\right)} \exp \left\{-\frac{1}{2}\left[\frac{2 d}{\sigma_{x}^{2}}+\frac{2 g}{\sigma^{2}}\right.\right. \\
\left.\left.+\frac{\sum_{i=1}^{n}\left(X_{i}-x_{i}\right)^{2}}{\sigma^{2}}+\frac{\sum_{i=1}^{n}\left(Y_{i}-\beta x_{i}\right)^{2}-n(\bar{Y}-\beta \bar{x})^{2}}{\lambda \sigma^{2}}+\frac{\sum_{i}^{n}\left(x_{i}-\bar{x}\right)^{2}}{\sigma_{x}^{2}}\right]\right\} .
\end{aligned}
$$

A seguir, integramos em relação a $\beta$ (ver seção 2.8 do Apêndice A),

$$
\begin{aligned}
\int_{-\infty}^{\infty} \int_{-\infty}^{\infty} \int_{-\infty}^{\infty} p\left(\underset{\sim}{\theta}, \underset{\sim}{x} \mid D_{\mathrm{obs}}\right) \mathrm{d} \alpha \mathrm{d} \mu \mathrm{d} \beta \propto\left(\sigma_{x}^{2}\right)^{-\left(\frac{n}{2}+c+\frac{1}{2}\right)}\left(\sigma^{2}\right)^{-(n+f)}\left(S_{x x}\right)^{-\frac{1}{2}} \\
\cdot \exp \left\{-\frac{1}{2}\left[\frac{2 d}{\sigma_{x}^{2}}+\frac{2 g}{\sigma^{2}}+\frac{\sum_{i=1}^{n}\left(X_{i}-x_{i}\right)^{2}}{\sigma^{2}}+\frac{S_{x x}}{\sigma_{x}^{2}}+\frac{S_{Y Y} S_{x x}-S_{x Y}^{2}}{\lambda \sigma^{2} S_{x x}}\right]\right\}
\end{aligned}
$$

onde $S_{x x}=\sum_{i=1}^{n}\left(x_{i}-\bar{x}\right)^{2}, S_{Y Y}=\sum_{i=1}^{n}\left(Y_{i}-\bar{Y}\right)^{2}, S_{x Y}=\sum_{i=1}^{n}\left(x_{i}-\bar{x}\right)\left(Y_{i}-\bar{Y}\right)$.

Agora, integrando com relação a $\sigma_{x}^{2}$ (ver seção 2.8 do Apêndice A),

$$
\begin{array}{r}
\int_{-\infty}^{\infty} \int_{-\infty}^{\infty} \int_{-\infty}^{\infty} \int_{0}^{\infty} p\left(\underset{\sim}{\theta} \underset{\sim}{x} \mid D_{\mathrm{obs}}\right) \mathrm{d} \alpha \mathrm{d} \mu \mathrm{d} \beta \mathrm{d} \sigma_{x}^{2} \propto \frac{\left(\sigma^{2}\right)^{-(n+f)}}{S_{x x}^{\frac{1}{2}}\left(2 d+S_{x x}\right)^{\frac{n+2 c-1}{2}}} . \\
\cdot \exp \left\{-\frac{1}{2}\left[\frac{\sum_{i}^{n}\left(X_{i}-x_{i}\right)^{2}}{\sigma^{2}}+\frac{\left(S_{Y Y} S_{x x}-S_{x Y}^{2}\right)}{\lambda \sigma^{2} S_{x x}}+\frac{2 g}{\sigma^{2}}\right]\right\} .
\end{array}
$$

Finalmente, integrando com respeito a $\sigma^{2}$ (ver seção 2.8 do Apêndice A), obtemos

$$
\begin{aligned}
& \int_{-\infty}^{\infty} \int_{-\infty}^{\infty} \int_{-\infty}^{\infty} \int_{0}^{\infty} \int_{0}^{\infty} p\left(\underset{\sim}{\theta}, \underset{\sim}{x} \mid D_{\mathrm{obs}}\right) \mathrm{d} \alpha \mathrm{d} \mu \mathrm{d} \beta \mathrm{d} \sigma_{x}^{2} \mathrm{~d} \sigma^{2} \\
& \propto \frac{1}{S_{x x}^{\frac{1}{2}}\left(2 d+S_{x x}\right)^{\frac{n+2 c-1}{2}}\left[\sum_{i}^{n}\left(X_{i}-x_{i}\right)^{2}+\frac{\left(S_{Y Y} S_{x x}-S_{x y}^{2}\right)}{\lambda S_{x x}}+2 g\right]^{n+f-1}}
\end{aligned}
$$


Portanto, a integral

$$
\int_{\Theta} p\left(\underset{\theta}{\theta} \mid D_{\mathrm{obs}}\right) \mathrm{d} \underset{\sim}{\theta}=\int_{\circledast} \int_{\Theta} p\left(\underset{\sim}{\theta} \underset{\sim}{x} \mid D_{\mathrm{obs}}\right) \mathrm{d} \underset{\sim}{\mathrm{d}} x
$$

é proporcional a

$$
\int_{x} \frac{1}{\left(S_{x x}\right)^{\frac{1}{2}}\left(2 d+S_{x x}\right)^{\frac{n+2 c-1}{2}}\left(\sum_{i=1}^{n}\left(X_{i}-x_{i}\right)^{2}+\frac{S_{Y Y} S_{x x}-S_{x Y}^{2}}{\lambda S_{x x}}+2 g\right)^{n+f-1}} \mathrm{~d} \underset{\sim}{x}
$$

onde $\underset{\sim}{x}=\left(\begin{array}{c}x_{1} \\ \vdots \\ x_{n}\end{array}\right), S_{x x}=\sum_{i=1}^{n}\left(x_{i}-\bar{x}\right)^{2}, S_{Y Y}=\sum_{i=1}^{n}\left(Y_{i}-\bar{Y}\right)^{2} \mathrm{e}$

$S_{x Y}=\sum_{i=1}^{n}\left(x_{i}-\bar{x}\right)\left(Y_{i}-\bar{Y}\right)$.

Notemos que para $n=1, \underset{\sim}{x}=x_{1}, S_{x_{1} x_{1}}=0$ e $S_{x_{1} Y_{1}}=0, \forall x_{1} \in \mathbb{R}$ e, portanto, o integrando não está definido, qualquer que seja $x_{1} \in \mathbb{R}$.

O cálculo dessa integral fica mais simples se, por exemplo, no lugar da soma de quadrados $S_{x x}=\sum_{i=1}^{n}\left(x_{i}-\bar{x}\right)^{2}$ tivermos uma soma de quadrados do tipo $\sum_{i} z_{i}^{2}$. Isso pode ser conseguido por meio de uma mudança de variáveis do tipo $\underset{\sim}{z}=H \underset{\sim}{x}$, onde

$$
\underset{\sim}{z}=\left(\begin{array}{c}
z_{1} \\
\vdots \\
z_{n}
\end{array}\right) \text { e } H_{n \times n}=\left[\begin{array}{ccccccc}
\frac{1}{\sqrt{2}} & -\frac{1}{\sqrt{2}} & 0 & 0 & 0 & \cdots & 0 \\
\frac{1}{\sqrt{6}} & \frac{1}{\sqrt{6}} & -\frac{2}{\sqrt{6}} & 0 & 0 & \ldots & 0 \\
\frac{1}{\sqrt{12}} & \frac{1}{\sqrt{12}} & \frac{1}{\sqrt{12}} & -\frac{3}{\sqrt{12}} & 0 & \cdots & 0 \\
\vdots & \vdots & \vdots & \vdots & \vdots & \vdots & \\
\frac{1}{\sqrt{n(n-1)}} & \frac{1}{\sqrt{n(n-1)}} & \frac{1}{\sqrt{n(n-1)}} & \frac{1}{\sqrt{n(n-1)}} & \frac{1}{\sqrt{n(n-1)}} & \cdots & -\frac{(n-1)}{\sqrt{n(n-1)}} \\
\frac{1}{\sqrt{n}} & \frac{1}{\sqrt{n}} & \frac{1}{\sqrt{n}} & \frac{1}{\sqrt{n}} & \frac{1}{\sqrt{n}} & \cdots & \frac{1}{\sqrt{n}}
\end{array}\right],
$$

onde a matriz $H$ é conhecida como a matriz de Helmert (Searle, 1971), que é ortogonal e está definida para $n \geq 2$.A transformação $\underset{\sim}{x} \mapsto H \underset{\sim}{x}$ é uma rotação de $\underset{\sim}{x}$ (no caso bidimensional, por exemplo, $H=\left(\begin{array}{cc}\cos (\theta) & -\operatorname{sen}(\theta) \\ \operatorname{sen}(\theta) & \cos (\theta)\end{array}\right)$ onde $\theta=\frac{\pi}{4}$ ).

No novo espaço (rotacionado), os dados observados $\underset{\sim}{X_{n \times 1}}$ e $\underset{\sim}{Y} \underset{n \times 1}{ }$ são representados, respectivamente, por $\underset{\sim}{b}=\left(\begin{array}{c}b_{1} \\ \vdots \\ b_{n}\end{array}\right)=H \underset{\sim}{X} \underset{\sim}{\underset{\sim}{c}}=\left(\begin{array}{c}c_{1} \\ \vdots \\ c_{n}\end{array}\right)=H \underset{\sim}{Y}$. 
Observação: No nosso contexto usaremos a notação $\underset{\sim}{b}$ para representar tanto o ponto $\left(b_{1}, \ldots, b_{n}\right)^{\top}$ ( no espaço $n$-dimensional) como ao vetor $\vec{b}$ (onde $\vec{b}$ pode ser representado geometricamente pelo segmento orientado que passa pela origem e tem

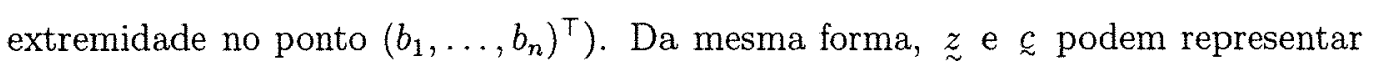
tanto pontos como vetores mas a notação $\vec{z}, \vec{c}$ é reservada apenas para vetores.

No novo espaço as quantidades $S_{x x}, S_{Y Y}, S_{x Y}$ e $\sum_{i=1}^{n}\left(X_{i}-x_{i}\right)^{2}$, que aparecem na integral (2.8.7), são iguais a $\sum_{i=1}^{n-1} z_{i}^{2}, \sum_{i=1}^{n-1} c_{i}^{2}, \sum_{i=1}^{n-1} z_{i} c_{i}$ e $\sum_{i=1}^{n-1}\left(b_{i}-z_{i}\right)^{2}+\left(\sqrt{n} \bar{X}-z_{n}\right)$, respectivamente. A justificativa segue mais abaixo.

\section{Justificativa}

$S_{x x}=\sum_{i=1}^{n} x_{i}^{2}=\frac{\left(\sum_{i=1}^{n} x_{i}\right)^{2}}{n}=\underset{\sim}{x} \underset{\sim}{x}-\frac{1}{n}\left(x^{\top} \underset{\sim}{1}\right)^{2}$, onde $\underset{\sim}{x}=H^{\top} z($ já que $\underset{\sim}{z}=H \underset{\sim}{x}$ e $H$ é ortogonal). Assim,

$$
S_{x x}={\underset{\sim}{z}}^{\top} H H^{\top} \underset{\sim}{z}-\frac{1}{n}\left(z^{\top} H \underset{\sim}{1}\right)^{2}={\underset{\sim}{z}}^{\top} \underset{\sim}{z}-\frac{1}{n}\left(z^{\top} H \underset{\sim}{1}\right)^{2}
$$

onde $H \underset{\sim}{1}=\left(\begin{array}{c}0 \\ \vdots \\ 0 \\ \sqrt{n}\end{array}\right)$ e, portanto,

$$
S_{x x}=\sum_{i=1}^{n} z_{i}^{2}-\frac{1}{n}\left(\sqrt{n} z_{n}\right)^{2}=\sum_{i=1}^{n-1} z_{i}^{2}
$$

Semelhantemente,

$$
S_{Y Y}=\sum_{i=1}^{n} Y_{i}^{2}-\frac{\left(\sum_{i=1}^{n} Y_{i}\right)^{2}}{n}={\underset{\sim}{Y}}^{\top} \underset{\sim}{Y}-\frac{1}{n}\left(\bar{Y}_{\sim}^{1}\right)^{2},
$$

onde $\underset{\sim}{Y}=H^{\top} \underset{\sim}{\mathcal{C}}$ (pois $\underset{\sim}{c}=H \underset{\sim}{Y}$ ). Portanto,

$$
S_{Y Y}=\mathcal{c}^{\top} \underset{\sim}{c}-\frac{1}{n}\left(c^{\top} H \underset{\sim}{1}\right)^{2}=\sum_{i=1}^{n} c_{i}^{2}-\frac{1}{n}\left(\sqrt{n} c_{n}\right)^{2}=\sum_{i=1}^{n-1} c_{i}^{2} .
$$


Também, semelhantemente,

$$
\begin{aligned}
& S_{x Y}=\sum_{i=1}^{n} x_{i} Y_{i}-\frac{\sum_{i=1}^{n} x_{i} \sum_{i=1}^{n} Y_{i}}{n}=\underset{\sim}{x} \underset{\sim}{Y}-\frac{1}{n}\left({\underset{\sim}{x}}^{\top} \underset{\sim}{1}\right)\left({\underset{\sim}{Y}}^{\top} \underset{\sim}{1}\right) \\
& ={\underset{\sim}{z}}^{\top} H H^{\top} \mathcal{\sim}-\frac{1}{n}\left({\underset{\sim}{z}}^{\top} H \underset{\sim}{1}\right)\left(c_{\sim}^{\top} H \underset{\sim}{1}\right)={\underset{\sim}{z}}^{\top} \mathcal{\sim}-\frac{\left(\sqrt{n} z_{n}\right)\left(\sqrt{n} c_{n}\right)}{n}=\sum_{i=1}^{n-1} z_{i} c_{i} .
\end{aligned}
$$

Finalmente,

$$
\begin{aligned}
\sum_{i=1}^{n}\left(X_{i}-x_{i}\right)^{2} & =(\underset{\sim}{X}-\underset{\sim}{x})^{\top}(\underset{\sim}{X}-\underset{\sim}{x})=\left(H^{\top} \underset{\sim}{b}-H^{\top} \underset{\sim}{z}\right)^{\top}\left(H^{\top} \underset{\sim}{b}-H^{\top} \underset{\sim}{z}\right) \\
& =(\underset{\sim}{b}-\underset{\sim}{z})^{\top} H H^{\top}(\underset{\sim}{b}-\underset{\sim}{z})=(\underset{\sim}{b}-\underset{\sim}{z})^{\top}(\underset{\sim}{b}-\underset{\sim}{z})=\sum_{i=1}^{n}\left(b_{i}-z_{i}\right)^{2} \\
& =\sum_{i=1}^{n-1}\left(b_{i}-z_{i}\right)^{2}+\left(b_{n}-z_{n}\right)^{2},
\end{aligned}
$$

onde

$$
b_{n}=\left(\frac{1}{\sqrt{n}}, \ldots, \frac{1}{\sqrt{n}}\right)\left(\begin{array}{c}
X_{1} \\
\vdots \\
X_{n}
\end{array}\right)=\sqrt{n} \bar{X}
$$

Portanto,

$$
\sum_{i=1}^{n}\left(X_{i}-x_{i}\right)^{2}=\sum_{i=1}^{n-1}\left(b_{i}-z_{i}\right)^{2}+\left(\sqrt{n} \bar{X}-z_{n}\right)^{2}
$$

Agora vamos chamar de $\underset{\sim}{z_{x}}$ (ou $\vec{z}_{x}$ ) o vetor formado pelas $n-1$ primeiras componentes de $\underset{\sim}{z} n \times 1=H \underset{\sim}{x}$ (isto é, $\underset{\sim}{z}=A \underset{\sim}{x}$, onde $A$ é a matriz formada pelas $n-1$ primeiras linhas da matriz $H$ ). Analogamente, vamos chamar de $\underset{\sim}{b} X\left(\right.$ ou $\vec{b}_{X}$ ) e de $c_{Y}$ (ou $\vec{c}_{Y}$ ) os vetores formados pelas primeiras $n-1$ componentes dos vetores $\underset{\sim}{b} \mathrm{e}$ $c$, respectivamente. Então,

$$
S_{x x}=\|\underset{\sim}{z}\|^{2}, S_{Y Y}=\|\underset{\sim}{c}\|^{2} \text { e } \sum_{i}^{n}\left(X_{i}-x_{i}\right)^{2}=(\underset{\sim}{b} X-\underset{\sim}{z})^{\top}(\underset{\sim}{b} X-\underset{\sim}{z})+\left(\sqrt{n} \bar{X}-z_{n}\right)^{2}
$$

Finalmente,

$$
S_{x Y}^{2}=\left(\sum_{i=1}^{n-1} z_{i} c_{i}\right)^{2}=\left({\underset{\sim}{x}}_{x}^{\top} c_{Y}\right)^{2}=\left|\overrightarrow{z_{x}} \cdot \vec{c}_{Y}\right|^{2}
$$

onde $\overrightarrow{z_{x}} \cdot \vec{c}_{Y}$ é o produto escalar (ou produto interno) dos vetores $\overrightarrow{z_{x}}$ e $\overrightarrow{c_{Y}}$, ou seja, $\overrightarrow{z_{x}} \cdot \overrightarrow{c_{Y}}=\left\|\overrightarrow{z_{x}}\right\| \cdot\left\|\vec{c}_{Y}\right\| \cos \theta$, onde $\theta$ é o ângulo entre $\overrightarrow{z_{x}}$ e $\vec{c}_{Y}$. Portanto,

$$
\frac{S_{x x} S_{Y Y}-S_{x Y}^{2}}{\lambda S_{x x}}=\frac{\left\|z_{x}\right\|^{2}\left\|c_{Y}\right\|^{2}-\left(z_{x}^{\top} c_{Y}\right)^{2}}{\lambda\left\|z_{x}\right\|^{2}}=\frac{\left\|z_{x}\right\|^{2}\left\|c_{Y}\right\|^{2}-\left\|z_{x}\right\|^{2}\left\|c_{Y}\right\|^{2} \cos ^{2} \theta}{\lambda\left\|z_{x}\right\|^{2}}
$$




$$
=\frac{\left\|c_{Y}\right\|^{2}\left(\operatorname{sen}^{2} \theta\right)}{\lambda}
$$

quando $\underset{\sim}{z} \neq \underset{\sim}{0}$.

Portanto, a quantidade $\frac{\left\|z_{x}\right\|\left\|^{2}\right\| c_{Y} \|^{2}-\left|z_{x} c_{y}\right|^{2}}{\lambda\left\|z_{x}\right\|^{2}}$ está bem definida para $\underset{\sim}{z_{x}} \neq 0$ e não depende de $\left\|z_{\sim}\right\|$ mas sim do ângulo $\theta$ entre $\overrightarrow{z_{x}}$ e $\vec{c}_{Y}$. Além disso, ela assume valores maiores ou iguais a zero, atingindo o zero se e somente se $\theta=0$ (isto é, quando os vetores $\overrightarrow{z_{x}}$ e $\overrightarrow{c_{Y}}$ forem linearmente dependentes).

Finalmente, o Jacobiano da transformação é 1 e, portanto, a integral em (2.8.7) é igual a

$$
\int_{Z=\left\{\underset{\sim}{z} \in \mathbb{R}^{n}\right\}} \frac{1}{{\underset{\sim}{z} x}_{x} \|\left(\left\|{\underset{\sim}{z}}_{x}\right\|^{2}+2 d\right)^{\frac{n+2 c-1}{2}}\left[\left\|z_{x}-\underset{\sim}{b} x\right\|^{2}+\frac{\left\|C_{r}\right\|^{2} \operatorname{sen}^{2} \theta}{\lambda}+2 g+\left(\sqrt{n} \bar{X}-z_{n}\right)^{2}\right]^{n+f-1}} \mathrm{~d} \underset{\sim}{z} .
$$

Para obter a integral dessa função, fazemos primeiramente a integração em relação à variável $z_{n}$ (com o objetivo de eliminar $z_{n}$ ), isto é, calculamos a seguinte integral:

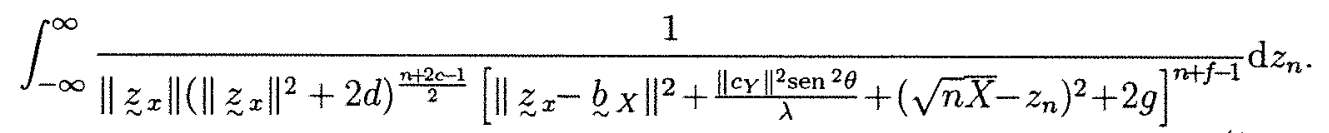

Fazendo

$$
k_{1}=\|\underset{\sim}{z}\|\left(\left\|z_{x}\right\|^{2}+2 d\right)^{\frac{n+2 c-1}{2}} \text { e } k_{2}=\left\|\underset{\sim}{z_{x}}-\underset{\sim}{b} x\right\|^{2}+\frac{\left\|c_{Y}\right\|^{2} \operatorname{sen}^{2} \theta}{\lambda}+2 g,
$$

temos que $k_{2}>0$ (mesmo quando $g=0$ ), pois as duas primeiras parcelas nunca se anulam simultaneamente, como veremos no Teorema 2.8.4.

Assim, a integral em (2.8.10) torna-se igual a

$$
\begin{aligned}
& \int_{-\infty}^{\infty} \frac{1}{k_{1}\left[k_{2}+\left(\sqrt{n} \bar{X}-z_{n}\right)^{2}\right]^{n+f-1}} \mathrm{~d} z_{n}=\frac{1}{k_{1} k_{2}^{n+f-1}} \int_{-\infty}^{\infty} \frac{1}{\left[1+\frac{\left(\sqrt{n} \bar{X}-z_{n}\right)^{2}}{k_{2}}\right]^{n+f-1}} \mathrm{~d} z_{n} \\
& =\frac{1}{k_{1} k_{2}^{n+f-1}} \int_{-\infty}^{\infty} \frac{1}{\left[1+\left(\frac{\sqrt{n} \bar{X}-z_{n}}{\sqrt{k_{2}}}\right)^{2}\right]^{n+f-1}} \mathrm{~d} z_{n}
\end{aligned}
$$


Com a mudança de variável $u=\frac{\sqrt{n} \bar{X}-z_{n}}{\sqrt{k_{2}}}$, a última expressão em (2.8.11) tornase igual a $\frac{1}{k_{1} k_{2}^{n+f-\frac{3}{2}}} \int_{-\infty}^{\infty} \frac{1}{\left[1+u^{2}\right]^{n+f-1}} \mathrm{~d} u$, onde $\int_{-\infty}^{\infty} \frac{1}{\left(1+u^{2}\right)^{n+f-1}} \mathrm{~d} u$ é finita se e somente se $2(n+f-1)>1$, isto é,

$$
n+f>\frac{3}{2}
$$

Portanto, a integral em (2.8.10) é proporcional a $\frac{1}{k_{1} k_{2}^{n+f-\frac{3}{2}}}$, ou seja, ela é proporcional à função

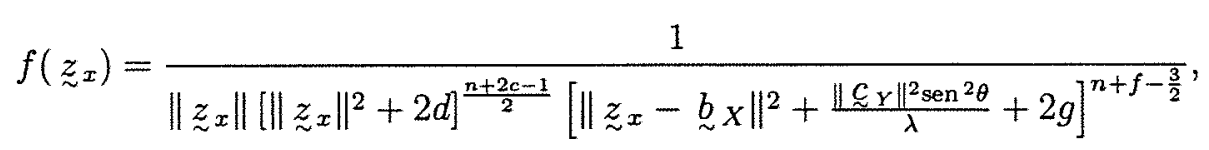

onde $d$ e $g$ são positivos. Portanto,

$$
\int_{\Theta} p\left(\underset{\sim}{\theta} \mid D_{\text {obs }}\right) \mathrm{d} \underset{\sim}{\theta} \propto \int_{Z_{x}=\left\{\underset{\sim}{z} \in \mathbb{R}^{n-1}\right\}} f(\underset{\sim}{z}) \mathrm{d} z_{1} \mathrm{~d} z_{2} \cdots \mathrm{d} z_{n-1}=\int_{Z_{x}} f(\underset{\sim}{z}) \mathrm{d}{\underset{\sim}{z}}_{x}
$$

Note que a função $f\left(\underset{\sim}{z_{x}}\right)$ em (2.8.13) é contínua e possui uma singularidade no ponto $\underset{\sim}{z} x_{x} \underset{\sim}{0}$. Esta singularidade pode ser tratada particionando-se a região de integração $Z_{x}$ em dois conjuntos $R_{1}$ e $R_{2}$, onde

$$
R_{1}=\left\{\underset{\sim}{z} \in \mathbb{R}^{n-1}:\|\underset{\sim}{z}\|<\varepsilon\right\} \quad \text { e } \quad R_{2}=\left\{\underset{\sim}{z_{x}} \in \mathbb{R}^{n-1}:\left\|\underset{\sim}{z_{x}}\right\| \geq \varepsilon\right\}
$$

com $\varepsilon$ suficientemente pequeno.

\section{Avaliação da integral de $f(\underset{\sim}{z})$ na região $R_{1}$}

É fácil ver que em $R_{1}$ a integral de $f\left(z_{x}\right)$ é finita se e somente se $n>2$. Uma forma de ver isso é notar primeiramente que nessa região o termo $\left[\left\|z_{x}\right\|^{2}+2 d\right]$, no denominador de $f\left(z_{x}\right)$, é "dominado" pela constante positiva $2 d$, isto é, satisfaz a desigualdade $2 d \leq\left\|z_{x}\right\|^{2}+2 d \leq 2 d+\varepsilon^{2}$ e portanto para $\varepsilon$ pequeno, $\left\|z_{x}\right\|^{2}+2 d \approx 2 d$ (isto é, para $\varepsilon \rightarrow 0$ (ou $\left\|z_{x}\right\| \rightarrow \underset{\sim}{0}$ ), temos que $\left\|z_{x}\right\|^{2}+2 d \rightarrow 2 d$ ). Da mesma forma, o termo $\left[\left\|{\underset{\sim}{z}}_{x}-{\underset{\sim}{b}}_{x}\right\|^{2}+\frac{\left\|c_{\gamma}\right\|^{2} \operatorname{sen}^{2} \theta}{\lambda}+2 g\right]$ é "dominado" por uma constante positiva, 
pois

$$
\begin{aligned}
{\left[\left\|b_{X}\right\|-\varepsilon\right]^{2} } & \leq\left[\left\|\underset{\sim}{z_{x}}\right\|-\|\underset{\sim}{b}\|\right]^{2} \leq\|\underset{\sim}{\underset{z}{z}}-\underset{\sim}{b} x\|^{2} \\
& \leq\left(\left\|z_{x}\right\|+\|\underset{\sim}{b} \underset{x}{ }\|\right)^{2} \leq(\varepsilon+\|\underset{\sim}{b} x\|)^{2}
\end{aligned}
$$

e portanto

$$
\lim _{\varepsilon \rightarrow 0}\left[\left\|z_{x}-\underset{\sim}{b} x\right\|^{2}+\frac{\left\|c_{Y}\right\|^{2} \operatorname{sen}^{2} \theta}{\lambda}+2 g\right]=\|\underset{\sim}{b}\|^{2}+\frac{\left\|c_{Y}\right\|^{2} \operatorname{sen}^{2} \theta}{\lambda}+2 g,
$$

onde $\|\underset{\sim}{b} X\| \geq 0, \frac{\left\|\mathcal{C}_{Y}\right\|^{2} \operatorname{sen}^{2} \theta}{\lambda} \geq 0$ e $g$ é uma constante positiva.

Portanto, a integral $\int_{R_{1}} f\left(\underset{\sim}{z_{x}}\right) \mathrm{d} \underset{\sim}{z}$ é finita se e somente se $\int_{R_{1}} \frac{1}{\left\|z_{\sim}\right\|} \mathrm{d} z_{x}<\infty$.

Mas, pelo Lema 2.8.1(a), a integral $\int_{R_{1}} \frac{1}{\left\|z_{x}\right\|} \mathrm{d} z_{x}$ é finita se e somente se $1<n-1$, isto é, $n>2$.

Avaliação da integral de $f(\underset{\sim}{z})$ na região $R_{2}$

Para analisar a integral de $f(\underset{\sim}{z})$ na região $R_{2}$ vamos particionar essa região em dois subconjuntos:

$$
R_{2 a}=\left\{\underset{\sim}{z} \in \mathbb{R}^{n-1}: \varepsilon \leq\|\underset{\sim}{z}\|<k\right\} \quad \text { e } \quad R_{2 b}=\left\{\underset{\sim}{z} \in \mathbb{R}^{n-1}:\left\|\underset{\sim}{z_{x}}\right\| \geq k\right\}
$$

onde $k$ é escolhido suficientemente grande.

Na região $R_{2 a}$ a integral $\int_{R_{2 a}} f\left({\underset{\sim}{x}}_{x}\right) \mathrm{d} \underset{\sim}{z}$ é finita pois $f(\underset{\sim}{z})$ é uma função limitada e o conjunto $R_{2 a}$ também é limitado. Na região $R_{2 b}$, a função $f\left(z_{x}\right)$ é limitada, porém $R_{2 b}$ não é um conjunto limitado, entretanto a quantidade $\left\|z_{x}\right\|^{2}$ domina os termos $\left[\left\|z_{x}\right\|^{2}+2 d\right]$ e $\left[\left\|z_{x}-\underset{\sim}{b} X\right\|^{2}+\frac{\|\mathcal{C} Y\|^{2} \operatorname{sen}^{2} \theta}{\lambda}+2 g\right]$ no denominador de $f\left(z_{x}\right)$ e portanto a integral

$$
\int_{R_{2 b}} f\left(\underset{\sim}{z_{x}}\right) \mathrm{d} z_{\sim} x<\infty \Leftrightarrow \int_{R_{2 b}} \frac{1}{\left\|z_{\sim}\right\|^{1+\frac{2(n+2 c-1)}{2}+2\left(n+f-\frac{3}{2}\right)}} \mathrm{d} z_{\sim} z_{x}<\infty .
$$

Portanto, $\int_{R_{2 b}} f\left(z_{\sim} x\right) \mathrm{d} z_{x}$ converge se e somente se $1+\frac{2(n+2 c-1)}{2}+2\left(n+f-\frac{3}{2}\right)>n-1$, isto é, $(n+f+c>1)$, o que é garantido pelo Lema 2.8.1(b). 
É fácil ver que na região $R_{2 b}$ o termo $\left[\left\|\underset{\sim}{z_{x}}\right\|^{2}+2 d\right]$ é dominado por $\|\underset{\sim}{z}\|^{2}$, pois nessa região vale a desigualdade

$$
k^{2} \leq\left\|z_{x}\right\|^{2} \leq\left[\left\|z_{x}\right\|^{2}+2 d\right]
$$

e, portanto, para $k$ suficientemente grande, $\left[\left\|z_{x}\right\|^{2}+2 d\right] \approx\left\|z_{x}\right\|^{2}$, isto é, para $k \rightarrow \infty, \Rightarrow\left\|z_{x}\right\| \rightarrow \infty$ e portanto $\lim _{k \rightarrow \infty}\left(\|\underset{\sim}{z}\|^{2}+2 d\right)=\lim _{k \rightarrow \infty}\left\|z_{x}\right\|^{2}$.

Analogamente, é simples verificar que na região $R_{2 b}$ o termo $\left[\left\|\underset{\sim}{z}{ }_{x}-\underset{\sim}{b} X\right\|^{2}+\frac{\|\mathcal{E}\|^{2} \|^{2} \operatorname{sen}^{2} \theta}{\lambda}+2 g\right]$ é dominado por $\|\underset{\sim}{z}\|^{2}$.

Finalmente, analisando-se simultaneamente todas as regiões $R_{1}, R_{2 a}$ e $R_{2 b}$ simultaneamente (levando em conta também a desigualdade em (2.8.12)), concluímos que $\int_{\Theta} p\left(\underset{\sim}{\theta} \mid D_{\text {obs }}\right) \mathrm{d} \theta$ é finita se e somente se $n>2$ e $n+f+c>1$, isto é, a posteriori é própria em $\Theta$ se e somente se $n \geq 3$.

Teorema 2.8.3 A condição necessária e suficiente para a existência da distribuição à posteriori do modelo Normal em (2.8.1) com priori dada por $\pi(\underset{\sim}{\theta}) \propto \pi\left(\sigma_{x}^{2}\right) \pi\left(\sigma^{2}\right)$, onde $\pi\left(\sigma_{x}^{2}\right) \propto\left(\sigma_{x}^{2}\right)^{-r}, r>0, e \sigma^{2} \sim I G(f, g)$, é que $0<r<\frac{1}{2}$ e $n \geq 2$.

Demonstração: Para demonstrar esse teorema podemos aproveitar os cálculos obtidos na demonstração do Teorema 2.8.2, já que a distribuição a priori especificada nesse teorema coincide com a priori do Teorema 2.8.2 quando substituímos os hiperparâmetros $c$ e $d$, respectivamente, por $(r-1)$ e zero.

Portanto, a integral em $\Theta$ da distribuição à posteriori

$$
\begin{aligned}
\int_{\Theta} p\left(\underset{\theta}{\theta} \mid D_{\mathrm{obs}}\right) \mathrm{d} \underset{\sim}{\theta} & =\int_{x} \int_{\Theta} p\left(\underset{\sim}{x}, \underset{\sim}{\theta} \mid D_{\mathrm{obs}}\right) \mathrm{d} \underset{\sim}{\theta} \mathrm{d} \underset{\sim}{x} \propto \int_{\mathfrak{x}} \int_{\Theta} L(\underset{\sim}{\theta} \mid D) \pi(\theta) \mathrm{d} \underset{\sim}{\theta} \mathrm{d} \underset{\sim}{x} \\
& \propto \int_{Z_{x}=\left\{\underset{\sim}{z_{x}} \in \mathbb{R}^{n-1}\right\}} g\left(\underset{\sim}{z_{x}}\right) \mathrm{d} \underset{\sim}{z},
\end{aligned}
$$

onde

$$
g\left(z_{\sim} x\right)=\frac{1}{\left\|z_{x}\right\|^{1+\frac{2(n+2 r-3)}{2}}\left[\left\|z_{x}-\underset{\sim}{b} X\right\|^{2}+\frac{\left\|c_{Y}\right\|^{2} \operatorname{sen}^{2} \theta}{\lambda}+2 g\right]^{n+f-\frac{3}{2}}}
$$




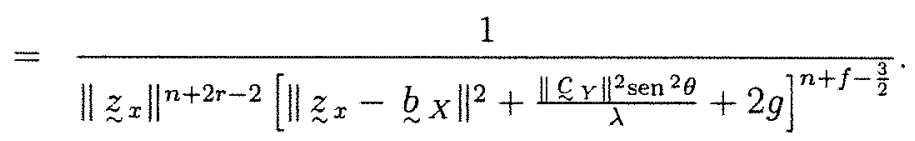

Note que a função $g\left(z_{x}\right)$ foi obtida a partir de $f\left(z_{x}\right)$ dada em (2.8.13) substituindo-se $c$ por $r-1$ e $d$ por zero e assumindo que a desigualdade em (2.8.12) é válida.

Para demonstrar esse teorema particionamos a região de integração $Z_{x}$ da mesma forma que no Teorema 2.8.2, e o objetivo é encontrar as condições para que a integral $\int_{Z_{x}} g(\underset{\sim}{z}) \mathrm{d} z_{\sim}$ seja finita.

\section{Avaliação da integral na região $R_{1}$}

Na região $R_{1}$ a integral $\int_{R_{1}} g\left(\underset{\sim}{z_{x}}\right) \mathrm{d} \underset{\sim}{z}$ é finita se e somente se $\int_{R_{1}} \frac{1}{\|\underset{\sim}{x}\|^{n+2 r-2}} \mathrm{~d} z_{x}$ for finita (pois o termo $\left[\left\|{\underset{\sim}{z}}_{x}-{\underset{\sim}{b} x}_{x}\right\|^{2}+\frac{\|\underset{c}{C}\|^{2} \operatorname{sen}^{2} \theta}{\lambda}+2 g\right]$ que aparece no denominador, é dominado por uma constante positiva (como vimos na demonstração do Teorema 2.8.2). Portanto, a integral em $R_{1}$ é finita se e somente se $0<r<\frac{1}{2}$ (pois pelo

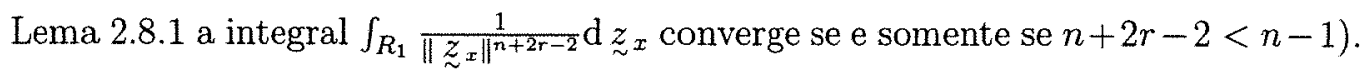

\section{Avaliação da integral na região $R_{2}$}

Na região $R_{2 a}$ não existe problema pois $g\left(z_{x}\right)$ é limitada e $R_{2 a}$ é limitado. Em $R_{2 b}$ a integral $\int_{R_{2 b}} g\left(z_{x}\right) \mathrm{d} z_{x}$ converge se e somente se $\int_{R_{2 b}} \frac{1}{\left\|z_{x}\right\|^{n+2 r-2+2\left(n+f-\frac{3}{2}\right)}} \mathrm{d} z_{x}$ convergir (pois $\|\underset{\sim}{z}\|^{2}$ domina o termo $\left[\left\|z_{x}-\underset{\sim}{b} x\right\|^{2}+\frac{\left\|C_{y}\right\|^{2} \operatorname{sen}^{2} \theta}{\lambda}+2 g\right]$ que aparece no denominador como já discutimos na demonstração do Teorema 2.8.2. Portanto, pelo Lema 2.8.1(b), a integral em $R_{2 b}$ é finita se e somente se $n+2 r-2+2\left(n+f-\frac{3}{2}\right)>$ $n-1$, isto é, $n+f+r>2$.

Observação: Se $n=1$, a integral

$$
\int_{\Theta} p\left(\underline{\theta} \mid D_{\mathrm{obs}}\right) \mathrm{d} \underline{\theta}=\int_{x=\mathbb{R}} \frac{\left(S_{x_{1} x_{1}}\right)^{f-r+\frac{1}{2}}}{\left[S_{x_{1} x_{1}}\left(X_{1}-x_{1}\right)^{2}+\frac{\left(S_{x_{1} x_{1}} S_{Y_{1} Y_{1}}-S_{x_{1} Y_{1}}^{2}\right)}{\lambda}+S_{x_{1} x_{1}} 2 g\right]^{f}} \mathrm{~d} x_{1}
$$


(que é igual a (2.8.7) quando substituímos $c$ por $(r-1), d$ por zero, $n$ por $1, S_{x x}$ por $S_{x_{1} x_{1}}, S_{x Y}$ por $S_{x_{1} Y_{1}}$ e $S_{Y Y}$ por $\left.S_{Y_{1} Y_{1}}\right)$ está bem definida e é finita se e somente se $f=0$ e $0<r<\frac{1}{2}$ (pois $S_{x_{1} x_{1}}$ e $S_{x_{1} Y_{1}}$ são nulos para todo $x_{1} \in \mathbb{R}$ ). Entretanto, por hipótese, $f$ é positivo e portanto $n$ deve ser necessariamente maior ou igual a 2 .

Analisando todos esses resultados simultaneamente, concluímos que a condição necessária e suficiente para a existência da posteriori é que $n \geq 2$ e $0<r<\frac{1}{2}$.

Teorema 2.8.4 Suponhamos que os vetores de dados $\underset{\sim}{X}=\left(\begin{array}{c}X_{1} \\ \vdots \\ X_{n}\end{array}\right)$ e $\underset{\sim}{Y}=\left(\begin{array}{c}Y_{1} \\ \vdots \\ Y_{n}\end{array}\right)$ são linearmente independentes (isto é, pelo menos um ponto $\left(X_{i}, Y_{i}\right)$ não é colinear com os demais) e que as componentes de $\underset{\sim}{X}$, bem como as de $\underset{\sim}{Y}$, não são todas iguais. Então se a distribuição à priori é dada por $\pi(\underset{\sim}{\theta})=\pi\left(\sigma_{x}^{2}\right) \pi\left(\sigma^{2}\right)$, onde $\sigma_{x}^{2} \sim I G(c, d)$ $e \pi\left(\sigma^{2}\right) \propto\left(\sigma^{2}\right)^{-s}, c, d, s>0$, a condição necessária e suficiente para que a posteriori do modelo Normal em (2.8.1) seja própria é que $n \geq 3$.

Demonstração: A demonstração desse teorema é muito semelhante à do Teorema 2.8.5.

Teorema 2.8.5 Suponha que $\underset{\sim}{X}$ e $\underset{\sim}{Y}$ são linearmente independentes e as componentes, tanto de $\underset{\sim}{X}$ como de $\underset{\sim}{Y}$, não são todas iguais. Então, se a distribuição à priori é dada por $\pi(\underset{\sim}{\theta})=\pi\left(\sigma_{x}^{2}\right) \pi\left(\sigma^{2}\right)$, onde $\pi\left(\sigma_{x}^{2}\right) \propto\left(\sigma_{x}^{2}\right)^{-r}$ e $\pi\left(\sigma^{2}\right) \propto\left(\sigma^{2}\right)^{-s}$, com $r, s>0 ;$ a condição necessária e suficiente para que a posteriori do modelo em (2.8.1) seja própria é que $n+r+s>3$ e $0<r<\frac{1}{2}$.

Demonstração: Seja $p\left(\underset{\theta}{\theta} \mid D_{\text {obs }}\right)$ a distribuição a posteriori. A integral em $\Theta$ de $p\left(\underset{\theta}{\theta} \mid D_{\text {obs }}\right)$ é dada por

$$
\begin{aligned}
& \int_{\Theta} p\left(\underset{\theta}{\theta} \mid D_{\mathrm{obs}}\right) \mathrm{d} \theta=\int_{\mathfrak{Z}} \int_{\Theta} p\left(\underset{\sim}{x}, \underset{\underset{\theta}{\theta}}{ } \mid D_{\mathrm{obs}}\right) \mathrm{d} \underset{\underset{\sim}{\theta}}{\mathrm{d}} \underset{\sim}{x} \propto \int_{\mathfrak{x}} \int_{\Theta} L(\underset{\theta}{\theta} \mid D) \pi(\underset{\theta}{\theta}) \mathrm{d} \underset{\sim}{\theta} \mathrm{d} \underset{\sim}{x}
\end{aligned}
$$

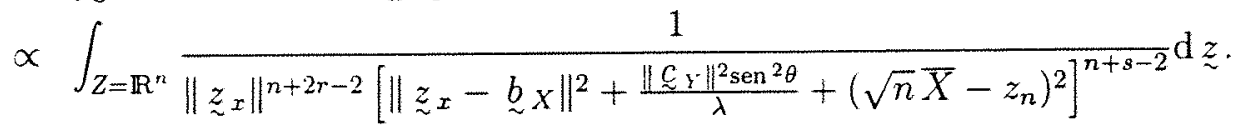


Essa integral foi obtida substituindo-se na integral em (2.8.9) as constantes $c$ por $(r-1), d$ por zero, $f$ por $(s-1)$ e $g$ por zero.

Para estudar essa integral, primeiramente fazemos a integração em relação à $z_{n}$, ou seja, calculamos

$$
\frac{1}{\|\underset{\sim}{z}\|^{n+2 r-2}} \int_{-\infty}^{\infty} \frac{1}{\left[\left\|z_{x}-\underset{\sim}{b} x\right\|^{2}+\frac{\left\|c_{\gamma}\right\|^{2} \operatorname{sen}^{2} \theta}{\lambda}+\left(\sqrt{n} \bar{X}-z_{n}\right)^{2}\right]^{n+s-2}} \mathrm{~d} z_{n}
$$

Fazendo agora $k=\left\|\underset{\sim}{z_{x}}-\underset{\sim}{b} x\right\|^{2}+\frac{\|\mathcal{C} y\|^{2} \operatorname{sen}^{2} \theta}{\lambda}$, a integral em $z_{n}$ acima é igual a

$$
\int_{-\infty}^{\infty} \frac{1}{\left(k+\left(\sqrt{n} \bar{X}-z_{n}\right)^{2}\right)^{n+s-2}} \mathrm{~d} z_{n}
$$

Mas como $k$ é positivo, já que os termos $\|\underset{\sim}{z} x-\underset{\sim}{b}\|^{2}$ e $\frac{\|\underline{C} Y\|^{2} \operatorname{sen}^{2} \theta}{\lambda}$ não se anulam simultaneamente (como justificaremos mais adiante), então

$$
\begin{aligned}
\int_{-\infty}^{\infty} \frac{1}{\left[k+\left(\sqrt{n} \bar{X}-z_{n}\right)^{2}\right]^{n+s-2}} \mathrm{~d} z_{n} & =\frac{1}{k^{n+s-2}} \int_{-\infty}^{\infty} \frac{1}{\left[1+\left(\frac{\sqrt{n} \bar{X}-z_{n}}{\sqrt{k}}\right)^{2}\right]^{n+s-2}} \mathrm{~d} z_{n} \\
& =\frac{1}{k^{n+s-\frac{5}{2}}} \int_{-\infty}^{\infty} \frac{1}{\left(1+u^{2}\right)^{n+s-2}} \mathrm{~d} u
\end{aligned}
$$

onde $\int_{-\infty}^{\infty} \frac{1}{\left(1+u^{2}\right)^{n+s-2}} \mathrm{~d} u<\infty$ se e somente se $2(n+s-2)>1$, isto é, $n+s>\frac{5}{2}$ (que é igual à desigualdade em (2.8.12) quando substituímos $f$ por $(s-1)$ ).

Assumindo que $n+s>\frac{5}{2}$ então $\int_{-\infty}^{\infty} \frac{1}{\left[k+\left(\sqrt{n} \bar{X}-z_{n}\right)^{2}\right]^{n+s-2}} \mathrm{~d} z_{n}$ é proporcional a $\frac{1}{k^{n+s-\frac{5}{2}}}$ e portanto

$$
\begin{aligned}
& \int_{\theta} p\left(\underset{\sim}{\theta} \mid D_{\text {obs }}\right) \mathrm{d} \underset{\sim}{\theta} \propto \int_{Z_{x}=\mathbb{R}^{n-1}} \frac{1}{\|\underset{\sim}{z} x\|^{n+2 r-2}\left[\left\|\sim_{\sim}^{z}-{\underset{\sim}{b} X}_{X}\right\|^{2}+\frac{\|\mathcal{C} Y\|^{2} \operatorname{sen}^{2} \theta}{\lambda}\right]^{n+s-\frac{5}{2}}} \mathrm{~d} \underset{\sim}{z} x \\
& =\int_{Z_{x}} h(\underset{\sim}{z}) \mathrm{d} z_{\sim} x, \quad \text { onde } \\
& h\left(\underset{\sim}{z_{x}}\right)=\frac{1}{\left\|\underset{\sim}{z_{x}}\right\|^{n+2 r-2}\left[\| \underset{\sim}{\left.z_{x}-\underset{\sim}{b} x \|^{2}+\frac{\|\underset{\gamma}{(}\|^{2} \operatorname{sen}^{2} \theta}{\lambda}\right]^{n+s-\frac{3}{2}}}\right.}
\end{aligned}
$$

Note que $h(\underset{\sim}{z})$ é contínua e possui uma única singularidade na origem (e outras duas singularidades removíveis). Para avaliar quais as condições em que a integral 
$\int_{\mathbb{R}^{n-1}} h(\underset{\sim}{z}) \mathrm{d} z_{\sim}$ converge, particionamos a região de integração em vários subconjuntos. A Figura 2.0 ilustra essa partição para o caso $n=3$.

A seguir definimos as partições da região de integração:

$R_{1}=\left\{\underset{\sim}{z_{x}} \in \mathbb{R}^{n-1}:\left\|\underset{\sim}{z_{x}}\right\|<\varepsilon_{1}\right\}$, onde $\varepsilon_{1}$ é suficientemente pequeno, por exemplo, $\varepsilon_{1}=\frac{\|b x\|}{10}\left(\mathbb{R}_{1}\right.$ é a bola de centro na origem e raio $\varepsilon_{1}$ e isola a singularidade na origem);

$R_{3}=\left\{\underset{\sim}{\underset{x}{z}} \in \mathbb{R}^{n-1}:\left\|\underset{\sim}{z_{x}}\right\| \geq k\right\}$

$R_{2}=\left\{\underset{\sim}{z_{x}} \in \mathbb{R}^{n-1}: \varepsilon_{1} \leq\|\underset{\sim}{z}\| \leq k\right\}$, onde $k$ é suficientemente grande, por exemplo, $k=2\|\underset{\sim}{b} x\|$. Esta região é particionada ainda em três sub-regiões, $R_{2 a}, R_{2 b}$ e $R_{2 c}$, que definimos a seguir:

$R_{2 a}=R_{2} \cap M$, onde $M$ é a região compreendida entre as retas $s$ e $t$ da Figura 2.0.

As retas $s$ e $t$ passam pela origem e formam um ângulo $\theta_{\varepsilon}$ com $\vec{c}_{Y}$, onde $\theta_{\varepsilon}$ é escolhido suficientemente pequeno (por exemplo, $\theta_{\varepsilon}=\varphi / 10$, onde $\varphi$ é o ângulo entre $\vec{c}_{Y}$ e $\left.\vec{b}_{X}\right)$. Portanto, a região $M$ pode ser escrita como $M=\left\{\underset{\sim}{z_{x}}=\left(z_{1}, \ldots, z_{n-1}\right)^{T} \in \mathbb{R}^{n-1} \mid \vec{\sim}_{x}\right.$ e $\vec{c}_{Y}$ formam um ângulo $\theta$ menor do que $\left.\theta_{\varepsilon}=\varphi / 10\right\}$.

A região $R_{2 b}$ é a bola de centro em $\underset{\sim}{b} X$ e raio $\varepsilon_{2}$,

$$
R_{2 b}=\left\{\underset{\sim}{z} x \in \mathbb{R}^{n-1}:\|\underset{\sim}{z} x-\underset{\sim}{b} X\|<\varepsilon_{2}\right\},
$$

onde, $\varepsilon_{2}$ é fixado arbitrariamente, tão pequeno quanto se queira, por exemplo, $\varepsilon_{2}=$ $\min \left\{\frac{\left\|b_{x}\right\|-\varepsilon_{1}}{10} \frac{d\left(b_{x}, r\right)-d(P, r)}{10}\right\}$, onde

$d\left({\underset{\sim}{b}}_{X}, r\right)$ é a distância perpendicular do ponto $\underset{\sim}{b} X$ à reta $r$, ou seja, $d(\underset{\sim}{b}, r)=$ $\frac{\left\|\vec{b}_{X} \times \vec{c}_{Y}\right\|}{\left\|\vec{c}_{Y}\right\|}$ onde $\vec{b}_{X} \times \vec{c}_{Y}=\left\|\vec{b}_{X}\right\| \operatorname{sen} \varphi$ (isto é, $\vec{b}_{X} \times \vec{c}_{Y}$ é o produto vetorial de $\vec{b}_{X}$ e $\vec{c}_{Y}$ ); 
$P$ é o ponto de interseção de duas retas: a reta $s$ e a reta perpendicular a $r$ que passa pelo ponto $\underset{\sim}{b}$;

$\mathrm{d}(P, r)=$ distância perpendicular do ponto $P$ à reta $r, d(P, r)=\frac{\left\|\overrightarrow{O P} \times \vec{c}_{Y}\right\|}{\left\|\vec{c}_{Y}\right\|}$

Finalmente, a região $R_{2 c}$ é a região complementar, isto é, $R_{2 c}=R_{2} \cap\left(R_{2 a} \cup R_{2 b}\right)$.

É importante observar que os vetores $\underset{\sim}{b}$ e e $\underset{\sim}{c} Y$ são distintos do vetor nulo, pois pela hipótese do Teorema 2.8.5, os vetores $\underset{\sim}{X}$ e $\underset{\sim}{Y}$ não têm todas as componentes iguais (lembrando que $\underset{\sim}{b} X=A \underset{\sim}{X}$ e ${\underset{\sim}{C} Y}_{Y}=A \underset{\sim}{Y}$, onde $A$ é formada pelas primeiras $n-1$ linhas da matriz de Helmert definida em (2.8.8)).

O ângulo $\varphi$ entre $\vec{b}_{X}$ e $\vec{c}_{Y}$ é diferente de zero pois por hipótese $\vec{X}$ e $\vec{Y}$ são linearmente independentes.

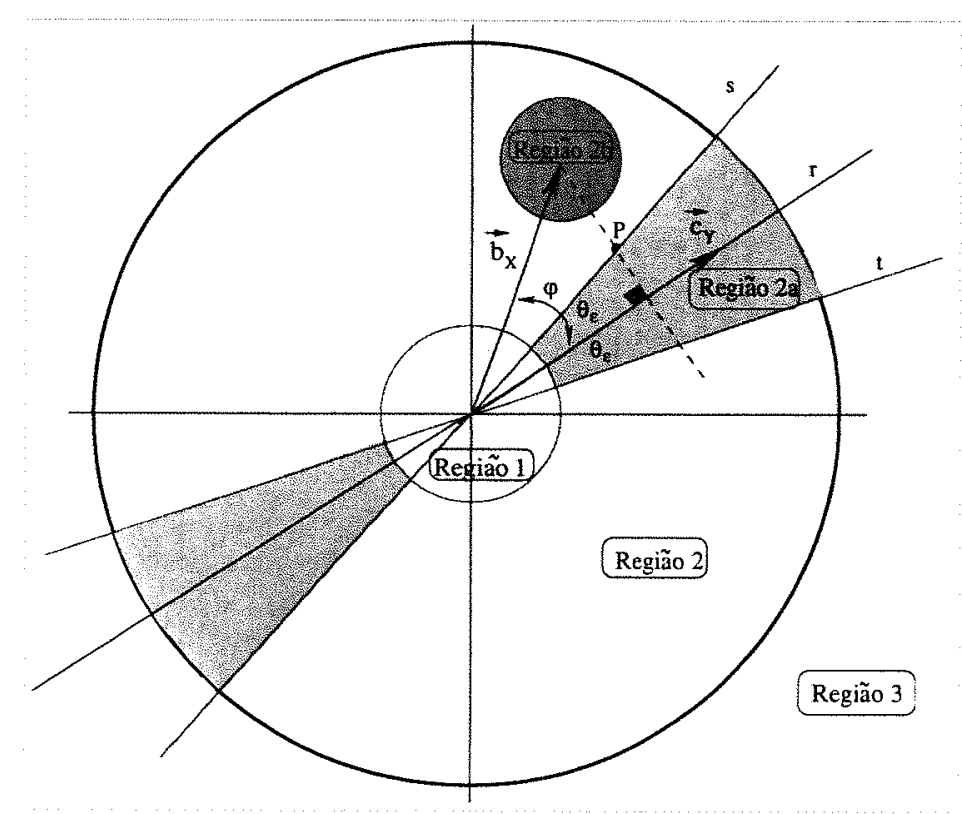

Figura 2.0: Região de integração da distribuição à posteriori no Teorema 2.8.4 quando $n=3$. Os vetores observados $\vec{b}_{X}$ e $\vec{c}_{Y}$ são representados pelos segmentos orientados que passam pela origem e tem extremidades nos pontos ${\underset{\sim}{b} X}_{X}=$ $\left(b_{1}, \ldots, b_{n-1}\right)^{\top}$ e $c_{Y}=\left(c_{1}, \ldots, c_{n-1}\right)^{\top}$ respectivamente. O ângulo formado por $\vec{b}_{X}$ e $\vec{c}_{Y}$ é $\varphi$. A reta $r$ passa pela origem e tem a direção de $\vec{c}_{Y}$. Cada uma das retas $s$ e $t$ forma um ângulo $\theta_{\varepsilon}$ com a reta $r$. 
AVALIAÇÃO DA INTEGRAL DE $h\left(\underset{\sim}{z_{x}}\right)$ NA REGIÃO $R_{1}$ (onde $h\left(z_{x}\right)$ é dada em $(2.8 .15))$

Na região $R_{1}$ o termo $\left[\left\|\underset{\sim}{z_{x}}-\underset{\sim}{b} x\right\|^{2}+\frac{\|C y\|^{2} \operatorname{sen}^{2} \theta}{\lambda}\right]$ no denominador de $h(\underset{\sim}{z})$ é dominado por $\left[\|\underset{\sim}{b} X\|^{2}+\frac{\|\mathcal{C} Y\|^{2} \operatorname{sen}^{2} \theta}{\lambda}\right]$, que é positivo. De fato,

$\left(\|\underset{\sim}{b} x\|-\varepsilon_{1}\right)^{2} \leq|\|\underset{\sim}{z}\|-\|\underset{\sim}{b} x\||^{2} \leq\left\|{\underset{\sim}{z}}_{x}-{\underset{\sim}{b}}_{x}\right\|^{2} \leq(\|\underset{\sim}{z}\|\|+\| \underset{\sim}{b} x \|)^{2} \leq\left(\|\underset{\sim}{b} x\|+\varepsilon_{1}\right)^{2}$ e, portanto, para $\varepsilon_{1}$ suficientemente pequeno, $\|\underset{\sim}{b}\|^{2} \cong\|\underset{\sim}{z} x-\underset{\sim}{b} x\|^{2}$. Note que embora $\frac{\left\|\varepsilon_{Y}\right\|^{2} \operatorname{sen}^{2} \theta}{\lambda}$ possa ser nulo, $\|\underset{\sim}{b}\|^{2}$ é diferente de zero pela hipótese do Teorema 2.8.4 e, portanto, $\left\|\underset{\sim}{b_{X}}\right\|^{2}+\frac{\left\|\varepsilon_{Y}\right\|^{2} \operatorname{sen}^{2} \theta}{\lambda}>0$.

Portanto, $\int_{R_{1}} h(\underset{\sim}{z}) \mathrm{d} z_{x}$ é finita se e somente se $\int_{R_{1}} \frac{1}{\|\underset{\sim}{z}\|^{\mid n+r-2}} \mathrm{~d} \underset{\sim}{z} x$ convergir. Pelo Lema 2.8.1 esta integral converge se e somente se $n+2 r-2<n-1$, isto é, $0<r<\frac{1}{2}$.

\section{AVALIAÇÃO DA INTEGRAL NA REGIÃO $R_{2 a}$}

Nessa região a função $h\left(z_{x}\right)$ é limitada e portanto a integral $\int_{R_{2 a}} h\left(z_{x}\right) \mathrm{d} z_{x}$ é finita já que o domínio de integração $R_{2 a}$ é um conjunto limitado. É fácil ver que $h(\underset{\sim}{z})$ é limitada pois nessa região vale a desigualdade

$$
0 \leq \frac{\left\|\varepsilon_{Y}\right\|^{2} \operatorname{sen}^{2} \theta}{\lambda}<\frac{\left\|\varepsilon_{Y}\right\|^{2} \operatorname{sen}^{2} \theta_{\varepsilon}}{\lambda}
$$

(pelo fato que o ângulo $\theta$ entre $\vec{z}_{x}$ e $\vec{C}_{Y}$ é menor que $\theta_{\varepsilon}$ ) e, além disso, quando $\underset{\sim}{z} \in R_{2 a}$ então $\underset{\sim}{z_{x}} \in R_{2}$ e $\underset{\sim}{z} \notin R_{2 b} \mathrm{e}$, portanto, as seguintes desigualdades também são válidas:

$$
\varepsilon_{1} \leq\|\underset{\sim}{z}\|<k \quad \text { e } \quad \varepsilon_{2}^{2} \leq\|\underset{\sim}{z} x-\underset{\sim}{b} x\|^{2} \leq(\|\underset{\sim}{z}\|+\|\underset{\sim}{b} x\|)^{2} \leq(k+\|\underset{\sim}{b}\|)^{2} .
$$

Isso implica que

$0<\frac{1}{k^{n+2 r-2}\left[(k+\|\underset{\sim}{b} X\|)^{2}+\frac{\|\underset{c}{c}\|^{2} \operatorname{sen}^{2} \theta_{\varepsilon}}{\lambda}\right]^{n+s-\frac{5}{2}}} \leq h(\underset{\sim}{z} x) \leq \frac{1}{\varepsilon_{1}^{n+2 r-2}\left[\varepsilon_{2}\right]^{2\left(n+s-\frac{5}{2}\right)}}<\infty$. 


\section{AVALIAÇÃO DA INTEGRAL EM $R_{2 b}$}

Nessa região a integral $\int_{R_{2 b}} h(\underset{\sim}{z}) \mathrm{d} \underset{\sim}{z} x$ também é finita pois $h(\underset{\sim}{z})$ é limitada e $R_{2 b}$ é um conjunto limitado. Aqui também é simples verificar que $h\left(\underset{\sim}{z_{x}}\right)$ é limitada pois quando $\underset{\sim}{z} \in R_{2 b}$, então $\underset{\sim}{z} \in R_{2}$ e $\underset{\sim}{z} \notin R_{2 a}$ e, portanto, valem as seguintes desigualdades:

$$
\varepsilon_{1} \leq\left\|{\underset{\sim}{z}}_{x}\right\|<k \quad \text { e } \quad \frac{\left\|c_{Y}\right\|^{2} \operatorname{sen}^{2} \theta_{\varepsilon}}{\lambda} \leq \frac{\left\|c_{Y}\right\|^{2} \operatorname{sen}^{2} \theta}{\lambda} \leq \frac{\left\|c_{Y}\right\|^{2}}{\lambda}
$$

Além disso, vale que $0 \leq\left\|\underset{\sim}{z} \underset{x}{b_{x}} x\right\|<\varepsilon_{2}$ e portanto

$$
\frac{1}{k^{n+2 r-2}\left[\varepsilon_{2}^{2}+\frac{\left\|\mathcal{C}_{\gamma}\right\|^{2}}{\lambda}\right]^{n+s-\frac{5}{2}}} \leq h\left(z_{x}\right) \leq \frac{1}{\varepsilon_{1}^{n+2 r-2}\left[\frac{\left\|\mathcal{C}_{\gamma}\right\|^{2} \operatorname{sen}^{2} \theta_{\varepsilon}}{\lambda}\right]^{n+s-\frac{5}{2}}}
$$

\section{AVALIAÇÃO DA INTEGRAL EM $R_{2 c}$}

Nesta região também a integral $\int_{R_{2 c}} h(\underset{\sim}{z}) \mathrm{d} z_{x}$ é finita pois a função $h(\underset{\sim}{z})$ é limitada e $R_{2 c}$ é limitada. De fato, se $\underset{\sim}{z} \in R_{2 c}$, então $\underset{\sim}{z} \in R_{2} \cap R_{2 a}^{c} \cap R_{2 b}^{c}$ e valem as seguintes desigualdades:

$$
\varepsilon_{1} \leq\left\|{\underset{\sim}{x}}_{x}\right\|<k, \quad \frac{\left\|c_{Y}\right\|^{2} \operatorname{sen}^{2} \theta_{\varepsilon}}{\lambda} \leq \frac{\left\|c_{Y}\right\|^{2} \operatorname{sen}^{2} \theta}{\lambda} \leq \frac{\left\|c_{Y}\right\|^{2}}{\lambda}
$$

e $\varepsilon_{2} \leq\|\underset{\sim}{z} x-\underset{\sim}{b} x\| \leq(k+\|\underset{\sim}{b} x\|)$ e portanto

$$
\frac{1}{k^{n+2 r-2}\left[(k+\|\underset{\sim}{\underset{\sim}{b}} x\|)^{2}+\frac{\|\underset{\sim}{C} \gamma\|^{2} \operatorname{sen}^{2} \theta}{\lambda}\right]^{n+s-\frac{5}{2}}} \leq h(\underset{\sim}{z}) \leq \frac{1}{\varepsilon_{1}^{n+2 r-2}\left[\varepsilon_{2}^{2}+\frac{\left\|C_{Y}\right\|^{2} \operatorname{sen}^{2} \theta_{\varepsilon}}{\lambda}\right]^{n+s-\frac{5}{2}}} .
$$

AVALIAÇÃO DA INTEGRAL EM $R_{3}$

$\operatorname{Em} R_{3}$ a função $h\left(\underset{\sim}{z_{x}}\right)$ é limitada, mas a região de integração não é um conjunto limitado, entretanto, o termo $\left[\left\|z_{x}-\underset{\sim}{b} x\right\|^{2}+\frac{\left\|C_{\gamma}\right\|^{2} \operatorname{sen}^{2} \theta}{\lambda}\right]$ do denominador de $h(\underset{\sim}{z})$ é dominado por $\left\|z_{x}\right\|^{2}$. (De fato, para $k$ suficientemente grande, por exemplo $k=$ $2\|\underset{\sim}{b} x\|$, vale que $\|\underset{\sim}{b} x\|<\frac{\|\underset{z}{z}\|}{2}$ e portanto valem as desigualdades

$$
\frac{k^{2}}{4} \leq\left(\frac{\left\|z_{x}\right\|}{2}\right)^{2} \leq\left(\|\underset{\sim}{z}\|-\left\|\underset{\sim}{b_{X}}\right\|\right)^{2} \leq\left[\left\|{\underset{\sim}{z} x}_{-} \underset{\sim}{b}\right\|^{2}+\frac{\left\|c_{Y}\right\|^{2} \operatorname{sen}^{2} \theta}{\lambda}\right]
$$


então, para $k$ suficientemente grande, $\frac{\left\|z_{x}\right\|^{2}}{2} \equiv\left[\left\|\underset{\sim}{z_{x}}-\underset{\sim}{b}\right\|^{2}+\frac{\left\|\mathcal{C}_{\gamma}\right\|^{2} \operatorname{sen}^{2} \theta}{\lambda}\right]$, isto é, para $k \rightarrow \infty \Rightarrow\left\|z_{x}\right\| \rightarrow \infty \Rightarrow \lim _{k \rightarrow \infty} \frac{\left\|z_{x}\right\|^{2}}{2}=\lim _{k \rightarrow \infty}\left[\left\|z_{\sim}-\underset{\sim}{b}\right\|^{2}+\frac{\left\|\mathcal{C}_{Y}\right\|^{2} \operatorname{sen}^{2} \theta}{\lambda}\right]$.) Portanto, a integral

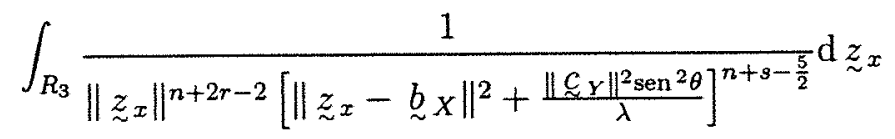

é finita se e somente se $\int_{R_{3}} \frac{1}{\left\|z_{\sim}\right\|^{n+2 r-2+2\left(n+s-\frac{5}{2}\right)}} \mathrm{d} z_{x}$ é finita. Mas isso ocorre se e somente se $n+2 r-2+2\left(n+s-\frac{5}{2}\right)>n-1$, isto é, $n+r+s>3$.

Conclusão da demonstração:

Analisando-se simultaneamente todas essas informações, concluímos que a condição necessária e suficiente para que a posteriori no Teorema 2.8 .5 seja própria é que $0<r<\frac{1}{2}$ e $n+r+s>3$.

\subsubsection{Condições necessárias e suficientes no caso do modelo não identificável}

É fato conhecido que mesmo um modelo não identificável com priori imprópria pode ter posteriori própria (Gelfand e Sahu, 1999, e Erickson, 1989). Nessa seção discutimos a existência da posteriori do modelo estrutural normal sem restrições de identificabilidade sob diferentes escolhas de prioris impróprias.

Na seção 2.6 definimos o modelo

$$
\begin{aligned}
& Y_{i}=\alpha+\beta x_{i}+e_{i} \\
& \quad X_{i}=x_{i}+e_{i}
\end{aligned}
$$

onde

$$
\left(\begin{array}{c}
e_{i} \\
u_{i} \\
x_{i}
\end{array}\right) \stackrel{\text { i.i.d. }}{\sim} N\left(\left(\begin{array}{l}
0 \\
0 \\
\mu
\end{array}\right) ;\left(\begin{array}{ccc}
\sigma_{e}^{2} & 0 & 0 \\
0 & \sigma_{u}^{2} & 0 \\
0 & 0 & \sigma_{x}^{2}
\end{array}\right)\right), i=1, \ldots, n,
$$

$\mathrm{e} \Theta=\left\{\underset{\sim}{\theta}=\left(\alpha, \beta, \mu, \sigma_{x}^{2}, \sigma_{u}^{2}, \sigma_{e}^{2}\right)^{T} \mid, \alpha, \beta, \mu \in \mathbb{R}\right.$ e $\left.\sigma_{x}^{2}, \sigma_{u}^{2}, \sigma_{e}^{2} \in \mathbb{R}^{+}\right\}$.

A distribuição à posteriori, baseada nos dados completos $(D)$ é dada por

$$
p\left(\underset{\theta}{\theta} \mid D_{\text {obs }}\right)=\int_{\mathfrak{X}=\mathbb{R}^{n}} p\left(\underset{\sim}{\theta}, \underset{\sim}{x} \mid D_{\text {obs }}\right) \mathrm{d} \underset{\sim}{x}=\int_{\mathfrak{X}} L(\underset{\sim}{\theta} \mid D) \pi(\underset{\sim}{\theta}) \mathrm{d} \underset{\sim}{x},
$$




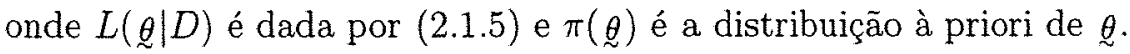

As prioris consideradas nessa seção serão da forma $\pi(\underset{\sim}{\theta})=\prod_{i=1}^{6} \pi(\underbrace{}_{i})$, onde $\pi(\alpha)$, $\pi(\beta)$ e $\pi(\mu)$ são proporcionais a $1, \sigma_{e}^{2} \sim I G(p, q)$ e as componentes $\sigma_{x}^{2}$ e $\sigma_{u}^{2}$ podendo ter distribuição própria ou imprópria de acordo com os teoremas abaixo.

A seguir apresentamos teoremas que garantem posteriori própria para quatro diferentes distribuições à priori.

\section{Teoremas:}

Teorema 2.8.6 No modelo Normal com erros nas variáveis, dado em (2.8.16), e

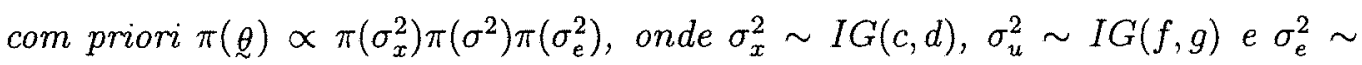
$I G(p, q)$, a condição necessária e suficiente para a existência da posteriori correspondente é que $n \geq 3$.

Demonstração: A integral da posteriori no espaço paramétrico $\Theta$ é

$$
\begin{aligned}
& \int_{\Theta} p\left(\underset{\sim}{\theta} \mid D_{\text {obs }}\right) \mathrm{d} \underset{\sim}{\theta}=\int_{\Theta} \int_{\mathscr{X}=\mathbb{R}^{n}} p\left(\underset{\sim}{x}, \underset{\sim}{\theta} \mid D_{\text {obs }}\right) \mathrm{d} \underset{\sim}{x} \mathrm{~d} \underset{\sim}{\theta} \\
& =\int_{x} \int_{\Theta} p\left(\underset{\sim}{x}, \underset{\theta}{\theta} \mid D_{\mathrm{obs}}\right) \mathrm{d} \underset{\sim}{\theta} \mathrm{d} \underset{\sim}{x} \propto \int_{\mathscr{X}} \int_{\Theta} L(\underset{\sim}{\theta} \mid D) \pi(\underset{\sim}{\theta}) \mathrm{d} \underset{\sim}{\underset{\mathrm{d}}{\mathrm{d}} \underset{\sim}{x}} \\
& \propto \int_{¥} \frac{1}{S_{x x}^{\frac{1}{2}}\left[2 d+S_{x x}\right]^{\frac{n+2 c-1}{2}}\left[2 g+\sum_{i=1}^{n}\left(X_{i}-x_{i}\right)^{2}\right]^{\frac{n}{2}+f}\left[2 q+\frac{S_{x x} S_{Y Y}-S_{x Y}^{2}}{S_{x x}}\right]^{\frac{n}{2}+p-1}} \mathrm{~d} \underset{\sim}{x},
\end{aligned}
$$

onde $S_{x x}=\sum_{i=1}^{n}\left(x_{i}-\bar{x}\right)^{2}, S_{Y Y}=\sum_{i=1}^{n}\left(Y_{i}-\bar{Y}\right)^{2}, S_{x Y}=\sum_{i=1}^{n}\left(x_{i}-\bar{x}\right)\left(Y_{i}-\bar{Y}\right)$.

Usando a transformação que a cada $(\underset{\sim}{x}, \underset{\sim}{X}, \underset{\sim}{Y})$ associa $(\underset{\sim}{z}, \underset{\sim}{b}, c)=(H \underset{\sim}{x}, H \underset{\sim}{X}, H \underset{\sim}{Y})$, onde $H$ é definida em (2.8.8), a integral acima torna-se igual a

$$
\begin{gathered}
\int_{Z=\mathbb{R}^{n}} 1 /\left\{\left\|\underset{\sim}{z_{x}}\right\|\left[2 d+\left\|\underset{\sim}{z_{x}}\right\|^{2}\right]^{\frac{n+2 c-1}{2}}\left[2 g+\|\underset{\sim}{\underset{\sim}{z} x}-\underset{\sim}{b} X\|^{2}+\left(\sqrt{n} \bar{X}-z_{n}\right)^{2}\right]^{\frac{n}{2}+f}\right. \\
\left..\left[2 q+\left\|c_{Y}\right\|^{2} \operatorname{sen}^{2} \theta\right]^{\frac{n}{2}+p-1}\right\} \mathrm{d} \underset{\sim}{z}
\end{gathered}
$$

onde $\underset{\sim}{z},{\underset{\sim}{b}}_{X}$ e $\underset{\sim}{c} Y$ são os vetores formados pelas primeiras $n-1$ componentes dos vetores $\underset{\sim}{z}, \underset{\sim}{b}$ e $\underset{\sim}{c}$, respectivamente, e $\underset{\sim}{z}=\left(z_{1}, z_{2}, \ldots, z_{n}\right)=\left(\underset{\sim}{z}, z_{n}\right)$. 
Agora, eliminando $z_{n}$ por integração e assumindo que a desigualdade $(n+2 f>1)$ é válida, temos que a integral acima é proporcional a

$$
\begin{gathered}
\int_{Z_{x}=\mathbb{R}^{n-1}} 1 /\left\{\|\underset{\sim}{z}\|\left[2 d+\|\underset{\sim}{z}\|^{2}\right]^{\frac{n+2 c-1}{2}}\left[2 g+\|\underset{\sim}{z} x-\underset{\sim}{b} X\|^{2}\right]^{\frac{n+2 f-1}{2}}\right. \\
\left..\left[2 q+\left\|c_{\sim}\right\|^{2} \operatorname{sen}^{2} \theta\right]^{\frac{n}{2}+p-1}\right\} \mathrm{d} \underset{\sim}{z_{x}} .
\end{gathered}
$$

Para analisar essa integral, particionamos $Z_{x}$ da mesma forma que no Teorema 2.8.2, e assim temos que

Na região $R_{1}=\left\{\underset{\sim}{z} \in Z_{x} \mid\|\underset{\sim}{z}\|<\varepsilon\right\}$, ela converge se e somente se a integral $\int_{R_{1}} \frac{1}{\left\|z_{\sim}\right\|} \mathrm{d} z_{x}<\infty$, isto é, $n \geq 3$. Na região complementar, a integral converge se e somente se $1+\frac{2(n+2 c-1)}{2}+\frac{2(n+2 f-1)}{2}>n-1$, isto é, sempre.

Analisando simultaneamente todas as informações, concluímos que a posteriori é própria se e somente se $n \geq 3$.

Teorema 2.8.7 No modelo Normal em (2.8.16) e com priori $\pi(\underset{\sim}{\theta}) \propto \pi\left(\sigma_{x}^{2}\right) \pi\left(\sigma_{u}^{2}\right)$. .$\pi\left(\sigma_{e}^{2}\right)$, onde $\pi\left(\sigma_{x}^{2}\right) \propto\left(\sigma_{x}^{2}\right)^{-r}, r>0, \sigma_{u}^{2} \sim I G(f, g)$ e $\sigma_{e}^{2} \sim I G(p, q)$, a condição necessária e suficiente para a existência da posteriori correspondente é que $0<r<\frac{1}{2}$ $e n+2(r+f)>2$.

Demonstração: Substituindo-se $d$ por zero e $c$ por $r-1$ em (2.8.18), temos que

$$
\begin{aligned}
& \int_{\Theta} p\left(\underset{\sim}{\theta} \mid D_{\mathrm{obs}}\right) \mathrm{d} \underset{\sim}{\theta} \propto \\
& \int_{Z_{x}=\mathbb{R}^{n-1}} \frac{1}{\left\|\underset{\sim}{z_{x}}\right\|^{n+2 r-2}\left[2 g+\|\underset{\sim}{\underset{\sim}{*}} x-\underset{\sim}{b} X\|^{2}\right]^{\frac{n+2 f-1}{2}}\left[2 q+\|\underset{\sim}{\mathcal{C}} Y\|^{2} \operatorname{sen}^{2} \theta\right]^{\frac{n}{2}+p-1}} \mathrm{~d} \underset{\sim}{\underset{\sim}{z}} x .
\end{aligned}
$$

Para analisar essa integral particionamos $Z_{x}$ da mesma forma que no Teorema 2.8 .3 e assim temos que:

Na região $R_{1}=\left\{\underset{\sim}{z_{x}} \in Z_{x} \mid\left\|\underset{\sim}{z_{x}}\right\|<\varepsilon\right\}$, ela converge se e somente se $n+2 r-2<$ $n-1$, isto é, $0<r<\frac{1}{2}$.

$\mathrm{Na}$ região complementar, ela converge se e somente se $n+2 r-2+2\left(\frac{n+2 f-1}{2}\right)>$ $n-1$, isto é, $n+2(r+f)>2$. 
Analisando simultaneamente essas informações, concluímos que a posteriori é própria se e somente se $0<r<\frac{1}{2}$ e $n+2(r+f)>2$.

Teorema 2.8.8 No modelo Normal com erros nas variáveis dado em (2.8.16) e com priori $\pi(\underset{\sim}{\theta}) \propto \pi\left(\sigma_{x}^{2}\right) \pi\left(\sigma_{u}^{2}\right) \pi\left(\sigma_{e}^{2}\right)$, onde $\sigma_{x}^{2} \sim I G(c, d), \pi\left(\sigma_{u}^{2}\right) \propto\left(\sigma_{u}^{2}\right)^{-s}$ e $\sigma_{e}^{2} \sim$ $I G(p, q)$, onde $\underset{\sim}{X}$ e $\underset{\sim}{Y}$ são linearmente independentes e as componentes de $\underset{\sim}{X}$, bem como as de $\underset{\sim}{Y}$, não são todas iguais, então a condição necessária e suficiente para que a posteriori seja própria é que $n \geq 3 e 0<s<1$.

Demonstração: A demonstração é bastante parecida com a do Teorema 2.8 .9 a seguir. Por este motivo a omitimos.

Teorema 2.8.9 No modelo Normal com erros nas variáveis dado em (2.8.16) e com priori para $\underset{\sim}{\theta}$ dada por $\pi(\underset{\theta}{\theta}) \propto \pi\left(\sigma_{x}^{2}\right) \pi\left(\sigma_{u}^{2}\right) \pi\left(\sigma_{e}^{2}\right)$, onde $\pi\left(\sigma_{x}^{2}\right) \propto\left(\sigma_{x}^{2}\right)^{-r}, \pi\left(\sigma_{u}^{2}\right) \propto$ $\left(\sigma_{u}\right)^{-s}, \pi\left(\sigma_{e}^{2}\right) \sim I G(p, q)$, onde $\underset{\sim}{X}$ e $\underset{\sim}{Y}$ são linearmente independentes e as componentes de $\underset{\sim}{X}$, bem como as de $\underset{\sim}{Y}$, não são todas iguais, então a condição necessária e suficiente para que a posteriori seja própria é que $n+2(r+s)>4,0<r<\frac{1}{2}$, $0<s<1$.

Demonstração: Substituindo $d$ e $g$ por zero, $c$ por $r-1$ e $f$ por $s-1$ em (2.8.17), temos que

$$
\begin{aligned}
& \int_{\Theta} p\left(\theta \mid D_{\mathrm{obs}}\right) \mathrm{d} \theta \propto \int_{x} \frac{1}{S_{x x}^{\frac{n+2 r-2}{2}}\left[\sum_{i=1}^{n}\left(X_{i}-x_{i}\right)^{2}\right]^{\frac{n+2 s-2}{2}}\left[2 q+\frac{S_{x x} S_{Y Y}-S_{x Y}^{2}}{S_{x x}}\right]^{\frac{n+2 p-2}{2}}} \mathrm{~d} \underset{\sim}{x} \\
& =\int_{Z} \frac{1}{\left\|{\underset{\sim}{x} x}_{x}\right\|^{n+2 r-2}\left[\left\|{\underset{\sim}{z} x}_{x}-\underset{\sim}{b} X\right\|^{2}+\left(\sqrt{n} \bar{X}-z_{n}\right)^{2}\right]^{\frac{n+2 s-2}{2}}\left[2 q+\left\|c_{Y}\right\|^{2} \operatorname{sen}^{2} \theta\right]^{\frac{n+2 p-2}{2}}} \mathrm{~d} \underset{\sim}{z} .
\end{aligned}
$$

Integrando a função dentro do sinal de integração em (2.8.20), com relação a $z_{n}$, obtemos a função

$$
\frac{1}{\left\|z_{\sim}\right\|^{n+2 r-2}\left[2 q+\|\subseteq Y\|^{2} \operatorname{sen}^{2} \theta\right]^{\frac{n+2 p-2}{2}}\left(\left\|z_{x}-\underset{\sim}{b} X\right\|^{2}\right)^{\frac{n+2 s+2}{2}}} \int_{-\infty}^{\infty} \frac{1}{\left(1+\left(\frac{\sqrt{n} \bar{X}_{-z_{n}}}{\left\|{\underset{\sim}{z} x^{-}}_{\sim}^{b}\right\|}\right)^{2}\right)^{\frac{n+2 s-2}{2}}} \mathrm{~d} z_{n} .
$$


Agora, fazendo $u=\frac{\sqrt{n} \bar{X}-z_{n}}{\|\underset{\sim}{Z} x-\underset{\sim}{b}\|}$, a integral

$$
\int_{-\infty}^{\infty} \frac{1}{\left(1+\left(\frac{\sqrt{n} \bar{X}-z_{n}}{\|\underset{\sim}{z}-\underset{\sim}{b} x\|}\right)^{2}\right)^{\frac{n+2 s-2}{2}}} \mathrm{~d} z_{n}=\|\underset{\sim}{\underset{\sim}{z}} \underset{x}{-\underset{\sim}{b} x}\| \int_{-\infty}^{\infty} \frac{1}{\left(1+u^{2}\right)^{\frac{n+2 s-2}{2}}} \mathrm{~d} u
$$

onde $\int_{-\infty}^{\infty} \frac{1}{\left(1+u^{2}\right)^{\frac{n+2 s-2}{2}}} \mathrm{~d} u<\infty$ se e somente se $2\left(\frac{n+2 s-2}{2}\right)>1$, isto é, $n+2 s>3$ (notemos que a função $\frac{1}{\left[1+u^{2}\right]^{\frac{n+2 s-2}{2}}}$ só não está definida num único ponto $u$ quando $\underset{\sim}{z}=\underset{\sim}{b} x$ e, portanto, não traz problemas para a integral de Riemann).

Portanto, a integral em (2.8.20) é proporcional a

$$
\int_{Z_{x}} \frac{1}{\left\|\underset{\sim}{z_{x}}\right\|^{n+2 r-2}\left[\left\|{\underset{\sim}{x}}_{x}-\underset{\sim}{b} X\right\|\right]^{n+2 s-3}\left[2 q+\left\|\mathcal{C}_{Y}\right\|^{2} \operatorname{sen}^{2} \theta\right]^{\frac{n+2 p-2}{2}}} \mathrm{~d}{\underset{\sim}{x}}_{x}
$$

Para analisar essa integral, particionamos a região de integração $Z_{x}$ nas regiões:

$R_{1}=\left\{\underset{\sim}{z_{x}} \in \mathbb{R}^{n-1}:\|\underset{\sim}{z}\|<\varepsilon_{1}\right\}$ para $\varepsilon_{1}$ suficientemente pequeno (por exemplo, $\left.\varepsilon_{1}=\frac{\left\|b_{x}\right\|}{10}\right)$

$R_{2}=\left\{\underset{\sim}{z} \in \mathbb{R}^{n-1}: \varepsilon_{1} \leq\|\underset{\sim}{z}\|<k\right\}$, onde $k$ é suficientemente grande (por exemplo, $k=2\|\underset{\sim}{b} x\|)$

$R_{2 a}=\left\{\underset{\sim}{z} \in R_{2}:\left\|\underset{\sim}{z} \varepsilon_{1}-\underset{\sim}{b}\right\|<\varepsilon_{2}\right\}$, onde $\varepsilon_{2}$ é suficientemente pequeno (por exemplo, $\left.\varepsilon_{2}=\frac{\left\|{\underset{\sim}{x}}_{X}\right\|-\varepsilon_{1}}{10}\right)$,

$R_{3}=\left\{\underset{\sim}{z_{x}} \in \mathbb{R}^{n-1}:\left\|{\underset{\sim}{x}}_{x}\right\| \geq k\right\}$

Em $R_{1}$, a integral é finita se e somente se $n+2 r-2<n-1$, isto é, $0<r<\frac{1}{2}$.

Em $R_{3}$, ela é finita se e somente se $n+2 r-2+n+2 s-3>n-1$, isto é, $n+2(r+s)>4$.

Em $R_{2 a}$, fazendo-se a transformação $\underset{\sim}{u}=\underset{\sim}{z} x-\underset{\sim}{b} x$, a integral acima é proporcional a

$$
\int_{\| u} \frac{1}{\| \varepsilon_{2}} \frac{1}{\|u+\underset{\sim}{b} x\|^{n+2 r-2}[\underset{\sim}{u}]^{n+2 s-3}} \mathrm{~d} \underset{\sim}{u}
$$

que é finita se e somente se $n+2 s-3<n-1$, isto é, $0<s<1$. 
Em $R_{2} \cap R_{2 a}^{c}$, a integral é finita pois a função dentro do sinal de integração é limitada e o domínio de integraç ao $R_{2} \cap R_{2 a}^{c}$ é um conjunto limitado.

Analisando simultaneamente todas essas informações, concluímos que a posteriori é própria se e somente se $0<r<\frac{1}{2}, n+2(r+s)>4$ e $0<s<1$.

Observação 1: As condições para a existência da posteriori nos modelos com e sem restrição de identificabilidade são bem distintas (principalmente entre os teoremas 2.8.4 e 2.8.8 e entre os teoremas 2.8 .5 e 2.8 .9 )

Observação 2: Os modelos dessa seção apresentam dois problemas distintos: envolvem uma verossimilhança não identificável e incorporam prioris impróprias. Embora tenhamos conseguido provar a existência das posterioris a partir de certas prioris impróprias, ainda há o problema da falta de identificabilidade que pode acarretar em falha na convergência do algoritmo de Gibbs especialmente porque a priori é pouco informativa. ( $\mathrm{Na}$ seção 2.12 , entretanto, exibimos um conjunto de dados onde aparentemente não ocorre problema de convergência do algoritmo de Gibbs, onde este foi baseado no modelo não identificável com priori praticamente não informativa).

\subsection{Estimação do modelo}

Para estimar os parâmetros do modelo, podemos usar vários estimadores que correspondem a diferentes funções de perda. É fato bastante conhecido que tomando-se a função perda quadrática, o estimador de Bayes de $\tau(\theta)$, que é o estimador que minimiza a perda esperada com relação à distribuição à posteriori (isto é, o estimador que minimiza o risco à posteriori) é a média da distribuição de $\tau(\underset{\sim}{\theta})$. No caso da função perda ser igual ao desvio absoluto, o estimador de Bayes é a mediana da posteriori e no caso da função perda 0-1 é a moda da posteriori.

Nos exemplos de aplicação vamos apresentar não somente as estimativas pontuais 
da média, mediana, quantis e variância da posteriori de cada um dos parâmetros, como também os intervalos de credibilidade de comprimento mínimo para cada parâmetro (que coincidem com os intervalos "Highest Posterior Density", ou HPD, quando as distribuições forem unimodais). Além das estimativas pontuais e por intervalo, vamos apresentar também as estimativas das densidades marginais à posteriori de cada um dos parâmetros do modelo.

Partindo do pressuposto que os $\left(T-T_{0}\right)$ vetores de observações gerados pelo algoritmo MCMC (onde $T_{0}=$ "burn in period") podem ser considerados como uma amostra da distribuição à posteriori que desejamos analisar, então as medidas acima podem ser usadas para fazer inferência sobre essa posteriori.

Se tivermos gerado $m$ cadeias paralelas, podemos utilizar essa informação na estimação. Na tabela abaixo apresentamos o diagrama das observações provenientes de $m$ cadeias paralelas geradas por um algoritmo MCMC.

\begin{tabular}{|c|c|c|c|}
\hline cadeia 1 & cadeia 2 & $\cdots$ & cadeia $m$ \\
\hline$\dot{\theta}_{1}^{(1)}$ & $\theta_{2}^{(1)}$ & $\cdots$ & ${\underset{\sim}{\theta}}_{m}^{(1)}$ \\
\hline$\vdots$ & & & : \\
\hline$\underline{\theta}_{1}^{\left(T_{0}\right)}$ & $\underset{\sim}{\theta} \underset{2}{\left(T_{0}\right)}$ & $\cdots$ & $\underline{\theta}_{m}^{\left(T_{0}\right)}$ \\
\hline$\vdots$ & & & $\vdots$ \\
\hline$\theta_{1}^{(T)}$ & $\underline{\theta}_{2}^{(T)}$ & $\cdots$ & $\underline{\theta}_{m}^{(T)}$ \\
\hline
\end{tabular}

No caso, por exemplo, do modelo estrutural com restrição de identificabilidade $\sigma_{e}^{2}=\lambda \sigma_{u}^{2}=\lambda \sigma^{2}, \theta_{i}^{(j)}$ é igual a $\left(\alpha_{i}^{(j)}, \beta_{i}^{(j)}, \mu_{i}^{(j)}, \sigma_{x(i)}^{(j)}, \sigma_{(i)}^{2^{(j)}}\right)$. Assim, por exemplo, uma estimativa simples da média e variância da posteriori de $\alpha$, é dada por

$$
\begin{aligned}
\hat{E}\left(\alpha \mid D_{\mathrm{obs}}\right) & =\frac{1}{m\left(T-T_{0}\right)} \sum_{i=1}^{m} \sum_{j=T_{0}+1}^{T} \alpha_{i}^{(j)} \\
\widehat{\operatorname{Var}}\left(\alpha \mid D_{\mathrm{obs}}\right) & =\frac{1}{m\left(T-T_{0}\right)-1} \sum_{i=1}^{m} \sum_{j=T_{0}+1}^{T}\left(\alpha_{i}^{(j)}-\hat{E}\left(\alpha \mid D_{\mathrm{obs}}\right)\right)^{2}
\end{aligned}
$$

e uma estimativa simples da densidade marginal à posteriori de $\alpha$ é obtida fazendo-se um histograma com os valores $\alpha_{i}^{(j)}, i=1, \ldots, m, j=T_{0}, \ldots, T$. 
Observação 1: No modelo estrutural com restrição de identificabilidade $\sigma_{e}^{2}=$ $\lambda \sigma^{2}$, a densidade condicional completa à posteriori de $\alpha, \pi\left(\alpha \mid \beta, \mu, \sigma_{x}^{2}, \sigma^{2}, \underset{\sim}{x}, D_{\text {obs }}\right)$ é conhecida (e tem esperança e variância finita) dada em (2.5.9) por

$$
\begin{aligned}
& \alpha \mid \beta, \mu, \sigma_{x}, \sigma^{2}, \underset{\sim}{x}, D_{\text {obs }} \sim N(A, B), \text { onde } \\
& A=\frac{n \sigma_{\alpha}^{2}(\bar{Y}-\beta \bar{x})+\lambda a \sigma_{\alpha}^{2}}{n+\sigma_{\alpha}^{2}+\lambda \sigma^{2}}, \text { onde } \bar{x}=\frac{1}{n} \sum_{t=1}^{n} x_{t}, \\
& \text { e } \quad B=\frac{\lambda \sigma^{2} \sigma_{\alpha}^{2}}{n \sigma_{\alpha}^{2}+\lambda \sigma^{2}} .
\end{aligned}
$$

Então a média da distribuição marginal de $\alpha, E\left(\alpha \mid D_{\text {obs }}\right)$, pode ser estimada pelo método de Monte Carlo por

$$
\frac{1}{m\left(T-T_{0}\right)} \sum_{i=1}^{m} \sum_{j=T_{0}+1}^{T} E\left(\alpha \mid \beta_{i}^{(j)}, \mu_{i}^{(j)}, \sigma_{x(i)}^{2^{(j)}}, \sigma_{i}^{2^{(j)}},{\underset{\sim}{x}}_{i}^{(j)}, D_{\mathrm{obs}}\right)=\frac{1}{m\left(T-T_{0}\right)} \sum_{i=1}^{m} \sum_{j=T_{0}+1}^{T} A_{i}^{(j)} .
$$

Analogamente, a variância, $\operatorname{Var}\left(\alpha \mid D_{\text {obs }}\right)$, pode ser estimada por

$$
\frac{1}{m\left(T-T_{0}\right)} \sum_{i=1}^{m} \sum_{j=T_{0}+1}^{T} B_{i}^{(j)}+\frac{1}{m\left(T-T_{0}\right)} \sum_{i=1}^{m} \sum_{j=T_{0}+1}^{T}\left(A_{i}^{(j)}\right)^{2}-\left(\frac{\sum_{i=1}^{m} \sum_{j=T_{0}+1}^{T} A_{i}^{(j)}}{m\left(T-T_{0}\right)}\right)^{2},
$$

onde

$$
\begin{aligned}
A_{i}^{(j)} & =\frac{n \sigma_{\alpha}^{2}\left(\bar{Y}-\beta_{i}^{(j)} \bar{\sim}_{(i)}^{(j)}\right)+\lambda a \sigma_{i}^{2(j)}}{n \sigma_{\alpha}^{2}+\lambda \sigma_{i}^{2(j)}}, \text { onde } \underset{\sim}{\bar{x}_{(i)}^{(j)}}=\frac{1}{n} \sum_{t=1}^{n} x_{t(i)}^{(j)}, \\
\mathrm{e} \quad B_{i}^{(j)} & =\frac{\lambda \sigma_{i}^{2^{(j)}} \sigma_{\alpha}^{2}}{n \sigma_{\alpha}^{2}+\lambda \sigma_{i}^{2(j)}} .
\end{aligned}
$$

Esses estimadores são conhecidos como "estimadores Rao-Blackwelizados".

Usando o mesmo raciocínio, estima-se a própria densidade marginal de $\alpha$ por

$$
\hat{p}\left(\alpha \mid D_{\text {obs }}\right)=\frac{1}{m\left(T-T_{0}\right)} \sum_{i=1}^{m} \sum_{j=T_{0}+1}^{T} \pi\left(\alpha \mid \beta_{i}^{(j)}, \mu_{i}^{(j)}, \sigma_{x(i)}^{(j)}, \sigma_{i}^{2^{(j)}}, x_{\sim}^{(j)}, D_{\text {obs }}\right),
$$

onde

$$
\pi\left(\alpha \mid \beta_{i}^{(j)}, \mu_{i}^{(j)}, \sigma_{x(i)}^{2^{(j)}}, \sigma_{i}^{2}, \underset{\sim}{x}(j)=\frac{1}{\sqrt{2 \pi B_{i}^{(j)}}} \exp \left\{-\frac{1}{2 B_{i}^{(j)}}\left(\alpha-A_{i}^{(j)}\right)^{2}\right\}\right.
$$


Observação 2: As distribuições marginais à posteriori dos parâmetros $\beta, \mu, \sigma_{x}^{2}$ e $\sigma^{2}$ no modelo estrutural dado em (2.3.1), bem como suas médias e variâncias podem ser estimadas de forma análoga à descrita na Observação 1, utilizando-se respectivamente as condicionais completas dadas em (2.5.9) a (2.5.13).

Observação 3: Para estimar a variância, por exemplo, de $\hat{E}\left(\alpha \mid D_{\text {obs }}\right)$, utilizando uma única e longa cadeia de comprimento $T-T_{0}$, podemos formar $n$ grupos de $k=\frac{\left(T-T_{0}\right)}{n}$ observações e calcular $\bar{\alpha}_{1}, \bar{\alpha}_{2}, \ldots, \bar{\alpha}_{n}$, onde $\bar{\alpha}_{i}$ é a média amostral do $i$-ésimo grupo ( $k$ deve ser suficientemente grande de tal modo que a autocorrelação amostral obtida com base nos $\bar{\alpha}_{i}$ é bem pequena, digamos menor que 0,1 ). Nesse caso, um estimador de $\operatorname{Var}\left(\hat{E}\left(\alpha \mid D_{\text {obs }}\right)\right)$ é dado por

$$
\frac{1}{n(n-1)} \sum_{i=1}^{n}\left(\bar{\alpha}_{i}-\bar{\alpha}\right)^{2}, \text { onde } \bar{\alpha}=\frac{1}{n} \sum_{i=1}^{n} \bar{\alpha}_{i} .
$$

Outro estimador (proposto por Kass et al., 1998) é dado por

$$
\widehat{\operatorname{Var}}(\hat{\alpha})=\frac{S_{\alpha}^{2}}{E S S}
$$

onde $S_{\alpha}^{2}=\sum_{i=T_{0}+1}^{T} \frac{\left(\alpha_{i}-\hat{\alpha}\right)}{\left(T-T_{0}\right)-1} \quad$ e $\quad E S S=\frac{N}{1+2 \sum_{k=1}^{\infty} \hat{\rho}_{k}(\alpha)}$, onde $\rho_{k}(\alpha)$ é a autocorrelação no lag $k$ para o parâmetro $\alpha$ (que pode ser estimada usando as autocorrelações amostrais a partir dos dados gerados da cadeia. A série $\sum_{k=1}^{\infty} \hat{\rho}_{k}(\alpha)$ é aproximada desprezando-se todos os termos a partir do $k_{0}+1$-ésimo termo, quando $\left.\left|\rho_{k_{0}}(\alpha)\right|<0,1\right)$.

As variâncias das médias dos demais parâmetros do modelo podem ser estimadas da mesma forma.

\subsection{Avaliação do ajuste e análise de sensibilidade}

Na subseção 2.10.1 analisamos a sensibilidade na estimação da média dos parâmetros do modelo estrutural a diferentes valores do hiperparâmetro $\lambda$, onde $\lambda=\sigma_{e}^{2} / \sigma_{u}^{2}$, e 
na sação 2.10 .2 avaliamos a qualidade do ajuste.

\subsubsection{Sensibilidade do modelo a diferentes valores da razão das variâncias residuais}

No modelo estrutural Normal com restrição de identificabilidade $\sigma_{e}^{2}=\lambda \sigma_{u}^{2}$, a constante $\lambda$ é tratada como hiperparâmetro conhecido, e é importante fazer uma avaliação da sensibilidade na estimação dos parâmetros quando diferentes valores de $\lambda$ são fixados. Nesse trabalho limitamo-nos apenas a uma análise exploratória, construindo gráficos das médias amostrais de cada parâmetro em função de $\lambda$ mantendo todos os demais parâmetros fixados ( isto é, variando apenas o valor de $\lambda$, onde $\lambda>0$ ) e usando amostras da posteriori obtidas por exemplo por algum método MCMC. Na seção 2.12 esse procedimento é ilustrado por meio de um exemplo com dados simulados. Um estudo mais completo sobre esse tópico deve ser conduzido futuramente.

\subsubsection{Avaliação da qualidade do ajuste}

Após ajustarmos o modelo aos dados devemos verificar se esse ajuste é adequado, isto é, se o modelo é compatível com os dados. A literatura bayesiana sobre o assunto é bem vasta e muitas das técnicas de diagnóstico utilizam a distribuição preditiva à posteriori de alguma forma, por exemplo, comparando os dados observados com os dados simulados pela priori preditiva (já que se o modelo está bem ajustados esses dois conjuntos de dados devem se assemelhar); um exemplo é o conjunto de técnicas denominado "posterior preditive checks" (Gelman et al., 1997, cap. 6). Outra possibilidade também é estudar os resíduos baseados na diferença entre os valores observados e os valores previstos a partir da posteriori preditiva. Para uma breve apresentação das técnicas de diagnóstico mais conhecidas, ver Carlin e Louis (2000), Chen et al. (2000), Gelman et al. (1997). Outras referências muito importantes são 
Spiegelhalter et al. (2002) e o relatório técnico de Dey e Maiti (2002).

Nesse capítulo vamos nos restringir somente ao cálculo da densidade condicional preditiva de validação cruzada e dos resíduos de validação cruzada para o modelo estrutural Normal.

Observação: Convém lembrar entretanto que além da avaliação da qualidade do ajuste ou adequação do modelo ("internal checking") onde são utilizadas medidas de diagnóstico para verificar se o modelo é compatível com os dados, isto é, se o modelo se ajusta adequadamente aos dados, é importante também avaliar o efeito nas inferências quando diferentes modelos alternativos são utilizados para o mesmo problema ("external checking") como por exemplo modelos com diferentes prioris, diferentes hiperparâmetros, diferentes verossimilhanças, diferentes estruturas hierárquicas, etc., já que esses modelos podem estar igualmente bem ajustados mas nos levarem a diferentes conclusões.

\section{(a) Densidade preditiva de validação cruzada}

Quando o tamanho da amostra for relativamente pequeno, que não possamos nos dar ao luxo de reservar uma parte dos dados (digamos 20 a 30\%) apenas para a validação do modelo (ou seja, usar somente 70 a $80 \%$ dos dados para o ajuste do modelo), então uma solução é utilizar a "densidade condicional preditiva de validação" cruzada, $\pi\left(Y_{i} \mid Y_{(-i)}\right), i=1, \ldots, n$, onde $\underset{\sim}{Y}=\left(Y_{1}, \ldots, Y_{n}\right)$ é a amostra dos dados e $Y_{(-i)}$ é a amostra resultante após a exclusão da observação $Y_{i}$.

Essa densidade é conhecida como " $\mathrm{CPO}_{i}$ " ("Conditional Predictive Ordinate"), e dá a "verossimilhança" de cada ponto $Y_{i}$ condicionada ao restante das observações $Y_{(-i)}$

$$
\begin{aligned}
& \mathrm{CPO}_{i}=\pi\left(Y_{i} \mid Y_{(-i)}\right)=\int_{\Theta} \pi\left(Y_{i}, \underset{\sim}{\theta} \mid Y_{(-i)}\right) \mathrm{d} \underset{\sim}{\theta} \\
& =\int_{\Theta} \pi\left(Y_{i} \mid \underset{\sim}{\theta}, Y_{(-i)}\right) \pi\left(\underset{\sim}{\theta} \mid Y_{(-i)}\right) \mathrm{d} \underset{\sim}{\theta},
\end{aligned}
$$


onde $\underset{\sim}{\theta}$ é o vetor de parâmetros do modelo considerado.

Essa medida foi proposta inicialmente por Geisser (1980) e estudada posteriormente por Gelfand et al. (1992). O gráfico dos valores da $\mathrm{CPO}_{i}$ versus $i$, por exemplo, pode ser usado como diagnóstico para detectar "outliers", já que um valor "alto" de $\mathrm{CPO}_{i}$ indica que a observação $Y_{i}$ está bem ajustada, e um valor "baixo", o contrário.

No modelo estrutural, a amostra das observações é $D_{\text {obs }}=\left(\underset{\sim}{U_{1}}, \ldots,{\underset{\sim}{n}}_{n}\right)$, onde $\underset{\sim}{U_{i}}=\left(\begin{array}{l}Y_{i} \\ X_{i}\end{array}\right)$ é a $i$-ésima observação e ${\underset{\sim}{(-i)}}_{(-1)}=D_{\text {obs(-i) }}$ é a amostra após a extração de $U_{i}$.

A seguir apresentamos três fórmulas alternativas de estimar a $\mathrm{CPO}_{i}$ para o modelo estrutural Normal, usando o fato que

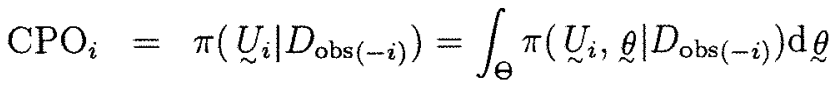

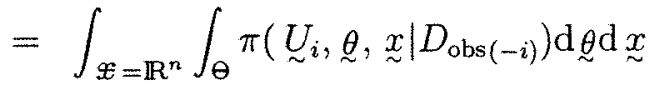

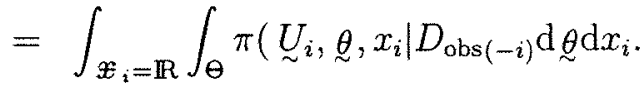

\section{Cálculo da $\mathrm{CPO}_{i}$}

As fórmulas a seguir referem-se ao modelo sem restrições de identificabilidade. No caso do modelo com a restrição $\sigma_{e}^{2}=\lambda \sigma_{u}^{2}=\lambda \sigma^{2}$, basta substituir nessas fórumlas essa restrição.

\section{(1) Fórmula 1}

$$
\begin{aligned}
& \mathrm{CPO}_{i}=\pi\left({\underset{\sim}{i}}_{i} \mid D_{\mathrm{obs}(-i)}\right)=\int_{\Theta} \pi\left(\underset{\sim}{U_{i}} \mid \underset{\sim}{\theta}\right) \pi\left(\underset{\sim}{\theta} \mid D_{\mathrm{obs}(-i)}\right) \mathrm{d} \underset{\sim}{\theta} \\
& =\left[\int_{\Theta} \frac{1}{\pi\left({\underset{\sim}{i} \mid}_{i} \mid \theta\right)} \pi\left(\underset{\sim}{\theta} \mid D_{\mathrm{obs}}\right) \mathrm{d} \theta\right]^{-1}, i=1, \ldots, n,
\end{aligned}
$$

onde ${\underset{\sim}{i}}_{i}=\left(\begin{array}{l}Y_{1}^{\prime} \\ X_{1}\end{array}\right)$ e $\pi\left(\underset{\sim}{\theta} \mid D_{\text {obs }}\right)$ é a posteriori de $\underset{\sim}{\theta}$.

$$
U_{i} \mid \underset{\sim}{\operatorname{iid}} \sim\left(\left(\begin{array}{c}
\alpha+\beta \mu \\
\mu
\end{array}\right) ; \quad\left(\begin{array}{cc}
\beta^{2} \sigma_{x}^{2}+\sigma_{e}^{2} & \beta \sigma_{x}^{2} \\
\beta \sigma_{x}^{2} & \sigma_{x}^{2}+\sigma_{u}^{2}
\end{array}\right)\right)
$$


isto é,

$$
\begin{aligned}
\pi\left(\underset{\sim}{\left.U_{i} \mid \underline{\theta}\right)=}\right. & (2 \pi|\Sigma|)^{-\frac{1}{2}} \exp \left\{-\frac{1}{2|\Sigma|}\left[\left(\beta^{2} \sigma_{x}^{2}+\sigma_{e}^{2}\right)\left(X_{i}-\mu\right)^{2}\right.\right. \\
& \left.\left.-2 \beta \sigma_{x}^{2}\left(Y_{i}-\alpha-\beta \mu\right)\left(X_{i}-\mu\right)+\left(\sigma_{x}^{2}+\sigma_{u}^{2}\right)\left(Y_{i}-\alpha-\beta \mu\right)^{2}\right]\right\}
\end{aligned}
$$

onde $|\Sigma|=\beta^{2} \sigma_{u}^{2} \sigma_{x}^{2}+\sigma_{e}^{2} \sigma_{x}^{2}+\sigma_{e}^{2} \sigma_{u}^{2}$

Demonstração: $\pi\left({\underset{\sim}{i}}_{i} \mid D_{\mathrm{obs}(-i)}\right)=\int_{\Theta} \pi\left({\underset{\sim}{i}}_{i}, \underset{\sim}{ } \mid D_{\mathrm{obs}(-i)}\right) \mathrm{d} \underset{\sim}{\theta}$

$$
=\int_{\Theta} \pi\left(\underset{\sim}{U_{i} \mid \theta}, D_{\mathrm{obs}(-i)}\right) \pi\left(\underset{\sim}{\theta} \mid D_{\mathrm{obs}(-i)}\right) \mathrm{d} \underset{\sim}{\theta}=\int_{\Theta} \pi\left(\underset{\sim}{U_{i}} \mid \underset{\sim}{\theta}\right) \pi\left(\underset{\sim}{\theta} \mid D_{\mathrm{obs}(-i)}\right) \mathrm{d} \underset{\sim}{\theta} .
$$

A última igualdade segue pelo fato de que dado $\underset{\sim}{\theta}$, então $\underset{\sim}{U_{i}}$ e $D_{\text {obs(-i) }}$ são independentes. Para completar a demonstração, usamos a seguinte igualdade:

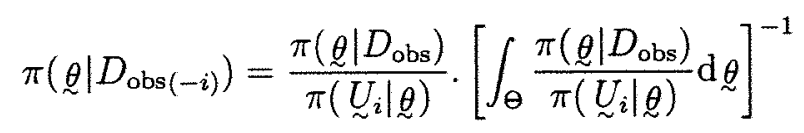

Portanto,

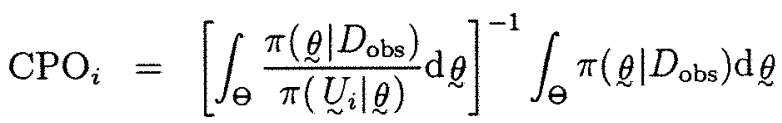

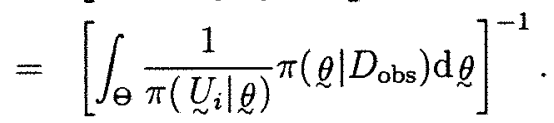

A igualdade em (2.10.3) sai da seguinte relação:

$$
\pi\left(\underset{\sim}{\theta} \mid D_{\text {obs }}\right)=\frac{\pi\left(\underset{\sim}{\theta},{\underset{\sim}{i}}_{i},{\underset{\sim}{(-i)}}_{(-i)}\right)}{\pi\left(D_{\text {obs }}\right)} \propto \pi\left(\underset{\sim}{U_{i} \mid \theta}, \underset{\sim}{U_{(-i)}}\right) \pi\left(\underset{\sim}{\theta} \mid \underset{\sim}{\left.U_{(-i)}\right)}\right)
$$

onde $\pi\left(\underset{\sim}{U_{i} \mid \theta,}, U_{(-i)}\right)=\pi\left(\underset{\sim}{U_{i}} \mid \underset{\sim}{\theta}\right)$ e portanto

$$
\pi\left(\underset{\sim}{\theta} \mid D_{\mathrm{obs}(-i)}\right) \propto \frac{\pi\left(\underset{\theta}{\theta} \mid D_{\mathrm{obs}}\right)}{\pi\left(\underset{\sim}{\left.U_{i} \mid \theta\right)} . \underset{\sim}{\theta} .\right.}
$$

Assim,

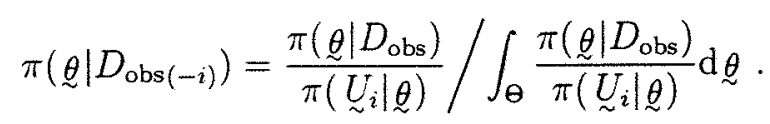

Observação: É importante ressaltar que o truque usado acima permite que o cálculo da $\mathrm{CPO}_{i}$ para todo $i=1, \ldots, n$ seja feito usando a mesma posteriori $\pi\left(\underset{\theta}{\theta} \mid D_{\mathrm{obs}}\right)$. 


\section{Estimativa de $\mathrm{CPO}_{i}$}

Dada uma amostra $\left({\underset{\sim}{(1)}}^{(1)}, \ldots,{\underset{\theta}{(G)}}^{(G)}\right.$ da posteriori $p\left(\underset{\sim}{\theta} \mid D_{\text {obs }}\right)$ do modelo estrutural Normal, obtida por exemplo por algum dos métodos MCMC descritos no Capítulo 2, então uma estimativa de Monte Carlo de $\mathrm{CPO}_{i}$ é dada por

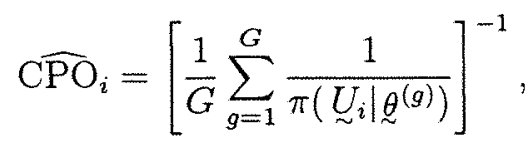

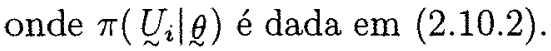

Observação: Computacionalmente esse método é muito eficiente pois a amostragem é feita de uma única posteriori. Caso contrário deveríamos obter para cada $i$ uma amostra $\left({\underset{\sim}{(i)}}_{(i)}^{(1)}, \ldots,{\underset{\sim}{(i)}}_{(G)}^{(G)}\right)$, da posteriori correspondente $p\left(\underset{\sim}{\theta} \mid D_{\text {obs }(-i)}\right)$ e uma estimativa de Monte Carlo de $\mathrm{CPO}_{i}$ seria

$$
\widehat{\mathrm{CPO}}_{i}=\frac{1}{G} \sum_{g=1}^{G} \pi\left({\underset{\sim}{i}}_{i} \mid \underset{(i)}{(g)}\right), \quad i=1, \ldots, n .
$$

O problema agora é que isso traria um alto "custo computacional", especialmente se $n$ é muito grande (já que amostramos $\mathrm{G}$ elementos de cada uma das n posterioris distintas $p\left(\underset{\sim}{\theta} \mid D_{\mathrm{obs}(-1)}\right), \ldots, p\left(\underset{\sim}{\theta} \mid D_{\mathrm{obs}(-n)}\right)$ para obter $\left.\widehat{\mathrm{COO}}_{1}, \ldots, \widehat{\mathrm{CO}}_{n}\right)$.

\section{(2) Fórmula 2}

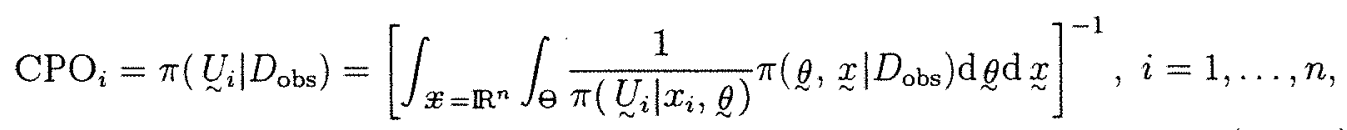

onde $\underset{\sim}{x}=\left(x_{1}, \ldots, x_{n}\right)^{T}, \pi\left(\underset{\sim}{\theta}, \underset{\sim}{x} \mid D_{\text {obs }}\right)$ é a distribuição conjunta de $\underset{\sim}{x}$ e $\underset{\sim}{\theta}$ à posteriori e

$$
U_{i} \mid x_{i}, \underset{g}{\stackrel{\mathrm{iid}}{\sim}} N\left(\left(\begin{array}{c}
\alpha+\beta x_{i} \\
x_{i}
\end{array}\right) ; \quad\left(\begin{array}{cc}
\sigma_{e}^{2} & 0 \\
0 & \sigma_{u}^{2}
\end{array}\right)\right)
$$

isto é,

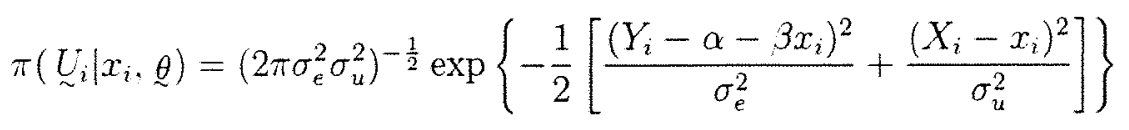




\section{Demonstração:}

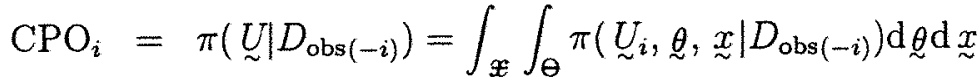

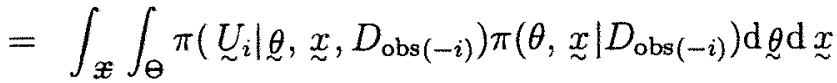

$$
\begin{aligned}
& =\int_{\Re} \int_{\Theta} \pi\left(\underset{\sim}{U_{i}} \mid x_{i}, \underset{\sim}{\theta}\right) \pi\left(\underset{\sim}{\theta} \underset{\sim}{x} \mid D_{\mathrm{obs}(-i)}\right) \mathrm{d} \underset{\sim}{\theta} \mathrm{d} \underset{\sim}{x},
\end{aligned}
$$

onde

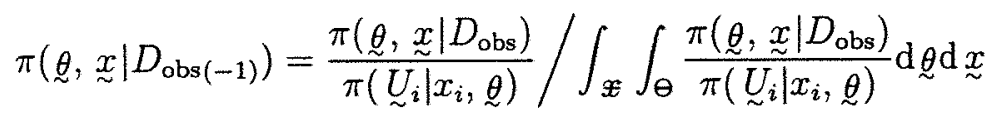

e portanto

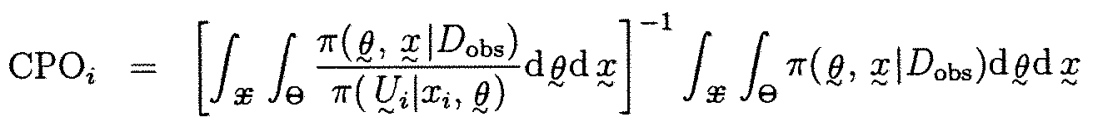

$$
\begin{aligned}
& =\left[\int_{\mathfrak{x}} \int_{\Theta} \frac{\pi\left(\underset{\sim}{\theta}, \underset{\sim}{x} \mid D_{\mathrm{obs}}\right)}{\pi\left(\underset{\sim}{\left.U_{i} \mid x_{i}, \theta\right)} \mathrm{d}\right)} \underset{\underset{\sim}{\theta}}{\mathrm{d}} \underset{\sim}{x}\right]^{-1} \text {. }
\end{aligned}
$$

A igualdade em (2.10.7) é obtida a partir da relação

$$
\pi\left(\underset{\sim}{\theta}, \underset{\sim}{x} \mid D_{\mathrm{obs}}\right) \propto \pi\left(\underset{\sim}{\theta} \underset{\sim}{x}, U_{i}, D_{\mathrm{obs}(-i)}\right)=\pi\left(\underset{\sim}{U_{i}} \mid x_{i}, \underset{\sim}{\theta}\right) \pi\left(\underset{\sim}{x}, \underset{\theta}{\theta} \mid D_{\mathrm{obs}(-i)}\right)
$$

e portanto

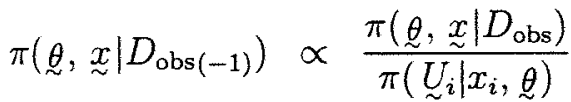

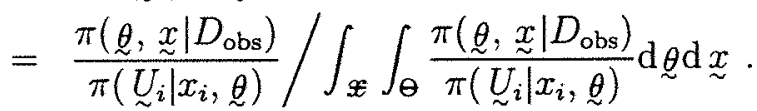

\section{Estimativa de $\mathrm{CPO}_{i}$}

Dada uma amostra $(\underbrace{(1)},{\underset{\sim}{x}}^{(1)}, \ldots,{\underset{\theta}{(G)}}^{(G)}{\underset{\sim}{(G)}}^{(G)}$ da distribuição $p\left(\underset{\sim}{\theta}, \underset{\sim}{x} \mid D_{\text {obs }}\right)$, obtida por exemplo pelo algoritmo de Gibbs (desde que ele convirja), então uma estimativa de Monte Carlo da $\mathrm{CPO}_{i}$ é dada por

$$
\widehat{\mathrm{CPO}}_{i}=\left[\frac{1}{G} \sum_{g=1}^{G} \frac{1}{\pi(\underbrace{}_{i} \mid x_{i}^{(g)}, \theta^{(g)})}\right]^{-1},
$$


onde $\pi\left({\underset{\sim}{i}}_{i} \mid x_{i}, \underset{\sim}{\theta}\right)$ é dada em (2.10.6) e onde $x_{i}^{(g)}$ é a $i$-ésima componente do vetor gerado ${\underset{\sim}{x}}^{(g)}$.

\section{(3) Fórmula 3}

$$
\begin{aligned}
\mathrm{CPO}_{i} & =\pi\left({\underset{\sim}{U}}_{i} \mid D_{\mathrm{obs}(-i)}\right) \\
& =\left[\int_{x_{i}=\mathbb{R}} \int_{\Theta} \frac{1}{\pi\left(U_{i} \mid x_{i}, \underset{\sim}{\theta}\right)} \pi\left(x_{i} \mid \underset{\sim}{\theta}\right) \pi\left(\underset{\sim}{\theta} \mid D_{\mathrm{obs}}\right) \mathrm{d} \underset{\sim}{\theta} \mathrm{d} x_{i}\right]^{-1},
\end{aligned}
$$

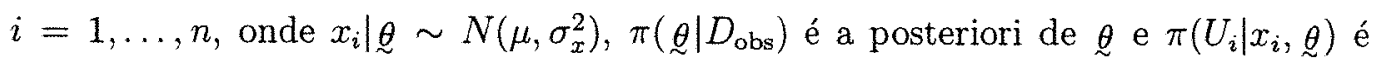
dada em (2.10.6).

\section{Demonstração}

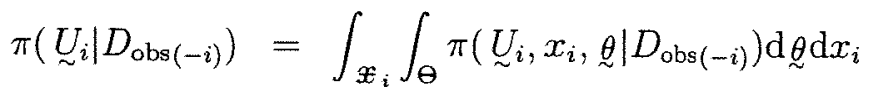

$$
\begin{aligned}
& =\int_{x_{i}} \int_{\Theta} \pi\left({\underset{\sim}{i}}_{i} \mid x_{i}, \underset{\sim}{\theta}, D_{\mathrm{obs}(-i)}\right) \pi\left(x_{i}, \underset{\sim}{\theta} \mid D_{\mathrm{obs}(-i)}\right) \mathrm{d} \underset{\sim}{\theta} \mathrm{d} x_{i},
\end{aligned}
$$

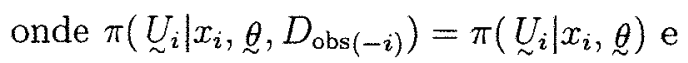

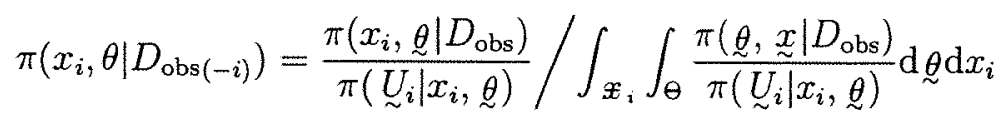

e portanto

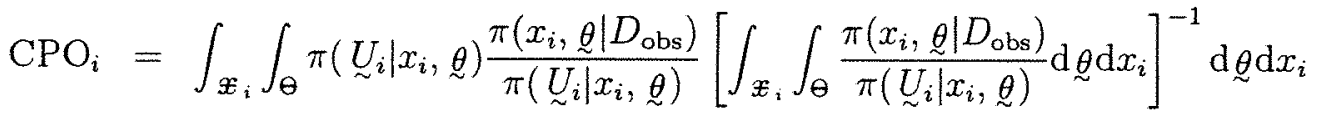

$$
\begin{aligned}
& =\left[\int_{x_{i}} \int_{\theta} \frac{\pi\left(x_{i}, \theta \mid D_{\text {obs }}\right)}{\pi\left({\underset{\sim}{i}}_{i} \mid x_{i}, \theta\right)} \mathrm{d} \underset{\sim}{\theta} \mathrm{d} x_{i}\right]^{-1}
\end{aligned}
$$

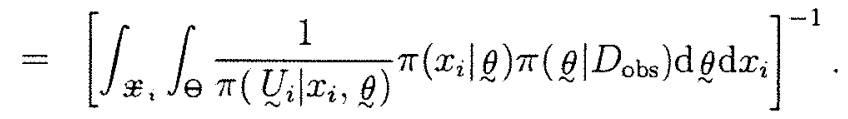

\section{Estimativa de $\mathrm{CPO}_{i}$}

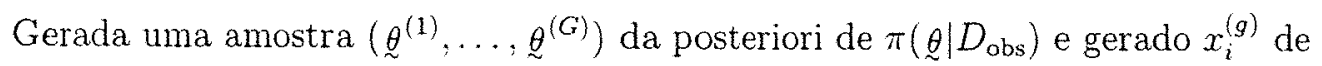
$\pi\left(x_{i} \mid \underline{\theta}^{(g)}\right)$, onde $x_{i} \mid \underline{\theta}^{(g)} \sim N\left(\mu^{(g)}, \sigma_{x}^{2(g)}\right)$, então uma estimativa de Monte Carlo de 
$\mathrm{CPO}_{i}$ é dada por

$$
\widehat{C P O}_{i}=\left(\frac{1}{G} \sum_{g=1}^{G} \frac{1}{\pi(\underbrace{}_{i} \mid x_{i}^{(g)}, \underbrace{(g)})}\right)^{-1}
$$

Notemos que, embora a fórmula (2.10.11) coincida com a fórmula (2.10.8), a diferença está basicamente no processo de geração de $x_{i}^{(g)}$; além de termos, em princípio, a opção de usar também outros algoritmos (além do Gibbs) para amostrar $\stackrel{\theta}{(g)}^{(g)}$ de $\pi\left(\theta \mid D_{\text {obs }}\right)$.

Observação: Podemos plotar $\widehat{\mathrm{COO}}_{i}$ versus $i$ ou versus $X_{i}$ (ou mesmo versus $Y_{i}$ ) para analisar o ajuste do modelo.

\section{(b) Resíduos de validação cruzada}

Se $\underset{\sim}{Y}=\left(Y_{1}, \ldots, Y_{n}\right)$ é a amostra de observações e $\underset{\sim}{Y}(-i)$ é a amostra após extrair $Y_{i}$, então o resíduo estimado de "validação cruzada" é a diferença entre o valor observado $Y_{i}$ e o valor ajustado ou previsto pelo modelo condicional aos dados $\underset{\sim}{Y}(-i)$. Gelfand et al. (1992) definiram este resíduo como $r_{i}=Y_{i}-E\left(Y_{i} \mid Y_{(-i)}\right), i=1, \ldots, n$, onde $E\left(Y_{i} \mid Y_{(-i)}\right)$ é a média com relação à distribuição preditiva de validação cruzada $\pi\left(Y_{i} \mid Y_{(-i)}\right)$. O resíduo padronizado então é definido por

$$
r_{i}^{\prime}=\frac{Y_{i}-E\left(Y_{i} \mid Y_{(-i)}\right)}{\sqrt{\operatorname{Var}\left(Y_{i} \mid Y_{(-i)}\right)}}, \quad i=1, \ldots, n
$$

Nessa seção, o objetivo é obter os resíduos de validação cruzada padronizados do modelo estrutural Normal.

No modelo estrutural Normal os resíduos bidimensionais são

$$
\left(\begin{array}{l}
e_{i} \\
u_{i}
\end{array}\right) \stackrel{\text { iid }}{\sim} N_{2}(\underset{\sim}{0}, V) \text { onde } V=\left(\begin{array}{cc}
\sigma_{e}^{2} & 0 \\
0 & \sigma_{u}^{2}
\end{array}\right), i=1, \ldots, n .
$$

Estes resíduos podem ser escritos como

$$
\left(\begin{array}{l}
e_{i} \\
u_{i}
\end{array}\right)=\left(\begin{array}{c}
Y_{i} \\
X_{i}
\end{array}\right)-\left(\begin{array}{c}
\alpha+\beta x_{i} \\
x_{i}
\end{array}\right)=\left(\begin{array}{c}
Y_{i} \\
X_{i}
\end{array}\right)-E\left[\left(\begin{array}{c}
Y_{i} \\
X_{i}
\end{array}\right) \mid \theta, x_{i}\right]
$$


e portanto, os resíduos padronizados são

$$
V^{-\frac{1}{2}}\left(\begin{array}{c}
e_{i} \\
u_{i}
\end{array}\right)=\operatorname{Var}^{-\frac{1}{2}}\left(\left(\begin{array}{c}
Y_{i} \\
X_{i}
\end{array}\right) \mid \underset{\theta}{\theta}, x_{i}\right)\left[\left(\begin{array}{c}
Y_{i} \\
X_{i}
\end{array}\right)-E\left(\left(\begin{array}{c}
Y_{i} \\
X_{i}
\end{array}\right) \mid \underset{\theta}{\theta}, x_{i}\right)\right] \stackrel{\mathrm{iid}}{\sim} N_{2}\left(\underset{\sim}{0}, I_{2 \times 2}\right),
$$

$i=1, \ldots, n$.

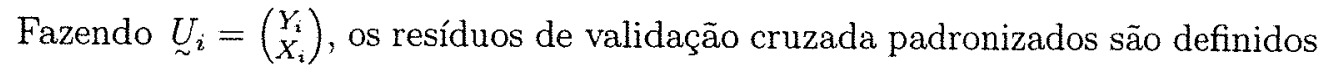
por

$$
\underset{\sim}{d}=\operatorname{Var}^{-\frac{1}{2}}\left(\underset{\sim}{U_{i}} \mid D_{\mathrm{obs}(-i)}\right)\left[\underset{\sim}{U_{i}}-E\left({\underset{\sim}{U}}_{i} \mid D_{\mathrm{obs}(-i)}\right)\right], i=1, \ldots, n
$$

onde $\left.D_{\text {obs }(-i)}={\underset{\sim}{U}(-i)}_{\left(\sim_{1}\right.}, \ldots, \underset{\sim}{U_{i-1}}, \underset{\sim}{U_{i+1}}, \ldots, \underset{\sim}{U_{n}}\right)$ e onde $E\left({\underset{\sim}{U}}_{i} \mid D_{\text {obs }(-i)}\right) \mathrm{e}$ $\operatorname{Var}\left({\underset{\sim}{i}}_{i} \mid D_{\mathrm{obs}(-i)}\right)$ são calculados a seguir.

- Cálculo de $E\left({\underset{\sim}{i}}_{i} \mid D_{\mathrm{obs}(-i)}\right)$

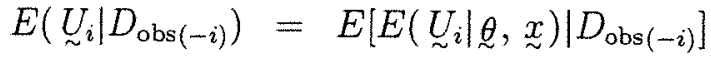

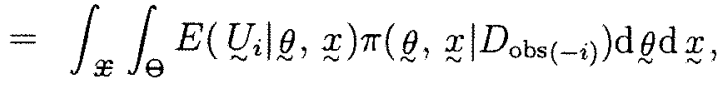

onde

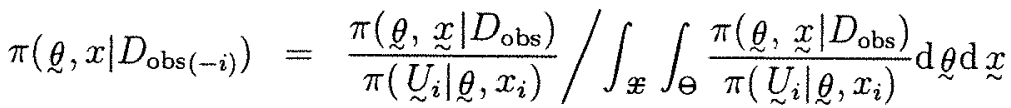

$$
\begin{aligned}
& =\mathrm{CPO}_{i} \frac{\pi\left(\underset{\sim}{\theta}, \underset{\sim}{x} \mid D_{\mathrm{obs}}\right)}{\pi\left(\underset{\sim}{U_{i} \mid} \underset{\sim}{\theta}, x\right)},
\end{aligned}
$$

onde

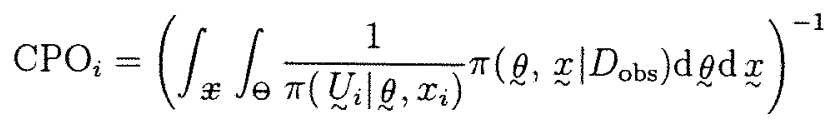

(como em (2.10.7)). Portanto,

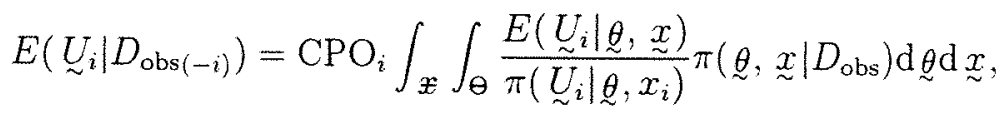

onde

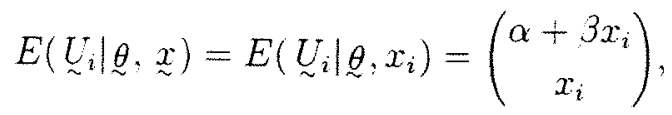


$\pi\left({\underset{\sim}{i}}_{i} \mid \theta, x_{i}\right)$ é dada em $(2.10 .6)$ e $\pi\left(\underset{\sim}{\theta}, \underset{\sim}{x} \mid D_{\text {obs }}\right)$ é a distribuição conjunta à posteriori de $\underset{\sim}{\theta}$ e $\underset{\sim}{x}$.

\section{- Estimação de $E\left({\underset{\sim}{i}}_{i} \mid D_{\mathrm{obs}(-i)}\right)$ por Monte Carlo}

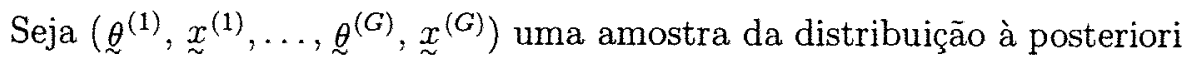
$p\left(\underset{\sim}{\theta} \underset{\sim}{x} \mid D_{\text {obs }}\right)$, obtida por exemplo pelo algoritmo de Gibbs.

Uma estimativa de Monte Carlo de $E\left({\underset{\sim}{i}}_{i} \mid D_{\mathrm{obs}(-i)}\right)$ é dada por

$$
\hat{E}\left({\underset{\sim}{i}}_{i} \mid D_{\mathrm{obs}(-i)}\right)=\widehat{\mathrm{CPO}}_{i} \frac{1}{G} \sum_{g=1}^{G} \frac{1}{\pi\left({\left.\underset{\sim}{i} \mid \underline{\sim}^{(g)}, x_{i}^{(g)}\right)}_{(}\right.}\left(\begin{array}{c}
\alpha^{(g)}+\beta^{(g)} x_{i}^{(g)} \\
x_{i}^{(g)}
\end{array}\right)
$$

onde

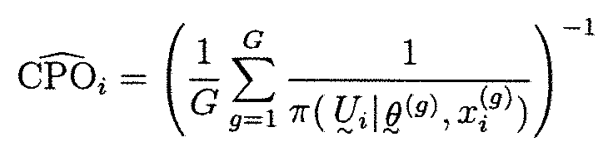

e $\pi\left({\underset{\sim}{i}}_{i} \mid \underset{\sim}{\theta}, x_{i}\right)$ é dada em (2.10.6).

- Cálculo da $\operatorname{Var}\left(\underset{\sim}{U_{i}} \mid D_{\mathrm{obs}(-i)}\right)$

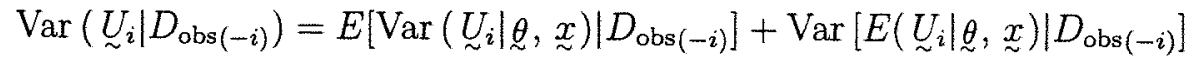

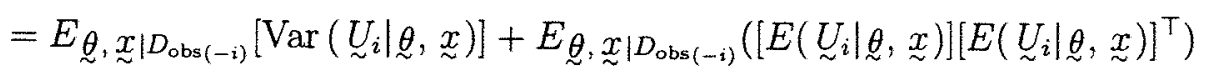

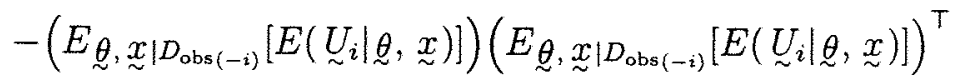

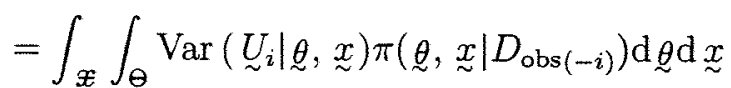

$$
\begin{aligned}
& +\int_{x} \int_{\Theta}\left[E\left(\underset{\sim}{U_{i} \mid \theta}, \underset{\sim}{x}\right)\right]\left[E\left(\underset{\sim}{U_{i}} \mid \underset{\sim}{\theta}, \underset{\sim}{x}\right)\right]^{\top} \pi\left(\underset{\sim}{\theta} \underset{\sim}{x} \mid D_{\mathrm{obs}(-i)}\right) \mathrm{d} \underset{\sim}{\theta} \mathrm{d} \underset{\sim}{x} \\
& -\left[\int_{\mathfrak{X}} \int_{\theta} E\left(\underset{\sim}{U_{i} \mid} \underset{\sim}{\theta}, \underset{\sim}{x}\right) \pi\left(\underset{\sim}{\theta}, \underset{\sim}{x} \mid D_{\mathrm{obs}(-i)}\right) \mathrm{d} \underset{\sim}{\theta} \mathrm{d} \underset{\sim}{x}\right] \text {. }
\end{aligned}
$$

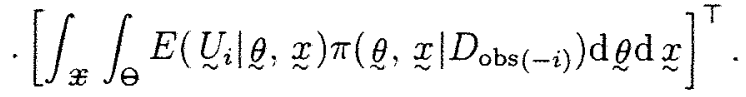

Mas

$$
\pi\left(\underset{\sim}{\theta}, \underset{\sim}{x} \mid D_{\mathrm{obs}(-i)}\right)=\mathrm{CPO}_{i} \frac{\pi\left(\underline{\theta}, x \mid D_{\mathrm{obs}}\right)}{\pi\left({\underset{\sim}{i} \mid}_{\sim}^{U_{\sim}}, x_{i}\right)}
$$

onde

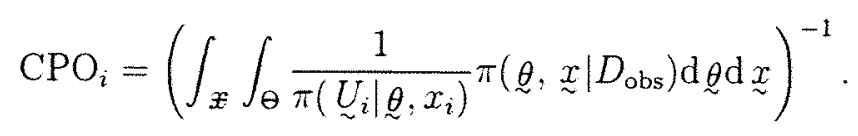


Portanto,

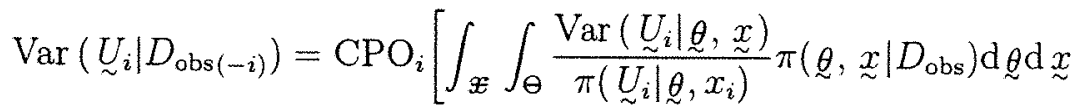

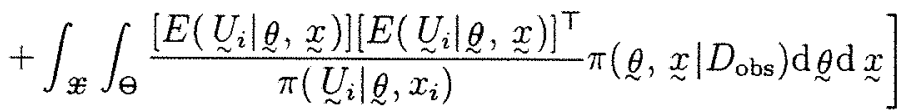

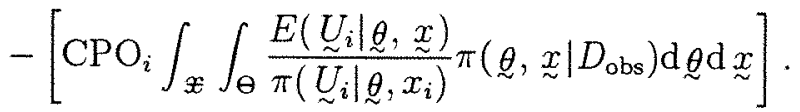

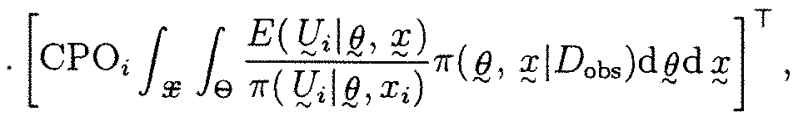

onde

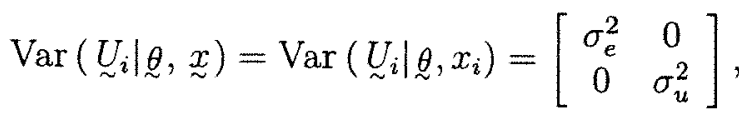

$$
\begin{aligned}
& E\left(\underset{\sim}{U_{i}} \mid \underset{\sim}{\theta}, \underset{\sim}{x}\right)=\left(\begin{array}{c}
\alpha+\beta x_{i} \\
x_{i}
\end{array}\right)
\end{aligned}
$$

e $\pi\left(\underset{\sim}{\theta}, \underset{\sim}{x} \mid D_{\text {obs }}\right)$ é a posteriori conjunta de $\underset{\sim}{\theta}$ e $\underset{\sim}{x}$.

- Estimação de $\operatorname{Var}\left(\underset{\sim}{U_{i}} \mid D_{\text {obs(-i) }}\right)$

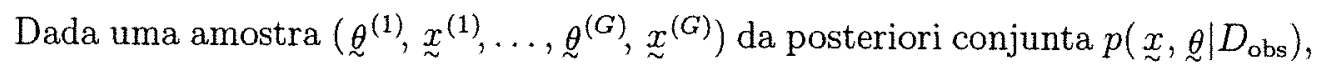
gerada por exemplo pelo algoritmo de Gibbs, então uma estimativa de Monte Carlo dessa matriz de covariâncias é dada por

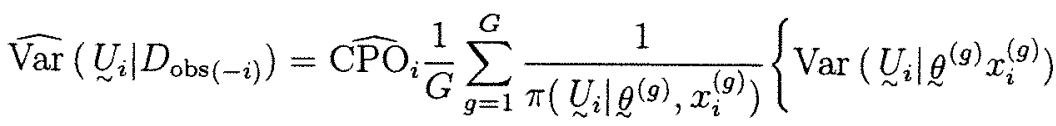

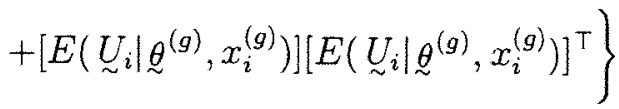

$$
\begin{aligned}
& -\widehat{\mathrm{CPO}}_{i}^{2}\left(\frac{1}{G} \sum_{g=1}^{G} \frac{E\left(U_{i} \mid \theta^{(g)}, x_{i}^{(g)}\right)}{\pi\left({\underset{\sim}{i}}_{i} \mid \underline{\sim}^{(g)}, x_{i}^{(g)}\right)}\right)\left(\frac{1}{G} \sum_{g=1}^{G} \frac{E\left({\underset{\sim}{i}}_{i} \mid \theta^{(g)}, x_{i}^{(g)}\right)}{\pi\left({\underset{\sim}{i}}_{i} \mid \underline{\theta}^{(g)}, x_{i}^{(g)}\right)}\right)^{\top}
\end{aligned}
$$

onde

$$
\widehat{\mathrm{PO}}_{i}=\left(\frac{1}{G} \sum_{g=1}^{G} \frac{1}{\pi(\underbrace{}_{i} \mid \underline{\theta}^{(g)}, x_{i}^{(g)})}\right)^{-1}
$$

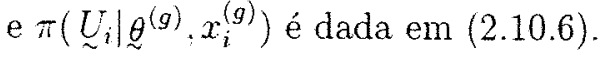


Observação 1: Uma maneira alternativa de estimar $E\left({\underset{\sim}{i}}_{i} \mid D_{\mathrm{obs}(-i)}\right)$ e $\operatorname{Var}\left({\underset{\sim}{i}}_{i} \mid D_{\mathrm{obs}(-i)}\right)$ é a seguinte:

(1) obter uma amostra $\left({\underset{\sim}{(1)}}^{(1)}, \ldots, \underset{\sim}{\theta^{(G)}}\right)$ da posteriori $p\left(\underset{\sim}{\theta} \mid D_{\mathrm{obs}}\right)$ (usando qualquer dos métodos MCMC deste capítulo),

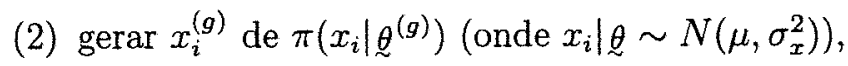

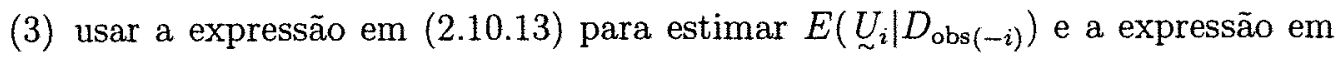
(2.10.15) para estimar $\operatorname{Var}\left(\underset{\sim}{U_{i}} \mid D_{\text {obs(-i) }}\right)$.

Esse estimador é útil quando não conseguimos amostrar de $p\left(\underset{\sim}{x}, \underset{\sim}{\sim} \mid D_{\text {obs }}\right)$ usando, por exemplo, o algoritmo de Gibbs, mas conseguimos amostrar da posteriori $p\left(\underset{\underset{ }{\theta} \mid D_{\mathrm{obs}}}{ }\right)$ por meio do algoritmo de Metropolis-Hastings em Gibbs por exemplo.

Observação 2: $\mathrm{Na}$ análise de resíduos do modelo linear estrutural Normal, nosso objetivo é checar a suposição de que

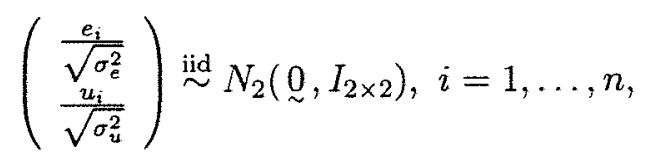

(isto é, $\frac{e_{i}}{\sqrt{\sigma_{e}^{2}}} \stackrel{\text { iid }}{\sim} N(0,1), \frac{u_{i}}{\sqrt{\sigma_{u}^{2}}} \stackrel{\text { iid }}{\sim} N(0,1)$, onde $e_{i}$ e $u_{i}$ são independentes para $i=$ $1, \ldots, n)$, como também a linearidade do modelo. Para essa análise, vamos usar os vetores de resíduos de validação cruzada padronizados

$$
\hat{d}_{i}=\left(\begin{array}{l}
\hat{d}_{i 1} \\
\hat{d}_{i 2}
\end{array}\right)=\widehat{\operatorname{Var}}^{-\frac{1}{2}}\left({\underset{\sim}{i}}_{i} \mid D_{\mathrm{obs}(-i)}\right)\left({\underset{\sim}{i}}_{i}-\hat{E}\left({\underset{\sim}{i}}_{i} \mid D_{\mathrm{obs}(-i)}\right)\right),
$$

lembrando que os $\hat{d}_{i}$ 's não são mais exatamente normalmente distribuídos e independentes, mesmo quando os resíduos $\left(\begin{array}{l}e_{i} \\ u_{i}\end{array}\right)$ são Normais e independentes.

Podemos analisar esses resíduos $\hat{d}_{i}, i=1, \ldots, n$, descritivamente por meio de gráficos, por exemplo, plotando $\hat{d}_{i 1}$ versus $Y_{i}$ (ou versus o valor ajustado $\hat{E}\left(Y_{i} \mid Y_{(-i)}\right)$ ) e $\hat{d}_{i 2}$ versus $X_{i}$ (ou $\hat{E}\left(X_{i} \mid X_{(-i)}\right)$ ), para $i=1, \ldots, n$. Esses gráficos nos ajudam a 
checar possíveis falhas na homogeneidade das variâncias e na linearidade (bem como a existência de "outliers").

Podemos plotar também $\hat{d}_{i 1}$ versus $i$ (e $\hat{d}_{i 2}$ versus $i$ ), para $i=1, \ldots, n$, para checar uma possível falha na independência entre os $\hat{d}_{i}$ 's (bem como a existência de "outliers").

Para checar a normalidade da primeira componente do resíduo, podemos plotar os resíduos ordenados $\hat{d}_{(1), 1} \leq \hat{d}_{(2), 1} \leq \cdots \leq \hat{d}_{(n), 1}$ versus $\Phi^{-1}\left(F_{1}\right), \Phi^{(-1)}\left(F_{2}\right), \ldots$, $\Phi^{-1}\left(F_{n}\right)$, respectivamente, onde $F_{i}$ é a proporção de observações menores ou iguais a $\hat{d}_{(i), 1}$ (e portanto $\hat{d}_{(i), 1}$ é o valor que deixa uma área igual a $F_{i}$ à sua esquerda na distribuição empírica e portanto se não houver empates $\left.F_{i}=i / n\right)$ e $\Phi^{-1}\left(F_{i}\right)$ é o valor do resíduo que deixa uma área igual à $F_{i}$ à sua esquerda na distribuição $N(0,1)$. Portanto, se os resíduos tiverem de fato uma distribuição aproximadamente Normal, espera-se que os pontos $\left(\hat{d}_{(i), 1}, \Phi^{-1}\left(F_{i}\right)\right)$, para $i=1, \ldots, n$, estejam mais ou menos próximos da reta $y=x$. O mesmo procedimento pode ser usado para a segunda componente dos resíduos.

Para checar a normalidade bivariada podemos usar o dispositivo gráfico descrito em Johnson e Wichern (1998), páginas 195 e 196. O gráfico é construído da seguinte maneira:

(1) Calcular as distâncias $d_{i}^{*}=\left({\underset{\sim}{d}}_{i}-\underset{\sim}{\bar{d}}\right)^{\top} S^{-1}\left({\underset{\sim}{d}}_{i}-\underset{\hat{d}}{\bar{d}}\right), i=1, \ldots, n$, onde $\overline{\hat{d}}=$ $\frac{1}{n} \sum_{i=1}^{n}{\underset{\sim}{d}}_{i}$ e $S$ é a matriz de covariâncias amostrais obtida da amostra $\left({\underset{\sim}{d}}_{1}, \ldots\right.$, $\left.\hat{d}_{n}\right)$

(2) Ordenar os valores de $d_{i}^{*}: d_{(1)}^{*} \leq d_{(2)}^{*} \leq \cdots \leq d_{(n)}^{*}$;

(3) Plotar os pontos $\left(d_{(i)}^{*}, q_{c, 2}\left(F_{i}\right)\right), i=1, \ldots, n$, onde $F_{i}=\frac{i-1 / 2}{n} \cong$ proporção de observações $\leq d_{(i)}^{*}\left(\operatorname{assim} d_{(i)}^{*}\right.$ é o valor cuja área à sua esquerda é igual à $F_{i}$, na distribuição empírica) e $q_{c, 2}\left(F_{i}\right)$ é o valor cuja área à sua esquerda, numa 
$\chi_{2}^{2}$, é igual à $F_{i}$.

Se de fato os $\hat{d}_{i}$ são aproximadamente normais, então espera-se que os pontos $\left(d_{(i)}^{*}, q_{c, 2}\left(F_{i}\right)\right), i=1, \ldots, n$, estejam próximos da reta $y=x$.

Observação: A idéia desse procedimento se baseia no fato de que se um vetor aleatório $\underset{\sim}{X_{p \times 1}}$ tem distribuição $N_{p}(\mu, \Sigma), \operatorname{com}|\Sigma|>0$, então

$$
(\underset{\sim}{X}-\mu)^{\top} \Sigma^{-1}(\underset{\sim}{X}-\mu) \sim \chi_{p}^{2}
$$

\subsection{Algumas medidas de comparação de mode- los}

Nessa seção o objetivo é apresentar alguns critérios de escolha de modelos aplicados aos modelos estruturais Normais com erros nas variáveis.

\section{(1) $\mathrm{O}$ critério da medida $L$}

A medida $L$ é um critério que serve tanto para a comparação de modelos, como para a avaliação do ajuste e tem a vantagem de não requerer priori própria em geral (ver Ibrahim et al., 2000, e Ibrahim et al., 2001).

A seguir, descrevemos a formulação geral da medida L aplicada a um modelo qualquer, apresentando primeiramente o caso em que as variáveis observadas são unidimensionais e em seguida o caso multidimensional.

- Definição da medida $L$ no caso unidimensional

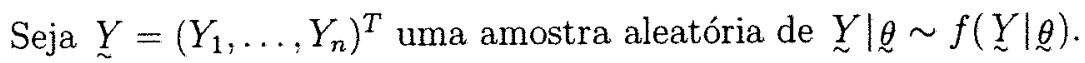

Seja ${\underset{\sim}{*}}^{*}=\left(Y_{1}^{*}, \ldots, Y_{n}^{*}\right)^{T}=\left(Y_{f_{1}}, \ldots, Y_{f_{2}}\right)^{T}, n$ observaçōes futuras com a mesma distribuição dos dados observados $\underset{\sim}{Y}$. 
Então a medida $L$ é definida como

$$
L_{2}(\underset{\sim}{Y}, \delta)=\sum_{i=1}^{n} \operatorname{Var}\left(Y_{i}^{*} \mid \underset{\sim}{Y}\right)+\delta \sum_{i=1}^{n}\left[E\left(Y_{i}^{*} \mid \underset{\sim}{Y}\right)-Y_{i}\right]^{2},
$$

onde $E\left(Y_{i}^{*} \mid \underset{\sim}{Y}\right)$ e $\operatorname{Var}\left(Y_{i}^{*} \mid \underset{\sim}{Y}\right)$ são, respectivamente, a esperança e a variância com relação à distribuição posteriori preditiva $\pi\left(Y_{i}^{*} \mid \underset{\sim}{Y}\right)$ e $0<\delta<1$, onde $\delta$ pode ser interpretado como um peso do viés quadrático e Ibrahim et al. (1998) estudaram o problema de encontrar um valor ótimo para $\delta$ e baseados nesse estudo concluíram que $\nu=\frac{1}{2}$ é um valor razoável.

Interpretação: Um "bom" modelo deveria ter uma pequena $\operatorname{Var}\left(Y_{i}^{*} \mid \underset{\sim}{Y}\right)$ e um viés $\left|Y_{i}-E\left(Y_{i}^{*} \mid \underset{\sim}{Y}\right)\right|$ também pequeno, isto é, um valor pequeno de $\left.L_{2} \underset{\sim}{Y}, \delta\right)$ e portanto entre dois modelos competitivos, o "melhor" será o que tiver a menor medida $L$.

- Definição da medida $L$ para variáveis multidimensionais

Seja $\underset{\sim}{Y}=(\underset{\sim}{Y} 1, \underset{\sim}{Y}, \ldots, \underset{\sim}{Y})$ uma amostra aleatória de $\underset{\sim}{Y} \mid \underset{\sim}{\theta} \sim f(\underset{\sim}{Y} \mid \underset{\sim}{\theta})$ e seja ${\underset{\sim}{Y}}^{*}=\left(\underset{\sim}{Y_{1}^{*}}, \underset{\sim}{Y}, \ldots, \underset{\sim}{Y *}\right)=\left(\underset{\sim}{Y} f_{1}, \underset{\sim}{Y} f_{2}, \ldots, \underset{\sim}{Y} f_{n}\right)$, o vetor formado de $n$ vetores de observações futuras com a mesma distribuição dos dados observados.

$$
L_{2}(\underset{\sim}{Y}, \delta)=\sum_{i=1}^{n} \operatorname{Var}\left(\underset{\sim}{Y_{i}^{*}} \mid \underset{\sim}{Y}\right)+\delta \sum_{i=1}^{n}\left[E\left(\underset{\sim}{Y}{ }_{i}^{*} \mid \underset{\sim}{Y}\right)-\underset{\sim}{Y} i\right][E(\underset{\sim}{Y} i \mid \underset{\sim}{Y})-\underset{\sim}{Y} i]^{\top},
$$

onde $0 \leq \delta \leq 1, \operatorname{Var}(\underset{\sim}{Y} \mid \underset{\sim}{Y})$ é a matriz de covariâncias e $E(\underset{\sim}{Y} \underset{i}{*} \mid \underset{\sim}{Y})$ é o vetor esperado com relação à distribuição posteriori preditiva.

Observação: $\left.L_{2} \underset{\sim}{Y}, \delta\right)$ é uma matriz e portanto uma saída é considerar medidas resumo como, por exemplo, o determinante dessa matriz (det $\left.L=\left|L_{2}(\underset{\sim}{Y}, \delta)\right|\right)$, ou o traço dela $\left(\operatorname{tr}\left(L_{2}(\underset{\sim}{Y} \mid \delta)\right)\right)$.

Calculo da Medida L no Modelo Estrutural Normal Simples: 
Nesse modelo os dados $\underset{\sim}{Y}$ são agora denotados por $D_{\text {obs }}=\left({\underset{\sim}{U}}_{1}, \ldots,{\underset{\sim}{n}}_{n}\right)$, onde $U_{\sim}=\left(\begin{array}{c}Y_{i} \\ X_{i}\end{array}\right)$, e os vetores de observações futuras, por $\underset{\sim}{U_{i}^{*}}=\left(\begin{array}{l}Y_{f_{i}} \\ X_{f_{i}}\end{array}\right), i=1,2, \ldots, n, \mathrm{e}$ $\underset{\sim}{\theta}=\left(\alpha, \beta, \mu, \sigma_{x}^{2}, \sigma_{u}^{2}, \sigma_{e}^{2}\right)^{T}$ (ou então $\underset{\sim}{\theta}=\left(\alpha, \beta, \mu, \sigma_{x}^{2}, \sigma^{2}\right)^{T}$ no modelo com restrição de identificabilidade $\sigma_{e}^{2}=\lambda \sigma_{u}^{2}=\lambda \sigma^{2}$ ).

Para calcular $E\left(\underset{\sim}{U_{i}^{*}} \mid D_{\text {obs }}\right)$ e $\operatorname{Var}\left(\underset{\sim}{U_{i}^{*}} \mid D_{\text {obs }}\right)$, usamos

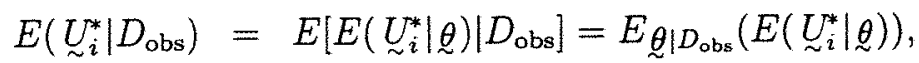

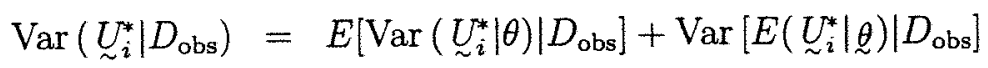

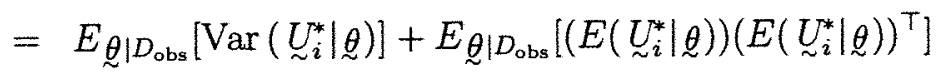

$$
\begin{aligned}
& -\left[E_{\underset{\theta}{\theta} \mid D_{\mathrm{obs}}}\left(E\left({\underset{\sim}{\sim}}_{i}^{*} \mid \theta\right)\right)\right]\left[E_{\theta \mid D_{\mathrm{obs}}}\left(E\left({\underset{\sim}{i}}_{i}^{*} \mid \theta\right)\right)\right]^{\top},
\end{aligned}
$$

onde

$$
E\left(\underset{\sim}{{\underset{\sim}{*}}_{i}^{*} \mid \theta}\right)=\left(\begin{array}{c}
\alpha+\beta \mu \\
\mu
\end{array}\right) \text { e } \operatorname{Var}\left(\underset{\sim}{{\underset{\sim}{i}}_{i}^{*} \mid \theta}\right)=\left[\begin{array}{cc}
\beta^{2} \sigma_{x}^{2}+\sigma_{e}^{2} & \beta \sigma_{x}^{2} \\
\beta \sigma_{x}^{2} & \sigma_{x}^{2}+\sigma_{u}^{2}
\end{array}\right]
$$

Portanto, a medida $L$ pode ser facilmente calculada usando o método simples de Monte Carlo da seguinte maneira:

Seja $\left({\underset{\theta}{(1)}}^{(1)}, \ldots, \underset{\sim}{(G)}\right)$ uma amostra da posteriori $\pi\left(\underset{\sim}{\theta} \mid D_{\text {obs }}\right)$ (gerada por exemplo pelo algoritmo de Gibbs da seção 4.2.2),

$$
\begin{aligned}
& \underset{\sim}{\mathcal{L}_{2}}\left(D_{\mathrm{obs}}, \delta\right)=n\left\{\frac{1}{G} \sum_{g=1}^{G}\left[\operatorname{Var}\left({\underset{\sim}{U}}_{i}^{*} \mid \Theta_{\sim}^{(g)}\right)+\left(E\left({\underset{\sim}{U}}_{i}^{*} \mid \theta^{(g)}\right)\right)\left(E\left({\underset{\sim}{U}}_{i}^{*} \mid \theta^{(g)}\right)\right)^{\top}\right]\right. \\
& \left.-\left(\frac{1}{G} \sum_{g=1}^{G} E\left({\underset{\sim}{i}}_{i}^{*} \mid \theta^{(g)}\right)\right)\left(\frac{1}{G} \sum_{g=1}^{G} E\left(\underset{\sim}{U_{i}^{*}}{\underset{\theta}{\theta}}^{(g)}\right)\right)^{\top}\right\}
\end{aligned}
$$

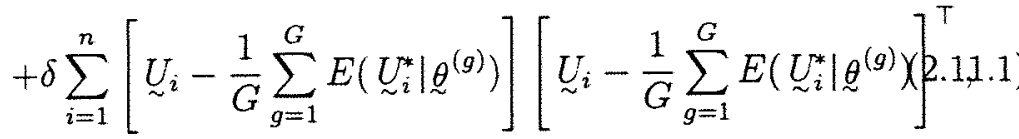

onde $\delta \in[0,1]$.

No caso de regressão simples, $L_{2}\left(D_{\text {obs }}, \delta\right)$ é uma matriz $2 \times 2$ e uma medida resumo pode ser o determinante $\operatorname{det}\left(\widehat{L_{2}}\left(D_{\mathrm{obs}}, \delta\right)\right)$ ou o traço $\left(\widehat{L_{2}}\left(D_{\mathrm{obs}}, \delta\right)\right)$.

(2) O critério da Informação Bayesiano (BIC) 
Essa medida é descrita na seção 4.4 .3 do Apêndice para o caso geral.

No modelo estrutural Normal $M_{i}$, temos que

$$
\mathrm{BIC}_{M_{i}}=-2 \log \left(\pi_{M_{i}}\left(\underset{\sim}{U_{i}} \ldots, \underset{\sim}{U_{n}} \mid \hat{\theta}_{M_{2}}\right)\right)
$$

onde $\underset{\sim}{U_{i}}=\left(\begin{array}{c}Y_{i} \\ X_{i}\end{array}\right)$ e $\pi_{M_{i}}\left(\underset{\sim}{U_{1}}, \ldots,{\underset{\sim}{n}}_{n} \mid \theta\right)$ é a densidade das observações $\left({\underset{\sim}{1}}_{1}, \ldots,{\underset{\sim}{n}}_{n}\right)$ do modelo $M_{i}, \hat{\theta}_{M_{i}}$ é o estimador de máxima verossimilhança de $\underset{\sim}{\theta}$ no modelo $M_{i}, p_{i}$ é o número de parâmetros do modelo $M_{i}$ e $n$ o tamanho da amostra (ver Fuller, 1980).

Num modelo normal com restrição de identificabilidade, essa medida é facilmente calculada pois nesse caso os estimadores de máxima verossimilhança são conhecidos explicitamente.

No modelo com restrição $\sigma_{e}^{2}=\lambda \sigma_{u}^{2}$, a densidade $\pi_{M_{i}}\left(\underset{\sim}{U_{i}} \mid \underset{\sim}{\theta}\right)$ é dada pela expressão em (2.1.4) multiplicada pela constante de proporcionalidade $(2 \pi)^{-\frac{1}{2}}$.

(3) Medidas informais alternativas para seleção de modelos

(a) O produto $\prod_{i=1}^{n} \mathrm{CPO}_{i}$

Para cada um dos modelos competitivos calcula-se o produto

$$
\prod_{i=1}^{n} \mathrm{CPO}_{i} \quad\left(\text { ou o } \log \left(\prod_{i=1}^{n} \mathrm{CPO}_{i}\right)\right)
$$

e escolhe-se o modelo que corresponder ao maior valor dessa medida. No caso do modelo estrutural Normal, podemos estimar $\mathrm{CPO}_{i}$, por exemplo, por meio das fórmulas (2.10.4), (2.10.8) e (2.10.11).

Quando o número de modelos competitivos é baixo (digamos, até 3), podemos também plotar os valores individuais de $\widehat{C P O}_{i}$ versus $i$ para cada modelo no mesmo sistema de coordenadas. No caso de exatamente dois modelos, o quociente dos valores de $\prod_{i=1}^{n} \widehat{\mathrm{CPO}}_{i}$ dá o estimador do Pseudo Fator de Bayes (ver o Apêndice B2). 
(b) A soma das normas (euclideanas) dos resíduos padronizados

$$
\sum_{i=1}^{n}\left|\frac{U_{i}-E\left(U_{i} \mid D_{\text {obs }}\right)}{\sqrt{\operatorname{Var}\left(U_{i} \mid D_{\text {obs }}\right)}}\right|
$$

onde

$$
\hat{E}\left({\underset{\sim}{i}}_{i} \mid D_{\mathrm{obs}}\right)=\frac{1}{G} \sum_{g=1}^{G}\left(\begin{array}{c}
\alpha^{(g)}+\beta^{(g)} \mu^{(g)} \\
\mu^{(g)}
\end{array}\right)
$$

$\mathrm{e}$

$$
\begin{aligned}
& \widehat{\operatorname{Var}}\left({\underset{\sim}{i}}_{i} \mid D_{\mathrm{obs}}\right)=\frac{1}{G}\left\{\sum_{g=1}^{G}\left(\begin{array}{cc}
\left(\beta^{(g)}\right)^{2} \sigma_{x}^{2^{(g)}}+\sigma_{e}^{2^{(g)}} & \beta^{(g)} \sigma_{x}^{2^{(g)}} \\
\beta^{(g)} \sigma_{x}^{2^{(g)}} & \sigma_{x}^{2^{(g)}}+\sigma_{u}^{2^{(g)}}
\end{array}\right)\right. \\
& \left.+\left(\begin{array}{c}
\alpha^{(g)}+\beta^{(g)} \mu^{(g)} \\
\mu^{(g)}
\end{array}\right)\left(\begin{array}{c}
\alpha^{(g)}+\beta^{(g)} \mu^{(g)} \\
\mu^{(g)}
\end{array}\right)^{\top}\right\}-\left(\hat{E}\left({\underset{\sim}{i}}_{i} \mid D_{\mathrm{obs}}\right)\right)\left(\hat{E}\left({\underset{\sim}{i}}_{i} \mid D_{\mathrm{obs}}\right)\right)^{\top} .
\end{aligned}
$$

Calcula-se para cada modelo o valor dessa soma e escolhe-se o modelo que corresponder ao menor valor dessa medida.

Outra alternativa é calcular a soma dos valores absolutos dos resíduos padronizados de validação cruzada

$$
\sum_{i=1}^{n}\left|\frac{\underset{i}{U_{i}-E\left(\sim_{i} \mid D_{\mathrm{obs}(-i)}\right)}}{\sqrt{\operatorname{Var}\left(U_{i} \mid D_{\mathrm{obs}(-i)}\right)}}\right|,
$$

onde a $E\left({\underset{\sim}{i}}_{i} \mid D_{\mathrm{obs}(-i)}\right)$ pode ser estimada, por exemplo, usando a fórmula em

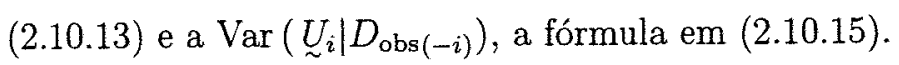




\subsection{EXEMPLOS}

Nessa seção, baseando-se em uma amostra de dados simulados, são analisados os modelos com e sem restrição de identificabilidade usando prioris próprias e impróprias; além disso são comparados tres algorítmos MCMC. Para uma melhor compreensão do texto é recomendável a leitura da seção 2.4 do Apêndice.

\subsubsection{Análise do modelo estrutural normal com restrição de identificabilidade}

\section{DADOS}

Nesta seção é utilizada uma amostra de tamanho $n$ de dados simulados a partir do modelo:

$$
\left\{\begin{array}{l}
Y_{i}=\alpha+\beta x_{i}+e_{i}, \quad i=1, \ldots, n \\
X_{i}=x_{i}+u_{i}
\end{array}\right.
$$

onde

$$
\left(\begin{array}{c}
e_{i} \\
u_{i} \\
x_{i}
\end{array}\right) \stackrel{\text { iid }}{\sim} N\left(\left(\begin{array}{c}
0 \\
0 \\
\mu
\end{array}\right) ;\left(\begin{array}{ccc}
\lambda \sigma^{2} & 0 & 0 \\
0 & \sigma^{2} & 0 \\
0 & 0 & \sigma_{x}^{2}
\end{array}\right)\right), i=1, \ldots, n,
$$

A Tabela 2.1 a seguir contém uma amostra de tamanho 30 de dados gerados a partir desse modelo utilizando-se um programa na linguagem OX ("Object-Oriented Matrix Programming Language")

Tabela 2.1: Amostra de tamanho $\mathrm{n}=30$ de dados simulados a partir do modelo (2.12.4) para $\alpha=2.0, \beta=1.0, \mu=2.0, \sigma_{x}^{2}=0.6, \sigma^{2}=0.1$ e $\lambda=1.0$.

\begin{tabular}{c|cc|c|cc|c|cc}
\hline$i$ & $Y_{i}$ & $X_{i}$ & $i$ & $Y_{i}$ & $X_{i}$ & $i$ & $Y_{i}$ & $X_{i}$ \\
\hline 1 & 3.6651 & 0.98589 & 11 & 3.2228 & 0.72437 & 21 & 4.8247 & 2.4804 \\
2 & 5.0580 & 2.4616 & 12 & 2.4887 & 0.75859 & 22 & 4.8126 & 2.6593 \\
3 & 3.8566 & 2.2892 & 13 & 3.2458 & 1.8808 & 23 & 3.5777 & 1.7877 \\
4 & 3.6561 & 1.1471 & 14 & 5.5650 & 3.5664 & 24 & 4.3372 & 1.6879 \\
5 & 4.9743 & 2.7713 & 15 & 4.1371 & 2.3534 & 25 & 3.6680 & 1.9640 \\
6 & 4.3220 & 2.2590 & 16 & 4.9251 & 2.5498 & 26 & 3.3724 & 1.3345 \\
7 & 3.7144 & 1.8731 & 17 & 3.8136 & 1.8851 & 27 & 4.3191 & 2.2530 \\
8 & 3.9894 & 2.3108 & 18 & 4.5970 & 2.5381 & 28 & 3.5498 & 1.0989 \\
9 & 4.5248 & 3.0514 & 19 & 4.5387 & 2.6756 & 29 & 2.7067 & 1.2432 \\
10 & 2.8640 & 0.90543 & 20 & 4.6773 & 2.9065 & 30 & 2.5472 & 0.84126 \\
\hline
\end{tabular}




\section{MODELO PROPOSTO}

Consideramos o modelo estrutural dado em (2.12.4 ou 2.3.1), com distribuição à priori $\pi\left(\alpha, \beta, \mu, \sigma_{x}^{2}, \sigma^{2}\right)=\pi(\alpha) \pi(\beta) \pi(\mu) \pi\left(\sigma_{x}^{2}\right) \pi\left(\sigma^{2}\right)$, onde $\alpha \sim N(0 ; 1000), \beta \sim$ $N(0 ; 1000), \mu \sim N(0 ; 1000), \sigma_{x}^{2} \sim I G(2.001 ; 1.0), \sigma^{2} \sim I G(2.001 ; 1.0)$ e $\lambda=1.0$.

\section{AJUSTE DO MODELO}

Nesse item vamos ajustar o modelo estrutural normal proposto acima aos dados da tabela 2.1, procedendo como se não conhecêssemos os verdadeiros valores dos parâmetros que foram usados para gerar esses dados.

Inicialmente utilizamos o algoritmo de Gibbs descrito na subseção $2.5 .3 \mathrm{com}$ o objetivo de amostrar da distribuição posteriori do modelo. São geradas $M=$ 4 seqüências paralelas de Gibbs de comprimento $T=10.000$ partindo de pontos iniciais gerados pelo procedimento descrito a seguir.

\section{(a) procedimento para gerar os dados iniciais}

(i) Localização de possíveis modas:

-Tomamos primeiramente uma grade de $20^{5}$ pontos igualmente espaçados na região do espaço paramétrico (aproximadamente em torno do estimador de máxima verossimilhança de $\underset{\sim}{\theta}, \underset{\sim}{\hat{\theta}}=(1.0465,1.9185,1.9748,0.4941,0.0659))$, dada pelo produto cartesiano $[-47.5,47.5] \times[-47.5,47.5] \times[-47.5,47.5] \times$ $[.01,47.51] \times[.01,47.51]$ e avaliamos o valor da função densidade à posteriori $p\left(\alpha, \beta, \mu, \sigma_{x}^{2}, \sigma^{2} \mid D_{\text {obs }}\right)$ em cada ponto dessa grade.

- Selecionamos os pontos que produzem os 10000 maiores valores da densidade à posteriori e utilizamos o algoritmo de otimização de Quase Newton denominado BFGS (Broyden, Fletcher, Goldfarb, Shanno) partindo de cada um desses pontos para localizar possíveis máximos locais (modas). (Para isso 
foi usado o comando MAXBFGS da linguagem OX).

- Repetimos os dois passos anteriores considerando agora uma grade mais fina de $20^{5}$ pontos igualmente espaçados na região dada pelo produto cartesiano: $[-4.75,4.75] . \times[-4.75,4.75] \times[-4.75,4.75] \times[.01,4.76] \times[.01,4.76]$. Desse procedimento foi encontrado apenas um único ponto de máximo:

$\hat{\theta}=(1.8927,1.0595,1.9748,0.43977,0.11100)$.

(ii) Geração dos valores iniciais:

- Geramos 10000 vetores, $\underset{\sim}{\theta_{1}}, \ldots, \underset{1}{\theta_{10000}}$, da distribuição $t_{4}(\hat{\theta}, \hat{V})$, onde $\hat{\theta}$ é a moda da posteriori dada acima e $\hat{V}=-\left.\left(\frac{\partial^{2} \log p\left(\theta \mid D_{\text {obs }}\right)}{\partial \theta \partial \theta^{\top}}\right)^{-1}\right|_{\theta=\hat{\theta}}$, utilizando a decomposição de Choleski de $\hat{V},\left(\hat{V}=L^{T} L\right)$ (ver seção 2.4 dõ Apêndice).

- Reamostramos dez vetores: $\underset{\sim}{\theta_{1}}, \ldots,{\underset{1}{10}}_{10}$ desses 10000 (usando o procedimento de Reamostragem Ponderada descrito na seção 2.4 do Apêndice), e pelo fato desses pontos estarem muito próximos resolvemos considerar apenas quatro deles:

$$
\begin{aligned}
& {\underset{\sim}{1}}_{1}^{(0)}=(1.36552,1.39858,1.62917,0.19871,0.12144)^{T} \text {, } \\
& \theta_{2}^{(0)}=(1.23000,1.40901,1.88743,0.61149,0.08740)^{T}, \\
& \theta_{3}^{(0)}=(1.77440,1.12877,2.26311,0.50376,0.03471)^{T} \text {, } \\
& \theta_{4}^{(0)}=(2.42116,0.68870,2.30602,0.35828,0.13245)^{T} \text {. }
\end{aligned}
$$

\section{(b) Diagnóstico de convergência das seqüências de Gibbs}

A seguir são apresentados alguns dispositivos gráficos (figuras 2.1 a 2.4) como também algumas medidas (tabela 2.2) para o diagnóstico de possíveis falhas na convergência do algoritmo de Gibbs (ver seção 2.4.6 do apêndice sobre diagnósticos de convergência). 


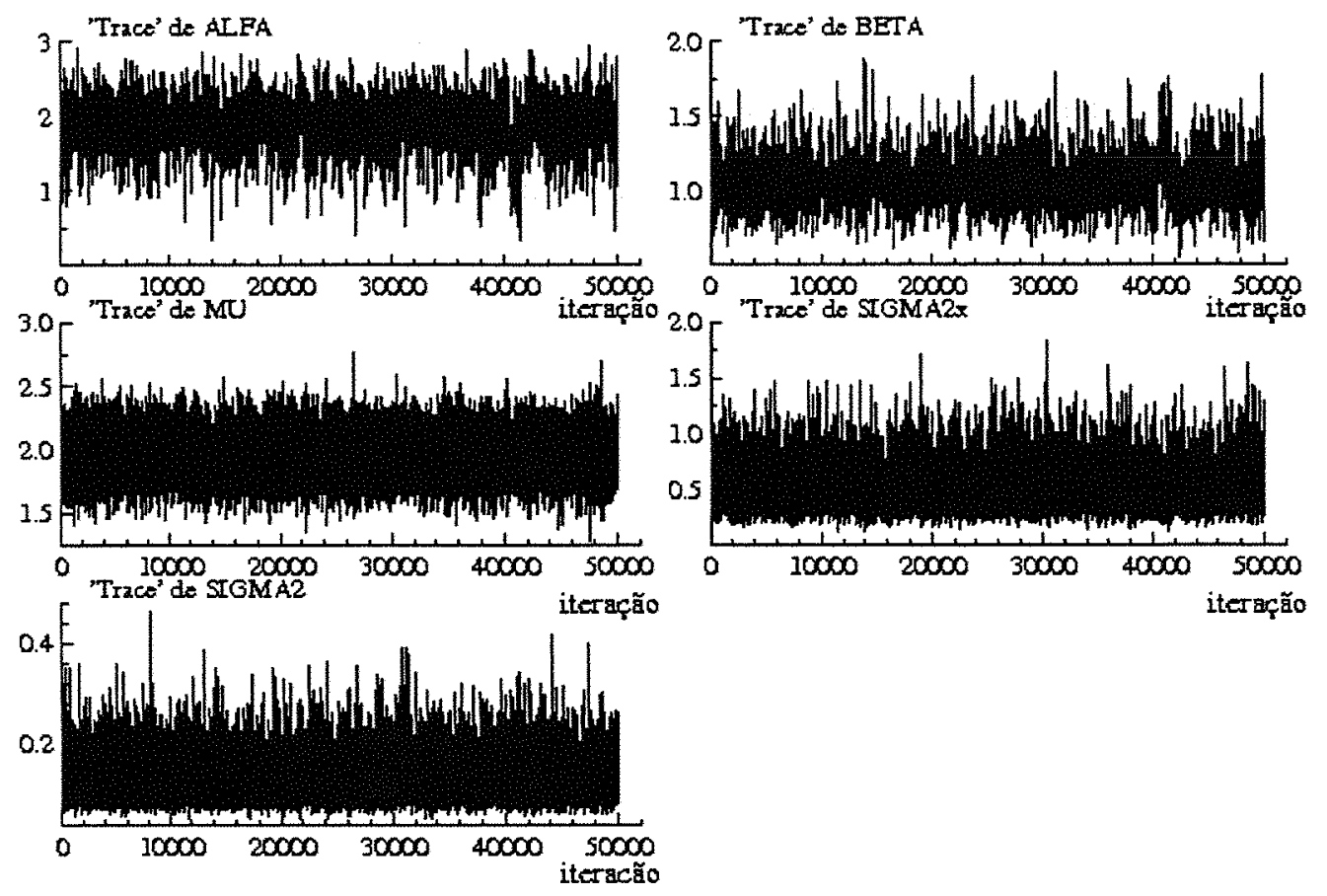

Figura 2.1: Gráficos das séries temporais ("Trace") de $\alpha, \beta, \mu, \sigma_{x}^{2}$ e $\sigma^{2}$, baseados em uma única seqüência de Gibbs de comprimento $T=50.000$ partindo de ${\underset{\sim}{1}}_{1}^{(0)}$.

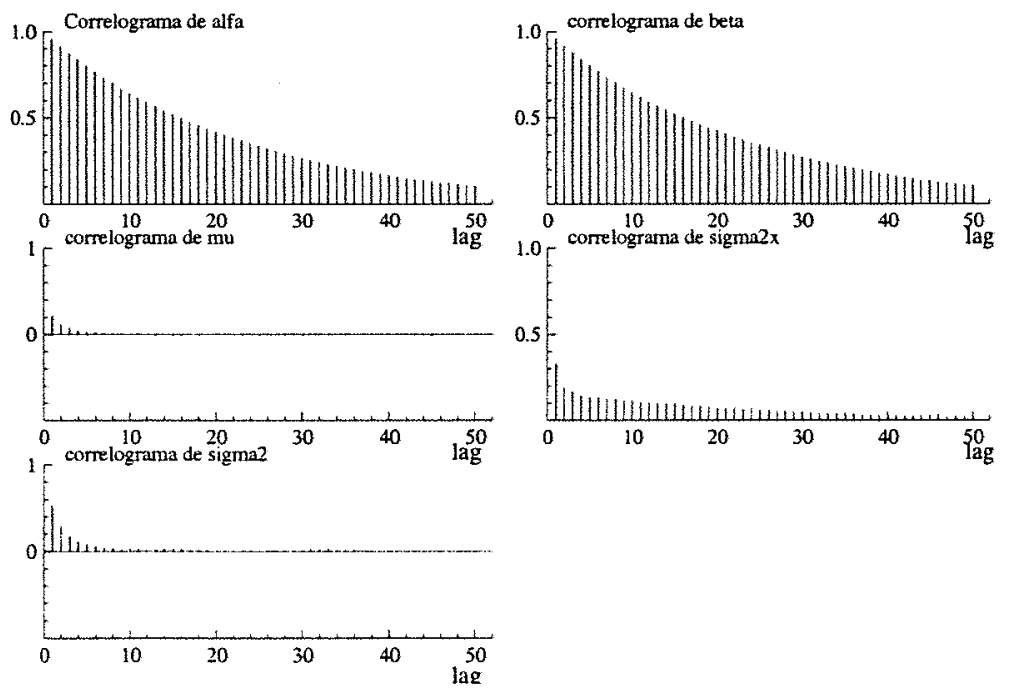

Figura 2.2: Gráficos das funções de auto-correlação de $\alpha, \beta, \mu, \sigma_{x}^{2}$ e $\sigma^{2}$, baseados em uma única seqüència de Gibbs de comprimento $T=50.000$ partindo de $\underline{\theta}_{1}^{(0)}$. 
Brooks \& Gelman Multivariate Shrink Factors

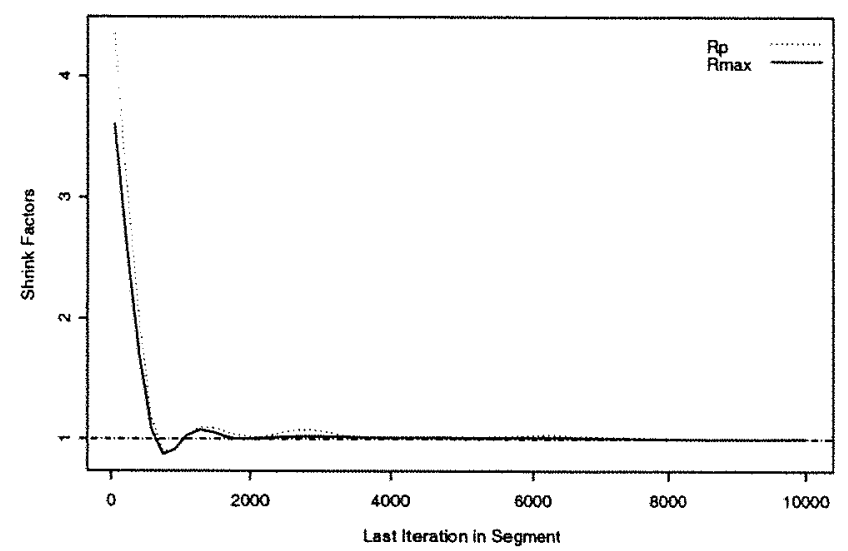

Figura 2.3: Gráfico da medida "Multivariate Potential Scale Reduction"(MPSRF) de Brooks e Gelman (1998) baseado em $M=4$ sequeencias de Gibbs de comprimento $T=10.000$ (Rmax é o maior valor entre as medidas univariadas de $\left.\alpha, \beta, \mu, \sigma_{x}^{2}, \sigma^{2}\right)$.

\section{Gelman \& Rubin Shrink Factors}
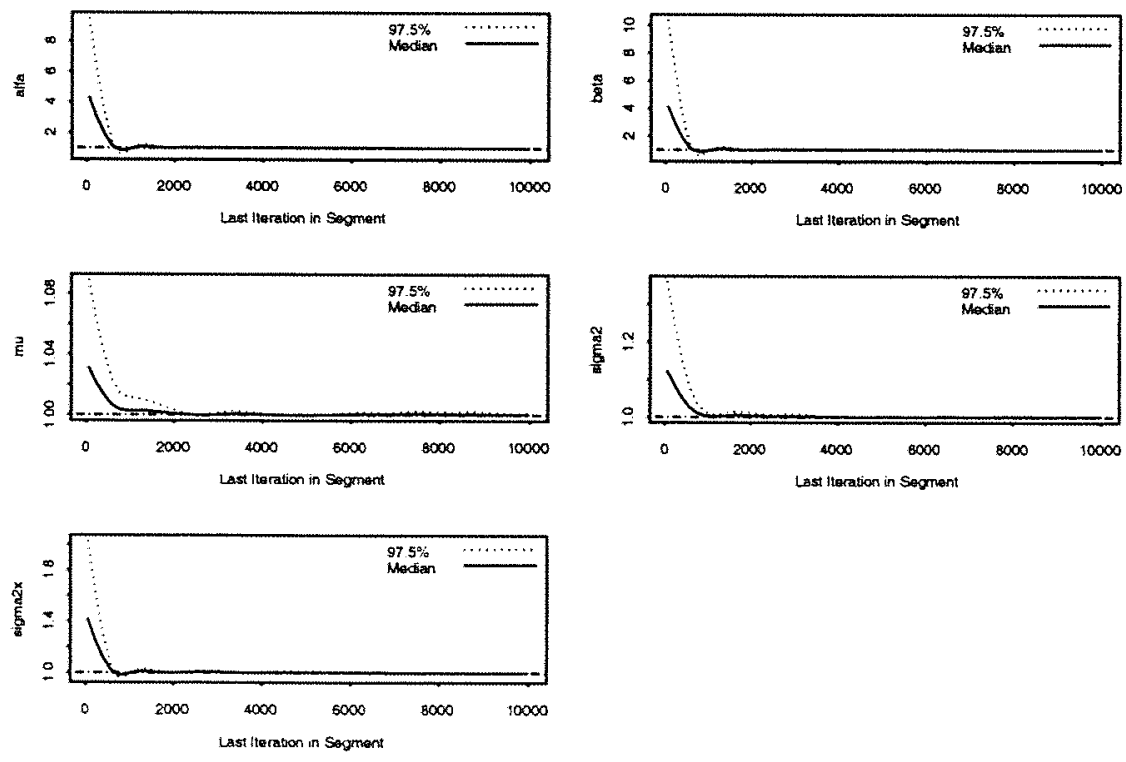

Figura 2.4: Gráfico da medida de Gelman e Rubin corrigida "Corrected potencial scale reduction factors" (CSRF) para cada parametro $\alpha, \beta, \mu, \sigma_{r}^{2}$ e $\sigma^{2}$, baseada em $M=4$ sequéncias de Gibbs de comprimento $T=10.000$. 
Tabela 2.2: Medidas de Diagnóstico de convergência de Brooks, Gelman e Rubin

\begin{tabular}{c|c|cc}
\hline & Medida de Gelman\&Rubin(PSRF) & Medida Corrigida(CSRF) & Quantil 0.975 \\
\hline$\alpha$ & 1.002184 & 1.003412 & 1.008271 \\
$\beta$ & 1.001965 & 1.002994 & 1.007383 \\
$\mu$ & 1.000334 & 1.000341 & 1.001258 \\
$\sigma_{x}^{2}$ & 1.000398 & 1.000408 & 1.001460 \\
$\sigma^{2}$ & 1.000705 & 1.000897 & 1.002601 \\
\hline
\end{tabular}

A medida multivariada (MPSRF) é igual a 1.0027.

Observação: Os dispositivos apresentados até aqui não nos sugerem que exista problema na convergência do algoritmo de Gibbs.

\section{(c) Estimação}

A tabela 2.3 apresenta as estimativas de várias medidas resumo da distribuição à posteriori. Essas estimativas foram obtidas utilizando-se a segunda metade de cada uma das $M=4$ seqüências de Gibbs de comprimento $T=10.000$ descritas anteriormente partindo-se dos pontos iniciais ${\underset{\sim}{1}}_{1}^{(0)}$ a ${\underset{\sim}{\theta}}_{4}^{(0)}$ apresentados anteriormente (lembrando que a amostra de dados utilizada é a da tabela 2.1 e a distribuição à priori é dada no item 2.).

Antes de partir para a análise dos resultados é importante salientar que neste modelo a distribuição à priori é praticamente não informativa e portanto, se de fato o procedimento de estimação é adequado, espera-se que as estimativas à posteriori dos parâmetros estejam próximas dos valores dos parâmetros que foram usados na geração dos dados. Além disso essas estimativas deveriam também estar próximas das estimativas de máximaverossimilhança.

$\mathrm{Na}$ tabela 2.3 observa-se que as estimativas do valor esperado da distr. à posteriori dos parâmetros, obtidas pela média aritmética e pela média Rao-Blackelizada, estão muito próximas, além disso, tanto a média como a mediana amostral estimam cada um dos parâmetros do modelo com "pequeno" viés(estimado) e somente o parâmetro $\sigma^{2}$ parece estar um pouco superestimado. Além disso, mais importante ainda. os valores verdadeiros dos parâmetros (aqueles usados para gerar a amostra 
de dados) pertencem aos intervalos de credibilidade apresentados. Notemos também que as estimativas de máximaveross. estão bem próximas das estimativas de Bayes.

A Figura 2.5 apresenta os gráficos das estimativas "Rao-Blackwelizadas" das densidades posterioris marginais dos parâmetros, obtidas de acordo com a seção 2.9, usando as sequiências de Gibbs descritas acima.

Tabela 2.3: Estimativas do valor esperado, quantis, desvio padrão, máximo, mínimo e intervalos de credibilidade de comprimento mínimo das distribuições posterioris marginais de $\alpha, \beta, \mu, \sigma_{x}^{2}, \sigma^{2}$.

\begin{tabular}{|c|c|c|c|c|c|}
\hline S RESUMO & & $\beta$ & & & \\
\hline $\begin{array}{l}\text { Média aritmética } \\
\text { Média "R-Blackweliz." }\end{array}$ & $\begin{array}{l}1.8652 \\
1.8653\end{array}$ & $\begin{array}{l}1.0743 \\
1.0743\end{array}$ & $\begin{array}{l}1.9718 \\
1.9732\end{array}$ & $\begin{array}{l}0.5045 \\
0.5043\end{array}$ & $\begin{array}{l}0.1340 \\
0.1337\end{array}$ \\
\hline $\begin{array}{r}0.025 \\
.05 \\
.1 \\
.2 \\
.25 \\
.3 \\
.4 \\
.5 \\
.6 \\
.7 \\
.75 \\
.8 \\
.9 \\
.975\end{array}$ & $\begin{array}{l}1.2142 \\
1.3461 \\
1.4766 \\
1.6241 \\
1.6783 \\
1.7287 \\
1.8102 \\
1.8846 \\
1.9576 \\
2.0315 \\
2.0713 \\
2.1160 \\
2.2335 \\
2.3282 \\
2.4072\end{array}$ & $\begin{array}{l}0.8116 \\
0.8518 \\
0.8973 \\
0.9537 \\
0.9746 \\
0.9937 \\
1.0296 \\
1.0641 \\
1.1011 \\
1.1407 \\
1.1636 \\
1.1896 \\
1.2610 \\
1.3301 \\
1.3966\end{array}$ & $\begin{array}{l}1.6819 \\
1.7316 \\
1.7875 \\
1.8513 \\
1.8757 \\
1.8977 \\
1.9361 \\
1.9717 \\
2.0078 \\
2.0472 \\
2.0686 \\
2.0924 \\
2.1554 \\
2.2127 \\
2.2586\end{array}$ & $\begin{array}{l}0.2663 \\
0.2924 \\
0.3275 \\
0.3731 \\
0.3906 \\
0.4084 \\
0.4431 \\
0.4793 \\
0.5171 \\
0.5607 \\
0.5886 \\
0.6199 \\
0.7151 \\
0.8064 \\
0.8896\end{array}$ & $\begin{array}{l}0.0808 \\
0.0868 \\
0.0941 \\
0.1041 \\
0.1082 \\
0.1123 \\
0.1200 \\
0.1278 \\
0.1368 \\
0.1471 \\
0.1537 \\
0.1609 \\
0.1823 \\
0.2015 \\
0.2204\end{array}$ \\
\hline $\begin{array}{l}p=0.95 \text { Lim. inf. } \\
p=0.90 \text { Lim sup. } \\
\text { Lim inf. } \\
\text { Lim sup. }\end{array}$ & $\begin{array}{l}1.2828 \\
2.4642 \\
1.3914 \\
2.3658\end{array}$ & & $\begin{array}{l}1.7300 \\
2.2105\end{array}$ & & \\
\hline $\begin{array}{l}\text { desvio padrão (dp) } \\
\text { d.p. Rao-Blackweliz. } \\
\text { Iaive SE" }=\frac{d p}{}\end{array}$ & $\begin{array}{c}0.3024 \\
0.3032 \\
0.002138\end{array}$ & $\begin{array}{c}0.1459 \\
0.1461 \\
0.001031\end{array}$ & $\begin{array}{c}0.1462 \\
0.1456 \\
0.001033\end{array}$ & & $\begin{array}{r}0.0360 \\
0.0361 \\
0.000255\end{array}$ \\
\hline "Batch & 0.010241 & 0.005016 & 0.001376 & 0.002418 & 0.000498 \\
\hline Estimat & & & & & \\
\hline & & & & 94 & 6599 \\
\hline
\end{tabular}

NOTA: Os valores dos paràmetros usados na geração dos dados são: $\alpha=2.0, \beta=1.0, \mu=2.0, \sigma_{x}^{2}=0.6$ e $\sigma^{2}=1.0$.

Os intervalos de credibilidade nesse exemplo coincidem com os intervalos HPD.

"Batch SE" é o desvio padrảo amostral das médias de grupos consecutivos de tamanho 50, dividido pela raiz quadrada do número de grupos (calculado por meio do BOA ("Bayesian Output Analysis")) $\mathrm{M}=$ número de cadeias paralelas.

$\mathrm{T}=$ número total de iteraçōes, $T_{0}=$ número de iteraçoes descartadas. 

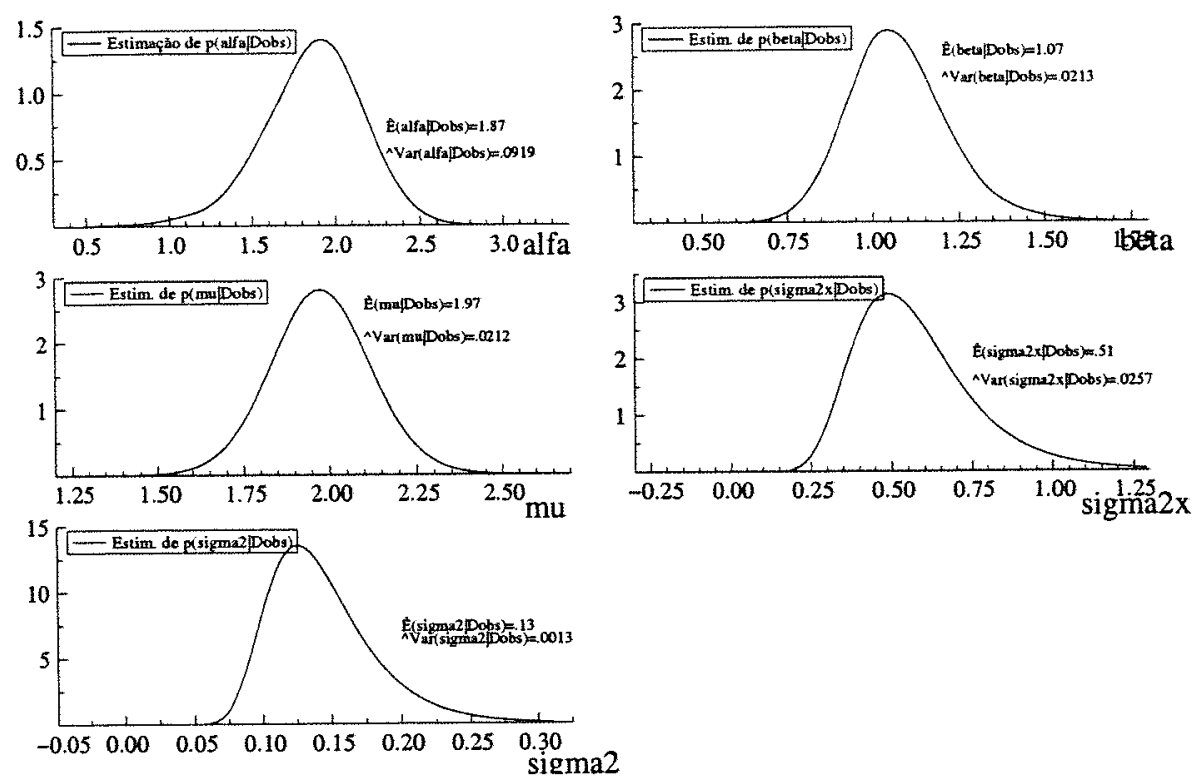

Figura 2.5: Gráficos das estimativas "Rao-Blackwelizadas" das densidades marginais à posteriori $p\left(\alpha \mid D_{\mathrm{obs}}\right), \ldots, p\left(\sigma^{2} \mid D_{\mathrm{obs}}\right)$, incluindo também suas médias e variâncias

\section{(d) Avaliação do ajuste}

A Figura 2.6 apresenta os gráficos dos valores da densidade condicional preditiva de validação cruzada, $\left[\mathrm{CPO}_{i}=\pi\left(\left(\begin{array}{c}Y_{i} \\ X_{i}\end{array}\right) \mid D_{\text {obs }}\right)\right]$, primeiramente em função de $i$, depois em função de $X_{i}$ e depois em função de $Y_{i}$, para $i=1, \ldots, 30$, onde $\mathrm{CPO}_{i}$ é estimada por meio da fórmula (2.10.8). As estimativas foram baseadas na segunda metade de uma única seqüência de comprimento $T=10.000$ do algoritmo de Gibbs que parte do ponto inicial ${\underset{\sim}{l}}_{1}^{(0)}$ dado anteriormente.

$\mathrm{Na}$ seção 2.10.2(b), os resíduos bayesianos bidimensionais de validação cruzada podiam ser estimados por

$$
d_{i}=\left(\begin{array}{c}
d_{i 1} \\
d_{i 2}
\end{array}\right)=\widehat{\operatorname{Var}}^{-\frac{1}{2}}\left(\left(\begin{array}{c}
Y_{i} \\
X_{i}
\end{array}\right) \mid D_{\mathrm{obs}(-i)}\right)\left(\left(\begin{array}{c}
Y_{i} \\
X_{i}
\end{array}\right)-\hat{E}\left(\left(\begin{array}{c}
Y_{i} \\
X_{i}
\end{array}\right) \mid D_{\mathrm{obs}(-i)}\right)\right),
$$

$i=1, \ldots, n$, onde $\hat{E}\left(\left(\begin{array}{c}Y_{2} \\ X_{i}\end{array}\right) \mid D_{\text {obs(-i) }}\right)$ é dada em $(2.10 .13)$ e $\widehat{\operatorname{Var}}^{-\frac{1}{2}}\left(\left(\begin{array}{l}Y_{2} \\ X_{i}\end{array}\right) \mid D_{\text {obs }(-i)}\right)$ é dada em (2.10.15), para $i=1, \ldots, n$. 
A Figura 2.7 apresenta os gráficos de cada uma das componentes $d_{1 i}$ e $d_{2 i}$ do vetor de resíduos.

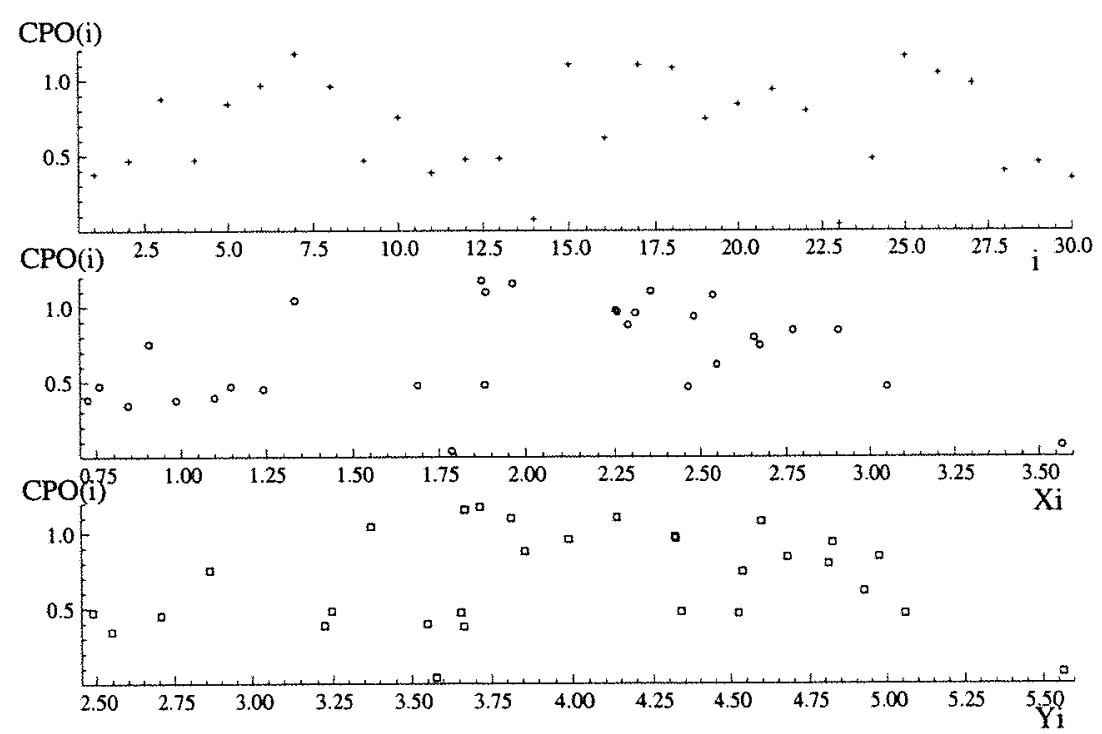

Figura 2.6: Gráficos dos valores da densidade condicional preditiva, (CPO(i)), versus $i$ (posição superior), versus $X_{i}$ (posiçào do meio) e versus $Y_{i}$ (posição inferior).

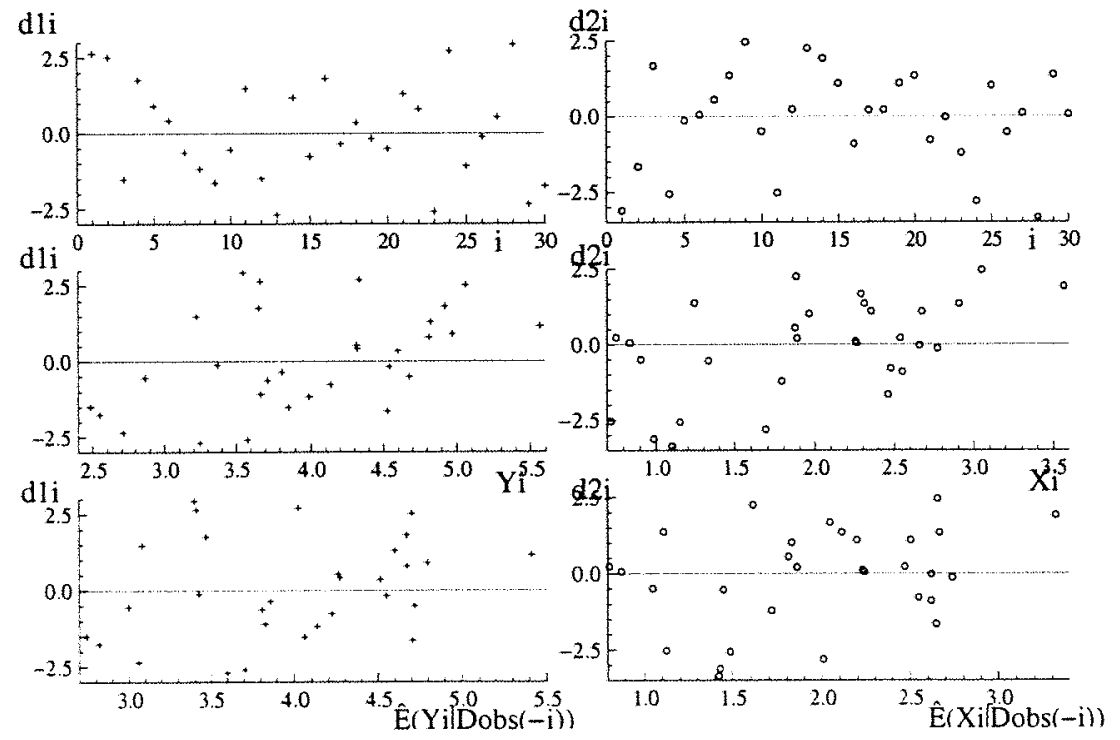

Figura 2.7: Do lado esquerdo os gráficos da componente $d_{1 i}$ versus $i$, versus $Y_{i}$ e versus $E\left(Y_{i} \mid \hat{D_{\mathrm{obs}}}(-i)\right)$. Do lado direito $\left(d_{2 i}\right)$ versus $i$, versus $X_{i}$ e versus $E\left(X_{i} \mid \hat{D_{\mathrm{obs}}}(-i)\right)$.. 
Os gráficos acima não sugerem que haja grandes problemas de falta de ajustamento. Entretanto na figura 2.7 existem 2 pontos nos gráficos de ordenada $d_{2_{i}}$ cujos valores são um pouquinho menores que -3 e que aparentemente poderiam ser vistos como pontos aberrantes, portanto uma análise de resídous mais aprofundada deve ser conduzida futuramente para examinar melhor o ajuste.

\section{SENSIBILIDADE $\grave{A} \lambda$ - quociente das variâncias residuais}

Examinamos descritivamente a variação nas estimativas dos valores esperados de $\alpha, \beta, \mu, \sigma_{x}^{2}$ e $\sigma^{2}$ à posteriori quando diferentes valores do hiperparâmetro $\lambda$ são considerados (mantendo-se os demais hiperparâmetros do modelo em (2.12.2) inalterados)

Ns figuras 2.8 e 2.9, são apresentados gráficos com as estimativas "Rao-Blackwelizadas" dos valores esperados dos parâmetros da distribuição à posteriori baseadas nos dados da Tabela 2.1. Para cada $\lambda$ fixado, foi gerada uma sequiência de Gibbs de comprimento $T=10.000$ (com vetor inicial igual ao estimador de máxima verossimilhança correspondente). Para calcular as estimativas considerou-se apenas a segunda metade de cada seqüência.

$\mathrm{Na}$ figura 2.10, comparamos os gráficos (das médias Rao-Blackelizadas versus $\lambda$ ) baseados em dois diferentes tamanhos de amostra $(n=50, n=500)$. Essas amostras foram geradas à partir do mesmo modelo em (2.12.1) usando a mesma semente (ranseed=17 da linguagem $O X$ ). Para cada $\lambda$ fixado, foi gerada uma seqüência de Gibbs partindo-se da estimativa de máxima verossimilhança correspondente. Para o cálculo das médias foram usadas apenas a segunda metade de cada seqüência de $\mathrm{T}=10000$ iterações . 


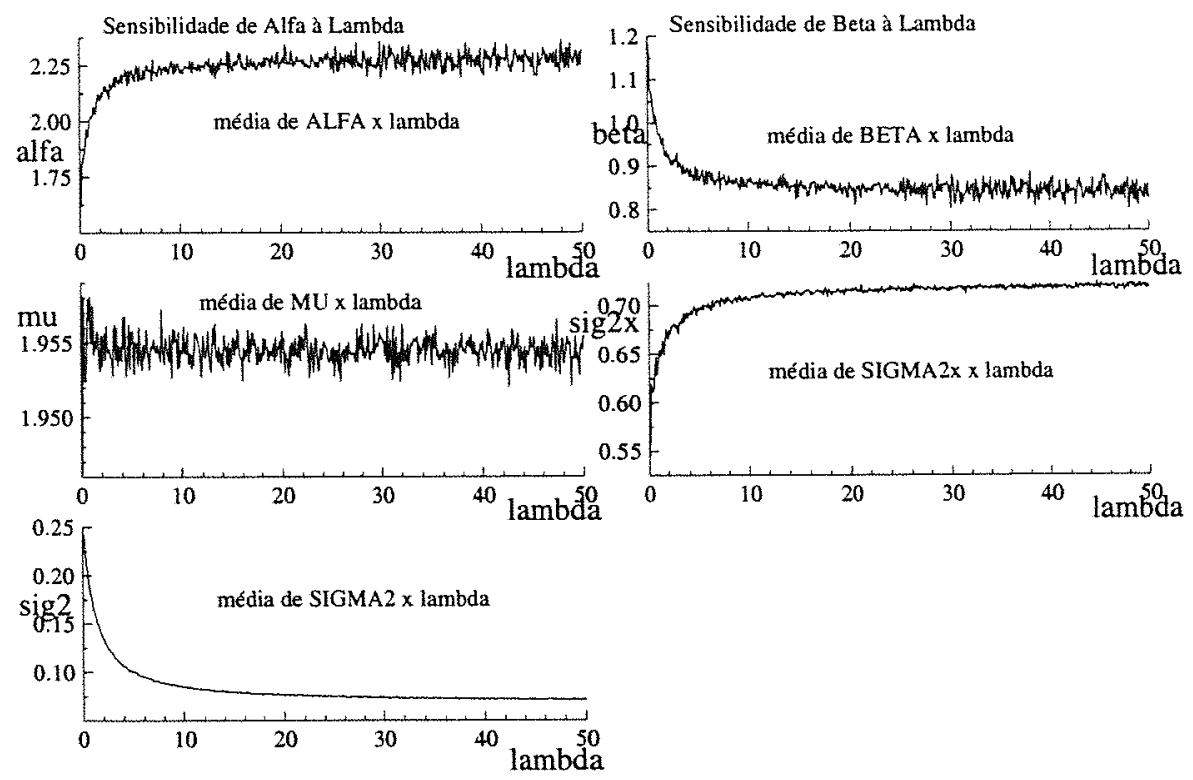

Figura 2.8: Gráficos das médias "R.-Blackwelizadas" de $\alpha, \beta, \mu, \sigma_{x}^{2}$ e $\sigma^{2}$ versus $\lambda$ baseadas na amostra de tamanho $n=30$ da tabela 2.1. A grade de pontos em $\lambda$ é formada por 500 pontos igualmente espaçados no intervalo $[0,50]$. (Os valores verdadeiros dos parâmetros são: $\alpha=2.0, \beta=1.0, \mu=1.0, \sigma_{x}^{2}=0.6$ e $\sigma^{2}=0.1$ e do hiperparâmetro $\lambda$ é 1.0.)
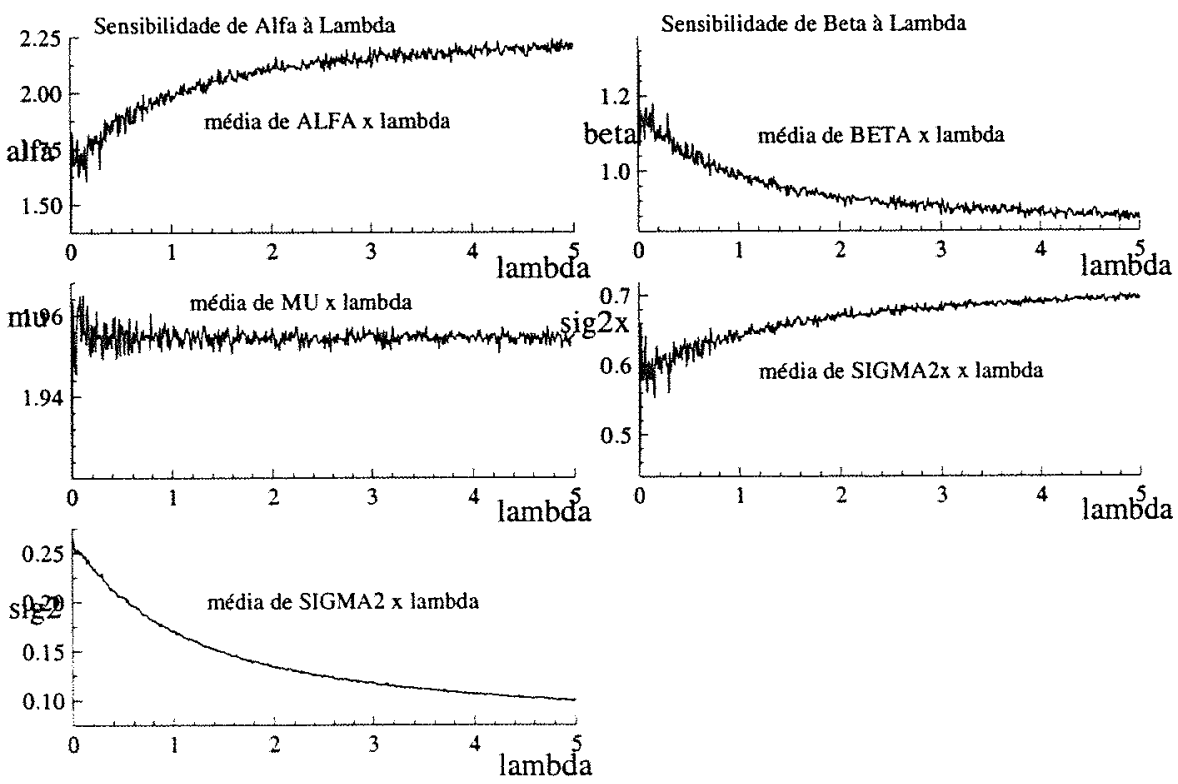

Figura 2.9: Idem à figura anterior, porém com grade de pontos em $\lambda$ formada por 500 pontos igualmente espaşados no intervalo $[0,5]$. 


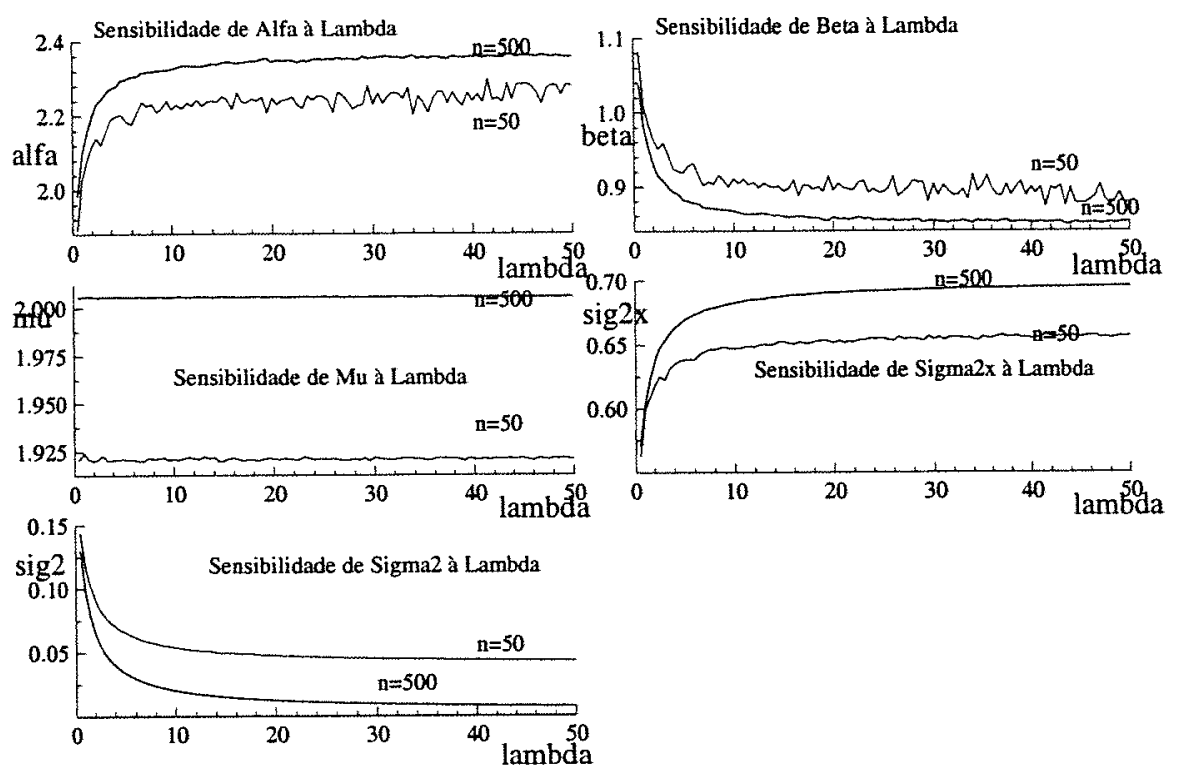

Figura 2.10: Gráfico das médias "Rao-Blackwelizadas" de $\alpha, \beta, \mu, \sigma_{x}^{2}$ e $\sigma^{2}$ versus $\lambda$, das posterioris baseadas nas amostras geradas de tamanho $n=50$ e $n=500$. A grade em $\lambda$ é formada de 100 pontos igualmente espaçados no intervalo $[0,50]$ (os valores verdadeiros dos parâmetros são $\alpha=2.0, \beta=1.0, \mu=2.0, \sigma_{x}^{2}=0.6$ e $\sigma^{2}=0.1$ e de $\lambda$ é 1.0$)$.

\section{Observações:}

Das figuras 2.8 e 2.9 observamos que:

(1) A média amostral do parâmetro $\mu$ parece não ser influenciada pelo valor de $\lambda$.

(2) Quando $\lambda$ é subestimado (isto é, quando $0<\lambda<1$ ), aparentemente, as médias amostrais de $\beta$ e $\sigma_{x}^{2}$ superestimam os valores verdadeiros de $\beta$ e $\sigma_{x}^{2}$ mas a média amostral de $\alpha$ subestima o verdadeiro valor de $\alpha$, entretanto a estimativa do viés de estimação não ultrapassa a $15 \%$ dos valores verdadeiros dos parâmetros.

(3) Quando $\lambda$ é superestimado $(\lambda>1)$, aparentemente ocorre o oposto ( $\alpha$ é superestimado e $\beta$ e $\sigma_{x}^{2}$ são subestimados) e o viés de estimação também não ultrapassa $15 \%$ (ou 20\%) dos valores verdadeiros dos parâmetros. Isso ocorre mesmo quando $\lambda$ é bem grande (da ordem de 50 vezes maior que o seu verdadeiro valor). Aparentemente as estimativas tendem a se estabilizar à medida que $\lambda$ cresce. 
(4) O valor da estimativa do viés na estimação de $\sigma^{2}$ é em geral bem grande. Além disso, quando o tamanho da amostra $(\mathrm{n})$ não é muito grande $(n \approx 30)$, o valor de $\sigma^{2}$ é muito superestimado quando $\lambda$ está próximo do verdadeiro valor $(0<\lambda<2)$, indicando que procedimento de estimação de $\sigma^{2}$ seria razoável apenas para amostras bem grandes .

Da figura 2.10 sugere que as estimativas dos parâmetros aparentemente tendem a se estabilizar independentemente do tamanho (n) da amostra, (mas o valor do viés de estimação depende de $n)$.

\section{COMPARAÇÃO ENTRE OS ALGORITMOS}

Para comparar os algoritmos de Gibbs, Grouped Gibbs e M.Collapsed Gibbs, apresentamos os gráficos das funções de autocorrelação amostral e das séries temporais de cada um dos parâmetros, como também as matrizes de correlação cruzada dos parâmetros do modelo dessa seção para uma particular amostra de dados(tabela 2.1), utilizando cada um dos algoritmos acima (partindo sempre do mesmo ponto inicial e tomando-se $\mathrm{T}=50.000$ iterações).

Foram obtidas também, a partir de cada algoritmo, as estimativas Rao-Blackwelizadas das densidades marginais à posteriori e as estimativas das medidas resumo da distr. à posteriori (com base em 4 seqüências de comprimento $T=10.000$ ). Entretanto serão apresentados apenas os gráficos da função de autocorrelação amostral (fig.2.11) e a tabela (2.4) com as matrizes de correlação cruzada sob os 3 algoritmos.

Da figura 2.11 a 2.13 podemos observar, com base nas autocorrelações amostrais, que aparentemente não há vantagem em usar o Grouped Gibbs em relação ao Gibbs simples, mas há um certo ganho (embora não muito grande) em utilizar o algoritmo M.Collapsed Gibbs sobre os demais para amostrar da distr. à posteriori do modelo. 


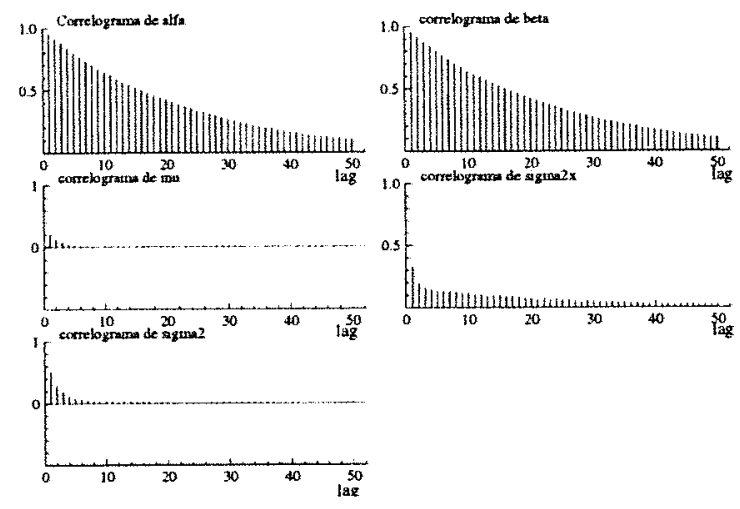

Figura 2.11: Função de autocorrelação amostral da seqüência do algoritmo de Gibbs.

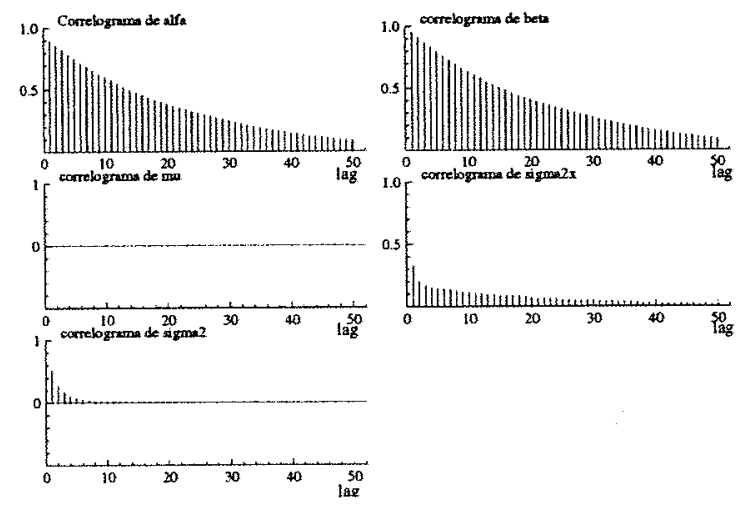

Figura 2.12: Função de autocorrelação amostral da seqüência do algoritmo "Grouped Gibbs".

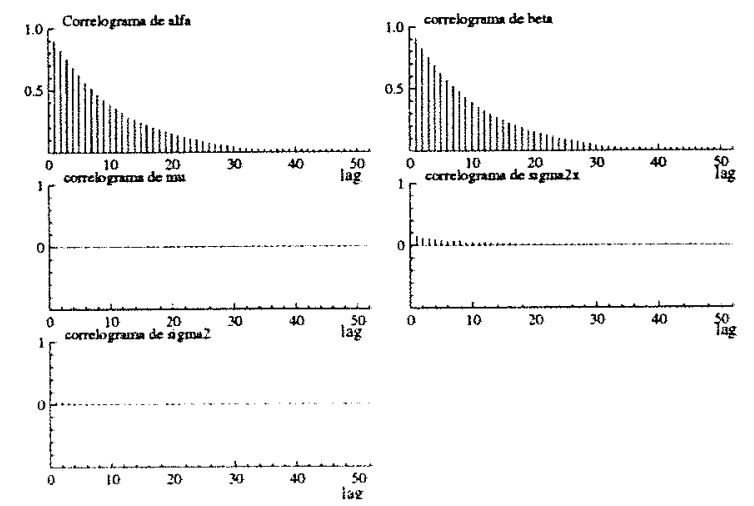

Figura 2.13: Função de autocorrelação amostral da seqüência do algoritmo "Modified and Collapsed Gibbs", com um mini Gibbs de comprimento $N=10$. 
Tabela 2.4: Matrizes de correlação de $\left(\alpha, \beta, \mu, \sigma_{x}^{2}, \sigma^{2}\right)$ obtidas apartir dos algoritmos de Gibbs, Grouped Gibbs e M.Collapsed Gibbs

\begin{tabular}{cc|ccccc}
\hline & & $\alpha$ & $\beta$ & $\mu$ & $\sigma^{2}$ & $\sigma^{2}$ \\
\hline Gibbs & $\alpha$ & 1.000 & -0.9509 & -0.0967 & 0.3688 & 0.0441 \\
& $\beta$ & & 1.000 & -0.0054 & -0.388 & 0.0459 \\
& $\mu$ & & & 1.000 & -0.0007 & -0.0039 \\
& $\sigma_{\tau}^{2}$ & & & & 1.000 & -0.1136 \\
& $\sigma^{2}$ & & & & & 1.0000 \\
\hline Group.Gibbs & $\alpha$ & 1.000 & -0.9484 & -0.0960 & 0.3746 & -0.0273 \\
& $\beta$ & & 1.000 & -0.0108 & -0.3961 & 0.0328 \\
& $\mu$ & & & 1.000 & 0.0135 & 0.0057 \\
& $\sigma_{x}^{2}$ & & & & 1.000 & -0.1108 \\
& $\sigma^{2}$ & & & & & 1.0000 \\
\hline M.Collap.Gibbs & $\alpha$ & 1.000 & -0.9494 & -0.0959 & 0.3678 & -0.0340 \\
& $\beta$ & & 1.000 & -0.0040 & -0.3853 & 0.0332 \\
& $\mu$ & & & 1.000 & 0.0212 & 0.0042 \\
& $\sigma_{x}^{2}$ & & & & 1.000 & -0.1107 \\
& $\sigma^{2}$ & & & & & 1.0000 \\
\hline
\end{tabular}

Da tabela 2.4 vemos, com base nos valores das correlações cruzadas, que aparentemente não há vantagem no uso do algoritmo Grouped Gibbs sobre o Gibbs simples, já que metade das correlações diminuiram e metade aumentaram. Entretanto o M.Collapsed Gibbs gerou uma seqüência cujas correlações cruzadas são em geral um pouquinho menores que as correlações obtidas pelo Gibbs simples.

\section{Observações:}

- Os gráficos das séries temporais obtidos pelos tres algoritmos, para esse particular exemplo, ficaram muito parecidos, não dando para distinguir nenhuma diferença marcante entre eles.

As estimativas das medidas resumo, obtidas pelos tres algoritmos ficaram muito próximas diferindo nos piores casos apenas pela segunda casa decimal. Os gráficos das estimativas Rao-Blackwelizadas das densidades marginais à posteriori ficaram praticamente indistinguiveis.

- Com base apenas no particular exemplo estudado acima, aparentemente não há grande vantagem em usar modificações do algoritmo de Gibbs. 


\subsubsection{Análise do modelo estrutural normal sem restrição de identificabilidade}

\section{DADOS}

A amostra de tamanho $\mathrm{n}=30$ de dados a ser usada nessa seção foi gerada a partir do modelo

$$
\left\{\begin{array}{l}
Y_{i}=\alpha+\beta x_{i}+e_{i}, \quad i=1, \ldots, n \\
X_{i}=x_{i}+u_{i}
\end{array}\right.
$$

onde

$$
\left(\begin{array}{c}
x_{i} \\
u_{i} \\
e_{i}
\end{array}\right) \stackrel{\mathrm{iid}}{\sim} N\left(\left(\begin{array}{c}
\mu \\
0 \\
0
\end{array}\right) ;\left(\begin{array}{ccc}
\sigma_{x}^{2} & 0 & 0 \\
0 & \sigma^{2} & 0 \\
0 & 0 & \sigma_{e}^{2}
\end{array}\right)\right), i=1, \ldots, n,
$$

para $\alpha=2.0, \beta=1.0, \mu=2.0, \sigma_{x}^{2}=0.6, \sigma^{2}=0.1, \sigma_{e}^{2}=0.1$.

Nesse caso, o conjunto de dados é o mesmo que o da Tabela 2.1.

\section{MODELO PROPOSTO}

Consideramos o modelo em (2.12.5) (ou (2.6.1), com distribuição à priori dada por $\pi\left(\alpha, \beta, \mu, \sigma_{x}^{2}, \sigma^{2}, \sigma_{e}^{2}\right)$ com componentes independentes, onde $\alpha, \beta$ e $\mu$ têm distribuição $N(0 ; 1000)$ e $\sigma_{x}^{2}, \sigma^{2}$ e $\sigma_{e}^{2}$ têm distribuição $I G(2.001 ; 1.0)$.

\section{AJUSTE DO MODELO}

(a) Pontos Iniciais: Geramos $M=4$ pontos iniciais usando procedimento semelhante ao da seção 2.12.1. Os pontos gerados foram:

$$
\begin{aligned}
& \underline{\sim}_{1}^{(0)}=(1.08372,1.30058,2.29445,0.23498,0.14854,0.17795) \\
& \underline{\theta}_{2}^{(0)}=(2.12682,1.03819,1.93648,0.33617,0.11543,0.10954) ; \\
& \underline{\theta}_{3}^{(0)}=(2.36979,0.83386,1.94213,0.43797,0.15633,0.12669) ; \\
& \underline{\theta}_{4}^{(0)}=(2.83504,0.65290,1.85471,0.57342 ; 0.17470,0.18693) ;
\end{aligned}
$$

Partindo desses pontos geramos $M=4$ seqüências de Gibbs de comprimento $T=$ 10.000 com o objetivo de amostrar da distribuição à posteriori. (Foi gerada também uma única seqüência de comprimento $T=50000$ para os gráficos de diagnóstico) 
(b) Diagnósticos de convergência das seqüências de Gibbs
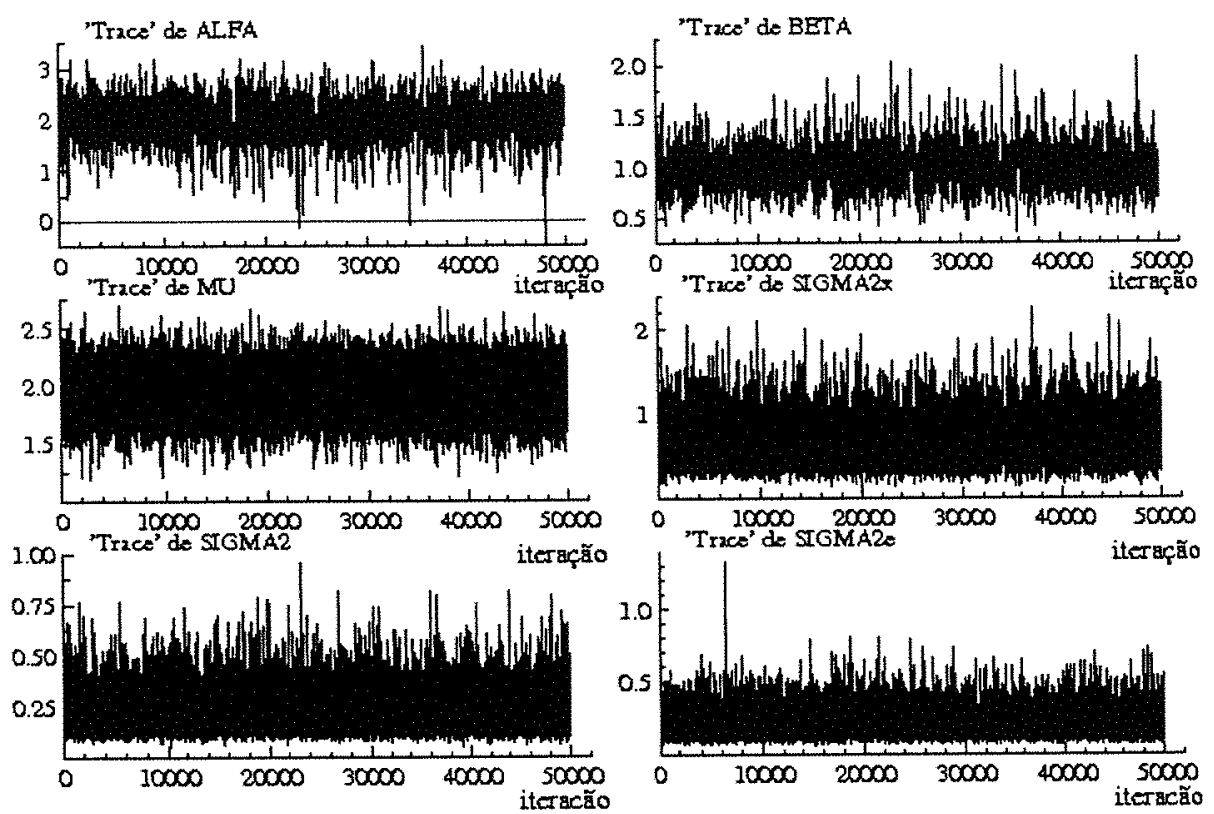

Figura 2.14: Gráficos das séries temporais ("trace") de $\alpha, \beta, \mu, \sigma_{x}^{2}, \sigma^{2}$ e $\sigma_{e}^{2}$, baseados em uma única seqüência de Gibbs de comprimento $T=50000$ partindo de ${\underset{1}{1}}_{1}^{(0)}$.

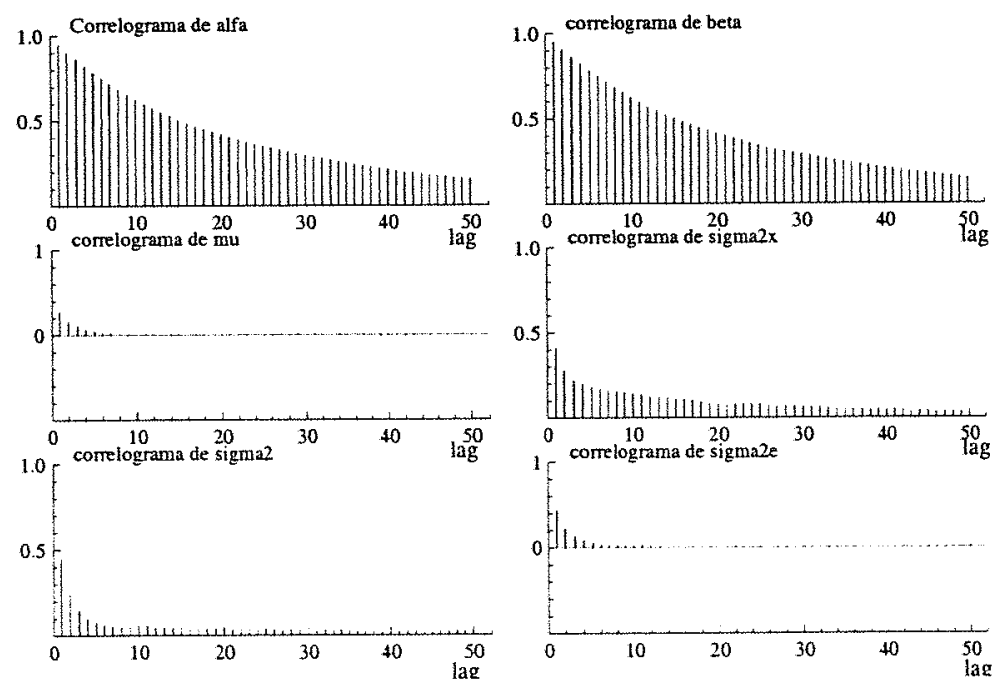

Figura 2.15: Gráficos das funçōes de auto-correlação de $\alpha, \beta, \mu, \sigma_{x}^{2}, \sigma^{2}$ e $\sigma_{e}^{2}$, baseados em uma única seqüência de Gibbs de comprimento $T=50000$ partindo de $\underset{\sim}{{ }_{1}^{(0)}}$. 


\section{Brooks \& Gelman Multivariate Shrink Factors}

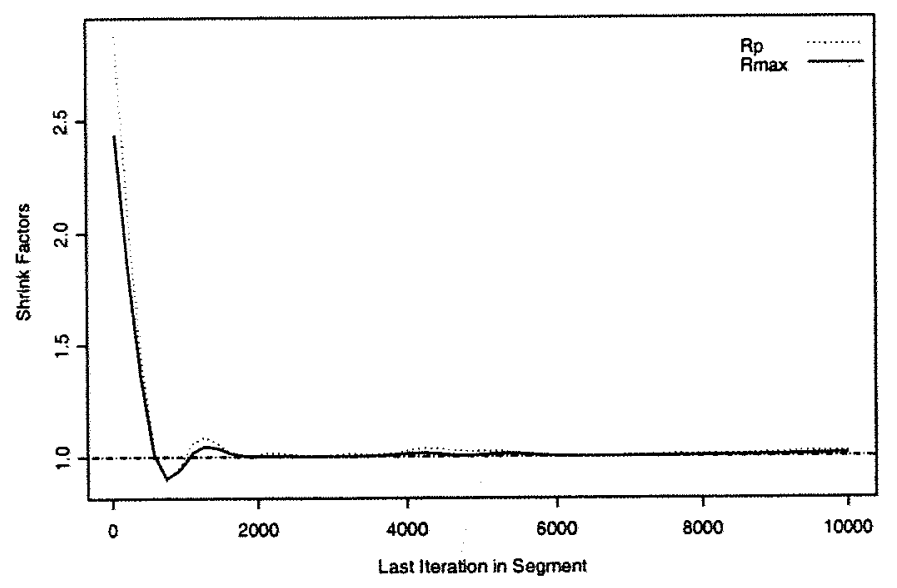

Figura 2.16: Gráfico da medida "Multivariate Potential Scale Reduction"(MPSRF) de Brooks e Gelman (1998) baseado em $M=4$ seqüências de Gibbs de comprimento $T=$ 10000 (Rmax é o maior valor entre as medidas univariadas de $\alpha, \beta, \mu, \sigma_{x}^{2}, \sigma^{2}, \sigma_{e}^{2}$ ).

\section{Gelman \& Rubin Shrink Factors}
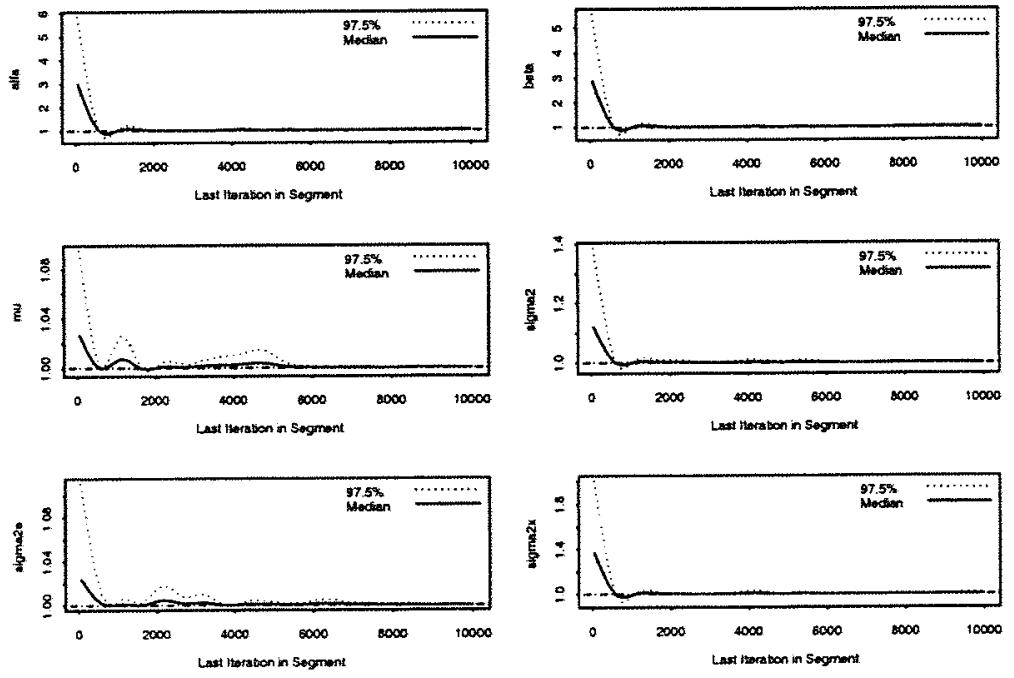

Figura 2.17: Gráfico da medida de Gelman e Rubin corrigida "Corrected potencial scale reduction factors" (CSRF) para cada parametro $\alpha, \beta, \mu, \sigma_{x}^{2}, \sigma^{2}, \sigma_{e}^{2}$, baseada em $M=4$ seqüências de Gibbs de comprimento $T=10.000$. 
Tabela 2.5: Medidas de Diagnóstico de convergência de Brooks, Gelman e Rubin

\begin{tabular}{c|c|cc}
\hline & Medida de Gelman\&Rubin(PSRF) & Medida Corrigida(CSRF) & Quantil 0.975 \\
\hline$\alpha$ & 1.008714 & 1.015572 & 1.035024 \\
$\beta$ & 1.008708 & 1.015500 & 1.034929 \\
$\mu$ & 0.9999645 & 1.000034 & 1.000171 \\
$\sigma_{x^{2}}$ & 1.000683 & 1.002484 & 1.006979 \\
$\sigma^{2}$ & 0.9999692 & 1.000794 & 1.002451 \\
$\sigma_{e}^{2}$ & 1.002024 & 1.000011 & 1.000157 \\
\hline
\end{tabular}

A medida multivariada (MPSRF) é igual a 1.014622 .

Obs. As figuras 2.14 a 2.17 e a tabela 2.5 não sugerem que exista problemas na convergência do algoritmo de Gibbs .

\section{(c) Estimação}

$\mathrm{Na}$ figura 2.18 apresentamos os gráficos das densidades posterioris marginais estimadas, calculadas de acordo com a seção 2.9 , e na tabela 2.6 as estimativas à posteriori de várias medidas resumo. Essas estimativas baseiam-se em $M=4$ seqüências de Gibbs de comprimento $T=10.000$, descartando-se as primeiras $T_{0}=$ 5.000 iterações.
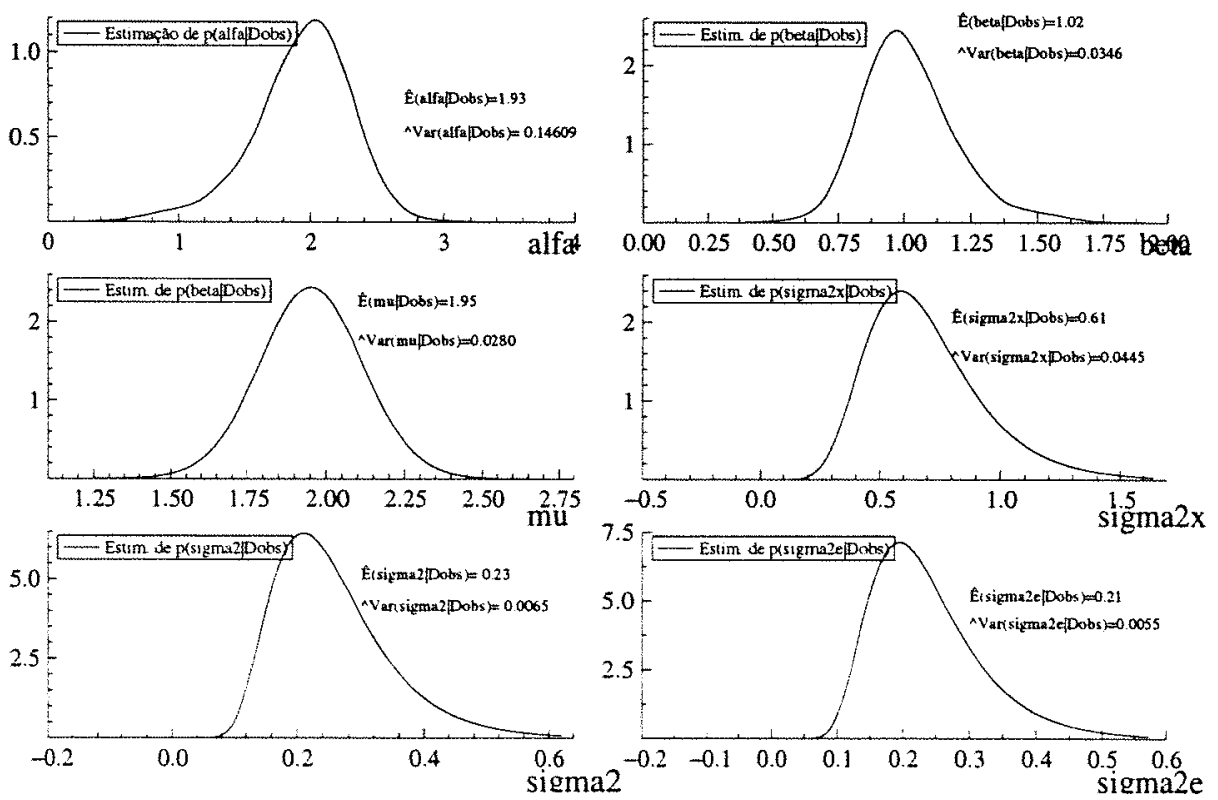

Figura 2.18: Gráficos das estimativas "Rao-Blackwelizadas" das densidades marginais à posteriori $p\left(\alpha \mid D_{\mathrm{obs}}\right), \ldots p\left(\sigma_{e}^{2} \mid D_{\mathrm{obs}}\right)$. 
Tabela 2.6: Estimativas do valor esperado, quantis, desvio padrão, máximo,mínimo e intervalos de credibilidade de comprimento mínimo das distribuições marginais à posteriori de $\alpha, \beta, \mu, \sigma_{x}^{2}, \sigma^{2}, \sigma_{e}^{2}$, usando os dados da tabela 2.1

\begin{tabular}{|c|c|c|c|c|c|c|}
\hline \multirow{3}{*}{$\begin{array}{r}\text { MEDIDAS RESUMO } \\
\text { Media aritmética } \\
\text { Média "R-Blackweliz." } \\
\text { Quantis amostrais } \\
0.025 \\
.05\end{array}$} & $\alpha$ & $\beta$ & & & & \\
\hline & $\begin{array}{l}1.9341 \\
1.9343\end{array}$ & $\begin{array}{l}1.0188 \\
1.0184\end{array}$ & $\begin{array}{l}1.9527 \\
1.9524\end{array}$ & $\begin{array}{l}0.6074 \\
0.6089\end{array}$ & $\begin{array}{l}0.2263 \\
0.2258\end{array}$ & $\begin{array}{l}0.2084 \\
0.2087\end{array}$ \\
\hline & $\begin{array}{l}1.0234 \\
1.2399 \\
1.4387 \\
1.6524 \\
1.7208 \\
1.7791 \\
1.8801 \\
1.9701 \\
2.0556 \\
2.1434 \\
2.1885 \\
2.2393 \\
2.3781 \\
2.4876 \\
2.5872\end{array}$ & $\begin{array}{l}0.7050 \\
0.7525 \\
0.8059 \\
0.8701 \\
0.8939 \\
0.9165 \\
0.9580 \\
0.9999 \\
1.0432 \\
1.0934 \\
1.0934 \\
1.1558 \\
1.254 \\
1.3499 \\
1.4631\end{array}$ & $\begin{array}{l}1.617 \\
1.6775 \\
1.7400 \\
1.8135 \\
1.842 \\
1.8658 \\
1.9108 \\
1.9522 \\
1.9945 \\
2.0399 \\
2.064 \\
2.0925 \\
2.1646 \\
2.229 \\
2.2851\end{array}$ & $\begin{array}{l}0.2917 \\
0.3270 \\
0.3724 \\
0.4334 \\
0.4584 \\
0.4825 \\
0.5284 \\
0.5749 \\
0.6254 \\
0.6844 \\
0.7181 \\
0.7609 \\
0.8805 \\
0.9984 \\
1.1204\end{array}$ & $\begin{array}{l}0.1122 \\
0.1232 \\
0.1388 \\
0.1598 \\
0.1691 \\
0.1776 \\
0.1944 \\
0.2123 \\
0.2313 \\
0.2539 \\
0.2674 \\
0.2830 \\
0.3325 \\
0.3776 \\
0.4217\end{array}$ & $\begin{array}{l}0.1032 \\
0.1144 \\
0.1289 \\
0.1478 \\
0.1559 \\
0.1641 \\
0.1791 \\
0.1949 \\
0.2126 \\
0.2336 \\
0.2461 \\
0.2608 \\
0.3039 \\
0.3467 \\
0.3887\end{array}$ \\
\hline $\begin{array}{l}\text { terv.Credib. HPD } \\
p=0.95 \text { Lim. inf. } \\
\text { Lim sup. } \\
p=0.90 \text { Lim. inf. } \\
\text { Lim sup. }\end{array}$ & $\begin{array}{l}1.1256 \\
2.6501 \\
1.313 \\
2.5362\end{array}$ & $\begin{array}{l}0.6678 \\
1.4027 \\
0.7240 \\
1.3086\end{array}$ & $\begin{array}{l}1.6253 \\
2.2916 \\
1.6811 \\
2.2305\end{array}$ & $\begin{array}{l}0.2487 \\
1.0250 \\
0.2828 \\
0.9145\end{array}$ & $\begin{array}{l}0.0993 \\
0.3901 \\
0.1090 \\
0.3471\end{array}$ & $\begin{array}{l}0.0881 \\
0.3554 \\
0.1001 \\
0.3162\end{array}$ \\
\hline $\begin{array}{l}\text { desvio padrão (dp) } \\
\text { d.p. Rao-Blackweliz. }\end{array}$ & $\begin{array}{l}0.3825 \\
0.3822\end{array}$ & $\begin{array}{l}0.1862 \\
0.1861\end{array}$ & $\begin{array}{l}0.1684 \\
0.1672\end{array}$ & $\begin{array}{l}0.2116 \\
0.2110\end{array}$ & & $\begin{array}{l}0.0744 \\
0.0807\end{array}$ \\
\hline "Naive SE" = & 0.002705 & 0 & م & 0.0014 & & 0.000020 \\
\hline $\begin{array}{l}\text { "Batch SE" } \\
\text { Mínimo } \\
\text { Máximo }\end{array}$ & $\begin{array}{l}0.014119 \\
0.3045 \\
3.4224 \\
\end{array}$ & $\begin{array}{l}0.0068 \\
0.3671 \\
1.9439 \\
\end{array}$ & $\begin{array}{l}0.00173 \\
1.2070 \\
2.6967 \\
\end{array}$ & $\begin{array}{l}0.0012 \\
0.1451 \\
2.2617 \\
\end{array}$ & $\begin{array}{l}0.0507 \\
0.8209 \\
\end{array}$ & $\begin{array}{l}0.055 \\
1.322 \\
\end{array}$ \\
\hline
\end{tabular}

NOTA: Os intervalos de credibilidade nesse exemplo coincidem com os intervalos HPD.

"Batch SE" é o desvio padrão amostral das médias de grupos consecutivos de tamanho 50, dividido pela raiz quadrada do número de grupos.

Os valores dos parâmetros usados na geração dos dados sāo: $\alpha=2.0, \beta=1.0, \mu=2.0, \sigma_{x}^{2}=0.6$, $\sigma^{2}=0.1$ e $\sigma_{e}^{2}=0.1$ )

$\mathrm{M}=$ número de cadeias paralelas.

$\mathrm{T}=$ número total de iteraçōes, $T_{0}=$ número de iteraçōes descartadas.

Na tabela 2.6 vemos que as estimativas do valor esperado da distribuição à posteriori dos parâmetros, obtidas pela média aritmética e pela média Rao-Blackelizada estão muito próximas, além disso, tanto a média como a mediana superestimam os valores verdadeiros dos parâmetros $\sigma^{2}$ e $\sigma_{e}^{2}$ (valores usados na geração dos dados), mas o valor do viés estimado de cada um dos demais parâmetros é pequeno. Mais importante ainda é que todas as estimativas dos intervalos de credibilidade de $95 \%$ contém os valores verdadeiros dos parâmetros. 


\section{(d) Avaliação do ajuste}

A Figura 2.19 a seguir apresenta os gráficos dos valores da densidade condicional preditiva de validação cruzada, $\left[\left(\mathrm{CPO}_{i}=\pi\left(\left(\begin{array}{l}Y_{i} \\ X_{i}\end{array}\right) \mid D_{\text {obs }}\right)\right)\right]$, primeiramente em função de $i$, depois em função de $X_{i}$ e também em função de $Y_{i}$, para $i=1, \ldots, 30$, onde $\mathrm{CPO}_{i}$ é estimada por meio da fórmula (2.10.8) adaptada para o caso do modelo sem restrições de identificabilidade. As estimativas basearam-se na segunda metade de uma sequência de comprimento $\mathrm{T}=10.000$ do algoritmo de Gibbs cujo ponto inicial é ${\underset{\sim}{1}}_{1}^{(0)}$.

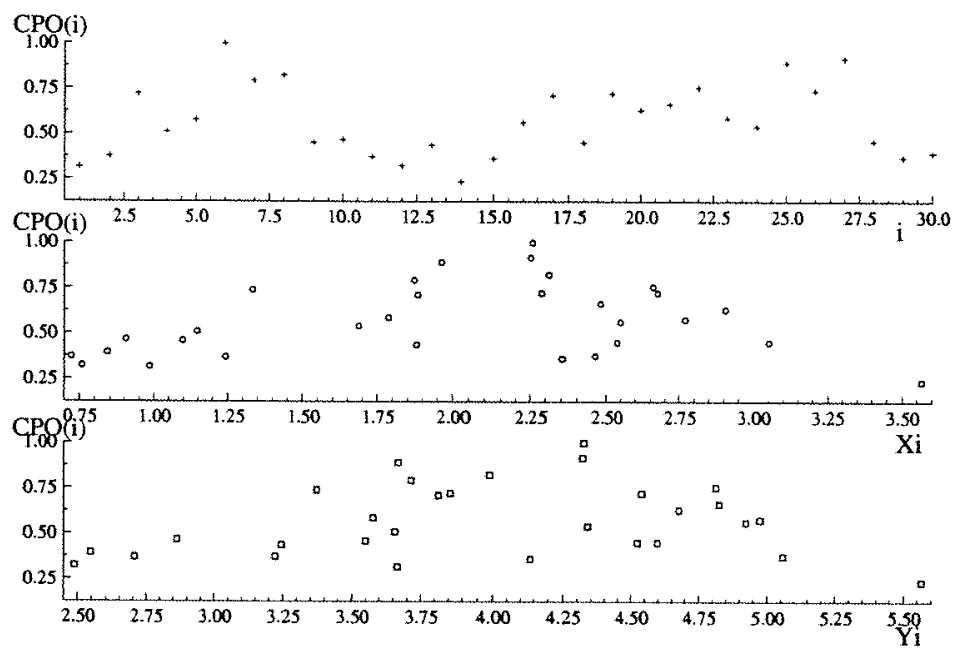

Figura 2.19: Gráficos dos valores da densidade condicional preditiva, (CPO(i)), versus $i$, versus $X_{i}$ e versus $Y_{i}$, baseados na segunda metade de uma única seqüência de Gibbs de comprimento $T=10.000$.

A figura 2.20 a seguir apresenta os gráficos das componentes $d_{1_{i}}$ e $d_{2_{i}}$ do vetor de resíduos bayesianos bidimensionais de validação cruzada

$$
d_{i}=\left(\begin{array}{c}
d_{1_{i}} \\
d_{2_{i}}
\end{array}\right)=\widehat{\operatorname{Var}}^{-\frac{1}{2}}\left(\left(\begin{array}{c}
Y_{i} \\
X_{i}
\end{array}\right) \mid D_{\mathrm{obs}(-i)}\right)\left(\left(\begin{array}{c}
Y_{i} \\
X_{i}
\end{array}\right)-\hat{E}\left(\left(\begin{array}{c}
Y_{i} \\
X_{i}
\end{array}\right) \mid D_{\mathrm{obs}(-i)}\right)\right),
$$

$i=1, \ldots, n$, dado na seção 2.10 .2 , onde as fórmulas de $\hat{E}\left(\left(\begin{array}{l}Y_{2} \\ X_{1}\end{array}\right) \mid D_{\text {obs }(-i)}\right)$ e de $\widehat{\operatorname{Var}}^{-\frac{1}{2}}\left(\left(\begin{array}{l}Y_{i} \\ X_{i}\end{array}\right) \mid D_{\mathrm{obs}(-i)}\right)$ foram adaptadas para o modelo sem restrição de identificabilidade a partir respectivamente das formulas (2.10.13) e (2.10.15). 


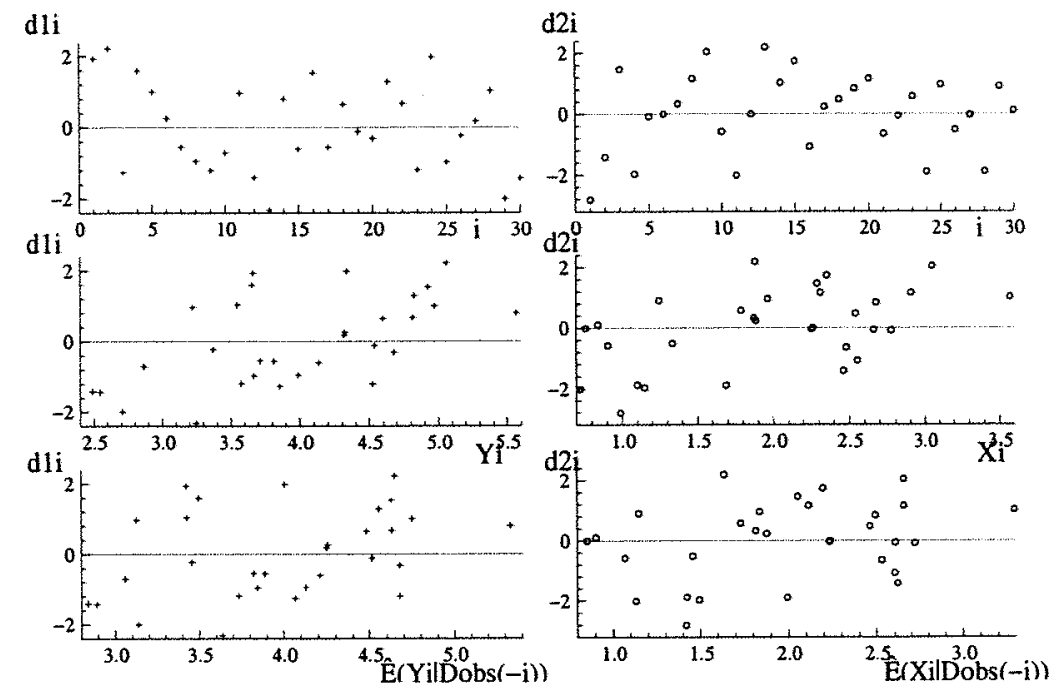

Figura 2.20: Os gráficos do lado esquerdo referem-se à primeira componente do vetor de resíduos $\left(d_{1 i}\right)$, e os do lado direito à segunda componente $\left(d_{2 i}\right)$.Eles estāo baseados na mesma sequencia de Gibbs utilizada na figura 2.19 .

As figuras 2.19 e 2.20 não sugerem que haja problemas de falta de ajustamento, além disso, aparentemente a qualidade do ajuste do modelo sem restrição é melhor do que a do modelo com restrição de identificabilidade (ver fig. 2.6 e 2.7).

\section{COMPARAÇÃO de MODELOS}

A figura 2.21 compara os valores das $\mathrm{CPO}(\mathrm{i})$ 's dos modelos com e sem restrição de identificabilidade. A fórmula em (2.10.8) foi usada para o cálculo desses valores (no caso sem restr. de identif. essa fórmula foi empregada com as devidas adaptações). Apenas a segunda metade de uma seqüência de Gibbs de comprimento $\mathrm{T}=10000$ foi utilizada nos cálculos.

A tabela 2.7 a apresenta, os valores estimados das seguintes medidas de comparação de modelos: a medida L e a soma dos resíduos padronizados em valor absoluto (ver seção 2.11) para os modelos com e sem restrições de identificabilidade.

$\mathrm{Na}$ figura 2.21 vemos que os valores das $\mathrm{CPO}$ (i)'s tendem a ser maiores no modelo com restr. de identificabilidade e na tabela 2.7 as estimativas da medida $L$ (baseadas no determinante) são consideravelmente menores no modelo com restr. de identif., 


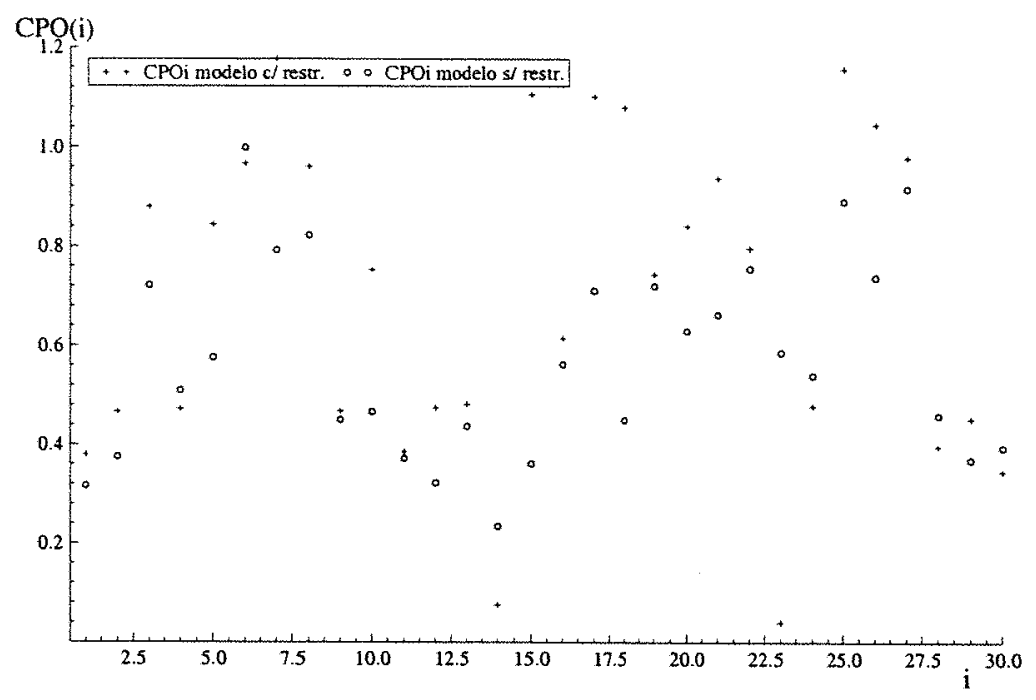

Figura 2.21: Gráfico dos valores da densidade condicional preditiva $(\mathrm{CPO}(\mathrm{i}))$ versus $i$, sob o modelo com restrição de identif. (+) e sob o modelo sem restrição de identif. (o)

Tabela 2.7: Estimativas da medida L e da soma das normas dos resíduos, nos modelos com e sem restrição de identificabilidade.

\begin{tabular}{l|r|cc}
\hline & & Modelo com Restr. & Modelo sem Restr. \\
\hline medida $L$ & det(L2) $\delta=0.0$ & 161.43 & 221.19 \\
& $\delta=0.1$ & 183.74 & 248.74 \\
& $\delta \equiv 0.5$ & 286.02 & 372.0 \\
& $\delta \equiv 0.9$ & 409.18 & 516.14 \\
& $\delta=1.0$ & 443.24 & 43.44 \\
& traço $\left(L_{2}\right) \delta=0.0$ & 41.73 & 43.39 \\
& $\delta \equiv 0.1$ & 45.23 & 46.89 \\
& $\delta \equiv 0.5$ & 59.24 & 60.89 \\
& $\delta \equiv 0.9$ & 73.24 & 74.90 \\
Soma das normas & $\delta \equiv 1.0$ & 76.74 & 78.40 \\
\hline dos residuos & & 57.65 & 46.56 \\
\hline
\end{tabular}

o que sugere que este modelo deve ser o escolhido. Entretanto se considerarmos apenas a soma dos módulos dos resíduos padronizados como critério, o modelo sem restrição deveria ser o escolhido (É interessante notar que as estimativas dos valores da medida L calculadas pelo traço, ficaram bem próximas nos dois modelos).

Assim, se os dados amostrais são os da tabela 2.1 e o valor de $\lambda$ não é conhecido, o modelo sem restr. de identif. (com distr. à priori vaga descrito nessa seção) não seria uma má escolha. 


\subsubsection{O modelo estrutural com restrição de identificabili- dade e priori imprópria}

\section{DADOS}

O conjunto de dados é o mesmo da Tabela 2.1.

\section{MODELO PROPOSTO}

Consideramos o modelo estrutural dado em (2.3.1) com distribuição à priori imprópria $\pi\left(\alpha, \beta, \mu, \sigma_{x}^{2}, \sigma^{2}\right) \operatorname{com} \alpha, \beta, \mu, \sigma_{x}^{2}$ e $\sigma^{2}$ "independentes", onde $\alpha, \beta$ e $\mu$ são proporcionais a $1, \pi\left(\sigma_{x}^{2}\right) \propto\left(\sigma_{x}^{2}\right)^{-r}$ e $\pi\left(\sigma^{2}\right) \propto\left(\sigma^{2}\right)^{-s}$, onde $r=.25$ e $s=1.0$.

\section{AJUSTE DO MODELO}

\section{(a) Pontos iniciais}

Geramos 4 pontos iniciais usando procedimento semelhante ao da seção 2.12.1: $\stackrel{\theta}{1}_{1}^{(0)}=(2.4012,0.8822,2.0033,0.1813,0.1507) ;{\underset{\sim}{2}}_{2}^{(0)}=(1.7943,1.1077,1.9321,0.6855$, $0.2603) ;{\underset{\sim}{3}}_{3}^{(0)}=(1.1561,1.3948,2.1476,0.2809,0.0832)$ e $\theta_{4}^{(0)}=(1.6981,1.1382$, $1.7934,0.3590,0.0731)$.

A partir dsses pontos geramos $M=4$ seqüências de Gibbs de comprimento $T=10000$ e uma seqüência mais longa de comprimento $T=50000$ partindo de ${\underset{\sim}{1}}_{1}^{(0)}$. (b) Diagnósticos de convergência das seqüências de Gibbs

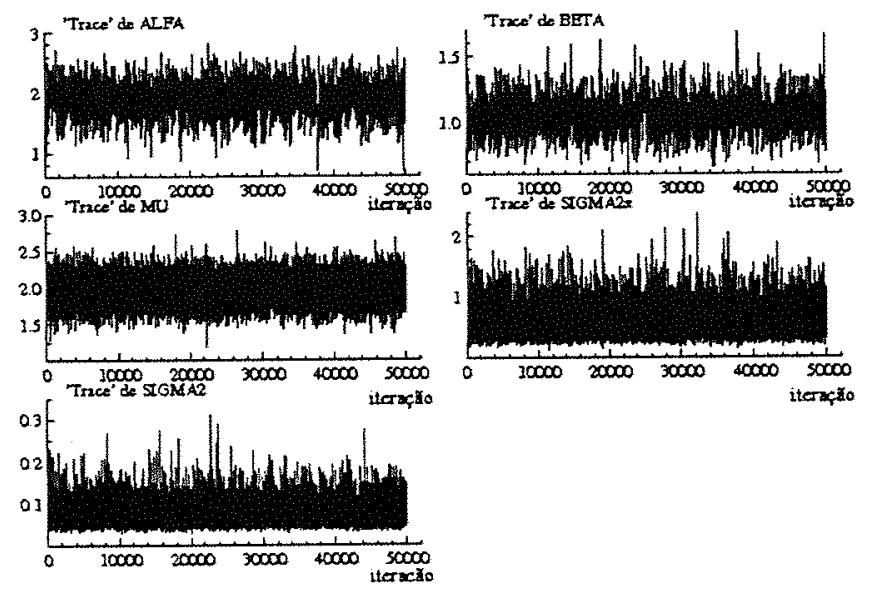

Figura 2.22: Gráficos das séries temporais de $\alpha, \beta, \mu, \sigma_{x}^{2}$ e $\sigma^{2}$. baseados na seq de Gibbs de compr. $T=50.000$. 


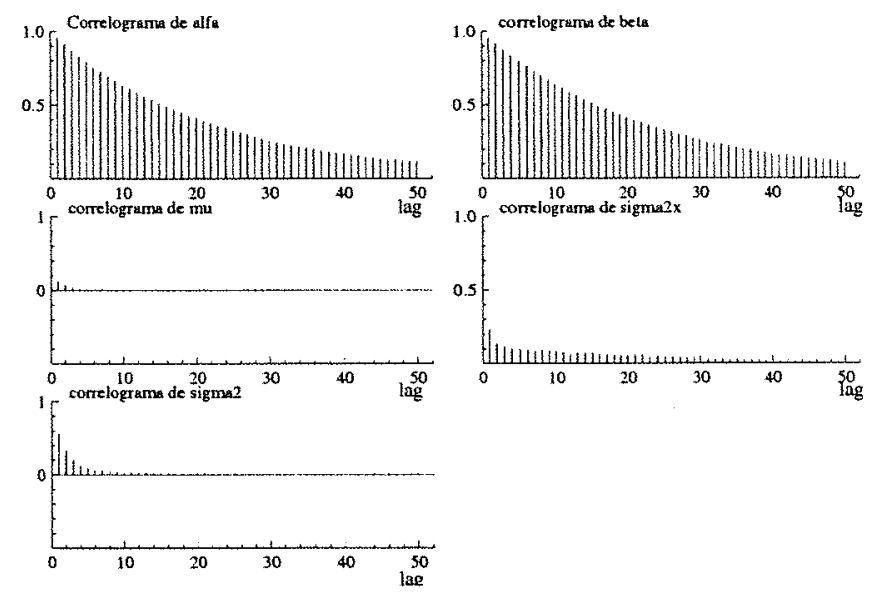

Figura 2.23: Gráficos das funçōes de auto-correlação de $\alpha, \beta, \mu, \sigma_{x}^{2}$ e $\sigma^{2}$, baseados em uma única seqüência de Gibbs de comprimento $T=50.000$.

Brooks \& Gelman Multivariate Shrink Factors

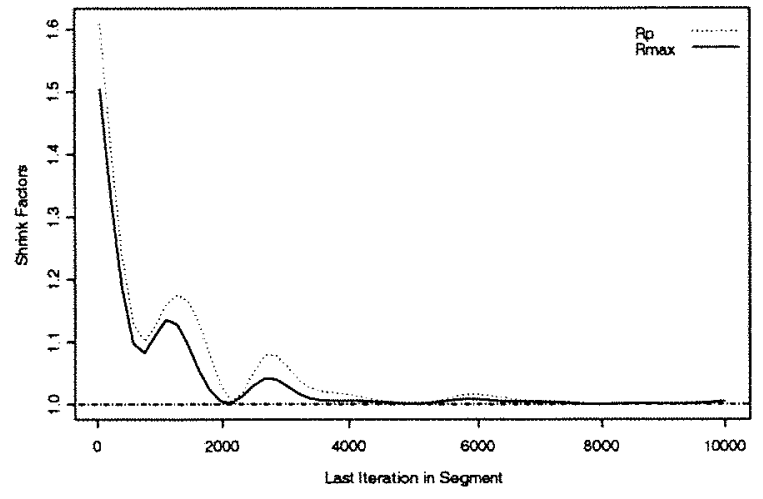

Figura 2.24: "Multivariate Potential Scale Reduction" (MPSRF) de Brooks e Gelman (1998) baseado em 4 seq. de Gibbs de compr. $T=10000$ (Rmax é o maior valor entre as medidas univariadas de $\alpha, \beta, \mu, \sigma_{x}^{2}, \sigma^{2}$ ).

Tabela 2.8: Medidas de Diagnóstico de convergência de Brooks, Gelman e Rubin

\begin{tabular}{c|c|cc}
\hline & Medida de Gelman\&Rubin(PSRF) & Medida Corrigida(CSRF) & Quantil 0.975 \\
\hline$\alpha$ & 1.004851 & 1.006815 & 1.017351 \\
$\beta$ & 1.004639 & 1.006362 & 1.016433 \\
$\mu$ & 0.9999868 & 1.000022 & 1.000206 \\
$\sigma_{x}^{2}$ & 1.000193 & 1.000693 & 1.001954 \\
$\sigma^{2}$ & 1.000496 & 1.000298 & 1.000919 \\
\hline
\end{tabular}

A medida multivariada (MPSRF) é igual a 1.0086177. 


\section{Gelman \& Rubin Shrink Factors}
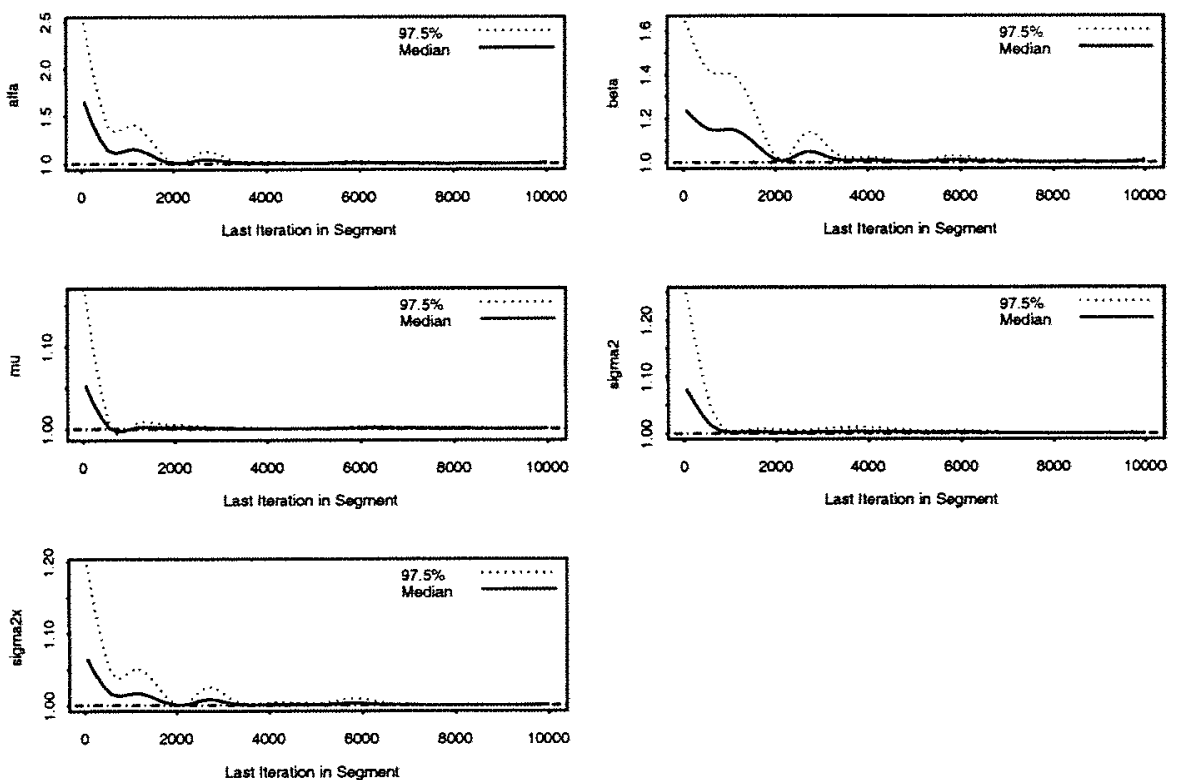

Figura 2.25: Gráfico da medida de Gelman e Rubin corrigida "Corrected potencial scale reduction factors" (CSRF) para cada parametro $\alpha, \beta, \mu, \sigma_{x}^{2}, \sigma^{2}$, baseada em $M=4$ seqüências de Gibbs de comprimento $T=10000$.

\section{(c) Estimação}

A tabela 2.9 apresenta as estimativas de várias medidas resumo da distribuição à posteriori e a figura 2.26 apresenta os gráficos das densidades posterioris marginais estimadas. Essas estimativas foram obtidas utilizando-se a segunda metade de cada uma das $M=4$ seqüências de Gibbs de comprimento $T=10.000$.

Antes de análisar os resultados, é importante lembrar que pelo fato da distribuição à priori ser imprópria (e portanto não informativa), as estimativas à posteriori dos parâmetros deveriam estar próximas dos valores dos parâmetros usados na geração dos dados se de fato o procedimento de estimação é adequado.

Na tabela 2.9 observa-se que a média aritmética e a média Rao-Blackelizada estão muito próximas, além disso, tanto a média como a mediana amostral estimam 
cada um dos parâmetros do modelo com "pequeno" viés(estimado) e somente o parâmetro $\sigma^{2}$ parece estar um pouquinho subestimado). Mais importante anda é que todos os intervalos estimados de credibilidade apresentados contém os valores verdadeiros dos parâmetros.

Tabela 2.9: Estimativas do valor esperado, quantis, desvio padrão, máximo,mínimo e intervalos de credibilidade de comprimento mínimo das distribuições marginais à posteriori de $\alpha, \beta, \mu, \sigma_{x}^{2}, \sigma^{2}$.

\begin{tabular}{|c|c|c|c|c|c|}
\hline MEDIDAS RESUMO & $\alpha$ & $\beta$ & & $\sigma_{r}^{2}$ & $\sigma^{2}$ \\
\hline $\begin{array}{l}\text { Média aritmética } \\
\text { Média "R-Blackweliz." }\end{array}$ & $\begin{array}{l}1.9050 \\
1.9047\end{array}$ & $\begin{array}{l}1.0542 \\
1.0543\end{array}$ & $\begin{array}{l}1.9731 \\
1.9735\end{array}$ & $\begin{array}{l}0.5792 \\
0.5785\end{array}$ & $\begin{array}{l}0.0766 \\
0.0765\end{array}$ \\
\hline $\begin{array}{r}0.025 \\
.05 \\
.1 \\
.25 \\
.3 \\
.4 \\
.5 \\
.6 \\
.75 \\
.89 \\
95 \\
975\end{array}$ & $\begin{array}{l}1.3910 \\
1.4853 \\
1.5925 \\
1.7144 \\
1.7977 \\
1.8671 \\
1.9259 \\
1.9821 \\
2.0372 \\
2.0677 \\
2.1887 \\
2.2607 \\
2.3217\end{array}$ & $\begin{array}{l}0.8500 \\
0.8827 \\
0.9198 \\
0.96167 \\
0.9905 \\
1.8169 \\
1.04717 \\
1.1062 \\
1.150 \\
1.1463 \\
1.2049 \\
1.3008\end{array}$ & $\begin{array}{l}1.6816 \\
1.7296 \\
1.8568 \\
1.8764 \\
1.8980 \\
1.9373 \\
2.0081 \\
2.0496 \\
2.0712 \\
2.0960 \\
2.1594 \\
2.2655\end{array}$ & $\begin{array}{l}0.3004 \\
8.3312 \\
0.3714 \\
0.4221 \\
0.4450 \\
8.5055 \\
0.5475 \\
0.5909 \\
0.6439 \\
0.6756 \\
0.7147 \\
0.886 \\
0.9422 \\
1.0453\end{array}$ & $\begin{array}{l}0.0447 \\
8.0481 \\
0.0525 \\
0.0583 \\
0.0607 \\
0.0632 \\
0.0679 \\
0.0728 \\
0.0781 \\
0.0843 \\
0.0843 \\
0.0924 \\
0.1057 \\
0.1180 \\
0.1302\end{array}$ \\
\hline $\begin{array}{l}\text { Credib. HPD } \\
0.95 \text { Lim. inf. }\end{array}$ & 1.4267 & 0.8428 & 1.6763 & 0.2532 & 0.0395 \\
\hline Lim sup. & & & & & \\
\hline$p=0.90$ Lim. inf. & 1.5215 & 0.8742 & 1.73 & 0.2 & 0.0432 \\
\hline Lim & $\begin{array}{l}2.2877 \\
02366\end{array}$ & $\begin{array}{l}1.2467 \\
0.11145\end{array}$ & $\begin{array}{l}2.2195 \\
0.1480\end{array}$ & $\begin{array}{l}0.8696 \\
0.1836\end{array}$ & $\begin{array}{l}0.1088 \\
0.0223\end{array}$ \\
\hline d.p. Rao-Blackweliz. & 0.2367 & 0.1143 & 0.1477 & 0.1924 & 0.0222 \\
\hline$"$ Naive SE" $=\frac{d p}{\sqrt{M\left(T-T_{0}\right)}}$ & 0.001673 & 0.000810 & 0.001047 & 0.001362 & 0.000157 \\
\hline "Bat & & 90 & 56 & 44 & 0. \\
\hline Mínil & $\begin{array}{l}0.7077 \\
2.6747\end{array}$ & $\begin{array}{l}0.6796 \\
1.6771\end{array}$ & $\begin{array}{l}1.3625 \\
2.7642\end{array}$ & $\begin{array}{l}0.1459 \\
2.1438\end{array}$ & $\begin{array}{l}0.0304 \\
0.2745\end{array}$ \\
\hline
\end{tabular}

NOTA: mesma nota da tabela 2.3 .

Comparando a tabela $2.9 \mathrm{com}$ a tabela 2.3 notamos que as esimativas de Bayes dos parâmetros do modelo com priori imprópria tem menor viés estimado do que as estimativas de Bayes no modelo com priori própria vaga (da secao 2.12.1) (Isso ja era esperado pelo fato de que no modelo com priori imprópria toda a informação vem somente dos dados). Observou-se também que os intervalos de credibilidade envolvendo o modelo com priori imprópria tendem a ter menor comprimento. 

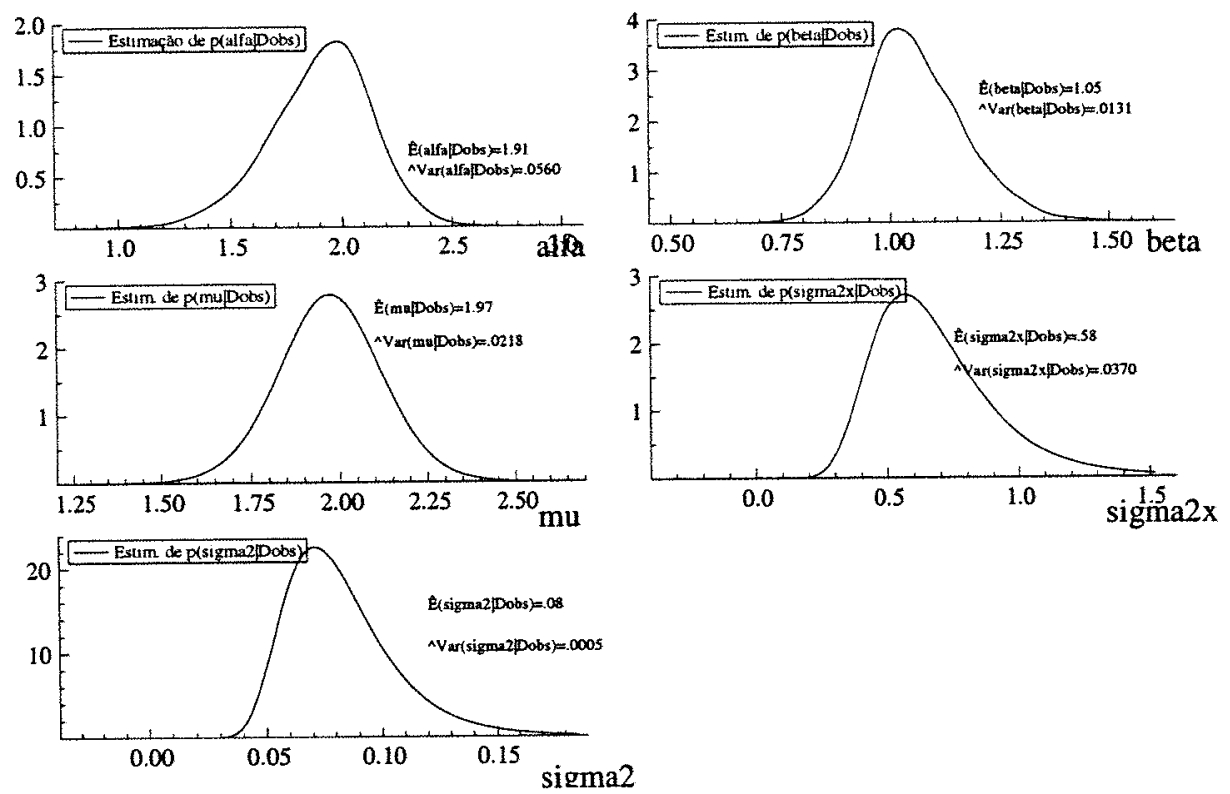

Figura 2.26: Gráficos das estimativas "Rao-Blackwelizadas" das densidades marginais à posteriori $p\left(\alpha \mid D_{\text {obs }}\right), \ldots, p\left(\sigma^{2} \mid D_{\text {obs }}\right)$.

\section{(d)Avaliação do ajuste}

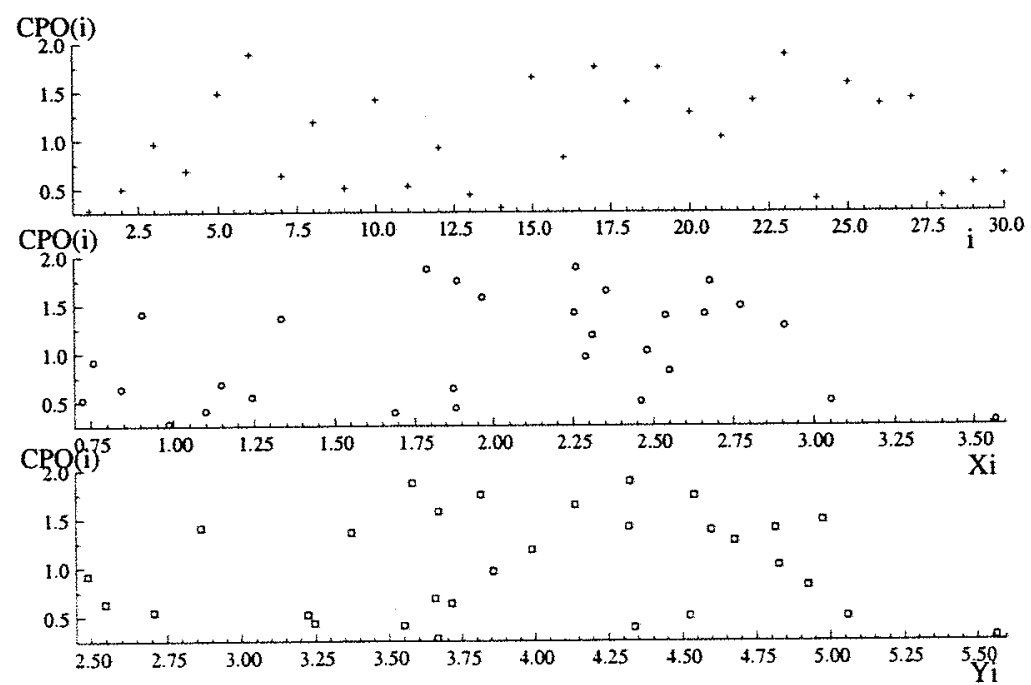

Figura 2.27: Gráficos dos valores da densidade condicional preditiva, $(\mathrm{CPO}(\mathrm{i}))$, versus $i$, versus $X_{i}$ e versus $Y_{i}$, baseados na segunda metade de uma única sequência de Gibbs de comprimento $T=10.000$ 
$\mathrm{Na}$ figura 2.27 temos os gráficos da densidade condicional preditiva de validação cruzada, $\left[\mathrm{CPO}_{i}=\pi\left(\left(\begin{array}{c}Y_{i} \\ X_{i}\end{array}\right) \mid D_{\text {obs }}\right)\right]$, em função de $i$, depois em função de $X_{i}$ e também em função de $Y_{i}$, para $i=1, \ldots, 30$, onde $\mathrm{CPO}_{i}$ é estimada por meio da fórmula (2.10.8).

A Figura 2.28 apresenta os gráficos dos valores estimados de cada uma das componentes do vetor de resíduos bayesianos de validação cruzada $\left(d_{1 i}\right.$ e $\left.d_{2 i}\right)$ da seção 2.10.2(b). Esses gráficos estão baseados na mesma sequencia de Gibbs utilizada na figura 2.27 .

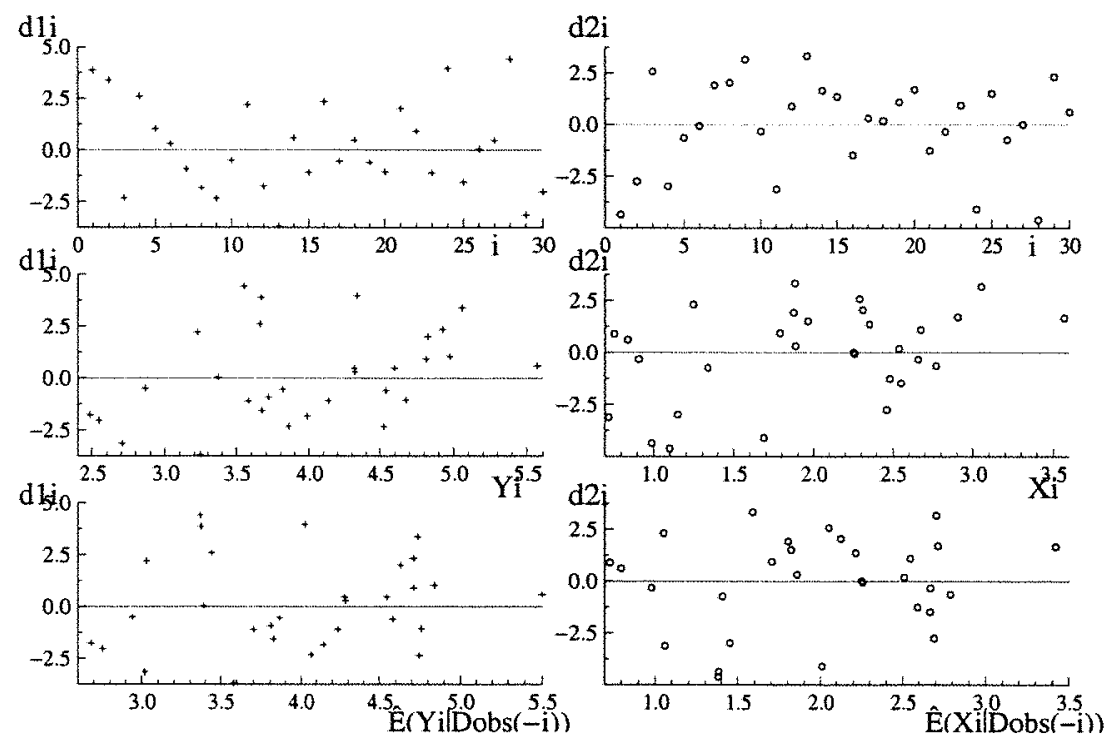

Figura 2.28: Do lado esquerdo estão os valores da componente $\left(d_{1 i}\right)$ dos resíduos bayesianos de validação cruzada em função de $i$, de $Y_{i}$ e de $\hat{E}\left(Y_{i} \mid D_{\text {obs-1 }}\right)$, e do lado direito os valores de $\left(d_{2 i}\right)$ em função de $i$, de $X_{i}$ e de $\hat{E}\left(X_{i} \mid D_{\text {obs(-i) }}\right)$.

$\mathrm{Na}$ figura 2.28 os gráficos à esquerda possuem 4 ou 5 possíveis pontos aberrantes (cujas ordenadas são maiores do que 3 em valor absoluto) o que sugere que uma análise de resídous mais detalhada deva ser realizada para examinar melhor o ajuste.

\section{SENSIBILIDADE $\grave{A} \lambda$ - a razão das variâncias residuais}

Nesse item analisamos descritivamente a variação nas estimativas dos valores es- 
perados de $\alpha, \beta, \mu, \sigma_{x}^{2}$ e $\sigma^{2}$ à posteriori quando diferentes valores do hiperparâmetro $\lambda$ são fixados (mantendo-se inalterados os demais hiperparâmetros do modelo).

Inicialmente nas figuras 2.29 e 2.30 exibimos gráficos com as médias "Rao-Blackwelizadas" dos parâmetros. (Para cada $\lambda$ foi gerada uma seqüência de Gibbs de comprimento $T=10.000$, cujo ponto inicial é o estimador de máxima verossimilhança correspondente, descartando-se a primeira metade de cada seqüência).

A seguir na figura 2.31 comparamos os gráficos baseados em dois diferentes tamanhos de amostra $(\mathrm{n}=50, n=500)$ geradas à partir do mesmo modelo e usando a mesma semente (ranseed $=17$ da linguagem OX). Para cada $\lambda$ fixado, a seqüência de Gibbs foi gerada partindo-se da estimativa de máxima verossim. correspondente.

Levando-se em conta que a distribuição à priori é imprópria (e que portanto, se o procedimento é adequado, as estimativas à posteriori devem estar próximas dos valores dos parâmetros usados na geração dos dados) então das figuras 2.29 e 2.30 concluimos que:

(1) A média amostral de $\mu$ parece não ser influenciada pelo valor de $\lambda$.

(2) Quando $\lambda$ é subestimado (isto é, quando $0<\lambda<1$ ), aparentemente, as médias amostrais de $\beta, \sigma_{x}^{2}$ e $\sigma^{2}$ superestimam respectivamente os valores verdadeiros de $\beta$, $\sigma_{x}^{2}$ e $\sigma^{2}$, mas a média amostral de $\alpha$ subestima o verdadeiro valor de $\alpha$.

(3) Quando $\lambda$ é superestimado $(\lambda>1)$, aparentemente $\alpha$ e $\sigma_{x}^{2}$ é superestimado e $\beta$ e $\sigma^{2}$ são subestimados.. Isso ocorre mesmo quando $\lambda$ é bem grande (da ordem de 50 vezes maior que o seu verdadeiro valor).

Da figura 2.31, temos também que as estimativas dos parâmetros aparentemente tendem a se estabilizar à medida que $\lambda$ cresce independentemente do tamanho da amostra (n), sendo que o valor do viés estimado em geral é diferente para cada $n$. 


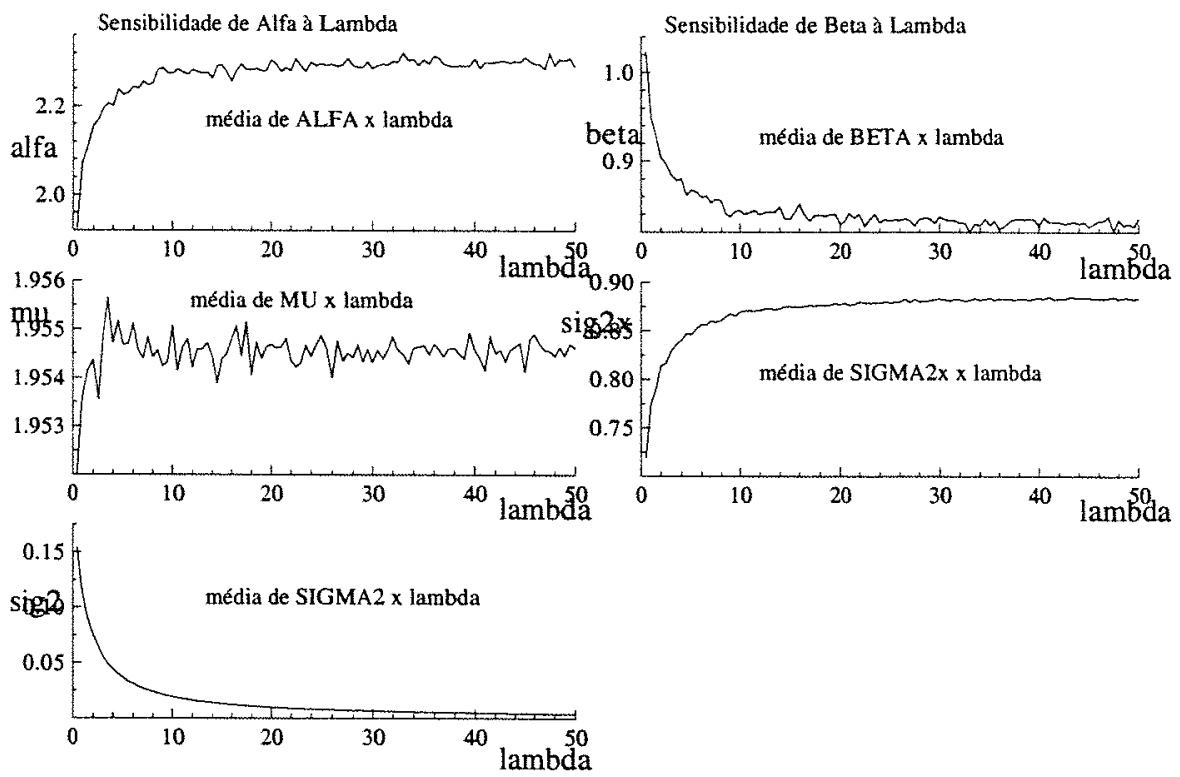

Figura 2.29: Gráficos das médias "R.-Blackwelizadas" de $\alpha, \beta, \mu, \sigma_{x}^{2}$ e $\sigma^{2}$ versus $\lambda$ da distr. posteriori baseada nos dados da Tabela 2.1. A grade de pontos em $\lambda$ é formada por 500 pontos igualmente espaçados no intervalo $[0,50]$.
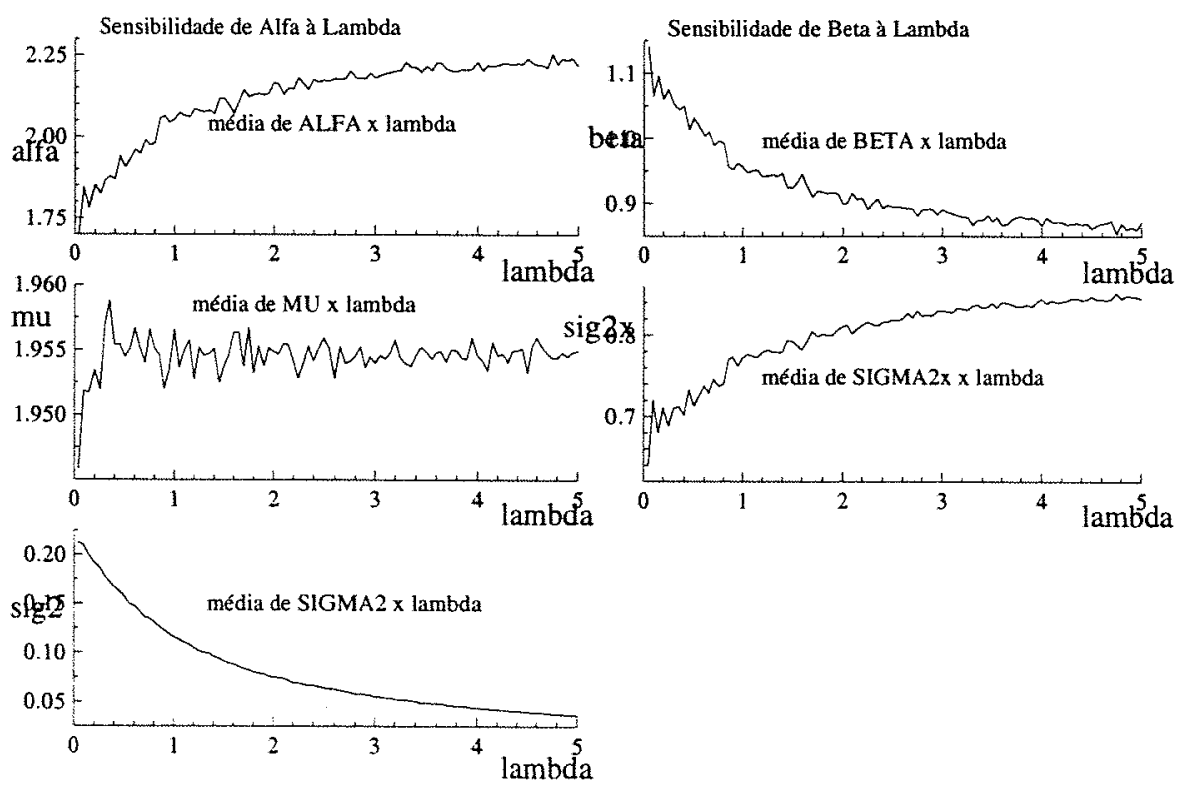

Figura 2.30: Idem à figura anterior, porém com grade em $\lambda$ formada por 500 pontos igualmente espaçados no intervalo $[0,5]$. 

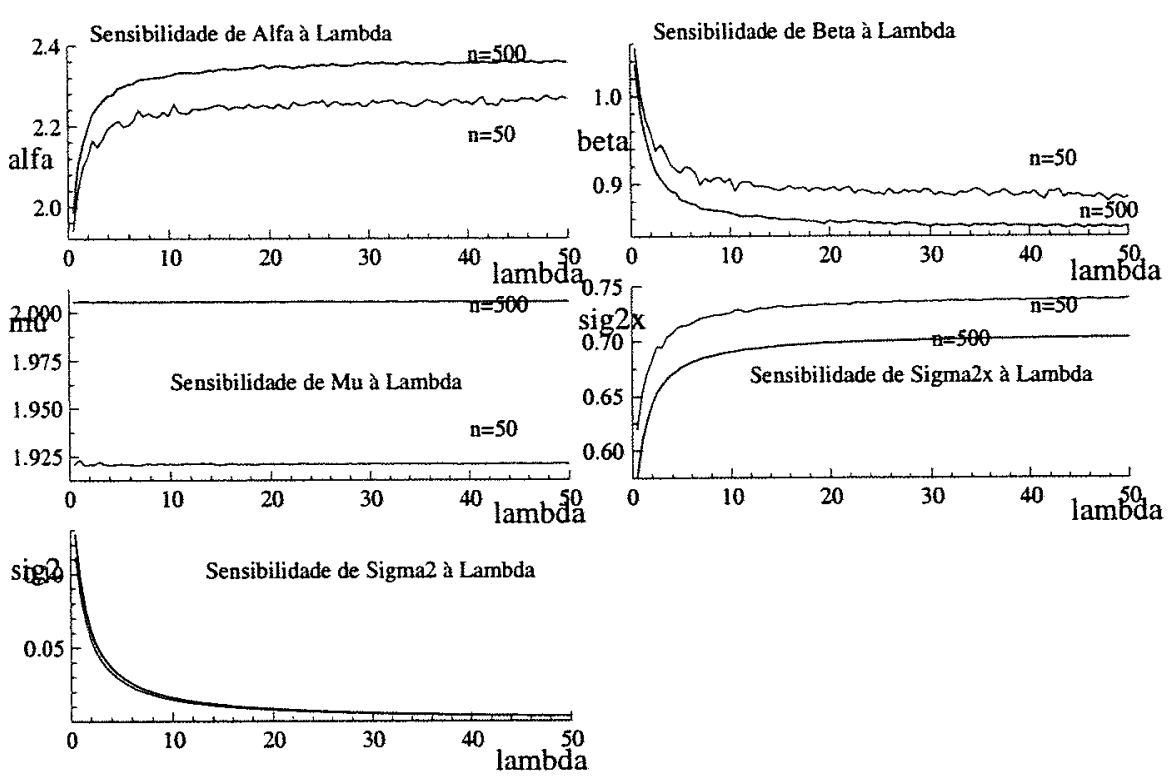

Figura 2.31: Gráfico das médias "Rao-Blackwelizadas" de $\alpha, \beta, \mu_{r} \sigma_{x}^{2}$ e $\sigma^{2}$ versus $\lambda$, das posterioris baseadas nas amostras geradas de tamanho $n=50$ e $n=500$. A grade em $\lambda$ é formada de 100 pontos igualmente espaçados no intervalo $[0,50]$ (os valores verdadeiros dos parâmetros são $\alpha=2.0, \beta=1.0, \mu=2.0, \sigma_{x}^{2}=0.6$ e $\sigma^{2}=0.1$ e de $\lambda$ é 1.0).

\section{COMPARAÇÃO de MODELOS}

$\mathrm{Na}$ figura 2.32 comparamos os valores estimados das $\mathrm{CPO}(\mathrm{i})$ 's no modelo com restrição de identificabilidade com priori própria e com priori imprópria (por meio da fórmula (2.10.8) e usando a segunda metade das seqüências de Gibbs de comprimento $\mathrm{T}=10000)$.

A tabela 2.10 apresenta os valores estimados das seguintes medidas de comparação de modelos: a medida $\mathrm{L}$ e a soma dos resíduos padronizados em valor absoluto (ver seção 2.11) para o modelo com priori própria e para o modelo com priori imprópria com restrição de identif. Os cálculos basearam-se sempre na segunda metade das seqüências de Gibbs de comprimento $T=10000$.

$\mathrm{Na}$ figura 2.32 vemos que os valores das $\mathrm{CPO}$ (i)'s tendem a ser maiores no modelo com priori imprópria e na tabela 2.10 as estimativas da medida $\mathrm{L}$, baseadas no determinante, são menores no modelo com priori imprópria, indicando que o 


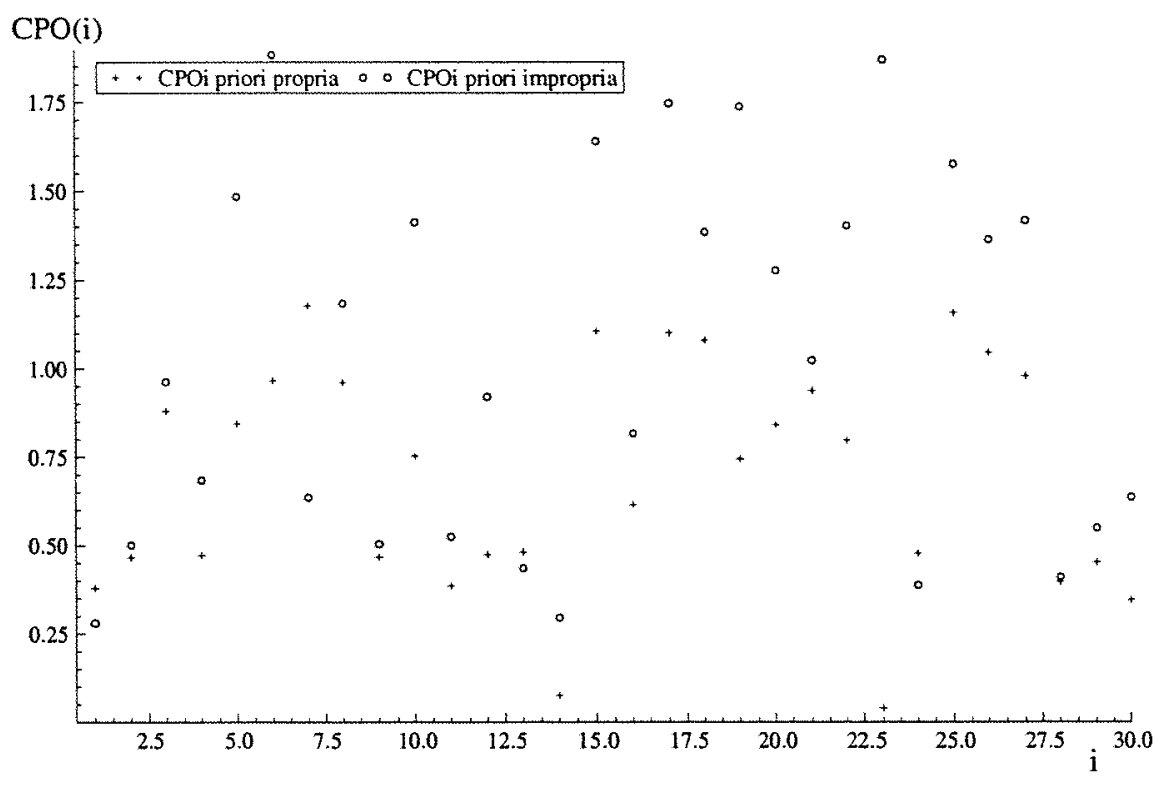

Figura 2.32: Valores da densidade condicional preditiva (CPO(i)) versus $i$, no modelo com priori própria $(+)$ e no modelo com priori imprópria $(0)$.

Tabela 2.10: As estimativas da medida $\mathrm{L}$ e da soma das normas dos resíduos, nos modelos com priori própria e com priori imprópria.

\begin{tabular}{r|r|cc}
\hline & & priori própria. & priori imprópria. \\
\hline medida L & $\operatorname{det}(\mathrm{L} 2) \delta=0.0$ & 161.43 & 98.94 \\
& $\delta=0.1$ & 183.74 & 115.67 \\
& $\delta=0.5$ & 286.02 & 195.67 \\
& $\delta=0.9$ & 409.18 & 296.49 \\
& $\delta=1.0$ & 443.24 & 324.96 \\
& $\operatorname{tr}\left(L_{2}\right) \delta=0.0$ & 41.73 & 42.40 \\
& $\delta=0.1$ & 45.23 & 45.9 \\
& $\delta=0.5$ & 59.24 & 59.9 \\
& $\delta=0.9$ & 73.24 & 73.91 \\
\hline Soma das normas & $\delta=1.0$ & 76.74 & 77.41 \\
\hline dos residuos & & & \\
\hline
\end{tabular}

modelo com priori imprópria deve ser o escolhido. Entretanto se considerarmos a soma dos módulos dos resíduos como critério, o modelo com priori própria deveria ser o escolhido (É interessante notar também que os valores da medida L calculados pelo traço, embora numericamente menores no modelo com priori própria, estão muito próximos nos dois modelos). 


\subsubsection{O modelo estrutural sem restrição de identificabili- dade e priori imprópria}

\section{DADOS}

O conjunto de dados é o mesmo da Tabela 2.1

\section{MODELO PROPOSTO}

Consideramos o modelo estrutural dado em (2.6.1) com distribuição à priori $\pi\left(\alpha, \beta, \mu, \sigma_{x}^{2}, \sigma^{2}, \sigma_{e}^{2}\right)$ com componentes "independentes", onde $\alpha, \beta$ e $\mu$ são proporcionais a $1, \pi\left(\sigma_{x}^{2}\right) \propto\left(\sigma_{x}^{2}\right)^{-\tau}, \pi\left(\sigma^{2}\right) \propto\left(\sigma^{2}\right)^{-s}$, com $r=.25$ e $s=0.5$, e $\sigma_{e}^{2} \sim I G(2.001 ; 1.0)$

\section{AJUSTE DO MODELO}

(a) Pontos iniciais

Usamos os mesmos pontos iniciais da seção 2.12.2, e partindo desses pontos geramos $M=4$ sequiências de Gibbs de comprimento $T=10.000$.

(b) Diagnósticos de convergência das seqüências de Gibbs

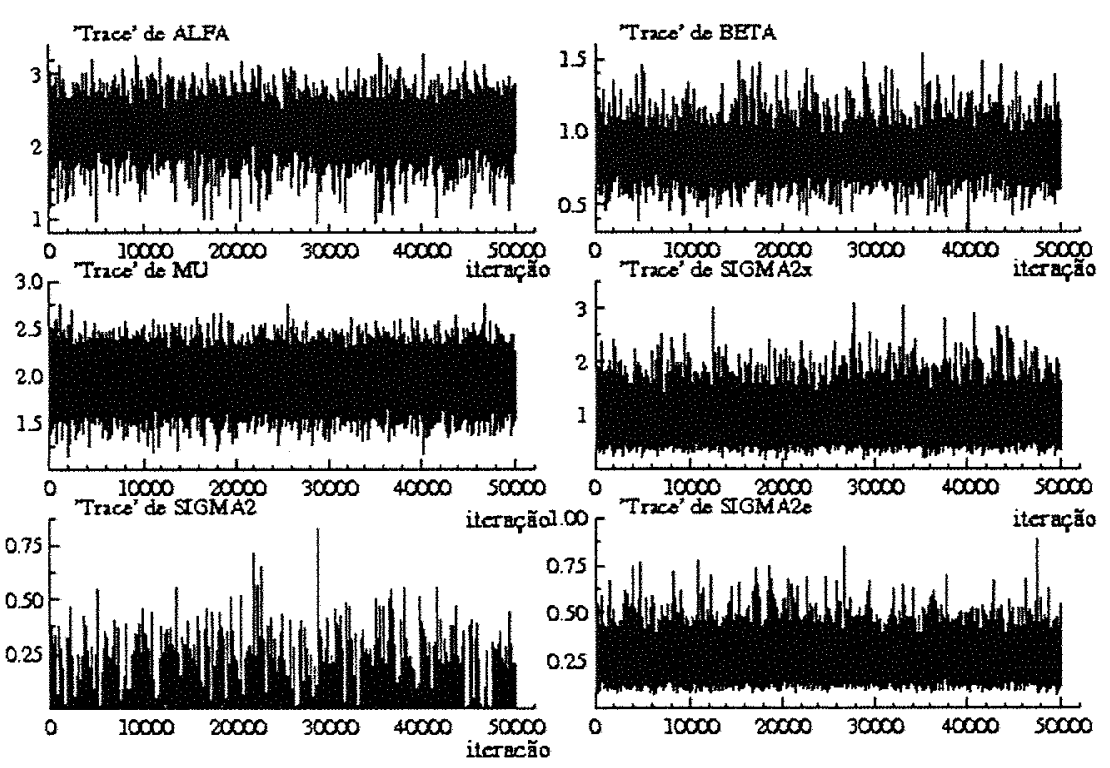

Figura 2.33: Gráficos das séries temporais ("trace") de $\alpha, \beta, \mu, \sigma_{x}^{2}, \sigma^{2}$ e $\sigma_{e}^{2}$, baseados em uma única seqüência de Gibbs de comprimento $T=50.000$. 


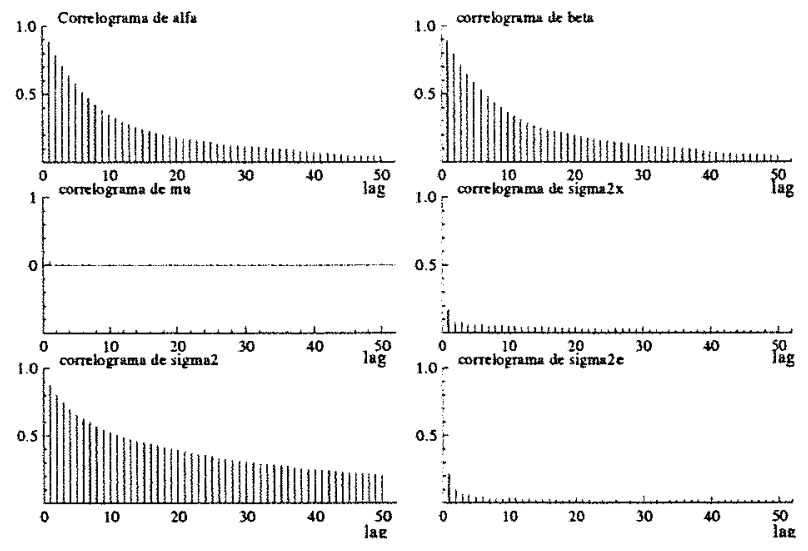

Figura 2.34: Gráficos das funçōes de auto-correlação de $\alpha, \beta, \mu, \sigma_{x}^{2}, \sigma^{2}$ e $\sigma_{e}^{2}$, baseados em uma única seqüência de Gibbs de comprimento $T=50.000$.

Brooks \& Gelman Multivariate Shrink Factors

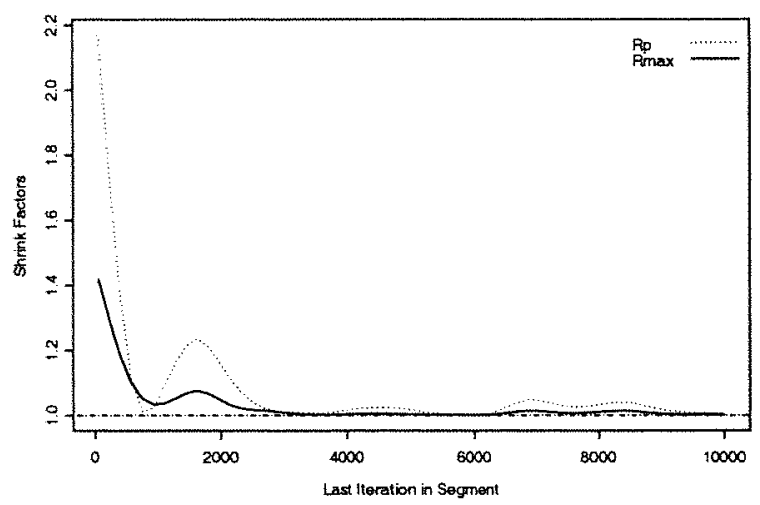

Figura 2.35: "Multivariate Potential Scale Reduction" (MPSRF) de Brooks e Gelman (1998) baseado em $M=4$ seq. de Gibbs de comprimento $T=10.000$ (Rmax é o maior valor entre as medidas univariadas de $\alpha, \beta, \mu, \sigma_{x}^{2}, \sigma^{2}, \sigma_{e}^{2}$ ).

Tabela 2.11: Medidas de Diagnóstico de convergência de Brooks, Gelman e Rubin

\begin{tabular}{c|c|cc}
\hline & Medida de Gelman\&Rubin(PSRF) & Medida Corrigida(CSRF) & Quantil 0.975 \\
\hline$\beta$ & 1.001738 & 1.002581 & 1.006482 \\
$\beta$ & 1.001903 & 1.002636 & 1.006882 \\
$\mu$ & 1.000015 & 1.000045 & 1.000289 \\
$\sigma_{\ddagger}^{2}$ & 1.000338 & 1.000524 & 1.001450 \\
$\sigma_{2}^{2}$ & 1.003152 & 1.003986 & 1.010873 \\
$\sigma_{e}^{2}$ & 1.00017 & 1.000260 & 1.000831 \\
\hline
\end{tabular}

A medida multivariada (MPSRF) é igual a 1.0053917. 


\section{Gelman \& Rubin Shrink Factors}
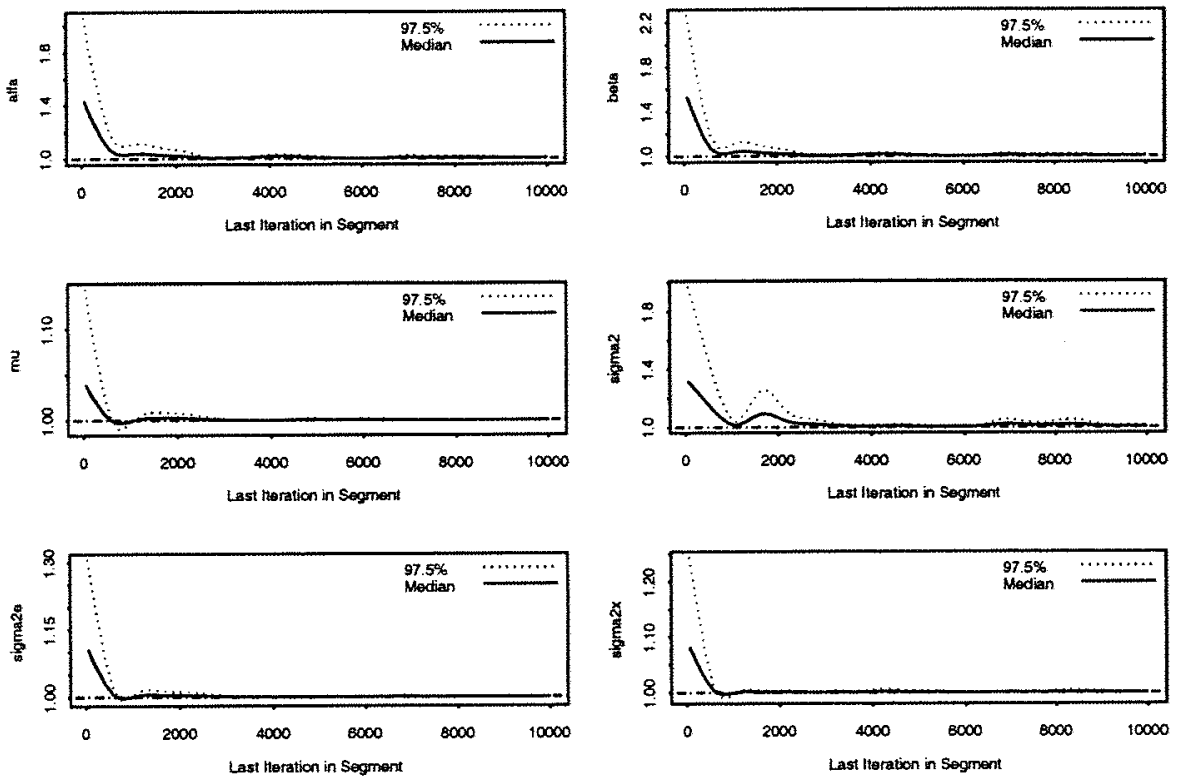

Figura 2.36: Gráfico da medida de Gelman e Rubin corrigida "Corrected potencial scale reduction factors" (CSRF) para cada parametro $\alpha, \beta, \mu, \sigma_{x}^{2}, \sigma^{2}, \sigma_{e}^{2}$, baseada em $M=4$ seqüencias de Gibbs de comprimento $T=10.000$.

\section{(c) Estimação}

Na tabela 2.12 apresentamos as estimativas de várias medidas resumo da distribuição à posteriori e na figura 2.37, os gráficos das densidades posterioris marginais estimadas. Essas estimativas baseiam-se em $M=4$ seqüências de Gibbs de comprimento $T=10.000$, descartando-se a primeira metada de cada uma.

Observa-se na tabela 2.12 que a média aritmética e a média Rao-Blackelizada estão muito próximas. A média e a mediana também estão bastante próximas (exceto no caso do parâmetro $\sigma^{2}$ ), além disso, elas superestimam o verdadeiro valor de $\sigma_{\epsilon}^{2}$ e subestimam o valor de $\sigma^{2}$. Nesse modelo, entretanto, os intervalos estimados de credibilidade de $95 \%$ e $90 \%$ para o parâmetro $\sigma^{2}$ nāo contém o verdadeiro valor de $\sigma^{2}$. 
Comparando a tabela 2.12 com a tabela 2.6 nota-se que embora as estimativas de Bayes no modelo com priori imprópria têm maior viés do que as estimativas de Bayes no modelo com priori própria vaga (da secao 2.12.2), os intervalos de credibilidade de $\alpha, \beta, \mu$ e $\sigma_{x}^{2}$, no modelo com priori imprópria tem menor comprimento. O problema é que $\sigma^{2}$ aparentemente não foi bem estimado (como já foi comentado).

Tabela 2.12: Estimativas do valor esperado, quantis, desvio padrão, máximo,mínimo e intervalos de credibilidade de comprimento mínimo das distribuições marginais à posteriori de $\alpha, \beta, \mu, \sigma_{x}^{2}, \sigma^{2}, \sigma_{e}^{2}$.

\begin{tabular}{|c|c|c|c|c|c|c|}
\hline MEDIDAS RESUMO & $\alpha$ & $\beta$ & $\mu$ & $\sigma_{x}^{2}$ & $\sigma^{2}$ & \\
\hline $\begin{array}{l}\text { Média aritmética } \\
\text { Média "R-Blackweliz." }\end{array}$ & $\begin{array}{l}2.2181 \\
2.2186\end{array}$ & $\begin{array}{l}0.8722 \\
0.8717\end{array}$ & $\begin{array}{l}1.9529 \\
1.9544\end{array}$ & $\begin{array}{l}0.8375 \\
0.8390\end{array}$ & $\begin{array}{l}0.0477 \\
0.0476\end{array}$ & $\begin{array}{l}0.2322 \\
0.2315\end{array}$ \\
\hline $\begin{array}{r}\text { Quantis amostras } \\
0.025 \\
.05 \\
.1 \\
.2 \\
.25 \\
.3 \\
.4 \\
.5 \\
.6 \\
.7 \\
.75 \\
.8 \\
9 \\
.95 \\
.975\end{array}$ & $\begin{array}{l}1.6050 \\
1.7499 \\
1.8886 \\
2.0198 \\
2.0666 \\
2.1069 \\
2.1745 \\
2.2361 \\
2.2953 \\
2.3586 \\
2.3923 \\
2.4312 \\
2.5348 \\
2.6215 \\
2.7048\end{array}$ & $\begin{array}{l}0.6410 \\
0.6789 \\
0.7211 \\
0.7713 \\
0.7887 \\
0.8044 \\
0.8349 \\
0.8632 \\
0.8924 \\
0.9251 \\
0.9432 \\
0.9648 \\
1.0315 \\
1.0954 \\
1.1668\end{array}$ & $\begin{array}{l}1.6061 \\
1.6681 \\
1.7340 \\
1.8105 \\
1.8416 \\
1.8654 \\
1.9119 \\
1.9533 \\
1.9961 \\
2.0406 \\
2.0672 \\
2.0949 \\
2.1704 \\
2.2368 \\
2.2940\end{array}$ & $\begin{array}{l}0.4355 \\
0.4883 \\
0.5497 \\
0.6251 \\
0.6547 \\
0.6844 \\
0.7396 \\
0.7956 \\
0.8585 \\
0.9317 \\
0.9757 \\
1.0257 \\
1.1776 \\
1.3232 \\
1.4683\end{array}$ & $\begin{array}{l}0.0000 \\
0.0003 \\
0.0009 \\
0.0032 \\
0.0050 \\
0.0072 \\
0.0132 \\
0.0225 \\
0.0355 \\
0.0546 \\
0.0674 \\
0.0822 \\
0.1305 \\
0.1785 \\
0.2224\end{array}$ & $\begin{array}{l}0.1264 \\
0.1387 \\
0.1544 \\
0.1751 \\
0.1840 \\
0.1917 \\
0.2070 \\
0.2222 \\
0.2387 \\
0.2573 \\
0.2694 \\
0.2821 \\
0.3212 \\
0.3597 \\
0.3953\end{array}$ \\
\hline $\begin{array}{l}p=0.95 \mathrm{Lim} \text {. inf. } \\
\text { Lim sup. } \\
p=0.90 \mathrm{Lim} . \text { inf. } \\
\text { Lim sup. }\end{array}$ & $\begin{array}{l}1.6487 \\
2.7330 \\
1.8043 \\
2.6711\end{array}$ & $\begin{array}{l}0.6188 \\
1.1334 \\
0.6624 \\
1.0736\end{array}$ & $\begin{array}{l}1.6122 \\
2.2982 \\
1.6701 \\
2.2376\end{array}$ & $\begin{array}{l}0.3929 \\
1.3853 \\
0.4290 \\
1.2331\end{array}$ & $\begin{array}{l}0.0000 \\
0.1785 \\
0.0000 \\
0.1305\end{array}$ & $\begin{array}{l}0.1128 \\
0.3692 \\
0.1208 \\
0.3319\end{array}$ \\
\hline $\begin{array}{l}\text { desvio padrão (dp) } \\
\text { d.p. Rao-Blackweliz. }\end{array}$ & $\begin{array}{l}0.2721 \\
0.2721\end{array}$ & $\begin{array}{l}0.1304 \\
0.1303\end{array}$ & $\begin{array}{l}0.1731 \\
0.1718\end{array}$ & $\begin{array}{l}0.2641 \\
0.2659\end{array}$ & $\begin{array}{l}0.0629 \\
0.0623\end{array}$ & $\begin{array}{l}0.0695 \\
0.0682\end{array}$ \\
\hline $\begin{array}{r}\text { "Naive SE" }=\frac{\frac{d p}{\sqrt{M\left(T-T_{0}\right)}}}{} \\
\text { Batch SE" }\end{array}$ & $\begin{array}{l}0.001924 \\
0.008153\end{array}$ & $\begin{array}{l}0.000922 \\
0.003976\end{array}$ & $\begin{array}{r}20.001224 \\
0.001393\end{array}$ & $\begin{array}{l}0.001867 \\
0.003726\end{array}$ & $\begin{array}{l}0.00044 \\
0.00218\end{array}$ & $\begin{array}{l}0.000491 \\
0.000822\end{array}$ \\
\hline & $\begin{array}{l}0.9371 \\
3.2785\end{array}$ & $\begin{array}{l}0.4045 \\
1.5371\end{array}$ & $\begin{array}{l}1.2028 \\
2.7565\end{array}$ & $\begin{array}{l}0.1684 \\
3.0913\end{array}$ & $\begin{array}{l}0.0000 \\
0.8244\end{array}$ & $\begin{array}{l}0.0735 \\
1.8424\end{array}$ \\
\hline
\end{tabular}

NOTA: Mesma nota da tabela 2.6 . 

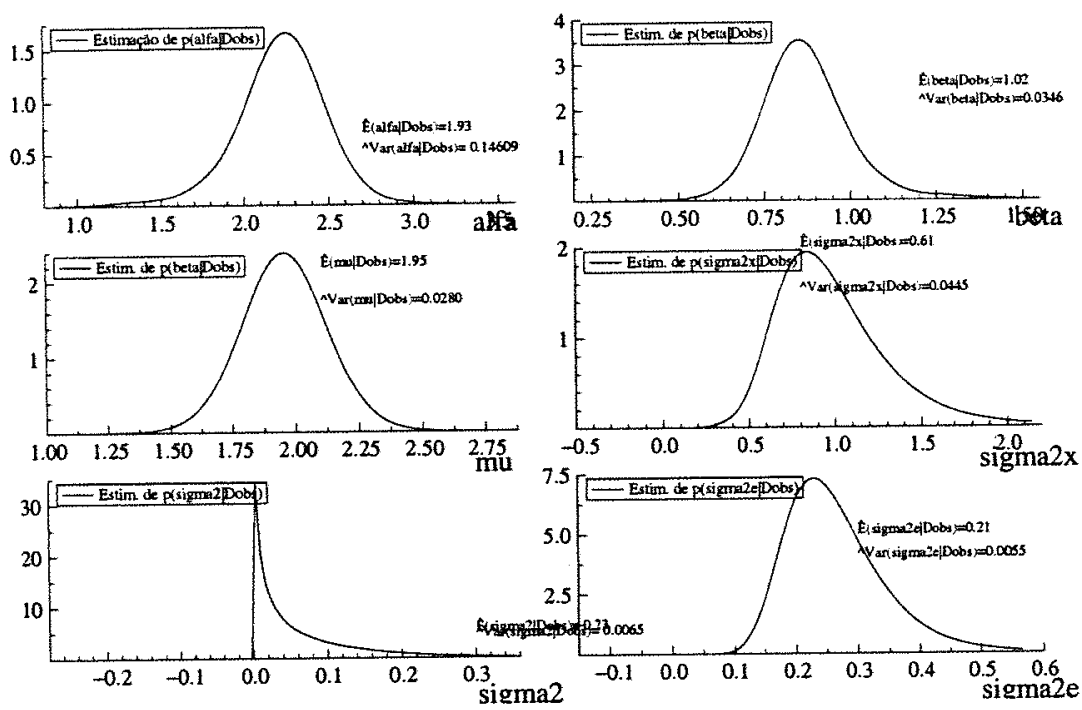

Figura 2.37: Gráficos das estimativas "Rao-Blackwelizadas" das densidades marginais à posteriori $p\left(\alpha \mid D_{\text {obs }}\right), \ldots, p\left(\sigma_{e}^{2} \mid D_{\text {obs }}\right)$.

\section{(d) Avaliação do ajuste}

A Figura 2.38 a seguir apresenta os gráficos dos valores da densidade condicional preditiva de validação cruzada $\left(\mathrm{CPO}_{i}=\pi\left(\left(\begin{array}{l}Y_{i} \\ X_{i}\end{array}\right) \mid D_{\mathrm{obs}}\right)\right)$ primeiramente em função de $i$, depois em função de $X_{i}$ e também em função de $Y_{i}$, para $i=1, \ldots, 30$, onde $\mathrm{CPO}_{i}$ é estimada por meio da fórmula (2.10.8) adaptada para o modelo dessa subseção. As estimativas basearam-se na segunda metade de uma seqüência de comprimento $\mathrm{T}=10.000$ do algoritmo de Gibbs cujo ponto inicial é ${\underset{\theta}{1}}_{1}^{(0)}$.

A figura 2.39 a seguir apresenta os gráficos das componentes $d_{1_{i}}$ e $d_{2_{i}}$ do vetor de resíduos bayesianos bidimensionais de validação cruzada

$$
d_{i}=\left(\begin{array}{c}
d_{1_{i}} \\
d_{2_{i}}
\end{array}\right)=\widehat{\operatorname{Var}}^{-\frac{1}{2}}\left(\left(\begin{array}{c}
Y_{i} \\
X_{i}
\end{array}\right) \mid D_{\mathrm{obs}(-i)}\right)\left(\left(\begin{array}{c}
Y_{i} \\
X_{i}
\end{array}\right)-\hat{E}\left(\left(\begin{array}{c}
Y_{i} \\
X_{i}
\end{array}\right) \mid D_{\mathrm{obs}(-i)}\right)\right),
$$

$i=1, \ldots, n$, dado na seção 2.10 .2 , onde as fórmulas de $\hat{E}\left(\left(\begin{array}{l}Y_{i} \\ X_{i}\end{array}\right) \mid D_{\mathrm{obs}(-i)}\right)$ e de $\widehat{\operatorname{Var}}^{-\frac{1}{2}}\left(\left(\begin{array}{l}Y_{2} \\ X_{1}\end{array}\right) \mid D_{\text {obs }(-i)}\right)$ foram adaptadas para o modelo dessa subseçãos a partir respectivamente das formulas (2.10.13) e (2.10.15). 


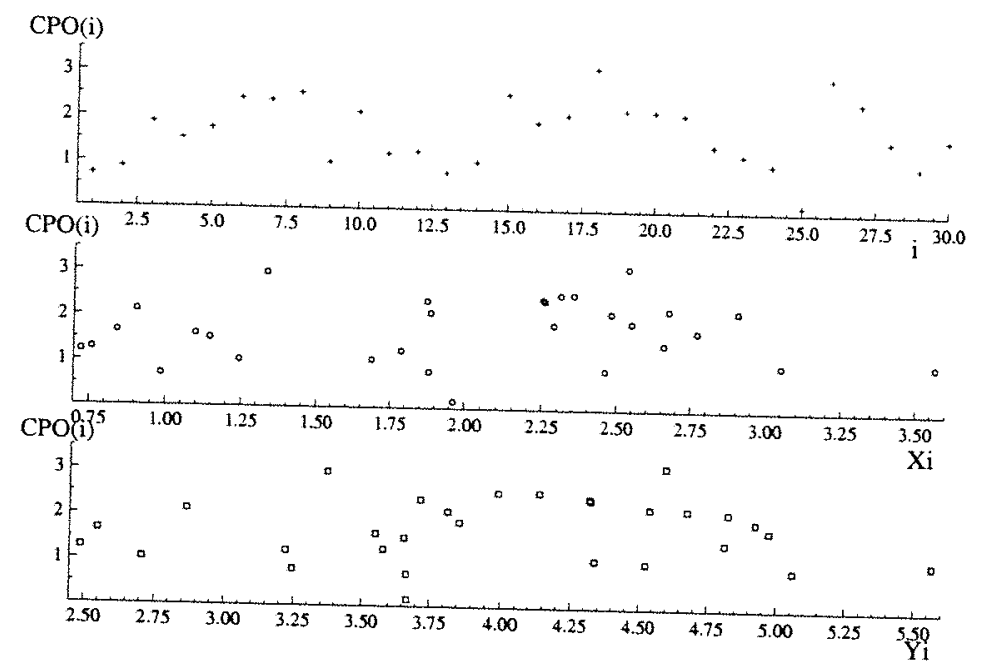

Figura 2.38: Gráficos dos valores da densidade condicional preditiva, (CPO(i)), versus $i$, versus $X_{i}$ e versus $Y_{i}$.

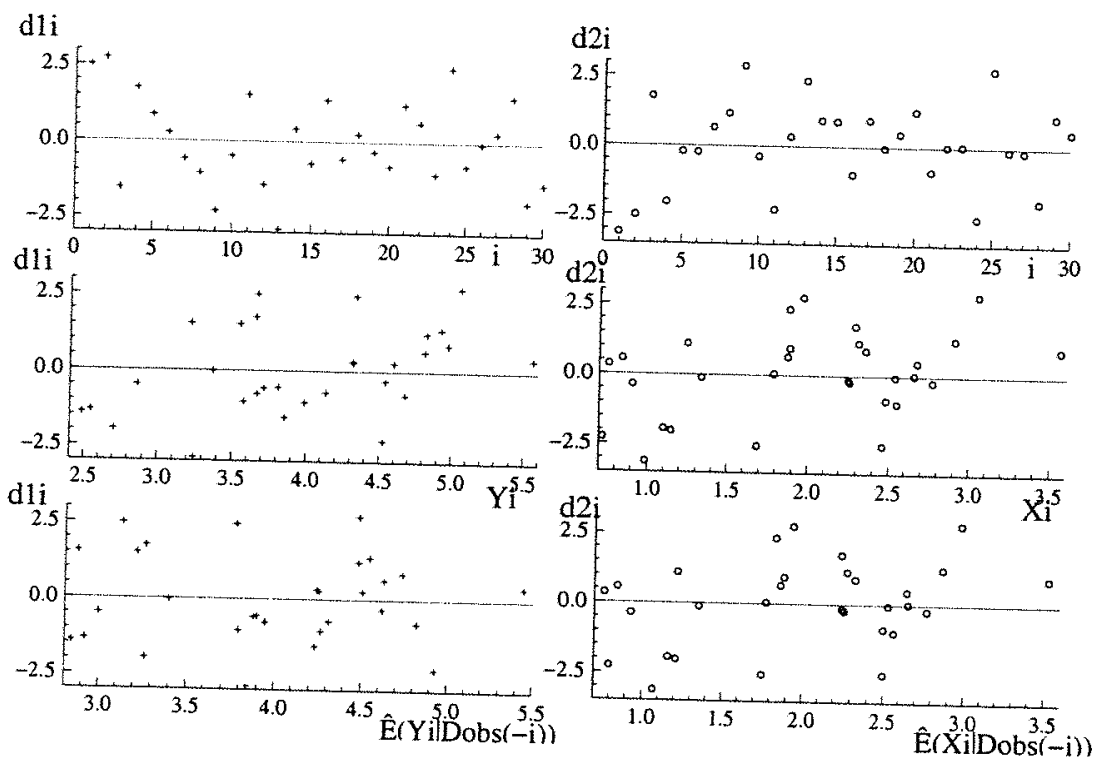

Figura 2.39: Os gráficos do lado esquerdo referem-se à primeira componente do vetor de resíduos $\left(d_{1 i}\right)$, e os 2.38 .
a direito à segunda componente $\left(d_{2 i}\right)$. Eles estão baseados na mesma sequencia de Gibbs utilizada na figura
a

Obs: As figuras 2.38 e 2.39 não sugerem que haja problemas de falta de ajustamento.

\section{COMPARAÇÃO de MODELOS}

A figura 2.40 compara a densidade condicional preditiva nos modelos com priori própria e com priori imprópria sem restriçāo de identif. (usando a fórmula (2.10.8) 
com as devidas adaptações). Para os cálculos, apenas a segunda metade de uma seqüência de Gibbs de comprimento $T=10000$ foi utilizada.

A tabela 2.13 apresenta, os valores estimados da medida $L$ e da soma dos resíduos padronizados em valor absoluto nos modelos com priori pópria e com priori imprópria sem restr.de identif.

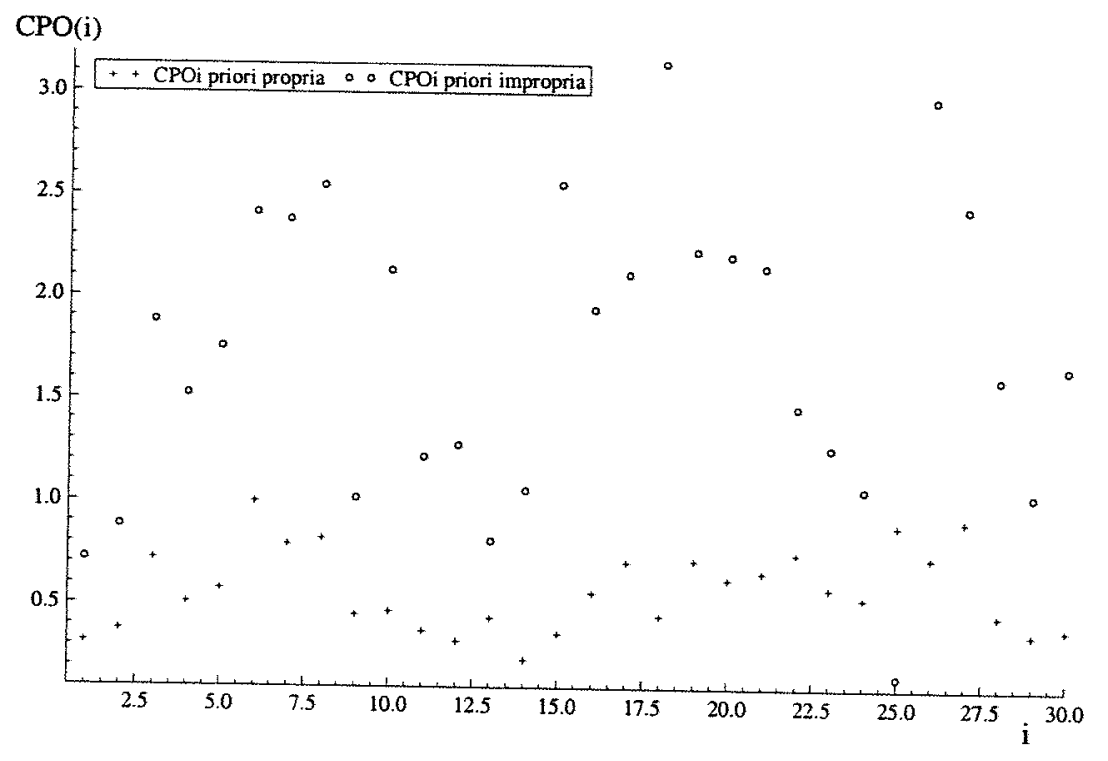

Figura 2.40: Densidade condicional preditiva $(\mathrm{CPO}(\mathrm{i}))$ versus $i$, no modelo com priori própria $(+)$ e no modelo com priori imprópria $(0)$.

Tabela 2.13: A medida L, o produto $\prod_{i=0}^{n} C P O_{i}$ e a soma das normas dos resíduos, nos modelos c/ priori própria e c/ priori imprópria.

\begin{tabular}{r|r|cc}
\hline & & priori propria. & priori imprópria. \\
\hline medida $L$ & $\operatorname{det}(\mathrm{L} 2) \delta=0.0$ & 221.19 & 137.66 \\
& $\delta=0.1$ & 248.74 & 157.58 \\
& $\delta=0.5$ & 372.0 & 250.30 \\
& $\delta=0.9$ & 516.14 & 363.91 \\
& $\delta=1.0$ & 555.44 & 395.68 \\
& $\operatorname{tr}\left(L_{2}\right) \delta=0.0$ & 43.39 & 44.12 \\
& $\delta=0.1$ & 46.89 & 47.62 \\
& $\delta=0.5$ & 60.89 & 61.63 \\
& $\delta=0.9$ & 74.90 & 75.63 \\
\hline Soma das normas & $\delta=1.0$ & 78.40 & 79.13 \\
\hline dos residuos & & & 52.25 \\
\hline
\end{tabular}


Na figura 2.40 vemos que os valores das $\mathrm{CPO}(\mathrm{i})$ 's tendem a ser maiores no modelo com priori imprópria e na tabela 2.14 as estimativas da medida $\mathrm{L}$, baseadas no determinante, são consideravelmente menores no modelo com priori imprópria . Isso sugere que o modelo com priori imprópria deva ser o escolhido. Entretanto se considerarmos como critério, apenas a soma dos módulos dos resíduos padronizados então o modelo com priori própria deveria ser o escolhido. Observou-se também que os valores da medida $L$ calculados pelo traço ficaram bem próximos nos dois modelos.

Assim, se o valor de $\lambda$ (quociente das variâncias residuais) não é conhecido e os dados amostrais são os da tabela 2.1, o modelo (sem restr. de identif.) com priori imprópria não seria uma má escolha.

\subsubsection{Resumo dos resultados:}

Na seção 2.12 foram ajustados 4 modelos bayesianos estruturais normais utilizando o particular conjunto de dados da tabela 2.1. Os modelos são:

1) modelo com restr. de identif. e priori própria vaga da subseção(2.12.1),

(2) modelo sem restr. de identif. com priori própria vaga (2.12.2),

(3) modelo com restr. de identif. e priori imprópria (2.12.3)

(4) modelo sem restr. com priori imprópria (2.12.4).

\section{1 - comparação dos algoritmos:}

Foram utilizados 3 diferentes algorítmos para amostrar da posteriori do modelo da subseção 2.12.1: O algoritmo de Gibbs simples, o algoritmo "Grouped Gibbs" e o algoritmo "M. Collapsed Gibbs". Aparentemente o "Grouped Gibbs" não traz vantagens sobre o primeiro algoritmo e existe apenas um pequeno ganho (em termos da função de autocorrelação e da matriz de correlações cruzadas) em usar o "M.Collap. Gibbs" sobre os demais, não justificando fortemente o seu uso (nesse 
particular exemplo).

2 - convergência

Os dispositivos de diagnóstico de convergência apresentados nessa seção não nos sugeriram que haja problemas na convergência do algoritmo de Gibbs em nenhum dos modelos considerados.

\section{3 - comparação dos modelos:}

- Comparando os modelos (1) e (3):

Levando em conta que no modelo (3) os intervalos de credibilidade tem menor comprimento, as estimativas dos parâmetros tem um menor viés de estimação, as estimativas da medida $L$ (calculadas pelo determinante) são menores e os valores das $\mathrm{CPO}(\mathrm{i})$ 's tendem a ser maiores do que no modelo (1), entao o modelo (3) é o escolhido.

- Comparando os modelos (2) e (4):

Do ponto de vista de estimação os dois tem vantagens e desvantagens (o modelo (4) tem intervalos de credibilidade de menor comprimento, porém maior viés de estimação e além disso o verdadeiro valor do parâmetro $\sigma^{2}$ não pertence ao intervalo de credibilidade estimado correspondente). Entretanto no modelo (4) as estimativas da medida $L$ (calculadas pelo determinante) são menores do que no modelo (2) e os valores das $\mathrm{CPO}(\mathrm{i})$ 's tendem a ser maiores do que no modelo (2). Por estes motivos uma possivel escolha seria o modelo (4).

Do exposto acima vemos que o uso de prioris impróprias (das seçõe 2.12 .3 e 2.12.4) traz benefícios tanto no caso do modelo com restrição como no caso sem restrição de identificabilidade.

- Comparando os modelos (1) e (2) e os modelos (3) e (4):

Usando a medida $L$ e os valores das CPO(i)'s vemos que o modelo (1) é escolhido sobre o modelo (2); além disso o modelo (3) é preferido ao (4). 


\section{4 - qualidade do ajuste}

Nos modelos sem restrição de identificabilidade (com priori própria ou imprópria), os gráficos das $\mathrm{CPO}(\mathrm{i})$ 's e das componentes dos vetores de resíduos, não nos sugeriram que haja algum problema de falta de ajuste. Entretanto nos modelos com restr. de identif. (especialmente o modelo com priori imprópria), ocorrem alguns pontos aberrantes, o que sugere que uma análise mais completa deva ser realizada.

\section{5 - Sensibilidade das estimativas dos parâmetros ao valor de $\lambda$}

Quando a amostra de dados é a da tabela 2.1 e o modelo escolhido é o modelo (1), o valor de $\lambda$ fornecido pelo usuário não afeta muito as estimativas de $\alpha, \beta, \mu$ e $\sigma_{x}^{2}$ em termos do viés (já que o valor máximo observado do viés estimado, foi da ordem de 15 a $20 \%$ do verdadeiro valor do parâmetro correspondente). Entretanto a escolha do $\lambda$ pode afetar bastante as estimativas de $\sigma^{2}$ (também em termos do viés de estimação) e nesse caso observou-se que mesmo que $\lambda$ seja escolhido corretamente, ainda assim esse parânmetro fica bastante superestimado, principalmente se o tamanho da amostra não for bem grande.

\section{Observaçōes gerais:}

- As médias aritméticas e as médias R-Blackwelizadas ficaram sempre muito próximas independentemente do modelo estudado.

- A medida L baseada no traço aparentemente não serviu para diferenciar os modelos.

- O critério da medida L (calculada pelo determinante) e o critério da comparação dos CPO(i)'s concordaram quanto à escolha dos modelos, entretanto discordaram do critério da soma dos resíduos padronizados na escolha dos modelos estudados nessa seção. 


\section{Capítulo 3}

\section{O modelo linear estrutural identificável $t$-Student com erros nas variáveis}

\section{Introdução}

É fato bastante conhecido que os modelos que usam a distribuição Normal não são robustos à presença de valores discrepantes, no sentido de que as inferências sob tais modelos podem mudar consideravelmente na presença de uma ou mais observaçōes discrepantes.

Uma maneira de reduzir a influência desses valores discrepantes é trocar o modelo Normal por um modelo com caudas mais pesadas como por exemplo o modelo $t$ Student.

A distribuição $t$-Student com $\nu$ graus de liberdade, $t_{\nu}(\mu, \Sigma)$, tem caudas mais longas que a Normal $(N(\mu, \Sigma))$ e além disso, quando o número de graus de liberdade tende a infinito $(\nu \rightarrow \infty)$, ela se aproxima da Normal e quando $\nu=1$ ela coincide com a distribuição de Cauchy. Assim, na literatura, uma alternativa robusta à Normal tem sido a distribuição $t$, fixando-se um valor pequeno para $\nu$. Gelman et al. (1997) sugerem utilizar $\nu=4$ para assegurar a existência dos momentos até terceira ordem e recomendam também que a distribuição Normal não deve ser usada 
quando os dados são "muito dispersos" (isto é, quando $10 \%$ dos pontos estiverem há uma distância superior a $1,5 d$ da mediana, onde $d$ é a distância entre o primeiro e o terceiro quartil, e o tamanho da amostra é grande). Além disso, segundo esses autores, nos modelos de regressão, a distribuição $t$ com um número pequeno de graus de liberdade fixado, trata os "outliers" como observações de alta variância e produz resultados semelhantes aos obtidos na regressão ponderada (em que os pesos são os inversos das variâncias).

A distribuição $t$ pode ser empregada não somente para acomodar eventuais pontos discrepantes (ou dados com alta dispersão), mas também situações em que os hiperparâmetros das prioris têm valores muito extremos ou então quando temos um modelo com várias hierarquias.

Nesse capítulo estudamos os modelos lineares estruturais $t$-independente e $t$ dependente com restrição de identificabilidade no caso em que o número de graus de liberdade é conhecido, considerando uma particular distribuição à priori própria com componentes independentes.

Nesse capítulo também são demonstrados quatro teoremas que estabelecem condições suficientes para a existência da distribuição posteriori no modelo $t$ - dependente para um conjunto particular de prioris impróprias.

Uma avaliação da qualidade do ajuste e uma análise de sensibilidade do número de graus de liberdade do modelo $t$ (quando este é assumido conhecido) também serão feitas nesse capítulo. Finalmente, descreveremos algumas técnicas de comparação de modelos.

Antes porém de apresentar os modelos de regressão estrutural $t$-dependente $e$ $t$-independente, apresentamos a seguir duas das formas de definir a distribuição $t$ multivariada descritas na literatura. Um estudo bastante abrangente sobre a distribuição $t$, situando-a também como membro da família de distribuições elípticas. 
é encontrado em Arellano-Valle (1994).

Definição 1: Dizemos que um vetor aleatório $\underset{\sim}{X_{n \times 1}}$ tem distribuição $t n$-variada e não-singular com $\nu$ graus de liberdade $(\nu>0)$, parâmetro de locação $\mu_{n \times 1}$ e parâmetro de escala $\underset{\sim}{\Sigma_{n \times n}}\left(\sum_{\sim}\right.$ positiva definida), isto é, $\underset{\sim}{X} \sim t_{n}(\underset{\sim}{\mu}, \underset{\sim}{\Sigma}, \nu)$, se a função densidade de $\underset{\sim}{X}$ é dada por

$$
f_{\underset{J}{X}}\left(\underset{\sim}{x} \mid \underset{\sim}{\mu}, \underset{\sim}{\sum}, \nu\right)=k(n, \nu)\left|\sum\right|^{-\frac{1}{2}}\left[\nu+(\underset{\sim}{X}-\underset{\sim}{\mu})^{\mathrm{T}} \Sigma^{-1}(\underset{\sim}{X}-\mu)\right]^{-\frac{(n+\nu)}{2}},
$$

$\underset{\sim}{x} \in \mathbb{R}^{n}, \mu \in \mathbb{R}^{n}, \sum_{\sim}>0$ (isto é, $\sum$ positiva definida), onde $k(n, \nu)=\frac{\Gamma\left(\frac{n+\nu}{2}\right) \nu^{\nu / 2}}{\Gamma\left(\frac{\nu}{2}\right) \pi^{n / 2}}$.

Observação 1: Quando $\nu=1$ a distribuição acima se reduz à distribuição de Cauchy $n$-variada (não central).

Observação 2: Quando $\nu \rightarrow \infty$ essa distribuição se aproxima de uma Normal $N(\mu, \Sigma)$

Observação 3: Essa definição assume explicitamente a existência de uma função densidade e portanto não permite incorporar uma versão singular da distribuição $t$ multivariada.

Definição 2: Dizemos que um vetor aleatório $\underset{\sim}{X}{ }_{n \times 1}$ segue uma distribuição $t n$ variada com $\nu$ graus de liberdade $(\nu>0)$, parâmetro de locação $\mu$ e parâmetro de escala $\underset{\sim}{\Sigma}$, isto é, $\underset{\sim}{X} \sim t_{n}\left(\mu, \underset{\sim}{\sum}, \nu\right)$, se $\underset{\sim}{X}=\mu+w^{\frac{1}{2}} \underset{\sim}{Z}$, onde $\underset{\sim}{Z} \sim N_{n}\left(\underset{\sim}{0}, \sum_{\sim}\right) \mathrm{e}$ $w \sim \frac{\nu}{\chi_{\nu}^{2}}$ são independentes, isto é, $\underset{\sim}{X} \mid w \sim N\left(\mu, w \Sigma_{\sim}\right)$ e $w \sim I G\left(\frac{\nu}{2}, \frac{\nu}{2}\right)$.

Observação 1: A distribuição incondicional de $\underset{\sim}{X}$ nesse caso é

$$
f_{X}(x)=\int_{0}^{\infty} f(\underset{\sim}{x} \mid w) f(w) \mathrm{d} w
$$

que é uma mistura de escala da distribuição normal. 
Observação 2: No caso em que $\underset{\sim}{ }$ é positiva definida, a Definição 1 e a Definição 2 são equivalentes.

Nesse capítulo apresentaremos o modelo de regressão estrutural $t$-independente e o modelo $t$-dependente, tanto no caso em que $\nu$ é conhecido como também no caso em que $\nu$ é desconhecido.

\subsection{O modelo estrutural $t$-independente com o número de graus de liberdade conhecido}

Nessa seção apresentamos o modelo de regressão linear simples $t$-independente com erros nas variáveis, considerando duas formulações equivalentes. Além disso, discutimos a implementação de algoritmos MCMC para amostrar da posteriori desses modelos.

\subsubsection{Definição do modelo}

\section{Formulação 1}

Considere as equações

$$
\left\{\begin{array}{l}
Y_{i}=\alpha+\beta x_{i}+e_{i} \\
X_{i}=x_{i}+u_{i}
\end{array}\right.
$$

onde $Y_{i}$ e $X_{i}$ são as variáveis aleatórias observáveis, $x_{i}$ a variável aleatória não observável e $u_{i}$ o erro na medição de $x_{i}$, com a suposição

$$
\left(\begin{array}{c}
e_{i} \\
u_{i} \\
x_{i}
\end{array}\right) \mid \theta \stackrel{\text { iid }}{\sim} t_{3}\left(\mu^{\prime}, \Sigma_{\sim}^{\prime}, \nu\right), i=1, \ldots, n,
$$

onde

onde $\sigma_{e}^{2}=\lambda \sigma_{u}^{2}=\lambda \sigma^{2} \operatorname{com} \lambda$ conhecido, $\nu>0$ e $\left|\Sigma^{\prime}\right| \neq 0$.

$$
\mu^{\prime}=\left(\begin{array}{c}
0 \\
0 \\
\mu
\end{array}\right) \quad \text { e } \quad \Sigma_{\sim}^{\prime}=\left(\begin{array}{ccc}
\sigma_{e}^{2} & 0 & 0 \\
0 & \sigma_{u}^{2} & 0 \\
0 & 0 & \sigma_{x}^{2}
\end{array}\right),
$$


O vetor de parâmetros é $\quad \underset{\sim}{\theta}=\left(\alpha, \beta, \mu, \sigma_{x}^{2}, \sigma^{2}\right)^{T}$

e o espaço paramétrico é $\underset{\sim}{\Theta}=\left\{\underset{\sim}{\theta}: \alpha, \beta, \mu \in \mathbb{R}\right.$ e $\sigma_{x}^{2}$ e $\left.\sigma^{2} \in \mathbb{R}_{+}\right\}$.

Observação: Quando $\nu \geq 3$, a distribuição acima tem matriz de covariâncias dada por $\operatorname{Var}\left(\left(\begin{array}{c}e_{i} \\ u_{i} \\ x_{i}\end{array}\right) \mid \theta\right)=\Sigma_{\sim}^{\prime} \frac{\nu}{\nu-2}$, e vetor de médias $\mu^{\prime}$. Quando $\nu=1$ caimos na distribuição de Cauchy não central com parâmetro de locação igual a $\mu^{\prime}$ e parâmetro de escala igual a $\Sigma^{\prime}$. que

Tomando-se $\underset{\sim}{Z}{ }_{i}=\left(\begin{array}{c}Y_{i} \\ X_{i} \\ x_{i}\end{array}\right), i=1, \ldots, n$, então a suposição em (3.1.1) implica

$$
Z_{i} \mid \underset{Q}{\operatorname{iid}} \sim t_{3}\left(\underset{\sim}{\mu}, \sum, \nu\right), \quad i=1, \ldots, n
$$

onde

$$
\mu=\left(\begin{array}{c}
\alpha+\beta \mu \\
\mu \\
\mu
\end{array}\right), \quad \Sigma=\left(\begin{array}{ccc}
\beta^{2} \sigma_{x}^{2}+\sigma_{e}^{2} & \beta \sigma_{x}^{2} & \beta \sigma_{x}^{2} \\
\beta \sigma_{x}^{2} & \sigma_{x}^{2}+\sigma_{u}^{2} & \sigma_{x}^{2} \\
\beta \sigma_{x}^{2} & \sigma_{x}^{2} & \sigma_{x}^{2}
\end{array}\right)
$$

onde $\sigma_{e}^{2}=\lambda \sigma_{u}^{2}=\lambda \sigma^{2}, \nu>0$ e $\sum_{\sim}$ é positiva definida.

Notemos que se a suposição em (3.1.2)vale, então

$$
\underset{\sim}{Z}=\left(\begin{array}{c}
Z_{1} \\
\vdots \\
Z_{n}
\end{array}\right) \sim t_{3 n}\left({\underset{\sim}{n}}_{n} \otimes \mu ; I_{n \times n} \otimes \sum_{\sim}, \nu\right)
$$

(mas a recíproca não é verdadeira).

A distribuição à priori de $\underset{\sim}{\theta}$ nesse modelo será denotada por $\pi(\underset{\sim}{\theta})$.

\section{Observações}

1. Arellano-Valle (1994) define o modelo estrutural $t$-independente no contexto da estatística clássica por meio da distribuição das variáveis observadas $\underset{\sim}{U_{i}}$ 
definidas por

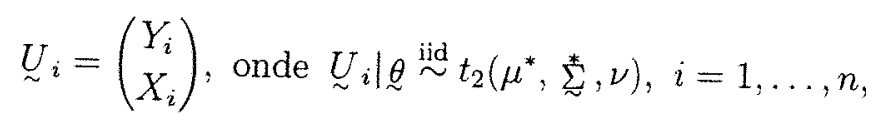

onde

$$
\mu^{*}=\left(\begin{array}{c}
\alpha+\beta \mu \\
\mu
\end{array}\right), \quad \Sigma^{*}=\left(\begin{array}{cc}
\beta^{2} \sigma_{x}^{2}+\sigma_{e}^{2} & \beta \sigma_{x}^{2} \\
\beta \sigma_{x}^{2} & \sigma_{x}^{2}+\sigma_{u}^{2}
\end{array}\right) .
$$

2. O modelo $t$-independente é não identificável e uma maneira de torná-lo identificável é incorporar por exemplo a restrição $\sigma_{e}^{2}=\lambda \sigma_{u}^{2}=\lambda \sigma^{2}$ (Arellano-Valle, 1994).

3. Mesmo no caso em que o número de graus de liberdade $(\nu)$ é desconhecido, o modelo se torna identificável quando adotamos por exemplo a restrição acima.

4. Em estatística bayesiana, como já discutimos no Capítulo 2, não há necessidade de que os modelos sejam identificáveis quando escolhemos convenientemente a distribuição à priori. Entretanto, nesse capítulo vamos estudar apenas o modelo $t$ identificável.

\section{A função de verossimilhança baseada nos dados completos}

A função de verossimilhança baseada nos dados completos, $D=\underset{\sim}{Z}=\left(\begin{array}{c}Z_{1} \\ \vdots \\ Z_{n}\end{array}\right)$,
é dada por

$$
L(\underset{\sim}{\theta} \mid D) \propto \prod_{i=1}^{\infty}\left|\sum_{i}\right|^{-\frac{1}{2}}\left[\nu+\left({\underset{\sim}{Z}}_{i}-\mu\right) \sum_{\sim}^{-1}(\underset{\sim}{Z}-\mu)\right]^{-\left(\frac{3+\nu}{2}\right)}
$$

onde $\underset{\sim}{\mu}$ e $\sum$ são definidos em (3.1.2).

\section{A função de verossimilhança baseada nos dados observados}

A função de verossimilhança baseada nos dados observados $D_{\text {obs }}=\left(\begin{array}{c}U_{1} \\ \vdots \\ U_{n}\end{array}\right)$ é 
dada por

$$
L\left(\underset{\sim}{\theta} \mid D_{\mathrm{obs}}\right) \propto \prod_{i=1}^{n}\left|\Sigma_{\sim}^{*}\right|^{-\frac{1}{2}}\left[\nu+\left({\underset{\sim}{U}}_{i}-\mu_{\sim}^{*}\right) \Sigma_{\sim}^{*^{-1}}\left(\underset{\sim}{U_{i}}-\mu^{*}\right)\right]^{-\left(\frac{2+\nu}{2}\right)},
$$

onde $\mu^{*}$ e $\Sigma^{*}$ são definidos em (3.1.3).

\section{A distribuição à posteriori}

A distribuição à posteriori $p\left(\theta \mid D_{\text {obs }}\right)$ é dada por

$$
p\left(\underset{\sim}{\theta} \mid D_{\mathrm{obs}}\right)=\int_{\mathfrak{X}} p\left(\underset{\sim}{\theta} \underset{\sim}{x} \mid D_{\mathrm{obs}}\right) \mathrm{d} \underset{\sim}{x} \propto \int_{\mathfrak{X}} L(\underset{\theta}{\theta} \mid D) \pi(\underset{\sim}{\theta}) \mathrm{d} \underset{\sim}{x},
$$

onde $L(\underset{\sim}{\theta} \mid D)$ é dada por (3.1.4) e $\pi(\underset{\sim}{\theta})$ é a distribuição à priori de $\underset{\sim}{\theta}$.

Outra forma de escrever a posteriori é

$$
p\left(\underset{\sim}{\theta} \mid D_{\mathrm{obs}}\right) \propto L\left(\underset{\sim}{\theta} \mid D_{\mathrm{obs}}\right) \pi(\underset{\sim}{\theta}),
$$

onde $L\left(\underset{\sim}{\theta} \mid D_{\text {obs }}\right)$ é dada por (3.1.5) e $\pi(\underset{\sim}{\theta})$ é a distribuição à priori de $\underset{\sim}{\theta}$.

\section{Formulação 2 - o modelo de mistura de escala da distribuição} Normal

Uma especificação equivalente do modelo acima é dada a seguir.

Seja a equação de regressão

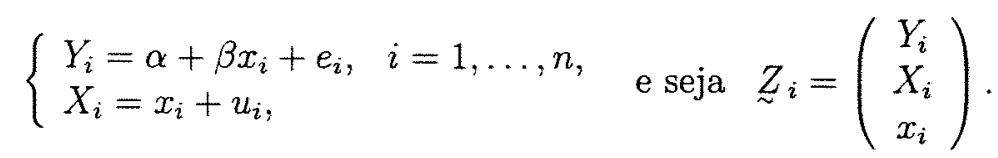

O modelo assume que

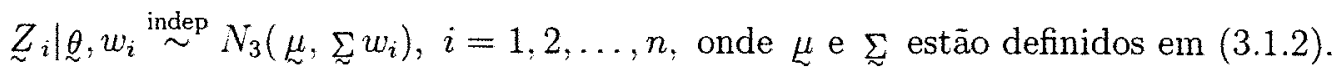

A distribuiçāo à priori adotada para $\underset{\sim}{\theta}$ e $\underset{\sim}{w}$ é da forma

$$
\pi(\underset{\theta}{\theta}, \underset{\sim}{w})=\pi(\underset{\sim}{\theta}) \pi(\underset{\sim}{w})
$$


onde $\underset{\sim}{w}=\left(w_{1}, \ldots, w_{n}\right)^{T}$ e $w_{i} \stackrel{\text { iid }}{\sim} I G\left(\frac{\nu}{2}, \frac{\nu}{2}\right), i=1, \ldots, n$.

O espaço paramétrico é $\Theta^{*}=\left\{(\underset{\sim}{\theta} \underset{\sim}{w})^{T}: \underset{\sim}{\theta} \in \Theta\right.$ e $\left.\underset{\sim}{w} \in \mathbb{R}_{+}^{n}\right\}$.

\section{A função de verossimilhança baseada nos dados completos}

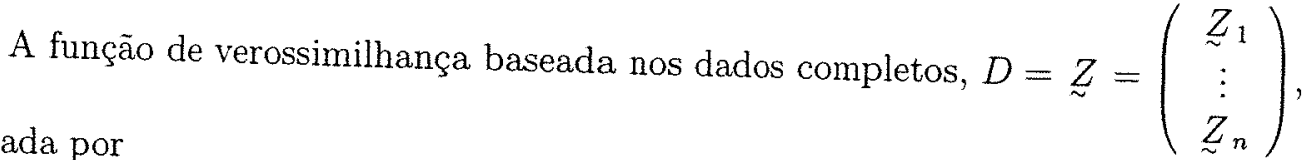

$$
L(\underset{\sim}{\theta} \underset{\sim}{w} \mid D) \propto \prod_{i=1}^{n}\left|\sum w_{i}\right|^{-\frac{1}{2}} \exp \left\{-\frac{1}{2}(\underset{\sim}{Z}-\mu)^{\top}\left(\sum_{\sim} w_{i}\right)^{-1}(\underset{\sim}{Z}-\mu)\right\},
$$

ou seja, ela é proporcional a

$$
\begin{aligned}
& \frac{1}{\left(\prod_{i=1}^{n} w_{i}^{3 / 2}\right)\left[\left(\sigma^{2}\right)^{2} \sigma_{x}^{2}\right]^{n / 2}} \exp \left\{-\frac{1}{2}\left[\sum_{i=1}^{n} \frac{\left(Y_{i}-\alpha-\beta x_{i}\right)^{2}}{\lambda \sigma^{2} w_{i}}+\sum_{i=1}^{n} \frac{\left(X_{i}-x_{i}\right)^{2}}{\sigma^{2} w_{i}}\right.\right. \\
& \left.\left.+\sum_{i=1}^{n} \frac{\left(x_{i}-\mu\right)^{2}}{\sigma_{x}^{2} w_{i}}\right]\right\} .
\end{aligned}
$$

\section{A função de verossimilhança baseada nos dados observados} onde $\underset{\sim}{U_{i}}=\left(\begin{array}{l}Y_{i} \\ X_{i}\end{array}\right)$ é

$$
L\left(\underset{\sim}{\theta}, \underset{\sim}{w} \mid D_{\mathrm{obs}}\right) \propto \prod_{i=1}^{n}\left|\Sigma_{\sim}^{*} w_{i}\right|^{-\frac{1}{2}} \exp \left\{-\frac{1}{2}\left({\underset{\sim}{U}}_{i}-\mu^{*}\right)^{\top}\left(\Sigma_{\sim}^{*} w_{i}\right)^{-1}\left(\underset{\sim}{\left.U_{i}-\mu^{*}\right)}\right\},\right.
$$

onde $\mu^{*}$ e $\underset{\sim}{\Sigma^{*}}$ são definidos em (3.1.3).

\section{Distribuição à posteriori}

A posteriori $p\left(\theta \underset{\sigma}{\theta} \mid D_{\text {obs }}\right)$ é dada por

$$
p\left(\underset{\sim}{\theta} \mid D_{\text {obs }}\right)=\int_{\mathfrak{X}=\mathbb{R}^{n}} \int_{W=\mathbb{R}_{+}^{n}} p\left(\underset{\sim}{\theta}, \underset{\sim}{w}, \underset{\sim}{x} \mid D_{\text {obs }}\right) \mathrm{d} \underset{\sim}{w} \mathrm{~d} \underset{\sim}{x},
$$


onde $p\left(\underset{\sim}{\theta} \underset{\sim}{w}, \underset{\sim}{x} \mid D_{\text {obs }}\right) \propto L(\underset{\sim}{\theta}, \underset{\sim}{w} \mid D) \pi(\theta) \pi(\underset{\sim}{w})$ e onde $L(\underset{\sim}{\theta}, \underset{\sim}{w} \mid D)$ é dada em (3.1.9). A posteriori é dada também por

$$
p\left(\underset{\sim}{\theta} \mid D_{\mathrm{obs}}\right) \propto \int_{\underset{\sim}{w}=\mathbb{R}_{+}^{n}} p\left(\underset{\sim}{\theta}, \underset{\sim}{w} \mid D_{\mathrm{obs}}\right) \mathrm{d} \underset{\sim}{w},
$$

onde $p\left(\underset{\sim}{\theta}, \underset{\sim}{w} \mid D_{\mathrm{obs}}\right) \propto L\left(\underset{\sim}{\theta}, \underset{\sim}{w} \mid D_{\mathrm{obs}}\right) \pi(\underset{\sim}{\theta}, \underset{\sim}{w})$ e onde $L\left(\underset{\sim}{\theta}, \underset{\sim}{w} \mid D_{\mathrm{obs}}\right)$ é dada em (3.1.10).

Observação 1: A formulação 1 e a formulação 2 do modelo $t$-independente são equivalentes, já que a posteriori de $\underset{\sim}{\theta}$, dada em (3.1.11), coincide com a posteriori de $\underset{\sim}{\theta}$ dada em (3.1.6). A justificativa dessa afirmação é dada a seguir.

Demonstração: $p\left(\underset{\sim}{\theta} \mid D_{\text {obs }}\right)=\int_{\mathfrak{X}=\mathbb{R}^{n}} \int_{W=\mathbb{R}_{+}^{n}} p\left(\underset{\theta}{w} w \underset{\sim}{x} \mid D_{\text {obs }}\right) \mathrm{d} \underset{\sim}{\underset{\sim}{w}} \underset{\sim}{x}$

$$
\begin{aligned}
& \propto \int_{\circledast} \int_{W=\mathbb{R}_{+}^{n}} L(\underset{\sim}{\theta}, w \mid D) \pi(\underset{\theta}{\theta}) \pi(\underset{\sim}{w}) \mathrm{d} \underset{\sim}{w} \mathrm{~d} \underset{\sim}{x} \\
& =\int_{x} \int_{W} \prod_{i=1}^{n}\left|\Sigma w_{i}\right|^{-\frac{1}{2}} \exp \left\{-\frac{1}{2}\left[\left(\underset{\sim}{Z_{i}}-\mu\right)^{\top}\left(\Sigma w_{i}\right)^{-1}\left(\underset{\sim}{Z_{i}}-\mu\right)\right]\right\} \pi(\theta) \prod_{i=1}^{n} w_{i}^{-\left(\frac{\nu}{2}+1\right)} \\
& \exp \left\{-\frac{\nu / 2}{w_{i}}\right\} \mathrm{d} \underset{\sim}{w} \mathrm{~d} \underset{\sim}{x}
\end{aligned}
$$

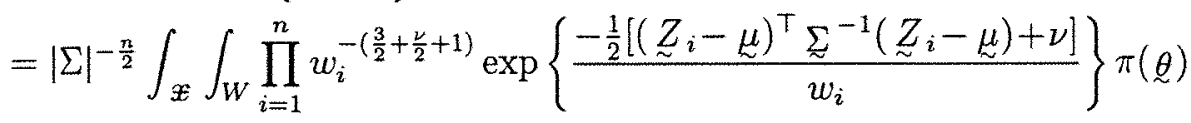

$$
\begin{aligned}
& \mathrm{d} \underset{\sim}{w} \mathrm{~d} \underset{\sim}{x} \\
& =|\Sigma|^{-\frac{n}{2}} \pi(\underset{\sim}{\theta}) \int_{\boxplus} \int_{W} \prod_{i=1}^{n} w_{i}^{-\left(\frac{3+\nu}{2}+1\right)} \exp \left\{\frac{-\frac{1}{2}\left[\left({\underset{\sim}{i}}_{i}-\mu\right)^{\top} \Sigma_{\left.\underset{\tau}{-1}\left({\underset{\sim}{i}}_{i}-\mu\right)+\nu\right]}\right.}{w_{i}}\right\} \\
& \mathrm{d} \underset{\sim}{w} \mathrm{~d} \underset{\sim}{x} \\
& =|\Sigma|^{-\frac{n}{2}} \pi(\underset{\sim}{\theta}) \int_{\mathscr{X}} \prod_{i=1}^{n} \int_{W_{i}=\mathbb{R}_{+}} w_{i}^{-\left(\frac{3+\nu}{2}+1\right)} \exp \left\{\frac{-\frac{1}{2}\left[\left(Z_{i}-\mu\right)^{\top} \sum^{-1}\left(\underset{\sim}{Z_{i}}-\mu\right)+\nu\right]}{w_{i}}\right\} \\
& \mathrm{d} w_{i} \mathrm{~d} \underset{\sim}{x},
\end{aligned}
$$

onde a função dentro da integral em $w_{i}$ é o núcleo de uma Gama Invertida

$$
I G\left(\frac{3+\nu}{2}, \frac{1}{2}\left[(\underset{\sim}{Z}-\mu)^{\top} \Sigma^{-1}\left(Z_{i}-\mu\right)+\nu\right]\right) .
$$

Portanto, (3.1.13) é igual a

$$
|\Sigma|^{-\frac{n}{2}} \pi(\theta) \int_{\nexists} \prod_{i=1}^{n} \frac{\Gamma\left(\frac{3+\nu}{2}\right)}{\left\{\frac{1}{2}\left[\left(Z_{i}-\mu\right)^{\top} \Sigma^{-1}\left(Z_{i}-\mu\right)+\nu\right]\right\}^{\frac{3+\nu}{2}}} \mathrm{~d} \underset{\sim}{x}
$$


e portanto

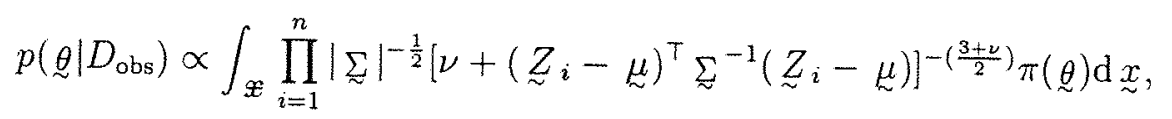

que coincide com (3.1.6).

\subsubsection{Implementação de algoritmos MCMC no modelo com particular priori própria de componentes independentes}

Tomando-se a distribuição à priori

$$
\pi(\underset{\sim}{\theta}, \underset{\sim}{w})=\pi(\underset{\theta}{\theta}) \pi(\underset{\sim}{w})=\pi(\alpha) \pi(\beta) \pi(\mu) \pi\left(\sigma_{x}^{2}\right) \pi\left(\sigma^{2}\right) \prod_{i=1}^{n} \pi\left(w_{i}\right)
$$

onde $\alpha \sim N\left(a, \sigma_{\alpha}^{2}\right), \beta \sim N\left(b, \sigma_{\beta}^{2}\right), \mu \sim N\left(m, \sigma_{\mu}^{2}\right), \sigma_{x}^{2} \sim I G(c, d), \sigma^{2} \sim I G(f, g) \mathrm{e}$ $w_{i} \sim \operatorname{IG}\left(\frac{\nu}{2}, \frac{\nu}{2}\right), i=1, \ldots, n$, e considerando a formulação 2 do modelo $t$-independente descrita na subseção anterior, podemos implementar o algoritmo de Gibbs, de forma semelhante à do modelo Normal do Capítulo 2, utilizando as distribuições condicionais completas exibidas abaixo, que são baseadas na distribuição à posteriori $p\left(\underset{\sim}{\theta}, \underset{\sim}{x}, \underset{\sim}{w} \mid D_{\text {obs }}\right)$ envolvida em (3.1.11).

É importante lembrar que todos os algoritmos MCMC descritos na seção 2.4 do Capítulo 2 para o modelo Normal podem ser implementados para esse modelo de forma semelhante quando usamos a formulação 2 .

É conveniente lembrar também que no caso do modelo $t$ sem restrição de identificabilidade a implementação dos algoritmos seria semelhante ao caso do modelo normal sem restrições definido na seção 2.6 (a mesma observação vale no caso do modelo $t$ com priori $\pi(\underset{\sim}{\theta}, \underset{\sim}{w})=\pi(\underset{\sim}{w}) \pi(\underset{\sim}{\theta})$, onde $\pi(\underset{\sim}{\theta})$ tem componentes condicionalmente independentes como em (2.7.1).

Nessa seção, entretanto, vamos nos restringir apenas à implementação do algoritmo original de Gibbs para o modelo $t$-independente com restrição de identificabilidade e com priori dada em (3.1.4), para não nos tornarmos muito repetitivos. 
Distribuições condicionais completas baseadas na distribuição $p\left(\underset{\sim}{\theta}, \underset{\sim}{w}, \underset{\sim}{x} \mid D_{\mathrm{obs}}\right)$ em (3.1.11)

$$
\begin{aligned}
& x_{i} \mid x_{(-i)}, \alpha, \beta, \mu, \sigma^{2}, \underset{\sim}{w}, D_{\text {obs }} \\
& \sim N\left(\frac{\sigma_{x}^{2}\left[\lambda X_{i}+\beta\left(Y_{i}-\alpha\right)\right]+\lambda \sigma_{\mu}^{2}}{\sigma_{x}^{2}\left[\lambda+\beta^{2}\right]+\lambda \sigma^{2}} ; \frac{\lambda \sigma^{2} \sigma_{x}^{2}\left(w_{i}\right.}{\sigma_{x}^{2}\left[\lambda+\beta^{2}\right]+\lambda \sigma^{2}}\right), \\
& i=1, \ldots, n \text {, } \\
& \alpha \mid \underset{\sim}{x}, \beta, \mu, \sigma_{x}^{2}, \sigma^{2}, \sigma^{2}, \underset{\sim}{w}, D_{\text {obs }} \\
& \sim N\left(\frac{\sigma_{\alpha}^{2} \sum_{i=1}^{n} \frac{\left(Y_{i}-\beta x_{i}\right)}{w_{i}}+\lambda \sigma^{2} a}{\sigma_{\alpha}^{2} \sum_{i=1}^{n} \frac{1}{w_{i}}+\lambda \sigma^{2}} ; \frac{\lambda \sigma^{2} \sigma_{\alpha}^{2}}{\sigma_{\alpha}^{2} \sum_{i=1}^{n} \frac{1}{w_{i}}+\lambda \sigma^{2}}\right), \\
& \beta \mid \underset{\sim}{x}, \alpha, \mu, \sigma_{x}^{2}, \sigma^{2}, \underset{\sim}{w}, D_{\mathrm{obs}} \\
& \sim N\left(\frac{\sigma_{\beta}^{2} \sum_{i=1}^{n} \frac{\left(Y_{i}-\alpha\right) x_{i}}{w_{i}}+\lambda b \sigma^{2}}{\sigma_{\beta}^{2} \sum_{i=1}^{n} \frac{x_{i}^{2}}{w_{i}}+\lambda \sigma^{2}} ; \frac{\lambda \sigma^{2} \sigma_{\beta}^{2}}{\sigma_{\beta}^{2} \sum_{i=1}^{n} \frac{x_{i}^{2}}{w_{i}}+\lambda \sigma^{2}}\right), \\
& \mu \mid \underset{\sim}{x}, \alpha, \beta, \sigma_{x}^{2}, \sigma^{2}, \underset{\sim}{w}, D_{\mathrm{obs}} \\
& \sim N\left(\frac{\sigma_{\mu}^{2} \sum_{i=1}^{n} \frac{x_{i}}{w_{i}}+\sigma_{x}^{2} m}{\sigma_{\mu}^{2} \sum_{i=1}^{n} \frac{1}{w_{i}}+\sigma_{x}^{2}} ; \frac{\sigma_{\mu}^{2} \sigma_{x}^{2}}{\sigma_{\mu}^{2} \sum_{i=1}^{n} \frac{1}{w_{i}}+\sigma_{x}^{2}}\right), \\
& \sigma_{x}^{2} \mid \underset{\sim}{x}, \alpha, \beta, \mu, \sigma^{2}, \underset{\sim}{w}, D_{\mathrm{obs}} \\
& \sim I G\left(\frac{n}{2}+c ; \frac{1}{2} \sum_{i=1}^{n} \frac{\left(x_{i}-\mu\right)^{2}}{w_{i}}+d\right), \\
& \sigma^{2} \mid \underset{\sim}{x}, \alpha, \beta, \mu, \sigma_{x}^{2}, \underset{\sim}{w}, D_{\mathrm{obs}} \\
& \sim I G\left(k+f ; \frac{1}{2}\left(\sum_{i=1}^{n} \frac{\left(Y_{i}-\alpha-\beta x_{i}\right)^{2}}{\lambda w_{i}}+\sum_{i=1}^{n} \frac{\left(X_{i}-x_{i}\right)^{2}}{w_{i}}\right)+g\right), \\
& u_{i} \mid u_{(-i)}, \underset{\sim}{x}, \alpha, \beta, \mu, \sigma_{x}^{2}, \sigma^{2}, D_{\mathrm{obs}} \\
& \sim I G\left(\frac{3+\nu}{2} ; \frac{1}{2}\left[\frac{\left(Y_{i}-\alpha-\beta x_{i}\right)^{2}+\lambda\left(X_{i}-x_{i}\right)^{2}}{\lambda \sigma^{2}}+\frac{\left(x_{i}-\mu\right)^{2}}{\sigma_{x}^{2}}+\nu\right]\right) . \\
& i=1, \ldots, n \text {, }
\end{aligned}
$$


Observação 1: Fazendo $w_{i}=1$ nas fórmulas (3.1.15) a (3.1.21) obtemos as fórmulas (2.5.9) a (2.5.14) do Capítulo 2.

Observação 2: No modelo dessa seção, o número de graus de liberdade, $\nu$, é suposto conhecido. Entretanto, nas aplicaçōes em geral não temos tal conhecimento. Uma maneira de tratar o problema é considerar o número de graus de liberdade $\nu$ como uma variável aleatória atribuindo uma priori para ela como por exemplo na seção 3.2 a seguir. Uma outra maneira é analisar o problema descritivamente tratando $\nu$ como um hiperparâmetro e calculando por exemplo a medida $L$ para vários valores de $\nu$ fixados e escolhendo o $\nu$ que corresponde ao menor valor dessa medida.

\subsubsection{Distribuição preditiva}

Seja $U_{f}=\left(\begin{array}{l}Y_{f} \\ X_{f}\end{array}\right)$ uma observação futura. A distribuição preditiva à posteriori no modelo $t$-independente definido em (3.1.8) é dada por

$$
\begin{aligned}
& \pi\left(\underset{\sim}{U_{f}} \mid D_{\mathrm{obs}}\right)=\int_{W_{f}} \int_{\Theta} \pi\left(\underset{\sim}{U_{f}}, \underset{\sim}{\theta}, w_{f} \mid D_{\mathrm{obs}}\right) \mathrm{d} \underset{\sim}{\theta} \mathrm{d} w_{f} \\
& =\int_{W_{f}} \int_{\Theta} \pi\left(\underset{\sim}{U_{f}} \mid \underset{\sim}{\theta}, w_{f}\right) \pi\left(w_{f}\right) \pi\left(\underset{\sim}{\theta} \mid D_{\mathrm{obs}}\right) \mathrm{d} \underset{\sim}{\theta} \mathrm{d} w_{f} \\
& \text { onde } \underset{\sim}{\theta}=\left(\alpha, \beta, \mu, \sigma_{x}^{2}, \sigma^{2}\right)
\end{aligned}
$$

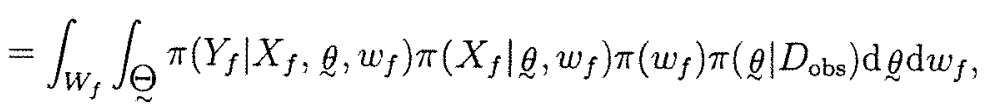

onde $\pi\left(\underset{\theta}{\theta} \mid D_{\text {obs }}\right)$ é a distribuição à posteriori,

$$
\begin{aligned}
& w_{f} \sim I G\left(\frac{\nu}{2}, \frac{\nu}{2}\right), \\
& X_{f} \mid \theta_{\sim}, w_{f} \sim N\left(\mu, \quad\left(\sigma_{x}^{2}+\sigma^{2}\right) w_{f}\right) \\
& \mathrm{e} Y_{f} \mid X_{f}, \theta \underset{\sim}{\theta}, w_{f} \sim N\left(\alpha+\beta \mu+\frac{\beta \sigma_{x}^{2}}{\sigma_{x}^{2}+\sigma^{2}}\left(X_{f}-\mu\right) ;\left(\beta^{2} \sigma_{x}^{2}+\lambda \sigma^{2}-\frac{\beta^{2}\left(\sigma_{x}^{2}\right)^{2}}{\sigma_{x}^{2}+\sigma^{2}}\right) w_{f}\right) .
\end{aligned}
$$

\section{Geração de uma observação futura}

Para gerar uma observação $\bigcup_{f}$, basta seguir os seguintes passos: 
(1) gerar ${\underset{\sim}{\theta}}^{(g)}$ de $\pi\left(\underset{\sim}{\theta} \mid D_{\text {obs }}\right)$ (ou de $\pi\left(\underset{\sim}{\theta}, \underset{\sim}{w} \mid D_{\text {obs }}\right)$ ou de $\pi\left(\underset{\sim}{\theta}, \underset{\sim}{w}, \underset{\sim}{x} \mid D_{\text {obs }}\right)$ );

(2) gerar $w_{f}^{(g)}$ de $\pi\left(w_{f}\right)$;

(3) gerar $X_{f}$ de $\pi\left(\left.X_{f}\right|_{\theta^{(g)}}, w_{f}^{(g)}\right)$ e

(4) gerar $Y_{f}$ de $\pi\left(Y_{f} \mid X_{f}^{(g)}, \underline{\theta}^{(g)}, w_{f}^{(g)}\right)$ (e portanto, dado que $X_{f}=X_{0}$, podemos gerar $Y_{f}$ de $\left.\pi\left(Y_{f} \mid X_{f}=X_{0}, \underline{\theta}^{(g)}, w_{f}^{(g)}\right)\right)$.

Semelhantemente, dado que $Y_{f}=Y_{0}$, podemos gerar $X_{f}$ de $\pi\left(X_{f} \mid Y_{f}=Y_{0}, \underline{\theta}^{(g)}\right.$, $\left.w_{f}^{(g)}\right)$, onde

$$
X_{f} \mid Y_{f}, \underset{\sigma}{\theta}, w_{f} \sim N\left(\mu+\frac{\beta \sigma_{x}^{2}}{\beta^{2} \sigma_{x}^{2}+\lambda \sigma^{2}}\left(Y_{f}-\alpha-\beta \mu\right) ;\left[\sigma_{x}^{2}+\sigma^{2}-\frac{\left(\beta \sigma_{x}^{2}\right)^{2}}{\beta^{2} \sigma_{x}^{2}+\lambda \sigma}\right] w_{f}\right)
$$

Observação: A distribuição preditiva $\pi\left({\underset{\sim}{f}}_{f} \mid D_{\text {obs }}\right)$ também pode ser escrita como

$$
\begin{aligned}
& \pi\left(\underset{\sim}{U_{f}} \mid D_{\mathrm{obs}}\right)=\int_{\mathscr{x}_{f}} \int_{\Theta} \int_{W_{f}} \pi\left(\underset{\sim}{U_{f}}, \underset{\theta}{\theta}, x_{f}, w_{f} \mid D_{\mathrm{obs}}\right) \mathrm{d} w_{f} \mathrm{~d} \underset{\sim}{\theta} \mathrm{d} x_{f} \\
& =\int_{\mathscr{x}_{f}} \int_{\theta} \int_{W_{f}} \pi\left({\underset{\sim}{f}}_{f} \mid x_{f}, w_{f}, \theta\right) \pi\left(x_{f} \mid \theta, w_{f}\right) \pi\left(w_{f}\right) \pi\left(\underset{\sim}{\theta} \mid D_{\mathrm{obs}}\right) \mathrm{d} w_{f} \mathrm{~d} \theta \underset{\sim}{\mathrm{d}} \mathrm{d} x_{f}
\end{aligned}
$$

onde $w_{f} \sim I G\left(\frac{\nu}{2}, \frac{\nu}{2}\right)$,

$$
\begin{aligned}
& x_{f} \mid \underset{\sim}{\theta}, w_{f} \sim N\left(\mu ; \sigma_{x}^{2} w_{f}\right) \\
& \bigcup_{\sim} \mid x_{f}, w_{f}, \theta \sim N\left[\left(\begin{array}{c}
\alpha+\beta x_{f} \\
x_{f}
\end{array}\right) ;\left(\begin{array}{cc}
\lambda \sigma & 0 \\
0 & \sigma^{2}
\end{array}\right) w_{f}\right] \text {, }
\end{aligned}
$$

isto é, $Y_{f}$ e $X_{f}$ são condicionalmente independentes dado $\underset{\sim}{\theta}, w_{f}, x_{f}$.

\subsubsection{Avaliação do ajuste}

Podemos fazer uma avaliação da qualidade do ajuste do modelo $t$-independente de forma semlhante à apresentada na seção 2.10. Para isso, basta obter as fórmulas de cálculo da densidade condicional preditiva de validação cruzada $\left(\mathrm{CPO}_{i}\right)$ e dos resíduos de validação cruzada para esse modelo. 


\section{(1) Densidade preditiva de validação cruzada}

A seguir apresentamos algumas fórmulas alternativas para estimar $\mathrm{CPO}_{i}=$

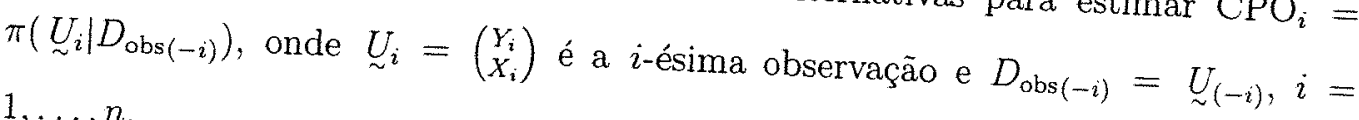
$1, \ldots, n$.

\section{Fórmula 1}

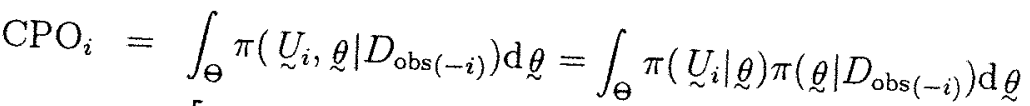

$$
\begin{aligned}
& =\left[\int_{\Theta} \frac{1}{\pi\left({\underset{\sim}{i}}_{i} \mid \theta\right)} \pi\left(\underset{\sim}{(\theta)} D_{\mathrm{obs}}\right) \mathrm{d} \theta \underset{\sim}{-1}\right.
\end{aligned}
$$

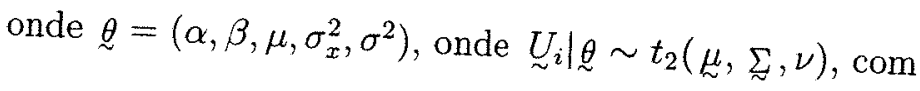

isto é,

$$
\mu=\left(\begin{array}{c}
\alpha+\beta \mu \\
\mu
\end{array}\right) \text { e } \sum=\left(\begin{array}{cc}
\beta^{2} \sigma_{x}^{2}+\lambda \sigma^{2} & \beta \sigma_{x}^{2} \\
\beta \sigma_{x}^{2} & \sigma_{x}^{2}+\sigma^{2}
\end{array}\right) \text {, }
$$

$$
\begin{aligned}
& \pi\left({\underset{\sim}{U}}_{i} \mid \theta\right)=k(n, \nu)|\Sigma|^{-\frac{1}{2}}\left[\nu+\left({\underset{\sim}{U}}_{i}-\mu\right)^{\top} \Sigma^{-1}\left({\underset{\sim}{i}}_{i}-\mu\right)\right]^{\frac{-(2+\nu)}{2}}, \\
& \text { onde } k(n, \nu)=\frac{\Gamma\left(\frac{2+\nu}{2}\right) \nu^{\nu / 2}}{\Gamma\left(\frac{\nu}{2}\right) \pi} \text { e } \nu \text { é o número de graus de liberdade (conhecido). }
\end{aligned}
$$

\section{Estimação}

Uma estimativa de Monte Carlo de $\mathrm{CPO}_{i}$ é dada por

$$
\widehat{\mathrm{PO}}_{i}=\left(\frac{1}{G} \sum_{g=1}^{G} \frac{1}{\pi\left({\left.\underset{\sim}{i} i \underline{\theta}^{(g)}\right)}^{-1}\right.}\right)^{-1}
$$

onde $\left({\underset{\theta}{ }}^{(1)}, \ldots, \underline{\theta}^{(G)}\right)$ é uma amostra da posteriori $p\left(\underset{\sim}{\theta} \mid D_{\text {obs }}\right)$, gerada por exemplo de $p\left(\underset{\sim}{\theta} \underset{\sim}{x}, \underset{\sim}{w} \mid D_{\text {obs }}\right)$ pelo algoritmo de Gibbs da seção 3.1 .2 , ou de $p\left(\underset{\sim}{\theta}, \underset{\sim}{w} \mid D_{\text {obs }}\right)$ pelo algoritmo de $\mathrm{M}-\mathrm{H}$ em Gibbs (desde que estes algoritmos convirjam).

\section{Fórmula 2}

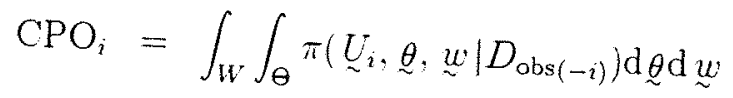




$$
\begin{aligned}
& =\int_{\Theta} \int_{\underset{\sim}{w}} \pi\left({\underset{\sim}{i}}_{i} \mid \underset{\sim}{\theta} \underset{\sim}{w}\right) \pi\left(\underset{\sim}{\theta}, \underset{\sim}{w} \mid D_{\mathrm{obs}(-1)}\right) \mathrm{d} \underset{\sim}{\theta} \mathrm{d} \underset{\sim}{w}
\end{aligned}
$$

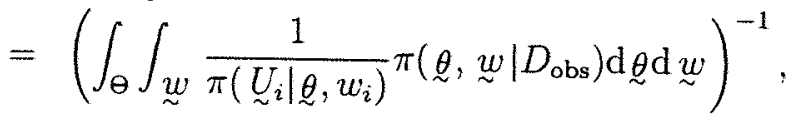

onde $\underset{\sim}{w}=\left(w_{1}, \ldots, w_{n}\right)^{T}$, e onde

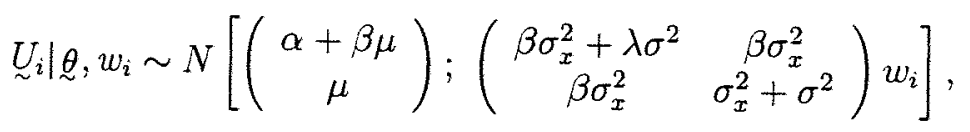

\section{Estimação}

$$
\widehat{\mathrm{PO}}_{i}=\left(\frac{1}{G} \sum_{g=1}^{G} \frac{1}{\pi\left({\underset{\sim}{i}}_{i} \mid \underline{\theta}^{(g)}, w_{i}^{(g)}\right)}\right)^{-1}
$$

onde $\left({\underset{\theta}{(1)}}^{(1)}, w_{i}^{(1)}, \ldots,{\underset{\theta}{(G)}}^{(G)}, w_{i}^{(G)}\right)^{T}$ é uma amostra da posteriori conjunta de $\underset{\sim}{\theta}$ e $\underset{\sim}{w}$, gerada por exemplo de $p\left(\underset{\sim}{\theta} \underset{\sim}{x}, \underset{\sim}{w} \mid D_{\text {obs }}\right)$ pelo algoritmo de Gibbs da seção 3.1 .2 ou de $p\left(\theta, \underset{\sim}{w} \mid D_{\text {obs }}\right)$ pelo algoritmo de M-H em Gibbs (desde que eles convirjam).

\section{Fórmula 3}

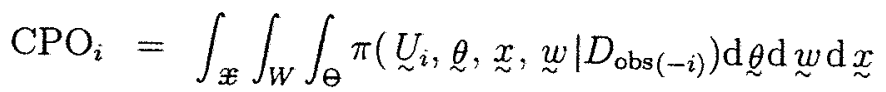

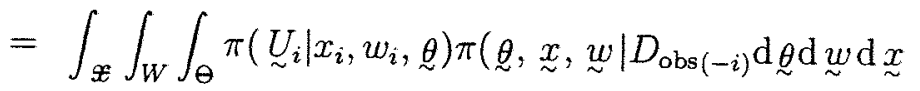

$$
\begin{aligned}
& \left.=\left(\int_{\mathfrak{X}} \int_{W} \int_{\Theta} \frac{1}{\pi\left(U_{i} \mid x_{i}, w_{i}, \theta\right.}\right) \pi\left(\underset{\sim}{\theta}, \underset{\sim}{x}, \underset{\sim}{w} \mid D_{\mathrm{obs}}\right) \mathrm{d} \underset{\sim}{\theta} \mathrm{d} \underset{\sim}{w} \mathrm{~d} \underset{\sim}{x}\right)^{-1}
\end{aligned}
$$

onde

$$
{\underset{\sim}{i}}_{i} \mid x_{i}, w_{i}, \underset{\theta}{\theta} \sim N\left[\left(\begin{array}{c}
\alpha+\beta x_{i} \\
x_{i}
\end{array}\right) ;\left(\begin{array}{cc}
\lambda \sigma^{2} & 0 \\
0 & \sigma^{2}
\end{array}\right) w_{i}\right]
$$

\section{Estimação}

$$
\widehat{\mathrm{COO}}_{i}=\left(\frac{1}{G} \sum_{g=1}^{G} \frac{1}{\pi\left(U_{i} \mid x_{i}^{(g)}, w_{i}^{(g)}, \theta^{(g)}\right)}\right)^{-1},
$$

onde $\left(\underline{\theta}^{(1)},{\underset{\sim}{x}}^{(1)}, w^{(1)}, \ldots,{\underset{\theta}{(G)}}^{x}{\underset{\sim}{(G)}}^{(G)} w^{(G)}\right)$ é uma amostra da posteriori conjunta

$p\left(\underset{\theta}{x} \underset{\sim}{x} \underset{\sim}{w} \mid D_{\text {obs }}\right)$ gerada por exemplo pelo algoritmo de Gibbs da seção 3.1.2, desde que haja convergência. 
Observação: Note que o cálculo de $\widehat{\mathrm{CPO}}_{i}$ nesse caso poderia eventualmente ser obtido gerando $\left({\underset{\theta}{(g)}}^{(g)}{\underset{\sim}{w}}^{(g)}\right)$ de $\pi\left(\underset{\theta}{\theta}, w \mid D_{\text {obs }}\right)$ pelo algoritmo de M-H em Gibbs (desde que este convirja) e depois gerando $x_{i}^{(g)}$ de $\pi\left(x_{i} \mid \theta_{\sim}^{(g)}, w_{i}^{(g)}\right) \sim N\left(\mu^{(g)}, \sigma_{x}^{2^{(g)}} w_{i}^{(g)}\right)$.

\section{(2) Resíduo de validação cruzada}

No modelo estrutural $t$-independente (com restrição de identificabilidade $\sigma_{e_{i}}^{2}=$ $\left.\lambda \sigma_{i}^{2}\right)$, formulado como mistura de escala da distribuição Normal, temos que os resíduos bidimensionais $\left(\begin{array}{l}e_{i} \\ u_{i}\end{array}\right)$ são tais que

$$
\left(\begin{array}{c}
e_{i} \\
u_{i}
\end{array}\right) \mid \underset{\sim}{\theta}, x_{i}, w_{i} \sim N_{2}(\underset{\sim}{0}, V) \text {, onde } V=\left(\begin{array}{cc}
\lambda \sigma^{2} & 0 \\
0 & \sigma^{2}
\end{array}\right) w_{i}, \text { para } i=1, \ldots, n .
$$

Os resíduos padronizados são iguais a

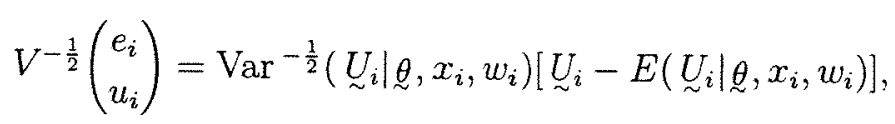

$i=1, \ldots, n$, e portanto os resíduos padronizados de validação cruzada nesse modelo são iguais a

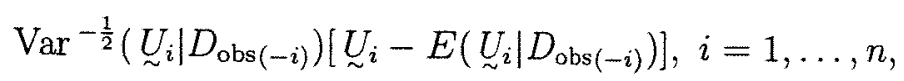

onde

- Cálculo de $E\left(\underset{\sim}{\left.U_{i} \mid D_{\mathrm{obs}(-i)}\right)}\right.$

$$
\begin{aligned}
& E\left[{\underset{\sim}{U}}_{i} \mid D_{\mathrm{obs}(-i)}\right]=E\left[E\left({\underset{\sim}{i}}_{i} \mid \underset{\sim}{\theta} \underset{\sim}{x}, \underset{\sim}{w}\right) \mid D_{\mathrm{obs}(-i)}\right] \\
& =\int_{\not} \int_{W} \int_{\Theta} E\left(\underset{\sim}{U_{i}} \mid \underset{\sim}{\theta}, \underset{\sim}{x}, \underset{\sim}{w}\right) \pi\left(\underset{\sim}{\theta}, \underset{\sim}{x}, \underset{\sim}{w} \mid D_{\mathrm{obs}(-i)}\right) \mathrm{d} \underset{\sim}{\theta} \mathrm{d} \underset{\sim}{w} \mathrm{~d} \underset{\sim}{x} \\
& =\mathrm{CPO}_{i} \int_{\circledast} \int_{W} \int_{\Theta} \frac{E\left({\underset{\sim}{U}}_{i} \mid \underset{\sim}{\theta}, \underset{\sim}{x}, \underset{\sim}{w}\right)}{\pi\left({\underset{\sim}{i}}_{i} \mid \underset{\sim}{\theta}, x_{i}, w_{i}\right)} \pi\left(\underset{\sim}{\theta} \underset{\sim}{x}, \underset{\sim}{w} \mid D_{\mathrm{obs}}\right) \mathrm{d} \underset{\sim}{\theta} \mathrm{d} \underset{\sim}{w} \mathrm{~d} \underset{\sim}{x},
\end{aligned}
$$

onde

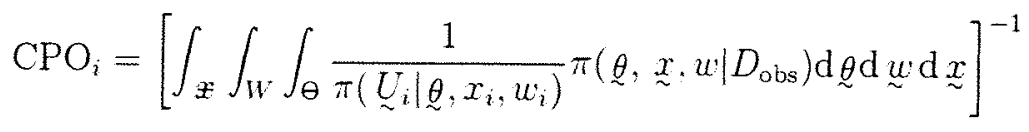


e onde

$$
\underset{\sim}{U_{i}} \mid \theta, x_{i}, w_{i} \sim N\left[\left(\begin{array}{c}
\alpha+\beta x_{i} \\
x_{i}
\end{array}\right) ;\left(\begin{array}{cc}
\lambda \sigma^{2} & 0 \\
0 & \sigma^{2}
\end{array}\right) w_{i}\right]
$$

Portanto,

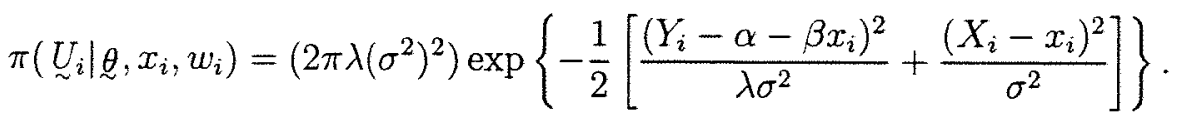

\section{Estimação}

Dada uma amostra de tamanho $G$ de $\pi\left(\underset{\sim}{\theta}, \underset{\sim}{w}, \underset{\sim}{x} \mid D_{\text {obs }}\right)$, obtida por exemplo por meio do algoritmo de Gibbs da seção 3.1 .2 (se houver convergência),

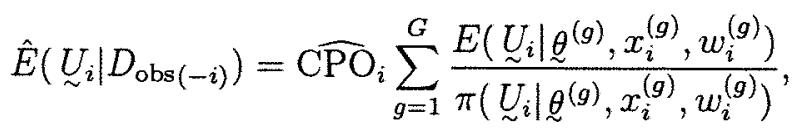

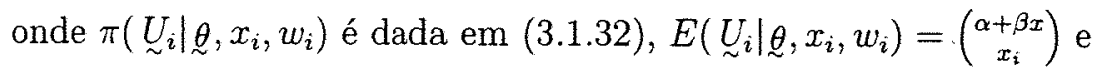

$$
\widehat{\mathrm{CPO}}_{i}=\left(\frac{1}{G} \sum_{j=1}^{G} \frac{1}{\pi\left({\underset{\sim}{i}}_{i} \mid \underline{\theta}^{(g)}, x_{i}^{(g)}, w_{i}^{(g)}\right)}\right)^{-1} .
$$

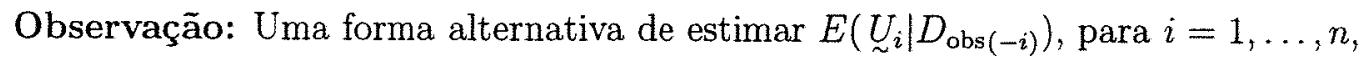

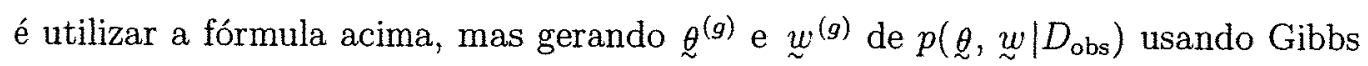
ou M-H em Gibbs (desde que convirjam) e depois gerando $x_{i}^{(g)}$ de $\pi\left(x_{i} \mid \theta^{(g)}, w_{i}^{(g)}\right)$, onde $x_{i} \mid \underset{\theta}{\theta}, w_{i} \sim N\left(\mu ; \sigma_{x}^{2} w_{i}\right)$.

Cálculo da $\operatorname{Var}\left(\underset{\sim}{U_{i}} \mid D_{\mathrm{obs}(-i)}\right)$

$$
\begin{aligned}
& \operatorname{Var}\left(\underset{\sim}{U_{i}} \mid D_{\mathrm{obs}}\right)=E\left[\operatorname{Var}\left(\underset{\sim}{U_{i}} \mid \underset{\sim}{\theta}, \underset{\sim}{x}, \underset{\sim}{w}\right) \mid D_{\mathrm{obs}(-i)}\right]+\operatorname{Var}\left[E\left(\underset{\sim}{U_{i}} \mid \underset{\sim}{\theta}, \underset{\sim}{x} \underset{\sim}{w}\right) \mid D_{\mathrm{obs}(-i)}\right]
\end{aligned}
$$

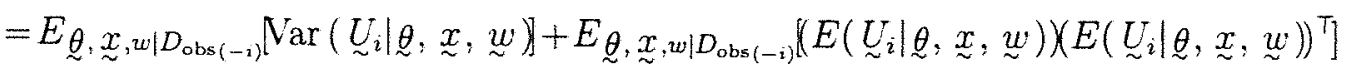

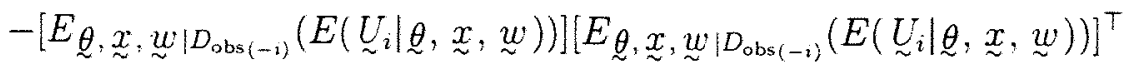

$$
\begin{aligned}
& =\int_{W} \int_{x} \int_{\theta} \operatorname{Var}\left(\underset{\sim}{U_{i} \mid} \mid \underset{\sim}{\mid} \underset{\sim}{x}, \underset{\sim}{w}\right) \pi\left(\underset{\sim}{\theta}, \underset{\sim}{x}, \underset{\sim}{w} \mid D_{\mathrm{obs}(-i)}\right) \mathrm{d} \underset{\sim}{\theta} \mathrm{d} \underset{\sim}{x} \mathrm{~d} \underset{\sim}{w} \\
& +\int_{W} \int_{\mathfrak{z}} \int_{\Theta}\left(E\left({\underset{\sim}{i}}_{i} \mid \underset{\sim}{\theta}, \underset{\sim}{x}, w\right)\right)\left(E\left({\underset{\sim}{U}}_{i} \mid \underset{\sim}{\theta} \underset{\sim}{x} ; w\right)\right)^{\top} \pi\left(\underset{\sim}{\theta} \underset{\sim}{x}, \underset{\sim}{w} \mid D_{\mathrm{obs}(-i)}\right) \mathrm{d} \underset{\sim}{\theta} \mathrm{d} \underset{\sim}{x} \mathrm{~d} \underset{\sim}{w} \\
& -\left[E\left({\underset{\sim}{U}}_{i} \mid D_{\mathrm{obs}(-i)}\right)\right]\left[E\left({\underset{\sim}{U}}_{i} \mid D_{\mathrm{obs}(-i)}\right)\right]^{\top}
\end{aligned}
$$




$$
\begin{aligned}
& =\mathrm{CPO}_{i} \int_{W} \int_{\Re} \int_{\Theta} \frac{\operatorname{Var}\left({\underset{\sim}{i}}_{i} \mid \theta, x_{i}, w_{i}\right)}{\pi\left(\underset{\sim}{U_{i} \mid}, \underline{\theta}, x_{i}, w_{i}\right)} \pi\left(\underset{\sim}{\theta}, \underset{\sim}{x}, \underset{\sim}{w} \mid D_{\mathrm{obs}}\right) \mathrm{d} \underset{\sim}{\theta} \mathrm{d} \underset{\sim}{x} \mathrm{~d} \underset{\sim}{w} \\
& +\mathrm{CPO}_{i} \int_{W} \int_{\mathfrak{x}} \int_{\theta}\left[E\left({\underset{\sim}{i}}_{i} \mid \underset{\sim}{\theta}, x_{i}, w_{i}\right)\right]\left[E\left(\underset{\sim}{U_{i} \mid} \underset{\sim}{\theta}, x_{i}, w_{i}\right)\right]^{\top} \pi\left(\underset{\sim}{\theta}, \underset{\sim}{w}, \underset{\sim}{x} \mid D_{\mathrm{obs}}\right) \mathrm{d} \underset{\sim}{\theta} \mathrm{d} \underset{\sim}{x} \mathrm{~d} \underset{\sim}{w} \\
& -\left[E\left(U_{i} \mid D_{\mathrm{obs}(-i)}\right)\right]\left[E\left(U_{i} \mid D_{\mathrm{obs}(-i)}\right)\right]^{\top}
\end{aligned}
$$

onde $\mathrm{CPO}_{i}$ é dada por (3.1.31), $E\left({\underset{\sim}{i}}_{i} \mid D_{\mathrm{obs}(-i)}\right)$ é dada por (3.1.30).

\section{Estimação}

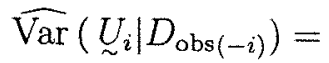

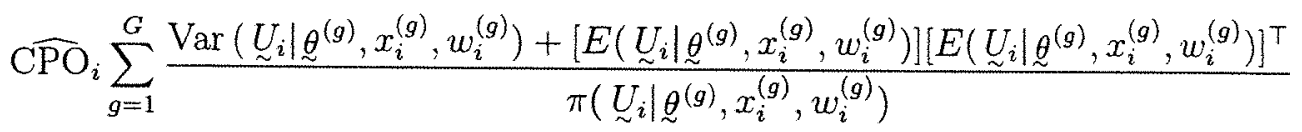

$$
\begin{aligned}
& -\left[\hat{E}\left(\underset{\sim}{U_{i} \mid} D_{\mathrm{obs}(-i)}\right)\right]\left[\hat{E}\left({\underset{\sim}{U}}_{i} \mid D_{\mathrm{obs}(-i)}\right)\right]^{\top},
\end{aligned}
$$

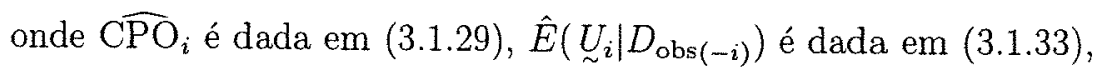

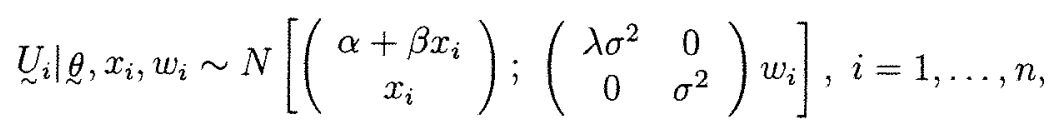

$\mathrm{e}\left({\underset{\sim}{\theta}}^{(1)},{\underset{\sim}{w}}^{(1)},{\underset{\sim}{x}}^{(1)}, \ldots,{\underset{\theta}{ }}^{(G)},{\underset{\sim}{w}}^{(G)},{\underset{\sim}{x}}^{(G)}\right)$ é uma amostra da posteriori $p\left(\underset{\sim}{\theta}, \underset{\sim}{w}, \underset{\sim}{x} \mid D_{\mathrm{obs}}\right)$.

\subsection{O modelo estrutural $t$-independente com graus de liberdade desconhecidos}

Nessa seção estudamos o modelo $t$-independente onde $\nu$ é desconhecido. Nesse caso escolhemos uma priori para $\nu$, incorporando-a ao modelo, e com base na distribuição marginal à posteriori de $\nu$ obtemos um estimador de Baves de $\nu$. Na subseção 3.2.2 analisamos o modelo considerando duas prioris próprias para $\nu$, a primeira é uma distribuição uniforme discreta e a segunda é a distribuição exponencial. O modelo de regressão $t$-independente com $\nu$ desconhecido no caso clássico (ou seja quando a variável independente é medida sem erros) foi estudado por Geweke (1993)). 


\subsubsection{Definição do modelo}

Considere o modelo identificável formulado como mistura de escala da distribuição Normal

$$
\left\{\begin{array}{l}
Y_{i}=\alpha+\beta x_{i}+e_{i}, \quad i=1, \ldots, n \\
X_{i}=x_{i}+u_{i}
\end{array}\right.
$$

onde

$$
\underset{\sim}{Z}=\left(\begin{array}{c}
Y_{i} \\
X_{i} \\
x_{i}
\end{array}\right), \text { com a suposição } \underset{\sim}{Z}{ }_{i} \mid \underset{\sim}{\theta}, \underset{\sim}{w}, \nu \stackrel{\text { indep }}{\sim} N_{3}\left(\underset{\sim}{\mu} ; \underset{\sim}{\Sigma} w_{i}\right)
$$

onde $\underset{\sim}{\mu}$ e $\sum_{\sim}$ são dadas em (3.1.2).

O vetor de parâmetros é $\underset{\sim}{*}=\left(\begin{array}{c}\underset{v}{w} \\ \underset{\nu}{\nu}\end{array}\right)$, onde $\underset{\sim}{\theta}=\left(\alpha, \beta, \mu, \sigma_{x}^{2}, \sigma^{2}\right)^{T}$ e $\underset{\sim}{w}=$ $\left(w_{1}, \ldots, w_{n}\right)^{T}$.

Vamos tomar a seguinte distribuição à priori:

$$
\pi(\underset{\sim}{\theta}, \underset{\sim}{w}, \nu)=\pi(\underset{\sim}{\theta}) \pi(\underset{\sim}{w} \mid \nu) \pi(\nu)
$$

onde $w_{i} \mid \nu \stackrel{\text { iid }}{\sim} I G\left(\frac{\nu}{2}, \frac{\nu}{2}\right), \pi(\underset{\theta}{\theta})$ é a priori de $\underset{\sim}{\theta}$ e $\pi(\nu)$ a priori de $\nu$.

\section{Função de verossimilhança}

A função de verossimilhança,$L(\underset{\sim}{\theta}, \underset{\sim}{w}, \nu \mid D)$, baseada nos dados completos $D$, é proporcional à função em (3.1.9), e a verossimilhança baseada nos dados observados, $L\left(\underset{\sim}{\theta}, \underset{\sim}{w}, \nu \mid D_{\mathrm{obs}}\right)$, é proporcional à função em (3.1.10).

\section{Distribuição à posteriori}

A distribuição conjunta à posteriori de $(\underset{\sim}{\theta}, \underset{\sim}{w}, \nu)$ é dada por

$$
p\left(\underset{\sim}{\theta}, \underset{\sim}{w}, \nu \mid D_{\mathrm{obs}}\right)=\int_{\mathcal{X}=\mathbb{R}^{n}} p\left(\underset{\sim}{x}, \underset{\sim}{\theta}, \underset{\sim}{w}, \nu \mid D_{\mathrm{obs}}\right) \mathrm{d} \underset{\sim}{x} .
$$

e portanto a distribuição à posteriori

$$
p\left(\underset{\sim}{\theta}, \nu \mid D_{\mathrm{obs}}\right)=\int_{\mathfrak{x}} \int_{W=\mathbb{R}_{+}^{n}} p\left(\underset{\sim}{x}, \underset{\sim}{\theta}, \underset{\sim}{w}, \nu \mid D_{\mathrm{obs}}\right) \mathrm{d} \underset{\sim}{\underset{\sim}{w} \mathrm{~d} \underset{\sim}{x},}
$$


onde

$$
p\left(\underset{\sim}{x}, \underset{\sim}{\theta}, \underset{\sim}{w}, \nu \mid D_{\mathrm{obs}}\right) \propto L\left(\underset{\sim}{\theta} \underset{\sim}{w}, \nu \mid D_{\mathrm{obs}}\right) \pi(\underset{\sim}{\theta}, \underset{\sim}{w}, \nu),
$$

onde $L\left(\underset{\sim}{\theta}, \underset{\sim}{w}, \nu \mid D_{\text {obs }}\right)$ é proporcional à função em (3.1.10).

\subsubsection{Implementação de algoritmos MCMC no modelo $t$-in- dependente para duas particulares escolhas de priori própria}

Caso 1: Considere a distribuição à priori

$$
\begin{aligned}
\pi(\underset{\sim}{\theta}, \underset{\sim}{w}, \nu) & =\pi(\underset{\sim}{\theta}) \pi(\underset{\sim}{w} \mid \nu) \pi(\nu) \\
& =\pi(\alpha) \pi(\beta) \pi(\mu) \pi\left(\sigma_{x}\right) \pi\left(\sigma^{2}\right)\left(\prod_{i=1}^{n} \pi\left(w_{i} \mid \nu\right)\right) \pi(\nu)
\end{aligned}
$$

onde $\alpha \sim N\left(a, \mu_{\alpha}\right), \mu \sim N\left(m, \sigma_{\mu}^{2}\right), \sigma_{x}^{2} \sim I G(c, d), \sigma^{2} \sim I G(f, g), w_{i} \mid \nu \stackrel{\text { iid }}{\sim} I G\left(\frac{\nu}{2}, \frac{\nu}{2}\right)$, $i=1, \ldots, n, \mathcal{V}$ é uma v.a. uniforme discreta no intervalo $\left[c-n_{0}+1, c\right]$, isto é, $\pi(\nu)=p[\mathcal{V}=\nu]=\left\{\begin{array}{ll}\frac{1}{n_{0}}, & c-n_{0}+1 \leq \nu \leq c, \\ 0, & \text { caso contrário, }\end{array} \quad\right.$ onde $n_{0}=$ número de elementos do domínio da variável aleatória $\mathcal{V}$ com probabilidade positiva e $c$ é uma constante inteira $(c>0)$. ( No caso particular em que $n_{0}=1$ e $c=\nu_{0}$, então $\mathcal{V}$ é uma variável degenerada no ponto $\nu_{0}$ ).

Nesse modelo podemos implementar o algoritmo de Gibbs para amostrar da posteriori usando as condicionais completas baseadas na distribuição conjunta à posteriori em (3.2.3). Nesse modelo, as condicionais completas univariadas de $x_{i}, \alpha$, $\beta, \mu, \sigma_{x}^{2}, w_{i}(i=1, \ldots, n)$ coincidem, respectivamente, com as distribuições dadas em (3.1.15) a (3.1.21); a única diferença é que agora devemos acrescentar a cada ciclo do algoritmo de Gibbs a distribuição

$$
\pi\left(\nu \mid \underset{\sim}{x}, \alpha, \beta, \mu, \sigma_{x}^{2}, \sigma^{2}, \underset{\sim}{w}, D_{\text {obs }}\right)=P\left(\mathcal{V}=\nu \mid \underset{\sim}{x}, \underset{\sim}{\theta}, \underset{\sim}{w}, D_{\text {obs }}\right)=\frac{L(\underset{\theta}{\theta}, \underset{\sim}{w}, \nu \mid D)}{\sum_{\nu=c-n_{0}-1}^{c} L(\underset{\theta}{\theta}, \underset{\sim}{w}, \nu \mid D)},
$$

onde $L(\underset{\sim}{\theta}, \underset{\sim}{w}, \nu \mid D)$ é proporcional à função em (3.1.9). 
Caso 2: Tomando a distribuição à priori em $(3.2 .5)$ e trocando $\pi(\nu)$ pela distribuição exponencial com parâmetro $\lambda_{0}$, então as distribuições condicionais completas univariadas à posteriori de $x_{i}, \alpha, \beta, \mu, \sigma_{x}^{2}, \sigma^{2}$ e $w_{i}$, baseadas em (3.2.3) coincidem com as do caso 1 e a condicional completa de $\nu$ é também exponencial de parâmetro $\lambda_{0}$.

\subsection{O modelo estrutural $t$-dependente com o nú- mero de graus de liberdade conhecido}

Nessa seção apresentamos o modelo de regressão linear simples $t$-dependente com erros nas variáveis. Da mesma forma que na seção 3.1 vamos considerar duas formulações equivalentes para esse modelo e além disso, discutiremos a implentação de algoritmos MCMC.

\subsubsection{Definição do modelo}

Formulação 1: Sejam as equações

$$
\left\{\begin{array}{l}
Y_{i}=\alpha+\beta x_{i}+e_{i}, \quad i=1, \ldots, n, \\
X_{i}=x_{i}+u_{i}
\end{array}\right.
$$

onde $\left(Y_{i}, X_{i}\right)$ são as variáveis aleatórias observáveis, $x_{i}$ a variável latente medida com erro e $u_{i}$ o erro na medição de $x_{i}$ com a suposição de que

$$
\underset{\sim}{Z} \underset{\sim}{\theta} \sim t_{3 n}\left(\underset{\sim}{1}{ }_{n} \otimes \mu ; I_{n \times n} \otimes \underset{\sim}{\Sigma}, \nu\right)
$$

onde

$$
\underset{\sim}{Z}=\left(\begin{array}{c}
{\underset{\sim}{1}}_{1} \\
\vdots \\
\underset{\sim}{Z}
\end{array}\right) \quad \text { e } \quad \underset{\sim}{Z}{ }_{i}=\left(\begin{array}{c}
Y_{i} \\
X_{i} \\
x_{i}
\end{array}\right)
$$

e onde

$$
\mu=\left(\begin{array}{c}
\alpha+\beta \mu \\
\mu \\
\mu
\end{array}\right), \quad \Sigma=\left(\begin{array}{ccc}
\beta^{2} \sigma_{x}^{2}+\sigma_{e}^{2} & \beta \sigma_{x}^{2} & \beta \sigma_{x}^{2} \\
3 \sigma_{x}^{2} & \sigma_{x}^{2}+\sigma_{u}^{2} & \sigma_{x}^{2} \\
\beta \sigma_{x}^{2} & \sigma_{x}^{2} & \sigma_{x}^{2}
\end{array}\right)
$$

com $\sigma_{e}^{2}=\lambda \sigma_{u}^{2}$ para $\lambda$ conhecida, $\nu>0$ conhecido, e $|\Sigma| \neq 0$. 
O espaço paramétrico nesse caso é

$$
\Theta=\left\{\underset{\sim}{\theta}=\left(\alpha, \beta, \mu, \sigma_{x}^{2}, \sigma^{2}\right)^{T}: \alpha, \beta, \mu \in \mathbb{R} \quad \text { e } \quad \sigma_{x}^{2}, \sigma^{2} \in \mathbb{R}_{+}\right\}
$$

A distribuição à priori de $\underset{\sim}{\theta}$ nesse modelo é denotada por $\pi(\underset{\sim}{\theta})$.

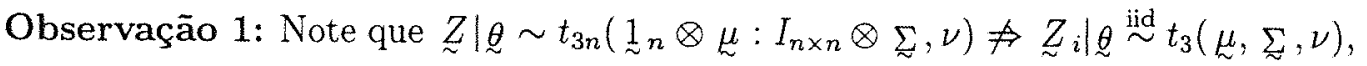
para $i=1, \ldots, n$.

Observação 2: O modelo $t$-dependente é não identificável pela mesma razão que o modelo $t$-independente também é.

Observação 3: Se $\nu \geq 3, \underset{\sim}{Z}$ tem matriz de covariâncias $\left(\operatorname{Var}(\underset{\sim}{Z})=I_{n \times n} \otimes \underset{\sim}{\sum} \frac{\nu}{\nu-2}\right)$ finita.

\section{Função de verossimilhança baseada nos dados completos} dada por

A função de verossimilhança baseada nos dados completos $D=\underset{\sim}{Z}=\left(\begin{array}{c}{\underset{\sim}{1}}_{1} \\ \vdots \\ Z_{n}\end{array}\right)$ é

$$
\begin{aligned}
& L(\underset{\sim}{\theta} \mid D) \propto\left|I_{n \times n} \otimes \sum_{\sim}\right|^{-\frac{1}{2}}\left[\nu+(\underset{\sim}{Z}-\underset{\sim}{1} n \otimes \underset{\sim}{\mu})^{\top}\left(I_{n \times n} \otimes \sum_{\sim}\right)^{-1}(\underset{\sim}{Z}-\underset{\sim}{1} \otimes \mu)^{\top}\right]^{-\left(\frac{3 n+\nu}{2}\right)} \\
& =|\Sigma|^{-\frac{n}{2}}\left[\nu+\sum_{i=1}^{n}\left(Z_{i}-\mu\right)^{\top} \Sigma^{-1}\left(Z_{i}-\mu\right)\right]^{-\left(\frac{3 n+\nu}{2}\right)},
\end{aligned}
$$

onde $\underset{\sim}{\mu}$ e $\sum_{\sim}$ são dados em (3.3.1).

Função de verossimilhança baseada nos dados observados

A função de verossimilhança baseada nos dados observados $D_{\text {obs }}=\left(\begin{array}{c}U_{1} \\ \vdots \\ U_{n}\end{array}\right)$, onde $\underset{\sim}{U_{i}}=\left(\begin{array}{l}Y_{i} \\ X_{i}\end{array}\right)$, é dada por

$$
L\left(\underset{\sim}{\theta} \mid D_{\mathrm{obs}}\right) \times\left|\sum_{i}^{*}\right|^{-\frac{n}{2}}\left[\nu+\sum_{i=1}^{n}\left({\underset{\sim}{U}}_{i}-\mu^{*}\right)^{\top} \sum_{\sim}^{*}-1\left({\underset{\sim}{i}}_{i}-\mu^{*}\right)\right]^{-\left(\frac{2 n+\nu}{2}\right)} .
$$


onde

$$
\mu^{*}=\left(\begin{array}{c}
\alpha+\beta \mu \\
\mu
\end{array}\right) \quad \text { e } \quad \sum_{\sim}^{*}=\left(\begin{array}{cc}
\beta^{2} \sigma_{x}^{2}+\lambda \sigma^{2} & \beta \sigma_{x}^{2} \\
\beta \sigma_{x}^{2} & \sigma_{x}^{2}+\sigma_{u}^{2}
\end{array}\right) .
$$

Distribuição à posteriori

A posteriori $p\left(\underset{\sim}{\theta} \mid D_{\mathrm{obs}}\right)$ é dada por

$$
p\left(\underset{\sim}{\theta} \mid D_{\mathrm{obs}}\right)=\int_{\mathfrak{X}=\mathbb{R}^{n}} p\left(\underset{\sim}{\theta}, \underset{\sim}{x} \mid D_{\mathrm{obs}}\right) \mathrm{d} \underset{\sim}{x}=\int_{\mathfrak{X}} L(\underset{\sim}{\theta} \mid D) \pi(\underset{\sim}{\theta}) \mathrm{d} \underset{\sim}{x},
$$

onde $L(\underset{\sim}{\theta} \mid D)$ é dada por (3.3.2), ou então

$$
p\left(\underset{\sim}{\theta} \mid D_{\text {obs }}\right) \propto L\left(\underset{\sim}{\theta} \mid D_{\text {obs }}\right) \pi(\underset{\sim}{\theta}),
$$

onde $L\left(\theta \mid D_{\text {obs }}\right)$ é dada por (??).

Formulação 2: o modelo de mistura de escala da distribuição Normal

Sejam as equações

$$
\left\{\begin{array}{l}
Y_{i}=\alpha+\beta x_{i}+e_{i}, \quad i=1, \ldots, n \\
X_{i}=x_{i}+e_{i}
\end{array}\right.
$$

e seja $\underset{\sim}{Z}=\left(\begin{array}{c}Z_{1} \\ \vdots \\ Z_{n}\end{array}\right)$, onde $\underset{\sim}{Z} i=\left(\begin{array}{c}Y_{i} \\ X_{i} \\ x_{i}\end{array}\right)$, com a suposição

$$
\underset{\sim}{Z} \mid \underset{\sim}{\theta}, w \sim N_{3 n}\left(\underset{\sim}{1} \otimes \underset{\sim}{\mu} ; \underset{n \times n}{I} \otimes \sum_{\sim} w\right)
$$

onde $\underset{\sim}{\mu}$ e $\sum$ são definidas em (3.3.1) e priori dada por $\pi(\underset{\sim}{\theta}, w)=\pi(\underset{\sim}{\theta}) \pi(w)$, onde $w \sim I G\left(\frac{\nu}{2}, \frac{\nu}{2}\right)$, isto é,

$$
\pi(w)=w^{-\left(\frac{\nu}{2}+1\right)} \exp \left\{-\frac{\nu}{2 w}\right\}, w>0
$$

Essa suposição é equivalente a

$$
Z_{i} \mid \theta, w, \stackrel{\mathrm{iid}}{\sim} N(\mu, \Sigma, w), \quad \text { para } i=1, \ldots, n,
$$


onde $\pi(\underline{\theta}, w)=\pi(\theta) \pi(w)$, com $w \sim I G\left(\frac{\nu}{2}, \frac{\nu}{2}\right)$.

\section{Função de verossimilhança baseada nos dados completos}

A função de verossimilhança baseada nos dados completos $D=\underset{\sim}{Z}$ é dada por

$$
\begin{aligned}
L(\underset{\sim}{\theta}, w \mid D) \propto & \left|\sum_{\sim}\right|^{-\frac{n}{2}} w^{-\frac{3 n}{2}} \exp \left\{-\frac{1}{2 w} \sum_{i=1}^{n}\left(\underset{\sim}{Z_{i}}-\mu\right)^{\top} \sum_{\sim}^{-1}\left(Z_{i}-\mu\right)\right\} \\
\propto & w^{-\frac{3 n}{2}}\left(\left(\sigma^{2}\right)^{2} \sigma_{x}^{2}\right)^{-\frac{n}{2}} \exp \left\{-\frac{1}{2 w}\left[\sum_{i=1}^{n} \frac{\left(Y_{i}-\alpha-\beta x_{i}\right)^{2}}{\lambda \sigma^{2}}\right.\right. \\
& \left.\left.+\sum_{i=1}^{n} \frac{\left(X_{i}-x_{i}\right)^{2}}{\sigma^{2}}+\sum_{i=1}^{n} \frac{\left(x_{i}-\mu\right)^{2}}{\sigma_{x}^{2}}\right]\right\} .
\end{aligned}
$$

Vemos que (3.3.8) coincide com (3.1.9) quando fazemos $w_{i}=w, i=1, \ldots, n$.

\section{Função de verossimilhança baseada nos dados observados}

A função de verossimilhança baseada nos dados observados $D_{\mathrm{obs}}=\left(\begin{array}{c}U_{1} \\ \vdots \\ {\underset{\sim}{n}}_{n}\end{array}\right)$, onde $\underset{\sim}{U_{i}}=\left(\begin{array}{c}Y_{i} \\ X_{i}\end{array}\right)$, é dada por

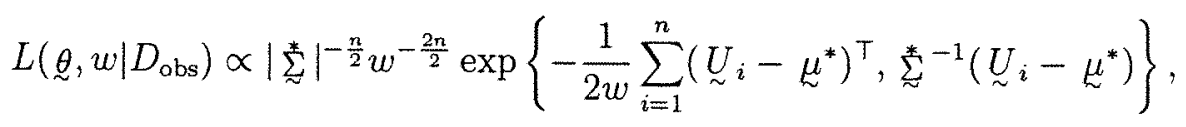

onde $\mu^{*}$ e ${ }_{z}^{*}$ são definidos em (3.1.3) (temos que (3.3.9) coincide com (3.1.10) quando $\left.w_{i}=w, i=1, \ldots, n\right)$.

\section{Distribuição à posteriori}

A posteriori $p\left(\underset{\sim}{\theta} \mid D_{\text {obs }}\right)$ é dada por

$$
\begin{aligned}
p\left(\underset{\sim}{\theta} \mid D_{\mathrm{obs}}\right) & =\int_{\mathfrak{X}=\mathbb{R}^{n}} \int_{W=\mathbb{R}_{+}} p\left(\underset{\sim}{\theta}, w, \underset{\sim}{x} \mid D_{\mathrm{obs}}\right) \mathrm{d} w \mathrm{~d} \underset{\sim}{x} \\
& \propto \int_{\mathfrak{X}=\mathbb{R}^{n}} \int_{W=\mathbb{R}_{+}} L(\underset{\sim}{\theta}, w \mid D) \pi(\underset{\sim}{\theta}, w) \mathrm{d} w \underset{\sim}{\underset{\sim}{x}},
\end{aligned}
$$

onde $L(\underset{\theta}{\theta}, w \mid D)$ é dada em $(3.3 .8)$ e $\pi(\underset{\sim}{\theta}, w)$ é a priori de $(\underset{\sim}{\theta}, w)$ dada em (3.3.6) (vemos que (3.3.10) coincide com (3.1.11) quando $w_{i}=w, i=1, \ldots, n$ ). 
Outra forma de escrever a posteriori é

$$
p\left(\underset{\sim}{\theta} \mid D_{\mathrm{obs}}\right) \propto \int_{W=\mathbb{R}_{+}} L\left(\underset{\sim}{\theta}, w \mid D_{\mathrm{obs}}\right) \pi(\underset{\sim}{\theta}, w) \mathrm{d} w
$$

onde $L\left(\underset{\theta}{\theta}, w \mid D_{\text {obs }}\right)$ é dada em (3.3.9) e $\pi(\underset{\sim}{\theta}, w)$ é dada em (3.3.6) (vemos que (3.3.11) coincide com (3.1.12) quando $\left.w_{i}=w, i=1, \ldots, n\right)$.

Observação: A posteriori de $\underset{\sim}{\theta}$ em (3.3.10), obtida a partir do modelo formulado como mistura de escala da distribuição normal coincide com a posteriori em (3.3.4), obtida sob o modelo na formulação 1 (o que implica que as duas formulações são equivalentes). A justificativa desse fato segue abaixo.

Demonstração:

$$
\begin{aligned}
& p\left(\underset{\sim}{\theta} \mid D_{\text {obs }}\right)=\int_{\mathfrak{X}=\mathbb{R}^{n}} \int_{W=\mathbb{R}_{+}} p\left(\underset{\sim}{\theta}, w, \underset{\sim}{x} \mid D_{\text {obs }}\right) \mathrm{d} w \mathrm{~d} \underset{\sim}{\underset{x}{x}} \\
& \propto \int_{\mathscr{x}} \int_{W} L(\underset{\theta}{\theta}, w \mid D) \pi(\underset{\sim}{\theta}) \pi(w) \mathrm{d} w \mathrm{~d} \underset{\sim}{x} \\
& =\int_{\mathscr{X}} \int_{W}|\Sigma|^{-\frac{n}{2}} w^{-\frac{3 n}{2}} \exp \left\{-\frac{1}{2 w} \sum_{i=1}^{n}\left(Z_{i}-\mu\right)^{\top} \Sigma^{-1}\left(\underset{\sim}{Z_{i}}-\mu\right)\right\} . \\
& \pi(\underset{\sim}{\theta}) w^{-\left(\frac{\nu}{2}+1\right)} \exp \left\{-\frac{\nu}{2 w}\right\} \mathrm{d} w \mathrm{~d} \underset{\sim}{x} \\
& =|\Sigma|^{-\frac{n}{2}} \pi(\underset{\sim}{\theta}) \int_{\circledast} \int_{W} w^{-\left(\frac{3 n}{2}+\frac{\nu}{2}+1\right)} \exp \left\{\frac{-\frac{1}{2}\left[\sum_{i=1}^{n}\left({\underset{\sim}{i}}_{i}-\mu\right)^{\top} \sum^{-1}\left({\underset{\sim}{i}}_{i}-\mu\right)+\nu\right]}{w}\right\} \mathrm{d} w \mathrm{~d} \underset{\sim}{x} \\
& =|\Sigma|^{-\frac{n}{2}} \pi(\theta) \int_{\mathfrak{X}} \frac{\Gamma\left(\frac{3 n+\nu}{2}\right)}{\frac{1}{2}\left[\sum_{i=1}^{n}\left(Z_{i}-\mu\right)^{\top} \Sigma^{-1}\left(Z_{i}-\mu\right)+\nu\right]^{\frac{3 n+\nu}{2}}} \mathrm{~d} \underset{\sim}{x} \\
& \propto \int_{X}|\Sigma|^{-\frac{n}{2}}\left[\sum_{i=1}^{n}\left({\underset{\sim}{Z}}_{i}-\mu\right)^{\top} \sum_{\sim}^{-1}\left(\underset{\sim}{Z_{i}}-\mu\right)+\nu\right]^{-\left(\frac{3 n+\nu}{2}\right)} \pi(\underset{\sim}{\theta}) \mathrm{d} \underset{\sim}{x},
\end{aligned}
$$

que coincide com (3.3.4).

\subsubsection{Implementação de algoritmos MCMC no modelo com particular priori própria de componentes independentes}

Tomando-se a distribuição à priori própria

$$
\pi(\theta, w)=\pi(\alpha) \pi(\beta) \pi(\mu) \pi\left(\sigma_{x}^{2}\right) \pi\left(\sigma^{2}\right) \pi(w)
$$


onde $\alpha \sim N\left(a, \sigma_{x}^{2}\right), \beta \sim N\left(b, \sigma_{\beta}^{2}\right), \mu \sim N\left(m, \sigma_{\mu}^{2}\right), \sigma_{x}^{2} \sim I G(c, d), \sigma^{2} \sim I G(f, g)$, $w \sim I G\left(\frac{\nu}{2}, \frac{\nu}{2}\right)$ e utilizando o modelo $t$-dependente formulado como mistura de escala da distribuição normal dado em (3.3.6) podemos implementar o algoritmo de Gibbs usando as distribuições condicionais completas baseadas na posteriori conjunta $p\left(\underset{\sim}{\theta} \underset{\sim}{x}, w \mid D_{\text {obs }}\right)$ dada em (3.3.10). Observamos entretanto que as condicionais completas à posteriori de $\alpha, \beta, \mu, \sigma_{x}^{2}$ e $\sigma^{2}$ coincidem com as do modelo $t$-independente da seção 3.1.2 quando fazemos $w_{i}=w, i=1, \ldots, n$.

A distribuição condicional completa de $w$, entretanto, é dada por

$$
\begin{aligned}
w \mid \underset{\sim}{x}, \alpha, \beta, \mu, \sigma_{x}^{2}, \sigma^{2}, D_{\mathrm{obs}} \sim & I G\left[\frac{3 n+\nu}{2} ; \frac{\nu}{2(\nu-2)}\left(\frac{\sum_{i=1}^{n}\left(Y_{i}-\alpha-\beta x_{i}\right)^{2}}{\lambda \sigma_{1}^{2}}\right.\right. \\
& \left.\left.+\frac{\sum_{i=1}^{n}\left(X_{i}-x_{i}\right)^{2}}{\sigma_{1}^{2}}+\frac{\sum_{i=1}^{n}\left(x_{i}-\mu\right)^{2}}{\sigma_{x}^{2}}+\nu\right)\right] .
\end{aligned}
$$

É importante lembrar que qualquer algoritmo utilizado para o modelo $t$-independente (na formulação 2) pode ser utilizado para o modelo $t$-dependente, bastando que $w_{i}=w$, para $i=1, \ldots, n$.

\subsubsection{Distribuição preditiva}

Seja $\underset{\sim}{U}=\left(\begin{array}{l}Y_{f} \\ X_{f}\end{array}\right)$ uma observação futura de $\left(\begin{array}{l}Y \\ X\end{array}\right)$. Então a distribuição preditiva à posteriori no modelo $t$-dependente é dada por

$$
\begin{aligned}
& \pi\left(\underset{\sim}{U_{f}} \mid D_{\mathrm{obs}}\right)=\int_{\Theta} \int_{W=\mathbb{R}_{+}} \pi\left(\underset{\sim}{U_{f}}, \underset{\sim}{\theta}, w \mid D_{\mathrm{obs}}\right) \mathrm{d} w \mathrm{~d} \underset{\sim}{\theta} \\
& =\int_{\theta} \int_{W=\mathbb{R}_{+}} \pi\left({\underset{\sim}{U} f}_{f} \mid \underline{\sim}, w\right) \pi\left(\underset{\sim}{\theta}, w \mid D_{\mathrm{obs}}\right) \mathrm{d} w \mathrm{~d} \theta \\
& =\int_{\Theta} \int_{W=\mathbb{R}_{+}} \pi\left(Y_{f} \mid X_{f}, \underset{\sim}{\theta}, w\right) \pi\left(X_{f} \mid \underset{\sim}{\theta}, w\right) \pi\left(\underset{\sim}{\theta}, w \mid D_{\mathrm{obs}}\right) \mathrm{d} w \mathrm{~d} \underset{\sim}{\theta},
\end{aligned}
$$

onde $\pi\left(\underset{\theta}{\theta}, w \mid D_{\text {obs }}\right)$ é a distribuição à posteriori de $\underset{\sim}{\theta}$ e $w$,

$$
\begin{gathered}
X_{f} \mid \theta, w \sim N\left(\mu ;\left(\sigma_{x}^{2}+\sigma^{2}\right) w\right) \\
\mathrm{e} Y_{f} \mid X_{f}, \underset{\theta}{\theta}, w \sim N\left(\alpha+\beta \mu+\frac{\beta \sigma_{x}^{2}}{\sigma_{x}^{2}+\sigma^{2}}\left(X_{f}-\mu\right) ;\left[\beta^{2} \sigma_{x}^{2}+\lambda \sigma^{2}-\frac{\beta^{2}\left(\sigma_{x}^{2}\right)^{2}}{\sigma_{x}^{2}+\sigma^{2}}\right] w\right) .
\end{gathered}
$$


Para gerar uma observação $\underset{\sim}{U_{f}}$ basta gerar $(\underset{\underset{ }{\theta}}{\theta}, w)$ da posteriori $\pi\left(\underset{\sim}{\theta}, w \mid D_{\text {obs }}\right)$, depois gerar $X_{f}$ de $\pi\left(X_{f} \mid \underset{\theta}{\theta}, w\right)$ e finalmente $Y_{f}$ de $\pi\left(Y_{f} \mid X_{f}, \underset{\theta}{\theta}, w\right)$.

Observação: A posteriori preditiva pode ser escrita também de outro modo:

$$
\begin{aligned}
& \pi\left(\underset{\sim}{U_{f}} \mid D_{\mathrm{obs}}\right)=\int_{\mathfrak{X}_{f}} \int_{\Theta} \int_{W=\mathbb{R}_{+}} \pi\left(\underset{\sim}{U_{f}}, \underset{\sim}{\theta}, w, x_{f} \mid D_{\mathrm{obs}}\right) \mathrm{d} w \mathrm{~d} \underset{\sim}{\theta} \mathrm{d} x_{f} \\
& =\int_{x_{f}} \int_{W} \int_{\Theta} \pi\left(U_{f} \mid \theta, w, x_{f}\right) \pi\left(x_{f} \mid \theta, w\right) \pi\left(\theta, w \mid D_{\text {obs }}\right) \mathrm{d} \theta \underset{\sim}{ } \mathrm{d} w \mathrm{~d} x_{f} \\
& =\int_{\mathfrak{x}_{f}} \int_{W} \int_{\Theta} \pi\left(Y_{f} \mid X_{f}, \underset{\sim}{\theta}, w, x_{f}\right) \pi\left(X_{f} \mid \underset{\sim}{\theta}, x_{f}, w\right) \pi\left(x_{f} \mid \underset{\sim}{\theta}, w\right) \pi\left(\underset{\sim}{\theta}, w \mid D_{\mathrm{obs}}\right) \mathrm{d} \underset{\theta}{\theta} \mathrm{d} w \mathrm{~d} x_{f}, \\
& \text { onde } \quad x_{f}\left|\underset{\sim}{\theta}, w \sim N\left(\mu, \sigma_{x}^{2} w\right), \quad X_{f}\right| x_{f}, \underset{\sim}{\theta}, w \sim N\left(x_{f}, \sigma_{u}^{2} w\right) \\
& Y_{f} \mid X_{f}, x_{f}, \underset{\sim}{\theta}, w \sim N\left(\alpha+\beta x_{f}, \sigma_{e}^{2} w\right) \text {. }
\end{aligned}
$$

\subsubsection{Avaliação do ajuste}

A avaliação da qualidade do ajuste no modelo t-dependente, definido por (3.3.1) ou por (3.3.6), pode ser feita de forma semelhante à do modelo $t$-independente descrita na seção 3.1.4, fazendo-se as devidas adaptações nas fórmulas de $\mathrm{CPO}_{i}$, $E\left(\underset{\sim}{U_{i}} \mid D_{\mathrm{obs}(-i)}\right)$ e $\operatorname{Var}\left(\underset{\sim}{U_{i}} \mid D_{\mathrm{obs}(-i)}\right)$.

\subsection{O modelo estrutural $t$-dependente com graus de liberdade desconhecidos}

Nessa seção definimos o modelo $t$-dependente com erros nas variáveis e número de graus de liberdade desconhecido, formulado como mistura de escala da distribuição Normal.

Considere as equações

$$
\left\{\begin{array}{l}
Y_{i}=\alpha+\beta x_{i}+e_{i}, \\
X_{i}=x_{i}+u_{i},
\end{array}\right.
$$

e seja $\underset{\sim}{Z}=\left(\begin{array}{c}Z_{1} \\ \vdots \\ Z_{n}\end{array}\right)$, onde $Z_{i}=\left(\begin{array}{c}Y_{i} \\ X_{i} \\ x_{i}\end{array}\right)$ com a suposição de que

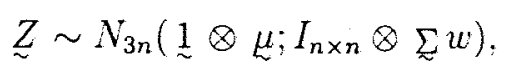


onde $\mu$ e $\sum_{\sim}$ são dadas em (3.2.1), com priori $\pi(\theta, w, \nu)$ dada por

$$
\pi(\theta, w, \nu)=\pi(\theta) \pi(w \mid \nu) \pi(\nu), \text { onde } w \mid \nu \sim I G\left(\frac{\nu}{2}, \frac{\nu}{2}\right)
$$

Esta suposição é equivalente a $\underset{\sim}{Z} \underset{i}{\stackrel{\text { iid }}{\sim}} N_{3}\left(\underset{\sim}{\mu}, \sum_{\sim} w\right)$, com priori $\pi(\underset{\sim}{\theta}, w, \nu)=$ $\pi(\underset{\sim}{\theta}) \pi(w \mid \nu) \pi(\nu)$, onde $w \mid \nu \sim I G\left(\frac{\nu}{2}, \frac{\nu}{2}\right)$.

Vemos que no modelo $t$-dependente temos um único $w$ onde $w \mid \nu \sim I G\left(\frac{\nu}{2}, \frac{\nu}{2}\right)$ (enquanto que no modelo $t$-independente $\left.w_{i} \mid \nu \stackrel{\text { iid }}{\sim} I G\left(\frac{\nu}{2}, \frac{\nu}{2}\right), i=1, \ldots, n\right)$ e portanto a análise do modelo e implementação dos algoritmos pode ser feita de forma semelhante à descrita na seção 3.2 .

\subsection{Condições para a existência da posteriori no modelo estrutural $t$-Student com erros depen- dentes e número de graus de liberdade conhe- cido}

Inicialmente apresentamos um resultado que será útil para demonstrar os teoremas.

Lema 3.5.1 Sejam a e p constantes positivas. Então a condição necessária e suficiente para que a integral $\int_{0}^{\infty} \frac{e^{-a / x}}{x^{p}} \mathrm{~d} x$ seja finita é que $p>1$.

Demonstração: Se $0<p \leq 1$, então

$$
\int_{0}^{\infty} \frac{e^{-a / x}}{x^{p}} \mathrm{~d} x>\int_{1}^{\infty} \frac{e^{-a / x}}{x^{p}} \mathrm{~d} x>\int_{1}^{\infty} \frac{e^{-a}}{x^{p}} \mathrm{~d} x \propto \int_{1}^{\infty} \frac{1}{x^{p}} \mathrm{~d} x=\infty
$$

e portanto, $\int_{0}^{\infty} \frac{e^{-a / x}}{x^{p}} \mathrm{~d} x$ diverge se $0<p \leq 1$.

Se $p>1$, usamos a transformação $t=\frac{a}{x}$ e portanto a integral

$$
\int_{0}^{\infty} \frac{e^{-a / x}}{x^{p}} \mathrm{~d} x=a^{1-p} \int_{0}^{\infty} e^{-t} t^{p-2} \mathrm{~d} t=a^{1-p} \int_{0}^{\infty} e^{-t} t^{(p-1)-1} \mathrm{~d} t
$$

Fazendo $r=p-1$, então para todo $p>1$ (isto é, para todo $r>0$ ), temos que

$$
\int_{0}^{\infty} \frac{e^{-a / x}}{x^{p}} \mathrm{~d} x=a^{-r} \int_{0}^{\infty} e^{-t} t^{r-1} \mathrm{~d} t=a^{-r} \Gamma(r),
$$


onde $\Gamma(r)$ é a função Gama com parâmetro $r$. Portanto,

$$
\begin{aligned}
\int_{0}^{\infty} \frac{e^{-a / x}}{x^{p}} \mathrm{~d} x & =a^{-r}(r-1) \Gamma(r-1)=a^{-r}(r-1)(r-2) \Gamma(r-2)=\cdots= \\
& =a^{-r}(r-1)(r-2) \cdots(r-k) \int_{0}^{\infty} e^{-t} t^{(r-k)-1} \mathrm{~d} t
\end{aligned}
$$

onde $k$ é o maior inteiro menor que $r$ e portanto $0<r-k<1$. Além disso,

$$
\begin{aligned}
\Gamma(r-k) & =\int_{0}^{\infty} e^{-t} t^{(r-k)-1} \mathrm{~d} t=\int_{0}^{\infty} \frac{e^{-t}}{t^{1-(r-k)}} \mathrm{d} t=\int_{0}^{1} \frac{e^{-t}}{t^{1-(r-k)}} \mathrm{d} t+\int_{1}^{\infty} \frac{e^{-t}}{t^{1-(r-k)}} \mathrm{d} t \\
& \leq \int_{0}^{1} \frac{1}{t^{1-(r-k)}} \mathrm{d} t+\int_{1}^{\infty} e^{-t} \mathrm{~d} t=\frac{1}{r-k}+e^{-1}<\infty .
\end{aligned}
$$

Portanto, para todo $r>0$, isto é, para todo $p>1, \int_{0}^{\infty} \frac{e^{-a / x}}{x^{p}} \mathrm{~d} x<\infty$.

Concluímos então que $\int_{0}^{\infty} \frac{e^{-a / x}}{x^{p}} \mathrm{~d} x$ é finita se e somente se $p>1$.

\section{Teoremas}

Teorema 3.5.2 No modelo t-Student com erros dependentes descrito na seção 3.4, com priori $\pi(\underset{\sim}{\theta}, w)=\pi(w) . \pi(\underset{\sim}{\theta})$, onde $\pi(w) \sim I G\left(\frac{\nu}{2}, \frac{u}{2}\right)$ e $\pi(\underset{\sim}{\theta}) \propto \pi\left(\sigma_{x}^{2}\right) . \pi\left(\sigma^{2}\right)$, onde $\pi\left(\sigma_{x}^{2}\right) \sim I G(c, d), \pi\left(\sigma^{2}\right) \sim I G(f, g)$, então uma condição suficiente para a existência da posteriori é que $n \geq 3$ e $0<c<n+\frac{(\nu-3)}{2}$ (outra condição suficiente é que $n \geq 3$ $\left.e 0<f<\frac{n+\nu-1}{2}\right)$.

Demonstração: O objetivo é mostrar que a integral $\int_{W} \int_{\Theta} p\left(\underset{\underset{\theta}{\theta}}{\theta}, w \mid D_{\text {obs }}\right) \mathrm{d} \underset{\sim}{\theta} \mathrm{d} w$ é finita, onde $\int_{W} \int_{\Theta} p\left(\underset{\sim}{\theta}, w \mid D_{\text {obs }}\right) \mathrm{d} \underset{\sim}{\theta} \mathrm{d} w=\int_{W} \int_{x} \int_{\theta} p\left(\underset{\sim}{\theta}, w, \underset{\sim}{x} \mid D_{\text {obs }}\right) \mathrm{d} \underset{\sim}{\theta} \mathrm{d} \underset{\sim}{x} \mathrm{~d} w, \quad$ onde $p\left(\underset{\sim}{\theta}, w, \underset{\sim}{x} \mid D_{\text {obs }}\right) \propto L(\underset{\theta}{\theta}, w \mid D) \pi(\underset{\sim}{\theta}, w)$, que é proporcional a

$$
\begin{aligned}
& w^{-\left(\frac{3 n+\nu}{2}+1\right)}\left(\sigma_{x}^{2}\right)^{-\left(\frac{n}{2}+c+1\right)}\left(\sigma^{2}\right)^{-(n+f+1)} \cdot \exp \left\{-\frac{1}{2}\left[\frac{\nu}{w}+\frac{2 d}{\sigma_{x}^{2}}+\frac{2 g}{\sigma^{2}}+\frac{\sum_{i=1}^{n}\left(Y_{i}-\alpha-\beta x_{i}\right)^{2}}{\lambda \sigma^{2} w}\right.\right. \\
& \left.\left.+\frac{\sum_{i=1}^{n}\left(X_{i}-x_{i}\right)^{2}}{\sigma^{2} w}+\frac{\sum_{i=1}^{n}\left(x_{i}-\mu\right)^{2}}{\sigma_{x}^{2} w}\right]\right\} .
\end{aligned}
$$

Primeiramente, fazemos a integração em relação a $\Theta$, que é bastante semelhante à do modelo Normal da seção anterior, e obtemos

$$
\int_{\Theta} p\left(\underline{\theta}, w, \underset{\sim}{x} \mid D_{\mathrm{obs}}\right) \mathrm{d} \theta
$$




$$
\propto \frac{w^{-\left(\frac{3 n+\nu-1}{2}\right)} \exp \left\{-\frac{\nu}{2 w}\right\}}{S_{x x}^{\frac{1}{2}}\left[2 d+\frac{S_{x x}}{w}\right]^{\frac{n+2 c-1}{2}}\left[\frac{\sum_{i=1}^{n}\left(X_{i}-x_{i}\right)^{2}}{w}+\frac{\left(S_{Y Y} S_{x x}-S_{x Y}^{2}\right)}{\lambda S_{x x} w}+2 g\right]^{n+f-1}} .
$$

Portanto,

$$
\begin{aligned}
& \int_{\mathscr{X}} \int_{\Theta} p\left(\underset{\sim}{\theta}, w, \underset{\sim}{x} \mid D_{\mathrm{obs}}\right) \mathrm{d} \underset{\sim}{\underset{\sim}{x} \underset{\sim}{x} \propto} \\
& \int_{\mathscr{X}} \frac{w^{-\frac{(3 n+\nu-1)}{2}} \exp \left\{-\frac{\nu}{2 w}\right\}}{S_{x x}^{\frac{1}{2}}\left[2 d+\frac{S_{x x}}{w}\right]^{\frac{n+2 c-1}{2}}\left[\frac{\sum_{i=1}^{n}\left(X_{i}-x_{i}\right)^{2}}{w}+\frac{\left(S_{Y Y} S_{x x}-S_{\tau Y}^{2}\right)}{\lambda S_{x x} w}+2 g\right]^{n+f-1}} \mathrm{~d} \underset{\sim}{x} .
\end{aligned}
$$

Fazendo a transformação que a cada $(\underset{\sim}{x}, \underset{\sim}{X} \underset{\sim}{Y})$ associa $\left(\underset{\sim}{z}{ }_{n \times 1}, \underset{\sim}{b}{ }_{n \times 1},{\underset{\sim}{n \times 1}}_{n \times 1}\right)=$ $\left(H_{n \times n} \underset{\sim}{x}, H_{n \times n} \underset{\sim}{X}, H_{n \times n} \underset{\sim}{Y}\right)$, onde $H$ é a matriz de Helmert (Searle, 1971) e definindo $\underset{\sim}{z}, \underset{\sim}{b} X, c_{\sim}$ como sendo os vetores formados pelas primeiras $n-1$ componentes de $\underset{\sim}{z} \underset{\sim}{b}$ e $\underset{\sim}{c}$, respectivamente, então a integral em (3.5.2) torna-se igual à integral

$$
\int_{\mathcal{Z}=\left\{z_{\sim} \in \mathbb{R}^{n}\right\}} \frac{w^{-\frac{(3 n+\nu-1)}{2}} \exp \left\{-\frac{\nu}{2 w}\right\}}{\left\|z_{x}\right\|\left[\| \frac{\left\|z_{x}\right\|^{2}}{w}+2 d\right]^{\frac{n+2 c-1}{2}}\left[\frac{\left\|z_{x}-b_{x}\right\|^{2}}{w}+\frac{\left\|\mathcal{C}_{Y}\right\|^{2} \operatorname{sen}^{2} \theta}{\lambda w}+\frac{\left(\sqrt{n} \bar{X}-z_{n}\right)^{2}}{w}+2 g\right]^{n+f-1}} \mathrm{~d} z,
$$

onde $z=\left(z_{1}, \ldots, z_{n}\right)^{T}$.

Para eliminar a variável $z_{n}$, fazemos o cálculo da integral apenas em relação a $z_{n}$ de forma semelhante à apresentada da demonstração do Teorema 2.8.2. Portanto, se a desigualdade $n+f>\frac{3}{2}$ é válida, então a integral em (3.5.3) torna-se proporcional a

$$
\int_{\mathcal{Z}_{x}} \frac{w^{-\left(\frac{3 n+\nu}{2}-1\right)} \exp \left\{-\frac{\nu}{2 w}\right\}}{\left\|z_{x}\right\|\left[\frac{\left\|z_{x}\right\|^{2}}{w}+2 d\right]^{\frac{n+2 c-1}{2}}\left[\frac{\left\|z_{x}-b_{x}\right\|^{2}}{w}+\frac{\|\mathcal{C} Y\|^{2} \operatorname{sen}^{2} \theta}{\lambda w}+2 g\right]^{n+f-\frac{3}{2}}} \mathrm{~d} z_{\sim},
$$

onde $\mathcal{Z}_{x}=\left\{z_{x}=\left(z_{1}, \ldots, z_{n-1}\right)^{T} \in \mathbb{R}^{n-1}\right\}$,

A função dentro do sinal de integral é

$$
f\left({\underset{\sim}{x}}_{x}, w\right)=\frac{w^{-\left(\frac{3 \pi+\nu}{2}-1\right)} \exp \left\{-\frac{\nu}{2 w}\right\}}{\left\|z_{x}\right\|\left[\frac{\left\|z_{x}\right\|^{2}}{w}+2 d\right]^{\frac{n+2 c-1}{2}}\left[\frac{\left\|z_{x}-b x\right\|^{2}}{w}+\frac{\left\|\mathcal{C}_{Y}\right\|^{2} \operatorname{sen}^{2} \theta}{\lambda w}+2 g\right]^{n+f-\frac{3}{2}}}
$$

que é igual a

$$
\frac{\exp \left\{\frac{-\nu}{2 w}\right\}}{w^{\frac{\nu}{2}+1-c-f}\left\|\underset{\sim}{z_{x}}\right\|\left[2 d w+\|\underset{\sim}{\underset{x}{z}}\|^{2}\right]^{\frac{n+2 c-1}{2}}\left[2 g w+\| \underset{\sim}{\left.z_{x}-{\underset{\sim}{b}}_{X} \|^{2}+\frac{\left\|\mathcal{C}_{\gamma}\right\|^{2} \operatorname{sen}^{2} \theta}{\lambda}\right]^{n+f-\frac{3}{2}}} .\right.}
$$




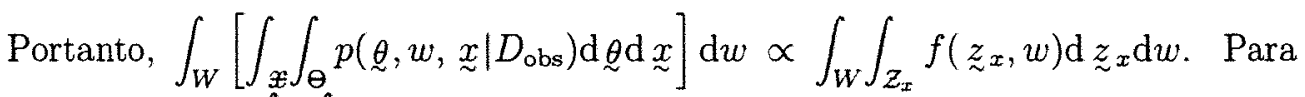
analisar a integral $\int_{W} \int_{Z_{x}} f\left({\underset{\sim}{x}}_{x}, w\right) \mathrm{d} z_{\sim} \mathrm{d} w$, vamos particionar a região de integração $W \times \mathcal{Z}_{x}$ em dois conjuntos, $W \times R_{1}=\mathbb{R}^{+} \times\left\{z_{x} \in \mathbb{R}^{n-1}:\left\|z_{x}\right\|<\varepsilon\right\}$ e $W \times R_{2}=$ $\mathbb{R}^{+} \times\left\{\underset{\sim}{z} \in \mathbb{R}^{n-1}:\left\|{\underset{\sim}{x}}_{x}\right\| \geq \varepsilon \|\right.$, onde $\varepsilon$ é fixado arbitrariamente tão pequeno quanto se queira.

\section{AVALIAÇÃO DA INTEGRAL NA REGIÃO $W \times R_{1}$}

Nessa região a integral

$$
\int_{W} \int_{R_{1}} f(\underset{\sim}{z} x, w) \mathrm{d}{\underset{\sim}{x}}_{x} \mathrm{~d} w=\int_{\mathbb{R}^{+}} \int_{\left\{\sim_{x} \in \mathbb{R}^{n-1}:\left\|z_{x}\right\|<\varepsilon\right\}} f\left(\underset{\sim}{z_{x}}, w\right) \mathrm{d}{\underset{\sim}{z} x}_{x} \mathrm{~d} w
$$

pode ser majorada pela seguinte integral

$$
\begin{aligned}
& \int_{W} \int_{\left\|z_{\sim} x\right\|<\varepsilon} \frac{\exp \left\{-\frac{\nu}{2 w}\right\}}{w^{\frac{\nu}{2}+1-c-f}\left\|z_{\sim}\right\|[2 d w+0]^{\frac{n+2 c-1}{2}}[2 g w+0]^{n+f-\frac{3}{2}}} \mathrm{~d} \underset{\sim}{z}{ }_{x} \mathrm{~d} w \\
& \propto \int_{W} \frac{\exp \left\{-\frac{\nu}{2 w}\right\}}{w^{\frac{3 n+\nu}{2}-1}}\left[\int_{\left\|z_{\sim} x\right\|<\varepsilon\left\|z_{\sim}\right\|} \frac{1}{2} z_{\sim}\right] \mathrm{d} w=\int_{W} \frac{\exp \left\{-\frac{\nu}{2 w}\right\}}{w^{\frac{3 n+\nu}{2}-1}} \mathrm{~d} w \int_{\left\|z_{x}\right\|<\varepsilon} \frac{1}{\left\|z_{\sim} x\right\|} \mathrm{d} z_{\sim} x
\end{aligned}
$$

onde pelo Lema 3.5.1, a integral $\int_{0}^{\infty} \frac{\exp \left\{-\frac{\nu}{2 w}\right\}}{w^{\frac{3 n+\nu}{2}-1}} \mathrm{~d} w$ é finita se e somente se $\frac{3 n+\nu}{2}-1>$ 1 (ou seja, $3 n+\nu>4$ ) e, pelo Lema 2.8.1, a integral $\int_{\left\|z_{x}\right\|<\varepsilon} \frac{1}{\left\|z_{x}\right\|} \mathrm{d} z_{x}$ é finita se é somente se $1<n-1$.

Portanto, na região $W \times R_{1}$ uma condição suficiente para que a integral convirja é que $n \geq 3$ e $3 n+\nu>4$, ou seja, simplesmente $n \geq 3$.

\section{AVALIAÇÃO DA INTEGRAL NA REGIÃO $W \times R_{2}$}

Nessa região, a integral $\int_{W} \int_{R_{2}} f\left({\underset{\sim}{x}}_{x}, w\right) \mathrm{d} \underset{\sim}{z} \mathrm{~d} w=\int_{W=\mathbb{R}^{+}} \int_{\left\|z_{x}\right\| \geq \varepsilon} f(\underset{\sim}{z}, w) \mathrm{d} \underset{\sim}{z} x \mathrm{~d} w$, pode ser majorada pela seguinte integral:

$$
\begin{aligned}
& \int_{W} \int_{\left\|z_{x}\right\| \geq \varepsilon} \frac{\exp \left\{-\frac{\nu}{2 w}\right\}}{w^{\frac{\nu}{2}+1-c-f}\left\|z_{x}\right\|\left[0+\left\|z_{x}\right\|^{2}\right]^{\frac{n+2 r-1}{2}}[2 g w+0]^{n+f-\frac{3}{2}}} \mathrm{~d} z_{\sim} \mathrm{d} w \\
& =\int_{W} \frac{\exp \left\{-\frac{\nu}{2 w}\right\}}{w^{\frac{\nu}{2}+1-c-f}[2 g w+0]^{n+f-\frac{3}{2}}} \mathrm{~d} w \cdot \int_{\left\|z_{\sim}\right\| \geq \Xi} \frac{1}{\left\|z_{\sim}\right\|\left[0+\left\|z_{\sim}\right\|^{2}\right]^{\frac{n+2 c-1}{2}}} \mathrm{~d} z_{x},
\end{aligned}
$$


que é proporcional a

$$
\int_{W} \frac{\exp \left\{-\frac{\nu}{2 w}\right\}}{w^{\frac{2 n+\nu-2 c-1}{2}}} \mathrm{~d} w \cdot \int_{\|{\underset{\sim}{z} \| \geq \varepsilon}_{\|}} \frac{1}{\left\|z_{x}\right\|^{n+2 c}} \mathrm{~d}{\underset{\sim}{z}}_{x} .
$$

Pelo Lema 3.5.1, a integral em $w$ é finita se e somente se $\frac{2 n+\nu-2 c-1}{2}>1$ (isto é, $2 n+\nu>2 c+3$ ). E pelo Lema 2.8.1 a integral em $\underset{\sim}{z}$ é finita se e somente se $n+2 c>n-1$ (ou seja, sempre).

Portanto, na região $W \times R_{2}$ uma condição suficiente para haver convergência da integral $\int_{W} \int_{R_{2}} f(\underset{\sim}{z}, w) \mathrm{d} \underset{\sim}{z} \mathrm{~d} w$ é que $2 n+\nu>2 c+3$.

Conclusão: Analisando conjuntamente todas as informações, concluímos que uma condição suficiente para a existência da distribuição a posteriori é que $n \geq 3$ e $0<c<n+\frac{\nu-3}{2}$.

Observação: Uma outra condição suficiente igualmente interessante é obtida quando na região $W \times R_{2}$ a integral $\int_{W=\mathbb{R}} \int_{R_{2}} f(\underset{\sim}{z}, w) \mathrm{d} \underset{\sim}{z} \mathrm{~d} w$ é majorada pela seguinte integral:

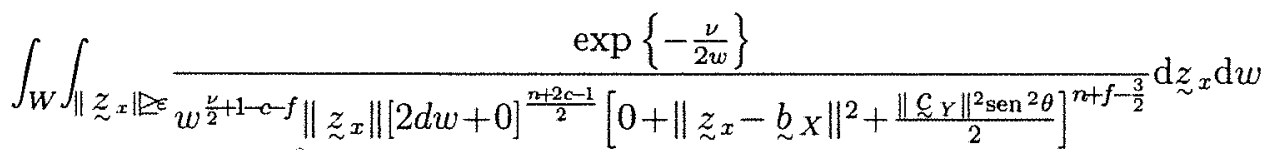

$$
\begin{aligned}
& \propto \int_{0}^{\infty} \frac{\exp \left\{-\frac{\nu}{2 w}\right\}}{w^{\frac{n+\nu-2 f+1}{2}}} \mathrm{~d} w \cdot \int_{\left\|z_{x}\right\| \geq \varepsilon} \frac{1}{\left\|z_{x}\right\|\left[\left\|z_{x}-{\underset{\sim}{b}}_{X}\right\|^{2}+\frac{\left\|\mathcal{C r}_{Y}\right\|^{2} \operatorname{sen}^{2} \theta}{\lambda}\right]^{n+f-\frac{3}{2}}} \mathrm{~d}{\underset{\sim}{x}}_{x} .
\end{aligned}
$$

Pelo Lema 3.5.1, a integral em $w$ é finita se e somente se $\frac{n+\nu-2 f+1}{2}>1$ (isto é, $n+\nu>2 f+1$ ). A integral em $\underset{\sim}{z}$ (que é semelhante à integral em $R_{3}$ na demonstração do Teorema 2.8.5) converge se e somente se $1+2\left(n+f-\frac{3}{2}\right)>n-1$, isto é, $n+2 f>1$ e portanto vale sempre. Assim, a integral $\int_{W} \int_{R_{2}} f(\underset{\sim}{z}, w) \mathrm{d} \underset{\sim}{z} \mathrm{~d} w<\infty$ se $n+\nu>2 f+1$.

Portanto, uma outra condição suficiente para que a posteriori seja própria é que $n \geq 3, n+\nu>2 f+1$ e $n+f>\frac{3}{2}$ (ou seja, $n \geq 3$ e $0<f<\frac{n+\nu-1}{2}$ ). 
Teorema 3.5.3 No modelo do Teorema 3.5.2, se substituirmos a distribuição $\pi\left(\sigma_{x}^{2}\right)$ por $\pi\left(\sigma_{x}^{2}\right) \propto\left(\sigma_{x}^{2}\right)^{-r}, r>0$, então uma condição suficiente para a existência da posteriori correspondente é que $n \geq 2,0<r<\frac{1}{2}$ e $0<f<\frac{\nu}{2}+1-r$.

Demonstração: Como a distribuição à priori neste teorema é igual à priori no Teorema 3.5.2 quando substituímos os hiperparâmetros $c$ e $d$, respectivamente, por $(r-1)$ e zero, então os cálculos feitos na demonstração do Teorema 3.5.2 podem ser reaproveitados. De fato,

$$
\begin{aligned}
& \int_{W} \int_{\Theta} p\left(\underset{\theta}{\theta}, w \mid D_{\mathrm{obs}}\right) \mathrm{d} \theta \underset{\sim}{\mathrm{d} w} \propto \int_{W} \int_{\mathcal{Z}_{x}=\left\{z_{\sim} \in \mathbb{R}^{n-1}\right\}} g\left(\underset{\sim}{z_{x}}, w\right) \mathrm{d}{\underset{\sim}{z}}_{x} \mathrm{~d} w \text {, onde } \\
& g(\underset{\sim}{z}, w)=\frac{\exp \left\{-\frac{\nu}{2 w}\right\}}{w^{\frac{\nu}{2}-r-f+2}\left\|{\underset{\sim}{z} x}_{x}\right\|^{n+2 r-2}\left[\left\|\underset{\sim}{z_{x}}-\underset{\sim}{b}\right\|^{2}+\frac{\|\underset{\sim}{\underline{c}}\|^{2} \operatorname{sen}^{2} \theta}{\lambda}+2 g w\right]^{n+f-\frac{3}{2}}}
\end{aligned}
$$

e $g(\underset{\sim}{z}, w)$ coincide com a função $f(\underset{\sim}{z}, w)$ dada em (3.5.4) quando substituímos $c$ por $r-1$ e $d$ por zero (desde que a desigualdade $n+f>\frac{3}{2}$ seja válida).

Do mesmo modo que no Teorema 3.5.2, a região de integração $W \times \mathcal{Z}_{x}$ é particionada em duas regiões: $W \times R_{1}$ e $W \times R_{2}$, onde

$$
W \times R_{1}=\mathbb{R}^{+} \times\left\{\underset{\sim}{z_{x}} \in \mathbb{R}^{n-1}:\left\|z_{x}\right\|<\varepsilon\right\} \quad \text { e } W \times R_{2}=W \times R_{1}^{c} .
$$

AVALIAÇÃO DA INTEGRAL NA REGIÃO $W \times R_{1}$

$$
\int_{W \times R_{1}} g\left({\underset{\sim}{x}}_{x}, w\right) \mathrm{d} z_{x} \mathrm{~d} w=\int_{W} \int_{\left\|z_{\sim}\right\|<\varepsilon} g\left({\underset{\sim}{z} x}_{x}, w\right) \mathrm{d}{\underset{\sim}{x}}_{x} \mathrm{~d} w
$$

que pode ser majorada pela integral

$$
\int_{W} \int_{\left\|z_{\sim}\right\|<\varepsilon} \frac{\exp \left\{-\frac{\nu}{2 w}\right\}}{w^{\frac{\nu}{2}-r+2-f}\left\|z_{\sim}\right\|^{n+2 r-2}[0+0+2 g w]^{n+f-\frac{3}{2}}} \mathrm{~d} z_{\sim} \mathrm{d} w
$$

que é proporcional a

$$
\int_{0}^{\infty} \frac{\exp \left\{-\frac{\nu}{2 w}\right\}}{w^{\frac{2 n-\nu-2 r+1}{2}}} \mathrm{~d} w \int_{\left\|z_{x}\right\|<\varepsilon} \frac{1}{\left\|z_{\sim}\right\|^{n+2 r-2}} \mathrm{~d} z_{\sim} x
$$


onde a integral em $\underset{\sim}{z}$ é finita se e somente se $n+2 r-2<n-1$ (isto é, $0<r<\frac{1}{2}$ ). Além disso, pelo Lema 3.5.1 a integral em $w$ é finita se e somente se $\frac{2 n+\nu-2 r+1}{2}>1$ (ou seja, $2 n+\nu>2 r+1$ ). Mas, como esta última condição está satisfeita sempre que $0<r<\frac{1}{2}$, concluímos que uma condição suficiente para que a integral na região $W \times R_{1}$ convirja é que $0<r<\frac{1}{2}$.

\section{AVALIAÇÃO DA INTEGRAL NA REGIÃO $W \times R_{2}$}

Nessa região, a integral $\int_{W} \int_{R_{2}} g(\underset{\sim}{z}, w) \mathrm{d} \underset{\sim}{z} \mathrm{~d} w$ pode ser majorada por

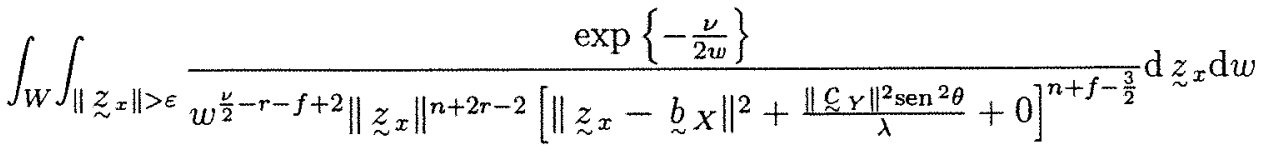

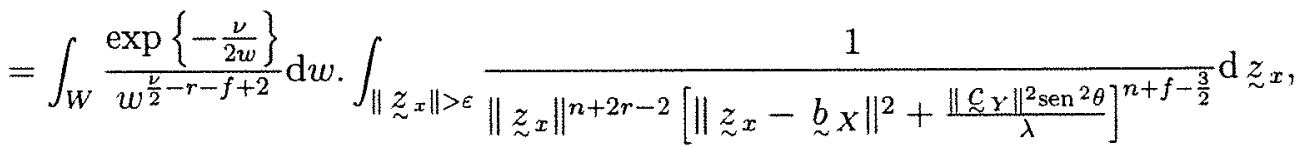

onde a integral em $w$ converge se e somente se $\frac{\nu}{2}-r-f+2>1$ (isto é, $f<\frac{\nu}{2}+1-r$ ) e a integral em $\underset{\sim}{z}$ converge se e somente se $n+2 r-2+2\left(n+f-\frac{3}{2}\right)>n-1$ (isto é, $n+r+f>2$ ). Entretanto, pelo fato que $n$ deve ser maior ou igual a 2 (ver observação abaixo), a condição $n+r+f>2$ é sempre verdadeira e portanto nessa região, uma condição suficiente para que a integral $\int_{W} \int_{R_{2}} g(\underset{\sim}{z}, w) \mathrm{d} \underset{\sim}{z_{x}} \mathrm{~d} w$ seja finita é que $f<\frac{\nu}{2}+1-r$ e $n \geq 2$.

Analisando simultaneamente essas informações, concluímos então que uma condição suficiente para que exista a posteriori nesse teorema é que $0<r<\frac{1}{2}, 0<f<\frac{\nu}{2}+1-r$ e $n \geq 2$.

Observação: Quando $n=1$, a distribuição a posteriori nesse modelo não está bem definida.

De fato, substituindo na integral em (3.5.2) os valores $d=0$ e $c=r-1$ e fazendo $S_{x x}=S_{x_{1} x_{1}}, S_{x Y}=S_{x_{1} Y_{1}}$ e $S_{Y Y}=S_{Y_{1} Y_{1}}$, então a integral $\int_{\mathscr{X}=\mathbb{R} \int_{\Theta}} p\left(\underset{\sim}{\theta}, w, x_{1} \mid D_{\text {obs }}\right) \mathrm{d} \underset{\sim}{\theta} \mathrm{d} x_{1}$ 
é proporcional a

$$
\int_{\mathscr{X}=\mathbb{R}} \frac{\left\{-\frac{\nu}{2 w}\right\}}{w^{\frac{\nu+2}{2}} S_{x_{1} x_{1}}^{\frac{1}{2}}\left[\frac{S_{x_{1} x_{1}}}{w}\right]^{r-1}\left[\frac{\left(X_{1}-x_{1}\right)^{2}}{w}+\frac{S_{x_{1} x_{1}} S_{Y_{1} Y_{1}}-S_{x_{1} Y_{1}}^{2}}{\lambda S_{x_{1} x_{1}} w}+2 g\right]^{f}} \mathrm{~d} x_{1} .
$$

Portanto, $\int_{W=\mathbb{R}^{+}} \int_{\mathfrak{X}=\mathbb{R}^{+}} \int_{\Theta} p\left(\underset{\sim}{\theta}, w, x_{1} \mid D_{\text {obs }}\right) \mathrm{d} \underset{\underset{ }{2}}{ } \mathrm{~d} x_{1} \mathrm{~d} w$ é proporcional a

$$
\int_{W=\mathbb{R}^{+}} \int_{\mathfrak{X}=\mathbb{R}} \frac{\exp \left\{-\frac{\nu}{2 w}\right\} S_{x_{1} x_{1}}^{f-r+\frac{1}{2}}}{w^{\frac{\nu}{2}-r-f+2}\left[S_{x_{1} x_{1}}\left(X_{1}-x_{1}\right)^{2}+\frac{\left(S_{x_{1} x_{1}} S_{\left.Y_{1} Y_{1}-S_{x_{1} Y_{1}}^{2}\right)}\right.}{\lambda}+2 g w\right]^{f}} \mathrm{~d} x_{1} \mathrm{~d} w .
$$

Como $S_{x_{1} x_{1}}$ e $S_{x_{1} Y_{1}}$ são nulos, $\forall x_{1} \in \mathbb{R}$, esta integral está bem definida e é finita se e somente se $f=0$ e $r<\frac{1}{2}$ (e nesse caso ela é nula), mas por hipótese $f$ é positivo e portanto a posteriori não está bem definida para $n=1$.

Teorema 3.5.4 No modelo do Teorema 3.5.2, se substituirmos a distribuição $\pi\left(\sigma^{2}\right)$ por $\pi\left(\sigma^{2}\right) \propto\left(\sigma^{2}\right)^{-s}, s>0$, e se os vetores de dados $\underset{\sim}{X} e \underset{\sim}{Y}$ são linearmente independentes e as componentes de $\underset{\sim}{X}$ (como as de $\underset{\sim}{Y}$ ) não são todas iguais então uma condição suficiente para a existência da posteriori é que $n+\nu>2 s-1$ e $n \geq 3$ :

Demonstração: A demonstração desse teorema é semelhante à do Teorema 3.5.5 a seguir e por este motivo não a apresentamos aqui.

Teorema 3.5.5 No modelo do Teorema 3.5.2 se substituirmos as distribuições $\pi\left(\sigma_{x}^{2}\right)$ e $\pi\left(\sigma^{2}\right)$, respectivamente, por $\pi\left(\sigma_{x}^{2}\right) \propto\left(\sigma_{x}^{2}\right)^{-r}$ e $\pi\left(\sigma^{2}\right) \propto\left(\sigma^{2}\right)^{-s}, r, s>0$, e se os vetores de dados $\underset{\sim}{X}$ e $\underset{\sim}{Y}$ são linearmente independentes e as componentes de $\underset{\sim}{X}$ (como também as de $\underset{\sim}{Y}$ ) não são todas iguais, então a condição necessária $e$ suficiente para que a posteriori correspondente seja própria é que $n+r+s>3$, $0<r<\frac{1}{2}$ e $0<s<\frac{\nu}{2}+2-r$.

Demonstração: Como a distribuição à posteriori coincide com a posteriori do Teorema 3.j.2 quando substituímos $c$ por $r-1, f$ por $s-1, d$ por zero e $g$ por zero, então 
muito dos cálculos necessários para demonstrar esse teorema são reaproveitados, fazendo-se as devidas substituições. De fato, a integral $\int_{W=\mathbb{R}^{+}} \int_{\Theta} p\left(\theta, w \mid D_{\text {obs }}\right) \mathrm{d} \theta \underset{\theta}{ } \mathrm{d} w$ é proporcional a $\int_{W=\mathbb{R}_{+}} \int_{Z_{x}=\left\{\underset{\sim}{z} \in \mathbb{R}^{n-1}\right\}} h(\underset{\sim}{z}, w) \mathrm{d} \underset{\sim}{z}{ }_{x} \mathrm{~d} w$, onde

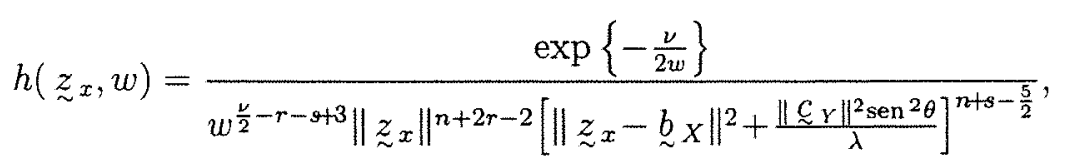

desde que a condição $n+s>\frac{5}{2}$ (que é igual à (2.8.12) quando $f=s-1$ ) seja válida. (Note que $h(\underset{\sim}{z}, w)$ é igual à função em (3.5.4) quando são feitas as substituições acima.)

Para analisar a integral dessa função, vamos particionar a região de integração $\mathcal{Z}_{x}$ de forma semelhante à usada na demonstração do Teorema 2.8.5 para o modelo Normal, isto é, $W \times \mathcal{Z}_{x}=W \times\left[R_{1} \cup R_{2} \cup R_{3}\right]$, onde $R_{2}=R_{2 a} \cup R_{2 b} \cup R_{2 c}$ (onde os conjuntos $R_{1}, R_{2}, R_{3}, R_{2 a}, R_{2 b}$ e $R_{2 c}$ são definidos da mesma forma que na demonstração do Teorema 2.8.5.

\section{AVALIAÇÃO DA INTEGRAL NA REGIÃO $W \times R_{1}$}

A integral de $h\left(z_{x}, w\right)$ em $W \times R_{1}$ é igual ao produto

$$
\int_{\mathbb{R}^{+}} \frac{\exp \left\{-\frac{\nu}{2 w}\right\}}{w^{\frac{\nu}{2}-r-s+3}} \mathrm{~d} w \cdot \int_{\|{\underset{\sim}{z} x}_{x<\varepsilon_{1}}} \frac{1}{\left\|\underset{\sim}{z_{x}}\right\|^{n+2 r-2}\left[\left\|{\underset{\sim}{x}}_{x}-\underset{\sim}{b} X\right\|^{2}+\frac{\|\underbrace{}_{Y}\|^{2} \operatorname{sen}^{2} \theta}{\lambda}\right]^{n+s-\frac{5}{2}}} \mathrm{~d}{\underset{\sim}{x}}_{x},
$$

onde pelo Lema 3.5.1 a integral em $w$ é finita se e somente se $\frac{\nu}{2}-r-s+3>1$ (isto é, $s<\frac{\nu}{2}+2-r$ ). A integral em $\underset{\sim}{z}$ (que coincide com a integral da função $g(\underset{\sim}{z}$ ) em (2.8.14) na região $R_{1}$ ) é finita se e somente se $n+2 r-2<n-1$ (isto é, $r<\frac{1}{2}$ ) (ver demonstração do Teorema 2.8.5 para o modelo Normal). Portanto, em $W \times R_{1}$, a integral de $h\left(z_{x}, w\right)$ é finita se e somente se $0<r<\frac{1}{2}$ e $0<s<\frac{\nu}{2}+2-r$. AVALIAÇÃO DA INTEGRAL NA REGIÃO $W \times R_{2}$ 


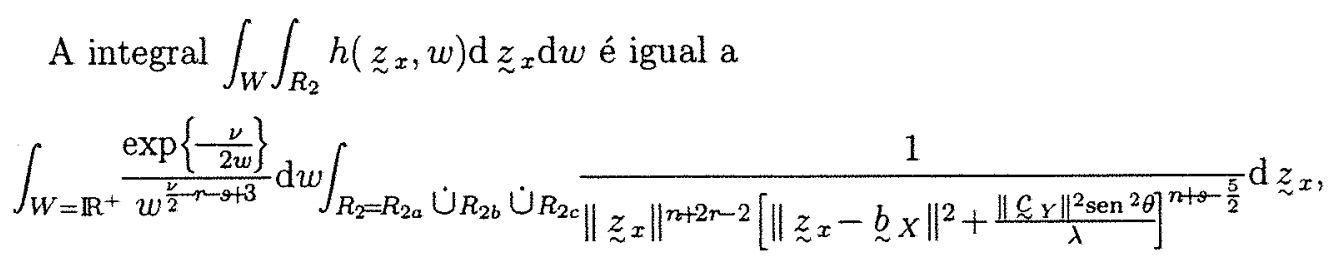
onde a integral em $w$ é finita se e somente se $\frac{\nu}{2}-r-s+3>1$ (isto é, $0<s<$ $\frac{\nu}{2}+2-r$ ). As integrais em $\underset{\sim}{z}$ nas regiões $R_{2 a}, R_{2 b}$ e $R_{2 c}$ são sempre finitas, como já foi demonstrado no Teorema 2.8 .5 da seção anterior. Portanto, em $W \times R_{2}$ a integral de $h(\underset{\sim}{z}, w)$ é finita se e somente se $0<s<\frac{\nu}{2}+2-r$.

AVALIAÇÃO DA INTEGRAL NA REGIÃO $W \times R_{3}$

$$
\begin{aligned}
& \int_{w=\mathbb{R}^{+}} \int_{R_{3}} h\left({\underset{\sim}{z} x}_{x}, w\right) \mathrm{d} \underset{\sim}{z_{x}} \mathrm{~d} w \\
& =\int_{\mathbb{R}^{+}} \frac{\exp \left\{-\frac{\nu}{2 w}\right\}}{w^{\frac{\nu}{2}-r-s+3}} \mathrm{~d} w \cdot \int_{\left\|z_{x}\right\|>k} \frac{1}{\left\|z_{x}\right\|^{n+2 r-2}\left[\left\|z_{x}-\underset{\sim}{b}\right\|^{2}+\frac{\|\mathcal{C} \gamma\|^{2} \operatorname{sen}^{2} \theta}{\lambda}\right]^{n+s-\frac{5}{2}}} \mathrm{~d} z_{\sim} x,
\end{aligned}
$$

onde a integral em $w$ converge se e somente se $0<s<\frac{\nu}{2}+2-r$ e a integral em $\underset{\sim}{z_{x}}$ (na região $R_{3}$ ) é finita se e somente se $n+2 r-2+2\left(n+s-\frac{5}{2}\right)>n-1$ (isto é, $n+r+s>3$ ), como já foi demonstrado no Teorema 2.8.5 para o modelo Normal. Portanto, a integral $\int_{W} \int_{R_{3}} h(\underset{\sim}{z}, w) \mathrm{d} \underset{\sim}{z} \mathrm{~d} w$ é finita se e somente se $0<s<\frac{\nu}{2}+2-r$ e $n+r+s>3$.

Conclusão: Analisando-se simultaneamente todas as informações, concluímos que a condição necessária e suficiente para que a posteriori seja própria é que $0<r<\frac{1}{2}$, $0<\frac{\nu}{2}+2-r$ e $n+r+s>3$.

\subsection{Sensibilidade do modelo a diferentes valores do número de graus de liberdade}

Nessa seção estudamos o efeito nas inferências à posteriori à medida que fixamos diferentes valores para o número de graus de liberdade do modelo estrutural $t$ Student com $\nu$ conhecido (mantendo-se todos os demais hiperparâmetros fixados). 
Sabemos que a distribuição $t$ aproxima-se da Normal quando $\nu \rightarrow \infty$ e portanto uma maneira de avaliar a sensibilidade à suposição de Normalidade (ou seja, à verossimilhança Normal) é verificar se as inferências à posteriori mudam muito quando o inverso do número de graus de liberdade, $\frac{1}{\nu}$, varia entre 0 e 1 . Uma maneira descritiva de fazer isso é construir um gráfico onde no eixo das abscissas colocamos os valores de $\frac{1}{\nu}$ e no das ordenadas, os valores correspondentes da média (ou mediana, moda, variância, quartis, etc.) da posteriori marginal de cada parâmetro.

$O$ procedimento acima pode ser feito para os modelos $t$-dependente e $t$-independente, com ou sem restrição de identificabilidade e com priori própria ou imprópria (desde que a posteríori seja própria).

\subsection{Algumas medidas de comparação de modelos}

\section{(1) O critério da medida $L$}

A seguir apresentamos a medida $L$ para o modelo de regressão estrutural simples $t$-independente com número de graus de liberdade fixado e com restrição de identificabilidade $\sigma_{e}^{2}=\lambda \sigma_{u}^{2}=\lambda \sigma^{2}$.

$$
L_{2}\left(D_{\mathrm{obs}}, \delta\right)=\sum_{i=1}^{n} \operatorname{Var}\left({\underset{\sim}{U}}_{i}^{*} \mid D_{\mathrm{obs}}\right)+\delta \sum_{i=1}^{n}\left(E\left(\underset{\sim}{U_{i}^{*}} \mid D_{\mathrm{obs}}\right)-\underset{\sim}{U_{i}}\right)\left(E\left({\underset{\sim}{U}}_{i}^{*} \mid D_{\mathrm{obs}}\right)-{\underset{\sim}{U}}_{i}\right)^{\mathrm{T}}
$$

onde $D_{\mathrm{obs}}=\left(\begin{array}{c}{\underset{\sim}{1}}_{1} \\ \vdots \\ {\underset{\sim}{U}}_{n}\end{array}\right)$ com ${\underset{\sim}{i}}_{i}=\left(\begin{array}{l}Y_{i} \\ X_{i}\end{array}\right)$.

Seja ${\underset{\sim}{1}}_{1}^{*}, \ldots, \widetilde{\sim}_{n}^{*} n$ vetores de observações futuras com a mesma distribuição dos $\operatorname{dados}\left({\underset{\sim}{i}}_{i}^{*}=\left(\begin{array}{l}Y_{f_{1}} \\ X_{f_{2}}\end{array}\right)\right)$. Seja $\underset{\sim}{\theta}=\left(\alpha, 3, \mu, \sigma_{x}^{2}, \sigma^{2}\right)^{T}$ e $\underset{\sim}{w}=\left(w_{1}, \ldots, w_{n}^{T}\right.$, onde

$$
\begin{aligned}
& E\left({\underset{\sim}{U}}_{i}^{*} \mid D_{\mathrm{obs}}\right)=E\left[E\left({\underset{\sim}{U}}_{i}^{*} \mid \underline{\sim}, \underset{\sim}{w}\right) \mid D_{\mathrm{obs}}\right]=E_{\underset{\theta}{\theta} \underset{\sim}{w} \mid D_{\mathrm{obs}}}\left[E\left({\underset{\sim}{i}}_{i}^{*} \mid \underline{\sim}, \underset{\sim}{w}\right)\right],
\end{aligned}
$$

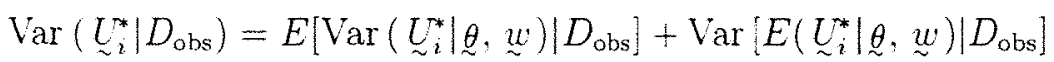




$$
\begin{aligned}
& \left.=E_{\underset{\theta}{\theta}, \underset{\sim}{w} \mid D_{\mathrm{obs}}}\left[\operatorname{Var}\left({\underset{\sim}{U}}_{i}^{*} \mid \underset{\sim}{\theta}, \underset{\sim}{w}\right)\right]+E_{\underset{\theta}{\theta}, \underset{\sim}{w} \mid D_{\mathrm{obs}}}\left[\left(E\left({\underset{\sim}{U}}_{i}^{*} \mid \underset{\sim}{\theta}, \underset{\sim}{w}\right)\right)\left(E\left({\underset{\sim}{i}}_{i}^{*} \mid \underset{\sim}{\theta}, \underset{\sim}{w}\right)\right)^{\top}\right)\right]
\end{aligned}
$$

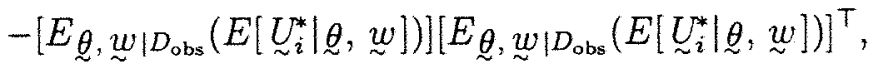

onde

$$
\begin{aligned}
E\left({\underset{\sim}{U}}_{i}^{*} \mid \theta, \underset{\sim}{w}\right) & =\left(\begin{array}{c}
\alpha+\beta \mu \\
\mu
\end{array}\right), \\
\operatorname{Var}\left({\underset{\sim}{\sim}}_{i}^{*} \mid \theta, \underset{\sim}{w}\right) & =\left[\begin{array}{cc}
\beta^{2} \sigma_{x}^{2}+\lambda \sigma^{2} & \beta \sigma_{x}^{2} \\
\beta \sigma_{x}^{2} & \sigma_{x}^{2}+\sigma^{2}
\end{array}\right] w_{i}, \quad i=1, \ldots, n .
\end{aligned}
$$

Dada uma amostra $\left({\underset{\sim}{\theta}}^{(1)},{\underset{w}{(1)}}^{(1)}, \ldots,{\underset{\theta}{\theta}}^{(G)}, \underset{\sim}{w}(G)\right)$ da posteriori $\pi\left(\underset{\sim}{\theta}, \underset{\sim}{w}, \underset{\sim}{x} \mid D_{\text {obs }}\right)$ definida em (3.1.11), podemos estimar a medida $L$ usando o método simples de Monte Carlo por

$$
\begin{aligned}
& \widehat{L_{2}}\left(D_{\mathrm{obs}}, \delta\right)=n\left\{\frac{1}{G} \sum_{g=1}^{G}\left(\operatorname{Var}\left({\underset{\sim}{U}}_{i}^{*} \mid \underline{\theta}^{(g)}, \underset{\sim}{w^{(g)}}\right)+\left[E\left({\underset{\sim}{U}}_{i}^{*} \mid \underline{\sim}^{(g)},{\underset{\sim}{w}}^{(g)}\right)\right]\left[E\left({\underset{\sim}{i}}_{i}^{*} \mid \theta^{(g)},{\underset{\sim}{w}}^{(g)}\right)\right]^{\top}\right)\right. \\
& \left.-\left[\frac{1}{G} \sum_{g=1}^{G} E\left({\underset{\sim}{i}}_{i}^{*} \mid \underline{\theta}^{(g)}, \underset{\sim}{w^{(g)}}\right)\right]\left[\frac{1}{G} \sum_{g=1}^{G} E\left({\underset{\sim}{i}}_{i}^{*} \mid{\underset{\theta}{(g)}}^{\underset{\sim}{w}}{ }^{(g)}\right)\right]^{\top}\right\} \\
& +\delta \sum_{i=1}^{n}\left[U_{i}-\frac{1}{G} \sum_{g=1}^{G} E\left({\underset{\sim}{i}}_{i}^{*} \mid \dot{\theta}^{(g)}, \underset{\sim}{w^{(g)}}\right)\right]\left[\bigcup_{\sim}-\frac{1}{G} \sum_{g=1}^{G} E\left({\underset{\sim}{i}}_{i}^{*} \mid{\underset{\sim}{(g)}}^{(g)}{\underset{\sim}{w}}^{(g)}\right)\right]^{\top},
\end{aligned}
$$

onde $\delta \in[0,1]$.

Observação: No caso do modelo $t$-dependente, o cálculo da medida $L$ é semelhante, bastando substituir $\underset{\sim}{w}$ por $w$. Portanto, se tomarmos uma amostra de tamanho $G$, $\left(\stackrel{\theta}{(1)}^{(1)}, w^{(1)}, \ldots, \underline{\theta}^{(G)}, w^{(G)}\right)$ da posteriori $\pi\left(\underset{\sim}{\theta}, w, \underset{\sim}{x} \mid D_{\text {obs }}\right)$ definida em (3.3.10) podemos obter $\widehat{L_{2}}\left(D_{\mathrm{obs}}, \delta\right)$ de forma semelhante (substituindo ${\underset{\sim}{w}}^{(g)}$ por $w^{(g)}$ ).

\section{(2) O critério da informação (BIC)}

$$
\mathrm{BIC}_{M_{i}}=-2 \log \left(\sup \pi_{M_{i}}\left(\underset{\sim}{U_{1}}, \ldots, \underset{\sim}{U_{n}} \mid \underset{\sim}{\theta}\right)\right)+p_{i} \log n,
$$

onde

$$
\begin{aligned}
& p_{i}=\text { número de parâmetros do modelo } M_{i}, \\
& n=\text { tamanho da amostra, } \\
& U_{\sim}=\left(\begin{array}{l}
Y_{2} \\
X_{i}
\end{array}\right) \text { é a } i \text {-ésima observação, } i=1, \ldots, n .
\end{aligned}
$$


No modelo estrutural $t$-dependente com número de graus de liberdade, $\nu$, conhecido,

$$
\pi_{M_{i}}\left(\underset{\sim}{U_{1}}, \ldots, \underset{\sim}{\left.U_{n} \mid \theta\right)}=K(2 n, \nu)\left|\sum_{\sim}^{*}\right|^{-\frac{1}{2}}\left(\nu+\left(\underset{\sim}{U}-\sim_{\sim}^{*}\right)^{\top} \sum_{\sim}^{*-1}\left(\underset{\sim}{U}-\mu_{\sim}^{*}\right)\right)^{-\frac{(2 n+\nu)}{2}}\right.
$$

onde $K(2 n, \nu)=\frac{\Gamma\left(\frac{2 n+\nu}{2}\right) \nu^{\nu / 2}}{\Gamma\left(\frac{\nu}{2}\right) \pi^{2 n / 2}}, \underset{\sim}{U}=\left(\begin{array}{c}{\underset{\sim}{1}}_{1} \\ \vdots \\ {\underset{\sim}{U}}_{n}\end{array}\right), \mu^{*}={\underset{\sim}{n} n}_{n} \otimes$ e ${\underset{\sim}{\Sigma}}^{*}=I_{n \times n} \otimes \sum$ e onde $\underset{\sim}{\mu}$ e $\sum_{\sim}$ são definidas em (3.3.1). Portanto,

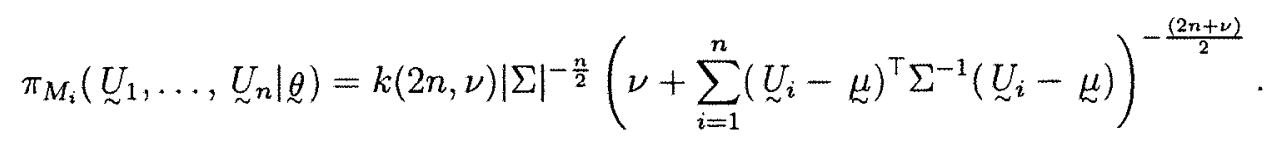

Os estimadores de máxima verossimilhança de $\mu$ e $\sum_{\sim}$ são dados, respectivamente, por

$$
\hat{\mu}=\frac{1}{n} \sum_{i=1}^{n}{\underset{\sim}{i}}_{i} \quad \text { e } \quad \hat{\Sigma}=\frac{1}{n} \sum_{i=1}^{n}\left(U_{i}-\hat{\mu}\right)\left({\underset{\sim}{i}}_{i}-\hat{\mu}\right)^{\top}
$$

(ver Arellano-Valle, 1994, página 200).

No modelo $t$-independente com graus de liberdade conhecidos,

$$
\begin{aligned}
& \pi_{M_{i}}\left(\underset{\sim}{U_{1}}, \ldots, \underset{\sim}{\left.U_{n} \mid \theta\right)}=\prod_{i=1}^{n} K(2, \nu)|\Sigma|^{-\frac{1}{2}}\left(\nu+\left({\underset{\sim}{i}}_{i}-\mu\right)^{\top} \Sigma^{-1}\left({\underset{\sim}{i}}_{i}-\mu\right)\right)\right. \\
& =(2 \pi)^{-n} \nu^{n\left(\frac{\nu}{2}+1\right)} \prod_{i=1}^{n}\left(\nu+\left(\underset{\sim}{U_{i}}-\underset{\sim}{\mu}\right)^{\top} \Sigma^{-1}\left({\underset{\sim}{i}}_{i}-\underset{\sim}{\mu}\right)\right)^{-\left(1+\frac{\nu}{2}\right)} .
\end{aligned}
$$

Os EMV de $\mu$ e $\sum_{\sim}$ podem ser obtidos por meio do algoritmo EM aplicado no modelo reparametrizado ortogonalmente (ver Arellano-Valle, 1994, página 206).

\section{(3) Algumas medidas alternativas para a seleção de modelos}

(a) O produto $\prod_{i=1}^{n} \mathrm{CPO}_{i}$.

Para cada modelo competitivo $M$ calculamos o produto $\prod_{i=1}^{n} \mathrm{CPO}_{i}^{(M)}$ e escolhemos o modelo cujo produto é máximo.

No caso do modelo estrutural $t$-independente com número de graus de liberdade conhecido, podemos estimar $\mathrm{CPO}_{i}$ por meio das fórmulas (3.1.25) ou (3.1.27) ou 
(3.1.29). No caso do modelo $t$-dependente, o cálculo do $\mathrm{CPO}_{i}$ pode ser obtido de forma semelhante fazendo-se as devidas adaptações.

(b) A soma dos resíduos de validação cruzada $\sum_{i=1}^{n}\left|\frac{\underset{i}{U_{i}-E\left({\underset{i}{i}}_{i} \mid D_{\mathrm{obs}(-i)}\right)}}{\sqrt{\operatorname{Var}\left({\underset{\sim}{i}}_{i} \mid D_{\mathrm{obs}(-i)}\right)}}\right|$.

Para cada modelo $M$ calculamos essa soma e escolhemos aquele cuja soma é mínima. No modelo estrutural $t$-independente com número de graus de liberdade conhecido, o valor esperado $E\left(\underset{\sim}{U_{i}} \mid D_{\mathrm{obs}(-i)}\right)$ e a variância, $\operatorname{Var}\left(\underset{\sim}{U_{i}} \mid D_{\mathrm{obs}(-i)}\right)$, podem ser estimados, respectivamente, pelas fórmulas (3.1.33) e (3.1.35).

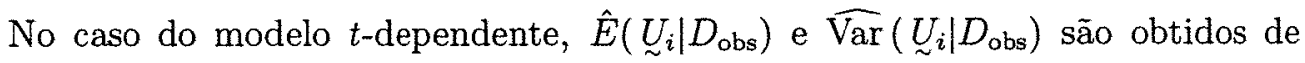
forma semelhante. 


\section{Capítulo 4}

\section{Uma análise do modelo estrutural Normal com erros nas variáveis com um ponto de mudança desconhecido}

\section{Introdução}

Neste capítulo vamos propor uma metodologia bayesiana para analisar o modelo de regressão linear simples com erros nas variáveis e mudança nos parâmetros após um determinado ponto da sequência de observações indexadas pelo tempo (ou espaço ou outra variável qualquer); as mudanças podendo ocorrer em todos os parâmetros, como também em qualquer subconjunto dos parâmetros.

Nesse capítulo estudamos o modelo onde os erros (e a covariável) são normalmente distribuídos e no Capítulo 5, estudaremos o caso em que os erros seguem uma distribuição $t$-Student.

$\mathrm{Na}$ literatura, Chang e Huang (1997) estudaram o modelo estrutural Normal com mudança somente na média da covariável e sob o ponto de vista da estatística clássica. Sua metodologia, entretanto, aparentemente pode ser estendida somente para testar mudança apenas no intercepto da reta de regressão no caso dos erros normais e nada mais. 
A análise inferencial desenvolvida nesse capítulo engloba tanto o problema de detectar a existência de mudança, como também o de estimar a posição do(s) ponto(s) de mudança (caso haja) e estimar os demais parâmetros de interesse (como por exemplo a magnitude da diferença entre os parâmetros antes e depois do ponto de mudança), como também as próprias distribuições marginais de cada parâmetro, além disso engloba também a análise preditiva.

O problema de detectar mudança pode ser formulado como um problema de teste de hipóteses ou um problema de escolha de modelos. No teste de hipótese testamos a hipótese nula de que não há mudança ao longo da seqüência de $n$ observações, isto é, $H_{0}: k=n$ (onde $k$ é o ponto de mudança), versus a hipótese alternativa de que ocorre mudança após o $k$-ésimo ponto da seqüência, isto é, $H_{1}: 1 \leq k \leq n-1$ (ou equivalentemente $H_{0}: \alpha_{1}=\alpha_{2}, \beta_{1}=\beta_{2}, \mu_{1}=\mu_{2}, \sigma_{x_{1}}^{2}=\sigma_{x_{2}}^{2}, \sigma_{1}^{2}=\sigma_{2}^{2}$ versus $H_{1}$ :pelo menos uma diferença).

O problema de detetar múltiplos pontos de mudança pode ser resolvido de forma muito simples aplicando-se o método da "segmentação binária" utilizado em Chen e Gupta (1997). Este método consiste, no primeiro passo, em testar a hipótese nula $\left(H_{0}\right)$ de que não há mudança versus a hipótese alternativa de que há uma única mudança. Se não há mudança, $H_{0}$ é aceita. Caso contrário, o ponto de mudança divide a seqüência original de observações em duas subseqüências. Para cada subseqüência repetimos o processo de testar $H_{0}$ versus $H_{1}$ da mesma forma que no primeiro passo, e continuamos o processo até que nenhuma mudança seja encontrada em quaisquer das subseqüências.

Nas seçōes 4.1 a 4.6 aparece a fórmulação envolvendo apenas o modelo identificável onde $\sigma_{e}^{2}=\lambda \sigma_{u}^{2}$, já que a formulação para o caso não identificável é muito semelhante e tornaria o estudo muito repetitivo. Entretanto na seção 4.7 temos um exemplo de aplicação envolvendo o modelo não identificv́el e que utilizada toda a a 
metodologia da seção.

\subsection{O modelo estrutural com um ponto de mu- dança e priori com componentes independen- tes}

Nessa seção inicialmente apresentamos o modelo linear estrutural que possue um único ponto de mudança (desconhecido) e que permite que a mudança possa ocorrer em todos os parâmetros após esse ponto. Em seguida apresentamos o modelo que permite mudança apenas em um subconjunto estritamente contido no conjunto de parâmetros.

\section{O modelo que permite mudança em todos os parâmetros}

O modelo que permite mudança em todos os parâmetros (e pelo menos um deles muda) após a $k$-ésima observação da seqüência ordenada de dados (onde $k$ é desconhecido) e tem a restrição de identificabilidade $\sigma_{e_{i}}^{2}=\lambda \sigma_{i}^{2}$, (onde $\sigma_{i}^{2}=\sigma_{u_{i}}^{2}$, para $i=1,2, \operatorname{com} \lambda$ conhecido), é definido por

$$
\left\{\begin{array}{l}
Y_{i}= \begin{cases}\alpha_{1}+\beta_{1} x_{i}+e_{i}, & i=1, \ldots, k, \quad 1 \leq k \leq n-1, \\
\alpha_{2}+\beta_{2} x_{i}+e_{i}, & i=k+1, \ldots, n,\end{cases} \\
X_{i}=x_{i}+u_{i}, \quad i=1, \ldots, n,
\end{array}\right.
$$

onde $Y_{i}$ e $X_{i}$ são as variáveis aleatórias observáveis, $x_{i}$ é a variável aleatória latente e $e_{i}, u_{i}$, os erros aleatórios, com a suposição que $\left(\begin{array}{c}e_{i} \\ u_{i} \\ x_{i}\end{array}\right)$ são independentes, para $i=1, \ldots, n, \mathrm{e}$

$$
\left(\begin{array}{c}
e_{i} \\
u_{i} \\
x_{i}
\end{array}\right) \sim\left\{\begin{array}{l}
N\left(\left(\begin{array}{c}
0 \\
0 \\
\mu_{1}
\end{array}\right) ;\left(\begin{array}{ccc}
\lambda \sigma_{1}^{2} & 0 & 0 \\
0 & \sigma_{1}^{2} & 0 \\
0 & 0 & \sigma_{x(1)}^{2}
\end{array}\right)\right), i=1, \ldots, k, \\
N\left(\left(\begin{array}{c}
0 \\
0 \\
\mu_{2}
\end{array}\right) ;\left(\begin{array}{ccc}
\lambda \sigma_{2}^{2} & 0 & 0 \\
0 & \sigma_{2}^{2} & 0 \\
0 & 0 & \sigma_{x(2)}^{2}
\end{array}\right)\right), i=k+1, \ldots, n,
\end{array}\right.
$$


Nesse modelo o vetor de parâmetros é $\underset{\theta}{\theta}=\left(\begin{array}{c}k \\ \theta_{1} \\ {\underset{\sim}{2}}_{2}\end{array}\right)$, onde $\underset{i}{\theta_{i}}=\left(\alpha_{i}, \beta_{i}, \mu_{i}, \sigma_{x(i)}^{2}, \sigma_{i}^{2}\right)^{T}$, para $i=1,2$ e o espaço paramétrico é $\Theta^{*}=\left\{\underline{\theta}: k \in \mathcal{K},\left(\alpha_{i}, \beta_{i}, \mu_{i}\right) \in \mathbb{R}^{3} \mathrm{e}\right.$ $\left.\left(\sigma_{x(i)}^{2}, \sigma_{i}^{2}\right) \in \mathbb{R}_{+}^{2}, i=1,2\right\}$.

Nesse modelo estamos assumindo que $\left(\alpha_{1}, \beta_{1}, \mu_{1}, \sigma_{x(1)}^{2}, \sigma_{1}^{2}\right) \neq\left(\alpha_{2}, \beta_{2}, \mu_{2}, \sigma_{x(2)}^{2}, \sigma_{2}^{2}\right)$, isto é, existe pelo menos uma diferença.

\section{Distribuição à priori}

A distribuição à priori de $\theta^{*}$ escolhida tem a forma

$$
\begin{gathered}
\pi(\underset{\sim}{*})=p(k) \pi\left(\underset{\sim}{\theta_{1}}\right) \pi\left({\underset{\sim}{2}}_{2}\right)=p(k) \prod_{i=1}^{2} \pi\left(\alpha_{i}\right) \pi\left(\beta_{i}\right) \pi\left(\mu_{i}\right) \pi\left(\sigma_{x(i)}^{2}\right) \pi\left(\sigma_{i}^{2}\right), \\
\text { onde } p(k)= \begin{cases}P(K=k), & k \in \mathcal{K}=\{1, \ldots, n-1\}, \\
0, & \text { caso contrário. }\end{cases}
\end{gathered}
$$

\section{Observações:}

- Nesse modelo estamos assumindo à priori que existe mudança em pelo memos um parâmetro. Entretanto se à priori não sabemos se existe mudança em algum parâmetro, então devemos desconsiderar a restrição ${\underset{\sim}{1}}_{1} \neq \underline{\sim}_{2}$ acima.

- Na estatística bayesiana, os parâmetros ${\underset{\sim}{\theta}}_{1}$ e ${\underset{\sim}{2}}_{2}$ podem ser estimados qualquer que seja $k,(1 \leq k \leq n-1)$. Entretanto para permitir estimativas "um pouco melhores" dos parâmetros $\underset{\sim}{\theta_{1}}$ e $\underset{\sim}{\theta_{2}}$, principalmente quando a distribuição à priori não for "muito informativa", podemos considerar $k$ variando por exemplo no conjunto $\mathcal{K}=\{3, \ldots, n-3\}$, ou seja podemos assumir à priori que a probabilidade de $k$ pertencer ao conjunto $\{1,2, n-2, n-1, n\}$ é zero.

\section{A função de verossimilhança baseada nos dados observados}

Seja $D_{\text {obs }}=\left\{\left(Y_{i}, X_{i}\right)^{\top}, i=1, \ldots, n\right\}$ o conjunto dos dados observados e $D_{\text {obsi }}{ }^{(k)}=$ $\left\{\left(Y_{i}, X_{i}\right)^{\top}, i=1, \ldots, k\right\}, D_{\mathrm{obs} 2}^{(n-k)}=\left\{\left(Y_{i}, X_{i}\right)^{\top}, i=k+1, \ldots, n\right\}$, respectivamente, os dados observados até a mudança e os dados observados após a mudança. 
A função de verossimilhança $L\left(\underset{\theta}{\theta} \mid D_{\text {obs }}\right)=L\left(k, \underset{\theta_{1}}{\theta_{2}} \underset{2}{ } \mid D_{\text {obs }}\right)$ é proporcional a

$$
\begin{aligned}
& \frac{1}{\left|\Sigma_{1}\right|^{k / 2}} \exp \left\{-\frac{1}{2\left|\Sigma_{1}\right|}\left[\left(\beta_{1}^{2} \sigma_{x(1)}^{2}+\lambda \sigma_{1}^{2}\right) \sum_{i=1}^{k}\left(X_{i}-\mu_{1}\right)^{2}\right.\right. \\
& \left.\left.-2 \beta_{1} \sigma_{x(1)}^{2} \sum_{i=1}^{k}\left(Y_{i}-\alpha_{1}-\beta_{1} \mu_{1}\right)\left(X_{i}-\mu_{1}\right)+\left(\sigma_{x(1)}^{2}+\sigma_{1}^{2}\right) \sum_{i=1}^{k}\left(Y_{i}-\alpha_{1}-\beta_{1} \mu_{1}\right)^{2}\right]\right\} \\
& \cdot \frac{1}{\left|\Sigma_{2}\right|^{(n-k) / 2}} \exp \left\{-\frac{1}{2\left|\Sigma_{2}\right|}\left[\left(\beta_{2}^{2} \sigma_{x(2)}^{2}+\lambda \sigma_{2}^{2}\right) \sum_{i=k+1}^{n}\left(X_{i}-\mu_{2}\right)^{2}\right.\right. \\
& -2 \beta_{2} \sigma_{x(2)}^{2} \sum_{i=k+1}^{n}\left(Y_{i}-\alpha_{2}-\beta_{2} \mu_{2}\right)\left(X_{i}-\mu_{2}\right) \\
& \left.\left.+\left(\sigma_{x(2)}^{2}+\sigma_{2}^{2}\right) \sum_{i=k+1}^{n}\left(Y_{i}-\alpha_{2}-\beta_{2} \mu_{2}\right)^{2}\right]\right\}
\end{aligned}
$$

onde $\left|\Sigma_{i}\right|=\beta_{i}^{2} \sigma_{x(i)}^{2} \sigma_{i}^{2}+\lambda \sigma_{i}^{2}\left(\sigma_{x(i)}^{2}+\sigma_{i}^{2}\right), i=1,2$.

Ou seja,

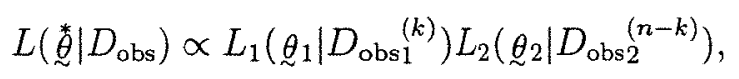

onde $L_{1}\left(\underset{\theta_{1}}{\theta_{1}} D_{\text {obsi }}^{(k)}\right)$ é a verossimilhança baseada nas $k$ primeiras observações e $L_{2}\left({\underset{\theta}{2}}_{2} \mid D_{\text {obs2 }}^{(n-k)}\right)$ a verossimilhança baseada nas observações após o ponto de mudança.

\section{A função de verossimilhança baseada nos dados completos}

Sejam $D=\left\{\left(Y_{i}, X_{i}, x_{i}\right)^{T}, i=1, \ldots, n\right\}, D^{(k)}=\left\{\left(Y_{i}, X_{i}, x_{i}\right)^{T}, i=1, \ldots, k\right\} \mathrm{e}$ $D^{(n-k)}=\left\{\left(Y_{i}, X_{i}, x_{i}\right)^{T}, i=k+1, \ldots, n\right\}$. A função de verossimilhança baseada nos dados completos, $L(\underset{\underset{\mid}{*}}{\stackrel{*}{\mid}} \mid D)=L\left(k, \underset{\sim}{\theta_{1}}, \underset{\underset{ }{\theta}}{\theta_{2}} \mid D\right)$ é proporcional a

$$
\begin{aligned}
& {\left[\sigma_{x(1)}^{2}\left(\sigma_{1}^{2}\right)^{2}\right]^{-k / 2} \exp \left\{-\frac{1}{2}\left[\frac{\sum_{i=1}^{k}\left(Y_{i}-\alpha_{1}-\beta_{1} x_{i}\right)^{2}}{\lambda \sigma_{1}^{2}}+\frac{\sum_{i=1}^{k}\left(X_{i}-x_{i}\right)^{2}}{\sigma_{1}^{2}}\right.\right.} \\
& \left.\left.+\frac{\sum_{i=1}^{k}\left(x_{i}-\mu_{1}\right)^{2}}{\sigma_{x(1)}^{2}}\right]\right\}\left[\sigma_{x(2)}^{2}\left(\sigma_{2}\right)^{2}\right]^{-(n-k) / 2} \exp \left\{-\frac{1}{2}\left[\frac{\sum_{i=k+1}^{n}\left(Y_{i}-\alpha_{2}-\beta_{2} x_{i}\right)^{2}}{\lambda \sigma_{2}^{2}}\right.\right. \\
& \left.\left.+\frac{\sum_{i=k+1}^{n}\left(X_{i}-x_{i}\right)^{2}}{\sigma_{2}^{2}}+\frac{\sum_{i=k+1}^{n}\left(x_{i}-\mu_{2}\right)^{2}}{\sigma_{x(2)}^{2}}\right]\right\},
\end{aligned}
$$

ou seja,

$$
L(\underset{\tilde{\theta}}{\tilde{*}} \mid D) \propto L_{1}\left(\underset{\theta_{1}}{ } \mid D^{(k)}\right) L_{2}\left({\underset{\sim}{2}}_{2} \mid D^{(n-k)}\right),
$$


onde $L_{1}(\underbrace{}_{1} \mid D^{(k)})$ é a verossimilhança baseada nos dados completos até a mudança e $L_{2}\left(\theta_{2} \mid D^{(n-k)}\right)$ a verossimilhança baseada nos dados completos após a mudança.

\section{Distribuição à posteriori}

A distribuição à posteriori pode ser expressa de duas formas (ou usando a verossimilhança baseada nos dados observados, ou a verossimilhança baseada nos dados completos), da mesma forma que no Capítulo 2. Ou seja,

$$
p\left(\stackrel{\underset{\sim}{*}}{\mid} \mid D_{\mathrm{obs}}\right) \propto L\left(\stackrel{\sim}{\stackrel{*}{*}} \mid D_{\mathrm{obs}}\right) \pi(\stackrel{*}{\stackrel{*}{*}}),
$$

onde $L\left(\underset{\underset{\sim}{*}}{\stackrel{*}{\mid}} \mid D_{\mathrm{obs}}\right)$ é proporcional a (4.1.3) e $\pi(\underset{\underset{\sim}{*})}{)}$ é dada por (4.1.2), isto é,

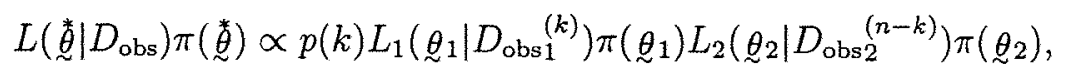

ou então

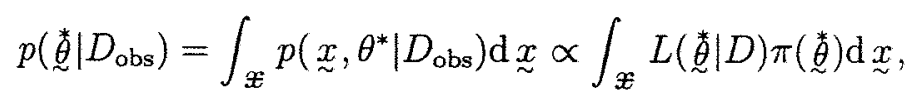

onde $\mathscr{X}=\left\{\underset{\sim}{x}=\left(x_{1}, \ldots, x_{n}\right)^{T}: x_{i} \in \mathbb{R}\right\}$ e $L(\underset{\sim}{\stackrel{*}{*} \mid D)}$ é proporcional à expressão (4.1.4) e $\pi(\underset{*}{*})$ é dada em (4.1.2).

\section{O modelo que permite mudanças num subconjunto de parâmetros}

O modelo que permite mudança em apenas um subconjunto fixado, estritamente contido no conjunto de parâmetros, pode ser definido usando o modelo em (4.1.1) como referência, bastando incorporar as devidas restrições. Notemos que existem $\left(\begin{array}{l}5 \\ 1\end{array}\right)+\left(\begin{array}{l}5 \\ 2\end{array}\right)+\left(\begin{array}{l}5 \\ 3\end{array}\right)+\left(\begin{array}{l}5 \\ 4\end{array}\right)=30$ possíveis modelos desse tipo. Um caso particular é o do modelo que permite mudança somente na média $\mu$, isto é, quando assumimos que os demais parâmetros $\left(\alpha, \beta, \sigma_{x}^{2}, \sigma^{2}\right)$ não mudam. Nesse caso, devemos impor ao modelo em (4.1.1) as seguintes restriçōes: $\alpha_{1}=\alpha_{2}=\alpha, \beta_{1}=\beta_{2}=\beta, \sigma_{x(1)}^{2}=\sigma_{x(2)}^{2}=\sigma_{x}^{2} \mathrm{e}$ $\sigma_{1}^{2}=\sigma_{2}^{2}=\sigma^{2}$. Assim, este modelo é dado por

$$
\left\{\begin{array}{l}
Y_{i}=\alpha+\beta x_{i}+e_{i} \\
X_{i}=x_{i}+u_{i}
\end{array}\right.
$$


onde

$$
\left(\begin{array}{c}
e_{i} \\
u_{i} \\
x_{i}
\end{array}\right) \stackrel{\text { iid }}{\sim}\left\{\begin{array}{l}
N\left(\left(\begin{array}{c}
0 \\
0 \\
\mu_{1}
\end{array}\right) ;\left(\begin{array}{ccc}
\lambda \sigma^{2} & 0 & 0 \\
0 & \sigma^{2} & 0 \\
0 & 0 & \sigma_{x}^{2}
\end{array}\right)\right), i=1, \ldots, k, \\
N\left(\left(\begin{array}{c}
0 \\
0 \\
\mu_{2}
\end{array}\right) ;\left(\begin{array}{ccc}
\lambda \sigma^{2} & 0 & 0 \\
0 & \sigma^{2} & 0 \\
0 & 0 & \sigma_{x}^{2}
\end{array}\right)\right), i=k+1, \ldots, n,
\end{array}\right.
$$

onde o espaço paramétrico é dado por

$$
\Theta^{*}=\left\{\stackrel{*}{\stackrel{*}{*}}=\left(k, \alpha, \beta, \mu_{1}, \mu_{2}, \sigma_{x}^{2}, \sigma^{2}\right)^{T}: k \in \mathcal{K},\left(\alpha, \beta, \mu_{1}, \mu_{2}\right) \in \mathbb{R}^{4},\left(\sigma_{x}^{2}, \sigma^{2}\right) \in \mathbb{R}_{+}^{2}\right\}
$$

e onde a distribuição à priori é dada por

$$
\pi(\stackrel{*}{\theta})=p(k) \pi(\alpha) \pi(\beta) \pi\left(\mu_{1}\right) \pi\left(\mu_{2}\right) \pi\left(\sigma_{x}^{2}\right) \pi\left(\sigma^{2}\right)
$$

com

$$
p(k)= \begin{cases}P[K=k], & k \in \mathcal{K}, \\ 0, & \text { caso contrário. }\end{cases}
$$

A função de verossimilhança $L\left(\underset{\sim}{Q} \mid D_{\text {obs }}\right)$ é proporcional à expressão em (4.1.3) quando substituímos $\alpha_{i}$ por $\alpha, \beta_{i}$ por $\beta, \sigma_{x(i)}^{2}$ por $\sigma_{x}^{2}$ e $\sigma_{i}^{2}$ por $\sigma^{2}$, para $i=1,2$, ou seja,

$$
\begin{aligned}
L\left(\underset{*}{*} \mid D_{\text {obs }}\right) \propto & \frac{1}{|\Sigma|^{n / 2}} \exp \left\{-\frac{1}{2|\Sigma|}\left[( \beta ^ { 2 } \sigma _ { x } ^ { 2 } + \lambda \sigma ^ { 2 } ) \left(\sum_{i=1}^{k}\left(X_{i}-\mu_{1}\right)^{2}\right.\right.\right. \\
& \left.+\sum_{i=k+1}^{n}\left(X_{i}-\mu_{2}\right)^{2}\right)-2 \beta^{2} \sigma_{x}^{2}\left(\sum_{i=1}^{k}\left(Y_{i}-\alpha-\beta \mu_{1}\right)\left(X_{i}-\mu_{1}\right)\right. \\
& \left.+\sum_{i=k+1}^{n}\left(Y_{i}-\alpha-\beta \mu_{2}\right)\left(X_{i}-\mu_{2}\right)\right)+\left(\sigma_{x}^{2}+\sigma^{2}\right)\left(\sum_{i=1}^{k}\left(Y_{i}-\alpha-\beta \mu_{1}\right)^{2}\right. \\
& \left.\left.\left.+\sum_{i=k+1}^{n}\left(Y_{i}-\alpha-\beta \mu_{2}\right)^{2}\right)\right]\right\}
\end{aligned}
$$

onde $|\Sigma|=\beta^{2} \sigma_{x}^{2} \sigma^{2}+\lambda \sigma^{2}\left(\sigma_{x}^{2}+\sigma^{2}\right)$.

Da mesma forma a funçāo de verossimilhança baseada nos dados completos $L(\stackrel{\leftrightarrow}{\theta} \mid D)$ é proporcional à expressão dada em (4.1.4), fazendo $\left(\alpha_{i}, \beta_{i}, \sigma_{x(i)}^{2}, \sigma_{i}^{2}\right)=$ $\left(\alpha, 3, \sigma_{x}^{2}, \sigma^{2}\right)$, para $i=1,2$. 
De forma semelhante define-se qualquer modelo que permite mudança apenas em um subconjunto de parâmetros.

Observação: Poderíamos considerar o conjunto $\mathcal{K}$ (dos possíveis valores de $k$ ) variando dependendo do modelo. Por exemplo, no modelo que permite mudança apenas em $\alpha$ e $\beta$ então $\mathcal{K}=\{2, \ldots, n-2\}$ e no modelo que permite mudança apenas em $\mu, \mathcal{K}=\{1, \ldots, n-1\}$ (já que o tamanho mínimo de amostra para estimar $\mu$ na estatística clássica é 1). Entretanto, como já mencionamos anteriormente, na estatística bayesiana isso não é necessário.

\subsection{A análise do modelo que permite mudança em todos os parâmetros com particular priori de componentes independentes}

Nessa seção o objetivo é fazer inferência sobre o modelo estrutural normal com priori própria de componentes independentes supondo que há mudança (em pelo menos um dos parâmetros) e esta mudança pode eventualmente ocorrer em todos eles.

O modelo é dado em (4.1.1) e a priori escolhida é da forma

$$
\pi\left(\stackrel{*}{\stackrel{*}{*})}=p(k) \prod_{i=1}^{2} \pi\left(\alpha_{i}\right) \pi\left(\beta_{i}\right) \pi\left(\mu_{i}\right) \pi\left(\sigma_{x(i)}^{2}\right) \pi\left(\sigma_{i}^{2}\right)\right.
$$

onde

$$
p(k)= \begin{cases}P[K=k]=\frac{1}{\# \mathcal{K}}, & k \in \mathcal{K}=\{1, \ldots, n-1\}, \\ 0, & \text { caso contrário, }\end{cases}
$$

onde $\# \mathcal{K}$ é o número de elementos do conjunto $\mathcal{K}$

$\pi\left(\alpha_{i}\right) \sim N\left(a_{i}, \sigma_{\alpha_{i}}^{2}\right), \pi\left(\beta_{i}\right) \sim N\left(b_{i}, \sigma_{\beta_{i}}^{2}\right), \pi\left(\mu_{i}\right) \sim N\left(m_{i}, \sigma_{\mu_{i}}^{2}\right), \pi\left(\sigma_{x(i)}^{2}\right) \sim I G\left(c_{i}, d_{i}\right) \mathrm{e}$ $\pi\left(\sigma_{i}^{2}\right) \sim I G\left(f_{i}, g_{i}\right)$, onde $a_{i}, b_{i}, m_{i}, c_{i}, d_{i}, f_{i}, g_{i}, \sigma_{\alpha_{i}}^{2}, \sigma_{\beta_{i}}^{2}, \sigma_{\mu_{i}}^{2}$ são hiperparâmetros conhecidos para $i=1.2$. 


\subsubsection{Distribuição à posteriori}

Como já vimos, a distribuição à posteriori pode ser escrita como

$$
p\left(\stackrel{*}{\theta} \mid D_{\mathrm{obs}}\right)=\int_{\mathfrak{X}} p\left(\underset{\sim}{x}, \stackrel{*}{\theta} \mid D_{\mathrm{obs}}\right) \mathrm{d} \underset{\sim}{x} \propto \int_{\mathfrak{X}} L\left(\theta^{*} \mid D\right) \pi\left(\theta^{*}\right) \mathrm{d} \underset{\sim}{x}
$$

e nesse caso

$$
\begin{aligned}
& p\left(\underset{\sim}{x}, \underset{\underset{\theta}{*}}{\stackrel{*}{*}} \mid D_{\mathrm{obs}}\right) \propto\left(\sigma_{1}^{2}\right)^{-\left(k+f_{1}+1\right)}\left(\sigma_{x(1)}^{2}\right)^{-\left(\frac{k}{2}+c_{1}+1\right)}\left(\sigma_{2}^{2}\right)^{-\left(n-k+f_{2}+1\right)}\left(\sigma_{x(2)}^{2}\right)^{-\left(\frac{n-k}{2}+c_{2}+1\right)} \\
& . \exp \left\{-\frac{1}{2}\left[\frac{\sum_{i=1}^{k}\left(Y_{i}-\alpha_{1}-\beta_{1} x_{i}\right)^{2}+\lambda\left(X_{i}-x_{i}\right)^{2}}{\lambda \sigma_{1}^{2}}+\sum_{i=1}^{k} \frac{\left(x_{i}-\mu_{1}\right)^{2}}{\sigma_{x(1)}^{2}}\right.\right. \\
& +\frac{\sum_{i=k+1}^{n}\left(Y_{i}-\alpha_{2}-\beta_{2} x_{i}\right)^{2}+\lambda\left(X_{i}-x_{i}\right)^{2}}{\lambda \sigma_{2}^{2}}+\frac{\sum_{i=k+1}^{n}\left(x_{i}-\mu_{2}\right)^{2}}{\sigma_{x(2)}^{2}}+\frac{\left(\alpha_{1}-a_{1}\right)^{2}}{\sigma_{\alpha_{1}}^{2}} \\
& +\frac{\left(\alpha_{2}-a_{2}\right)^{2}}{\sigma_{\alpha_{2}}^{2}}+\frac{\left(\beta_{1}-b_{1}\right)^{2}}{\sigma_{\beta_{1}}^{2}}+\frac{\left(\beta_{2}-b_{2}\right)^{2}}{\sigma_{\beta_{2}}^{2}}+\frac{\left(\mu_{1}-m_{1}\right)^{2}}{\sigma_{\mu_{1}}^{2}}+\frac{\left(\mu_{2}-m_{2}\right)^{2}}{\sigma_{\mu_{2}}^{2}} \\
& \left.\left.+\frac{2 d_{1}}{\sigma_{x(1)}^{2}}+\frac{2 d_{2}}{\sigma_{x(2)}^{2}}+\frac{2 g_{1}}{\sigma_{1}^{2}}+\frac{2 g_{2}}{\sigma_{2}^{2}}\right]\right\} p(k) \text {. }
\end{aligned}
$$

\subsubsection{Distribuições condicionais completas à posteriori ba- seadas nos dados completos}

Com base em $p\left(\underset{\sim}{x}, \stackrel{*}{\underset{*}{*}} \mid D_{\text {obs }}\right)$ em $(4.2 .2)$ (onde $\underset{\sim}{\theta}=\left(\begin{array}{c}k \\ \theta_{1} \\ \tilde{\theta}_{2}\end{array}\right)$ ), obtemos as condicionais completas de $x_{1}, x_{2}, \ldots, x_{n}, \alpha_{i}, \beta_{i}, \mu_{i}, \sigma_{x(i)}^{2}, \sigma_{i}^{2}$ e $k$, para $i=1,2$, dadas pelas fórmulas (4.2.3) a (4.2.14) a seguir.

- $x_{i} \mid x_{(-i)}, \alpha_{1}, \alpha_{2}, \beta_{1}, \beta_{2}, \mu_{1}, \mu_{2}, \sigma_{x(1)}^{2}, \sigma_{x(2)}^{2}, \sigma_{1}^{2}, \sigma_{2}^{2}, k, D_{\text {obs }}$

$$
\sim\left\{\begin{array}{c}
N\left(\frac{\sigma_{x(1)}^{2}\left[\lambda X_{i}+\beta_{1}\left(Y_{i}-\alpha_{1}\right)+\lambda \sigma_{1}^{2} \mu_{1}\right]}{\sigma_{x(1)}^{2}\left[\lambda+\beta_{1}^{2}\right]+\lambda \sigma_{1}^{2}} ; \frac{\lambda \sigma_{1}^{2} \sigma_{x(1)}^{2}}{\sigma_{x(1)}^{2}\left[\lambda+\beta_{1}^{2}\right]+\lambda \sigma_{1}^{2}}\right), \\
i=1, \ldots, k \\
N\left(\frac{\sigma_{x(2)}^{2}\left[\lambda X_{i}+\beta_{2}\left(Y_{i}-\alpha_{2}\right)+\lambda \sigma_{2}^{2} \mu_{2}\right]}{\sigma_{x(2)}^{2}\left[\lambda+\beta_{2}^{2}\right]+\lambda \sigma_{2}^{2}} ; \frac{\lambda \sigma_{2}^{2} \sigma_{x(2)}^{2}}{\sigma_{x(2)}^{2}\left[\lambda+\beta_{2}^{2}\right]+\lambda \sigma_{2}^{2}}\right), \\
i=k+1, \ldots, n
\end{array}\right.
$$


- $\alpha_{1} \mid \underset{\sim}{x}, \alpha_{2}, \beta_{1}, \beta_{2}, \mu_{1}, \mu_{2}, \sigma_{x(1)}^{2}, \sigma_{x(2)}^{2}, \sigma_{1}^{2}, \sigma_{2}^{2}, k, D_{\text {obs }}$

$\sim N\left(\frac{\sigma_{\alpha_{1}}^{2} \sum_{i=1}^{k}\left(Y_{i}-\beta_{1} x_{i}\right)+\lambda \sigma_{1}^{2} a_{1}}{k \sigma_{\alpha_{1}}^{2}+\lambda \sigma_{1}^{2}} ; \frac{\lambda \sigma_{1}^{2} \sigma_{\alpha_{1}}^{2}}{k \sigma_{\alpha_{1}}^{2}+\lambda \sigma_{1}^{2}}\right)$,

- $\alpha_{2} \mid \underset{\sim}{x}, \alpha_{1}, \beta, \beta_{2}, \mu, \mu_{2}, \sigma_{x(1)}^{2}, \sigma_{x(2)}^{2}, \sigma_{1}^{2}, \sigma_{2}^{2}, k, D_{\text {obs }}$

$\sim N\left(\frac{\sigma_{\alpha_{2}}^{2} \sum_{i=k+1}^{n}\left(Y_{i}-\beta_{2} x_{i}\right)+\lambda \sigma_{2}^{2} a_{2}}{(n-k) \sigma_{\alpha_{2}}^{2}+\lambda \sigma_{2}^{2}} ; \frac{\lambda \sigma_{2}^{2} \sigma_{\alpha_{2}}^{2}}{(n-k) \sigma_{\alpha_{2}}^{2}+\lambda \sigma_{2}^{2}}\right)$,

- $\beta_{1} \mid \underset{\sim}{x}, \alpha_{1}, \alpha_{2}, \beta_{2}, \mu_{1}, \mu_{2}, \sigma_{x(1)}^{2}, \sigma_{x(2)}^{2}, \sigma_{1}^{2}, \sigma_{2}^{2}, k, D_{\mathrm{obs}}$

$\sim N\left(\frac{\sigma_{\beta_{1}}^{2} \sum_{i=1}^{k}\left(Y_{i}-\alpha_{1}\right) x_{i}+\lambda b_{1} \sigma_{1}^{2}}{\sigma_{\beta_{1}}^{2} \sum_{i=1}^{k} x_{i}^{2}+\lambda \sigma_{1}^{2}} ; \frac{\lambda \sigma_{1}^{2} \sigma_{\beta_{1}}^{2}}{\sigma_{\beta_{1}}^{2} \sum_{i=1}^{k} x_{i}^{2}+\lambda \sigma_{1}^{2}}\right)$,

- $\beta_{2} \mid \underset{\sim}{x}, \alpha_{1}, \alpha_{2}, \beta_{1}, \mu_{1}, \mu_{2}, \sigma_{x(1)}^{2}, \sigma_{x(2)}^{2}, \sigma_{1}^{2}, \sigma_{2}^{2}, k, D_{\text {obs }}$

$\sim N\left(\frac{\sigma_{\beta_{2}}^{2} \sum_{i=k+1}^{n}\left(Y_{i}-\alpha_{2}\right) x_{i}+\lambda b_{2} \sigma_{2}^{2}}{\sigma_{\beta_{2}}^{2} \sum_{i=k+1}^{n} x_{i}^{2}+\lambda \sigma_{2}^{2}} ; \frac{\lambda \sigma_{2}^{2} \sigma_{\beta_{2}}^{2}}{\sigma_{\beta_{2}}^{2} \sum_{i=k+1}^{n} x_{i}^{2}+\lambda \sigma_{2}^{2}}\right)$,

- $\mu_{1} \mid \underset{\sim}{x}, \alpha_{1}, \alpha_{2}, \beta_{1}, \beta_{2}, \mu_{2}, \sigma_{x(1)}^{2}, \sigma_{x(2)}^{2}, \sigma_{1}^{2}, \sigma_{2}^{2}, k, D_{\text {obs }}$

$\sim N\left(\frac{\sigma_{\mu_{1}}^{2} \sum_{i=1}^{k} x_{i}+\sigma_{x(1)}^{2} m_{1}}{k \sigma_{\mu_{1}}^{2}+\sigma_{x(1)}^{2}} ; \frac{\sigma_{\mu_{1}}^{2} \sigma_{x(1)}^{2}}{k \sigma_{\mu_{1}}^{2}+\sigma_{x(1)}^{2}}\right)$,

- $\mu_{2} \mid \underset{\sim}{x}, \alpha_{1}, \alpha_{2}, \beta_{1}, \beta_{2}, \mu_{2}, \sigma_{x(1)}^{2}, \sigma_{x(2)}^{2}, \sigma_{1}^{2}, \sigma_{2}^{2}, k, D_{\text {obs }}$

$\sim N\left(\frac{\sigma_{\mu_{2}}^{2} \sum_{i=k+1}^{n} x_{i}+\sigma_{x(2)}^{2} m_{2}}{(n-k) \sigma_{\mu_{2}}^{2}+\sigma_{x(2)}^{2}} ; \frac{\sigma_{\mu_{2}}^{2} \sigma_{x(2)}^{2}}{(n-k) \sigma_{\mu_{2}}^{2}+\sigma_{x(2)}^{2}}\right)$

- $\sigma_{x(1)}^{2} \mid \underset{\sim}{x}, \alpha_{1}, \alpha_{2}, \beta_{1}, \beta_{2}, \mu_{1}, \mu_{2}, \sigma_{x(2)}^{2}, \sigma_{1}^{2}, \sigma_{2}^{2}, k, D_{\text {obs }}$

$\sim I G\left(\frac{k}{2}+c_{1} ; \frac{1}{2} \sum_{i=1}^{k}\left(x_{i}-\mu_{1}\right)^{2}+d_{1}\right)$,

- $\sigma_{x(2)}^{2} \mid \underset{\sim}{x}, \alpha_{1}, \alpha_{2}, \beta_{1}, \beta_{2}, \mu_{1}, \mu_{2}, \sigma_{x(1)}^{2}, \sigma_{1}^{2}, \sigma_{2}^{2}, k, D_{\mathrm{obs}}$ 


$$
\sim I G\left(\frac{n-k}{2}+c_{2} ; \frac{1}{2} \sum_{i=k+1}^{n}\left(x_{i}-\mu_{2}\right)^{2}+d_{2}\right)
$$

- $\sigma_{1}^{2} \mid \underset{\sim}{x}, \alpha_{1}, \alpha_{2}, \beta_{1}, \beta_{2}, \mu_{1}, \mu_{2}, \sigma_{x(1)}^{2}, \sigma_{x(2)}^{2}, \sigma_{2}^{2}, k, D_{\mathrm{obs}}$

$$
\sim I G\left(k+f_{1} ; \frac{1}{2}\left[\frac{\sum_{i=1}^{k}\left(Y_{i}-\alpha_{1}-\beta_{1} x_{i}\right)^{2}}{\lambda}+\sum_{i=1}^{k}\left(X_{i}-x_{i}\right)^{2}\right]+g_{1}\right),
$$

- $\sigma_{2}^{2} \mid \underset{\sim}{x}, \alpha_{1}, \alpha_{2}, \beta_{1}, \beta_{2}, \mu_{1}, \mu_{2}, \sigma_{x(1)}^{2}, \sigma_{x(2)}^{2}, \sigma_{1}^{2}, k, D_{\text {obs }}$

$$
\begin{aligned}
& \sim I G\left(n-k+f_{2} ; \frac{1}{2}\left[\frac{\sum_{i=k+1}^{n}\left(Y_{i}-\alpha_{2}-\beta_{2} x_{i}\right)^{2}}{\lambda}+\sum_{i=k+1}^{n}\left(X_{i}-x_{i}\right)^{2}\right]+g_{2}\right), \\
& P\left[K=k \mid \underset{\sim}{x}, \alpha_{1}, \alpha_{2}, \beta_{1}, \beta_{2}, \mu_{1}, \mu_{2}, \sigma_{x(1)}^{2}, \sigma_{x(2)}^{2}, \sigma_{1}^{2}, \sigma_{2}^{2}, k, D_{\text {obs }}\right]=\frac{L\left(k, \underset{\theta_{1}}{\theta_{2}} \mid D\right) p(k)}{\sum_{k=1}^{n-1} L\left(k \underset{\theta_{1}}{\theta_{2}} \mid D\right) p(k)}, \\
& =\frac{L\left(k, \underline{\theta}_{1}, \underline{\theta}_{2} \mid D\right)}{\sum_{k \in \mathcal{K}} L\left(k, \underline{\sim}_{1}, \underline{\sim}_{2} \mid D\right)}, k \in \mathcal{K},
\end{aligned}
$$

onde $L\left(k, \underset{\sim}{\theta_{1}}, \underset{2}{\theta_{2}} \mid D\right)$ é dada por (4.1.4)

Observação 1: Usando as distribuições condicionais completas acima podemos implementar o algoritmo de Gibbs descrito na seção 2.5 .3 com o objetivo de obter uma amostra da distribuição à posteriori cujo núcleo é dado em (4.2.2).

Observação 2: A análise do modelo com priori de componentes condicionalmente independentes, como as do Capítulo 2, pode ser feita de forma semelhante.

\subsubsection{Estimação do modelo que permite mudança em todos os parâmetros}

Semelhantemente à seção 2.9 , podemos obter as estimativas das densidades marginais à posteriori de cada parâmetro do modelo em (4.1.1). bem como as estimativas pontuais da média, mediana, quantis e variância dessas distribuições e os intervalos 
de densidade máxima (HPD).

A distribuição marginal à posteriori de $K, P\left[K=k \mid D_{\text {obs }}\right]$ será estimada por

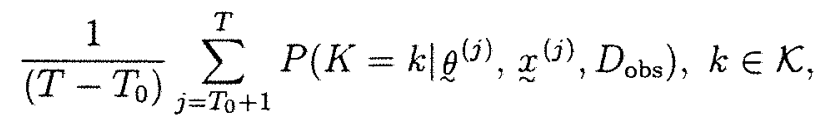

onde ${\underset{\theta}{(j)}}^{(j)}=\left(\begin{array}{c}\theta_{1}^{(j)} \\ {\underset{\sim}{\theta}}_{2}^{(j)}\end{array}\right),{\underset{\theta}{i}}_{i}^{(j)}=\left(\alpha_{i}, \beta_{i}, \mu_{i}, \sigma_{x(i)}^{2}, \sigma_{i}^{2}\right)^{T}$ e ${\underset{\sim}{x}}^{(j)}=\left(x_{1}^{(j)}, \ldots, x_{n}^{(j)}\right)^{T}$,

$T=$ número de iterações,

$T_{0}=$ número de iterações iniciais descartadas referentes ao período "burn in",

$$
\text { e } P\left(K=k \mid{\underset{\theta}{\theta}}^{(j)},{\underset{\sim}{x}}^{(j)}, D_{\text {obs }}\right)=\frac{L(k, \underbrace{(j)} \mid D^{(j)})}{\sum_{k \in \mathcal{K}} L\left(k,{\underset{\theta}{(j)}}^{(j)} \mid D^{(j)}\right)}, k \in \mathcal{K},
$$

onde $D^{(j)}=\left\{\left(Y_{i}, X_{i}, x_{i}^{(j)}\right)^{T}, i=1, \ldots, n\right\}$ e $L\left(k,{\underset{\theta}{\theta}}^{(j)} \mid D^{(j)}\right)$ é dada em (4.1.4).

No caso de $m$ cadeias paralelas o estimador será

$$
\frac{1}{m\left(T-T_{0}\right)} \sum_{i=1}^{m} \sum_{j=T_{0}+1}^{T} P\left(K=k \mid{\underset{\sim}{(i)}}_{(j)}^{(j)} \underset{\sim}{(j)}\left(D_{\mathrm{obs}}\right)\right.
$$

onde $P\left(K=k \mid \underset{(i)}{\theta_{(j)}^{(j)}}, \underset{\sim}{x}(j), D_{\mathrm{obs}}\right)=P\left(K=k \mid{\underset{\sim}{(i)}}_{(j)}^{(j)}, D_{(i)}^{(j)}\right)$

e $D_{(i)}^{(j)}=\left\{\left(Y_{k}, X_{k}, x_{k(i)}^{(j)}\right), k=1, \ldots, n\right\}$

O ponto de mudança pode ser estimado também pela moda da distribuição acima (que corresponde ao estimador de Bayes quando se adota a função perda 0-1).

A média da posteriori de $k$ pode ser estimada por

$$
\frac{1}{m\left(T-T_{0}\right)} \sum_{i=1}^{m} \sum_{j=T_{0}+1}^{T} E\left[K \mid \theta_{i}^{(j)},{\underset{\sim}{x}}_{i}^{(j)}, D_{\text {obs }}\right],
$$

onde

$$
E\left(K \mid{\underset{\theta}{i}}_{i}^{(j)}, \underset{\sim}{x_{i}^{(j)}}, D_{\mathrm{obs}}\right]=\sum_{k \in \mathcal{K}} k P\left[K=k \mid{\underset{\sim}{\theta}}_{i}^{(j)}, \underset{\sim}{x}{ }_{i}^{(j)}, D_{\mathrm{obs}}\right]
$$

e a variância da posteriori pode ser estimada por

$$
\begin{aligned}
& \frac{1}{m\left(T-T_{0}\right)} \sum_{i=1}^{m} \sum_{j=T_{0}+1}^{T} \operatorname{Var}\left(K \mid \underline{\sim}_{i}^{(j)},{\underset{x}{i}}_{i}^{(j)}, D_{\text {obs }}\right) \\
& +\frac{1}{m\left(T-T_{0}\right)} \sum_{i=1}^{m} \sum_{j=T_{0}+1}^{T}\left(E\left[K \mid \theta_{i}^{(j)}, x_{i}^{(j)}, D_{\mathrm{obs}}\right]\right)^{2} \\
& -\left(\frac{1}{m\left(T-T_{0}\right)} \sum_{i=1}^{m} \sum_{j=T_{0}+1}^{T} E\left(K \mid \theta_{i}^{(j)}, \underset{\sim}{x_{i}^{(j)}}, D_{\mathrm{obs}}\right)\right)^{2} \text {, }
\end{aligned}
$$


onde

$$
\begin{aligned}
& \operatorname{Var}\left(K \mid{\underset{\theta}{i}}_{i}^{(j)},{\underset{\sim}{x}}_{i}^{(j)}, D_{\text {obs }}\right)= \\
& \sum_{k \in \mathcal{K}} k^{2} P\left[K=k \mid{\underset{\sim}{i}}_{i}^{(j)}, \underset{\sim}{x_{i}^{(j)}}, D_{\mathrm{obs}}\right]-\left(\sum_{k \in \mathcal{K}} k P\left[K=k \mid \underset{\sim}{\theta_{i}^{(j)}}, \underset{\sim}{x_{i}^{(j)}}, D_{\mathrm{obs}}\right]\right)^{2} .
\end{aligned}
$$

As distribuições marginais à posteriori dos demais parâmetros do modelo, bem como suas médias e variâncias, podem ser estimados de forma semelhante à da seção 2.9 utilizando as distribuições condicionais completas dadas em (4.2.4) a (4.2.13).

\subsection{Implementação do algoritmo de Gibbs no mo- delo que permite mudança em um subcon- junto fixado de parâmetros}

Nesta seção o objetivo é implementar o algoritmo de Gibbs para amostrar da posteriori do modelo estrutural com priori própria de componentes independentes e que permite mudança apenas em um subconjunto não vazio contido estritamente em $\left\{\alpha, \beta, \mu, \sigma_{x}^{2}, \sigma^{2}\right\}$.

Temos 30 possíveis modelos diferentes $\left(\left(\begin{array}{l}5 \\ 1\end{array}\right)+\left(\begin{array}{l}5 \\ 2\end{array}\right)+\left(\begin{array}{l}5 \\ 3\end{array}\right)+\left(\begin{array}{l}5 \\ 4\end{array}\right)\right)$ que permitem mudanças em até quatro parâmetros simultaneamente. Entretanto percebemos que se as distribuições condicionais completas do modelo que permite mudança em todos os cinco parâmetros forem conhecidas, isto é, se conhecermos primeiramente as seguintes distribuições de $\left(\mathrm{a}_{1}\right)$ a $(\mathrm{g})$ abaixo:

(a $)\left[x_{i} \mid x_{(-i)}, \alpha_{1}, \alpha_{2}, \beta_{1}, \beta_{2}, \mu_{1}, \mu_{2}, \sigma_{x(1)}^{2}, \sigma_{x(2)}^{2}, \sigma_{1}^{2}, \sigma_{2}^{2}, k, D_{\text {obs }}\right]$, para $i=1, \ldots, k$,

$\left(\mathrm{a}_{2}\right)\left[x_{i} \mid x_{(-i)}, \alpha_{1}, \alpha_{2}, \beta_{1}, \beta_{2}, \mu_{1}, \mu_{2}, \sigma_{x(1)}^{2}, \sigma_{x(2)}^{2}, \sigma_{1}^{2}, \sigma_{2}^{2}, k, D_{\mathrm{obs}}\right]$, para $i=k+1, \ldots, n$,

$\left(\mathrm{b}_{1}\right)$ e $\left(\mathrm{b}_{2}\right)\left[\alpha_{i} \mid \underset{\sim}{x}, \alpha_{(-i)}, \beta_{1}, \beta_{2}, \mu_{1}, \mu_{2}, \sigma_{x(1)}^{2}, \sigma_{x(2)}^{2}, \sigma_{1}^{2}, \sigma_{2}^{2}, k, D_{\mathrm{obs}}\right], i=1,2$,

$\left(c_{1}\right)$ e $\left(c_{2}\right)\left[\beta_{i} \mid \underset{\sim}{x}, \alpha_{1}, \alpha_{2}, \beta_{(-i)}, \mu_{1}, \mu_{2}, \sigma_{x(1)}^{2}, \sigma_{x(2)}^{2}, \sigma_{1}^{2}, \sigma_{2}^{2}, k, D_{\text {obs }}\right], i=1.2$. 
$\left(\mathrm{d}_{1}\right)$ e $\left(\mathrm{d}_{2}\right)\left[\mu_{i} \mid \underset{\sim}{x}, \alpha_{1}, \alpha_{2}, \beta_{1}, \beta_{2}, \mu_{(-i)}, \sigma_{x(1)}^{2}, \sigma_{x(2)}^{2}, \sigma_{1}^{2}, \sigma_{2}^{2}, k, D_{\text {obs }}\right], i=1,2$

$\left(\mathrm{e}_{1}\right)$ e $\left(\mathrm{e}_{2}\right)\left[\sigma_{x(i)}^{2} \mid \underset{\sim}{x}, \alpha_{1}, \alpha_{2}, \beta, \beta_{2}, \mu_{1}, \mu_{2}, \sigma_{x(-i)}^{2}, \sigma_{1}^{2}, \sigma_{2}^{2}, k, D_{\mathrm{obs}}\right], i=1,2$,

$\left(\mathrm{f}_{1}\right)$ e $\left(\mathrm{f}_{2}\right)\left[\sigma_{i}^{2} \mid \underset{\sim}{x}, \alpha_{1}, \alpha_{2}, \beta_{1}, \beta_{2}, \mu_{1}, \mu_{2}, \sigma_{x(1)}^{2}, \sigma_{x(2)}^{2}, \sigma_{(-i)}^{2}, k, D_{\mathrm{obs}}\right], i=1,2$,

(g) $P\left[K=k \mid \underset{\sim}{x}, \alpha_{1}, \alpha_{2}, \beta_{1}, \beta_{2}, \mu_{1}, \mu_{2}, \sigma_{x(1)}^{2}, \sigma_{x(2)}^{2}, \sigma_{1}^{2}, \sigma_{2}^{2}, k, D_{\text {obs }}\right], k \in \mathcal{K}$

e além disso, se conhecermos também as distribuições condicionais completas de (h) a (l) abaixo,

(h) $\left[\alpha \mid \beta_{1}, \beta_{2}, \mu_{1}, \mu_{2}, \sigma_{x(1)}^{2}, \sigma_{x(2)}^{2}, \sigma_{1}^{2}, \sigma_{2}^{2}, \underset{\sim}{x}, k, D_{\text {obs }}\right]$ do modelo que assume que o intercepto $\alpha$ não muda (mas os demais parâmetros podem mudar),

(i) $\left[\beta \mid \alpha_{1}, \alpha_{2}, \mu_{1}, \mu_{2}, \sigma_{x(1)}^{2}, \sigma_{x(2)}^{2}, \sigma_{1}^{2}, \sigma_{2}^{2}, \underset{\sim}{x}, k, D_{\text {obs }}\right]$ do modelo que assume que $\beta$ não muda (mas os demais parâmetros podem mudar),

(j) $\left[\mu \mid \alpha_{1}, \alpha_{2}, \beta_{1}, \beta_{2}, \sigma_{x(1)}^{2}, \sigma_{x(2)}^{2}, \sigma_{1}^{2}, \sigma_{2}^{2}, \underset{\sim}{x}, k, D_{\text {obs }}\right]$ do modelo que assume que $\mu$ não muda (mas os demais parâmetros podem mudar),

(k) $\left[\sigma_{x}^{2} \mid \alpha_{1}, \alpha_{2}, \beta_{1}, \beta_{2}, \mu_{1}, \mu_{2}, \sigma_{1}^{2}, \sigma_{2}^{2}, \underset{\sim}{x}, k, D_{\text {obs }}\right]$ do modelo que assume que $\sigma_{x}^{2}$ não muda (mas os demais podem mudar),

(1) $\left[\sigma^{2} \mid \alpha_{1}, \alpha_{2}, \beta_{1}, \beta_{2}, \mu_{1}, \mu_{2}, \sigma_{x(1)}^{2}, \sigma_{x(2)}^{2}, \underset{\sim}{x}, k, D_{\text {obs }}\right]$ do modelo que assume que $\sigma^{2}$ não muda (mas os demais podem mudar),

então seremos capazes de obter quaisquer distribuições condicionais completas que quisermos, bastando fazer as restrições convenientes, e portanto do ponto de vista computacional teremos apenas o trabalho de escrever basicamente um único programa que servirá para todos os 31 possíveis modelos.

Por exemplo, para implementar o algoritmo de Gibbs no modelo que permite mudança em pelo menos um dos parâmetros $\left\{\alpha, \beta, \sigma^{2}\right\}$, as distribuiçōes condicionais 
completas necessárias são obtidas a partir de $\left(a_{1}\right),\left(a_{2}\right),\left(b_{1}\right),\left(b_{2}\right),\left(f_{1}\right),\left(f_{2}\right),(g),(j)$ e (k) impondonse as restrições $\mu_{i}=\mu \mathrm{e} / \mathrm{ou} \sigma_{x(i)}^{2}=\sigma_{x}^{2}$ nas distribuições que envolverem os parâmetros $\mu_{i} \mathrm{e} /$ ou $\sigma_{x(i)}^{2}$, para $i=1,2$, como também no valor inicial do algoritmo.

As distribuições condicionais de $\left(\mathrm{a}_{1}\right)$ a $(\mathrm{g})$ são dadas, respectivamente, por (4.2.3), $(4.2 .4), \ldots,(4.2 .14)$ e as distribuições de (h) até (l) são dadas respectivamente por (4.3.1) a (4.3.5) a seguir:

$$
\begin{aligned}
& \left.\alpha \mid \beta_{1}, \beta_{2}, \mu_{1}, \mu_{2}, \sigma_{x(1)}^{2}, \sigma_{x(2)}^{2}, \sigma_{1}^{2}, \sigma_{2}^{2}, k, \underset{\sim}{x}, D_{\mathrm{obs}}\right) \sim \\
& N\left(\frac{\sigma_{2}^{2} \sigma_{\alpha}^{2} \sum_{i=1}^{k}\left(Y_{i}-\beta_{1} x_{i}\right)+\sigma_{1}^{2} \sigma_{\alpha}^{2} \sum_{i=k+1}^{n}\left(Y_{i}-\beta_{2} x_{i}\right)+\lambda \sigma_{1}^{2} \sigma_{2}^{2} a}{k \sigma_{2}^{2} \sigma_{\alpha}^{2}+(n-k) \sigma_{1}^{2} \sigma_{\alpha}^{2}+\lambda \sigma_{1}^{2} \sigma_{2}^{2}} ;\right. \\
& \left.\frac{\lambda \sigma_{1}^{2} \sigma_{2}^{2} \sigma_{\alpha}^{2}}{k \sigma_{2}^{2} \sigma_{\alpha}^{2}+(n-k) \sigma_{1}^{2} \sigma_{\alpha}^{2}+\lambda \sigma_{1}^{2} \sigma_{2}^{2}}\right),
\end{aligned}
$$

$$
\begin{aligned}
& \beta \mid \alpha_{1}, \alpha_{2}, \mu_{1}, \mu_{2}, \sigma_{x(1)}^{2}, \sigma_{x(2)}^{2}, \sigma_{1}^{2}, \sigma_{2}^{2}, k, \underset{\sim}{x}, D_{\text {obs }} \sim \\
& N\left(\frac{\sigma_{2}^{2} \sigma_{\beta}^{2} \sum_{i=1}^{k}\left(Y_{i}-\alpha_{1}\right) x_{i}+\sigma_{1}^{2} \sigma_{\beta}^{2} \sum_{i=k+1}^{n}\left(Y_{i}-\alpha_{2}\right) x_{i}+\lambda \sigma_{1}^{2} \sigma_{2}^{2} b}{\sigma_{2}^{2} \sigma_{\beta}^{2} \sum_{i=1}^{k} x_{i}^{2}+\sigma_{2}^{2} \sigma_{\beta}^{2} \sum_{i=k+1}^{n} x_{i}^{2}+\lambda \sigma_{1}^{2} \sigma_{2}^{2}} ;\right. \\
& \left.\frac{\sigma_{1}^{2} \sigma_{2}^{2} \sigma_{\beta}^{2}}{\sigma_{2}^{2} \sigma_{\beta}^{2} \sum_{i=1}^{k} x_{i}^{2}+\sigma_{2}^{2} \sigma_{\beta}^{2} \sum_{i=k+1}^{n} x_{i}^{2}+\lambda \sigma_{1}^{2} \sigma_{2}^{2}}\right) \text {, } \\
& \left.\mu \mid \alpha_{1}, \alpha_{2}, \beta_{1}, \beta_{2}, \sigma_{x(1)}^{2}, \sigma_{x(2)}^{2}, \sigma_{1}^{2}, \sigma_{2}^{2}, k, \underset{\sim}{x}, D_{\mathrm{obs}}\right) \sim \\
& N\left(\frac{\sigma_{x(2)}^{2} \sigma_{\mu}^{2} \sum_{i=1}^{k} x_{i}+\sigma_{x(1)}^{2} \sigma_{\mu}^{2} \sum_{i=k+1}^{n} x_{i}+\sigma_{x(1)}^{2} \sigma_{x(2)}^{2} m}{k \sigma_{x(2)}^{2} \sigma_{\mu}^{2}+(n-k) \sigma_{x(1)}^{2} \sigma_{\mu}^{2}+\sigma_{x(1)}^{2} \sigma_{x(2)}^{2}} ;\right. \\
& \left.\frac{\sigma_{x(1)}^{2} \sigma_{x(2)}^{2} \sigma_{\mu}^{2}}{k \sigma_{x(2)}^{2} \sigma_{\mu}^{2}+(n-k) \sigma_{x(1)}^{2} \sigma_{\mu}^{2} \Sigma+\sigma_{x(1)}^{2} \sigma_{x(2)}^{2}}\right), \\
& \sigma_{x}^{2} \mid \alpha_{1}, \alpha_{2}, \beta_{1}, \beta_{2}, \mu_{1}, \mu_{2}, \sigma_{1}^{2}, \sigma_{2}^{2}, k, \underset{\sim}{x}, D_{\mathrm{obs}} \sim \\
& I G\left(\frac{n}{2}+c ; \frac{1}{2}\left(\sum_{i=1}^{k}\left(x_{i}-\mu_{1}\right)^{2}+\sum_{i=k+1}^{n}\left(x_{i}-\mu_{2}\right)^{2}\right)+d\right) \text {, } \\
& \sigma^{2} \mid a_{1}, \alpha_{2}, \beta_{1}, \beta_{2}, \mu, \mu_{2}, \sigma_{x(1)}^{2}, \sigma_{x(2)}^{2}, k, \underset{\sim}{x}, D_{\mathrm{obs}} \sim
\end{aligned}
$$




$$
I G\left(n+f ; \frac{1}{2 \lambda}\left(\sum_{i=1}^{k}\left(Y_{i}-\alpha_{1}-\beta_{1} x_{i}\right)^{2}+\sum_{i=k+1}^{n}\left(Y_{i}-\alpha_{2}-\beta_{2} x_{i}\right)^{2}+\lambda \sum_{i=1}^{n}\left(X_{i}-x_{i}\right)^{2}\right)+g\right) .
$$

\section{Observações:}

1. As distribuições em (4.3.1) a (4.3.5) coincidem com as distribuições em (5.1.22) a (5.1.26) quando substituímos $w_{i}$, por 1 , para $i=1, \ldots, n$.

2. Na distribuição de $K$ dada em (g), o conjunto $\mathcal{K}$ pode diferir dependendo do modelo, entretanto $\mathcal{K}$ pode ser sempre igual a $\{1, \ldots, n-1\}$, como já foi discutido anteriormente.

3. A estimação dos parâmetros do modelo que admite mudança apenas em um subconjunto de parâmetros pode ser feita de forma semelhante à da seção 4.2.3.

4. A análise do modelo com priori análoga à da seção 2.7 (isto é priori de componentes condicionalmente independentes), pode ser feita também de forma análoga.

\subsection{Detecção de mudança}

Nas seções anteriores foram considerados modelos com um único ponto de mudança e o problema era estimar a localização desse ponto bem como estimar os demais parâmetros. Entretanto, em muitas situações nas aplicações não sabemos se há ou não mudança nos parâmetros. Nessa seção apresentamos três procedimentos distintos para analisar o problema, que serão descritos nas subseções 4.4.1, 4.4.2 e 4.4.3, respectivamente. 


\subsubsection{Procedimento 1 - Um teste baseado na distribuição à posteriori de $k$}

Uma maneira de analisar o problema é reformular o modelo permitindo que $k$ possa assumir o valor $n$, ou seja, permitir que o modelo inclua a possibilidade de que não haja mudança nos parâmetros (via $k=n$ ) e então testar a hipótese nula de que não há mudança $\left(H_{0}: k=n\right)$ versus a alternativa $\left(H_{1}\right)$ de que há exatamente um ponto de mudança (isto é, $H_{1}: k \neq n$ ).

Nessa subseção, apresentamos um teste bayesiano informal baseado na distribuição marginal à posteriori de $k$, que consiste simplesmente em comparar a probabilidade à posteriori $P\left[k=n \mid D_{\text {obs }}\right]$ de que não há mudança com a probabilidade $1-P\left(k=n \mid D_{\text {obs }}\right)$ de que há uma mudança. (Broemeling e Choy, 1981, desenvolveram esse teste para o modelo de regressão clássico que permite mudança no coeficiente angular e no intercepto.

É interessante notar que nesse caso o teste de hipóteses e a estimação dos parâmetros, são feitos simultaneamente.

A seguir, apresentamos o modelo estendido que nos permite realizar o teste bayesiano mencionado, e depois apresentamos os passos para implementar o algoritmo de Gibbs, com o objetivo de amostrar da posteriori desse modelo estendido.

O modelo estendido que permite mudança em todos os parâmetros:

$$
\left\{\begin{array}{l}
Y_{i}= \begin{cases}\left\{\begin{array}{l}
\alpha_{1}+\beta_{1} x_{i}+e_{i}, \quad i=1,2, \ldots, k, \\
\alpha_{2}+\beta_{2} x_{i}+e_{i}, \quad i=k+1, \ldots, n, \\
\alpha_{1}+\beta_{1} x_{i}+e_{i}, i=1, \ldots, n,
\end{array}\right. & \text { se } 1 \leq k \leq n-1\end{cases} \\
X_{i}=x_{i}+u_{i}, \quad i=1, \ldots, n,
\end{array}\right.
$$

onde $\left(e_{i}, u_{i}, x_{i}\right)^{T}$ são independentes $\mathrm{e}$ 


$$
\left(\begin{array}{c}
e_{i} \\
u_{i} \\
x_{i}
\end{array}\right) \sim\left\{\begin{array}{l}
\left(\begin{array}{l}
N\left(\left(\begin{array}{c}
0 \\
0 \\
\mu_{1}
\end{array}\right) ;\left(\begin{array}{ccc}
\lambda \sigma_{1}^{2} & 0 & 0 \\
0 & \sigma_{1}^{2} & 0 \\
0 & 0 & \sigma_{x(1)}^{2}
\end{array}\right)\right) i=1, \ldots, k, \\
N\left(\left(\begin{array}{c}
0 \\
0 \\
\mu_{2}
\end{array}\right) ;\left(\begin{array}{ccc}
\lambda \sigma_{2}^{2} & 0 & 0 \\
0 & \sigma_{2}^{2} & 0 \\
0 & 0 & \sigma_{x(2)}^{2}
\end{array}\right)\right) i=k+1, \ldots, n, \\
N\left(\left(\begin{array}{c}
0 \\
0 \\
\mu_{1}
\end{array}\right) ;\left(\begin{array}{ccc}
\lambda \sigma_{1}^{2} & 0 & 0 \\
0 & \sigma_{1}^{2} & 0 \\
0 & 0 & \sigma_{x(1)}^{2}
\end{array}\right)\right) i=1, \ldots, n, \text { se } k=n
\end{array}\right.
\end{array}\right.
$$

Notemos que para $1 \leq k \leq n-1$ então $\underset{\sim}{\theta_{1}} \neq \underset{\sim}{\theta_{2}}$, onde $\underset{\sim}{\theta_{i}}=\left(\alpha_{i}, \beta_{i}, \mu_{i}, \sigma_{x(i)}^{2}, \sigma_{i}^{2}\right)^{T}$ e para $k=n$ então $\underset{\sim}{\theta_{1}}={\underset{\sim}{2}}_{2}$.

Portanto, esse modelo permite que haja mudança em todos os parâmetros como também que não haja mudança em nenhum.

Nesse modelo o vetor de parâmetros é $\underset{\stackrel{*}{*}}{*}=\left(\begin{array}{c}\theta_{1} \\ \ddot{\theta}_{2} \\ k\end{array}\right)$ e, se $1 \leq k \leq n-1$, a função de verossimilhança $L(\underset{\stackrel{*}{\theta}}{\mid} D)$ é proporcional a

$$
\begin{aligned}
& \left(\sigma_{x(1)}^{2}\right)^{-k / 2}\left(\sigma_{1}^{2}\right)^{-k} \exp \left\{-\frac{1}{2}\left[\frac{\sum_{i=1}^{k}\left(Y_{i}-\alpha_{1}-\beta_{1} x_{i}\right)^{2}}{\lambda \sigma_{1}^{2}}+\frac{\sum_{i=1}^{k}\left(X_{i}-x_{i}\right)^{2}}{\sigma_{1}^{2}}\right.\right. \\
& \left.\left.+\frac{\sum_{i=1}^{k}\left(x_{i}-\mu_{1}\right)^{2}}{\sigma_{x(1)}^{2}}\right]\right\}\left(\sigma_{x(2)}^{2}\right)^{-(n-k) / 2}\left(\sigma_{2}^{2}\right)^{-(n-k)} \exp \left\{-\frac{1}{2}\left[\frac{\sum_{i=k+1}^{n}\left(Y_{i}-\alpha_{2}-\beta_{2} x_{i}\right)^{2}}{\lambda \sigma_{2}^{2}}\right.\right. \\
& \left.\left.+\frac{\sum_{i=1}^{n}\left(X_{i}-x_{i}\right)^{2}}{\sigma_{2}^{2}}+\frac{\sum_{i=k+1}^{n}\left(x_{i}-\mu_{2}\right)^{2}}{\sigma_{x(2)}^{2}}\right]\right\} .
\end{aligned}
$$

Se $k=n$, a função de verossimilhança é proporcional a

$$
\begin{aligned}
& \left(\sigma_{x(1)}^{2}\right)^{-n / 2}\left(\sigma_{1}^{2}\right)^{-n} \exp \left\{-\frac{1}{2}\left[\frac{\sum_{i=1}^{n}\left(Y_{i}-\alpha_{1}-\beta_{1} x_{i}\right)^{2}}{\lambda \sigma_{1}^{2}}+\frac{\sum_{i=1}^{n}\left(X_{i}-x_{i}\right)^{2}}{\sigma_{1}^{2}}\right.\right. \\
& \left.\left.\quad+\frac{\sum_{i=1}^{n}\left(x_{i}-\mu_{1}\right)^{2}}{\sigma_{x(1)}^{2}}\right]\right\} .
\end{aligned}
$$

Uma priori conveniente nesse caso é dada por

$$
\pi(\stackrel{*}{\theta})= \begin{cases}p(k) \pi\left(\underline{\theta}_{1}\right), & \text { se } k=n \\ p(k) \pi\left(\underline{\theta}_{1}\right) \pi\left(\underline{\theta}_{2}\right), & \text { se } k \in \mathcal{K}=\{1, \ldots, n-1\}, \\ 0, & \text { caso contrário }\end{cases}
$$


onde

$$
p(k)= \begin{cases}c, & \text { se } k=n \\ (1-c) \frac{1}{\# \mathcal{K}}, & \text { se } k \in \mathcal{K} \\ 0, & \text { caso contrário }\end{cases}
$$

$\mathrm{e}$

$$
\pi\left(\theta_{i}\right)=\pi\left(\alpha_{i}\right) \pi\left(\beta_{i}\right) \pi\left(\mu_{i}\right) \pi\left(\sigma_{x(i)}^{2}\right) \pi\left(\sigma_{i}^{2}\right),
$$

onde $\alpha_{i} \sim N\left(a_{i}, \sigma_{\alpha_{i}}^{2}\right), \beta_{i} \sim N\left(b_{i}, \sigma_{\beta_{i}}^{2}\right), \mu_{i} \sim N\left(m_{i}, \sigma_{\mu_{i}}^{2}\right), \sigma_{x(i)}^{2} \sim I G\left(c_{i}, d_{i}\right), \sigma_{i}^{2} \sim$ $I G\left(f_{i}, g_{i}\right), i=1,2$.

Na próxima seção apresentamos as distribuições condicionais completas à posteriori que serão utilizadas para implementar o algoritmo de Gibbs.

\section{Implementação do algoritmo de Gibbs:}

A seguir apresentamos as distribuições condicionais completas baseadas na posteriori do modelo estendido usadas para implementar o algoritmo de Gibbs.

Passo 0: Fornecer um vetor inicial $\underset{\sim}{(0)}=\left(k^{(0)}, \underset{\sim}{\theta_{1}^{(0)}}, \underset{2}{\theta_{2}^{(0)}}\right)^{T}$, onde $\stackrel{\theta}{i}_{i}^{(0)}=\left(\alpha_{i}^{(0)}, \beta_{i}^{(0)}, \mu_{i}^{(0)}, \sigma_{x(i)}^{(0)}, \sigma_{i}^{2^{(0)}}\right)^{T}, i=1,2$.

Passo 1:

- Se $k=n$ :

então gere $\underset{\sim}{x}=\left(x_{1}, \ldots, x_{n}\right)^{T}, \alpha_{1}, \beta_{1}, \mu_{1}, \sigma_{x(1)}^{2}$ e $\sigma_{1}^{2}$, onde:

$$
\begin{aligned}
& x_{i} \mid x_{(-i)}, \underset{\sim}{*}, D_{\mathrm{obs}} \sim \\
& N\left(\sigma_{x(1)}^{2}\left[\frac{\lambda X_{i}+\beta_{1}\left(Y_{i}-\alpha_{1}\right)+\lambda \sigma_{1}^{2} \mu_{1}}{\sigma_{x(1)}^{2}\left(\lambda+\beta_{1}^{2}\right)+\lambda \sigma_{1}^{2}}\right] ; \frac{\lambda \sigma_{1}^{2} \sigma_{x(1)}^{2}}{\sigma_{x(1)}^{2}\left(\lambda+\beta_{1}^{2}\right)+\lambda \sigma_{1}^{2}}\right) \\
& \quad \operatorname{para} i=1, \ldots, n, \\
& \alpha_{1} \mid \underset{\sim}{x}, \alpha_{2}, \beta_{1}, \beta_{2}, \mu_{1}, \mu_{2}, \sigma_{x(1)}^{2}, \sigma_{x(1)}^{2}, \sigma_{1}^{2}, \sigma_{2}^{2}, D_{\mathrm{obs}} \sim \\
& N\left(\frac{\sigma_{\alpha_{1}}^{2} \sum_{i=1}^{k}\left(Y_{i}-\beta_{1} x_{i}\right)+\lambda \sigma_{1}^{2} a_{1}}{n \sigma_{\alpha_{1}}^{2}+\lambda \sigma_{1}^{2}} ; \frac{\lambda \sigma_{1}^{2} \sigma_{\alpha_{1}}^{2}}{n \sigma_{\alpha_{1}}^{2}+\lambda \sigma_{1}^{2}}\right)
\end{aligned}
$$




$$
\begin{aligned}
& \beta_{1} \mid \underset{\sim}{x}, \alpha_{1}, \alpha_{2}, \beta_{2}, \mu_{1}, \mu_{2}, \sigma_{x(1)}^{2}, \sigma_{x(2)}^{2}, \sigma_{1}^{2}, \sigma_{2}^{2}, D_{\mathrm{obs}} \sim \\
& N\left(\frac{\sigma_{\beta_{1}}^{2} \sum_{i=1}^{n}\left(Y_{i}-\alpha_{1}\right) x_{i}+\lambda b_{1} \sigma_{1}^{2}}{\sigma_{\beta_{1}}^{2} \sum_{i=1}^{n} x_{i}^{2}+\lambda \sigma_{1}^{2}} ; \frac{\lambda \sigma_{1}^{2} \sigma_{\beta_{1}}^{2}}{\sigma_{\beta_{1}}^{2} \sum_{i=1}^{n} x_{i}^{2}+\lambda \sigma_{1}^{2}}\right) \\
& \mu_{1} \mid \underset{\sim}{x}, \alpha_{1}, \alpha_{2}, \beta_{1}, \beta_{2}, \sigma_{x(1)}^{2}, \sigma_{x(2)}^{2}, \sigma_{1}^{2}, \sigma_{2}^{2}, D_{\mathrm{obs}} \sim \\
& N\left(\frac{\sigma_{\mu_{1}}^{2} \sum_{i=1}^{n} x_{i}+\sigma_{x(1)}^{2} m_{1}}{n \sigma_{\mu_{1}}^{2}+\sigma_{x(1)}^{2}} ; \frac{\sigma_{\mu_{1}}^{2} \sigma_{x(2)}^{2}}{n \sigma_{\mu_{1}}^{2}+\sigma_{x(1)}^{2}}\right) \\
& \sigma_{x(1)}^{2} \mid \underset{\sim}{x}, \alpha_{1}, \alpha_{2}, \beta_{1}, \beta_{2}, \mu_{1}, \mu_{2}, \sigma_{x(2)}^{2}, \sigma_{1}^{2}, \sigma_{2}^{2}, D_{\mathrm{obs}} \sim \\
& I G\left(\frac{n}{2}+c_{1} ; \frac{1}{2} \sum_{i=1}^{n}\left(x_{i}-\mu_{1}\right)^{2}+d_{1}\right) \\
& \quad \sigma_{1}^{2} \mid \underset{\sim}{x}, \alpha_{1}, \alpha_{2}, \beta_{1}, \beta_{2}, \mu_{1}, \mu_{2}, \sigma_{x(1)}^{2}, \sigma_{x(2)}^{2}, \sigma_{2}^{2}, D_{\mathrm{obs}} \sim \\
& I G\left(n+f_{1} ; \frac{1}{2}\left[\frac{\sum_{i=1}^{n}\left(Y_{i}-\alpha_{1}-\beta_{1} x_{i}\right)^{2}}{\lambda}+\sum_{i=1}^{n}\left(X_{i}-x_{i}\right)^{2}\right]+g_{1}\right),
\end{aligned}
$$

Faça:

$$
\alpha_{2}=\alpha_{1}, \beta_{2}=\beta_{1}, \mu_{2}=\mu_{1}, \sigma_{x(2)}^{2}=\sigma_{x(1)}^{2}, \sigma_{2}^{2}=\sigma_{1}^{2} .
$$

- Caso contrário, se $1 \leq k \leq n-1$ :

então gere $\underset{\sim}{x}, \alpha_{1}, \alpha_{2}, \beta_{1}, \beta_{2}, \mu_{1}, \mu_{2}, \sigma_{x(1)}^{2}, \sigma_{x(2)}^{2}, \sigma_{1}^{2}$ e $\sigma_{2}^{2}$ usando as fórmulas (4.2.3) a (4.2.13), respectivamente.

Passo 2: Gerar um valor de $K$ da distribuição discreta

$$
\begin{aligned}
& P\left(K=k \mid \underset{\sim}{x}, \alpha_{1}, \alpha_{2}, \beta_{1}, \beta_{2}, \mu_{1}, \mu_{2}, \sigma_{x(1)}^{2}, \sigma_{x(2)}^{2}, \sigma_{1}^{2}, \sigma_{2}^{2}, D_{\mathrm{obs}}\right) \\
& =\left\{\begin{array}{c}
\frac{L_{1}\left(\underline{\theta}_{1} \mid D\right) p(n) \pi\left(\underline{\theta}_{1}\right)}{L_{1}\left(\underline{\theta}_{1} \mid D\right) p(n) \pi\left(\theta_{1}\right)+\sum_{k=1}^{n-1} L\left(k, \theta_{1}, \theta_{2} \mid D\right) p(k) \pi\left(\underline{\theta}_{1}\right) \pi\left(\underline{\theta}_{2}\right)}, \text { se } k=n, \\
\frac{L\left(k, \underline{\theta}_{1}, \theta_{2} D\right) p(k) \pi\left(\underline{\theta}_{1}\right) \pi\left(\underline{\theta}_{2}\right)}{L_{1}\left(\underline{\theta}_{1} \mid D\right) p(n) \pi\left(\underline{\theta}_{1}\right)+\sum_{k=1}^{n-1} L\left(k, \underline{\sim}_{1}, \underline{\sim}_{2} \mid D\right) p(k) \pi\left(\underline{\sim}_{1}\right) \pi\left(\underline{\theta}_{2}\right)}, 1 \leq k \leq n-1,
\end{array}\right.
\end{aligned}
$$

onde:

$$
p(k)=\text { probabilidade à priori de que } K=k(k=1,2, \ldots, n) .
$$


dada por exemplo por (4.4.4) e (4.4.5),

$L\left(k,{\underset{\theta}{1}}_{1}, \theta_{2} \mid D\right)$ é proporcional a (4.4.2),

$L_{1}\left(\theta_{1} \mid D_{\text {obs }}\right)$ é proporcional a $(4.4 .3)$,

$$
\begin{aligned}
\pi\left(\underline{\sim}_{2}\right)= & M\left(\sigma_{x(2)}^{2}\right)^{-\left(c_{2}+1\right)}\left(\sigma_{2}^{2}\right)^{-\left(f_{2}+1\right)} \exp \left\{-\frac{1}{2}\left[\frac{\left(\alpha_{2}-a_{2}\right)^{2}}{\sigma_{\alpha_{2}}^{2}}+\frac{\left(\beta_{2}-b_{2}\right)^{2}}{\sigma_{\beta_{2}}^{2}}+\frac{\left(\mu_{2}-m_{2}\right)^{2}}{\sigma_{\mu_{2}}^{2}}\right.\right. \\
& \left.\left.+\frac{2 d_{2}}{\sigma_{x(2)}^{2}}+\frac{2 g_{2}}{\sigma_{2}^{2}}\right]\right\},
\end{aligned}
$$

A constante de proporcionalidade $M$ é igual a

$$
\frac{d_{2}^{c_{2}} g_{2}^{f_{2}}}{\Gamma\left(c_{2}\right) \Gamma\left(f_{2}\right) 2 \pi \sqrt{2 \pi \sigma_{\alpha_{2}}^{2} \sigma_{\beta_{2}}^{2} \sigma_{\mu_{2}}^{2}}} .
$$

Passo 3: volte ao Passo 1.

Usando as distribuições condicionais completas acima podemos implementar o algoritmo de Gibbs com o objetivo de amostrar da posteriori do modelo dado por (4.4.1), (4.4.4), (4.4.5) e (4.4.6).

Se observações geradas pelo algoritmo constituem de fato uma amostra da posteriori, podemos estimar a distribuição à posteriori marginal de $k$ da mesma forma que na subseção 4.2 .3 (só que agora $k$ pode assumir o valor $n$ ) e se $P\left(k=n \mid D_{\text {obs }}\right)>$ $1-P\left(k=n \mid D_{\text {obs }}\right)$, então aceitamos $H_{0}$; caso contrário, rejeitamos $H_{0}$.

Observação 1: No caso do modelo que permite mudança em um subconjunto de até quatro parâmetros podemos testar a hipótese $H_{0}$ versus $H_{1}$ usando a distribuição marginal à posteriori de $k$ de forma semelhante à descrita acima, fazendo uma analogia com o procedimento da seção 4.3 .

Observação 2: É importante que se faça uma análise de sensibilidade da distribuição à posteriori de $k$ a diferentes prioris. Uma idéia bem simples é considerar uma grade de vários possíveis valores para o hiperparâmetro $c$ (ond $c=P(k=n)$ à priori) e ver como isso afeta a posteriori marginal de $k$. 
Segundo Kim (1991) que estudou o modelo de regressão clássico (cuja covariável não tem erros de medida), o teste de Bayes tradicional que usa a posteriori de $k$ para detectar mudança é bastante sensível à escolha da priori na maioria dos casos.

\subsubsection{Procedimento 2 - Uso da distribuição à posteriori da diferença (ou quociente) entre os parâmetros.}

Uma outra forma de detetar se existe mudança nos parâmetros é examinar as distribuições marginais à posteriori da diferença (ou quociente) entre os parâmetros "antes e depois da mudança" no modelo que permite mudança em todos os parâmetros. A hipótese $H_{0}$ pode ser dividida nas sub-hipóteses: $H_{01}: \alpha_{1}-\alpha_{2}=0, H_{02}: \beta_{1}-\beta_{2}=$ $0, H_{03}: \mu_{1}-\mu_{2}=0, H_{04}: \frac{\sigma_{x(2)}^{2}}{\sigma_{x(1)}^{2}}=1, H_{05}: \frac{\sigma_{2}^{2}}{\sigma_{1}^{2}}=1$, sendo que $H_{0}$ é rejeitada quando pelo menos uma das sub-hipóteses é rejeitada.

Em princípio a separação da hipótese nula $H_{0}$ nas cinco sub-hipóteses $H_{01}, \ldots, H_{05}$ é útil para determinarmos quais parâmetros mudam (isto é, quais parâmetros são responsáveis pela mudança).

Conceitualmente a decisão, por exemplo, de aceitar a sub-hipótese $H_{02}$ seria razoável se $P\left(\beta_{2}-\beta_{1}>0 \mid D_{\text {obs }}\right)=P\left(\beta_{2}-\beta_{1}<0 \mid D_{\text {obs }}\right)=0.5$. Assim se observarmos por exemplo que $\hat{P}\left(\beta_{2}-\beta_{1}>0 \mid D_{\text {obs }}\right) \approx 0.80$, ficaríamos razoavelmente confiantes de "rejeitar" essa hipótese.

Obs: A análise fica bastante simplificada quando a distribuição da diferença de $\theta_{2}-\theta_{1}$ é analisada apenas marginalmente (em vez de conjuntamente) entretanto o preço é que esta aproximação pode ser bem ruim.

\subsubsection{Procedimeto 3 - Uso de critérios de seleção ou escolha de modelos}

Outra forma de detectar a existência de mudança é tratar o problema de ponto de mudança como um problema de seleção de modelos, isto é utilizar métodos 
bayesianos de comparação (ou escolha) de modelos, para comparar o modelo sem nenhuma mudança com o modelo que permite mudança.

Existem vários critérios de escolha de modelos descritos na literatura para essa finalidade. Alguns dos mais populares são, por exemplo, o método do "Fator de Bayes", o "Pseudo Fator de Bayes", a "Medida L" e o BIC ("Bayesian Information Criterion").

Uma maneira de tratar o problema é comparar o modelo com mudança após o ponto $k$ desconhecido (aleatório) com o modelo sem nenhuma mudança (usando obviamente o mesmo conjunto de dados). Nesse caso, checa-se a existência de mudança e estima-se o ponto de mudança simultâneamente.

Uma alternativa para tratar o problema é para cada $k$ fixado, comparar o modelo com mudança em $k$ com o modelo sem mudança.

A seguir, apresentamos os critérios de escolha mencionados acima para o problema de mudança no modelo estrutural normal com erros nas variáveis.

\section{$\left(1^{\circ}\right) \mathrm{O}$ critério do Fator de Bayes}

O Fator de Bayes é um critério de comparação de dois modelos que se aplica somente no caso de prioris próprias. Na seção [4.4.3] do Apêndice, apresentamos a definição dessa medida.

O Fator de Bayes para comparar os modelos $M_{1, k}$ e $M_{2}$ (onde $M_{1, k}$ é o modelo com mudança nos parâmetros após a $k$-ésima observação e $M_{2}$ é o modelo sem mudanças) é definido por

$$
\mathrm{BF}_{(1, k), 2}=\frac{\pi\left({\underset{\sim}{U}}_{1}, \ldots,{\underset{\sim}{n}}_{n} \mid M_{1, k}\right)}{\pi\left({\underset{\sim}{1}}_{1}, \ldots, \underset{\sim}{U_{n}} \mid M_{2}\right)}
$$

onde $\underset{\sim}{U_{i}}=\left(\begin{array}{l}Y_{2} \\ X_{2}\end{array}\right)$ e onde $\pi\left({\underset{\sim}{1}}_{1}, \ldots, \underset{\sim}{U_{n}} \mid M_{1, k}\right)$ e $\pi\left({\underset{\sim}{1}}_{1}, \ldots, \underset{\sim}{U_{n}} \mid M_{2}\right)$ são as densidades marginais dos dados sob os modelos $M_{1 . k}$ e $M_{2}$, respectivamente. 
Na literatura existem várias técnicas que podem ser usadas para estimar o fator individual de Bayes $B_{(1, k), 2}$. Um método direto proposto por Newton e Raftery (1994), estima o numerador $\pi\left(\underset{\sim}{U_{1}}, \ldots,{\underset{\sim}{U}}_{n} \mid M_{1, k}\right)$ por

$$
\hat{\pi}\left(\underset{\sim}{U_{1}}, \ldots, \underset{\sim}{U_{n} \mid} M_{1, k}\right)=\left(\frac{1}{G} \sum_{g=1}^{G} \frac{1}{\pi\left({\underset{\sim}{1}}_{1}, \ldots,{\underset{\sim}{n}}_{n} \mid \underline{\sim}_{1}^{(g)},{\underset{\sim}{2}}_{2}^{(g)}, k^{(g)}\right)}\right)^{-1},
$$

onde $\left(\left(\begin{array}{c}\theta_{1}^{(1)} \\ \theta_{2}^{(1)} \\ \tilde{k}^{(1)}\end{array}\right), \ldots,\left(\begin{array}{c}\theta_{1}^{(G)} \\ \theta_{2}^{(G)} \\ k^{(g)}\end{array}\right)\right)$ é uma amostra da posteriori sob o modelo $M_{1, k}$ e $\pi\left(\underset{\sim}{U_{1}}, \ldots,{\underset{\sim}{n}}_{n} \mid \theta_{1}, \theta_{2}, k\right)$ é a densidade das observações sob o modelo $M_{1, k}$, descrito em (4.4.1).

O denominador $\pi\left(\underset{\sim}{U_{1}}, \ldots,{\underset{\sim}{n}}_{n} \mid M_{2}\right)$ é estimado por

$$
\hat{\pi}\left({\underset{\sim}{1}}_{1}, \ldots,{\underset{\sim}{n}}_{n} \mid M_{2}\right)=\left(\frac{1}{G} \sum_{g=1}^{G} \frac{1}{\pi\left({\underset{\sim}{1}}_{1}, \ldots,{\underset{\sim}{n}}_{n} \mid \theta^{(g)}\right)}\right)^{-1},
$$

onde $\left({\underset{\sim}{(1)}}^{(1)}, \ldots,{\underset{\theta}{(G)}}^{(G)}\right)$ é uma amostra da posteriori sob o modelo $M_{2}$ definido em (2.3.1) e $\pi\left(U_{\sim}, \ldots,{\underset{\sim}{2}}_{2} \mid \theta\right)$ é a densidade das observações sob esse modelo.

Outra maneira de tratar o problema é usar o método de Dey (1997).

Dey (1997) mostrou que o Fator de Bayes que compara um modelo sem mudança $\left(M_{2}\right)$ com um modelo com mudança $\left(M_{1}\right)$, onde o ponto de mudança $k$ não é especificado, pode ser escrito como uma combinação linear convexa de fatores de Bayes individuais, isto é,

$$
\mathrm{BF}_{1,2}=\sum_{k=1}^{n-1} p_{k} \mathrm{BF}_{(1, k), 2} \text { onde } p_{k}= \begin{cases}(1-c) p[K=k], & 1 \leq k \leq n-1 \\ c, & k=n\end{cases}
$$

é a distribuição à priori de $k$.

Esse resultado pode ser usado, por exemplo, para comparar o modelo estrutural Normal que permite mudança em todos os parâmetros (que está definido em (4.1.1) e tem priori em (4.1.2)) com o modelo estrutural Normal sem mudança que está definido em (2.3.1) e tem priori em (2.3.2). Nesse exemplo, o Fator de Bayes é dado 
por

$$
\mathrm{BF}_{1,2}=\sum_{k \in \mathcal{K}=\{1, \ldots, n-1\}} p_{k} \mathrm{BF}_{(1, k), 2}
$$

onde $p_{k}=\frac{1}{n-1}(1-c)$ (onde $\left.c=P(K=n)=0\right)$ e $\mathrm{BF}_{(1, k), 2}$ é o fator individual de Bayes que compara o modelo com mudança após o ponto $k$ fixado com o modelo sem mudança,

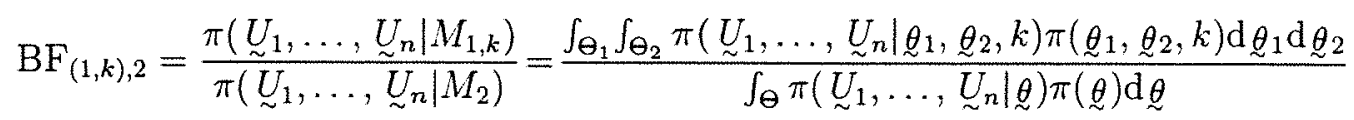
onde $\underset{\sim}{\theta}=\left(\alpha, \beta, \mu, \sigma_{x}^{2}, \sigma^{2}\right)^{T}$ e $\underset{\sim}{\theta_{i}}=\left(\alpha_{i}, \beta_{i}, \mu_{i}, \sigma_{x(i)}^{2}, \sigma_{i}^{2}\right)^{T}, i=1,2$, $\pi\left({\underset{\sim}{1}}_{1}, \ldots,{\underset{\sim}{n}}_{n} \mid \theta_{1},{\underset{\sim}{2}}_{2}, k\right)$ é a densidade das observações sob o modelo $M_{1, k}$ definido em (4.1.1) (com $k$ fixado),

$\pi\left({\underset{\sim}{1}}_{1},{\underset{\sim}{2}}_{2}, k\right)=\pi\left(\underset{\sim}{\theta_{1}}\right) \pi\left({\underset{\sim}{2}}_{2}\right)$, é a distribuição à priori dada em (4.2.1) quando $p(k)=1$, $\pi\left(\underset{\sim}{U_{1}}, \ldots, \underset{\sim}{\left.U_{n} \mid \theta\right)}\right.$ é a densidade das observações sob o modelo $M_{2}$ definido em (2.3.1) e $\pi(\underset{\sim}{\theta})$ é a priori dada em (2.3.2).

O resultado em (4.4.14) será verificado abaixo usando a justificativa dada em Chung e Dey (1996).

Justificativa: O Fator de Bayes para comparar o modelo $M_{1}$ com o modelo $M_{2}$ é

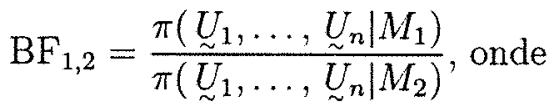

$$
\pi\left({\underset{\sim}{1}}_{1}, \ldots,{\underset{\sim}{U}}_{n} \mid M_{1}\right)=\pi\left({\underset{\sim}{1}}_{1}, \ldots, \underset{\sim}{U_{n}} \mid \text { mudança }\right)=\frac{\pi\left({\underset{\sim}{1}}_{1}, \ldots,{\underset{\sim}{n}}_{n}, 1 \leq k \leq n-1\right)}{P(1 \leq k \leq n-1)},
$$

onde $P(1 \leq k \leq n-1)=1-c$ (onde $c=p[K=n])$ e portanto

$$
\begin{aligned}
& \pi\left(\underset{\sim}{U_{1}}, \ldots, \underset{\sim}{U_{n}} \mid M_{1}\right)=\frac{1}{1-c} \int_{\Theta_{1}} \int_{\Theta_{2}} \pi\left({\underset{\sim}{1}}_{1}, \ldots, \underset{\sim}{U_{n}}, \underset{\sim}{\theta_{1}},{\underset{\sim}{2}}_{2}, 1 \leq k \leq n-1\right) \mathrm{d} \theta_{1} \mathrm{~d} \theta_{2} \\
& =\frac{1}{1-c} \int_{\Theta_{1}} \int_{\Theta_{2}} \pi\left(\underline{\sim}_{1}, \ldots,{\underset{\sim}{n}}_{n} \mid{\underset{\sim}{1}}_{1},{\underset{\sim}{2}}_{2}, 1 \leq k \leq n-1\right) \pi\left({\underset{\sim}{1}}_{1},{\underset{\sim}{2}}_{2}, 1 \leq k \leq n-1\right) \mathrm{d}{\underset{\sim}{1}}_{1} \mathrm{~d}{\underset{\sim}{2}}_{2} \\
& \frac{1}{1-c} \sum_{k=1}^{n-1} \int_{\Theta_{1}} \int_{\Theta_{2}} \pi\left({\underset{\sim}{1}}_{1}, \ldots, \underset{\sim}{U_{n}} \mid{\underset{\sim}{1}}_{1}, \underset{\sim}{\theta_{2}}, k\right) \pi\left(\underset{\underbrace{}_{1}}{\theta_{2}} \underset{2}{\theta_{2}}, k\right) \mathrm{d} \underset{\sim}{\theta_{1}} \mathrm{~d} \underset{\sim}{\theta_{2}} \\
& =\frac{1}{1-c} \sum_{k=1}^{n-1} \frac{(1-c)}{n-1} \int_{\theta_{1}} \int_{\theta_{2}} \pi\left({\underset{\sim}{1}}_{1}, \ldots, \underset{\sim}{U_{n}} \mid{\underset{\sim}{1}}_{1},{\underset{\theta}{2}}_{2}, k\right) \pi\left({\underset{\theta}{1}}_{1},{\underset{\sim}{\theta}}_{2}\right) \mathrm{d}{\underset{\sim}{1}}_{1} \mathrm{~d}{\underset{\sim}{2}}_{2}
\end{aligned}
$$




$$
=\frac{1}{n-1} \sum_{k=1}^{n-1} \pi\left(\underset{\sim}{U_{1}}, \ldots, \underset{\sim}{U_{n}} \mid M_{1, k}\right)
$$

Portanto,

$$
\mathrm{BF}_{1,2}=\frac{1}{n-1} \sum_{k=1}^{n-1} \frac{\pi\left(\underset{\sim}{U_{1}}, \ldots, \underset{\sim}{U_{n}} \mid M_{1, k}\right)}{\pi\left({\underset{\sim}{1}}_{1}, \ldots, \underset{\sim}{U_{n}} \mid M_{2}\right)}=\sum_{k=1}^{n-1} \frac{1}{n-1} \mathrm{BF}_{(1, k), 2}
$$

Observação: Utilizando o resultado de Dey (1997) podemos checar se existe mudança e obter um estimativa do ponto de mudança simultaneamente. Se houver mudança, o ponto de mudança será o valor de $k$ cujo Fator de Bayes individual é máximo.

O Fator de Bayes individual nesse caso é dado por

$$
\widehat{\mathrm{BF}}_{(1, k), 2}=\frac{\hat{\pi}\left({\underset{\sim}{1}}_{1}, \ldots, \underset{\sim}{\left.U_{n} \mid M_{1, k}\right)}\right.}{\hat{\pi}\left(\underset{\sim}{U_{1}}, \ldots, \underset{\sim}{\left.U_{n} \mid M_{2}\right)}\right.}
$$

que pode ser estimado pelo método de Newton e Raftery descrito anteriormente fazendo $k^{(g)}=k$.

Outro método relativamente simples de ser implementado é o método de Chib (1995), descrito na seção [4.4.3a] do Apêndice. A seguir, vamos apresentar os cálculos para a estimação do fator individual de Bayes pelo método de Chib (1995).

Estimação de $\pi\left({\underset{\sim}{1}}_{1}, \ldots,{\underset{\sim}{n}}_{n} \mid M_{2}\right)=\pi\left(D_{\text {obs }} \mid M_{2}\right)$

Usando o método de Chib descrito na seção [4.4.3 a] do apêndice, temos que

$$
\log \hat{\pi}\left(\underset{\sim}{U_{1}}, \ldots, \underset{\sim}{U_{n}} \mid M_{2}\right)=\log \pi\left({\underset{\sim}{1}}_{1}, \ldots,{\underset{\sim}{n}}_{n} \mid \underline{\theta}^{\prime}\right)+\log \pi\left(\underline{(}^{\prime}\right)-\log \hat{\pi}\left(\underline{\underline{\theta}}^{\prime} \mid D_{\mathrm{obs}}\right)
$$

onde $\underline{\sim}^{\prime}$ pode ser uma estimativa da média da posteriori $p\left(\underset{\sim}{\theta} \mid D_{\text {obs }}\right)$. Além disso,

$$
\begin{aligned}
\hat{\pi}\left(\underline{\theta}^{\prime} \mid D_{\mathrm{obs}}\right)= & \pi\left(\sigma^{2^{\prime}} \mid \underset{\sim}{x^{\prime}}, \alpha^{\prime}, \beta^{\prime}, \mu^{\prime}, \sigma_{x}^{2^{\prime}}, D_{\mathrm{obs}}\right) \hat{\pi}\left(\sigma_{x}^{2^{\prime}} \mid{\underset{\sim}{x}}^{\prime}, \alpha^{\prime}, \beta^{\prime}, \mu^{\prime}, D_{\mathrm{obs}}\right) . \\
& . \hat{\pi}\left(\mu^{\prime} \mid{\underset{\sim}{x}}^{\prime}, \alpha^{\prime}, \beta^{\prime}, D_{\mathrm{obs}}\right) \hat{\pi}\left(\beta^{\prime} \mid{\underset{\sim}{\prime}}^{\prime}, \alpha^{\prime}, D_{\mathrm{obs}}\right) \pi\left(\alpha^{\prime} \mid \underline{x}^{\prime}, D_{\mathrm{obs}}\right) \pi\left(\underset{\sim}{x^{\prime}} \mid D_{\mathrm{obs}}\right) .
\end{aligned}
$$

A seguir damos os passos para a estimação dessas quantidades. 
(a) $\hat{\pi}\left(\underset{\sim}{x} \mid D_{\text {obs }}\right)$ é calculada usando o algoritmo de Gibbs seguinte:

Passo 1: fornecer um valor inicial $\left(\alpha^{(0)}, \beta^{(0)}, \mu^{(0)}, \sigma_{x}^{2(0)}, \sigma^{2(0)}\right)$, faça $i=0$.

Passo 2: gerar $x_{i}^{(i+1)}$ de $\pi\left(x_{i} \mid \alpha^{(i)}, \beta^{(i)}, \mu^{(i)}, \sigma_{x}^{2^{(i)}}, \sigma^{2^{(i)}}, D_{\mathrm{obs}}\right), i=1,2, \ldots, n$, dada em (2.5.14),

gerar $\alpha^{(i+1)}$ de $\pi\left(\alpha \mid \underset{\sim}{x^{(i+1)}}, \beta^{(i)}, \mu^{(i)}, \sigma_{x}^{(i)}, \sigma^{2^{(i)}}, D_{\text {obs }}\right)$, dada em (2.5.9),

gerar $\beta^{(i+1)}$ de $\pi\left(\beta \mid x_{\sim}^{(i+1)}, \alpha^{(i+1)}, \mu^{(i)}, \sigma_{x}^{2^{(i)}}, \sigma^{2^{(i)}}, D_{\text {obs }}\right)$, dada em (2.5.10),

$\operatorname{gerar} \mu^{(i+1)} \operatorname{de} \pi\left(\mu \mid \underset{\sim}{x^{(i+1)}}, \alpha^{(i+1)}, \beta^{(i+1)}, \sigma_{x}^{2^{(i)}}, \sigma^{2^{(i)}}, D_{\text {obs }}\right)$, dada em (2.5.11),

gerar $\sigma_{x}^{2^{(i+1)}}$ de $\pi\left(\sigma_{x}^{2} \mid{\underset{\sim}{(i+1)}}^{(i+1)}, \beta^{(i+1)}, \mu^{(i+1)}, \sigma^{2^{(i)}}, D_{\text {obs }}\right)$, dada em (2.5.12),

gerar $\sigma^{2^{(i+1)}} \operatorname{de} \pi\left(\sigma^{2} \mid{\underset{x}{x}}^{(i+1)}, \alpha^{(i+1)}, \beta^{(i+1)}, \mu^{(i+1)}, \sigma_{x}^{2^{(i+1)}}, D_{\text {obs }}\right)$, dada em (2.5.13),

Passo 3: fazer $i=i+1$ e voltar ao passo 2, até convergir. Calcule

$$
\hat{\pi}\left(\underset{\sim}{x^{\prime}} \mid D_{\mathrm{obs}}\right)=\frac{1}{G} \sum_{g=1}^{G} \prod_{i=1}^{n} \pi\left(x_{i}^{\prime} \mid \alpha^{(g)}, \beta^{(g)}, \mu^{(g)}, \sigma_{x}^{2^{(g)}}, \sigma^{2^{(g)}}, D_{\mathrm{obs}}\right),
$$

onde $\left(\stackrel{\theta}{(1)}^{(1)}, \ldots,{\underset{\theta}{(G)}}^{(G)}\right)$ é uma amostra de $G$ elementos gerados pelo algoritmo.

(b) $\hat{\pi}\left(\alpha^{\prime} \mid x^{\prime}, D_{\text {obs }}\right)$ é calculada usando o seguinte algoritmo de Gibbs:

Passo 1: fornecer um valor inicial $\left(\beta^{(0)}, \mu^{(0)}, \sigma_{x}^{2^{(0)}}, \sigma^{2^{(0)}}\right)$, fazer $i=0$,

Passo 2: gerar

$$
\begin{aligned}
& \alpha^{(i+1)} \text { de } \pi\left(\alpha \mid \underset{\sim}{x}=\underset{\sim}{x^{\prime}}, \beta^{(i)}, \mu^{(i)}, \sigma_{x}^{2^{(i)}}, \sigma^{2^{(i)}}, D_{\mathrm{obs}}\right), \\
& \beta^{(i+1)} \text { de } \pi\left(\beta \mid \underset{\sim}{x}=\underset{\sim}{x^{\prime}}, \alpha^{(i+1)}, \mu^{(i)}, \sigma_{x}^{2^{(i)}}, \sigma^{2^{(i)}}, D_{\mathrm{obs}}\right), \\
& \mu^{(i+1)} \text { de } \pi\left(\mu \mid \underset{\sim}{x}=\underset{\sim}{x^{\prime}}, \alpha^{(i+1)}, \beta^{(i+1)}, \sigma_{x}^{2^{(i)}}, \sigma^{2^{(i)}}, D_{\mathrm{obs}}\right), \\
& \sigma_{x}^{2^{(i+1)}} \text { de } \pi\left(\sigma_{x}^{2} \mid \underset{\sim}{x}=\underset{\sim}{x^{\prime}}, \alpha^{(i+1)}, \beta^{(i+1)}, \mu^{(i+1)}, \sigma^{2^{(i)}}, D_{\mathrm{obs}}\right), \\
& \sigma^{2^{(i+1)}} \text { de } \pi\left(\sigma_{x}^{2} \mid \underset{\sim}{x}=\underset{\sim}{x^{\prime}}, \alpha^{(i+1)}, \beta^{(i+1)}, \mu^{(i+1)}, \sigma_{x}^{2^{(i+1)}}, D_{\mathrm{obs}}\right),
\end{aligned}
$$

Passo 3: fazer $i=i+1$ e voltar ao passo 2, até convergir. Calcule

$$
\hat{\pi}\left(\alpha^{\prime} \mid{\underset{\sim}{x}}^{\prime}, D_{\mathrm{obs}}\right)=\frac{1}{G} \sum_{g=1}^{G} \pi\left(\alpha^{\prime} \mid \underset{\sim}{x}={\underset{\sim}{x}}^{\prime}, 3^{(g)}, \mu^{(g)}, \sigma_{x}^{2^{(g)}}, \sigma^{2^{(g)}}, D_{\mathrm{obs}}\right) .
$$


(c) $\hat{\pi}\left(\beta^{\prime} \mid \underset{\sim}{\underset{\sim}{x}}, \alpha^{\prime}, D_{\text {obs }}\right)$ é calculada usando o seguinte algoritmo de Gibbs:

Passo 1: fornecer um valor inicial $\left(\mu^{(0)}, \sigma_{x}^{2^{(0)}}, \sigma^{2^{(0)}}\right)$, fazer $i=0$,

Passo 2: gerar

$$
\begin{aligned}
& \beta^{(i+1)} \text { de } \pi\left(\beta \mid \underset{\sim}{x}=\underset{\sim}{x}, \alpha=\alpha^{\prime}, \mu^{(i)}, \sigma_{x}^{2^{(i)}}, \sigma^{2^{(i)}}, D_{\text {obs }}\right), \\
& \mu^{(i+1)} \text { de } \pi\left(\mu \mid \underset{\sim}{x}={\underset{\sim}{x}}^{\prime}, \alpha=\alpha^{\prime}, \beta^{(i+1)}, \sigma_{x}^{2^{(i)}}, \sigma^{2^{(i)}}, D_{\text {obs }}\right), \\
& \sigma_{x}^{2^{(i+1)}} \text { de } \pi\left(\sigma_{x}^{2} \mid \underset{\sim}{x}=\underset{\sim}{x^{\prime}}, \alpha=\alpha^{\prime}, \beta^{(i+1)}, \mu^{(i+1)}, \sigma^{2^{(i)}}, D_{\text {obs }}\right), \\
& \sigma^{2^{(i+1)}} \text { de } \pi\left(\sigma^{2} \mid \underset{\sim}{x}=\underset{\sim}{x}, \alpha=\alpha^{\prime}, \beta^{(i+1)}, \mu^{(i+1)}, \sigma^{2^{(i+1)}}, D_{\text {obs }}\right),
\end{aligned}
$$

Passo 3: fazer $i=i+1$ e voltar ao passo 2, até convergir. Calcule

$$
\hat{\pi}\left(\beta^{\prime} \mid \underset{\sim}{x^{\prime}}, \alpha^{\prime}, D_{\mathrm{obs}}\right)=\frac{1}{G} \sum_{g=1}^{G} \pi\left(\beta^{\prime} \mid \underset{\sim}{x}=\underset{\sim}{x^{\prime}}, \alpha=\alpha^{\prime}, \mu^{(g)}, \sigma_{x}^{2^{(g)}}, \sigma^{2^{(g)}}, D_{\mathrm{obs}}\right) .
$$

(d) $\hat{\pi}\left(\mu^{\prime} \mid x_{\sim}^{\prime}, \alpha^{\prime}, \beta^{\prime}, D_{\text {obs }}\right)$ é calculado usando o seguinte algoritmo de Gibbs:

Passo 1: fornecer um valor inicial $\left(\sigma_{x}^{2^{(0)}}, \sigma^{2^{(0)}}\right)$, fazer $i=0$,

Passo 2: gerar

$$
\begin{aligned}
& \mu^{(i+1)} \text { de } \pi\left(\mu \mid \underset{\sim}{x}=\underset{\sim}{x}, \alpha=\alpha^{\prime}, \beta=\beta^{\prime}, \sigma_{x}^{2^{(i)}}, \sigma^{2^{(i)}}, D_{\mathrm{obs}}\right), \\
& \sigma_{x}^{2^{(i+1)}} \text { de } \pi\left(\sigma_{x}^{2} \mid \underset{\sim}{x}={\underset{\sim}{x}}^{\prime}, \alpha=\alpha^{\prime}, \beta=\beta^{\prime}, \mu^{(i+1)}, \sigma^{2^{(i+1)}}, D_{\mathrm{obs}}\right), \\
& \sigma^{2^{(i+1)}} \text { de } \pi\left(\sigma^{2} \mid \underset{\sim}{x}=\underset{\sim}{x^{\prime}}, \alpha=\alpha^{\prime}, \beta=\beta^{\prime}, \mu^{(i+1)}, \sigma_{x}^{2^{(i+1)}}, D_{\mathrm{obs}}\right),
\end{aligned}
$$

Passo 3: fazer $i=i+1$ e voltar ao passo 2, até convergir. Calcule

$$
\hat{\pi}\left(\mu^{\prime} \mid \underset{\sim}{x^{\prime}}, \alpha^{\prime}, \beta^{\prime}, D_{\mathrm{obs}}\right)=\frac{1}{G} \sum_{g=1}^{G} \pi\left(\mu^{\prime} \mid \underset{\sim}{x}={\underset{\sim}{x}}^{\prime}, \alpha=\alpha^{\prime}, \beta=\beta^{\prime}, \sigma_{x}^{2^{(g)}}, \sigma^{2^{(g)}}, D_{\mathrm{obs}}\right) .
$$

(e) $\hat{\pi}\left(\sigma_{x}^{2^{\prime}} \mid \underset{\sim}{x^{\prime}}, \alpha^{\prime}, \beta^{\prime}, \mu^{\prime}, D_{\text {obs }}\right)$ é calculado usando o seguinte algoritmo de Gibbs:

Passo 1: fornecer um valor inicial $\sigma^{2^{(0)}}$, fazer $i=0$,

Passo 2: gerar

$$
\begin{aligned}
& \sigma_{x}^{2^{(i+1)}} \text { de } \pi\left(\sigma_{x}^{2} \mid \underset{\sim}{x}={\underset{\sim}{x}}^{\prime}, \alpha=\alpha^{\prime}, \beta=\beta^{\prime}, \mu=\mu^{\prime}, \sigma^{2^{(i)}}, D_{\mathrm{obs}}\right), \\
& \sigma^{2^{(i+1)}} \text { de } \pi\left(\sigma^{2} \mid \underset{\sim}{x}={\underset{\sim}{x}}^{\prime}, \alpha=\alpha^{\prime}, \beta=\beta^{\prime}, \mu=\mu^{\prime}, \sigma^{2^{(i+1)}}, D_{\mathrm{obs}}\right),
\end{aligned}
$$


Passo 3: fazer $i=i+1$ e voltar ao passo 2, até convergir. Calcule $\hat{\pi}\left(\sigma_{x}^{2^{\prime}} \mid{\underset{\sim}{x}}^{\prime}, \alpha^{\prime}, \beta^{\prime}, \mu^{\prime}, D_{\mathrm{obs}}\right)=\frac{1}{G} \sum_{g=1}^{G} \pi\left(\sigma_{x}^{2^{\prime}} \mid \underset{\sim}{x}=\underset{\sim}{x^{\prime}}, \alpha=\alpha^{\prime}, \beta=\beta^{\prime}, \mu=\mu^{\prime}, \sigma^{2^{(g)}}, D_{\mathrm{obs}}\right)$.

(f) Calcular $\pi\left(\sigma^{2^{\prime}} \mid \underset{\sim}{x^{\prime}}, \alpha^{\prime}, \beta^{\prime}, \mu^{\prime}, \sigma_{x}^{2^{\prime}}, D_{\text {obs }}\right)$ que é dada em (2.5.13) quando substituímos $\stackrel{\theta}{\sim}$ por $\underline{\theta}^{\prime}$.

Estimação de $\pi\left(\underset{\sim}{U_{1}}, \ldots,{\underset{\sim}{n}}_{n} \mid M_{1, k}\right)$, onde $M_{1, k}$ é o modelo definido em (4.1.1) que permite mudança em todos os parâmetros após o ponto $k$.

No modelo $M_{1, k}$ com $k$ fixado, o vetor de parâmetros é $\left(\begin{array}{c}x^{(1)} \\ \stackrel{\theta}{(1)}_{\tilde{x}^{(2)}} \\ \widetilde{\theta}^{(2)}\end{array}\right)$, onde $\theta_{i}=$ $\left(\alpha_{i}, \beta_{i}, \mu_{i}, \sigma_{x(i)}^{2}, \sigma_{i}^{2}\right)^{T},{\underset{\sim}{x}}^{(1)}=\left(x_{1}, \ldots, x_{k}\right)^{T},{\underset{\sim}{x}}^{(2)}=\left(x_{k+1}, \ldots, x_{n}\right)^{T}$

e $\hat{\pi}\left({\underset{\sim}{1}}_{1}, \ldots, \underset{\sim}{U_{n}} \mid M_{1, k}\right)$ pode ser obtido de forma totalmente semelhante a $\hat{\pi}\left(\underset{\sim}{U_{1}}, \ldots, \underset{\sim}{U_{n}} \mid M_{2}\right)$, descrito acima.

\section{$\left(2^{\circ}\right)$ O critério do Pseudo Fator de Bayes}

Na seção [4.4.3b] do Apêndice damos uma breve introdução sobre essa medida no caso geral.

O Pseudo Fator de Bayes para comparar o modelo $M_{1, k}$ com o modelo $M_{2}$ (onde $M_{1, k}$ é o modelo estrutural Normal que permite mudança em todos os parâmetros e $M_{2}$ é o modelo sem mudança) é dado por

$$
\operatorname{PBF}_{(1, k) 2}=\frac{\prod_{i=1}^{n} \mathrm{CPO}_{i}^{\left(M_{1, k}\right)}}{\prod_{i=1}^{n} \mathrm{CPO}_{i}^{\left(M_{2}\right)}}
$$

onde $\mathrm{CPO}_{i}^{\left(M_{2}\right)}=\pi_{M_{2}}\left(\underset{\sim}{U_{i}} \mid D_{\mathrm{obs}(-i)}\right)$ pode ser estimada usando as fórmulas (2.10.5) ou (2.10.9) ou (2.10.11) e $\mathrm{CPO}_{i}^{\left(M_{1, k}\right)}=\pi_{M_{1, k}}\left({\underset{\sim}{i}}_{i} \mid D_{\text {obs(-i) }}\right)$ pode ser estimada usando por exemplo as fórmulas (4.5.5) ou (4.5.8), dadas mais adiante, para todo $i=1, \ldots, n$.

Observação: Se o modelo $M_{1 . k}$ à priori permite mudança em apenas um subconjunto de parâmetros após o ponto $k$, os cálculos podem ser refeitos facilmente, de 
forma totalmente semelhante, apenas incorporando as devidas restrições.

\section{$\left(2^{\circ}\right)$ A medida $L$ no modelo com ponto de mudança}

Na seção 2.11, a medida $L$ foi obtida para o modelo estrutural Normal sem mudança. Nesta seção obtemos a medida $L$ aplicada ao modelo estrutural Normal que permite mudança em todos os parâmetros após o ponto $k$. O modelo escolhido será aquele com o menor valor dessa medida.

- Formulação

$$
\begin{aligned}
& \text { Seja } D_{\text {obs }}=\left(\begin{array}{c}
U_{1} \\
\vdots \\
{\underset{\sim}{n}}_{n}
\end{array}\right) \text { onde } U_{i}=\left(\begin{array}{c}
Y_{i} \\
X_{i}
\end{array}\right) \text { é tal que } \\
& {\underset{\sim}{i}}_{i} \mid \Theta \sim\left\{\begin{array}{c}
N\left[\left(\begin{array}{c}
\alpha_{1}+\beta_{1} \mu_{1} \\
\mu_{1}
\end{array}\right) ;\left(\begin{array}{cc}
\beta_{1}^{2} \sigma_{x(1)}^{2}+\lambda \sigma_{1}^{2} & \beta_{1} \sigma_{x(1)}^{2} \\
\beta_{1} \sigma_{x(1)}^{2} & \sigma_{x(1)}^{2}+\sigma_{u_{1}}^{2}
\end{array}\right)\right], i=1, \ldots, k, \\
N\left[\left(\begin{array}{c}
\alpha_{2}+\beta_{2} \mu_{2} \\
\mu_{2}
\end{array}\right) ;\left(\begin{array}{cc}
\beta_{2}^{2} \sigma_{x(2)}^{2}+\lambda \sigma_{2}^{2} & \beta_{2} \sigma_{x(2)}^{2} \\
\beta_{2} \sigma_{x(2)}^{2} & \sigma_{x(2)}^{2}+\sigma_{u_{2}}^{2}
\end{array}\right)\right], i=k+1, \ldots, n,
\end{array}\right.
\end{aligned}
$$

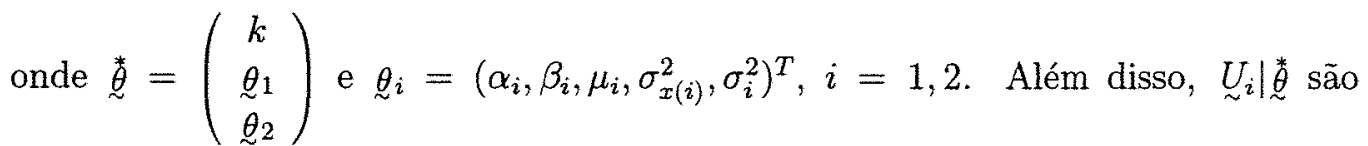
independentes para $i=1, \ldots, n$.

Seja $\underset{\sim}{U_{i}^{*}}, i=1, \ldots, n$, os vetores de observações futuras, com a mesma distribuição de ${\underset{\sim}{i}}_{i} \mid \stackrel{*}{*}$. Então a medida $L$ é dada por

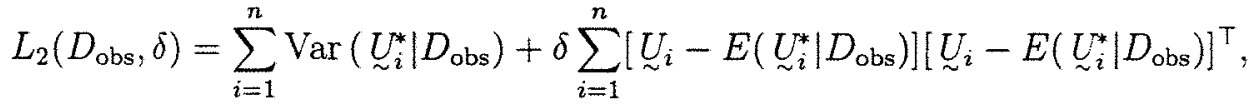

onde $0 \leq \delta \leq 1$ e onde

$$
\begin{aligned}
& E\left(\underset{\sim}{U_{i}^{*}} \mid D_{\mathrm{obs}}\right)=E_{\underset{\sim}{*} \mid D_{\mathrm{obs}}}\left[E\left(\underset{\sim}{U_{i}^{*}} \mid \underset{\sim}{*}\right)\right], \quad \mathrm{e}
\end{aligned}
$$

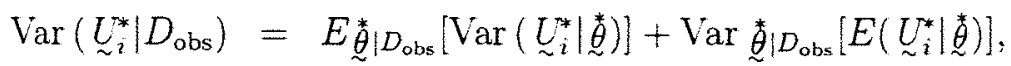

onde

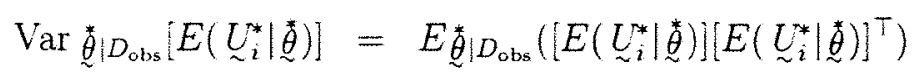




$$
-\left[E_{\underset{\theta}{*} \mid D_{\mathrm{obs}}}^{*}\left(E\left({\underset{\sim}{U}}_{i}^{*} \mid \stackrel{*}{*}\right)\right)\right]\left[E_{\underset{\sim}{*} \mid D_{\mathrm{obs}}}\left(E\left({\underset{\sim}{U}}_{i}^{*} \mid \stackrel{*}{\theta}\right)\right)\right]^{\top}
$$

Portanto, a medida $L$ pode ser facilmente calculada usando Monte Carlo da seguinte maneira.

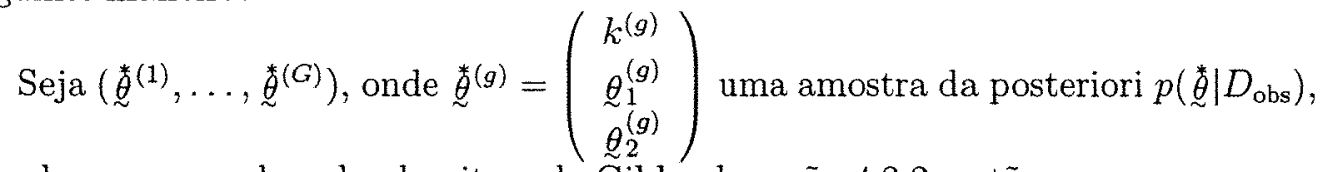
gerada por exemplo pelo algoritmo de Gibbs da seção 4.2.2, então

$$
\begin{aligned}
& \widehat{L_{2}}\left(D_{\mathrm{obs}}, \delta\right)=\sum_{i=1}^{n}\left\{\frac{1}{G} \sum_{g=1}^{G} \operatorname{Var}\left({\underset{\sim}{U}}_{i}^{*} \mid \stackrel{\sim}{\theta}^{(g)}\right)+\frac{1}{G} \sum_{g=1}^{G} E\left({\underset{\sim}{U}}_{i}^{*} \mid{\underset{\sim}{\ddot{\theta}}}^{(g)}\right) E\left({\underset{\sim}{U}}_{i}^{*} \mid{\underset{\sim}{\ddot{\theta}}}^{(g)}\right)^{\top}\right. \\
& \left.-\left(\frac{1}{G} \sum_{g=1}^{G} E\left({\underset{\sim}{U}}_{i}^{*} \mid \stackrel{*}{\theta}^{(g)}\right)\right)\left(\frac{1}{G} \sum_{g=1}^{G} E\left({\underset{\sim}{i}}_{i}^{*} \mid \stackrel{*}{\theta}^{(g)}\right)\right)^{\top}\right\}
\end{aligned}
$$

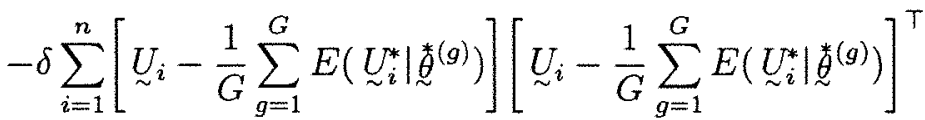

onde

$$
\begin{aligned}
& E\left({\underset{\sim}{i}}_{i}^{*} \mid \underset{\sim}{*}{ }^{(g)}\right)=\left\{\begin{array}{l}
E^{(1)}\left({\underset{\sim}{U}}_{i}^{*} \mid{\underset{\sim}{1}}_{1}^{(g)}\right)=\left(\begin{array}{c}
\alpha_{1}^{(g)}+\beta_{1}^{(g)} \mu_{1}^{(g)} \\
\mu_{1}^{(g)}
\end{array}\right), \quad \text { se } 1 \leq i \leq k^{(g)}, \\
E^{(2)}\left({\underset{\sim}{U}}_{i}^{*} \mid \Theta_{2}^{(g)}\right)=\left(\begin{array}{c}
\alpha_{2}^{(g)}+\beta_{2}^{(g)} \mu_{2}^{(g)} \\
\mu_{2}^{(g)}
\end{array}\right), \text { se } k^{(g)}+1 \leq i \leq n
\end{array}\right.
\end{aligned}
$$

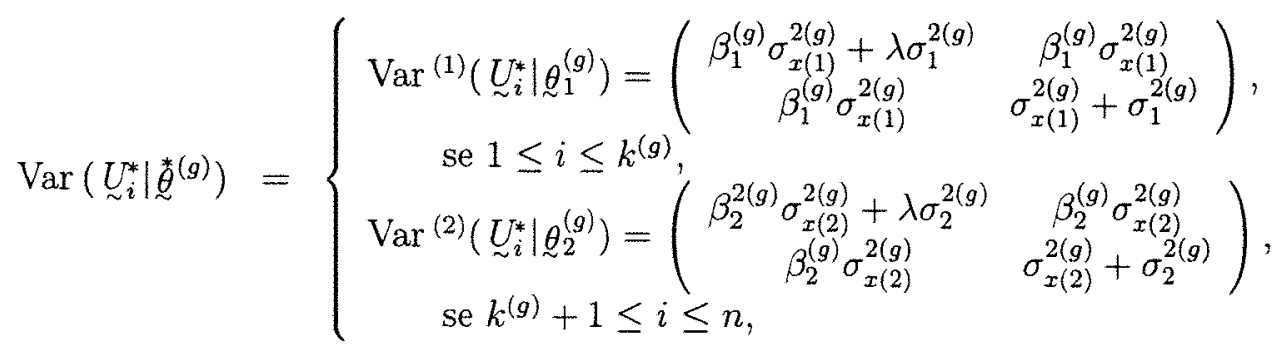

onde $\delta \in[0,1]$.

Note que $\widehat{L_{2}}\left(D_{\text {obs }}, \delta\right)$ é uma matriz $2 \times 2$ e uma possível medida resumo seria obter o determinante ou traço dessa matriz, isto é, $\operatorname{det}\left(\widehat{L_{2}}\left(D_{\text {obs }}, \delta\right)\right)$ ou $\operatorname{tr}\left(\widehat{L_{2}}\left(D_{\text {obs }}, \delta\right)\right)$.

Observação: No modelo com mudança apenas num subconjunto de parâmetros, o cálculo da medida $L$ é totalmente semelhante, bastando adaptar as fórmulas (incorporando-se as devidas restriçōes de igualdade). 


\section{$\left(4^{\circ}\right) \mathrm{O} \mathrm{BIC}$}

Seja $M_{1, k}$ o modelo estrutural Normal com restrição de identificabilidade e que permite mudança em todos os parâmetros após o ponto $k$ e seja $M_{2}$ o modelo sem mudança.

Na seção 2.11 vimos como calcular o $\mathrm{BIC}_{M_{2}}$. O cálculo do $\mathrm{BIC}_{M_{1, k}}$ também é simples já que no modelo identificável com ponto de mudança $k$, os estimadores de máxima verossimilhança são conhecidos explicitamente.

Observação: É interessante ressaltar que esses métodos podem ser utilizados para escolher o "melhor modelo" dentre os $2^{5}$ possíveis modelos (isto é, aqueles que permitem mudança em até 5 ou 4 ou 3 ou 2 ou 1 ou nenhum parâmetro).

\subsection{Distribuição preditiva e avaliação do ajuste}

A avaliação da qualidade do ajuste do modelo estrutural Normal com um ponto de mudança pode ser feita de forma análoga à apresentada na seção 2.10 para o modelo sem mudança. Para isso, vamos obter as fórmulas de cálculo da densidade condicional preditiva de validação cruzada $\mathrm{CPO}$ e dos resíduos de validação cruzada correspondentes.

Nessa seção vamos trabalhar apenas com o modelo que permite mudança em todos os parâmetros, lembrando que o procedimento para o caso de um modelo que permite mudança em apenas um subconjunto de parâmetros é totalmente semelhante, bastando fazer as devidas adaptações.

Na subseção 4.j.1, a seguir, apresentamos a distribuição preditiva, na subseção 4.5.2 as fórmulas de CPO e na subseção 4.5 .3 os resíduos de validação cruzada. 


\subsubsection{Distribuição preditiva}

A distribuição preditiva sob o modelo estrutural Normal que permite mudança em

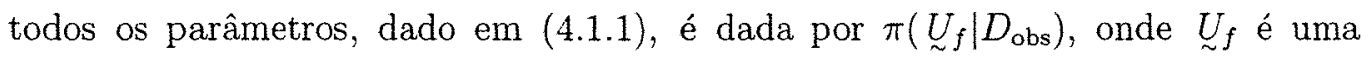
observação futura $\left(\begin{array}{l}Y_{f} \\ X_{f}\end{array}\right)$, e $D_{\text {obs }}$ é a amostra $\left(\underset{\sim}{U_{1}}, \underset{\sim}{U_{2}}, \ldots, \underset{\sim}{U_{n}}\right)$, onde $\underset{\sim}{U_{i}}=\left(\begin{array}{l}Y_{i} \\ X_{i}\end{array}\right)$.

Cálculo de $\pi\left(\underset{\sim}{U_{f}} \mid D_{\text {obs }}\right)$

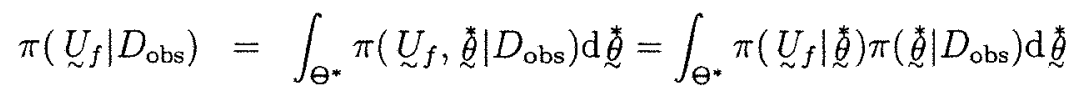

$$
\begin{aligned}
& =\int_{\Theta^{*}} \pi\left(Y_{f} \mid X_{f}, \stackrel{*}{\theta}\right) \pi\left(X_{f} \mid \stackrel{*}{\stackrel{*}{*}}\right) \pi\left(\underset{\theta}{\stackrel{*}{*}} \mid D_{\text {obs }}\right) \mathrm{d} \stackrel{*}{\theta}
\end{aligned}
$$

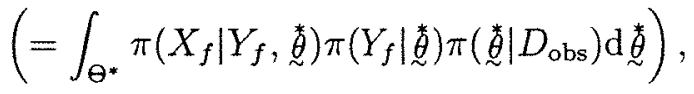

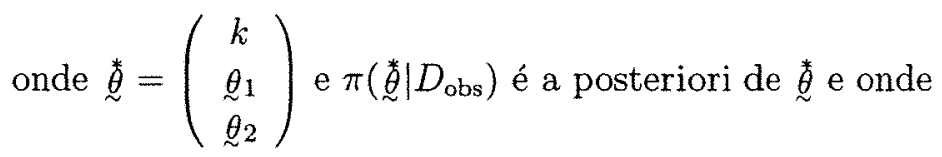

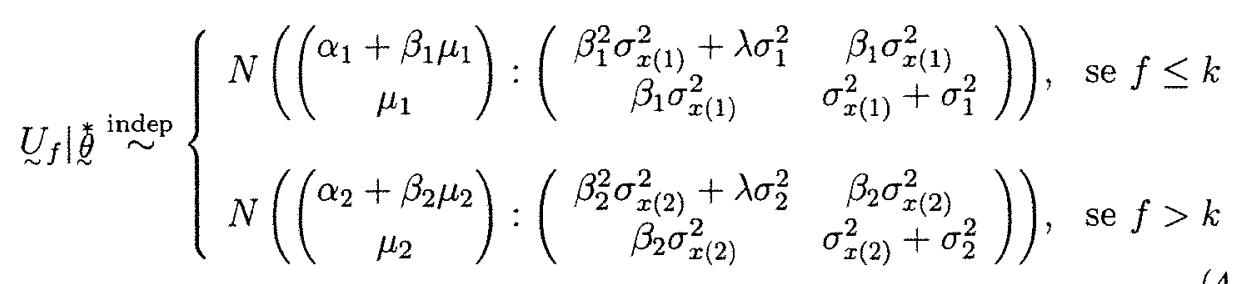

\section{Geração de uma observação futura}

Para gerar, por exemplo, uma observação ${\underset{\sim}{f}}_{f}$ após a mudança, basta seguir por exemplo os seguintes passos:

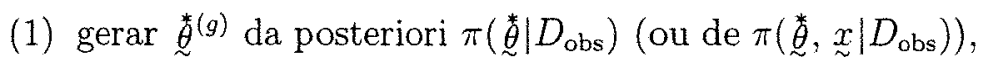

(2) gerar $X_{f}^{(g)} \operatorname{de} \pi\left(X_{f} \mid{ }_{\stackrel{*}{(}(g)}\right)$, onde $X_{f} \mid \underset{\sim}{\stackrel{*}{\sim}} \sim N\left(\mu_{2} ; \sigma_{x(2)}^{2}+\sigma_{2}^{2}\right)$,

(3) gerar $Y_{f}^{(g)}$ de $\pi\left(Y_{f} \mid X_{f}^{(g)}: \stackrel{*}{\theta}^{(g)}\right)$, onde

$$
Y_{f} \mid X_{f}, \stackrel{*}{*} \sim N\left(\alpha_{2}+\beta_{2} \mu_{2}+\frac{\beta_{2}^{2} \sigma_{x(2)}^{2}}{\sigma_{x(2)}^{2}+\sigma_{2}^{2}}\left(X_{f}-\mu_{2}\right) ; \beta_{2}^{2} \sigma_{x(2)}^{2}+\lambda \sigma_{2}^{2}-\frac{\beta_{2}^{2}\left(\sigma_{r(2)}^{2}\right)^{2}}{\sigma_{x(2)}^{2}+\sigma_{2}^{2}}\right) .
$$


Portanto, para gerar $Y_{f}$, após a mudança, dado que $X_{f}=X_{0}$, basta

(1) gerar ${\underset{\sim}{*}}^{(g)}$ de $\pi\left(\underset{\sim}{\stackrel{*}{\theta}} \mid D_{\text {obs }}\right)$ (ou $\left.\pi\left(\underset{\sim}{\stackrel{*}{*}} \underset{\sim}{x} \mid D_{\text {obs }}\right)\right)$,

(2) gerar $Y_{f}^{(g)}$ de $\pi\left(Y_{f} \mid X_{f}=X_{0}, \stackrel{*}{\theta}^{(g)}\right)$.

(b) Para gerar uma observação futura $\underset{\sim}{U_{f}}$ (ou $\underset{\sim}{Y_{f}} \mid X_{f}=X_{0}$ ), até a mudança, basta seguir os mesmos passos, porém agora

$$
\begin{aligned}
& X_{f} \mid \stackrel{*}{\theta} \sim N\left(\mu_{1} ; \sigma_{x(1)}^{2}+\sigma_{1}^{2}\right) \quad \mathrm{e} \\
& Y_{f} \mid X_{f}, \stackrel{*}{\ddot{\theta}} \sim N\left(\alpha_{1}+\beta_{1} \mu_{1}+\frac{\beta_{1}^{2} \sigma_{x(1)}^{2}}{\sigma_{x(1)}^{2}+\sigma_{1}^{2}}\left(X_{f}-\mu_{1}\right) ; \beta_{1}^{2} \sigma_{x(1)}^{2}+\lambda \sigma_{1}^{2}-\frac{\beta_{1}^{2}\left(\sigma_{x(1)}^{2}\right)^{2}}{\sigma_{x(1)}^{2}+\sigma_{1}^{2}}\right) .
\end{aligned}
$$

Observação: A distribuição preditiva $\pi\left(\underset{\sim}{U_{f}} \mid D_{\text {obs }}\right)$ também pode ser escrita como

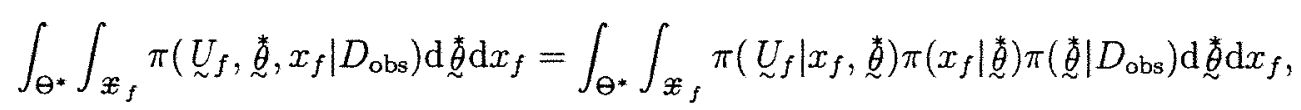

onde

$$
x_{f} \mid \stackrel{*}{\stackrel{\text { indep }}{\sim}} \begin{cases}N\left(\mu_{1}, \sigma_{x(1)}^{2}\right), & \text { se } f \leq k \\ N\left(\mu_{2}, \sigma_{x(2)}^{2}\right), & \text { se } f>k\end{cases}
$$

e

$$
U_{f} \mid x_{f}, \underset{\theta}{*} \stackrel{\text { indep }}{\sim}\left\{\begin{array}{c}
N\left[\left(\begin{array}{c}
\alpha_{1}+\beta_{1} x_{f} \\
x_{f}
\end{array}\right) ;\left(\begin{array}{cc}
\lambda \sigma_{1}^{2} & 0 \\
0 & \sigma_{1}^{2}
\end{array}\right)\right], f \leq k, \\
N\left[\left(\begin{array}{c}
\alpha_{2}+\beta_{2} x_{f} \\
x_{f}
\end{array}\right) ;\left(\begin{array}{cc}
\lambda \sigma_{2}^{2} & 0 \\
0 & \sigma_{2}^{2}
\end{array}\right)\right], f>k .
\end{array}\right.
$$

\subsubsection{Densidade condicional preditiva de validação cruzada}

A seguir apresentamos três maneiras alternativas de estimar a densidade preditiva de validação cruzada $\left(\mathrm{CPO}_{i}\right)$ para o modelo estrutural Normal com mudança em todos os parâmetros após a $k$-ésima observação.

Seja $\underset{\sim}{U_{i}}=\left(\begin{array}{l}Y_{i} \\ X_{i}\end{array}\right)$ a $i$-ésima observação da amostra, $D_{\text {obs }(-i)}=\underset{\sim}{L_{(-i)}}$. e $\mathrm{CPO}_{i}=$ $\pi\left(U_{i} \mid D_{\mathrm{obs}(-i)}\right)$. 
Fórmulas de cálculo da $\mathrm{CPO}_{i}$

Fórmula 1

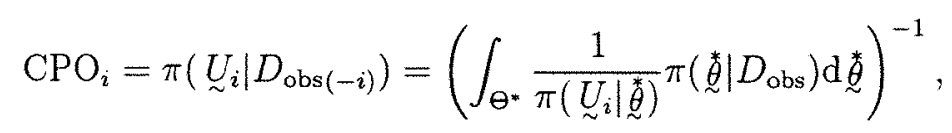

$i=1, \ldots, n$, onde $\underset{\sim}{\stackrel{*}{\theta}}=\left(\begin{array}{c}\theta_{1} \\ \underset{\theta_{2}}{k}\end{array}\right),{\underset{\sim}{i}}_{i}=\left(\alpha_{i}, \beta_{i}, \mu_{i}, \sigma_{x(i)}^{2}, \sigma_{i}^{2}\right)^{T}, i=1,2\left(\operatorname{com} \sigma_{i}^{2}=\sigma_{u_{i}}^{2} \mathrm{e}\right.$ $\left.\sigma_{e_{i}}^{2}=\lambda \sigma_{i}^{2}\right)$, onde

$$
\underset{\sim}{U_{i} \mid \stackrel{*}{\text { indep }} \sim}\left\{\begin{array}{l}
N\left(\left(\begin{array}{c}
\alpha_{1}+\beta_{1} \mu_{1} \\
\mu_{1}
\end{array}\right) ;\left(\begin{array}{cc}
\beta_{1}^{2} \sigma_{x(1)}^{2}+\lambda \sigma_{1}^{2} & \beta_{1}^{2} \sigma_{x(1)}^{2} \\
\beta_{1}^{2} \sigma_{x(1)}^{2} & \sigma_{x(1)}^{2}+\sigma_{1}^{2}
\end{array}\right)\right), \\
\text { se } 1 \leq i \leq k, \\
N\left(\left(\begin{array}{c}
\alpha_{2}+\beta_{2} \mu_{2} \\
\mu_{2}
\end{array}\right) ;\left(\begin{array}{cc}
\beta_{2}^{2} \sigma_{x(1)}^{2}+\lambda \sigma_{2}^{2} & \beta_{2}^{2} \sigma_{x(2)}^{2} \\
\beta_{2}^{2} \sigma_{x(2)}^{2} & \sigma_{x(2)}^{2}+\sigma_{2}^{2}
\end{array}\right)\right), \\
\text { se } k+1 \leq i \leq n .
\end{array}\right.
$$

\section{Estimação}

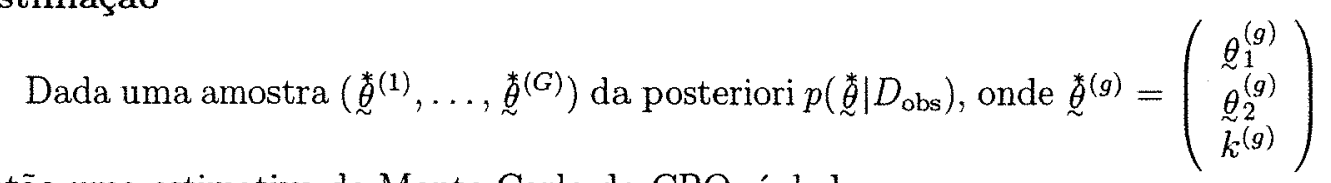
então uma estimativa de Monte Carlo de $\mathrm{CPO}_{i}$ é dada por

$$
\widehat{\mathrm{CO}}_{i}=\left(\frac{1}{G} \sum_{g=1}^{G} \frac{1}{\pi\left({\underset{\sim}{i}}_{i} \mid \stackrel{\theta}{\hat{\theta}}^{(g)}\right)}\right)^{-1}
$$

onde

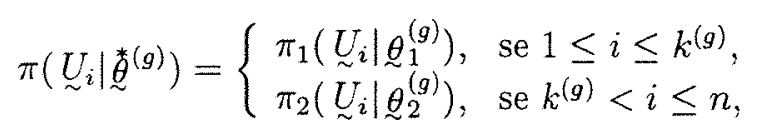

para $g=1, \ldots, G$, onde,

$$
\begin{gathered}
\pi_{j}\left({\underset{\sim}{\sim}}_{i} \mid \theta_{j}^{(g)}\right)=\left(2 \pi\left|\sum_{j}^{(g)}\right|\right)^{-\frac{1}{2}} \exp \left\{-\frac{1}{2\left|\sum_{j}^{(g)}\right|}\left[\left(\left(\beta_{j}^{(g)}\right)^{2} \sigma_{x(j)}^{2(g)}+\lambda \sigma_{j}^{2(g)}\right)\left(X_{i}-\mu_{j}^{(g)}\right)^{2}\right.\right. \\
\left.\left.-2 \beta_{j}^{(g)} \sigma_{x(j)}^{2(g)}\left(Y_{i}-\alpha_{j}^{(g)}-\beta_{j}^{(g)} \mu_{j}^{(g)}\right)\left(X_{i}-\mu_{j}^{(g)}\right)+\left(\sigma_{x(j)}^{2(g)}+\sigma_{j}^{2(g)}\right)\left(Y_{i}-\alpha_{j}^{(g)}-\beta_{j}^{(g)} \mu_{j}^{(g)}\right)^{2}\right]\right\} \\
e \sum_{j}^{(g)}=\left(\beta_{j}^{(g)}\right)^{2} \sigma_{j}^{2(g)} \sigma_{x(j)}^{2(g)}+\lambda \sigma_{x(j)}^{2(g)} \sigma_{(j)}^{2(g)}+\lambda\left(\sigma_{(j)}^{2(g)}\right)^{2}, j=1,2
\end{gathered}
$$


Observação: A amostra $\left(\stackrel{*}{\theta}^{(1)}, \ldots, \stackrel{*}{\theta}^{(G)}\right)$ pode ser gerada da posteriori $p\left(\underset{\sim}{\theta} \mid D_{\text {obs }}\right)$ por exemplo pelo algoritmo de Metropolis-Hastings em Gibbs (desde que este convirja), ou então da posteriori conjunta $p\left(\underset{\sim}{*}, \underset{\sim}{x} \mid D_{\text {obs }}\right)$, por exemplo pelo algoritmo de Gibbs (desde que este convirja).

\section{Fórmula 2}

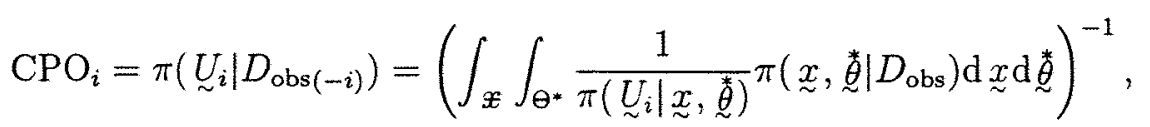

$i=1, \ldots, n$, onde

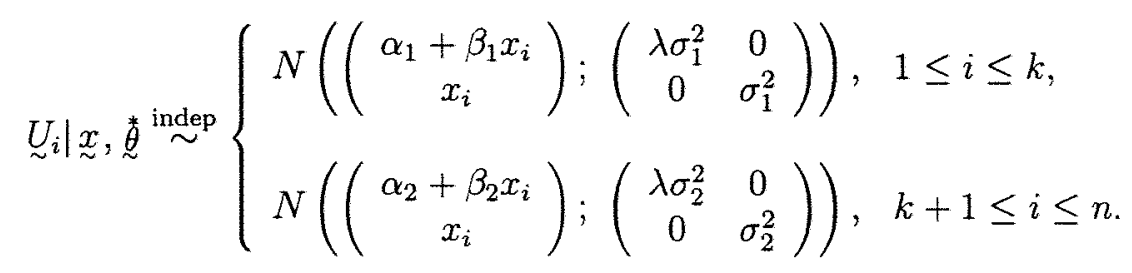

\section{Estimação}

Dada uma amostra $\left(\left({\underset{\sim}{\mathscr{\theta}^{(1)}}}_{\widetilde{*}^{(1)}}^{(1)}\right), \ldots,\left({\underset{\sim}{\tilde{X}^{(G)}}}^{(G)}\right)\right)$ da posteriori conjunta $\pi\left(\underset{\sim}{x}, \underset{\theta}{\theta} \mid D_{\text {obs }}\right)$, uma estimativa de Monte Carlo de $\mathrm{CPO}_{i}$ é dada por

$$
\widehat{C P O}_{i}=\left(\frac{1}{G} \sum_{g=1}^{G} \frac{1}{\pi(\underbrace{}_{i} \mid \underline{\vartheta}^{(g)}, x_{i}^{(g)}, k^{(g)})}\right)^{-1}
$$

onde

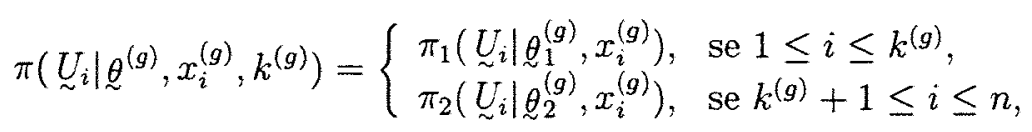

onde

$$
\pi_{j}\left({\underset{\sim}{i}}_{i} \mid \theta_{j}^{(g)}, x_{i}^{(g)}\right)=\left(2 \pi \lambda\left(\sigma_{j}^{2(g)}\right)^{2}\right)^{-\frac{1}{2}} \exp \left\{-\frac{1}{2}\left[\frac{\left(Y_{i}-\alpha_{j}^{(g)}-\beta_{j}^{(g)} x_{i}^{(g)}\right)^{2}}{\lambda \sigma_{j}^{2(g)}}+\frac{\left(X_{i}-x_{i}^{(g)}\right)^{2}}{\sigma_{j}^{2(g)}}\right]\right\}
$$

$g=1, \ldots, G, j=1,2$.

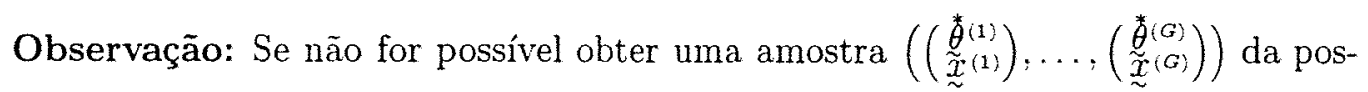
teriori $p\left(\underset{\theta}{*}, \underset{\sim}{x} \mid D_{\text {obs }}\right)$ pelo algoritmo de Gibbs (por exemplo, porque não há con-

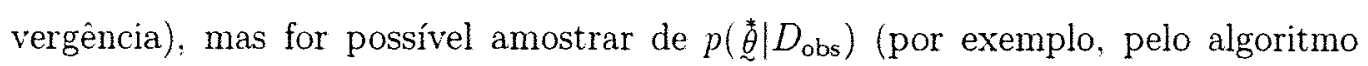


de M-H em Gibbs), então podemos ainda utilizar a expressão em (4.5.8) para estimar a $\mathrm{CPO}_{i}$. A diferença, entretanto, está no processo de geração. Nesse caso, dada

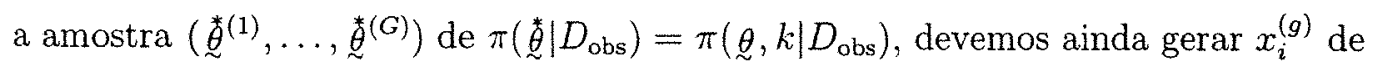
$\pi\left(x_{i} \mid \stackrel{*}{(g)}^{(g)}\right)$, onde

$$
x_{i} \mid \theta_{\sim}^{(g)}, k^{(g)} \sim \begin{cases}N\left(\mu_{1}^{(g)}, \sigma_{x(1)}^{2^{(g)}}\right), & 1 \leq k^{(g)} \leq n \\ N\left(\mu_{2}^{(g)}, \sigma_{x(2)}^{2(g)}\right), & k^{(g)}+1 \leq i \leq n .\end{cases}
$$

A justificativa desse procedimento segue do fato que

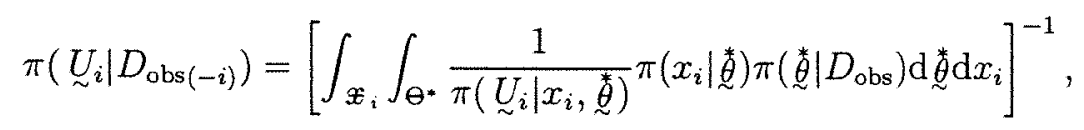

onde

$$
\left.x_{i}\right|_{\stackrel{*}{\theta}} \sim \begin{cases}N\left(\mu_{1}, \sigma_{x(1)}^{2}\right), & 1 \leq i \leq k, \\ N\left(\mu_{2}, \sigma_{x(2)}^{2}\right), & k+1 \leq i \leq n .\end{cases}
$$

\subsubsection{Resíduos de validação cruzada}

O resíduo padronizado de validação cruzada do modelo com mudança é dado por

$$
\underset{\sim}{d}=\operatorname{Var}^{-\frac{1}{2}}\left({\underset{\sim}{i}}_{i} \mid D_{\mathrm{obs}(-i)}\right)\left[{\underset{\sim}{i}}_{i}-E\left(\underset{\sim}{U_{i}} \mid D_{\mathrm{obs}(-i)}\right)\right], i=1, \ldots, n
$$

\section{Cálculo de $E\left(\underset{\sim}{\left.U_{i} \mid D_{\mathrm{obs}(-i)}\right)}\right.$}

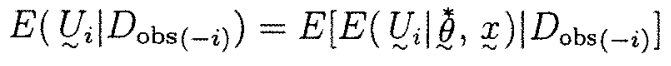

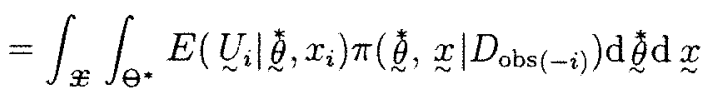

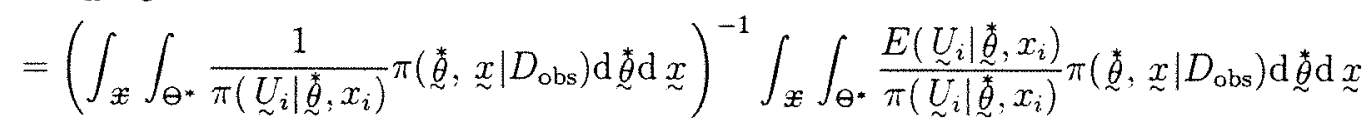

onde

$$
{\underset{\sim}{i} \mid}_{i} \mid x_{i} \stackrel{\text { indep }}{\sim}\left\{\begin{array}{l}
N\left(\left(\begin{array}{c}
\alpha_{1}+\beta_{1} x_{i} \\
x_{i}
\end{array}\right) ;\left(\begin{array}{cc}
\lambda \sigma_{1}^{2} & 0 \\
0 & \sigma_{1}^{2}
\end{array}\right)\right), i=1, \ldots, k, \\
N\left(\left(\begin{array}{c}
\alpha_{2}+\beta_{2} x_{i} \\
x_{i}
\end{array}\right) ;\left(\begin{array}{cc}
\lambda \sigma_{2}^{2} & 0 \\
0 & \sigma_{2}^{2}
\end{array}\right)\right), i=k+1, \ldots, n .
\end{array}\right.
$$


Estimação de $E\left({\underset{\sim}{i}}_{i} \mid D_{\mathrm{obs}(-i)}\right)$

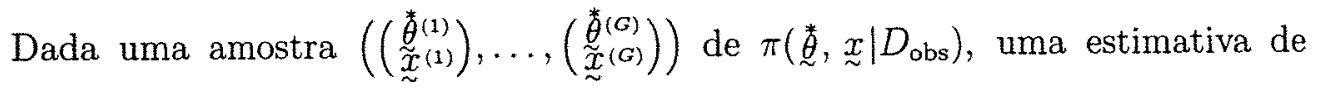
Monte Carlo de $E\left({\underset{\sim}{i}}_{i} \mid D_{\mathrm{obs}(-i)}\right)$ é dada por

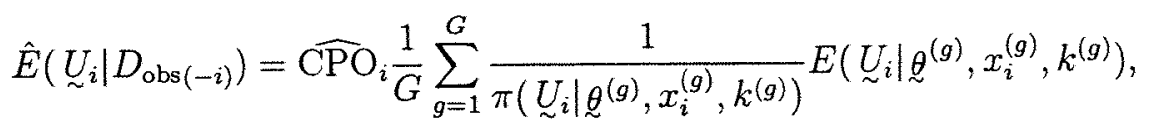

onde $\widehat{\mathrm{CPO}}_{i}$ é dada por (4.5.8), $\pi\left({\underset{\sim}{i}}_{i} \mid \Theta^{(g)}, x_{i}^{(g)}\right)$ é dada por (4.5.9),

$$
E\left({\underset{\sim}{i}}_{i} \mid \theta^{(g)}, x_{i}^{(g)}, k^{(g)}\right)=\left\{\begin{array}{c}
\left(\begin{array}{c}
\alpha_{1}^{(g)}+\beta_{1}^{(g)} x_{1}^{(g)} \\
x_{i}^{(g)}
\end{array}\right), i=1, \ldots, k^{(g)}, \\
\left(\begin{array}{c}
\alpha_{2}^{(g)}+\beta_{2}^{(g)} x_{i}^{(g)} \\
x_{i}^{(g)}
\end{array}\right), i=k^{(g)}+1, \ldots, n,
\end{array}\right.
$$

Estimação da $\operatorname{Var}\left(\underset{\sim}{U_{i}} \mid D_{\mathrm{obs}(-i)}\right)$

$$
\begin{aligned}
& \widehat{\operatorname{Var}}\left(\underset{\sim}{U_{i}} \mid D_{\mathrm{obs}(-i)}\right)=\widehat{\mathrm{CPO}}_{i}\left[\frac{1}{G} \sum_{g=1}^{G} \frac{\operatorname{Var}\left(U_{i} \mid \theta^{(g)}, x_{i}^{(g)}, k^{(g)}\right)}{\pi\left(U_{i} \mid \theta^{(g)}, x_{i}^{(g)}, k^{(g)}\right)}+\right. \\
& \left.\frac{1}{G} \sum_{j=1}^{G} \frac{E\left({\underset{\sim}{i}}_{i} \mid \theta^{(g)}, x_{i}^{(g)}, k^{(g)}\right) E\left(U_{i} \mid \theta^{(g)}, x_{i}^{(g)}, k^{(g)}\right)^{\top}}{\pi\left({\underset{\sim}{i}}_{i} \mid \Theta^{(g)}, x_{i}^{(g)}, k^{(g)}\right)}\right] \\
& -\left[\hat{E}\left(U_{i} \mid D_{\mathrm{obs}(-i)}\right)\right]\left[\hat{E}\left({\underset{\sim}{i}}_{i} \mid D_{\mathrm{obs}(-i)}\right)\right]^{\top},
\end{aligned}
$$

onde

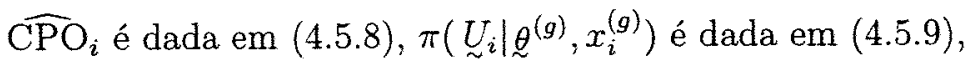
$\hat{E}\left({\underset{\sim}{i}}_{i} \mid D_{\mathrm{obs}(-i)}\right)$ é dada em (4.5.11), $E\left({\underset{\sim}{i}}_{i} \mid \underline{\theta}^{(g)}, x_{i}^{(g)}\right)$ é dada em (4.5.12), e

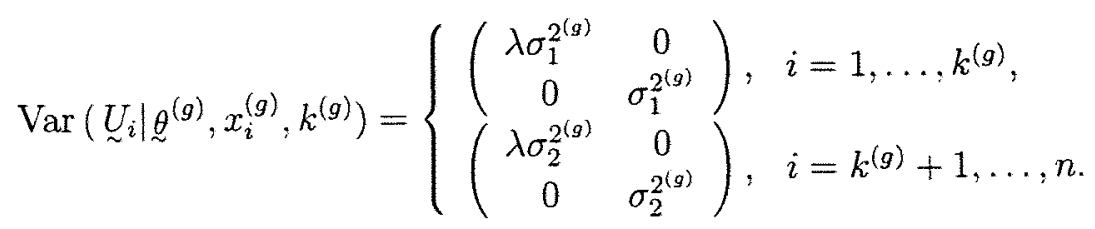




\subsection{Estabelecimento das condições para a existência da posteriori sob algumas escolhas de prioris impróprias}

Nessa seção vamos estudar a existência da distribuição à posteriori no modelo de regressão estrutural Normal com ponto de mudança e com restrição de identificabilidade (de que a razão das variâncias é conhecida) considerando prioris impróprias análogas as usadas na seção 2.8. Inicialmente, na subseção 4.6.1, estudamos o modelo que permite mudança em todos os parâmetros e depois, na subseção 4.6.2, o modelo que permite mudança apenas em $\sigma_{x}^{2}$ com priori do tipo $\pi(\underset{\sim}{\theta}, k) \propto p(k) \pi\left(\sigma^{2}\right) \prod_{i=1}^{2} \pi\left(\sigma_{x(i)}^{2}\right)$, onde $\pi\left(\sigma_{x(i)}^{2}\right) \sim I G\left(c_{i}, d_{i}\right)$, para $i=1,2$.

\subsubsection{Condições necessárias e suficientes no modelo que per- mite mudança em todos os parâmetros}

Nesta seção apresentamos alguns teoremas que garantem a existência da posteriori no modelo de regressão linear simples com erros normais que permite mudança em todos os parâmetros quando certas prioris impróprias do tipo $\pi(k, \underset{\sim}{\theta})=p(k) \pi\left(\underset{\sim}{\theta_{1}}\right) \pi\left(\underset{\sim}{\theta_{2}}\right)$ são utilizadas.

No modelo com mudança em todos os parâmetros e priori de componentes independentes, vimos em (4.1.5) que a posteriori $p\left(k, \underset{d}{\theta} \mid D_{\text {obs }}\right)$ é proporcional a

$$
p(k) \cdot L_{1}\left(\underset{\sim}{\theta_{1}} \mid D_{\mathrm{obs}} \stackrel{(k)}{1}\right) \cdot \pi\left({\underset{\sim}{\theta}}_{1}\right) \cdot L_{2}\left({\underset{\sim}{2}}_{2} \mid D_{\mathrm{obs} 2}^{(n-k)}\right) \pi\left({\underset{\sim}{2}}_{2}\right)
$$

onde $L_{1}\left(\underline{\theta}_{1} \mid D_{\mathrm{obs}}{ }_{1}^{(k)}\right)$ e $L_{2}\left(\underline{\sim}_{2} \mid D_{\mathrm{obs} 2}^{(n-k)}\right)$ são, respectivamente, as verossimilhanças antes e depois da mudança dadas em (4.1.3) e $\pi\left({\underset{\sim}{1}}_{1}\right)$ e $\pi\left(\underline{\theta}_{2}\right)$ são, respectivamente, as prioris antes e depois da mudança. $O$ espaço paramétrico é $\mathcal{K} \times \Theta$, onde $\mathcal{K}$ é um conjunto finito dos possíveis valores de $k$ e $\Theta=\left\{\underline{\theta}=\left(\begin{array}{l}\theta_{1} \\ \underline{\theta}_{2}\end{array}\right): \underline{\sim}_{i} \in \Theta_{i}\right\}$.

Portanto, para mostrar que a posteriori é própria (isto é, para mostrar que 
$\left.\sum_{k \in \mathcal{K}} \int_{\Theta} p\left(k, \underset{\theta}{\theta} \mid D_{\text {obs }}\right) \mathrm{d} \underset{\sim}{\theta}<\infty\right)$, devemos mostrar que

$$
\sum_{k \in \mathcal{K}} p(k) \int_{\Theta_{1}} L_{1}\left(\underset{\sim}{\theta_{1}} \mid D_{\text {obs }}{ }^{(k)}\right) \pi\left(\underset{\sim}{\theta_{1}}\right) \mathrm{d}{\underset{\sim}{\theta}}_{1} \cdot \int_{\theta_{2}} L_{2}\left(\underset{\sim}{\theta_{2}} \mid D_{\mathrm{obs}}^{(n-k)}\right) \pi\left({\underset{\sim}{2}}_{2}\right) \mathrm{d}{\underset{\sim}{\theta}}_{2}<\infty
$$

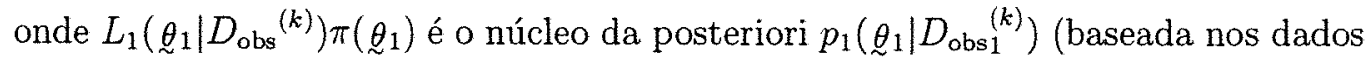
até a mudança $)$ e $L_{2}\left({\underset{\theta}{2}}_{2} \mid D_{\text {obs } 2}^{(n-k)}\right) \pi\left(\theta_{2}\right)$ é o núcleo da posteriori $p_{2}(\underbrace{}_{2} \mid D_{\text {obs2 }}{ }^{(n-k)})$ (baseada nos dados após a mudança).

Como $\mathcal{K}$ é um conjunto finito, então basta mostrar que qualquer parcela dessa soma é finita, ou seja, que as posterioris $p_{1}(\underbrace{}_{1} \mid D_{\text {obs } 1}^{(k)})$ e $p_{2}(\underbrace{}_{2} \mid D_{\text {obs } 2}^{(n-k)})$ são próprias para $k$ fixado $(k \in \mathcal{K})$.

Teorema 4.6.1 No modelo Normal com ponto de mudança dado em (4.1.1), com priori da forma $\pi(\underset{\underset{v}{*}}{)})=\pi\left(k,{\underset{\sim}{1}}_{1},{\underset{\sim}{2}}_{2}\right)=p(k) \prod_{i=1}^{2} \pi\left({\underset{\sim}{i}}_{i}\right)$, onde $p(k)=\left\{\begin{array}{ll}P[K=k], & k \in \mathcal{K}, \\ 0, & \text { caso contrário, }\end{array} \quad e \pi\left(\underline{\theta}_{i}\right) \propto \pi\left(\sigma_{x(i)}^{2}\right) \pi\left(\sigma_{i}^{2}\right)\right.$, onde $\pi\left(\sigma_{x(i)}^{2}\right) \sim I G\left(c_{i}, d_{i}\right)$ e $\pi\left(\sigma_{i}^{2}\right) \sim \operatorname{IG}\left(f_{i}, g_{i}\right), i=1,2$, então a condição necessária e suficiente para a existência da posteriori é que $k \geq 3$ e $n-k \geq 3$.

Demonstração: Para cada $k$ fixado, o Teorema 2.8.2 garante que a integral $\int_{\Theta_{1}} p_{1}\left({\underset{\sim}{1}}_{1} \mid D_{\mathrm{obs}}^{(k)}\right) \mathrm{d} \theta_{1}$ é finita se e somente se $k \geq 3$, e a integral $\int_{\Theta_{2}} p_{2}(\underbrace{}_{2} \mid D_{\mathrm{obs}}^{(n-k)}) \mathrm{d} \theta_{2}$ é finita se e somente se $(n-k) \geq 3$. Portanto, concluímos que a posteriori do modelo é própria se e somente se $3 \leq k \leq n-3$ e $n \geq 6$.

Teorema 4.6.2 No modelo do Teorema 4.6.1, se a distribuição $\pi\left(\sigma_{x(i)}^{2}\right)$ for substituída por $\pi\left(\sigma_{x(i)}^{2}\right) \propto\left(\sigma_{x(i)}^{2}\right)^{-r_{i}}, r_{i}>0, i=1,2$, então a condição necessária $e$ suficiente para que a posteriori correspondente seja própria é que $k \geq 2, n-k \geq 2$ $e 0<r_{i}<\frac{1}{2}, i=1,2$.

Demonstração: Para cada $k$ fixado, o Teorema 2.8 .3 garante que a integral $\int_{\theta_{1}} p_{1}(\underbrace{}_{1} \mid D_{\mathrm{obs}}{ }^{(k)}) \mathrm{d} \underline{\theta}_{1}$ é finita se e somente se $k \geq 2$ e $0<r_{1}<\frac{1}{2}$ e a integral 
$\int_{\Theta_{2}} p_{2}\left(\underset{\sim}{\theta_{2}} \mid D_{\mathrm{obs} 2}^{(n-k)}\right) \mathrm{d} \theta_{2}$ é finita se e somente se $(n-k) \geq 2$ e $0<r_{2}<\frac{1}{2}$. Portanto, a posteriori sob esse modelo é própria se e somente se $k \geq 2, n-k \geq 2$ e $0<r_{i}<\frac{1}{2}$, $i=1,2$.

Teorema 4.6.3 Se no modelo do Teorema 4.6.1 a distribuição de $\pi\left(\sigma_{i}^{2}\right)$ for substituída por $\pi\left(\sigma_{i}^{2}\right) \propto\left(\sigma_{i}^{2}\right)^{-s_{i}}, s_{i}>0, i=1,2$, e além disso os vetores de dados $\underset{\sim}{X^{(i)}}$ e $\underset{\sim}{Y^{(i)}}$ forem linearmente independentes e as componentes de $\underset{\sim}{X^{(i)}}$ (bem como as de ${\underset{\sim}{Y}}^{(i)}$ ) não são todas iguais (para $i=1,2$ ), então a condição necessária e suficiente para que a posteriori sob esse modelo seja própria é que $k \geq 3$ e $n-k \geq 3$.

Demonstração: A demonstração segue usando o mesmo argumento do teorema anterior e o Teorema 2.8.4 do Capítulo 2.

Teorema 4.6.4 No modelo do Teorema 4.6.1, se as distribuições de $\pi\left(\sigma_{x(i)}^{2}\right)$ e $\pi\left(\sigma_{i}^{2}\right)$ forem substituídas, respectivamente, por $\pi\left(\sigma_{x(i)}^{2}\right) \propto\left(\sigma_{x(i)}^{2}\right)^{-r_{i}}$ e $\pi\left(\sigma_{i}^{2}\right) \propto\left(\sigma_{i}^{2}\right)^{-s_{i}}$, $r_{i}, s_{i}>0, i=1,2$, e além disso $\underset{\sim}{X^{(i)}}$ e $Y^{(1)}$ forem linearmente independentes e tais que as componentes de $\underset{\sim}{X^{(i)}}$ (bem como as de ${\underset{\sim}{Y}}^{(i)}$ ) não são todas iguais, então a condição necessária e suficiente para que a distribuição à posteriori sob esse modelo seja própria é que $k+r_{1}+s_{1}>3 e(n-k)+r_{2}+s_{2}>3$ e $0<r_{i}<\frac{1}{2}, i=1,2$.

Demonstração: Usando o mesmo argumento do teorema anterior e o Teorema 2.8 .5 , concluímos que para cada $k$ fixado as integrais

$$
\int_{\Theta_{1}} p_{1}\left(\theta_{1} \mid D_{\mathrm{obs}_{1}}^{(k)}\right) \mathrm{d} \theta_{1} \quad \text { e } \int_{\Theta_{2}} p_{2}\left(\theta_{2} \mid D_{\mathrm{obs}_{2}}^{(n-k)}\right) \mathrm{d} \theta_{2}
$$

são finitas se e somente se $k+r_{1}+s_{1}>3,(n-k)+r_{2}+s_{2}>3$ e $0<r_{i}<\frac{1}{2}, i=1,2$, e portanto a posteriori existe se e somente se estas condições forem satisfeitas. 


\subsubsection{Condições suficientes no modelo que permite mudança apenas em $\sigma_{x}^{2}$}

Teorema 4.6.5 No modelo estrutural Normal que permite mudança apenas no parâmetro $\sigma_{x}^{2}$ após o ponto $k$, com priori $\pi(\underset{\sim}{\theta}, k) \propto p(k) \pi\left(\sigma^{2}\right) \prod_{i=1}^{2} \pi\left(\sigma_{x(i)}^{2}\right)$, onde $\theta=\left(\alpha, \beta, \mu, \sigma_{x(1)}^{2}, \sigma_{x(2)}^{2}, \sigma^{2}\right)^{T}, p(k)=\left\{\begin{array}{l}P[K=k], k \in \mathcal{K}, \text { onde } \mathcal{K} \text { é um conjunto finito, } \\ 0, \text { caso contrário, }\end{array}\right.$ $\pi\left(\sigma^{2}\right) \sim I G(f, g)$ e $\pi\left(\sigma_{x(i)}^{2}\right) \sim I G\left(c_{i}, d_{i}\right), i=1,2$, então uma condição suficiente para que a posteriori seja própria é que $n \geq 3$.

Demonstração: O objetivo é mostrar que $\sum_{k \in \mathcal{K}} \int_{\Theta} p\left(k, \underset{\sim}{\theta} \mid D_{\mathrm{obs}}\right) \mathrm{d} \underset{\sim}{\theta}<\infty$, onde $p\left(k, \underset{\sim}{\theta} \mid D_{\text {obs }}\right)=\int_{\Re} p\left(k, \underset{\sim}{\theta}, \underset{\sim}{x} \mid D_{\text {obs }}\right) \mathrm{d} \underset{\sim}{x}$ e $p\left(k, \underset{\sim}{\theta} \underset{\sim}{x} \mid D_{\text {obs }}\right) \propto L(k, \underset{\sim}{\theta} \mid D) \pi(k, \underset{\sim}{\theta})$, onde $D_{\text {obs }}=\left\{\left(Y_{i}, X_{i}\right)^{T}, i=1, \ldots, n\right\}, D=\left\{\left(Y_{i}, X_{i}, x_{i}\right)^{T}, i=1, \ldots, n\right\}, \Theta=$ $\left\{\underset{\theta}{\theta}=\left(\alpha, \beta, \mu, \sigma_{x(1)}^{2}, \sigma_{x(2)}^{2}, \sigma^{2}\right)^{T} \in \mathbb{R}^{3} \times \mathbb{R}_{+}^{3}\right\}, \mathcal{K}$ é o conjunto finito dos possíveis valores de $k$ e $\mathscr{X}=\left\{\underset{\sim}{x}=\left(x_{1}, \ldots, x_{n}\right) \in \mathbb{R}^{n}\right\}^{T}$.

Portanto, para mostrar que a posteriori é própria, basta mostrar que

$$
\begin{aligned}
& \int_{x} \int_{\Theta} L(k, \underset{\underset{ }{*}}{ } \mid D) \pi(\underset{\sim}{\theta}) \mathrm{d} \underset{\sim}{\theta} \mathrm{d} \underset{\sim}{x}<\infty \text { para } k \text { fixado, onde } \\
& L(k, \underset{\theta}{\theta} \mid D) \pi(\underset{\theta}{\theta}, k) \propto\left(\sigma^{2}\right)^{-(n+f+1)}\left(\sigma_{x(1)}^{2}\right)^{-\left(\frac{k}{2}+c_{1}+1\right)}\left(\sigma_{x(2)}^{2}\right)^{-\left(\frac{n-k}{2}+c_{2}+1\right)} . \\
& \cdot \exp \left\{-\frac{1}{2}\left[\frac{2 d_{1}}{\sigma_{x(1)}^{2}}+\frac{2 d_{2}}{\sigma_{x(2)}^{2}}+\frac{2 g}{\sigma^{2}}+\frac{\sum_{i=1}^{n}\left(Y_{i}-\alpha-\beta x_{i}\right)^{2}}{\lambda \sigma^{2}}+\frac{\sum_{i=1}^{n}\left(X_{i}-x_{i}\right)^{2}}{\sigma^{2}}\right.\right. \\
& \left.\left.+\frac{\sum_{i=1}^{k}\left(x_{i}-\mu\right)^{2}}{\sigma_{x(1)}^{2}}+\frac{\sum_{i=k+1}^{n}\left(x_{i}-\mu\right)^{2}}{\sigma_{x(2)}^{2}}\right]\right\} \text {. }
\end{aligned}
$$

Esta função pode ser majorada pela seguinte função:

$$
\begin{gathered}
f\left(k, \underset{\sim}{\theta} \underset{\sim}{x}, D_{\mathrm{obs}}\right)=\left(\sigma^{2}\right)^{-(n+f+1)}\left(\sigma_{x(1)}^{2}\right)^{-\left(\frac{k}{2}+c_{1}+1\right)}\left(\sigma_{x(2)}^{2}\right)^{-\left(\frac{n-k}{2}+c_{2}+1\right)} \cdot \exp \left\{-\frac{1}{2}\left[\frac{2 d_{1}}{\sigma_{x(1)}^{2}}\right.\right. \\
\left.\left.+\frac{2 d_{2}}{\sigma_{x(2)}^{2}}+\frac{2 g}{\sigma^{2}}+\frac{\sum_{i=1}^{n}\left(Y_{i}-\alpha-3 x_{i}\right)^{2}}{\lambda \sigma^{2}}+\frac{\sum_{i=1}^{n}\left(X_{i}-x_{i}\right)^{2}}{\sigma^{2}}+\frac{\sum_{i=1}^{k}\left(x_{i}-\mu\right)^{2}}{\sigma_{x(1)}^{2}}\right]\right\}
\end{gathered}
$$

e portanto

$$
\int_{\mathscr{X}} \int_{\Theta} L(k, \underline{\theta} \mid D) \pi(\underline{\theta}, k) \mathrm{d} \underset{\theta}{\mathrm{d}} \underset{\sim}{x} \leq \int_{\mathfrak{X}} \int_{\Theta} f\left(k, \underline{\theta}, \underset{\sim}{x}, D_{\mathrm{obs}}\right) \mathrm{d} \underset{\theta}{\mathrm{d}} \underset{\sim}{x} .
$$


Integrando inicialmente a função $f\left(k, \underset{\sim}{\theta}, \underset{\sim}{x}, D_{\text {obs }}\right)$ em relação a $\underset{\sim}{\theta}=$ $\left(\alpha, \beta, \mu, \sigma_{x(1)}^{2}, \sigma_{x(2)}^{2}, \sigma^{2}\right)^{T}$, obtemos que $\int_{\Theta} f\left(k, \underset{\sim}{\theta} \underset{\sim}{x}, D_{\mathrm{obs}}\right) \mathrm{d} \theta$ é proporcional a

$$
1 /\left[S_{x x}^{\frac{1}{2}}\left(2 d_{1}+S_{x^{(1)} x^{(1)}}\right)^{\frac{k+2 c_{1}+1}{2}}\left(2 g+\sum_{i=1}^{n}\left(X_{i}-x_{i}\right)^{2}+\frac{S_{x x} S_{Y Y}-S_{x Y}^{2}}{\lambda S_{x x}}\right)^{n+f-1}\right],
$$

onde $S_{x^{(1)} x^{(1)}}=\sum_{i=1}^{k}\left(x_{i}-\bar{x}_{1}\right)^{2}$.

$$
\begin{aligned}
& \text { Portanto, } \int_{\mathscr{X}} \int_{\Theta} f\left(k, \underset{\sim}{\theta}, \underset{\sim}{x}, D_{\text {obs }}\right) \mathrm{d} \underset{\sim}{\theta} \mathrm{d} \underset{\sim}{x} \\
& \leq\left(2 d_{1}\right)^{-\frac{\left(k+2 c_{1}+1\right)}{2}} \int_{\mathscr{X}} \frac{1}{S_{x x}^{\frac{1}{2}}\left(2 g+\sum_{i=1}^{n}\left(X_{i}-x_{i}\right)^{2}+\frac{S_{x x} S_{Y Y}-S_{x Y}^{2}}{\lambda S_{x x}}\right)^{n+f-1}} \mathrm{~d} \underset{\sim}{x} .
\end{aligned}
$$

A integral $\int_{\mathscr{X}} \frac{1}{S_{x x}^{\frac{1}{2}}\left(2 g+\sum_{i=1}^{n}\left(X_{i}-x_{i}\right)^{2}+\frac{S_{x x} S_{Y Y}-S_{x Y}^{2}}{\lambda S_{x x}}\right)^{n+f-1}} \mathrm{~d} \underset{\sim}{x}$ pode ser analisada mais facilmente fazendo uma transformação de variáveis (usada na seção 2.8) que a $\operatorname{cada}\left(\underset{\sim}{x} x_{n \times 1}, \underset{\sim}{X} X_{n \times 1}, Y_{n \times 1}\right)$ associa $\left(\underset{\sim}{z}{ }_{n \times 1}, \underset{\sim}{b}{ }_{n \times 1}, \underset{\sim}{c}{ }_{n \times 1}\right)=\left(H \underset{\sim}{x}{ }_{n \times 1}, H \underset{\sim}{X}{\underset{n \times 1}{ }}_{1}, H \underset{\sim}{Y}{ }_{n \times 1}\right)$, onde $H$ é a matriz de Helmert.

De fato, com esta mudança de variáveis, a integral acima fica igual a

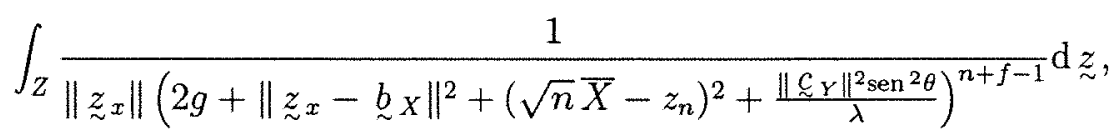

onde $\underset{\sim}{z}, \underset{\sim}{b}{ }_{x}$ e $\underset{\sim}{c} Y$ são os vetores formados pelas primeiras $n-1$ componentes de $\underset{\sim}{z} \underset{\sim}{b}$ e $\underset{\sim}{c}$, respectivamente. $\theta$ é o ângulo entre $\underset{\sim}{c_{Y}}$ e $\underset{\sim}{z}$ e $Z=\left\{\underset{\sim}{z}=\left(z_{1}, \ldots, z_{n}\right)^{T}\right.$ : $\left.z_{i} \in \mathbb{R}\right\}$.

Essa integral pode ser escrita como

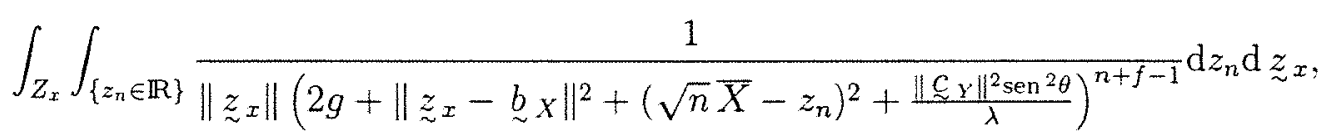

onde $Z_{x}=\left\{z_{x}=\left(z_{1}, \ldots, z_{n-1}\right): z_{i} \in \mathbb{R}\right\}$ e portanto, desde que $n+f>\frac{3}{2}$, ela é proporcional a

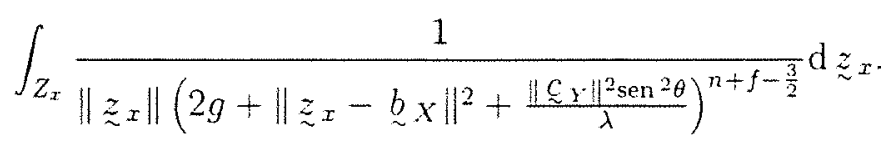


Essa última integral (que é totalmente semelhante àquela que aparece na demonstração do Teorema 2.8.4) é finita se $1<n-1$ e $1+2\left(n+f-\frac{3}{2}\right)>n-1$, isto é, se $n \geq 3$. Concluímos portanto que a posteriori é finita se $n \geq 3$.

Teorema 4.6.6 No modelo do Teorema 4.6.1, se trocarmos a distribuição $\pi\left(\sigma^{2}\right) \sim$ $I G(f, g)$ por $\pi\left(\sigma^{2}\right) \propto\left(\sigma^{2}\right)^{-s}, s>0$, e se $\underset{\sim}{X}$ e $\underset{\sim}{Y}$ forem linearmente independentes, onde as componentes de $\underset{\sim}{X}$, e as de $\underset{\sim}{Y}$, não são todas iguais, então uma condição suficiente para a existência da posteriori é que $n \geq 3$.

Demonstração: Substituindo $f$ por $(s-1)$ e $g$ por zero na demonstração anterior, vemos que $\int_{x} \int_{\Theta} p\left(\underset{\sim}{\theta}, k, \underset{\sim}{x} \mid D_{\text {obs }}\right) \mathrm{d} \underset{\sim}{\theta} \mathrm{d} \underset{\sim}{x}$ é majorada por uma integral que é proporcional a

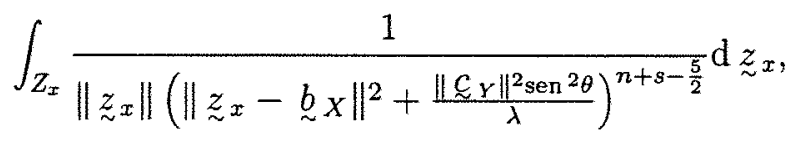

que é totalmente semelhante àquela que aparece na demonstração do Teorema 2.8.5 e portanto é finita se e somente se $1<n-1$ e $1+2\left(n+s-\frac{5}{2}\right)>n-1$. Concluímos que para cada $k$ fixado em $\mathcal{K}$, a integral $\int_{\mathcal{X}} \int_{\Theta} p\left(\underset{\sim}{\theta}, k \underset{\sim}{x} \mid D_{\text {obs }}\right) \mathrm{d} \underset{\sim}{\theta} \mathrm{d} \underset{\sim}{x}$ é finita se $n \geq 3$ e portanto, se $n \geq 3$, então

$$
\sum_{k \in \mathcal{K}} \int_{\mathscr{X}} \int_{\Theta} p\left(\underset{\sim}{\theta}, k, \underset{\sim}{x} \mid D_{\mathrm{obs}}\right) \mathrm{d} \underset{\sim}{\underset{\mathrm{d}}{\underset{\sim}{x}} \underset{\sim}{x}<\infty} .
$$




\subsection{EXEMPLOS}

Nessa seção são apresentados exemplos de aplicação da teoria das seções 4.1 a 4.4, envolvendo os modelos com restrição e sem restrição de identificabilidade, utilizando uma amostra de dados simulados.

\subsubsection{Análise do modelo estrutural normal identificável}

1. O MODELO que permite mudança em todos os parâmetros:

$$
\left\{\begin{array}{l}
Y_{i}=\left\{\begin{array}{l}
\alpha_{1}+\beta_{1} x_{i}+e_{i}, \quad i=1, \ldots, k, \quad 1 \leq k \leq n-1, \\
\alpha_{2}+\beta_{2} x_{i}+e_{i}, \quad i=k+1, \ldots, n,
\end{array}\right. \\
X_{i}=x_{i}+u_{i}, \quad i=1, \ldots, n
\end{array}\right.
$$

onde

$$
\left(\begin{array}{c}
e_{i} \\
u_{i} \\
x_{i}
\end{array}\right) \stackrel{\text { iid }}{\sim}\left\{\begin{array}{l}
N\left(\left(\begin{array}{c}
0 \\
0 \\
\mu_{1}
\end{array}\right) ;\left(\begin{array}{ccc}
\lambda \sigma_{1}^{2} & 0 & 0 \\
0 & \sigma_{1}^{2} & 0 \\
0 & 0 & \sigma_{x(1)}^{2}
\end{array}\right)\right), i=1, \ldots, k, \\
N\left(\left(\begin{array}{c}
0 \\
0 \\
\mu_{2}
\end{array}\right) ;\left(\begin{array}{ccc}
\lambda \sigma_{2}^{2} & 0 & 0 \\
0 & \sigma_{2}^{2} & 0 \\
0 & 0 & \sigma_{x(2)}^{2}
\end{array}\right)\right), i=k+1, \ldots, n,
\end{array}\right.
$$

Distribuição à priori:

$\pi\left({\underset{\sim}{1}}_{1}, \underline{\sim}_{2}, k\right)=\pi(k) \pi\left({\underset{\sim}{1}}_{1}\right) \pi\left({\underset{\sim}{2}}_{2}\right)$, onde $\pi\left({\underset{\sim}{i}}_{i}\right)=\pi\left(\alpha_{i}\right) \pi\left(\beta_{i}\right) \pi\left(\mu_{i}\right) \pi\left(\sigma_{x_{i}}^{2}\right) \pi\left(\sigma_{i}^{2}\right)$,

$i=1,2$, onde $\alpha_{i} \sim N(0 ; 1000), \beta_{i} \sim N(0 ; 1000), \mu_{i} \sim N(0 ; 1000)$,

$\sigma_{x_{i}}^{2} \sim I G(2.001 ; 1.0), \sigma_{i}^{2} \sim I G(2.001 ; 1.0) \mathrm{e}$

$$
p(k)= \begin{cases}P(K=k)=\frac{1}{\# \mathcal{K}}, & k \in \mathcal{K}=\{1, \ldots, n-1\}, \\ 0, & \text { caso contrário. }\end{cases}
$$

e $\lambda=1$ com probabilidade 1 .

\section{DADOS}

A Tabela 4.1 apresenta uma amostra de tamanho $n=50$ de dados gerados a partir do modelo em (4.7.1), com mudança em todos os parâmetros, onde $\alpha_{1}=2.0$, 
$\beta_{1}=1.0, \mu_{1}=2.0, \sigma_{x(1)}^{2}=0.6, \sigma_{1}^{2}=0.1, \alpha_{2}=1.0, \beta_{2}=2.0, \mu_{2}=4.0, \sigma_{x(2)}^{2}=1.2, \sigma_{2}^{2}=0.2$, $\lambda=1.0, k=20$, utilizando-se um programa elaborado na linguagem $\mathrm{OX}$ (usando a semente "ranseed=17").

Tabela 4.1: Amostra de tamanho $\mathrm{n}=50$ de dados simulados a partir do modelo (4.7.1), onde $\alpha_{1}=2.0, \beta_{1}=1.0, \mu_{1}=2.0, \sigma_{x(1)}^{2}=0.6, \sigma_{1}^{2}=0.1, \alpha_{2}=1.0, \beta_{2}=2.0, \mu_{2}=4.0$, $\sigma_{x(2)}^{2}=1.2, \sigma_{2}^{2}=0.2, \lambda=1.0, k=20$.

\begin{tabular}{llllllllllll}
$i$ & $Y_{i}$ & $X_{i}$ & $i$ & $Y_{i}$ & $X_{i}$ & $i$ & $Y_{i}$ & $X_{i}$ & $i$ & $Y_{i}$ & $X_{i}$ \\
\hline 1 & 4.3092 & 1.7503 & 14 & 4.1315 & 2.3448 & 27 & 8.7985 & 3.6264 & 40 & 11.355 & 5.4473 \\
2 & 3.4970 & 1.2028 & 15 & 4.6854 & 2.1908 & 28 & 13.962 & 6.3921 & 41 & 8.7974 & 2.9984 \\
3 & 3.6984 & 1.8785 & 16 & 3.0402 & 1.0815 & 29 & 7.0030 & 2.9641 & 42 & 7.9116 & 4.0154 \\
4 & 5.1943 & 2.4628 & 17 & 3.8688 & 2.0400 & 30 & 8.3874 & 4.1586 & 43 & 7.2250 & 4.0347 \\
5 & 5.1489 & 2.8678 & 18 & 5.5450 & 3.5886 & 31 & 11.844 & 6.2737 & 44 & 11.923 & 5.7843 \\
6 & 2.3622 & 0.7882 & 19 & 4.3375 & 1.6627 & 32 & 10.348 & 3.9149 & 45 & 5.3486 & 2.1688 \\
7 & 4.2616 & 1.8405 & 20 & 4.2863 & 2.1210 & 33 & 8.1851 & 3.7377 & 46 & 10.021 & 4.5871 \\
8 & 3.5888 & 2.5897 & 21 & 4.6501 & 2.8548 & 34 & 6.7018 & 2.7880 & 47 & 8.2339 & 3.5194 \\
9 & 3.6661 & 2.5529 & 22 & 9.2052 & 4.0509 & 35 & 9.3974 & 4.4571 & 48 & 3.2243 & 0.7888 \\
10 & 4.8814 & 3.6252 & 23 & 4.6988 & 1.9951 & 36 & 10.736 & 3.7249 & 49 & 8.8972 & 3.3560 \\
11 & 4.0650 & 2.5828 & 24 & 12.144 & 5.0546 & 37 & 8.3573 & 3.4238 & 50 & 9.8834 & 4.3421 \\
12 & 3.9926 & 2.5085 & 25 & 8.7343 & 3.4862 & 38 & 12.353 & 5.4157 & & & \\
13 & 4.2061 & 2.4555 & 26 & 5.2684 & 2.2406 & 39 & 7.8066 & 3.1820 & & & \\
\hline
\end{tabular}

OBSERVAÇÃO: Nesse estudo, pelo fato de que a distribuição à priori escolhida é praticamente não informativa e o conjunto de dados foi simulado a partir do modelo em (4.7.1), onde $\alpha_{1}=2.0, \beta_{1}=1.0, \mu_{1}=2.0, \sigma_{x(1)}^{2}=0.6, \sigma_{1}^{2}=0.1, \alpha_{2}=1.0, \beta_{2}=2.0$, $\mu_{2}=4.0, \sigma_{x(2)}^{2}=1.2, \sigma_{2}^{2}=0.2, \lambda=1.0, k=20$ então, se a metodologia empregada é adequada, devemos esperar que as estimativas e os resultados à posteriori sejam compatíveis com esse modelo.

\section{AJUSTE DO MODELO}

Os algoritmos de Gibbs (simples) e "Modified and Collapsed Gibbs" foram utilizados com o objetivo de amostrar da distribuição à posteriori do modelo.

De cada algorítmo geramos $M=4$ seqüências paralelas de comprimento $T=$ 10.000 partindo de pontos iniciais obtidos pelo seguinte procedimento:

Obtivemos as estimativas de máximaverossimilhança de $\theta=\left(\begin{array}{c}\theta_{1} \\ \sigma_{2}\end{array}\right)$. para cada valor de $k$ fixado (onde $k=3, \ldots, n-3$ ). Aplicamos um algorítmo de otimização 
de Quase-Newton (denominado BFGS) usando cada uma dessas estimativas como ponto inicial e localizamos n-5 pontos estacionários da posteriori. Depois disso geramos 1000 pontos $\underset{\sim}{\stackrel{*}{\theta}}=\left(\begin{array}{c}k \\ \theta_{1} \\ \underbrace{}_{2}\end{array}\right)$ da distribuição formada pela mistura de distribuições $t_{4}\left({\underset{\sim}{(}}_{i}^{*}, \hat{V}_{i}^{*}\right), i=1, \ldots, n-5$, onde ${\underset{\sim}{\hat{\theta}}}_{i}^{*}$ é o i-ésimo ponto estacionário e

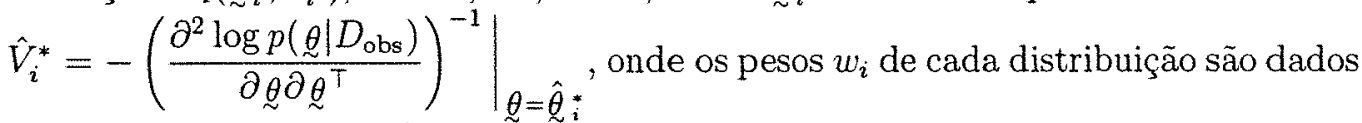
por $w_{i}=q\left(\hat{\theta}_{i}^{*} \mid D_{\text {obs }}\right)\left|\hat{V}_{i}^{*}\right|^{\frac{1}{2}}$, onde $q\left(\hat{\theta}_{i}^{*} \mid D_{\text {obs }}\right)$ é a posteriori não normalizada calculada no ponto $\hat{\theta}_{i}^{*}$.

Finalmente reamostramos apenas 10 pontos (utilizando re-amostragem ponderada), dos quais apenas os seguintes quatro pontos foram usados para inicializar o algorítmo de Gibbs:

$$
\begin{aligned}
& \stackrel{\sim}{\theta}_{1}^{(0)}=(21,2.153,0.828,2.608,0.393,0.216,0.265,2.281,3.335,0.862,0.215)^{T} \\
& \ddot{\theta}_{2}^{(0)}=(21,1.466,1.197,2.286,0.256,0.185,0.331,2.149,3.813,1.299,0.202)^{T} \\
& \stackrel{*}{\theta}^{(0)}=(21,3.140,0.393,2.522,0.582,0.102,2.299,1.716,3.998,1.726,0.084)^{T} \\
& \ddot{\theta}_{4}^{(0)}=(21,1.924,0.967,1.950,0.404,0.064,1.758,2.013,3.791,1.638,0.306)^{T}
\end{aligned}
$$

\section{3.a Diagnósticos de convergência}

A seguir são apresentados alguns dispositivos gráficos para o diagnóstico de possíveis falhas na convergência dos algoritmos de Gibbs e Modified Collapsed Gibbs.

Para cada um dos parâmetros do modelo proposto temos os gráficos das séries temporais, das funções de auto-correlação e da medida de Gelman e Rubin baseados nos algoritmos de Gibbs e Modified Collapsed Gibbs. Temos também os gráficos da medida de Brooks e Gelman obtidos a partir desses dois algoritmos.

As figuras 4.1 a 4.8 não sugerem que exista problema na convergência dos algoritmos de Gibbs e Modified Collapsed Gibbs. Além disso o algoritmo M.Collap.Gibbs parece ter uma melhor performance que o Gibbs (como pode ser visto principalmente pela comparação das funções de auto-correlação amostral correspondentes). 


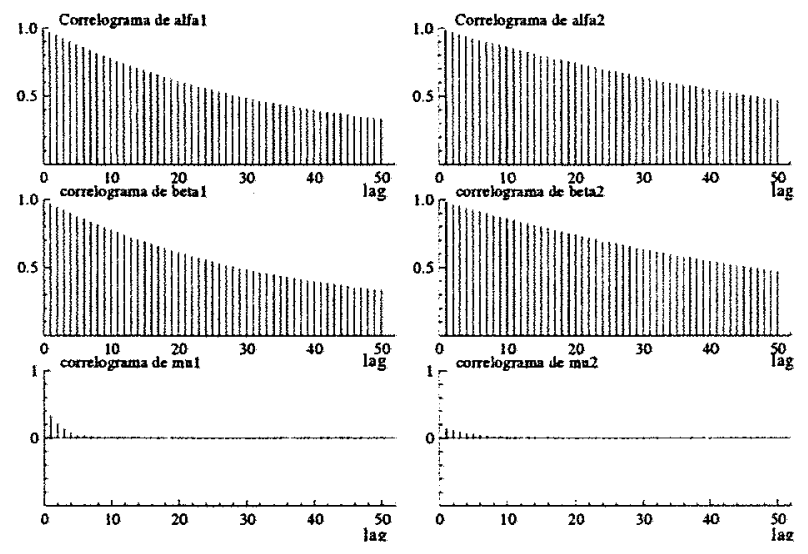

Figura 4.1: Gráficos das funçōes de auto-correlação de $\alpha_{1}, \alpha_{2}, \beta_{1}, \beta_{2}, \mu_{1}$ e $\mu_{2}$, baseados em uma única seqüencia de Gibbs de comprimento $T=50.000$, partindo do ponto inicial $\ddot{\theta}_{1}^{(0)}$.

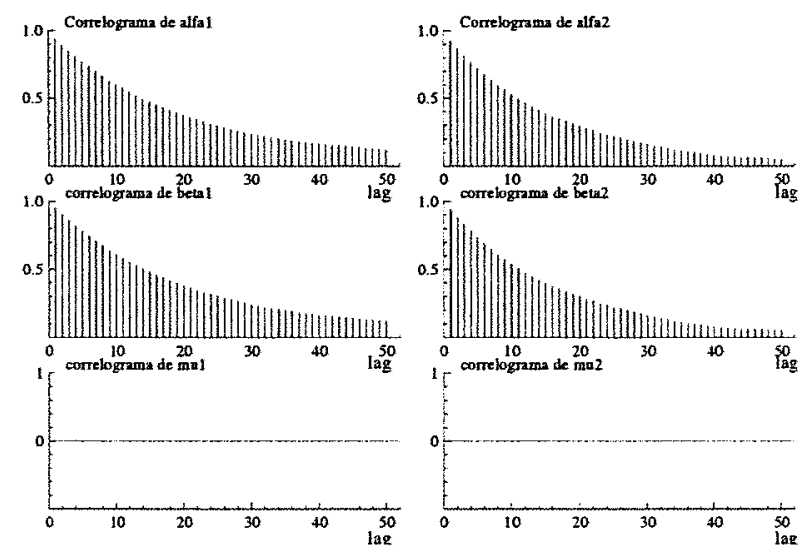

Figura 4.2: Gráficos das funções de auto-correlação de $\alpha_{1}, \alpha_{2}, \beta_{1}, \beta_{2}, \mu_{1}$ e $\mu_{2}$, baseados em uma única sequêencia do algoritmo M.Collapsed Gibbs de comprimento $T=50.000$.
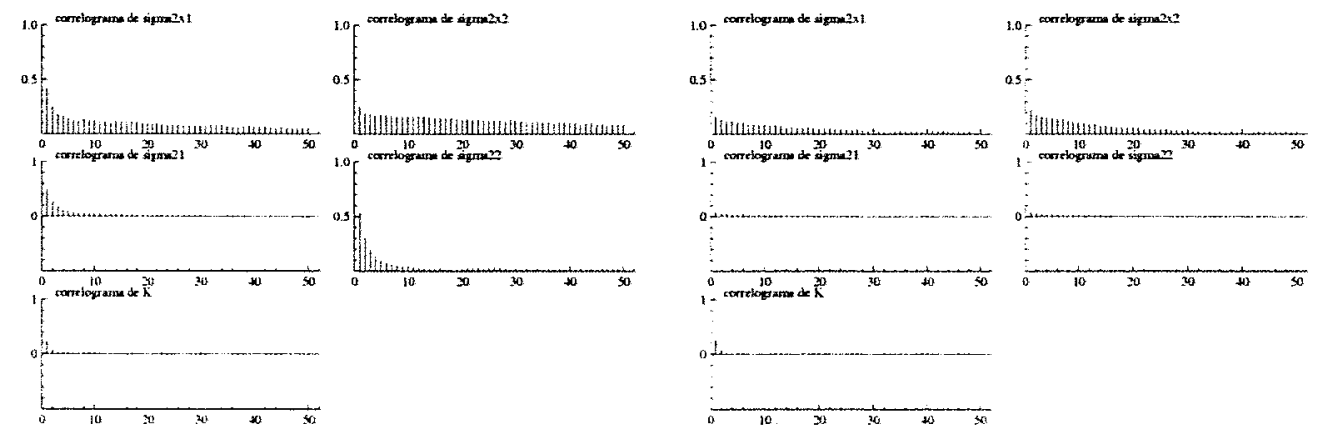

Figura 4.3: Gráficos das funçōes de auto-correlaçāo de $\sigma_{x_{1}}^{2}, \sigma_{x_{2}}^{2}, \sigma_{1}^{2}, \sigma_{2}^{2}$ e $k$. Os 4 do lado esquerdo baseiam-se na mesma seq. de Gibbs da figura 4.1 e os $\$$ do lado direito na mesma seq. do alg. "M.Collapsed Gibbs" da fig 4.2 . 


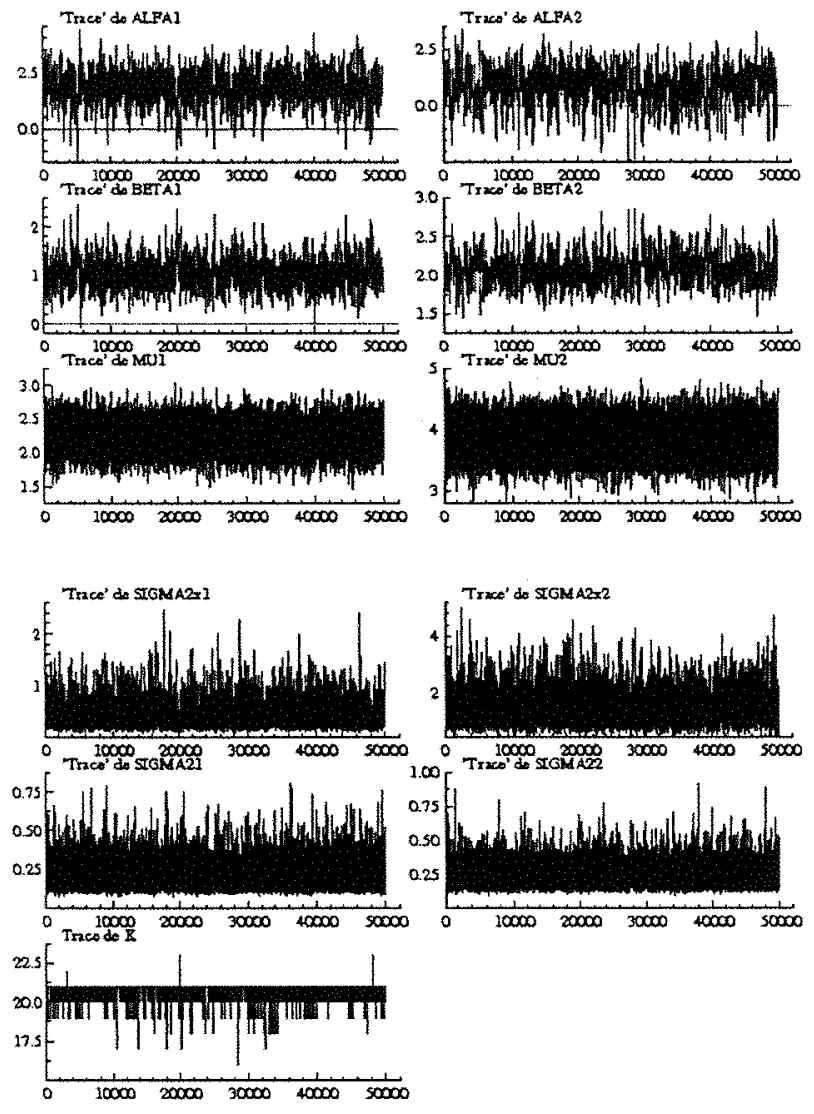

Figura 4.4: Gráficos das séries temporais ("trace") de $\alpha_{1}, \alpha_{2}, \beta_{1}, \beta_{2}, \mu_{1}, \mu_{2}, \sigma_{x_{1}}^{2}, \sigma_{x_{2}}^{2}, \sigma_{1}^{2}, \sigma_{2}^{2}$ e $k$ versus "número de iteraçóes "baseados em uma única seqüencia de comprimento $T=50.000$ do algoritmo de Gibbs .

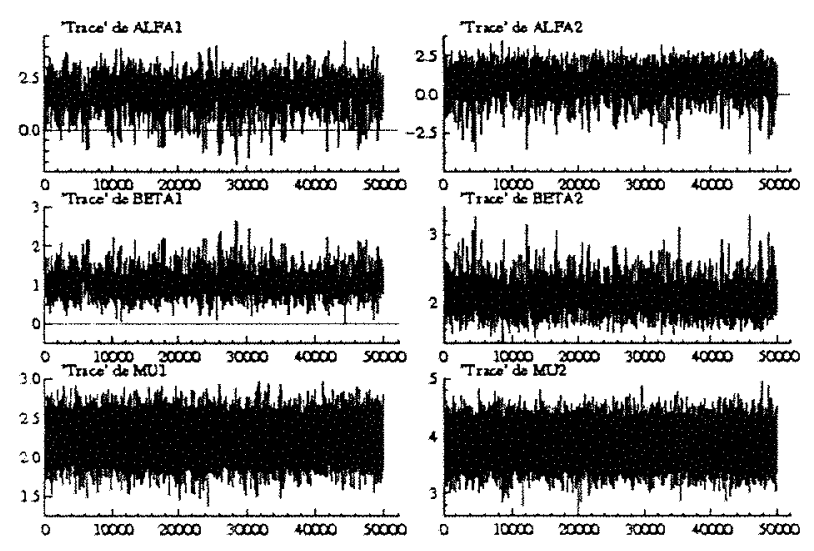

Figura 4.5: "Trace" de $\alpha_{1}, \alpha_{2}, \beta_{1}, \beta_{2}, \mu_{1}, \mu_{2}$ baseados em uma seq. de $\mathrm{T}=50000$ do alg. M.Collap.Gibbs. 


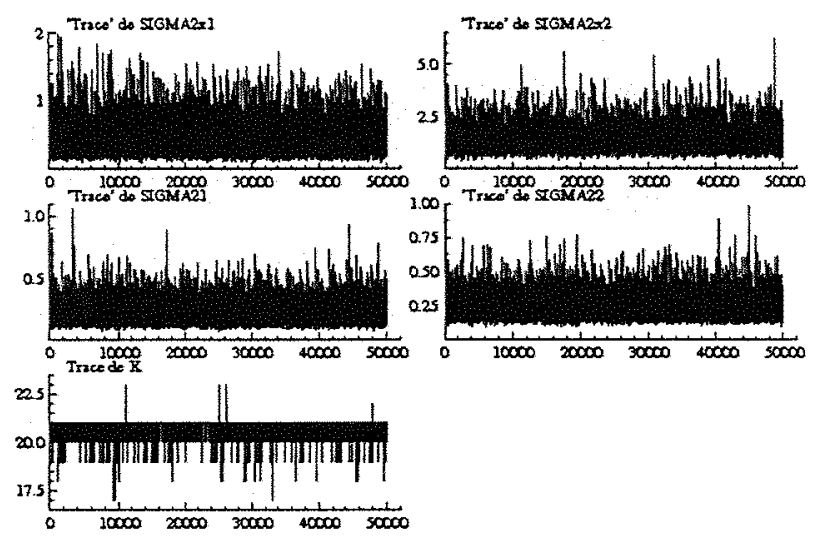

Figura 4.6: Gráficos das séries temporais ("Trace") de $\sigma_{x_{1}}^{2}, \sigma_{x_{2}}^{2}, \sigma_{1}^{2}, \sigma_{2}^{2}$ e $k$ versus " nro. de iteraçōes" baseados em uma única seqüência de comprimento $T=50.000$ do algoritmo M.Collapsed Gibbs .

Brooks \& Gelman Multivariate Shrink Factors

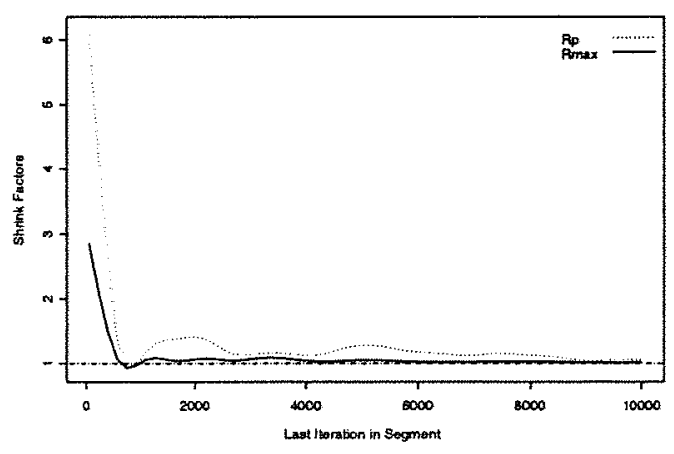

Brooks \& Gelman Multivariate Shrink Factors

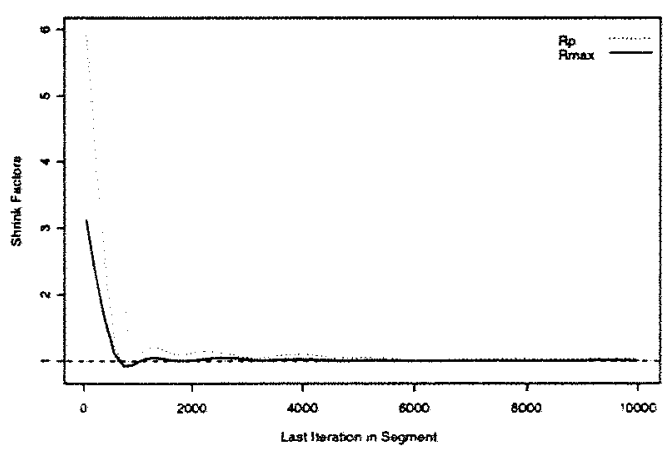

Figura 4.7: "Multiv. Potential Scale Reduction" de Brooks e Gelman (1998) baseados em $M=4$ sequiencias de comprimento $T=10.000$. O gráfico superior é baseado no alg. de Gibbs e o inferior no alg. M.Collapsed Gibbs. 
Gelman \& Rubin Shrink Factors
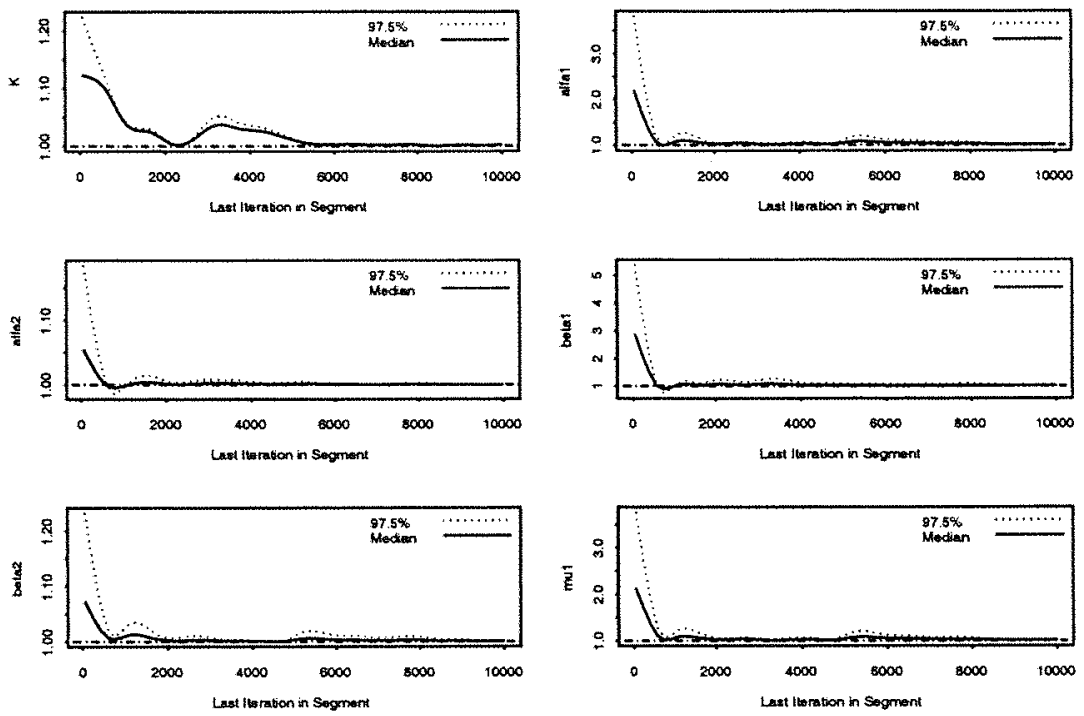

Gelman \& Rubin Shrink Factors
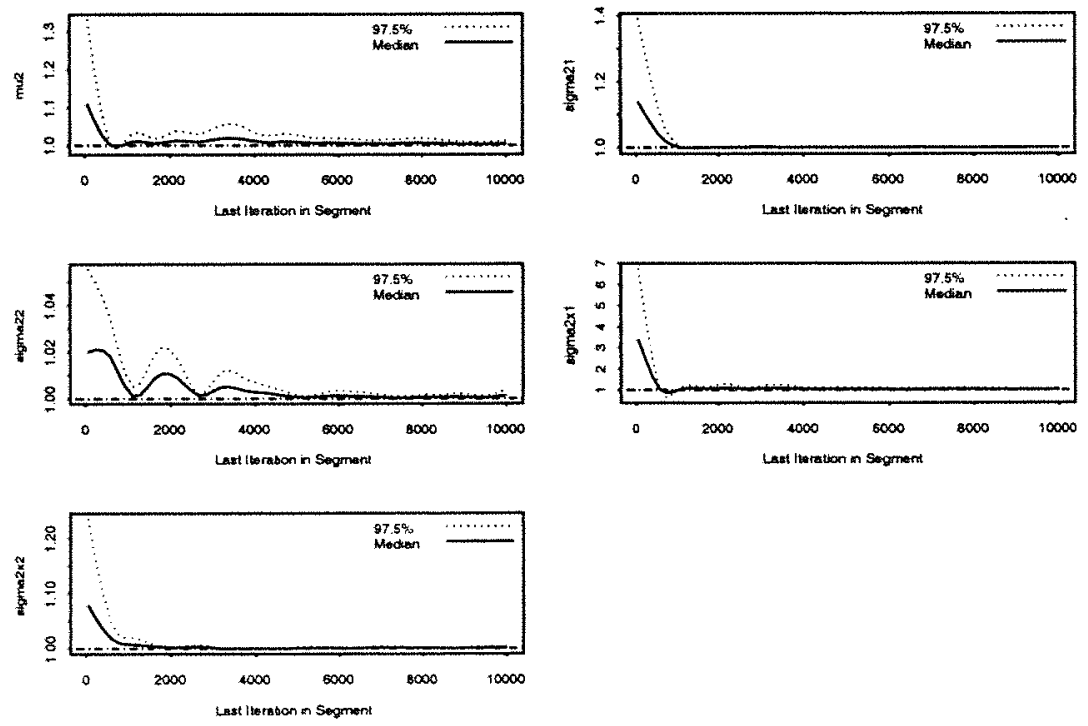

Figura 4.8: Gráfico da medida de Gelman e Rubin corrigida "Corrected potencial scale reduction factors" (CSRF) para cada parametro $k, \alpha_{1}, \alpha_{2}, \beta_{1}, \beta_{2}, \mu_{1}, \mu_{2}, \sigma_{x_{1}}^{2}, \sigma_{x_{2}}^{2}, \sigma_{1}^{2}, \sigma_{2}^{2}$, baseado em $M=4$ seqüéncias de comprimento $T=10.000$ de Gibbs. 
Gelman \& Rubin Shrink Factors
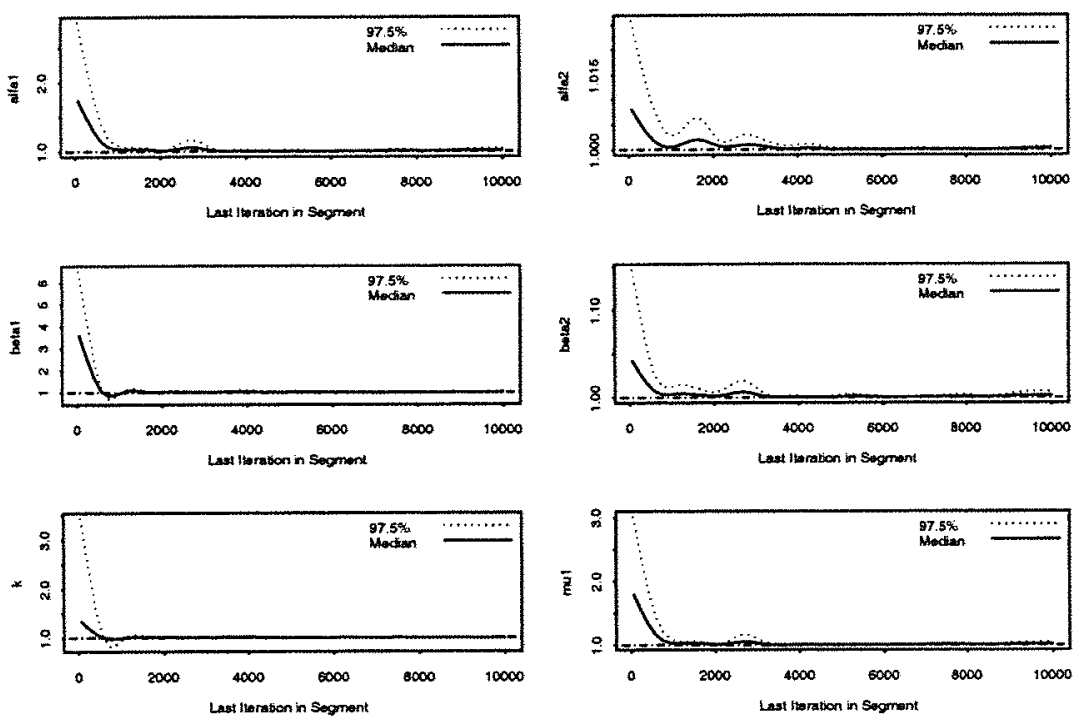

Gelman \& Rubin Shrink Factors
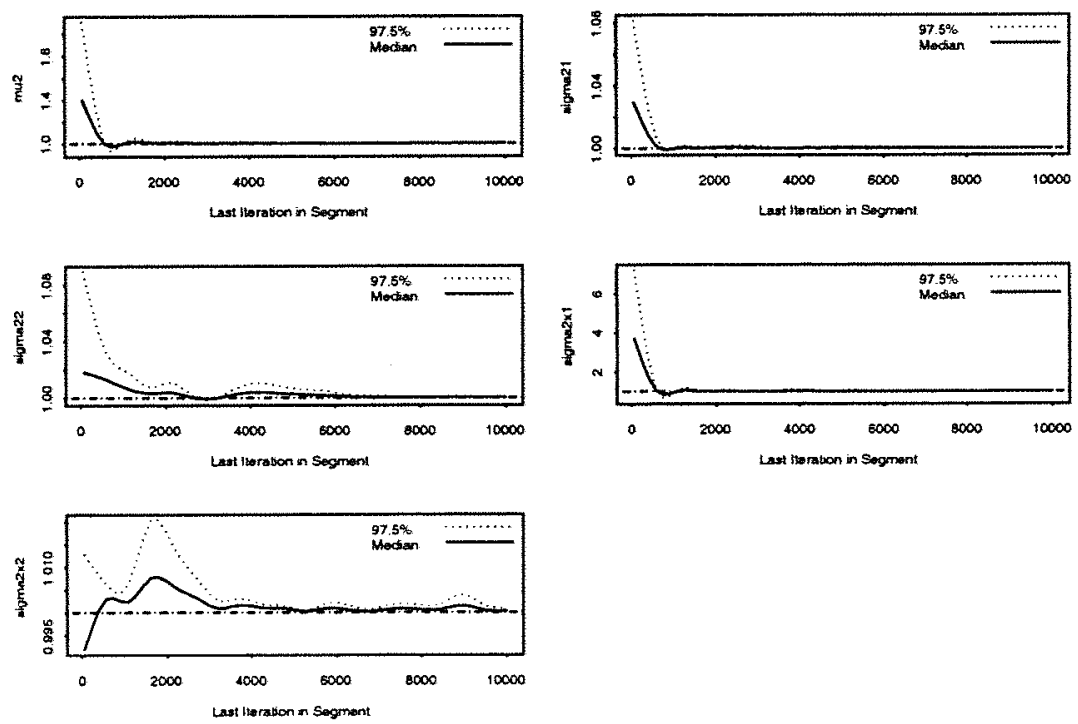

Figura 4.9: Gráfico da medida de Gelman e Rubin corrigida "Corrected potencial scale reduction factors " (CSRF) para cada parametro $k, \alpha_{1}, \alpha_{2}, \beta_{1}, \beta_{2}, \mu_{1}, \mu_{2}, \sigma_{x_{1}}^{2}, \sigma_{x_{2}}^{2}, \sigma_{1}^{2}, \sigma_{2}^{2}$, baseado em $M=4$ sequências de comprimento $T=10.000$ do algoritmo M.Collapsed Gibbs. 


\section{3.b Estimação:}

Para verificar se as estimativas à posteriori do ponto de mudança $k$ diferem muito do valor verdadeiro, aplicamos o algoritmo M.Collapsed Gibbs $n-1$ vezes, cada vez usando um conjunto diferente de dados simulados (cada conjunto foi gerado apartir do modelo em (4.7.1) com um valor de $\mathrm{k}$ diferente ( $\mathrm{k}$ entre 1 e $\mathrm{n}-1$ ), mas com os mesmos valores de $\underset{\sim}{\theta_{1}}$ e $\underset{\sim}{\theta_{2}}$ usados na geração da tabela 4.1).

A tabela 4.2 apresenta os valores verdadeiros de $\mathrm{k}$ e as estimativas correspondentes, $\hat{k}$, obtidas utilizando-se o algoritmo "M. Collapsed Gibbs" partindo-se do ponto inicial $\left(k^{(0)}, \underset{1}{\theta_{1}^{(0)}}, \underset{2}{(0)}\right)$, onde $k^{(0)}=25$ e $\theta_{i}^{(0)}=\left(\beta_{i}^{(0)}, \sigma_{x_{i}}^{2(0)}, \sigma_{i}^{2(0)}\right)=(1,1,1)$, $\mathrm{i}=1,2$, e descartando-se a primeira metade do total de 10.000 iterações.

Tabela 4.2: Valores verdadeiros de $k$ e respectivos valores estimados $(\hat{k})$.

\begin{tabular}{cc|cc|cc|cc|cc}
\hline$k$ & $\hat{k}$ & $k$ & $\hat{k}$ & $k$ & $\hat{k}$ & $k$ & $\hat{k}$ & $k$ & $\hat{k}$ \\
\hline 1 & 1.11 & 11 & 10.95 & 21 & 20.99 & 31 & 30.96 & 41 & 40.97 \\
2 & 13.97 & 12 & 11.97 & 22 & 22.84 & 32 & 31.97 & 42 & 41.90 \\
3 & 2.58 & 13 & 12.86 & 23 & 22.96 & 33 & 32.98 & 43 & 42.94 \\
4 & 3.03 & 14 & 14.95 & 24 & 23.88 & 34 & 33.94 & 44 & 43.70 \\
5 & 4.80 & 15 & 14.74 & 25 & 25.28 & 35 & 34.95 & 45 & 44.90 \\
6 & 5.89 & 16 & 15.97 & 26 & 25.95 & 36 & 35.71 & 46 & 45.33 \\
7 & 6.75 & 17 & 16.92 & 27 & 26.98 & 37 & 36.92 & 47 & 47.99 \\
8 & 8.87 & 18 & 17.95 & 28 & 27.91 & 38 & 37.96 & 48 & 47.90 \\
9 & 8.99 & 19 & 18.96 & 29 & 28.73 & 39 & 38.97 & 49 & 48.97 \\
10 & 10.00 & 20 & 20.97 & 30 & 29.93 & 40 & 39.64 & 50 & \\
\hline
\end{tabular}

Na tabela 4.2 observa-se que a única estimativa discrepante ocorre quando o verdadeiro valor de $\mathrm{k}$ é 2 .

Observou-se nesse exemplo que para valores de $k$ bem pequenos há uma tendência de que as estimativas de $\underset{\sim}{\theta_{1}}=\left(\alpha_{1}, \beta_{1}, \mu_{1}, \sigma_{x_{1}}^{2}, \sigma_{1}^{2}\right)$ não sejam muito boas (em termos do viés) quando comparadas com o verdadeiro valor de $\underline{\theta}_{1}$ e que as estimativas de $\underline{\theta}_{2}=\left(\alpha_{2}, \beta_{2}, \mu_{2}, \sigma_{x_{1}}^{2}, \sigma_{1}^{2}\right)$ sejam melhores (ou seja, mais próximas do verdadeiro valor 
de $\theta_{2}$ ). Semelhantemente, quando o verdadeiro valor de $\mathrm{k}$ está próximo de $\mathrm{n}$, as estimativas de ${\underset{\sim}{1}}_{1}$ são melhores do que as de $\theta_{2}$. Esse fato já era esperado pela própria estrutura do problema.

A seguir apresentamos as estimativas de várias medidas resumo da distribuição à posteriori bem como as estimativas das distribuições posterioris marginais. Essas estimativas basearam-se em $\mathrm{M}=4$ seqüências do algoritmo $\mathrm{M}$. Collapsed Gibbs de comprimento $T=10.000$, após descartar-se a primeira metade de cada uma. Os pontos iniciais dessas sequiências foram obtidos apartir dos pontos ${\underset{\sim}{1}}_{1}^{(0)}$ a $\theta_{4}^{(0)}$ (dados no início da seção) após descartar-se as componentes $\alpha_{1}^{(0)}, \alpha_{2}^{(0)}, \mu_{1}^{(0)}, \mu_{2}^{(0)}$ de cada um.

A tabela 4.4 apresenta as estimativas à posteriori de algumas medidas resumo da distribuição à posteriori (quando os dados observados são os da tabela 4.1 e a distribuição à priori é dada no inicio da seção). Observa-se nessa tabela que os valores da média aritmética e da média Rao-Blackelizada estão muito próximas. Observa-se também que tanto a média como a mediana amostral superestimam o verdadeiro valor do parâmetro $\sigma_{1}^{2}$, entretanto, de maneira geral, elas estimam cada um dos demais parâmetros apenas com um pequeno viés. Mais importante ainda é que os intervalos de credibilidade estimados contém os valores verdadeiros dos parâmetros (no caso de $\sigma_{1}^{2}$ apenas o I.C. de $95 \%$ com duas casas decimais satisfaz isso).

A tabela 4.3 apresenta a distribuição de freqüências à posteriori de $k$.

Tabela 4.3: Distribuição de freqüência relativa à posteriori de $k$ :

\begin{tabular}{c|ccccc|c}
\hline$k$ & 1 a 18 & 19 & 20 & 21 & 22 a 50 & \\
\hline$\hat{P}(K=k \mid$ Dobs $)$ & 0.000 & 0.003 & 0.029 & 0.968 & 0.000 & 1 \\
\hline
\end{tabular}

A Figura 4.10 apresenta os gráficos das estimativas "Rao-Blackwelizadas" das distribuições posterioris marginais de $\alpha_{1}, \alpha_{2}, \beta_{1}, \beta_{2}, \ldots, \sigma_{1}^{2}, \sigma_{2}^{2}$ e $k$. 
Tabela 4.4: Estimativas do valor esperado, quantis, desvio padrão, máximo, mínimo e intervalos de credibilidade de comprimento mínimo das distribuições marginais à posteriori de $\alpha_{1}, \alpha_{2}, \beta_{1}, \beta_{2}, \ldots, \sigma_{1}^{2}, \sigma_{2}^{2}$ e $k$, usando o algoritmo M.Collap.Gibbs. (Os dados simulados são os da tabela 4.1 e a distribuição à priori é dada em (4.7.1))

\begin{tabular}{|c|c|c|c|c|c|c|}
\hline ESTIMATIVAS & & & & & & \\
\hline $\begin{array}{l}\text { Média aritm. } \\
\text { Média R-Blackw. }\end{array}$ & $\begin{array}{l}1.7799 \\
1.7800\end{array}$ & $\begin{array}{l}0.8341 \\
0.8329\end{array}$ & $\begin{array}{l}1.0649 \\
1.0646\end{array}$ & $\begin{array}{l}2.0774 \\
2.0774\end{array}$ & $\begin{array}{l}2.2372 \\
2.2366\end{array}$ & $\begin{array}{l}3.8593 \\
3.8582\end{array}$ \\
\hline $\begin{array}{r}\text { Qudus } \\
0.025 \\
.05 \\
.25 \\
.5 \\
.75 \\
.95 \\
.975\end{array}$ & $\begin{array}{l}0.3712 \\
0.6654 \\
1.4302 \\
1.8420 \\
2.1985 \\
2.6901 \\
2.8424\end{array}$ & $\begin{array}{l}-0.7153 \\
-0.4000 \\
0.3768 \\
0.8873 \\
1.3374 \\
1.9396 \\
2.1221\end{array}$ & $\begin{array}{l}0.6040 \\
0.6673 \\
0.8828 \\
1.0406 \\
1.2176 \\
1.5450 \\
1.6810\end{array}$ & $\begin{array}{l}1.7608 \\
1.8051 \\
1.9534 \\
2.0649 \\
2.1911 \\
2.3865 \\
2.4640\end{array}$ & $\begin{array}{l}1.8894 \\
1.9503 \\
2.1218 \\
2.2367 \\
2.3532 \\
2.5238 \\
2.5814\end{array}$ & $\begin{array}{l}3.3937 \\
3.4707 \\
3.7050 \\
3.8604 \\
4.0149 \\
4.2427 \\
4.3267\end{array}$ \\
\hline $\begin{array}{ll}p=0.95 & \text { Lim. inf. } \\
& \text { Lim sup. } \\
p=0.90 & \text { Lim. inf. } \\
& \text { Lim sup. }\end{array}$ & $\begin{array}{l}0.5146 \\
2.9365 \\
0.7975 \\
2.7811\end{array}$ & $\begin{array}{r}-0.5797 \\
2.2255 \\
-0.3160 \\
2.0026\end{array}$ & $\begin{array}{l}0.5561 \\
1.6076 \\
0.6229 \\
1.4830\end{array}$ & $\begin{array}{l}1.7445 \\
2.4371 \\
1.7889 \\
2.3606\end{array}$ & $\begin{array}{l}1.8964 \\
2.5854 \\
1.9570 \\
2.5296\end{array}$ & $\begin{array}{l}1.8785 \\
4.3136 \\
1.9490 \\
4.2402\end{array}$ \\
\hline $\begin{array}{l}\text { desv.padrão (dp) } \\
\text { dp R-Blackweliz, } \\
\text { "Naive SE" } \\
\text { "Batch SE" }\end{array}$ & $\begin{array}{l}0.6201 \\
0.6198 \\
0.0044 \\
0.0207\end{array}$ & $\begin{array}{l}0.7277 \\
0.7307 \\
0.0051 \\
0.0017\end{array}$ & $\begin{array}{l}0.2689 \\
0.2687 \\
0.0019 \\
0.0232\end{array}$ & $\begin{array}{l}0.1811 \\
0.1811 \\
0.0013 \\
0.0026\end{array}$ & $\begin{array}{l}0.1751 \\
0.1742 \\
0.0012 \\
0.0090\end{array}$ & $\begin{array}{l}0.2349 \\
0.2345 \\
0.0017 \\
0.0062\end{array}$ \\
\hline $\begin{array}{l}\text { Mínimo } \\
\text { Máximo } \\
\end{array}$ & $\begin{array}{r}-1.6637 \\
4.0242 \\
\end{array}$ & $\begin{array}{r}-2.8830 \\
3.3749 \\
\end{array}$ & $\begin{array}{l}0.1122 \\
2.6588 \\
\end{array}$ & $\begin{array}{l}1.4197 \\
3.1043 \\
\end{array}$ & $\begin{array}{l}1.4755 \\
2.9229 \\
\end{array}$ & \\
\hline ESTIMATIVAS & $\sigma_{x}^{2}$ & $\sigma_{x}^{2}$ & $\sigma_{1}^{2}$ & $\sigma_{2}^{2}$ & k & \\
\hline $\begin{array}{l}\text { Media aritm. } \\
\text { Média R-Blackw. }\end{array}$ & $\begin{array}{l}0.41950 \\
0.4199\end{array}$ & $\begin{array}{l}1.3540 \\
1.3608\end{array}$ & $\begin{array}{l}0.2156 \\
0.2158\end{array}$ & $\begin{array}{l}0.2410 \\
0.2408\end{array}$ & $\begin{array}{l}20.9650 \\
20.9670\end{array}$ & \\
\hline $\begin{array}{r}0.025 \\
.05 \\
.25 \\
.5 \\
.75 \\
.95 \\
.975\end{array}$ & $\begin{array}{l}0.1828 \\
0.2059 \\
0.2981 \\
0.3878 \\
0.5037 \\
0.7417 \\
0.8412\end{array}$ & $\begin{array}{l}0.7446 \\
0.8114 \\
1.0601 \\
1.2860 \\
1.5760 \\
2.1165 \\
2.3523\end{array}$ & $\begin{array}{l}0.1189 \\
0.1291 \\
0.1678 \\
0.2034 \\
0.2495 \\
0.3422 \\
0.3815\end{array}$ & $\begin{array}{l}0.1434 \\
0.1544 \\
0.1944 \\
0.2306 \\
0.2756 \\
0.3633 \\
0.3989\end{array}$ & $\begin{array}{l}20 \\
21 \\
21 \\
21 \\
21 \\
21 \\
21\end{array}$ & \\
\hline $\begin{array}{l}p=0.95 \mathrm{Lim} \text {. inf. } \\
\text { Lim sup. } \\
p=0.90 \mathrm{Lim} \text {. inf. } \\
\text { Lim sup. }\end{array}$ & $\begin{array}{l}0.1575 \\
0.7629 \\
0.1632 \\
0.6613\end{array}$ & $\begin{array}{l}0.6745 \\
2.2009 \\
0.7479 \\
1.9915\end{array}$ & $\begin{array}{l}0.1040 \\
0.3502 \\
0.1164 \\
0.3172\end{array}$ & $\begin{array}{l}0.1346 \\
0.3766 \\
0.1418 \\
0.3402\end{array}$ & $\begin{array}{l}21 \\
21 \\
21 \\
21\end{array}$ & \\
\hline $\begin{array}{l}\text { desv.padrão (dp) } \\
\text { dp R-Blackweliz, } \\
\text { "Naive SE" } \\
\text { "Batch SE" }\end{array}$ & $\begin{array}{l}0.1722 \\
0.1736 \\
0.0012 \\
0.0059\end{array}$ & $\begin{array}{l}0.4161 \\
0.4192 \\
0.0030 \\
0.0007\end{array}$ & $\begin{array}{l}0.0685 \\
0.0685 \\
0.0005 \\
0.0012\end{array}$ & $\begin{array}{l}0.0663 \\
0.0663 \\
0.0005 \\
0.0005\end{array}$ & $\begin{array}{l}0.2117 \\
0.2044 \\
0.0014 \\
0.0022\end{array}$ & \\
\hline $\begin{array}{l}\text { Mínimo } \\
\text { Máximo }\end{array}$ & $\begin{array}{l}0.1043 \\
1.8396\end{array}$ & $\begin{array}{l}0.4343 \\
5.5860\end{array}$ & $\begin{array}{l}0.0642 \\
0.8925\end{array}$ & $\begin{array}{l}0.0915 \\
0.7710\end{array}$ & $\begin{array}{l}17 \\
23\end{array}$ & \\
\hline
\end{tabular}

NOTA: Os intervalos de credibilidade nesse exemplo coincidem com os intervalos HPD.

"Batch SE" é o desvio padrão amostral das médias de grupos consecutivos de tamanho 50, dividido pela raiz quadrada do número de grupos (calculado por meio do BOA ("Bayesian Output Analysis")). 

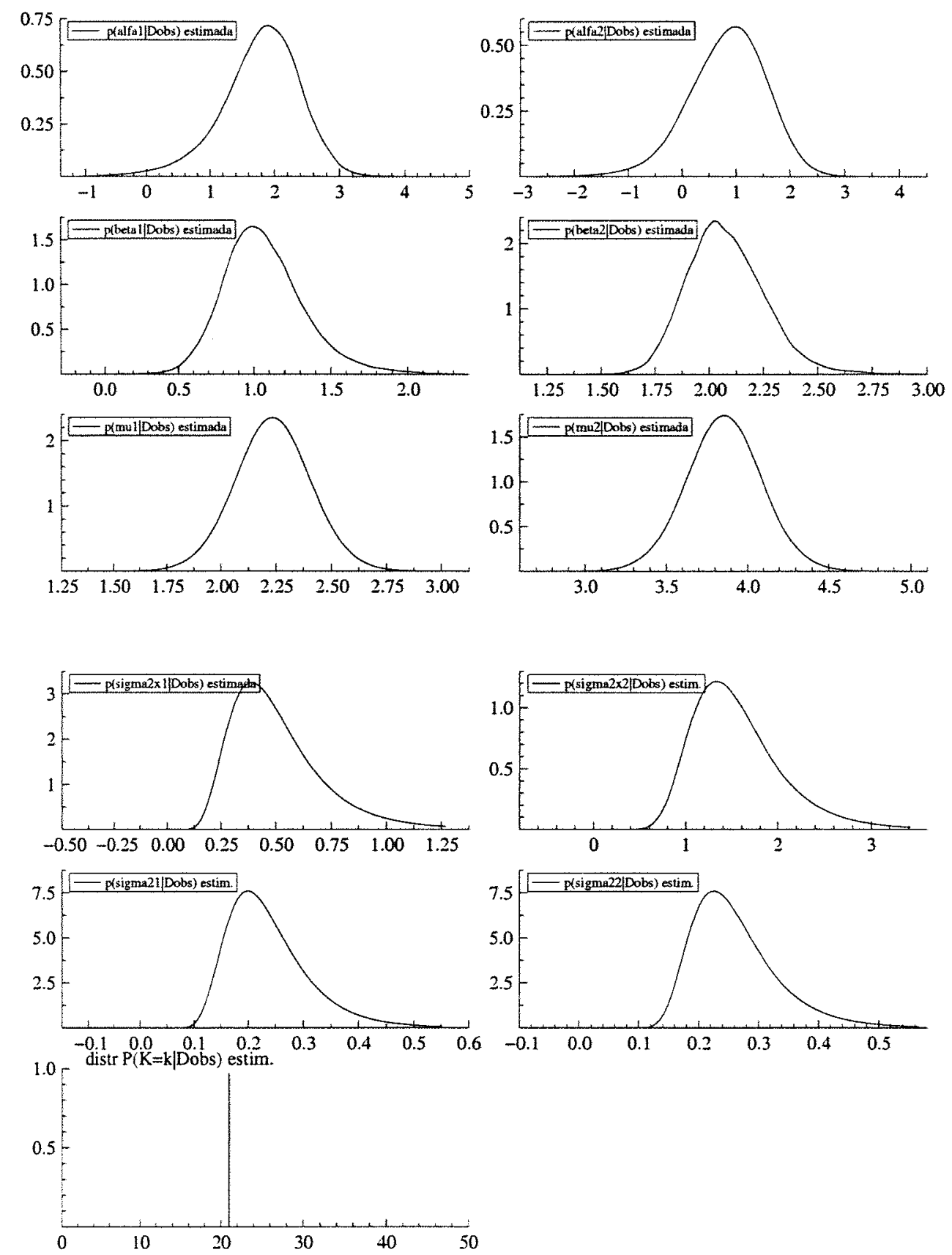

Figura 4.10: Gráficos das estimativas "Rao-Blackwelizadas" das densidades marginais à posteriori $p\left(\alpha_{1} \mid D_{\text {obs }}\right), p\left(\alpha_{2} \mid D_{\text {obs }}\right), \ldots, p\left(\sigma_{1}^{2} \mid D_{\text {obs }}\right), p\left(\sigma_{2}^{2} \mid D_{\text {obs }}\right)$ e $p\left(k \mid D_{\text {obs }}\right)$. 


\section{3.c Avaliação do ajuste}

Para avaliar a qualidade do ajuste primeiramente apresentamos a figura $4.11 \mathrm{com}$ os gráficos dos valores da densidade condicional preditiva de validação cruzada $\mathrm{CPO}_{i}$ (onde $\mathrm{CPO}_{i}=\pi\left(\left(\begin{array}{c}Y_{i} \\ X_{i}\end{array}\right) \mid D_{\text {obs }}\right)$ ) primeiramente em função de $\mathrm{i}$, depois em função de $X_{i}$, e depois em função de $Y_{i}$, para $i=1, \ldots, 50$, onde $\mathrm{CPO}_{i}$ é estimada por meio da fórmula (4.5.8). As estimativas foram baseadas na segunda metade de uma seqüência de comprimento $\mathrm{T}=10.000$ do algoritmo M.Collap.Gibbs que parte do ponto inicial $\Theta_{1}^{(0)}$ dado no início da seção (descartando as componentes $\left.\alpha_{1}^{(0)}, \alpha_{2}^{(0)}, \mu_{1}^{(0)}, \mu_{2}^{(0)}\right)$.

Em seguida apresentamos a figura 4.12 com os gráficos dos resíduos bayesianos de validação cruzada.

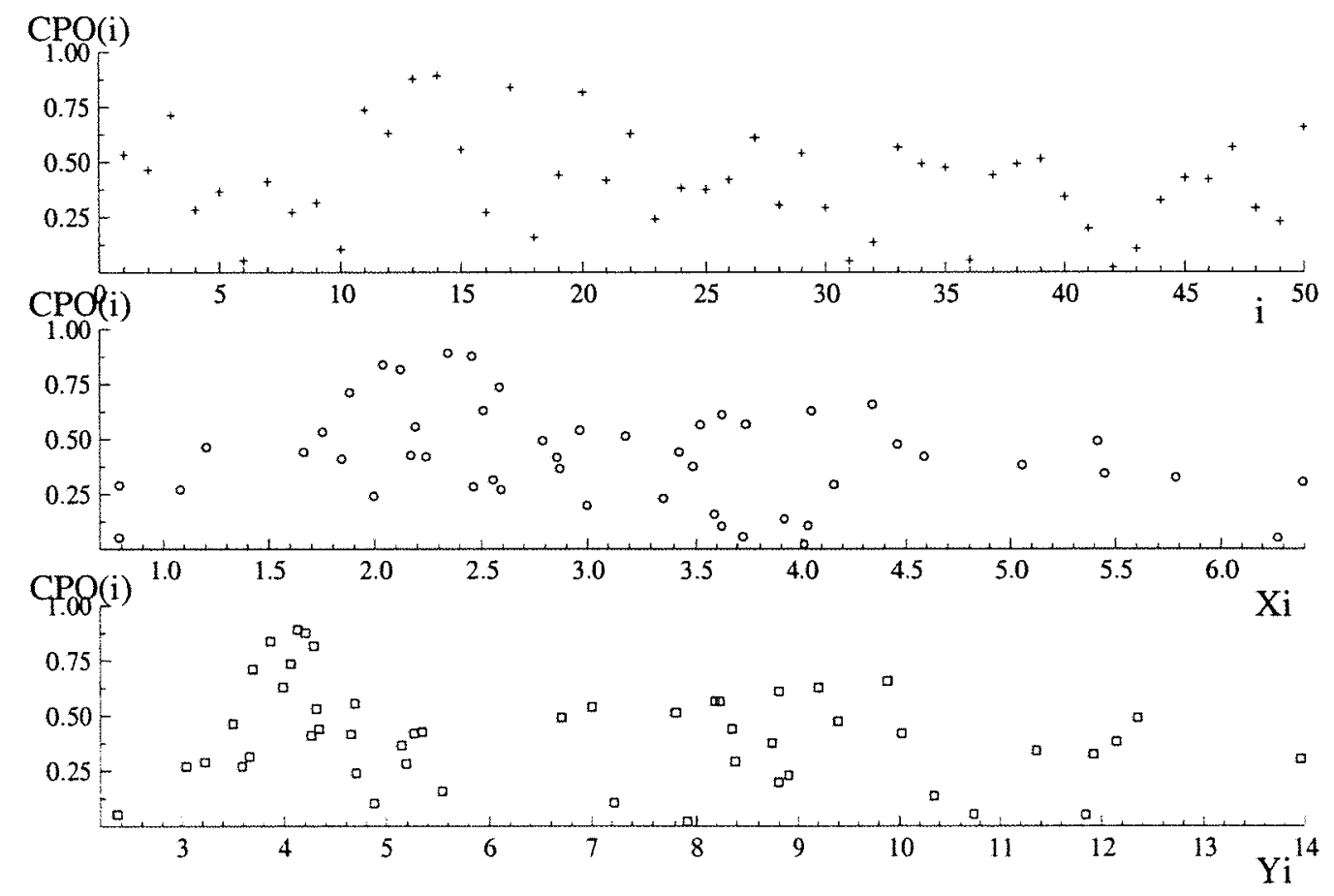

Figura 4.11: Gráfico dos valores da densidade condicional preditiva, $\mathrm{CPO}(\mathrm{i})$, versus $i$ (posição superior), versus $X_{i}$ (posição do meio) e versus $Y_{i}$ (posição inferior.) 
$\mathrm{Na}$ seção 4.5 .3 vimos que os resíduos bayesianos bidimensionais de validação cruzada podem ser estimados por

$$
d_{i}=\left(\begin{array}{c}
d_{1_{i}} \\
d_{2_{i}}
\end{array}\right)=\widehat{\operatorname{Var}}^{-\frac{1}{2}}\left(\left(\begin{array}{c}
Y_{i} \\
X_{i}
\end{array}\right) \mid D_{\mathrm{obs}(-i)}\right)\left(\left(\begin{array}{c}
Y_{i} \\
X_{i}
\end{array}\right)-\hat{E}\left(\left(\begin{array}{c}
Y_{i} \\
X_{i}
\end{array}\right) \mid D_{\mathrm{obs}(-i)}\right)\right),
$$

$i=1, \ldots, n$, onde $\hat{E}\left(\left(\begin{array}{l}Y_{i} \\ X_{i}\end{array}\right) \mid D_{\text {obs }(-i)}\right)$ é dada em (4.5.11)

e $\widehat{\operatorname{Var}}^{-\frac{1}{2}}\left(\left(\begin{array}{c}Y_{i} \\ X_{i}\end{array}\right) \mid D_{\text {obs(-i) }}\right)$ é dada em (4.5.12), para $i=1, \ldots, n$.

A Figura 4.12 apresenta do lado esquerdo os gráficos da componente $d_{1_{i}}$ e do lado direito os gráficos da componente $d_{2_{i}}$ do vetor de resíduos.

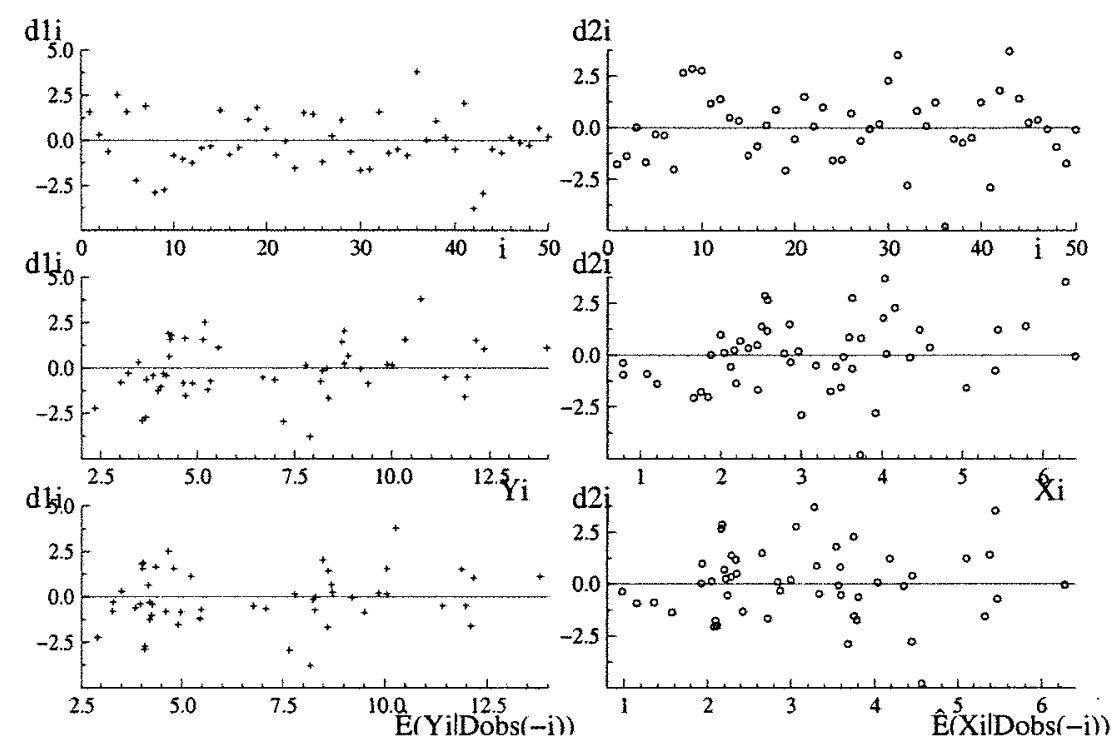

Figura 4.12: Do lado esquerdo os gráficos de $d_{1_{i}}$ versus $i$, versus $Y_{i}$ e versus $E\left(Y_{i} \mid \hat{D_{\text {obs }}}(-i)\right)$. Do lado direito os gráficos de $\left(d_{2_{i}}\right)$ versus $i$, versus $X_{i}$ e versus $E\left(X_{i} \mid \hat{D_{\text {obs }}}(-i)\right)$.

Os gráficos acima não sugerem que exista grandes problemas de falta de ajustamento. Entretanto na figura 4.12 existem 3 pontos nos gráficos de ordenada $d_{1}$ (do lado esquerdo) e 4 pontos nos gráficos de ordenada $d_{2_{i}}$ (do lado direito) cujos valores são maiores do que 3 e portanto, aparentemente, poderiam ser vistos como pontos aberrantes, entretanto uma análise de resídous mais aprofundada deve ser conduzida futuramente para examinar melhor a qualidade desse ajuste. 


\subsubsection{Análise do modelo estrutural não identificável}

Nesta seção utilizamos uma amostra de tamanho $n=50$ de dados gerados a partir do modelo que permite mudança em todos os parâmetros dado a seguir:

\section{MODELO}

$$
\left\{\begin{array}{l}
Y_{i}= \begin{cases}\alpha_{1}+\beta_{1} x_{i}+e_{i}, & i=1, \ldots, k, \quad 1 \leq k \leq n-1, \\
\alpha_{2}+\beta_{2} x_{i}+e_{i}, & i=k+1, \ldots, n\end{cases} \\
X_{i}=x_{i}+u_{i}, \quad i=1, \ldots, n
\end{array}\right.
$$

onde

$$
\left(\begin{array}{c}
e_{i} \\
u_{i} \\
x_{i}
\end{array}\right) \stackrel{\text { iid }}{\sim}\left\{\begin{array}{l}
N\left(\left(\begin{array}{c}
0 \\
0 \\
\mu_{1}
\end{array}\right) ;\left(\begin{array}{ccc}
\sigma_{e_{1}}^{2} & 0 & 0 \\
0 & \sigma_{1}^{2} & 0 \\
0 & 0 & \sigma_{x(1)}^{2}
\end{array}\right)\right), i=1, \ldots, k, \\
N\left(\left(\begin{array}{c}
0 \\
0 \\
\mu_{2}
\end{array}\right) ;\left(\begin{array}{ccc}
\sigma_{e_{2}}^{2} & 0 & 0 \\
0 & \sigma_{2}^{2} & 0 \\
0 & 0 & \sigma_{x(2)}^{2}
\end{array}\right)\right), i=k+1, \ldots, n,
\end{array}\right.
$$

\section{Distribuição à priori}

$$
\begin{aligned}
& \pi(\stackrel{*}{\stackrel{*}{)}})=\pi\left({\underset{\sim}{1}}_{1},{\underset{\sim}{\theta}}_{2}, k\right)=\pi(k) \pi\left(\underset{\theta_{1}}{\theta_{1}}\right) \pi\left({\underset{\sim}{2}}_{2}\right), \text { onde } \pi\left(\underset{\sim}{\theta_{i}}\right)=\pi\left(\alpha_{i}\right) \pi\left(\beta_{i}\right) \pi\left(\mu_{i}\right) \pi\left(\sigma_{x_{i}}^{2}\right) \pi\left(\sigma_{e_{i}}^{2}\right) \pi\left(\sigma_{i}^{2}\right), \\
& i=1,2 \text {, onde } \alpha_{i} \sim N(0 ; 1000), \beta_{i} \sim N(0 ; 1000), \mu_{i} \sim N(0 ; 1000), \sigma_{x_{i}}^{2} \sim I G(2.001 ; 1.0) \text {, } \\
& \sigma_{e_{i}}^{2} \sim I G(2.001 ; 1.0), \sigma_{i}^{2} \sim I G(2.001 ; 1.0) \mathrm{e} \\
& p(k)= \begin{cases}P(K=k)=\frac{1}{\# \mathcal{K}}, & k \in \mathcal{K}=\{1, \ldots, n-1\}, \\
0, & \text { caso contrário. }\end{cases}
\end{aligned}
$$

\section{DADOS}

Os dados foram simulados apartir do modelo em (4.7.2) com mudança em todos os parâmetros onde $\alpha_{1}=2.0, \beta_{1}=1.0, \mu_{1}=2.0, \sigma_{x(1)}^{2}=0.6, \sigma_{e_{1}}^{2}=0.1, \sigma_{1}^{2}=0.1, \alpha_{2}=1.0$, $\beta_{2}=2.0, \mu_{2}=4.0, \sigma_{x(2)}^{2}=1.2, \sigma_{2}^{2}=0.2, \sigma_{e_{2}}^{2}=0.2, k=20$. Usando a mesma semente da seção anterior, o conjunto de dados é o mesmo que o da Tabela 4.1.

OBSERVAÇÃO: Nesse estudo, como os dados utilizados foram simulados apartir do modelo em (4.7.2) e a distribuição à priori escolhida acima é praticamente não informativa, devemos esperar que as estimativas e conclusōes à posteriori sejam compatíveis com esse modelo (4.7.2). 


\section{AJUSTE DO MODELO}

Os algoritmos de Gibbs e "Modified and Collapsed Gibbs" foram utilizados com o objetivo de amostrar da distribuição à posteriori do modelo. De cada algorítmo foram geradas $M=4$ sequêencias paralelas de comprimento $T=10.000$ partindo de pontos iniciais obtidos pelo procedimento descrito a seguir:

Inicialmente para cada valor fixado de $k(3 \leq k \leq n-3)$ foram fixados 40 valores de $\eta$, onde $\eta$ é tal que $\sigma_{e_{i}}^{2}=\eta \sigma_{i}^{2}, i=1,2,\left(\eta=\frac{1}{20}, \ldots, \frac{1}{3}, \frac{1}{2}, 1,2,3, \ldots, 20\right)$. Para cada uma dessas possíveis combinações foram obtidas (analiticamente) as estimativas de máximaverossimilhança ${\underset{\sim}{\theta}}_{i}$ de ${\underset{\sim}{i}}_{i}=\left(\alpha_{i}, \beta_{i}, \mu_{i}, \sigma_{x_{i}}^{2}, \sigma_{i}^{2}, \sigma_{e_{i}}^{2}\right)$, para $i=1,2$.

A seguir foi aplicado o algorítmo de otimização BFGS tomando-se cada um dos pontos $\left(\begin{array}{c}k \\ \hat{\theta}_{1} \\ \hat{\theta}_{2}\end{array}\right)$ acima como ponto inicial do processo iterativo, tendo sido localizados $n-5$ pontos estacionários da distribuição à posteriori.

O próximo passo foi gerar 1000 pontos do tipo $\underset{\sim}{\theta}=\left(\begin{array}{c}k \\ \theta_{1} \\ \tilde{\theta}_{2}\end{array}\right)$ da distribuição formada pela mistura de distribuições $t_{4}\left(\hat{\theta}_{i}^{*}, \hat{V}_{i}^{*}\right), i=1, \ldots, n-5$, onde ${\underset{\sim}{\hat{\theta}}}_{i}^{*}$ é o i-ésimo

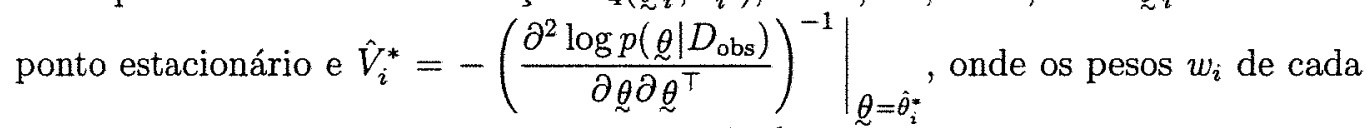
distribuição são dados por $w_{i}=q\left(\hat{\theta}_{i}^{*} \mid D_{\text {obs }}\right)\left|\hat{V}_{i}^{*}\right|^{\frac{1}{2}}$, onde $q\left(\hat{\theta}_{i}^{*} \mid D_{\text {obs }}\right)$ é a posteriori não normalizada calculada no ponto $\hat{\sim}_{i}^{*}$

Finalmente, (utilizando-se re-amostragem ponderada) foram reamostrados 10 pontos dos quais apenas os seguintes quatro foram usados para o algoritmo de Gibbs:

$$
\begin{aligned}
& \stackrel{\vec{\theta}}{1}_{1}^{(0)}=(21,2.26,0.85,2.38,0.37,0.19,0.214,0.10,2.37,3.66,1.54,0.25,0.25)^{T} \\
& \dot{\theta}_{2}^{(0)}=(21,0.92,1.21,2.54,0.37,0.23,0.19,0.98,2.11,3.35,0.62,0.36,0.17)^{T} \\
& \stackrel{*}{\theta}_{3}^{(0)}=(21,2.13,0.92,2.07,0.41,0.25,0.19,0.64,2.02,4.04,1.33,0.28,0.22)^{T} \\
& \ddot{\theta}_{4}^{(0)}=(21,0.35,1.63,2.31,-0.10,0.46,0.11,-2.08,2.69,4.33,0.49,0.24,0.35)^{T}
\end{aligned}
$$

A seguir são apresentados alguns dispositivos gráficos para o diagnóstico de possíveis falhas na convergência dos algoritmos de Gibbs e Modified Collapsed Gibbs. 


\section{3.a Diagnósticos de convergência}
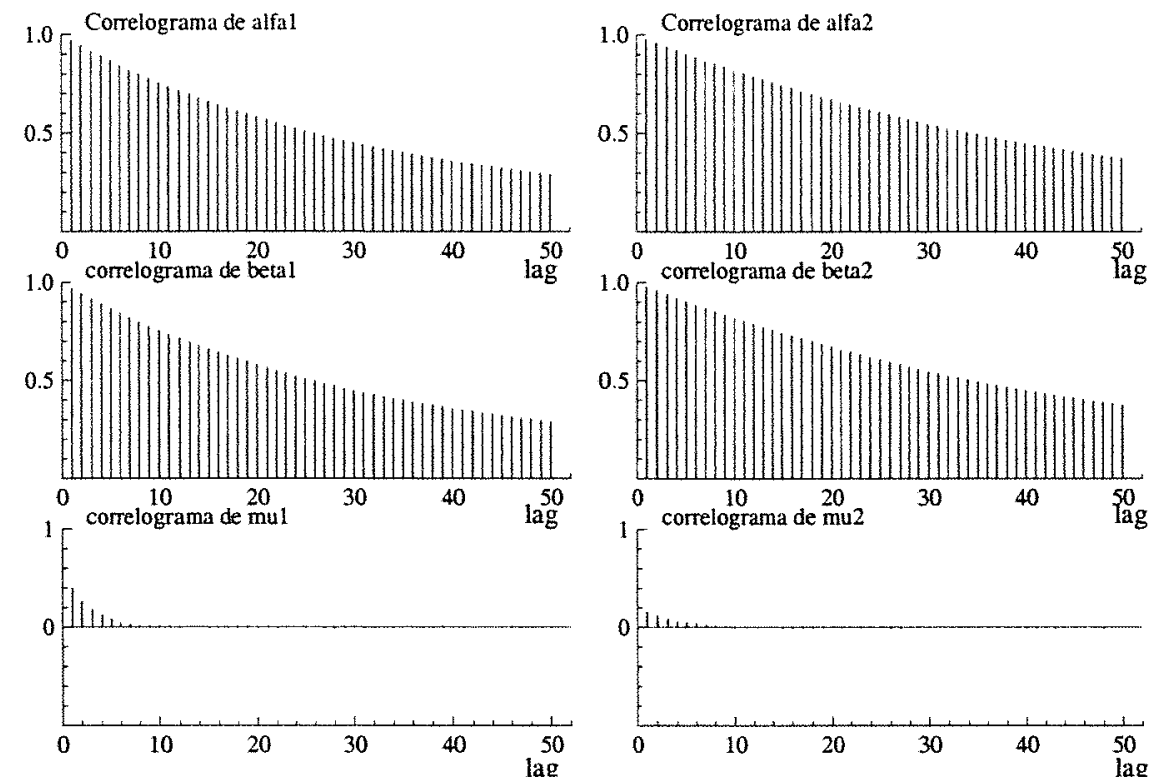

Figura 4.13: Gráficos das funçōes de auto-correlação de $\alpha_{1}, \alpha_{2} \beta_{1}, \beta_{2}, \mu_{1}$ e $\mu_{2,}$, baseados em uma única sequêencia de Gibbs de comprimento $T=50.000$, partindo do ponto inicial $\tilde{\theta}_{1}^{(0)}$.

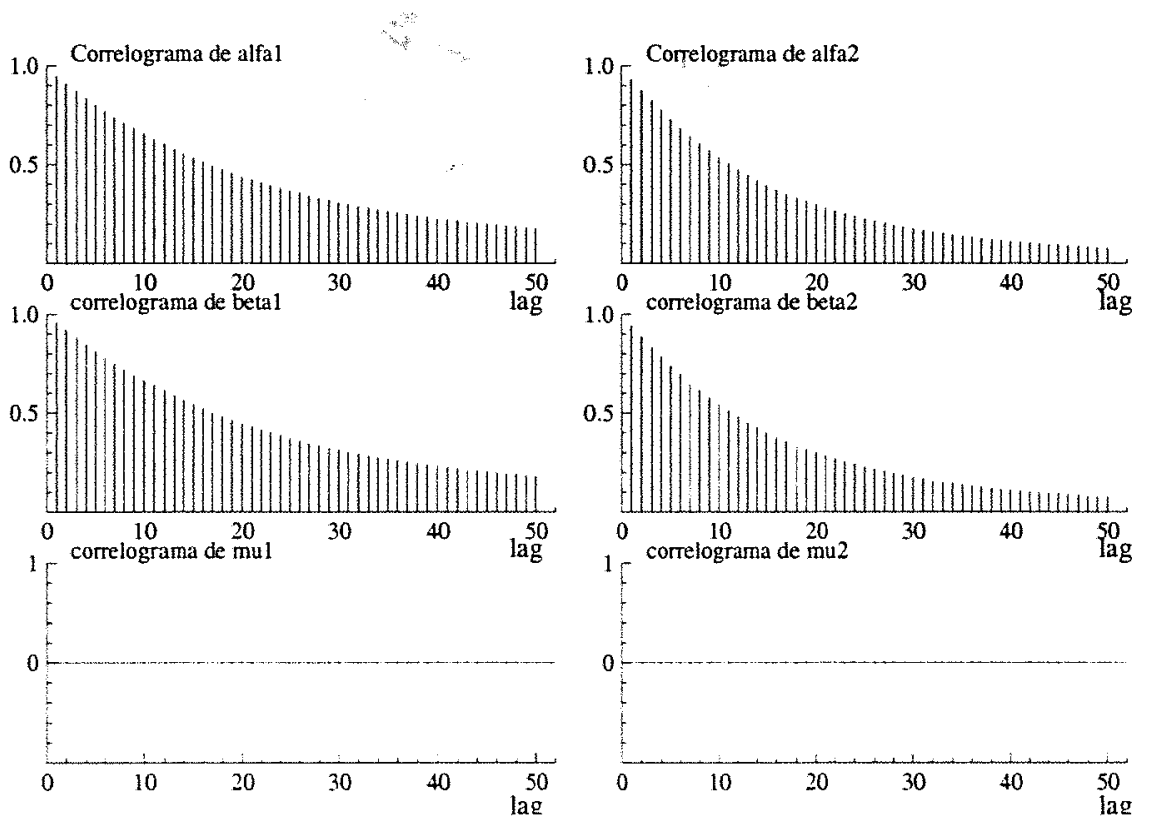

Figura 4.14: Gráficos das funçōes de auto-correlação de $\alpha_{1}, \alpha_{2}, \beta_{1}, \beta_{2}, \mu_{1}$ e $\mu_{2}$, baseados em uma única sequência do algoritmo M. Collap. Gibbs de comprimento $T=50.000$. 

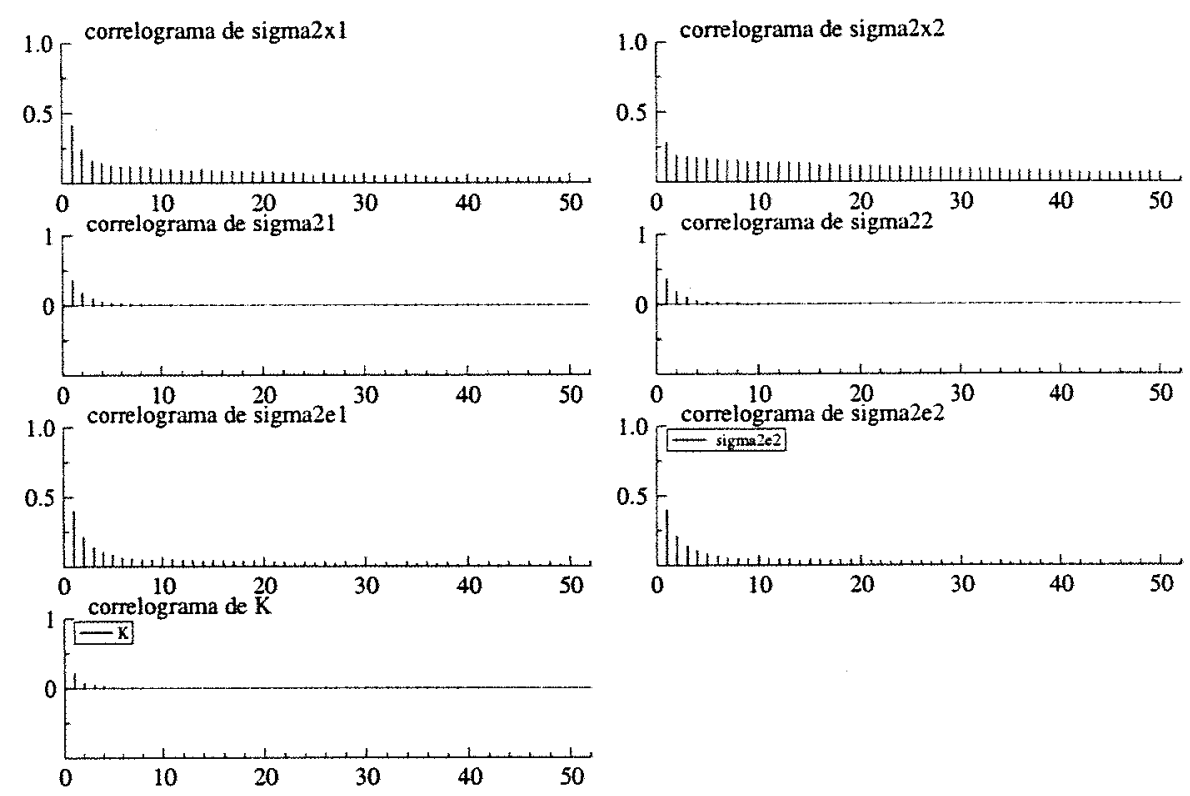

Figura 4.15: Gráficos das funções de auto-correlação de $\sigma_{x_{1}}^{2}, \sigma_{x_{2}}^{2}, \sigma_{e_{1}}^{2}, \sigma_{e_{2}}^{2}, \sigma_{1}^{2}, \sigma_{2}^{2}$ e $k$ baseados em uma sequiência de Gibbs de comprimento $\mathrm{T}=50.000$, partindo de $\ddot{\theta}_{1}^{(0)}$.
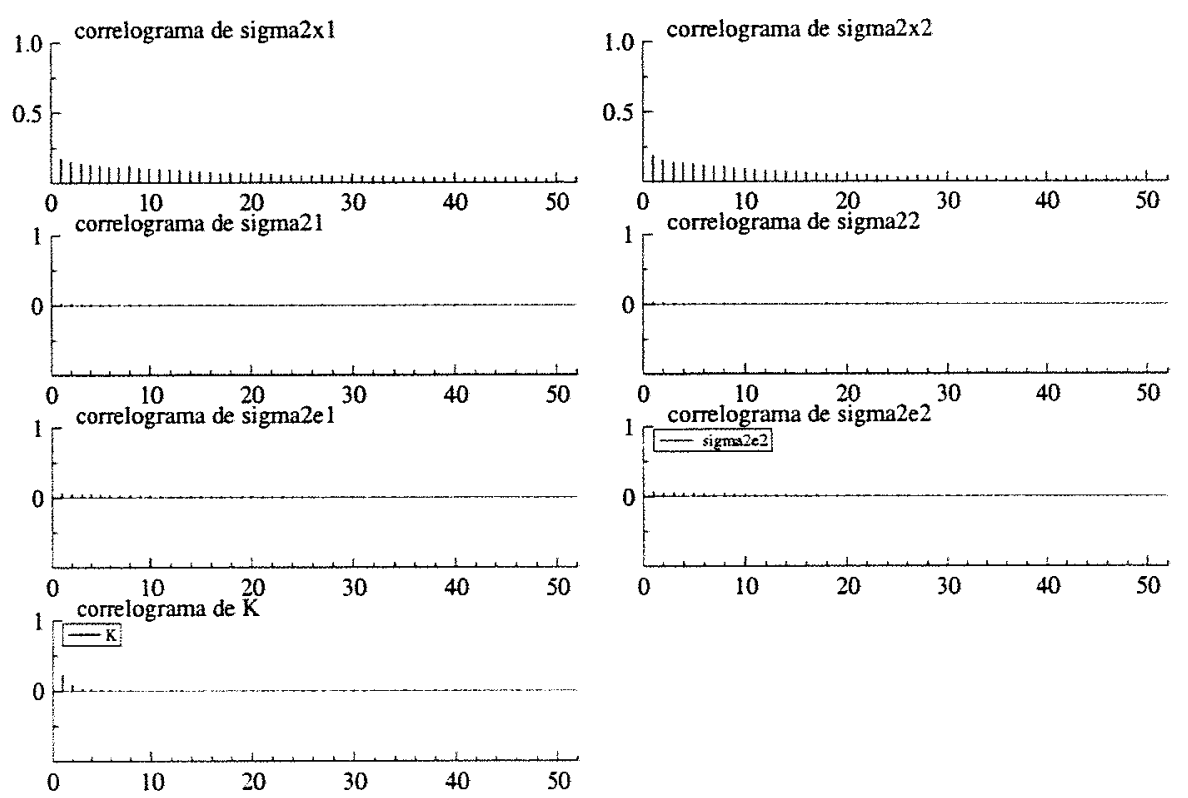

Figura 4.16: Gráficos das funçōes de auto-correlação de $\sigma_{x_{1}}^{2}, \sigma_{x_{2}}^{2}, \sigma_{e_{1}}^{2}, \sigma_{e_{2}}^{2}, \sigma_{1}^{2}, \sigma_{2}^{2}$ e $k$, baseados em uma sequiencia do algoritmo M.Collap. Gibbs de comprimento $\mathrm{T}=50000$. 

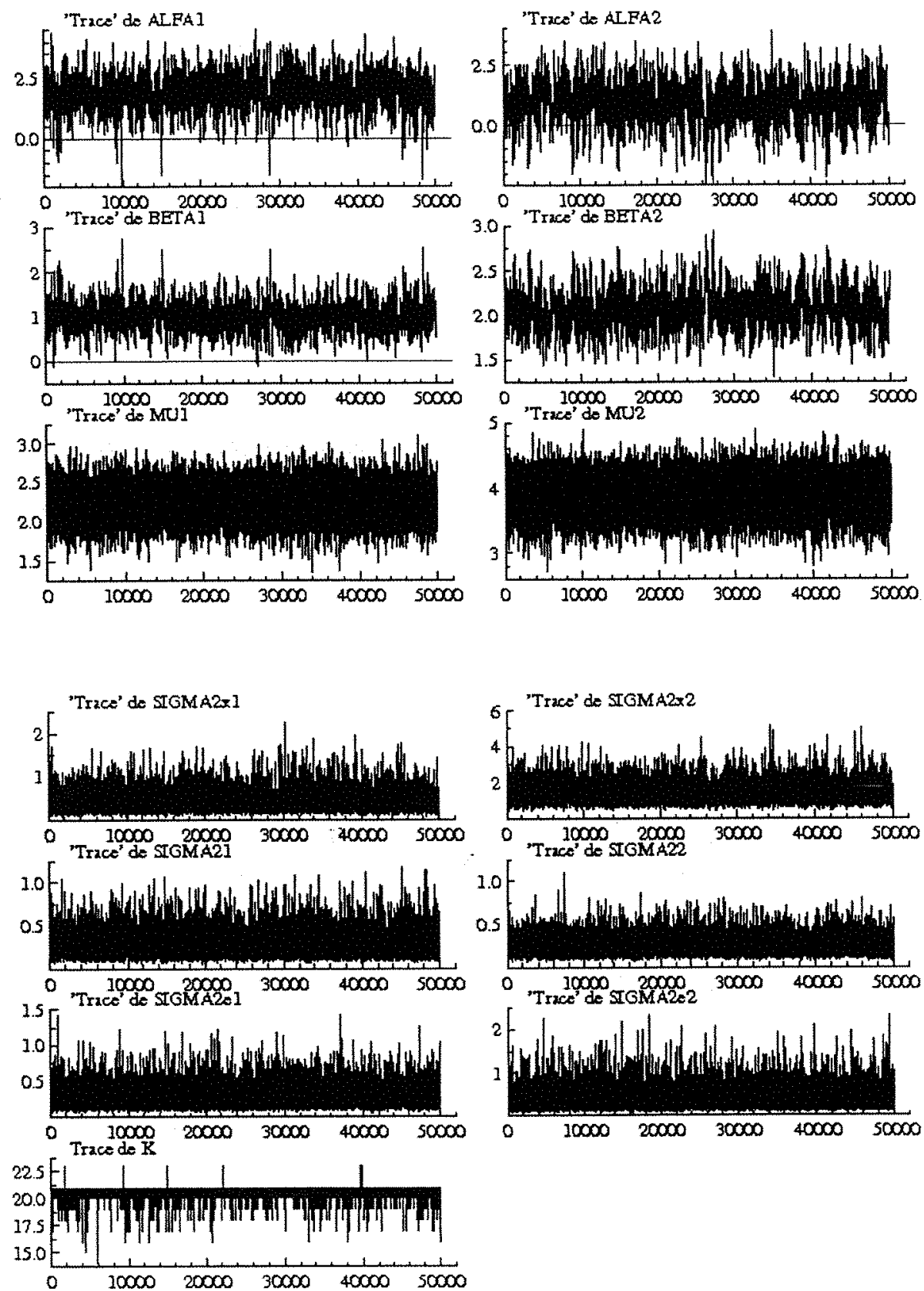

Figura 4.17: Gráficos das séries temporais de $\alpha_{1}, \alpha_{2}, \beta_{1}, \beta_{2}, \mu_{1}, \mu_{2}, \sigma_{x_{1}}^{2}$, $\sigma_{x_{2}}^{2}, \sigma_{e_{1}}^{2}, \sigma_{\varepsilon_{2}}^{2}, \sigma_{1}^{2}, \sigma_{2}^{2}$ e $k$ baseados em uma seqüencia de comprimento $T=50.000$ do algoritmo de Gibbs partindo de $\stackrel{*}{\theta}_{1}^{(0)}$. 

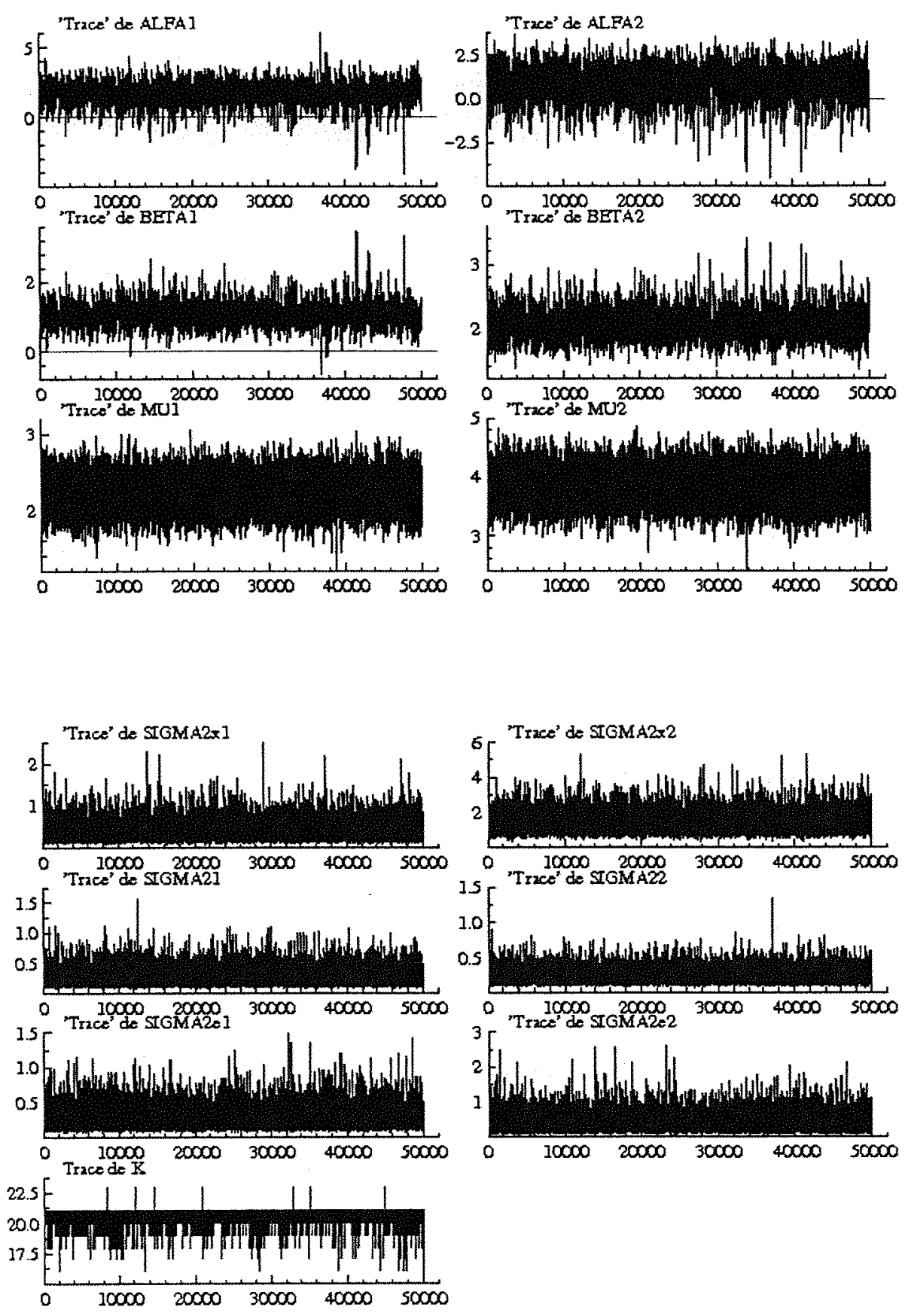

Figura 4.18: Gráficos das séries temporais de $\sigma_{x_{1}}^{2}, \sigma_{x_{2}}^{2}, \sigma_{e_{1}}^{2}, \sigma_{e_{2}}^{2}, \sigma_{1}^{2}, \sigma_{2}^{2}$ e $k$ baseados em uma seqüência de comprimento $T=50.000$ do algoritmo Modified Collapsed Gibbs. 
Brooks \& Gelman Multivariate Shrink Factors

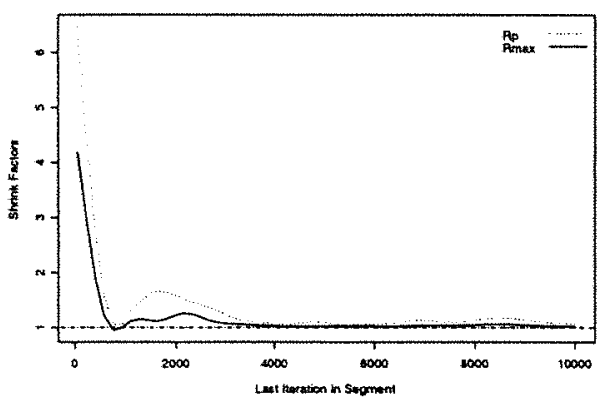

Brooks \& Gelman Multivariate Shrink Factors

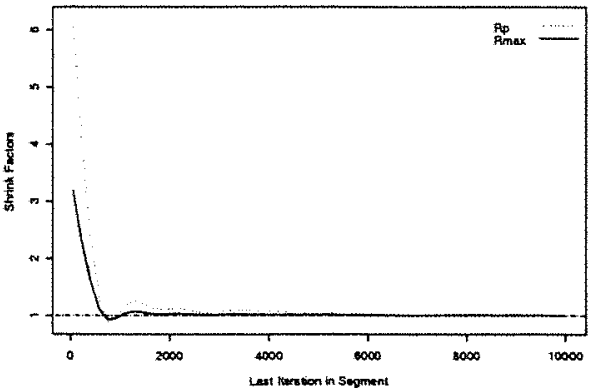

Figura 4.19: "Multiv. Potential Scale Reduction" de Brooks e Gelman (1998). O gráfico à esquerda é baseado em $M=4$ seqüéncias de comprimento $T=10.000$ do algoritmo de Gibbs e o da direita no algoritmo M. Collap. Gibbs.

\section{Gelman \& Rubin Shrink Factors}
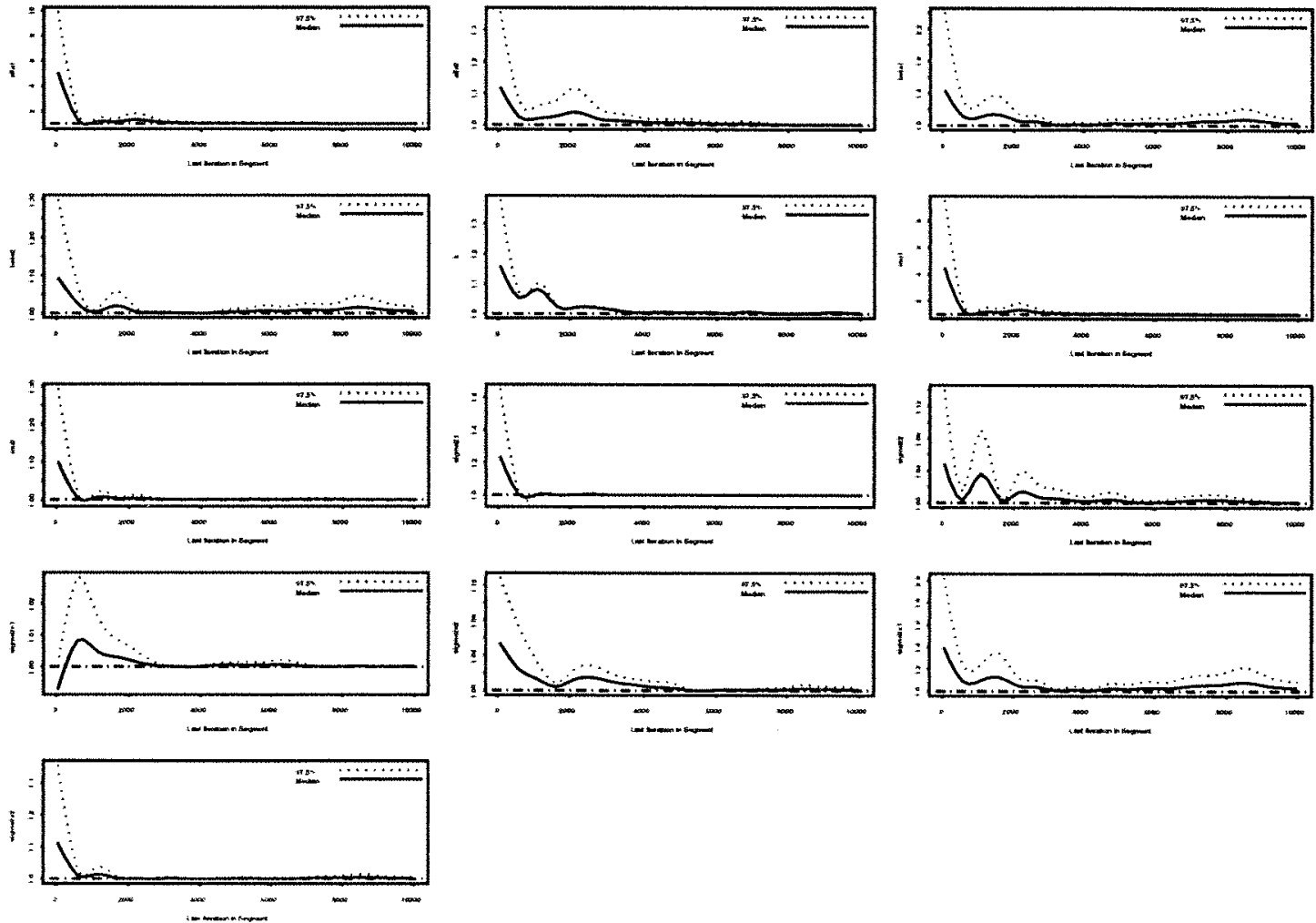

Figura 4.20: Gráfico da medida de Gelman e Rubin corrigida "Corrected potencial scale reduction factors" (CSRF) para cada un dos parametros $k, \alpha_{1}, \alpha_{2}, \beta_{1}, \beta_{2}, \ldots, \sigma_{1}^{2}, \sigma_{2}^{2}$, baseada em $M=4$ seqüencias de comprimento $T=10.000$ de Gibbs. 


\section{Gelman \& Rubin Shrink Factors}
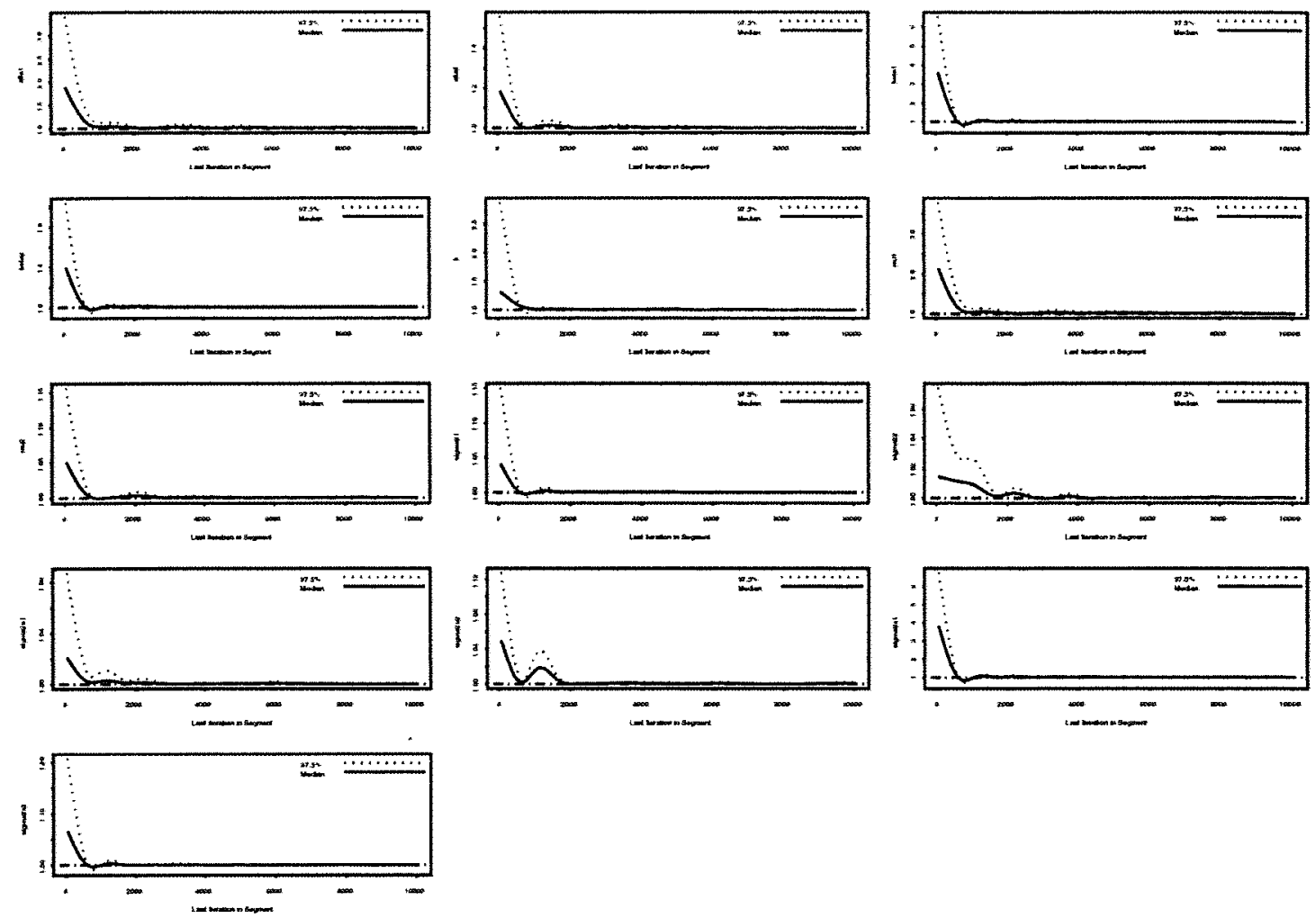

Figura 4.21: Gráfico da medida de Gelman e Rubin corrigida "Corrected potencial scale reduction factors " (CSRF) para cada um dos parametros $k, \alpha_{1}, \alpha_{2}, \beta_{1}, \ldots, \sigma_{1}^{2}, \sigma_{2}^{2}$, baseada em $M=4$ sequêencias de comprimento $T=10.000$ do algoritmo M.Collapsed Gibbs.

As figuras 4.13 a 4.21 não sugerem que exista problemas na convergência dos algoritmos de Gibbs e Modified Collapsed Gibbs. Além disso o algoritmo Modified Collapsed Gibbs parece ter uma melhor performance que o Gibbs (como pode ser visto principalmente pela comparação das funções de auto-correlação amostral correspondentes).

\section{3.b Estimação}

Semelhantemente à seção 4.7.1, e com o objetivo de examinar se a estimativa do ponto de mudança $\mathrm{k}$, do modelo em (4.7.2), (obtida por meio do algoritmo M. 
Collapsed Gibbs), difere muito do seu valor verdadeiro, apresentamos também nessa seção uma tabela com diferentes valores de $\mathrm{k}$ fixados e suas correspondentes estimativas $\hat{k}$ obtidas por esse algoritmo partindo-se sempre do ponto inicial $\left(k^{(0)},{\underset{\sim}{1}}_{1}^{(0)},{\underset{\sim}{\theta}}_{2}^{(0)}\right)$, onde $k^{(0)}=25$ e ${\underset{i}{i}}_{i}^{(0)}=\left(\beta_{i}^{(0)}, \sigma_{x_{i}}^{2(0)}, \sigma_{e_{i}}^{2(0)}, \sigma_{i}^{2(0)}\right)=(1,1,1,1), \mathrm{i}=1,2$, utilizando-se apenas a segunda metade do total de 10.000 iterações.

(Observemos que cada linha da tabela envolveu um conjunto de dados diferente, simulado apartir do modelo (4.7.2) com um valor de $k$ entre 1 e $n-1$ e com ${\underset{\sim}{1}}_{1}=(2.0,1.0,2.0,0.6,0.1,0.1)$ e $\left.\underset{\sim}{\theta_{2}}=(1.0,2.0,4.0,1.2,0.2,0.2)\right)$.

Tabela 4.5: Valores verdadeiros de $k$ e correspondentes valores estimados $(\hat{k})$.

\begin{tabular}{cc|cc|cc|cc|cc}
\hline$k$ & $\hat{k}$ & $k$ & $\hat{k}$ & $k$ & $\hat{k}$ & $k$ & $\hat{k}$ & $k$ & $\hat{k}$ \\
\hline 1 & 1.11 & 11 & 10.92 & 21 & 20.99 & 31 & 30.93 & 41 & 40.94 \\
2 & 2.67 & 12 & 11.97 & 22 & 22.80 & 32 & 31.95 & 42 & 41.76 \\
3 & 4.13 & 13 & 12.80 & 23 & 22.92 & 33 & 32.97 & 43 & 42.90 \\
4 & 16.37 & 14 & 14.93 & 24 & 23.79 & 34 & 33.91 & 44 & 43.45 \\
5 & 4.52 & 15 & 14.67 & 25 & 25.39 & 35 & 34.93 & 45 & 44.82 \\
6 & 5.83 & 16 & 15.95 & 26 & 25.93 & 36 & 35.46 & 46 & 38.17 \\
7 & 6.37 & 17 & 16.85 & 27 & 26.98 & 37 & 36.86 & 47 & 47.99 \\
8 & 8.76 & 18 & 17.91 & 28 & 27.77 & 38 & 37.95 & 48 & 47.93 \\
9 & 8.92 & 19 & 18.94 & 29 & 28.71 & 39 & 38.95 & 49 & 48.78 \\
10 & 10.00 & 20 & 20.93 & 30 & 29.90 & 40 & 39.56 & 50 & \\
\hline
\end{tabular}

A seguir apresentamos as estimativas de várias medidas resumo da distribuição à posteriori bem como as estimativas das distribuiçōes posterioris marginais. Essas estimativas basearam-se em $\mathrm{M}=4$ seqüências do algoritmo M. Collapsed Gibbs de comprimento $T=10.000$, após descartar-se a primeira metade de cada uma. Os pontos iniciais dessas sequêencias foram obtidos apartir dos pontos ${\underset{1}{\theta}}_{1}^{(0)}$ a ${\underset{4}{\theta_{4}}}^{(0)}$ (dados no início da seção) após descartar-se as componentes $\alpha_{1}^{(0)}, \alpha_{2}^{(0)}, \mu_{1}^{(0)}, \mu_{2}^{(0)}$ de cada um.

A tabela 4.6 apresenta a distribuição de freqüências à posteriori de $k$ e a tabela 4.7 exibe as estimativas de várias medidas resumo da distribuição à posteriori.

Tabela 4.6: Distribuição de freqüência relativa à posteriori de $k$ :

\begin{tabular}{c|l|l|l|l|l|l|l|l|}
\hline$k$ & 16 & 17 & 18 & 19 & 20 & 21 & 23 & outros \\
\hline$\hat{P}(K=k \mid$ Dobs $)$ & 0.0001 & 0.0006 & 0.0016 & 0.0050 & 0.0504 & 0.9414 & 0.0002 & 0.0000 \\
\hline
\end{tabular}


Tabela 4.7: Estimativas do valor esperado, quantis, desvio padrão, máximo, mínimo e intervalos de credibilidade de comprimento mínimo das distribuições marginais à posteriori de $\alpha_{1}, \alpha_{2}, \ldots, \sigma_{e_{1}}^{2}, \sigma_{e_{2}}^{2}, \sigma_{1}^{2}, \sigma_{2}^{2}$, usando o algoritmo M.Collap.Gibbs. (Os valores verdadeiros dos parâmetros são: $\alpha_{1}=2.0, \beta_{1}=1.0, \mu_{1}=2.0, \sigma_{x(1)}^{2}=0.6, \sigma_{1}^{2}=0.1$, $\sigma_{e_{1}}^{2}=0.1, \alpha_{2}=1.0, \beta_{2}=2.0, \mu_{2}=4.0, \sigma_{x(2)}^{2}=1.2, \sigma_{2}^{2}=0.2, \sigma_{e_{2}}^{2}=0.2, k=20$.)

\begin{tabular}{|c|c|c|c|c|c|c|c|}
\hline ESTIMATIVAS & $\omega_{1}$ & 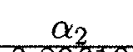 & $\beta_{1}$ & $\beta_{2}$ & 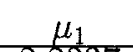 & & \\
\hline $\begin{array}{l}\text { Media aritm. } \\
\text { Média R-Blackw. } \\
\text { Quantis }\end{array}$ & $\begin{array}{l}1.8264 \\
1.8267\end{array}$ & $\begin{array}{l}0.89619 \\
0.89714\end{array}$ & $\begin{array}{l}1.0444 \\
1.0442\end{array}$ & $\begin{array}{l}2.0602 \\
2.0601\end{array}$ & $\begin{array}{l}2.2337 \\
2.2358\end{array}$ & $\begin{array}{l}3.8574 \\
3.8569\end{array}$ & \\
\hline $\begin{array}{r}0.025 \\
.05 \\
.25 \\
.75 \\
.95 \\
.975 \\
\end{array}$ & $\begin{array}{l}0.4356 \\
0.6979 \\
1.4138 \\
1.8574 \\
2.2632 \\
2.8488 \\
3.0496\end{array}$ & $\begin{array}{l}-0.9168 \\
-0.5635 \\
0.4030 \\
0.9589 \\
1.4670 \\
2.1315 \\
2.3402\end{array}$ & $\begin{array}{l}0.5101 \\
0.6044 \\
0.8546 \\
1.0327 \\
1.2229 \\
1.5333 \\
1.6495\end{array}$ & $\begin{array}{l}1.7019 \\
1.7538 \\
1.9184 \\
2.0438 \\
2.1828 \\
2.4200 \\
2.5132\end{array}$ & $\begin{array}{l}1.8835 \\
1.9414 \\
2.1152 \\
2.2329 \\
2.3523 \\
2.5262 \\
2.5859\end{array}$ & $\begin{array}{l}3.3929 \\
3.4698 \\
3.7016 \\
3.8597 \\
4.0117 \\
4.2366 \\
4.3145\end{array}$ & \\
\hline $\begin{array}{l}p=0.95 \mathrm{Lim} \text {. inf. } \\
p=0.90 \mathrm{Lim} \text { Lim. inf. } \\
\text { Lim sup. }\end{array}$ & $\begin{array}{l}0.5317 \\
3.1392 \\
0.7635 \\
2.8982\end{array}$ & $\begin{array}{l}-0.7548 \\
2.4604 \\
-0.3577 \\
2.2916\end{array}$ & $\begin{array}{l}0.4852 \\
1.6174 \\
0.5839 \\
1.5090\end{array}$ & $\begin{array}{l}1.6697 \\
2.4727 \\
1.7201 \\
2.3774\end{array}$ & $\begin{array}{l}1.8754 \\
2.5751 \\
1.9420 \\
2.5265\end{array}$ & $\begin{array}{l}3.4102 \\
4.3267 \\
3.4695 \\
4.2357\end{array}$ & \\
\hline $\begin{array}{l}\text { desv.padrão (dp) } \\
\text { dp R-Blackweliz; } \\
\text { "Naive SE" } \\
\text { "Batch SE" }\end{array}$ & $\begin{array}{l}0.6576 \\
0.6585 \\
0.0046 \\
0.0302\end{array}$ & $\begin{array}{l}0.8348 \\
0.8340 \\
0.0059 \\
0.0034\end{array}$ & $\begin{array}{l}.2848 \\
0.2857 \\
0.0020 \\
0.0351\end{array}$ & $\begin{array}{l}0.2079 \\
0.8340 \\
0.0015 \\
0.0082\end{array}$ & $\begin{array}{l}0.1791 \\
0.1802 \\
0.0013 \\
0.0130\end{array}$ & $\begin{array}{l}0.2349 \\
0.2356 \\
0.0017 \\
0.0014\end{array}$ & \\
\hline $\begin{array}{l}\text { Mínimo } \\
\text { Máximo }\end{array}$ & $\begin{array}{c}-1.3483 \\
5.9849\end{array}$ & $\begin{array}{l}-4.5125 \\
3.4845\end{array}$ & $\begin{array}{c}-0.6558 \\
2.4486\end{array}$ & $\begin{array}{l}1.4031 \\
3.3271\end{array}$ & $\begin{array}{l}1.2050 \\
3.0601\end{array}$ & $\begin{array}{l}2.7809 \\
4.8526\end{array}$ & \\
\hline ESTIMATIVAS & $\sigma_{x_{1}}^{2}$ & $\sigma_{x}^{2}$ & $\sigma_{1}^{2}$ & $\sigma_{2}^{2}$ & $\sigma_{e}^{2}$ & $\sigma_{0}^{2}$ & $\kappa$ \\
\hline $\begin{array}{l}\text { Média aritm. } \\
\text { Média R-Blackw. }\end{array}$ & $\begin{array}{l}0.41292 \\
0.41219\end{array}$ & $\begin{array}{l}1.3674 \\
1.3654\end{array}$ & $\begin{array}{l}0.26525 \\
0.26597\end{array}$ & $\begin{array}{l}0.24144 \\
0.24224\end{array}$ & $\begin{array}{l}0.27657 \\
0.27639\end{array}$ & $\begin{array}{l}0.39666 \\
0.39562\end{array}$ & $\begin{array}{l}20.93 \\
20.93\end{array}$ \\
\hline $\begin{array}{r}0.025 \\
.05 \\
.25 \\
.5 \\
.75 \\
.95 \\
.975\end{array}$ & $\begin{array}{l}0.1757 \\
0.1995 \\
0.2924 \\
0.3798 \\
0.4977 \\
0.7361 \\
0.8338\end{array}$ & $\begin{array}{l}0.7274 \\
0.8011 \\
1.0657 \\
1.2989 \\
1.5906 \\
2.1655 \\
2.4072\end{array}$ & $\begin{array}{l}0.1228 \\
0.1369 \\
0.1924 \\
0.2459 \\
0.3140 \\
0.4600 \\
0.5246\end{array}$ & $\begin{array}{l}0.1237 \\
0.1358 \\
0.1848 \\
0.2285 \\
0.2834 \\
0.3900 \\
0.4342\end{array}$ & $\begin{array}{l}0.1237 \\
0.1385 \\
0.1963 \\
0.2537 \\
0.3305 \\
0.4878 \\
0.5609\end{array}$ & $\begin{array}{l}0.1477 \\
0.1671 \\
0.2574 \\
0.3540 \\
0.4892 \\
0.7697 \\
0.8942\end{array}$ & $\begin{array}{l}20 \\
20 \\
21 \\
21 \\
21 \\
21 \\
21\end{array}$ \\
\hline $\begin{array}{l}p=0.95 \mathrm{Lim} \text {. inf. } \\
p=0.90 \mathrm{Lim} \text { Lim. inf. } \\
\text { Lim sup. }\end{array}$ & $\begin{array}{l}0.1409 \\
0.7509 \\
0.1625 \\
0.6584\end{array}$ & $\begin{array}{l}0.6280 \\
2.2114 \\
0.7182 \\
2.0073\end{array}$ & $\begin{array}{l}0.0978 \\
0.4671 \\
0.1078 \\
0.4056\end{array}$ & $\begin{array}{l}0.1099 \\
0.4032 \\
0.1222 \\
0.3613\end{array}$ & $\begin{array}{l}0.1044 \\
0.5013 \\
0.1154 \\
0.4375\end{array}$ & $\begin{array}{l}0.1085 \\
0.7796 \\
0.1204 \\
0.6592\end{array}$ & $\begin{array}{l}20 \\
21 \\
21 \\
21\end{array}$ \\
\hline $\begin{array}{l}\text { desv.padrão (dp) } \\
\text { dp R-Blackweliz; } \\
\text { "Naive SE", } \\
\text { "Batch SE" }\end{array}$ & $\begin{array}{l}0.1732 \\
0.1739 \\
0.0012 \\
0.0088\end{array}$ & $\begin{array}{l}0.4303 \\
0.4254 \\
0.0030 \\
0.0013\end{array}$ & $\begin{array}{l}0.1045 \\
0.1057 \\
0.0007 \\
0.0025\end{array}$ & $\begin{array}{l}0.0802 \\
0.0810 \\
0.0006 \\
0.0015\end{array}$ & $\begin{array}{l}0.1149 \\
0.1146 \\
0.0008 \\
0.0024\end{array}$ & $\begin{array}{l}0.1953 \\
0.1950 \\
0.0014 \\
0.0039\end{array}$ & $\begin{array}{l}0.3095 \\
0.3104 \\
0.0022 \\
0.0029\end{array}$ \\
\hline $\begin{array}{l}\text { Mínimo } \\
\text { Máximo }\end{array}$ & $\begin{array}{l}0.0799 \\
2.4958 \\
\end{array}$ & $\begin{array}{l}0.3990 \\
5.1758\end{array}$ & $\begin{array}{l}0.0663 \\
1.1225\end{array}$ & $\begin{array}{l}0.06571 \\
1.3458\end{array}$ & $\begin{array}{l}0.0689 \\
1.3485\end{array}$ & $\begin{array}{l}0.0777 \\
2.5940\end{array}$ & $\begin{array}{l}16 \\
23 \\
\end{array}$ \\
\hline
\end{tabular}

NOTA: Os intervalos de credibilidade nesse exemplo coincidem com os intervalos HPD. 
A Figura 4.22 apresenta os gráficos das estimativas "Rao-Blackwelizadas" das distribuições posterioris marginais de $\alpha_{1}, \alpha_{2}, \beta_{1}, \beta_{2}, \ldots, \sigma_{1}^{2}, \sigma_{2}^{2}$ e $k$.
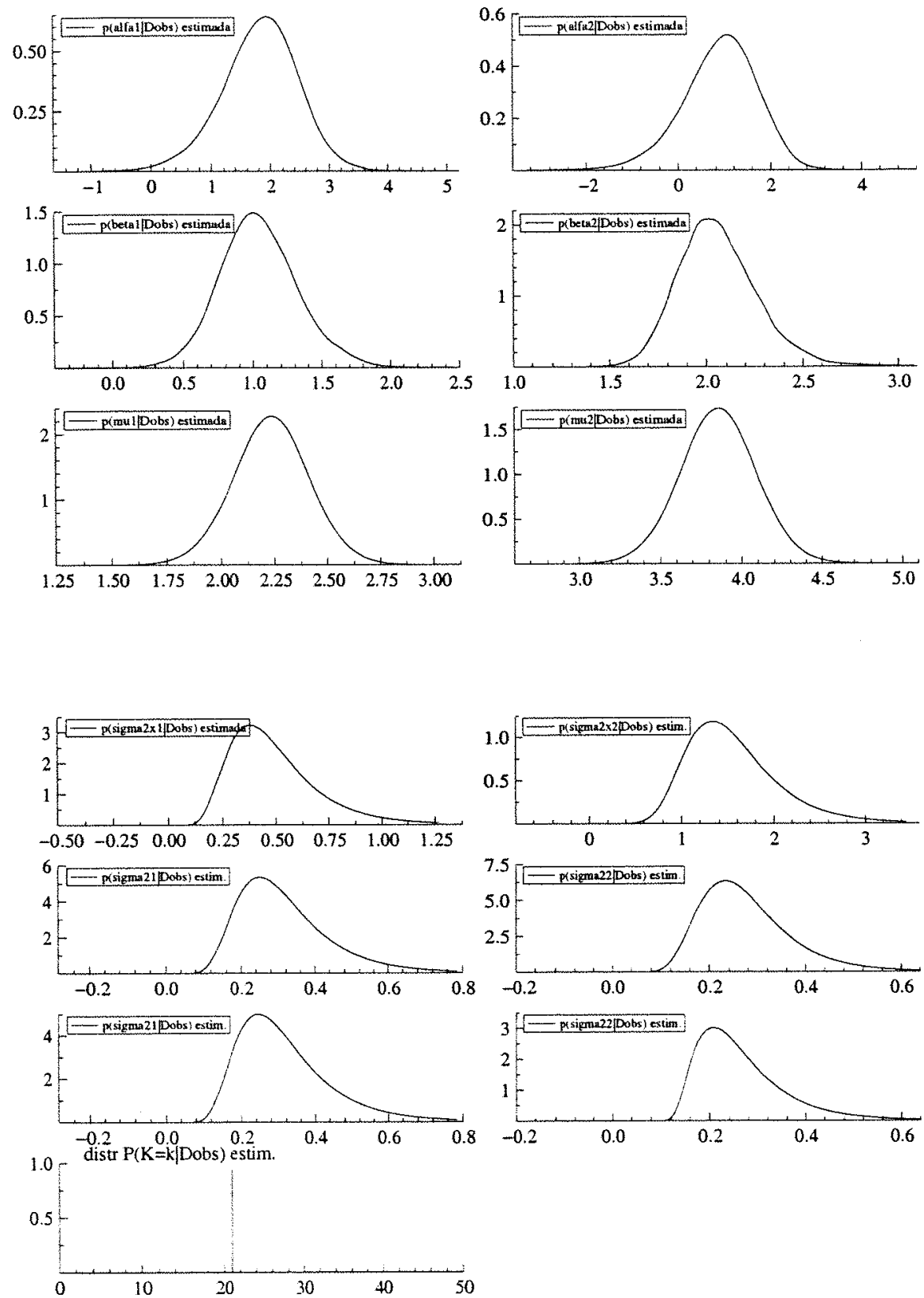

Figura 1.22: Gráficos das estimativas "Rao-Blackwelizadas" das densidades marginais à posteriori $p\left(\alpha_{1} \mid D_{\text {obs }}\right), p\left(\alpha_{2} \mid D_{o b s}\right), \ldots p\left(\sigma_{1}^{2} \mid D_{\text {obs }}\right), p\left(\sigma_{2}^{2} \mid D_{\text {obs }}\right)$ e $p\left(k \mid D_{\text {obs }}\right)$. 


\section{Observações:}

Na tabela 4.5 a única estimativa discrepante ocorre quando o verdadeiro valor de $\mathrm{k}$ é 4 . Semelhantemente a seção anterior, observou-se também que se o valor verdadeiro de k é bem pequeno, então o vetor de parâmetros $\underset{\sim}{\theta_{1}}$ (onde $\underset{\sim}{\theta_{1}}=$ $\left.\left(\alpha_{1}, \beta_{1}, \mu_{1}, \sigma_{x_{1}}^{2}, \sigma_{1}^{2}, \sigma_{e_{1}}^{2}\right)^{T}\right)$ tende a não ser bem estimado, e se $\mathrm{k}$ é grande (próximo de $\mathrm{n}$ ), então $\underset{\sim}{\theta_{2}}$ é que tende a ter estimativas pobres. Esse fato já era esperado pela própria estrutura do problema.

Na tabela 4.7 vemos que tanto a média como a mediana superestimam os valores dos parâmetros $\sigma_{1}^{2}, \sigma_{e_{1}}^{2}$ e $\sigma_{e_{2}}^{2}$, entretanto o valor do viés estimado é pequeno para os demais parâmetros. Mais importante ainda é que todos os intervalos estimados de credibilidade de $95 \%$ contém os verdadeiros valores dos parâmetros (no caso de $\sigma_{e_{1}}^{2}$ isso é verdade se considerarmos apenas 2 casas decimais).

É interessante observar que no caso particular em que o conjunto de dados é o da tabela 4.1, os comprimentos dos intervalos de credibilidade no modelo sem restrição de identificabilidade (com priori dada em (4.7.2)) tendem a ser ligeiramente maiores do que no modelo com restrição de identificabilidade (com priori dada em (4.7.1) ), entretanto em ambos os modelos esses intervalos estimados contiveram os verdadeiros valores dos parametros.

\section{3.c Avaliação do ajuste}

A figura 4.23 apresenta os gráficos dos valores da densidade condicional preditiva de validação cruzada $\mathrm{CPO}_{i}$ (onde $\mathrm{CPO}_{i}=\pi\left(\left(\begin{array}{l}Y_{i} \\ X_{i}\end{array}\right) \mid D_{\text {obs }}\right)$ ) primeiramente em função de i, depois em função de $X_{i}$, e também em função de $Y_{i}$, para $i=1, \ldots, 50$ (onde $\mathrm{CPO}_{i}$ é estimada por meio da fórmula (4.5.8) adaptada para o caso do modelo sem restriçōes de identificabilidade). As estimativas basearam-se na segunda metade de uma sequência de comprimento $T=10.000$ do algoritmo M.Collap.Gibbs cujo ponto inicial é formado pelas componentes de $\theta_{1}^{(0)}$, após descartar $\alpha_{1}^{(0)}: \alpha_{2}^{(0)}, \mu_{1}^{(0)}, \mu_{2}^{(0)}$. 


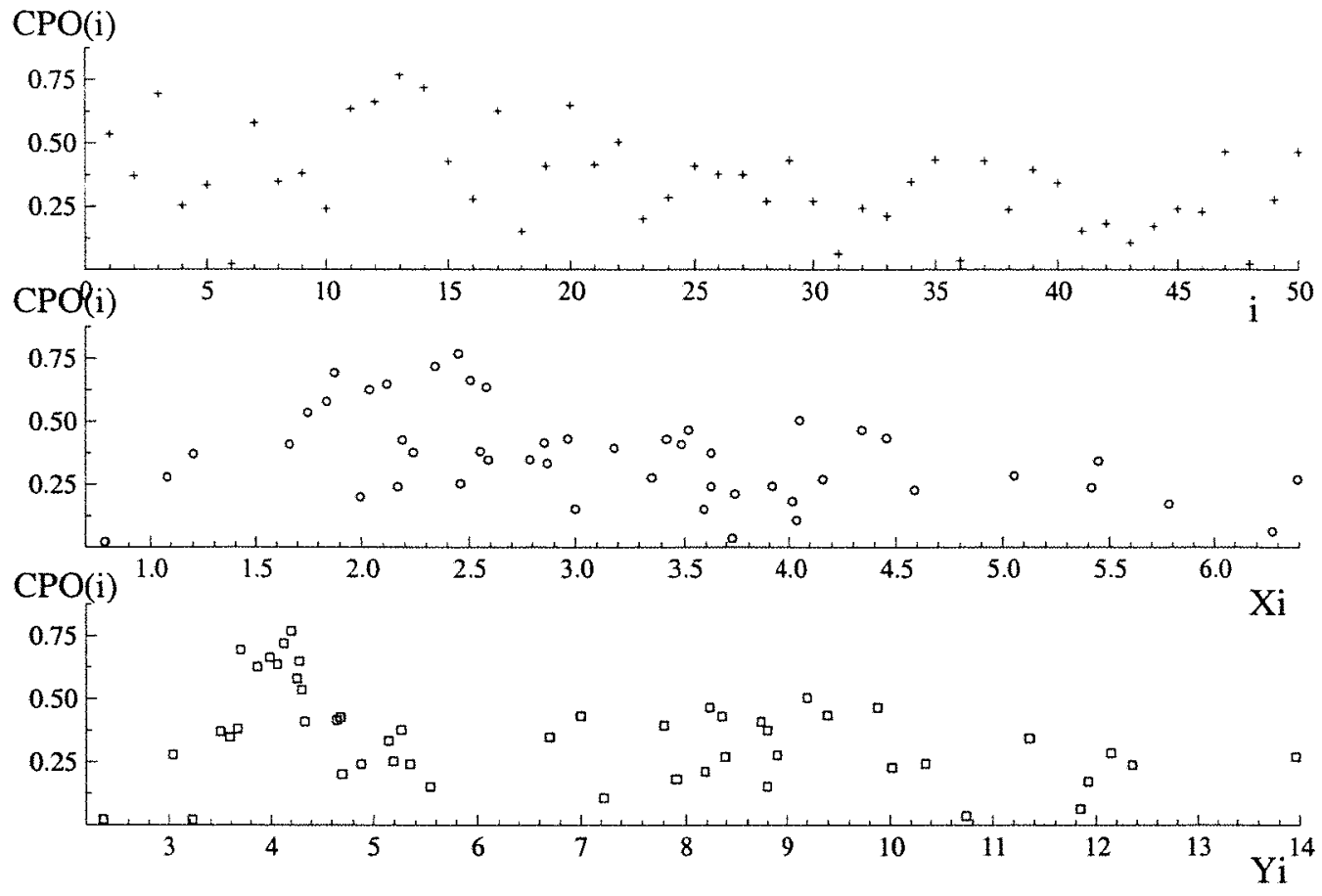

Figura 4.23: Gráficos dos valores da densidade condicional preditiva, $\mathrm{CPO}(\mathrm{i})$, versus $i$ (posição superior), versus $X_{i}$ (posiçãodo meio) e versus $Y_{i}$ (posição inferior).

A figura 4.24 a seguir apresenta os gráficos das componentes $d_{1_{i}}$ e $d_{2_{i}}$ do vetor de resíduos bayesianos bidimensionais de validação cruzada

$$
d_{i}=\left(\begin{array}{c}
d_{1_{i}} \\
d_{2_{i}}
\end{array}\right)=\widehat{\operatorname{Var}}^{-\frac{1}{2}}\left(\left(\begin{array}{c}
Y_{i} \\
X_{i}
\end{array}\right) \mid D_{\mathrm{obs}(-i)}\right)\left(\left(\begin{array}{c}
Y_{i} \\
X_{i}
\end{array}\right)-\hat{E}\left(\left(\begin{array}{c}
Y_{i} \\
X_{i}
\end{array}\right) \mid D_{\mathrm{obs}(-i)}\right)\right)
$$

$i=1, \ldots, n$, dado na seção 4.5 .3 , onde as fórmulas de $\hat{E}\left(\left(\begin{array}{l}Y_{i} \\ X_{i}\end{array}\right) \mid D_{\mathrm{obs}(-i)}\right)$ e de $\widehat{\operatorname{Var}}^{-\frac{1}{2}}\left(\left(\begin{array}{c}Y_{i} \\ X_{1}\end{array}\right) \mid D_{\text {obs }(-i)}\right)$ foram adaptadas para o modelo sem restrição de identificabilidade.

As figuras 4.23 e 4.24 não sugerem que exista problema de falta de ajustamento. Entretanto nos gráficos de ordenada $d_{1_{1}}$ da figura 4.24 existem 2 pontos cujos valores são maiores do que 3 (em valor absoluto) e nos gráficos de ordenada $d_{2_{1}}$ da mesma figura, existem 4 pontos nessas condições. Esses pontos aparentemente poderiam 

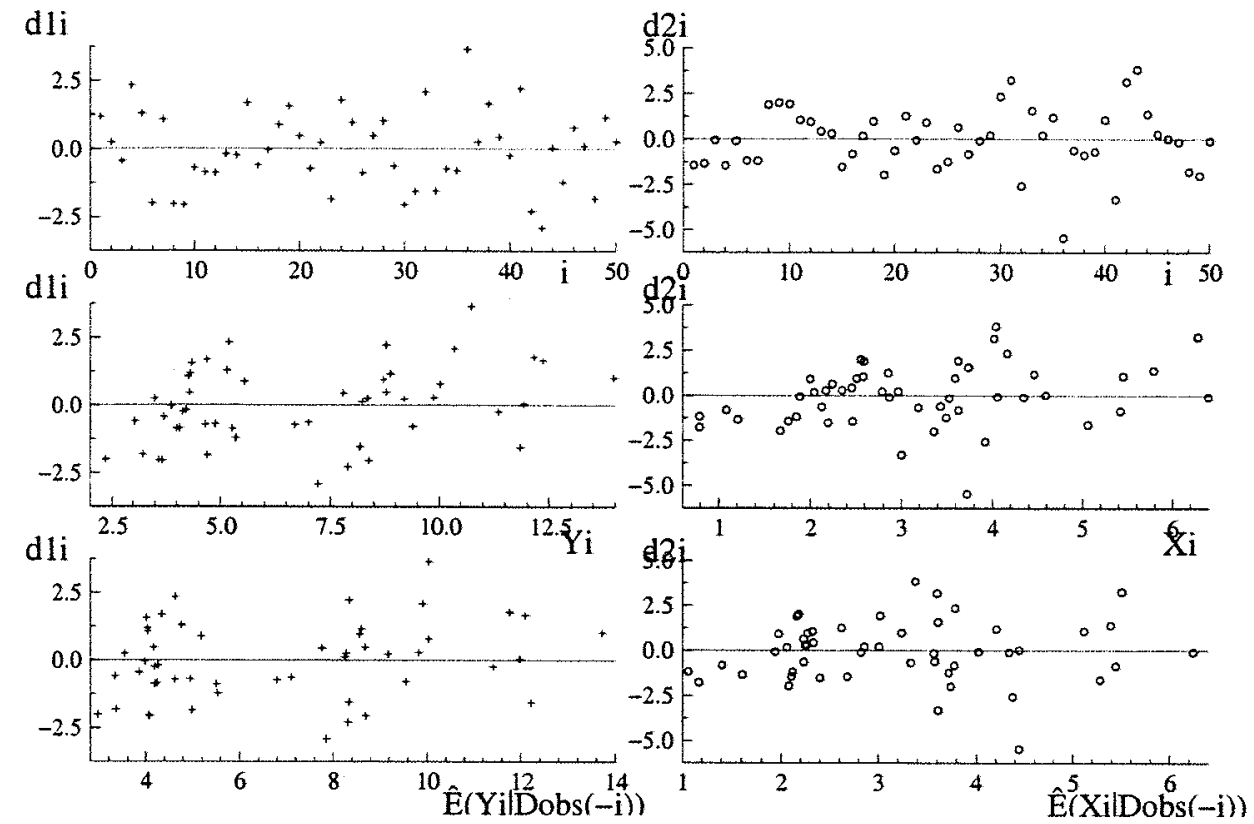

Figura 4.24: Os gráficos do lado esquerdo referem-se à primeira componente dos resíduos $\left(d_{i 1}\right)$, e os do lado direito à segunda componente $\left(d_{i 2}\right)$.

ser vistos como aberrantes, entretanto uma análise de resídous mais aprofundada deve ser conduzida futuramente para examinar melhor a qualidade do ajuste.

Quando o conjunto de dados observados é o da tabela 4.1, a qualidade do ajuste aparentemente não difere muito entre os modelos com e sem restrição de identificabilidade (com suas respectivas prioris dadas em (4.7.1) e (4.7.2)).

Levando em conta também que as estimativas dos parâmetros não diferem muito nos dois modelos, o modelo sem restrição aparentemente é mais vantajoso já que ele não requer o conhecimento do valor de lambda (o quociente entre as variâncias residuais). 


\subsubsection{Análise do procedimento 1 para detetar mudança ba- seada na distribuição à posteriori de $k$.}

\section{MODELO}

Nessa seção utilizamos o modelo com restricão de identificabilidade que permite mudança em todos os parâmetros e onde $\mathrm{k}$ pode assumir os valores de $1 \mathrm{a} \mathrm{n}$, definido na seção 4.4.1.

A distribuição à priori escolhida é

$$
\pi(\stackrel{*}{\theta})= \begin{cases}p(k) \pi\left(\underline{\theta}_{1}\right), & \text { se } k=n, \\ p(k) \pi\left(\underline{\theta}_{1}\right) \pi\left(\underline{\sim}_{2}\right), & \text { se } k \in \mathcal{K}=\{1, \ldots, n-1\}, \\ 0, & \text { caso contrário, }\end{cases}
$$

onde

$$
\begin{gathered}
\pi\left(\theta_{i}\right)=\pi\left(\alpha_{i}\right) \pi\left(\beta_{i}\right) \pi\left(\mu_{i}\right) \pi\left(\sigma_{x(i)}^{2}\right) \pi\left(\sigma_{i}^{2}\right), i=1,2 \mathrm{e} \\
p(k)= \begin{cases}c, & \text { se } k=n \\
(1-c) \frac{1}{\# \mathcal{K}}, & \text { se } k \in \mathcal{K} \\
0, & \text { caso contrário, }\end{cases}
\end{gathered}
$$

onde $c=P(K=n)=\frac{1}{n}, \alpha_{i} \sim N(0 ; 1000), \beta_{i} \sim N(0 ; 1000), \mu_{i} \sim N(0 ; 1000)$, $\sigma_{x_{i}}^{2} \sim I G(2.001 ; 1.0), \sigma_{e_{i}}^{2} \sim I G(2.001 ; 1.0), \sigma_{i}^{2} \sim I G(2.001 ; 1.0)$.

Nessa seção utilizaremos o conjunto de dados da tabela 4.1 .

\section{AJUSTE DO MODELO}

Para amostrar da distribuição à posteriori foi implementado o algoritmo de Gibbs descrito na seção 4.4.1. Foram geradas 4 seqüências paralelas de comprimento $T=$ 10.000 partindo-se dos mesmos pontos iniciais usados na seção 4.7.1.

A seguir nas figuras 4.25 a 4.28 e na tabela 4.8 apresentamos alguns gráficos e medidas de diagnóstico de convergência desse algoritmo.

Os dispositivos apresentados nessas figuras não nos sugeriram que exista algum problema na convergência do algoritmo de Gibbs. 

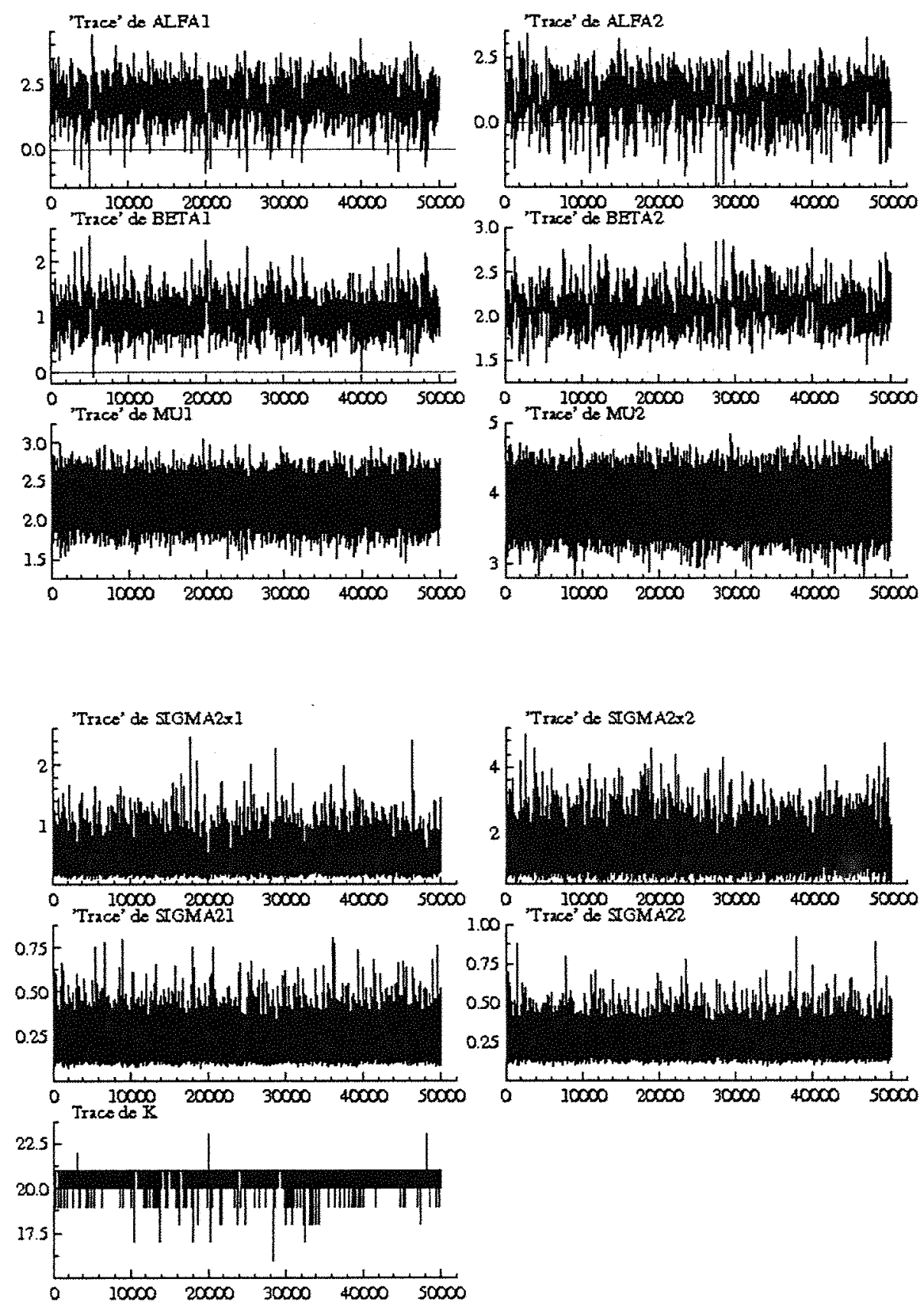

Figura 4.25: Séries temporais de $\alpha_{1}, \alpha_{2}, \beta_{1}, \beta_{2}, \mu_{1}, \mu_{2}, \sigma_{x_{1}}^{2}, \sigma_{x_{2}}^{2}, \sigma_{1}^{2}, \sigma_{2}^{2}$ e $k$ baseadas em uma sequiência de comprimento $T=50.000$ do algoritmo de Gibbs partindo do ponto $\stackrel{\sim}{*}_{(0)}$. 

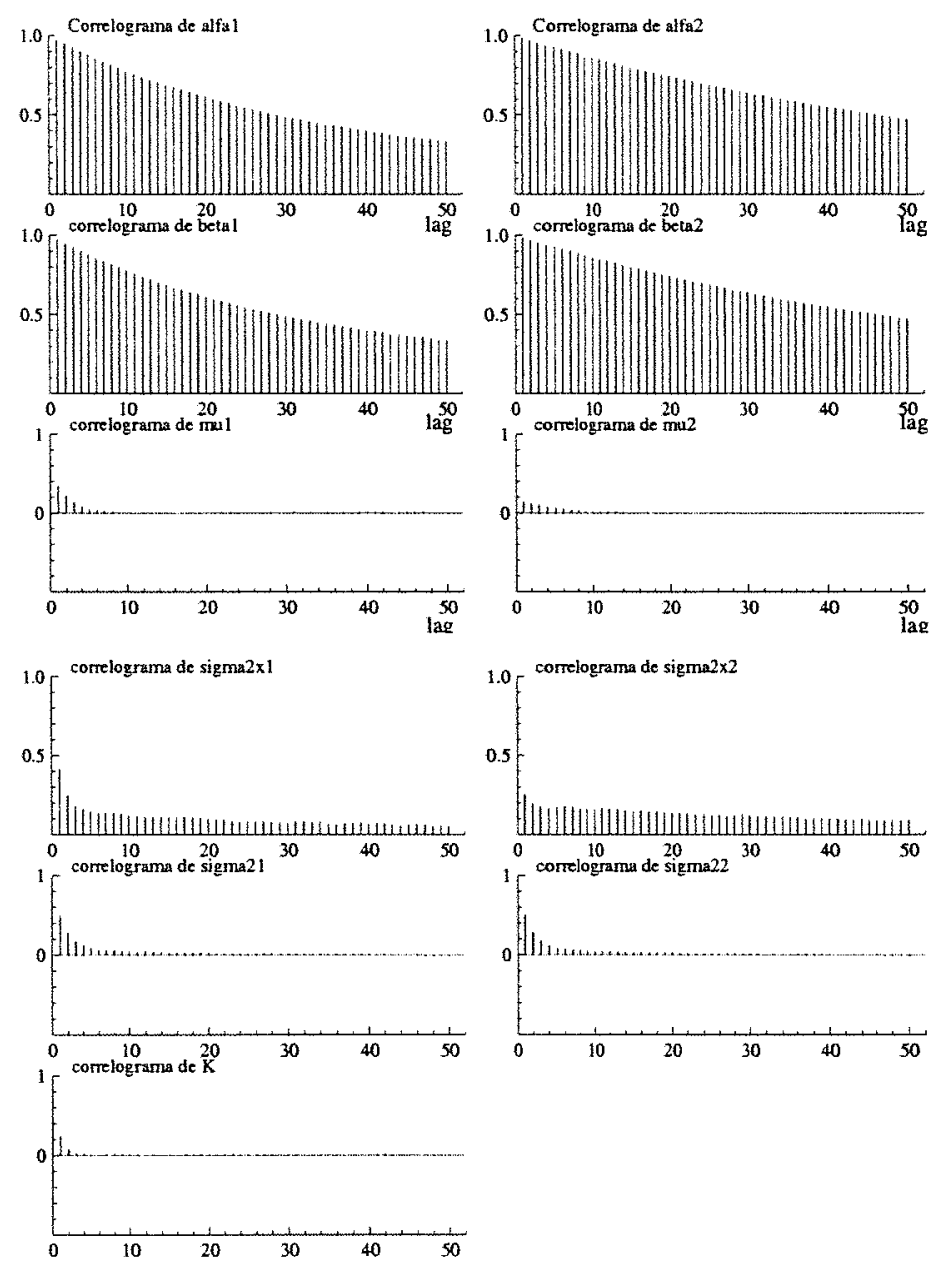

Figura 4.26: Gráficos das funções de auto-correlação de $\alpha_{1}, \alpha_{2}, \beta_{1}, \beta_{2}, \mu_{1}, \mu_{2}, \sigma_{x_{1}}^{2}, \sigma_{x_{2}}^{2}, \sigma_{1}^{2}, \sigma_{2}^{2}$ e $k$ baseados numa seguêencia de comprimento $T=50.000$ de Gibbs, partindo de ${\underset{\sigma}{(0)}}_{(0)}$.

Tabela 4.8: Medidas de Diagnóstico de convergência de Brooks, Gelman e Rubin

\begin{tabular}{c|c|cc|c|c|cc}
\hline & PSRF & CSRF & Quantil 0.975 & & PSRF & CSRF & Quantil 0.975 \\
\hline$\alpha_{1}$ & 1.003949 & 1.017607 & 1.027021 & $\alpha_{2}$ & 1.000187 & 1.000210 & 1.000818 \\
$\beta_{1}$ & 1.017694 & 1.030607 & 1.071077 & $\beta_{2}$ & 1.000654 & 1.000877 & 1.002472 \\
$\mu_{1}$ & 1.003834 & 1.017390 & 1.026530 & $\mu_{2}$ & 1.003358 & 1.004180 & 1.011505 \\
$\sigma_{x_{1}}^{2}$ & 1.018132 & 1.030291 & 1.071477 & $\sigma_{r_{2}}^{2}$ & 1.000985 & 1.001547 & 1.003849 \\
$\sigma_{1}^{2}$ & 1.000147 & 1.000228 & 1.000750 & $\sigma_{2}^{2}$ & 1.000961 & 1.001298 & 1.003543 \\
$k$ & 1.000149 & 1.003085 & 1.003623 & & & & \\
\hline
\end{tabular}

A medida multivariada (MPSRF) é igual a 1.0576164. 


\section{Brooks \& Gelman Multivariate Shrink Factors}

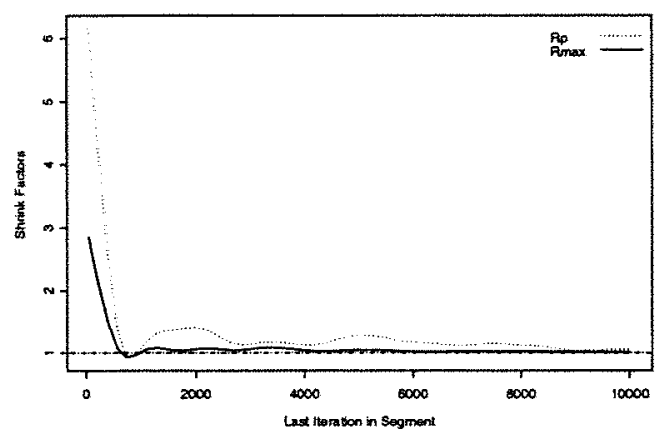

Figura 4.27: "Multiv. Pot.Scale Reduction" de Brooks e Gelman (1998) com base em 4 seg. onde $\mathrm{T}=10.000$.

\section{Gelman \& Rubin Shrink Factors}
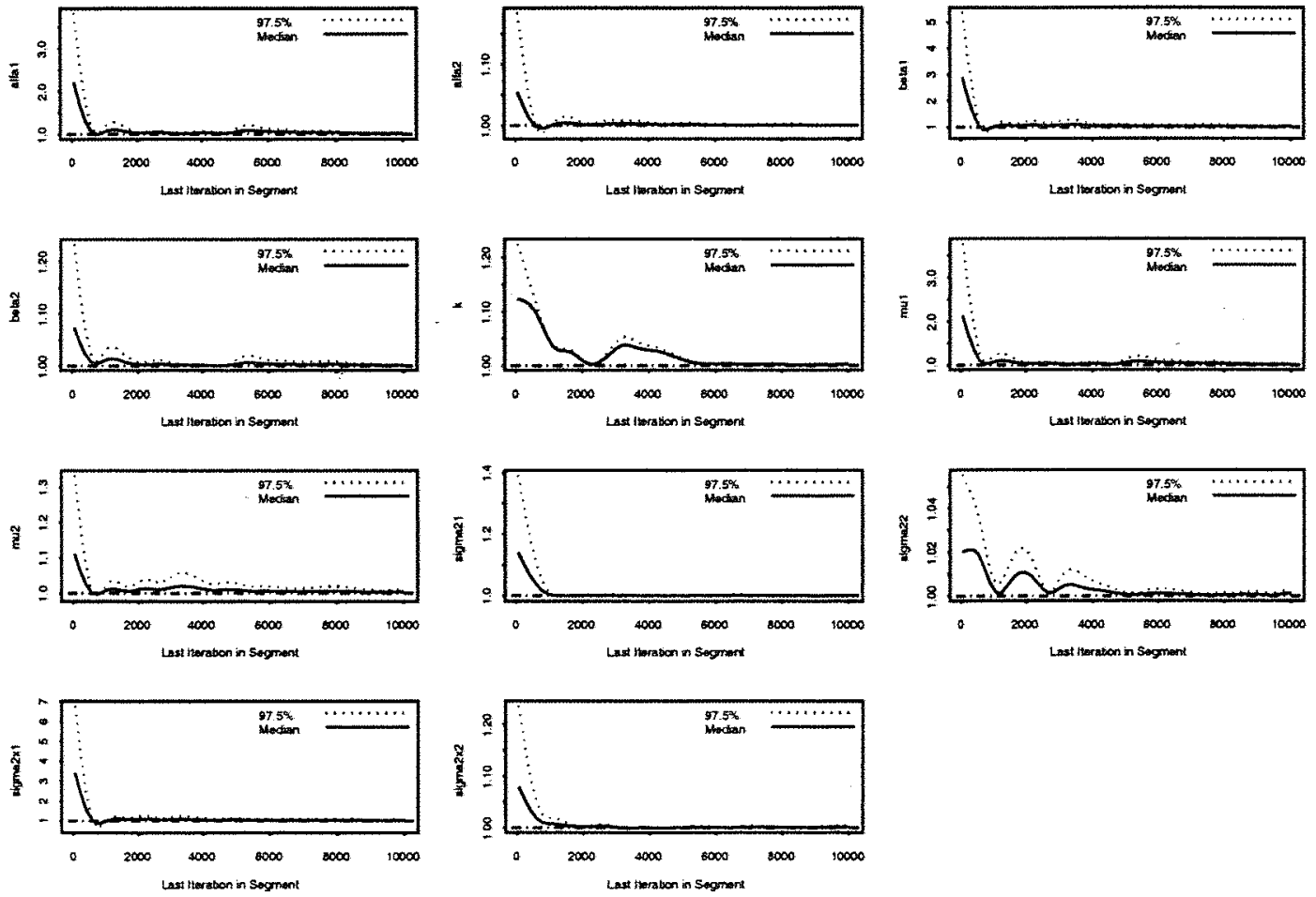

Figura 4.28: Gráfico da medida de Gelman e Rubin corrigida "Corrected potencial scale reduction factors" (CSRF) para cada um dos parâmetros $k, \alpha_{1}, \alpha_{2}, \beta_{1}, \beta_{2}, \ldots, \sigma_{1}^{2}, \sigma_{2}^{2}$, baseada em $M=4$ sequèencias de comprimento $T=10.000$ de Gibbs. 


\section{2.a Estimação}

A tabela 4.9 exibe para cada valor de $k=k_{0}$ (entre 1 e $\mathrm{n}$ ), a respectiva estimativa $(\hat{k})$ baseada nos dados gerados apartir do modelo (dessa seção) onde $k=k_{0}, \underset{\sim}{\theta_{1}}=(2.0,1.0,2.0,0.6,0.1)^{T}$ e $\underset{\sim}{\theta_{2}}=(1.0,2.0,4.0,1.2,0.2)^{T}$ utilizando-se o algoritmo de Gibbs partindo do ponto inicial $\left(k^{(0)}, \theta_{1}^{(0)},{\underset{\theta}{2}}_{2}^{(0)}\right)$, onde $k^{(0)}=1$ e ${\underset{\sim}{i}}_{i}^{(0)}=$ $\left(\alpha_{i}^{(0)}, \beta_{i}^{(0)}, \mu_{i}^{(0)}, \sigma_{x_{i}}^{2(0)}, \sigma_{i}^{2(0)}\right)=(1,1,1,1,1), i=1,2$.

Tabela 4.9: Valores verdadeiros de $k$ e respectivos valores estimados $(\hat{k})$ baseados na segunda metade da seqüência de Gibbs correspondente.

\begin{tabular}{cc|cc|cc|cc|cc}
\hline$k$ & $\hat{k}$ & $k$ & $\hat{k}$ & $k$ & $\hat{k}$ & $k$ & $\hat{k}$ & $k$ & $\hat{k}$ \\
\hline 1 & 12.57 & 11 & 10.95 & 21 & 20.99 & 31 & 30.96 & 41 & 40.96 \\
2 & 1.93 & 12 & 11.96 & 22 & 22.83 & 32 & 31.97 & 42 & 41.85 \\
3 & 50.00 & 13 & 12.87 & 23 & 22.95 & 33 & 32.98 & 43 & 42.94 \\
4 & 31.18 & 14 & 14.95 & 24 & 23.88 & 34 & 33.94 & 44 & 43.71 \\
5 & 4.82 & 15 & 14.72 & 25 & 25.25 & 35 & 34.95 & 45 & 44.91 \\
6 & 5.89 & 16 & 15.97 & 26 & 25.95 & 36 & 35.71 & 46 & 45.32 \\
7 & 6.72 & 17 & 16.93 & 27 & 26.98 & 37 & 36.92 & 47 & 49.73 \\
8 & 8.87 & 18 & 17.95 & 28 & 27.92 & 38 & 37.96 & 48 & 50.00 \\
9 & 8.99 & 19 & 18.96 & 29 & 28.73 & 39 & 38.97 & 49 & 50.00 \\
10 & 10.00 & 20 & 20.97 & 30 & 29.93 & 40 & 39.68 & 50 & 50.00 \\
\hline
\end{tabular}

Na tabela 4.9 estimativas extremamente discrepantes ocorreram para $k=1, k=3$ e $k=4$. Observou-se também que para valores de $k$ bem pequenos, as estimativas de cada uma das componentes de ${\underset{\sim}{1}}_{1}$, onde ${\underset{\sim}{\theta}}_{1}=\left(\alpha_{1}, \beta_{1}, \mu_{1}, \sigma_{x_{1}}^{2}, \sigma_{1}^{2}, \sigma_{e_{1}}^{2}\right)^{T}$, são muito viesadas. Também se $k$ é grande (próximo de $\mathrm{n}$ ), as componentes de ${\underset{\sim}{2}}_{2}=\left(\alpha_{2}, \beta_{2}, \mu_{2}, \sigma_{x_{1}}^{2}, \sigma_{1}^{2}, \sigma_{e_{2}}^{2}\right)^{T}$ tendem a ter um grande viés.

A tabela 4.10 apresenta as estimativas de várias medidas resumo da distribuição à posteriori e a tabela 4.11, a distribuição de freqüências à posteriori de $k$. A figura 4.24 apresenta os gráficos das estimativas "Rao-blackwelizadas" das distribuições marginais à posteriori. Essas estimativas baseiam-se em 4 seqüências de Gibbs de comprimento $T=10.000$ que partem respectivamente dos pontos iniciais $\stackrel{*}{\stackrel{*}{\theta}}_{1}^{(0)}, \stackrel{*}{\theta}_{2}^{(0)}$, $\stackrel{*}{\theta}_{3}^{(0)}$ e $\stackrel{*}{\theta}_{4}^{(0)}$, após descartar-se a primeira metade de cada uma. 
Tabela 4.10: Estimativas do valor esperado, quantis, desvio padrão, máximo, mínimo e intervalos de credibilidade de comprimento mínimo das distribuições marginais à posteriori de $\alpha_{1}, \alpha_{2}, \ldots, \sigma_{1}^{2}, \sigma_{2}^{2}$, usando o algoritmo de Gibbs. (Os valores verdadeiros dos parâmetros são: $\alpha_{1}=2.0, \beta_{1}=1.0, \mu_{1}=2.0, \sigma_{x(1)}^{2}=0.6, \sigma_{1}^{2}=0.1$, $\alpha_{2}=1.0, \beta_{2}=2.0, \mu_{2}=4.0, \sigma_{x(2)}^{2}=1.2, \sigma_{2}^{2}=0.2, k=20$.)

\begin{tabular}{|c|c|c|c|c|c|c|}
\hline ESTIMATIVAS & & & $\beta_{1}$ & $\beta_{2}$ & & \\
\hline $\begin{array}{l}\text { Média aritm. } \\
\text { Média R-Blackw. }\end{array}$ & $\begin{array}{l}1.8140 \\
1.8147\end{array}$ & $\begin{array}{l}0.8539 \\
0.8540\end{array}$ & $\begin{array}{l}1.0500 \\
1.0504\end{array}$ & $\begin{array}{l}2.0720 \\
2.0721\end{array}$ & $\begin{array}{l}2.2357 \\
2.2357\end{array}$ & $\begin{array}{l}3.8581 \\
3.8587\end{array}$ \\
\hline $\begin{array}{r}\text { Quanus } \\
0.025 \\
.05 \\
.25 \\
.5 \\
.75 \\
.95 \\
.975\end{array}$ & $\begin{array}{l}0.4568 \\
0.7484 \\
1.4138 \\
1.8645 \\
2.2251 \\
2.7121 \\
2.8967\end{array}$ & $\begin{array}{l}-0.9560 \\
-0.5399 \\
0.4030 \\
0.9417 \\
1.366 \\
1.9164 \\
2.1074\end{array}$ & $\begin{array}{l}0.5845 \\
0.6601 \\
0.8710 \\
1.0250 \\
1.2033 \\
1.5133 \\
1.6468\end{array}$ & $\begin{array}{l}1.7635 \\
1.8080 \\
1.9452 \\
2.0514 \\
2.1737 \\
2.4214 \\
2.5069\end{array}$ & $\begin{array}{l}1.8915 \\
1.9472 \\
2.1181 \\
2.2361 \\
2.3533 \\
2.5211 \\
2.5832\end{array}$ & $\begin{array}{l}3.3909 \\
3.4740 \\
3.7017 \\
3.8578 \\
4.0163 \\
4.2412 \\
4.3169\end{array}$ \\
\hline $\begin{array}{l}\text { Int.Credib. } \\
p=0.95 \text { Lim. inf. } \\
\text { Lim sup. } \\
p=0.90 \text { Lim. inf. } \\
\text { Lim sup. }\end{array}$ & $\begin{array}{l}0.5478 \\
2.9576 \\
0.8242 \\
2.7743\end{array}$ & $\begin{array}{c}-0.7254 \\
2.2576 \\
-0.3584 \\
2.0416\end{array}$ & $\begin{array}{l}0.5412 \\
1.5907 \\
0.6339 \\
1.4828\end{array}$ & $\begin{array}{l}1.7412 \\
2.4721 \\
1.7846 \\
2.3795\end{array}$ & $\begin{array}{l}1.8923 \\
2.5839 \\
1.9516 \\
2.5240\end{array}$ & $\begin{array}{l}1.8940 \\
4.3205 \\
1.9527 \\
4.2456\end{array}$ \\
\hline $\begin{array}{l}\text { desv.padrão (dp) } \\
\text { dp R-Blackweliz; } \\
\text { "Naive SE" }\end{array}$ & $\begin{array}{l}0.6219 \\
0.6212 \\
0.0044\end{array}$ & $\begin{array}{l}0.7461 \\
0.7467 \\
0.0053\end{array}$ & $\begin{array}{l}.2701 \\
0.2704 \\
0.0019\end{array}$ & $\begin{array}{l}0.1851 \\
0.1851 \\
0.0013\end{array}$ & $\begin{array}{l}0.1753 \\
0.1749 \\
0.0012\end{array}$ & $\begin{array}{l}0.2354 \\
0.2356 \\
0.0017\end{array}$ \\
\hline $\begin{array}{l}\text { Mínimo } \\
\text { Máximo }\end{array}$ & $\begin{array}{r}-1.4821 \\
4.3952 \\
\end{array}$ & $\begin{array}{r}-2.4096 \\
3.1949 \\
\end{array}$ & $\begin{array}{r}-0.0792 \\
2.4700 \\
\end{array}$ & $\begin{array}{l}1.4869 \\
2.8586 \\
\end{array}$ & $\begin{array}{l}1.5147 \\
3.0517 \\
\end{array}$ & $\begin{array}{l}2.8388 \\
4.8382 \\
\end{array}$ \\
\hline ESTIMATIVAS & $\sigma_{x}^{2}$ & $\sigma_{\pi n}^{2}$ & $\sigma_{1}^{2}$ & $\sigma_{2}^{2}$ & $k$ & \\
\hline $\begin{array}{l}\text { Média aritm. } \\
\text { Média R-Blackw. }\end{array}$ & $\begin{array}{l}0.4254 \\
0.4242\end{array}$ & $\begin{array}{l}1.3662 \\
1.3670\end{array}$ & $\begin{array}{l}0.2155 \\
0.2159\end{array}$ & $\begin{array}{l}0.2412 \\
0.2410\end{array}$ & $\begin{array}{l}20.970 \\
20.968\end{array}$ & \\
\hline $\begin{array}{r}0.025 \\
.05 \\
.25 \\
.5 \\
.75 \\
.95 \\
975 \\
\end{array}$ & $\begin{array}{l}0.1840 \\
0.2085 \\
0.3036 \\
0.3920 \\
0.5090 \\
0.7490 \\
0.8531\end{array}$ & $\begin{array}{l}0.7387 \\
0.8098 \\
1.0690 \\
1.3003 \\
1.5913 \\
2.1412 \\
2.3643\end{array}$ & $\begin{array}{l}0.1185 \\
0.1290 \\
0.1671 \\
0.2030 \\
0.2499 \\
0.3437 \\
0.38261\end{array}$ & $\begin{array}{l}0.1451 \\
0.1552 \\
0.1940 \\
0.2304 \\
0.2746 \\
0.3674 \\
0.4029\end{array}$ & $\begin{array}{l}20 \\
21 \\
21 \\
21 \\
21 \\
21 \\
21\end{array}$ & \\
\hline $\begin{array}{l}p=0.95 \mathrm{Lim} \text {. inf. } \\
p=0.90 \mathrm{Lim} \text {.im. inf. } \\
\text { Lim sup. }\end{array}$ & $\begin{array}{l}0.1465 \\
0.7674 \\
0.1643 \\
0.6661\end{array}$ & $\begin{array}{l}0.6547 \\
2.2156 \\
0.7316 \\
2.0020\end{array}$ & $\begin{array}{l}0.1047 \\
0.3507 \\
0.1137 \\
0.3133\end{array}$ & $\begin{array}{l}0.1324 \\
0.3770 \\
0.1422 \\
0.3396\end{array}$ & $\begin{array}{l}21 \\
21 \\
21 \\
21\end{array}$ & \\
\hline $\begin{array}{l}\text { desv.padrão (dp) } \\
\text { dp R-Blackweliz,; } \\
\text { "Naive SE" }\end{array}$ & $\begin{array}{l}0.1762 \\
0.1746 \\
0.0012\end{array}$ & $\begin{array}{l}0.4212 \\
0.4211 \\
0.0030\end{array}$ & $\begin{array}{l}0.0689 \\
0.0689 \\
0.0005\end{array}$ & $\begin{array}{l}0.0667 \\
0.0667 \\
0.0005\end{array}$ & $\begin{array}{l}0.1862 \\
0.1951 \\
0.0013\end{array}$ & \\
\hline $\begin{array}{l}\text { Mínimo } \\
\text { Máximo }\end{array}$ & $\begin{array}{l}0.0799 \\
2.4958\end{array}$ & $\begin{array}{l}0.3990 \\
5.1758\end{array}$ & $\begin{array}{l}0.0663 \\
1.1225\end{array}$ & $\begin{array}{l}0.06571 \\
1.3458\end{array}$ & $\begin{array}{l}16 \\
21\end{array}$ & \\
\hline
\end{tabular}

Tabela 4.11: Distribuição de frequêencia relativa à posteriori de $k$ :

\begin{tabular}{c|l|l|l|l|l|}
\hline$k$ & 18 & 19 & 20 & 21 & outro\$ \\
\hline$\hat{P}(K=k \mid$ Dobs $)$ & 0.0001 & 0.0019 & 0.0250 & 0.9720 & 0.0000 \\
\hline
\end{tabular}



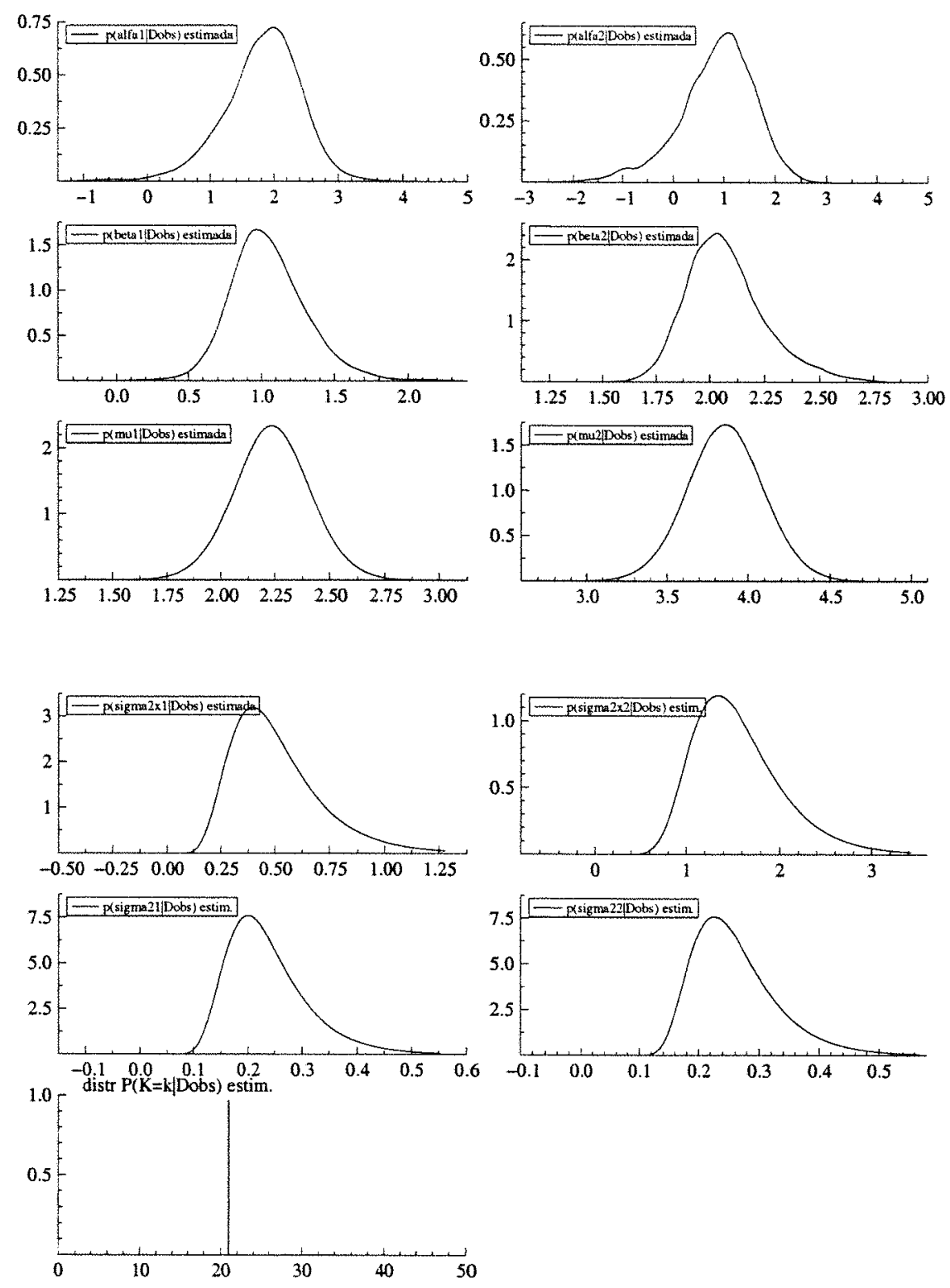

Figura 4.29: Gráficos das estimativas "Rao-Blackwelizadas" das densidades marginais à posteriori $p\left(\alpha_{1} \mid D_{\text {obs }}\right), p\left(\alpha_{2} \mid D_{\text {obs }}\right), \ldots, p\left(\sigma_{1}^{2} \mid D_{\text {obs }}\right), p\left(\sigma_{2}^{2} \mid D_{\text {obs }}\right)$ e $p\left(k \mid D_{\text {obs }}\right)$.

Observação 1: Da tabela 4.11 vemos que $\hat{P}(k=n)<\hat{P}(k \neq n)$ (já que $\hat{P}(k=$ $n) \approx 0$ ) e portanto a hipótese nula $H_{0}$ (de que não há mudança ) é rejeitada, o que era esperado já que a amostra de dados utilizada é a da tabela 4.1 e a distribuição 
à priori é praticamente não informativa.

Observação 2: Os comentários sobre a tabela 4.10 são semelhantes àqueles feitos sobre a tabela 4.3 .

\section{2.b Sensibilidade das estimativas ao valor do hiperparâmetro c}

A seguir analisamos a sensibilidade nas estimativas dos parâmetros do modelo estudado nessa seção quando variamos os valores do hiperparâmetro c à priori, onde $c=P(K=n)$. A tabela 4.12 apresenta, para cada valor de $c$ fixado, a estimativa da média e mediana de cada parâmetro e a tabela 4.13 a distribuição de freqüências à posteriori do ponto de mudança $\mathrm{k}$.

A tabela 4.14 apresenta também, para cada valor de c fixado, a estimativa da média de cada parâmetro primeiramente quando o conjunto de dados é gerado apartir do modelo com mudança no ponto $k=7$ e depois apartir do modelo com mudança em $k=43\left(\right.$ ambos com $\underset{\sim}{\theta_{1}}=(2.0,1.0,2.0,0.6,0.1)^{T}$ e $\left.\underset{\sim}{\underset{2}{*}}=(1.0,2.0,4.0,1.2,0.2)^{T}\right)$ e finalmente apartir do modelo sem nenhuma mudança $(k=50)$.

Os pontos iniciais das seqüências de Gibbs, em cada caso, foram obtidos pelo mesmo método usado anteriormente para o caso $\mathrm{k}=20$.

\section{Observações:}

- O algoritmo usado nessa seção pode não funcionar (convergir) dependendo dos valores iniciais. Para ilustrar fizemos a seguinte experiência: geramos 50 conjuntos de dados, cada um a partir do modelo com um valor de $k$ diferente $(1 \leq$ $k \leq 50)$ e $\underset{\sim}{\theta_{1}}$ e ${\underset{\theta}{2}}_{2}$ fixados $\left(\underset{\sim}{\theta_{1}}=(2.0,1.0,2.0,0.6,0.1)\right.$ e ${\underset{\sim}{\theta}}_{2}=(1.0,2.0,4.0,1.2,0.2))$. Para cada um desses conjuntos de dados, o algoritmo foi rodado partindo-se de pontos iniciais distintos da forma $(k^{(0)}, \underbrace{(0) T}_{1},{\underset{\theta}{2}}_{2}^{(0) T})$, onde $3 \leq k^{(0)} \leq 47 \mathrm{e}$ ${\underset{\theta}{1}}_{1}^{(0)}$ e ${\underset{\sim}{2}}_{2}^{(0)}$ são os estimadores de máximaveross. de $\underset{\sim}{\theta_{1}}$ e ${\underset{\sim}{2}}_{2}$ dado $k=k^{(0)}$.

Observou-se que se o valor verdadeiro de $k$ está próximo de $\frac{n}{2}$, então partindose de qualquer ponto inicial $\left(k^{(0)},{\underset{\sim}{1}}_{1}^{(0) T},{\underset{\sim}{2}}_{2}^{(0) T}\right)$ descrito acima, o algorítmo tende 
Tabela 4.12: Estimativas do valor esperado e da mediana dos parâmetros da distribuição à posteriori quando diferentes valores do hiperparâmetro $\mathrm{c}$, onde $\mathrm{c}=P(K=n)$, são fixados à priori. Os valores verdadeiros dos parâmetros são: $k=20, \alpha_{1}=2.0, \beta_{1}=1.0, \mu_{1}=2.0$, $\sigma_{x(1)}^{2}=0.6, \sigma_{1}^{2}=0.1, \alpha_{2}=1.0, \beta_{2}=2.0, \mu_{2}=4.0, \sigma_{x(2)}^{2}=1.2, \sigma_{2}^{2}=0.2$.

\begin{tabular}{|c|c|c|c|c|c|c|c|c|}
\hline \multirow[b]{2}{*}{$k$} & \multicolumn{2}{|c|}{$\begin{array}{c}c=0.02 \\
\text { media mediana }\end{array}$} & \multicolumn{2}{|c|}{$\begin{array}{c}c=0.5 \\
\text { media mediana }\end{array}$} & \multicolumn{2}{|c|}{$\begin{array}{c}c=0.95 \\
\text { media mediana }\end{array}$} & \multicolumn{2}{|c|}{$\begin{array}{c}c=0.99 \\
\text { media mediana }\end{array}$} \\
\hline & 20.97 & 21 & 20.97 & 21 & 20.97 & 21 & 20.97 & 21 \\
\hline $\begin{array}{l}\alpha_{1} \\
\alpha_{2}\end{array}$ & $\begin{array}{l}1.8140 \\
0.8539\end{array}$ & $\begin{array}{l}1.8645 \\
0.9417\end{array}$ & $\begin{array}{l}1.8140 \\
0.8539\end{array}$ & $\begin{array}{l}1.8645 \\
0.9417\end{array}$ & $\begin{array}{l}1.8147 \\
0.8540\end{array}$ & $\begin{array}{l}1.8645 \\
0.9417\end{array}$ & $\begin{array}{l}1.8147 \\
0.8540\end{array}$ & $\begin{array}{l}1.8645 \\
0.9417\end{array}$ \\
\hline $\begin{array}{l}\beta_{1} \\
\beta_{2}\end{array}$ & $\begin{array}{l}1.0500 \\
2.0720\end{array}$ & $\begin{array}{l}1.0250 \\
2.0514\end{array}$ & $\begin{array}{l}1.0500 \\
2.0720\end{array}$ & $\begin{array}{l}1.0250 \\
2.0514\end{array}$ & $\begin{array}{l}1.0504 \\
2.0721\end{array}$ & $\begin{array}{l}1.0250 \\
2.0514\end{array}$ & $\begin{array}{l}1.0504 \\
2.0721\end{array}$ & $\begin{array}{l}1.0250 \\
2.0514\end{array}$ \\
\hline $\begin{array}{l}\mu_{1} \\
\mu_{2}\end{array}$ & $\begin{array}{l}2.2357 \\
3.8581\end{array}$ & $\begin{array}{l}2.2361 \\
3.8578\end{array}$ & $\begin{array}{l}2.2357 \\
3.8581\end{array}$ & $\begin{array}{l}2.2361 \\
3.8578\end{array}$ & $\begin{array}{l}2.2357 \\
3.6567\end{array}$ & $\begin{array}{l}2.2361 \\
3.8578\end{array}$ & $\begin{array}{l}2.2357 \\
3.8587\end{array}$ & $\begin{array}{l}2.2361 \\
3.8578\end{array}$ \\
\hline $\begin{array}{l}\sigma_{x_{1}}^{2} \\
\sigma_{x_{2}}^{2}\end{array}$ & $\begin{array}{l}0.4254 \\
1.3662\end{array}$ & $\begin{array}{l}0.3920 \\
1.3003\end{array}$ & $\begin{array}{l}0.4254 \\
1.3662\end{array}$ & $\begin{array}{l}0.3920 \\
1.3003\end{array}$ & $\begin{array}{l}0.4242 \\
1.3670\end{array}$ & $\begin{array}{l}0.3920 \\
1.3003\end{array}$ & $\begin{array}{l}0.4242 \\
1.3670\end{array}$ & $\begin{array}{l}0.3920 \\
1.3003\end{array}$ \\
\hline $\begin{array}{l}\sigma_{1}^{2} \\
\sigma_{2}^{2}\end{array}$ & $\begin{array}{l}0.2155 \\
0.2412\end{array}$ & $\begin{array}{l}0.2030 \\
0.2304\end{array}$ & $\begin{array}{l}0.2155 \\
0.2412\end{array}$ & $\begin{array}{l}0.2030 \\
0.2304\end{array}$ & $\begin{array}{l}0.2159 \\
0.2410\end{array}$ & $\begin{array}{l}0.2030 \\
0.2304\end{array}$ & $\begin{array}{l}0.2159 \\
0.2410\end{array}$ & $\begin{array}{l}0.2030 \\
0.2304\end{array}$ \\
\hline
\end{tabular}

Tabela 4.13: Distribuição de frequiências à posteriori do ponto de mudança $k$ quando diferentes valores do hiperparâmetro $c$ são fixados.

\begin{tabular}{c|cccc}
\hline$k$ & $c=0.02$ & $c=0.5$ & $c=0.95$ & $c=0.99$ \\
\hline 18 & 0.0001 & 0.0001 & 0.0001 & 0.0001 \\
19 & 0.0019 & 0.0019 & 0.0019 & 0.0019 \\
20 & 0.0250 & 0.0250 & 0.0250 & 0.0250 \\
21 & 0.9729 & 0.9729 & 0.9729 & 0.9729 \\
outros & 0.0000 & 0.0000 & 0.0000 & 0.0000 \\
\hline
\end{tabular}

a convergir. Entretanto quando o verdadeiro valor de $k$ está próximo de 1 ou n, o algoritmo pode não convergir dependendo do ponto inicial $\left(k^{(0)}, \underset{\sim}{(0) T},{\underset{\sim}{\theta}}_{2}^{(0) T}\right)$.

Esse algoritmo aparentemente tem uma certa tendência de retornar ao estado $k=n$ (embora este não seja um ponto absorvente)se alguma vez ele entrou nesse estado, a não ser que o valor de $c(c=P(K=n)=p(n))$ à priori seja bem pequeno. Uma saida foi tomar como valor inicial de $k$ o menor valor possível $\left(k^{(0)}=3\right)$, e tomar ${\underset{\sim}{1}}_{1}^{(0)}$ e ${\underset{\sim}{\theta}}_{2}^{(0)}$ como sendo os estimadores de maximaverossimilhança de ${\underset{\sim}{1}}_{1}$ e $\theta_{2}$ respectivamente. Uma outra saida é tentar utilizar algum método para obter bons valores iniciais como por exemplo a 
Tabela 4.14: Estimativas do valor esperado dos parâmetros da distribuição à posteriori quando diferentes conjuntos de dados são utilizados (simulados a partir do modelo com $k=7, k=43$ e $k=50$ ), quando diferentes valores de c são utilizados ( $\mathrm{c}=0.02$, $c=0.5$ e $c=0.99$ ).

\begin{tabular}{|c|c|c|c|c|c|c|c|c|c|}
\hline & $c=0$ & $\begin{array}{l}k=7 \\
c=0.5\end{array}$ & $=0$ & 02 & $\begin{array}{l}k=43 \\
c=05\end{array}$ & & $=0,02$ & $\begin{array}{l}\mathrm{k}=50 \\
\mathrm{c}=05\end{array}$ & \\
\hline$\alpha_{1}$ & & & & 2.1052 & 2.1052 & 2.1052 & 2.0357 & $\begin{array}{l}c=0.0 \\
2.0245\end{array}$ & 2.0245 \\
\hline$\alpha_{2}$ & & 1.4291 & 1400 & 0.6667 & 0.6667 & 0.6667 & 2.0357 & $\overline{2} .0245$ & 2.0245 \\
\hline & 428 & 1.1428 & .1428 & 0.9904 & 0.9904 & 0.9904 & 1.0049 & 1.0104 & 1.010 \\
\hline & & 1.8845 & .8845 & 2.0705 & 2.0705 & & & & \\
\hline & & & & & & 1.00 & 1.9 & .9 & .9217 \\
\hline$\mu_{2}$ & 3.8306 & 3.8306 & 3.8306 & 3.6363 & 3.6363 & 3.6363 & 1.9215 & 1.9217 & 1.9217 \\
\hline$\sigma_{x_{1}}^{2}$ & 0.4980 & 0.4980 & 0.4980 & 0.6334 & 0.6334 & 0.6334 & 0.6021 & 0.6000 & 0.6000 \\
\hline$\sigma_{x_{2}}^{2^{+}}$ & 1.4209 & 1.4209 & 1.4209 & 1.5202 & 1.5202 & 1.5202 & 0.6021 & 0.6000 & 0.6000 \\
\hline$\sigma_{1}^{2}$ & 0.3831 & 0.3831 & 0.3831 & 0.1449 & 0.1449 & 0.1449 & 0.1 & 0.1179 & 0.1179 \\
\hline$\sigma_{2}^{2}$ & 0.1823 & 0.1823 & 0.1823 & 0.3746 & 0.3746 & 0.3746 & 0.1181 & 0.1179 & 0.1179 \\
\hline$k$ & 6.7099 & 6.7099 & 6.7095 & & 42.944 & 42.944 & 49.999 & 50.000 & 50.000 \\
\hline
\end{tabular}

técnica apresentada na seção 4.7.1 (vale lembrar entretanto que esta técnica não funcionou nesse exemplo quando o verdadeiro valor de $k$ é 5).

- Na tabela 4.14 observa-se que se o verdadeiro valor de $k$ é 7 , então a maioria dos parâmetros antes da mudança tende a ter estimativas mais pobres (em termos do viés) do que a maioria dos parâmetros depois da mudança. Semelhantemente quando $k=43$, os parâmetros após a mudança são pior estimados do que os parâmetros antes da mudança. Isso já era esperado pelo fato de que no primeiro caso o tamanho da amostra antes da mudança é pequeno e no segundo caso o tamanho da amostra depois da mudança é que é pequeno, além disso a distribuição à priori é pouco informativa (embora $k$ tenha uma distribuição de probabilidade discreta com domínio limitado, as distribuições $\pi\left(\underline{\sim}_{1}\right)$ e $\pi\left(\underline{\theta}_{2}\right)$ são muito pouco informativas).

- Os dados das tabelas 4.12 a 4.14 sugerem que as estimativas dos valores esperados dos parâmetros da distr. à posteriori, bem como a distrib. de freqüências à posteriori do ponto de mudança $k$, não são sensíveis a variação no valor hiperparâmetro $c$, onde c é a probabilidade à priori de não existir mudança. 


\subsubsection{Análise do procedimento 2 para detetar mudança ba- seada na distribuição à posteriori das diferenças (ou quocientes)}

\section{Caso (a): Modelo com restrição de identificabilidade}

Nessa seção vamos considerar inicialmente o modelo com restrição de identificabilidade e que permite mudança em todos os parâmetros definido na seção 4.7.1. (É importante lembrar que no caso em que $\alpha_{1}=\alpha_{2}, \beta_{1}=\beta_{2}, \mu_{1}=\mu_{2}, \sigma_{x_{1}}^{2}=\sigma_{x_{2}}^{2}$ e $\sigma_{1}^{2}=\sigma_{2}^{2}$ então caimos no modelo sem mudança, independentemente do valor de $\mathrm{k}$ )

Nesse estudo foram usados dados simulados e a distribuição à priori escolhida (dada na secão 4.7.1) é muito pouco informativa. Então é razoável esperar, se de fato o método utilizado é adequado, que as estimativas à posteriori (bem como os resultados e conclusões) sejam compatíveis com o modelo apartir do qual os dados são gerados.

As figuras 4.30 e 4.31 apresentam os histogramas das densidades marginais à posteriori de $\alpha_{i}, \beta_{i}, \mu_{i}, \sigma_{x_{i}}^{2}, \sigma_{i}^{2}$ e $k$ para $i=1,2$ e também os histogramas das diferenças $\alpha_{1}-\alpha_{2}, \beta_{2}-\beta_{1}, \mu_{2}-\mu_{1}$ e dos quocientes $\sigma_{x_{2}}^{2} / \sigma_{x_{1}}^{2}$ e $\sigma_{2}^{2} / \sigma_{1}^{2}$. Os cálculos são obtidos com base nos dados da tabela 4.1 e o algorítmo usado para amostrar da distribuição à posteriori é o M.Collapsed Gibbs (partindo do ponto inicial $\stackrel{\bullet}{(0)}^{(0)}$ dado na seção 4.7.1).

A partir dessas distribuições as seguintes estimativas foram obtidas: $\hat{P}\left(\alpha_{1}-\alpha_{2}>\right.$ $\left.0 \mid D_{\text {obs }}\right) \approx 0.842, \hat{P}\left(\beta_{2}-\beta_{1}>0 \mid D_{\text {obs }}\right) \approx 0.996, \hat{P}\left(\mu_{2}-\mu_{1}>0 \mid D_{\text {obs }}\right) \approx 0.993$, $\hat{P}\left(\sigma_{x_{2}}^{2} / \sigma_{x_{1}}^{2}>1 \mid D_{\text {obs }}\right) \approx 1.000$ e $\hat{P}\left(\sigma_{2}^{2} / \sigma_{1}^{2}>1 \mid D_{\text {obs }}\right) \approx 0.625$.

Esses resultados sugerem que todos os parâmetros do modelo mudam após o ponto $k=21$, o que está compatível com o esperado. 

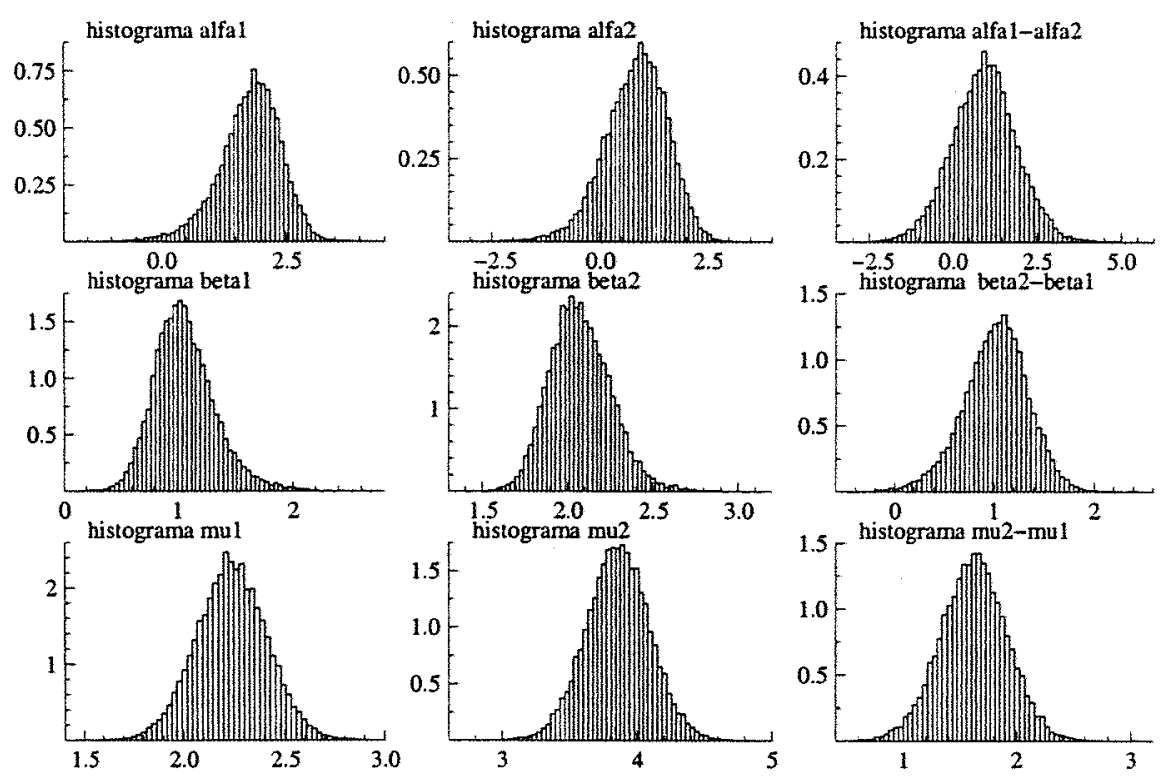

Figura 4.30: Histograma das densidades marginais à posteriori de $p\left(\alpha_{i} \mid D_{\text {obs }}\right), p\left(\beta_{i} \mid D_{\text {obs }}\right)$, $p\left(\mu_{i} \mid D_{\text {obs }}\right), i=1,2$ e $p\left(\alpha_{1}-\alpha_{2} \mid D_{\text {obs }}\right), p\left(\beta_{2}-\beta_{1} \mid D_{\text {obs }}\right), p\left(\mu_{2}-\mu_{1} \mid D_{\text {obs }}\right)$.
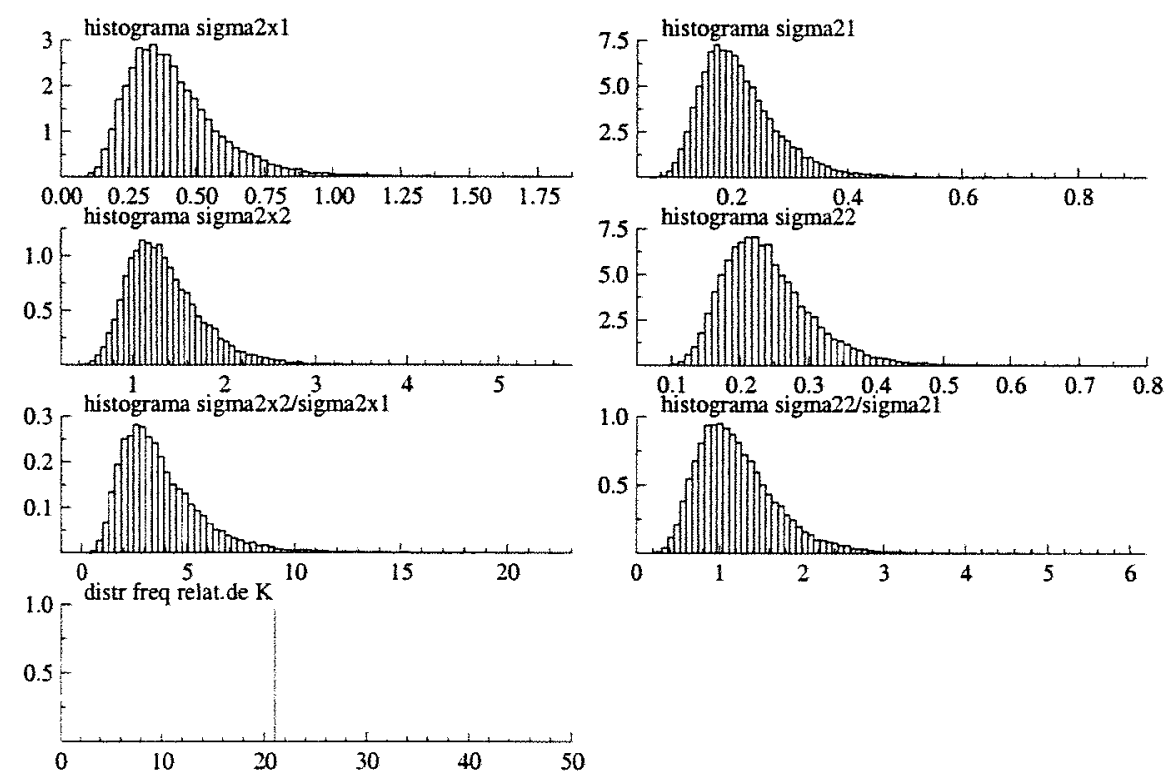

Figura 4.31: Histograma das densidades marginais à posteriori de $p\left(\sigma_{x_{1}}^{2} \mid D_{\mathrm{obs}}\right)$, $p\left(\sigma_{x_{2}}^{2} \mid D_{\text {obs }}\right)$ e $p\left(\sigma_{x_{2}}^{2} / \sigma_{x_{1}}^{2} \mid D_{\text {obs }}\right)$ e também de $p\left(\sigma_{1}^{2} \mid D_{\text {obs }}\right), p\left(\sigma_{2}^{2} \mid D_{\text {obs }}\right)$ e $p\left(\sigma_{2}^{2} / \sigma_{1}^{2} \mid D_{\text {obs }}\right)$. 
Observações: O mesmo modelo (modelo estrutural com restrição de identificabilidade e que permite mudança em todos os parâmetros com distribuição à priori dada em (4.7.1)) foi ajustado a outros dois conjuntos de dados. O primeiro conjunto foi gerado a partir do modelo (com restr. identif.) e sem nenhuma mudança (onde $\alpha_{1}=\alpha_{2}=2.0, \beta_{1}=\beta_{2}=1.0, \mu_{1}=\mu_{2}=2.0, \sigma_{x_{1}}^{2}=\sigma_{x_{2}}^{2}=0.6, \sigma_{1}^{2}=\sigma_{2}^{2}=0.1 \mathrm{e}$ $\lambda=1.0$ ), o segundo conjunto foi gerado a partir do modelo (com restr. identif.) com mudança apenas na média e na variância de $x$ (onde $\alpha_{1}=\alpha_{2}=2.0, \beta_{1}=\beta_{2}=1.0$, $\mu_{1}=2.0, \mu_{2}=4.0, \sigma_{x_{1}}^{2}=0.6, \sigma_{x_{2}}^{2}=2.2, \sigma_{1}^{2}=\sigma_{2}^{2}=0.1$ e $\lambda=1.0$ ).

Nos dois casos o algoritmo M.Collapsed Gibbs partiu do ponto inicial $\left(k^{(0)}=25\right.$, $\left.\beta_{i}^{(0)}=1, \sigma_{i}^{2(0)}=1, \sigma_{x_{i}}^{2(0)}=1, i=1,2\right)$. Do primeiro ajuste obtivemos as estimativas: $\hat{P}\left(\alpha_{2}-\alpha_{1}>0 \mid D_{\text {obs }}\right) \approx 0.51, \hat{P}\left(\beta_{2}-\beta_{1}>0 \mid D_{\text {obs }}\right) \approx 0.50, \hat{P}\left(\mu_{2}-\mu_{1}>0 \mid D_{\text {obs }}\right) \approx$ $0.51, \hat{P}\left(\sigma_{x_{2}}^{2} / \sigma_{x_{1}}^{2}>1 \mid D_{\text {obs }}\right) \approx 0.53$ e $\hat{P}\left(\sigma_{2}^{2} / \sigma_{1}^{2}>1 \mid D_{\text {obs }}\right) \approx 0.64$. Nesse caso em geral as estimativas estão dentro do esperado, apenas a estimativa da probabilidade $P\left(\sigma_{2}^{2} / \sigma_{1}^{2}>1 \mid D_{\text {obs }}\right)$ aparentemente está um pouco mais discrepante (em relação ao valor 0.5$)$.

Do segundo ajuste observamos que $\hat{P}\left(\alpha_{1}-\alpha_{2}>0 \mid D_{\text {obs }}\right) \approx 0.62, \hat{P}\left(\beta_{1}-\beta_{2}>\right.$ $\left.0 \mid D_{\text {obs }}\right) \approx 0.57, \hat{P}\left(\mu_{2}-\mu_{1}>0 \mid D_{\text {obs }}\right) \approx 1.00, \hat{P}\left(\sigma_{x_{2}}^{2} / \sigma_{x_{1}}^{2}>1 \mid D_{\text {obs }}\right) \approx 1.00 \mathrm{e}$ $\hat{P}\left(\sigma_{1}^{2} / \sigma_{2}^{2}>1 \mid D_{\text {obs }}\right) \approx 0.86$. Esses resultados não sugerem que a mudança ocorre somente na média e na variância de $x$ (o que não estaria compatível com o esperado).

Nesses particulares exemplos, vemos que, embora o método pode não detectar corretamente qual parâmetro está mudando (no modelo que permite mudança em todos os parâmetros), ele forneceu resultado mais ou menos razoável no caso em que não há mudança.

\section{Caso (b) Modelo sem restrição de identificabilidade}

Nesse item vamos usar procedimento semelhante ao do caso (a) porém agora 

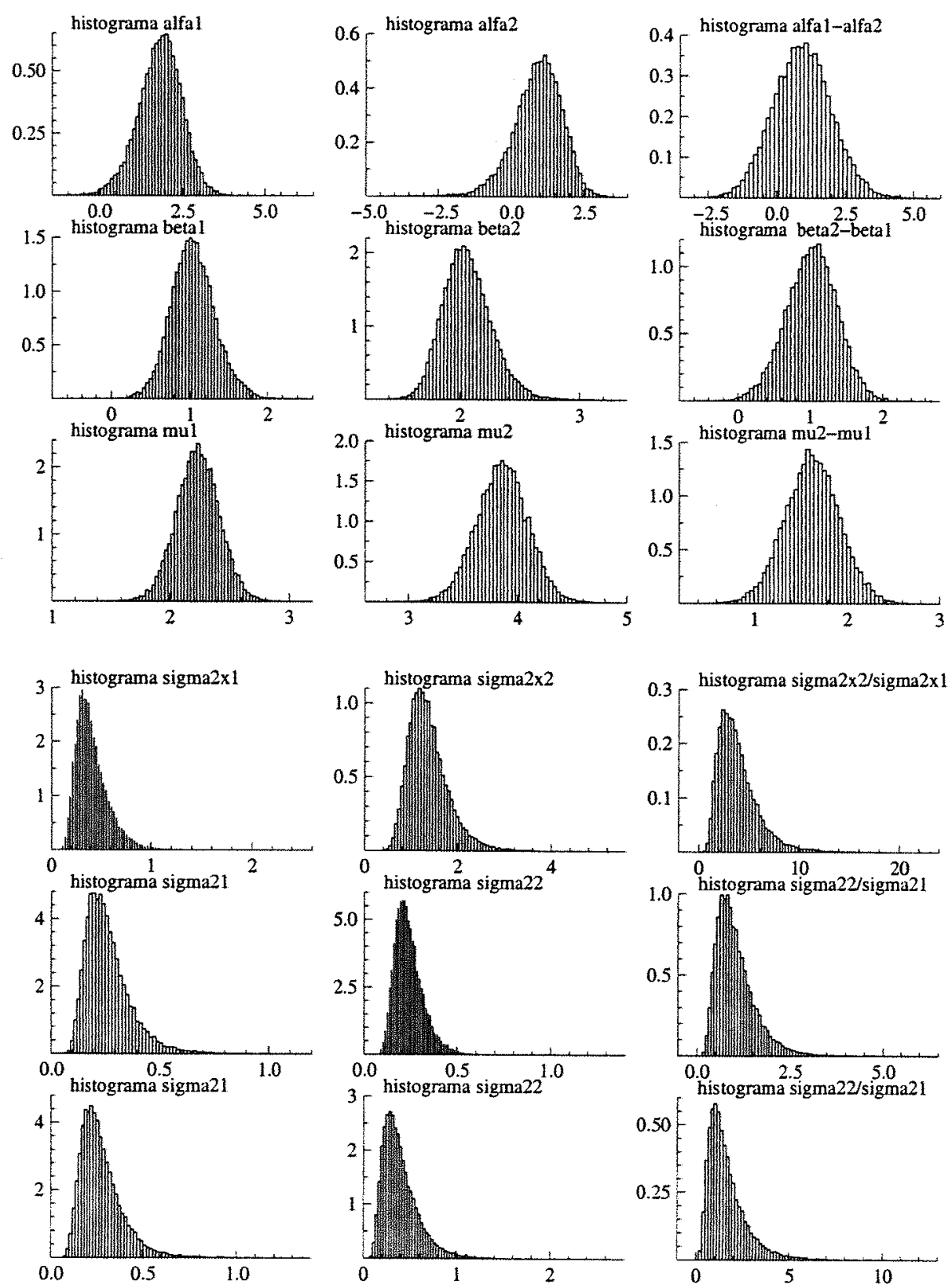

Figura 4.32: Histograma das densidades marginais à posteriori de $p\left(\alpha_{i} \mid D_{\mathrm{obs}}\right), p\left(\beta_{i} \mid D_{\mathrm{obs}}\right)$, $p\left(\mu_{i} \mid D_{\text {obs }}\right), p\left(\sigma_{x_{1}}^{2} \mid D_{\text {obs }}\right), p\left(\sigma_{i}^{2} \mid D_{\text {obs }}\right), p\left(\sigma_{e_{i}}^{2} \mid D_{\text {obs }}\right), p\left(K=k \mid D_{\text {obs }}\right)$ para $i=1,2, \mathrm{e}$, $p\left(\alpha_{1}-\alpha_{2} \mid D_{\mathrm{obs}}\right), p\left(\beta_{2}-\beta_{1} \mid D_{\mathrm{obs}}\right), p\left(\mu_{2}-\mu_{1} \mid D_{\mathrm{obs}}\right), p\left(\sigma_{x_{2}}^{2} / \sigma_{x_{1}}^{2} \mid D_{\mathrm{obs}}\right), p\left(\sigma_{2}^{2} / \sigma_{1}^{2} \mid D_{\mathrm{obs}}\right)$, $p\left(\sigma_{e_{2}}^{2} / \sigma_{e_{1}}^{2} \mid D_{\mathrm{obs}}\right)$. 
para o modelo sem restrição de identificabilidade e que permite mudança em todos os parâmetros definido na seção 4.7.2.

A figura 4.32 apresenta os histogramas das densidades marginais à posteriori de $\alpha_{i}, \beta_{i}, \mu_{i}, \sigma_{x_{i}}^{2}, \sigma_{e_{i}}^{2}, \sigma_{i}^{2}$ e $k$ e também os histogramas das diferenças $\alpha_{1}-\alpha_{2}, \beta_{2}-\beta_{1}$, $\mu_{2}-\mu_{1}$ e dos quocientes $\sigma_{x_{2}}^{2} / \sigma_{x_{1}}^{2}, \sigma_{e_{2}}^{2} / \sigma_{e_{1}}^{2}$ e $\sigma_{2}^{2} / \sigma_{1}^{2}$. Os cálculos foram obtidos com base nos dados da tabela 4.1 e o algorítmo usado para amostrar da distribuição à posteriori é o M.Collapsed Gibbs (o ponto inicial usado é o ${\underset{\sim}{*}}^{(0)}$ dado na seção 4.7.2).

A partir das distribuições das diferenças e quocientes acima obtivemos as seguintes estimativas : $\hat{P}\left(\alpha_{1}-\alpha_{2}>0 \mid D_{\text {obs }}\right) \approx 0.808, \hat{P}\left(\beta_{2}-\beta_{1}>0 \mid D_{\text {obs }}\right) \approx 0.997, \hat{P}\left(\mu_{2}-\mu_{1}>\right.$ $\left.0 \mid D_{\text {obs }}\right) \approx 1.000, \hat{P}\left(\sigma_{x_{2}}^{2} / \sigma_{x_{1}}^{2}>1 \mid D_{\text {obs }}\right) \approx 0.994, \hat{P}\left(\sigma_{e_{1}}^{2} / \sigma_{e_{2}}^{2}>1 \mid D_{\text {obs }}\right) \approx 0.559$ $\hat{P}\left(\sigma_{2}^{2} / \sigma_{1}^{2}>1 \mid D_{\text {obs }}\right) \approx 0.706$.

Os resultados acima sugerem que há mudança em todos os parâmetros do modelo (o que está dentro do esperado).

\section{Observações:}

O modelo sem restrição foi ajustado ainda a outros dois conjuntos de dados (o primeiro gerado a partir do modelo sem mudança (com $\alpha_{1}=\alpha_{2}=2.0, \beta_{1}=\beta_{2}=1.0$, $\mu_{1}=\mu_{2}=2.0, \sigma_{x_{1}}^{2}=\sigma_{x_{2}}^{2}=0.6, \sigma_{1}^{2}=\sigma_{2}^{2}=0.1$ e $\left.\sigma_{e_{1}}^{2}=\sigma_{e_{2}}^{2}=0.1\right)$ ) e o outro gerado a partir do modelo com mudança na média e na variância de $x$ (com $\alpha_{1}=\alpha_{2}=2.0$, $\beta_{1}=\beta_{2}=1.0, \mu_{1}=2.0, \mu_{2}=4.0, \sigma_{x_{1}}^{2}=0.6, \sigma_{x_{2}}^{2}=2.2, \sigma_{1}^{2}=\sigma_{2}^{2}=0.1 \mathrm{e}$ $\sigma_{e_{1}}^{2}=\sigma_{e_{2}}^{2}=0.1$ ). Os pontos iniciais em cada exemplo foram obtidos pelo mesmo procedimento descrito na seção 4.7.2.

Do primeiro ajuste obtivemos as estimativas: $\hat{P}\left(\alpha_{2}-\alpha_{1}>0 \mid D_{\text {obs }}\right) \approx 0.53, \hat{P}\left(\beta_{2}-\right.$ $\left.\beta_{1}>0 \mid D_{\text {obs }}\right) \approx 0.53, \hat{P}\left(\mu_{2}-\mu_{1}>0 \mid D_{\text {obs }}\right) \approx 0.59, \hat{P}\left(\sigma_{x_{1}}^{2} / \sigma_{x_{2}}^{2}>1 \mid D_{\text {obs }}\right) \approx 0.51$, $\hat{P}\left(\sigma_{1}^{2} / \sigma_{2}^{2}>1 \mid D_{\text {obs }}\right) \approx 0.64, \hat{P}\left(\sigma_{\varepsilon_{1}}^{2} / \sigma_{\varepsilon_{2}}^{2}>1 \mid D_{\text {obs }}\right) \approx 0.64$. Nesse caso os primeiros quatro valores estão dentro do esperado entretanto os dois últimos valores estão um pouco acima do esperado. 
Do segundo ajuste obtivemos: $\hat{P}\left(\alpha_{2}-\alpha_{1}>0 \mid D_{\text {obs }}\right) \approx 0.54, \hat{P}\left(\beta_{1}-\beta_{2}>0 \mid D_{\text {obs }}\right) \approx$ $0.51, \hat{P}\left(\mu_{2}-\mu_{1}>0 \mid D_{\text {obs }}\right) \approx 0.99, \hat{P}\left(\sigma_{x_{2}}^{2} / \sigma_{x_{1}}^{2}>1 \mid D_{\text {obs }}\right) \approx 0.99, \hat{P}\left(\sigma_{1}^{2} / \sigma_{2}^{2}>1 \mid D_{\text {obs }}\right) \approx$ $0.74 \hat{P}\left(\sigma_{e_{1}}^{2} / \sigma_{e_{2}}^{2}>1 \mid D_{\text {obs }}\right) \approx 0.75$. Esses resultados não sugerem que a mudança ocorre somente na média e na variância de $x$ (o que não está compatível com o esperado).

\section{Observação:}

Dos resultados acima sobre os particulares exemplos, vemos que o método de examinar as distribuições das diferenças ou quocientes dos parâmetros pode não detectar corretamente qual parâmetro está (ou não) mudando. 


\subsubsection{Análise do procedimento 3 para detetar mudança baseada nos critérios de seleção de modelos.}

\section{Uso de critérios de escolha de modelos em modelos identificáveis}

A tabela 4.15 a seguir contém as estimativas do Fator de Bayes e Pseudo-Fator de Bayes para comparar dois modelos com restrição de identificabilidade: o modelo que permite mudança em todos os parâmetros (estudado na seção 4.7.1) e o modelo sem nenhuma mudança (estudado na seção 2.12.1), quando os dados observados são os da tabela 4.1. Além disso contém as estimativas da Medida L e da soma das normas dos resíduos padronizados sob os dois modelos. Os cálculos envolvendo cada modelo foram obtidos sempre com base na segunda metade de uma seqüência de comprimento $\mathrm{T}=10000$ do algorítmo M.Collapsed Gibbs (partindo de um único ponto inicial, $\stackrel{*}{d}_{1}^{(0)}$, obtido pelo método descrito na seção 4.7.1).

Tabela 4.15: Estimativa do Fator de Bayes (pelo método de Newton e Raftery), do Pseudo-Fator de Bayes, da Medida L e da soma das normas dos resíduos sob cada um dos modelos, com e sem restrição de identificabilidade (utilizando os dados da tabela 4.1).

\begin{tabular}{|c|c|c|c|}
\hline medidas & $\begin{array}{l}\text { modelo com } \\
\text { mudança }\end{array}$ & $\begin{array}{l}\text { modelo sem } \\
\text { mudança }\end{array}$ & \\
\hline $\begin{aligned} \operatorname{det}(\text { L2) } & \delta=0.0 \\
\delta & =0.1 \\
\delta & =0.5 \\
\delta & =0.9 \\
\delta & =1.0\end{aligned}$ & $\begin{array}{l}3427.99 \\
3986.91 \\
6628.24 \\
9918.61 \\
10842.60\end{array}$ & $\begin{array}{l}7764.21 \\
9216.22 \\
16258.00 \\
25273.70 \\
27836.10\end{array}$ & \\
\hline $\begin{aligned} & \operatorname{tr}\left(L_{2}\right) \delta=0.0 \\
& \delta=0.1 \\
& \delta=0.5 \\
& \delta=0.9 \\
& \delta=1.0 \\
&\end{aligned}$ & $\begin{array}{l}256.63 \\
280.40 \\
375.46 \\
470.52 \\
494.29 \\
\end{array}$ & $\begin{array}{l}554.12 \\
607.91 \\
823.06 \\
1038.21 \\
1092.00 \\
\end{array}$ & \\
\hline $\begin{array}{l}\text { Soma das normas } \\
\text { dos resíduos }\end{array}$ & 92.47 & 104.87 & \\
\hline$\prod_{i=1}^{n} C P O_{i}^{M_{i}}$ & $5.16 \times 10^{-25}$ & $1.84 \times 10^{-33}$ & $\begin{array}{l}\text { Pseudo-Fator de } \\
\text { Bayes }=2.80 \times 10^{8}\end{array}$ \\
\hline$\hat{\pi}\left(U_{1}, \ldots, U_{n} \mid M_{i}\right)$ & $2.03 \times 10^{-40}$ & $1.60 \times 10^{-54}$ & $\begin{array}{c}\text { Fator de Bayes } \\
=1.27 \times 10^{14}\end{array}$ \\
\hline$\hat{k}$ & 20.97 & & \\
\hline
\end{tabular}

Na figura 4.33 temos um gráfico comparando os modelos com e sem mudança (com restrição de identificabilidade) por meio dos valores da densidade condicional 
preditiva (CPOi) versus $i$, quando os dados são os da tabela 4.1 e o algoritmo utilizado para amostrar das posterioris é o M.Collap.Gibbs.

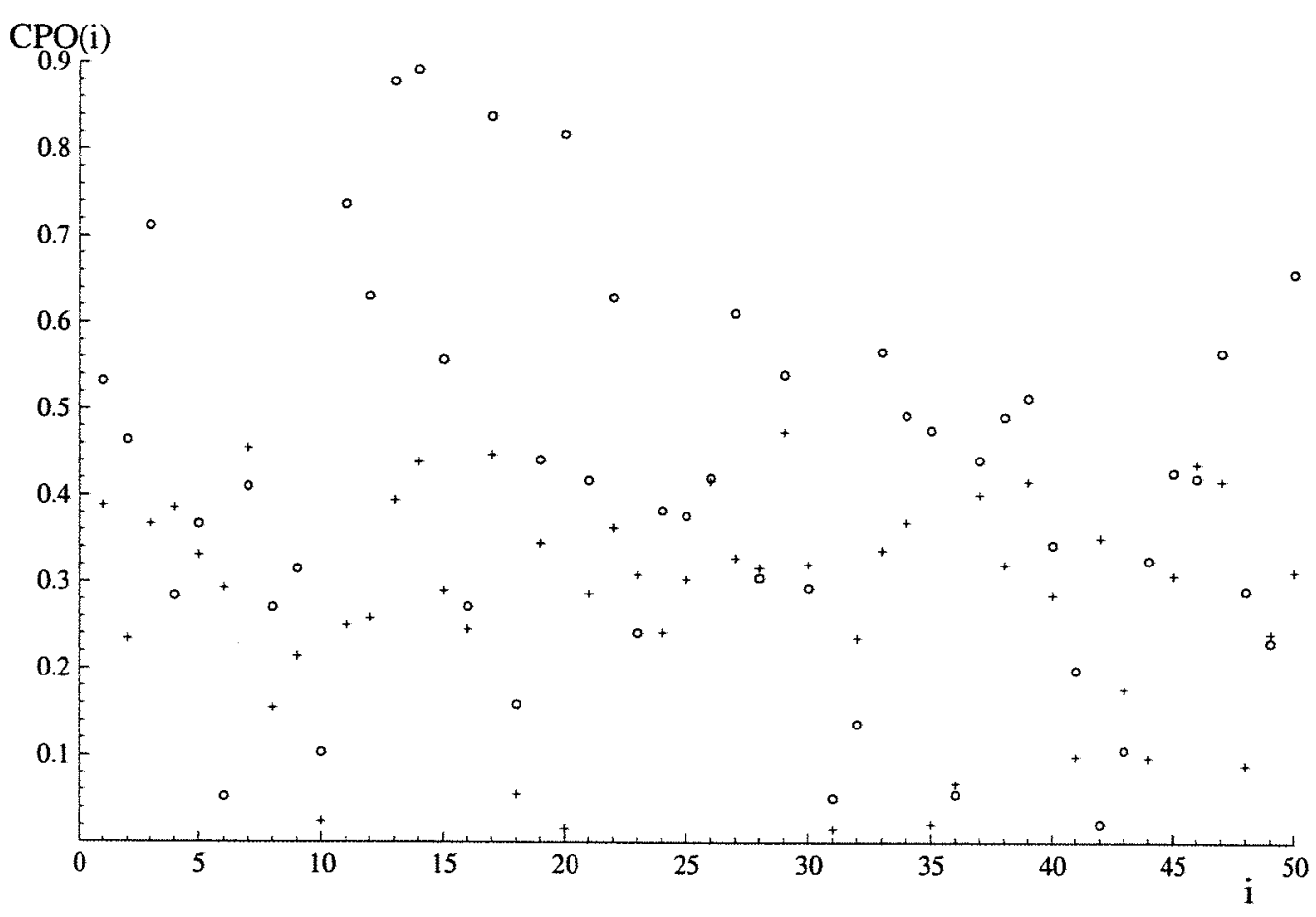

Figura 4.33: Valores da densidade condicional preditiva (CPO(i)) versus $i$, sob o modelo com mudança (símbolo o) e sob o modelo sem mudança (símbolo + ).

Uso de critérios de seleção de modelos em modelos sem restrição de identificabilidade

Da mesma forma que no caso anterior (com restrição de identificabilidade), os dados utilizados para ajustar cada um dos modelos foram os da tabela 4.1 e o algorítimo empregado foi o M.Collapsed Gibbs (partindo em cada caso de um único ponto inicial gerado pela técnica descrita na seção 4.7.2).

A tabela 4.16 descrita à seguir é semelhante à tabela 4.15 , porém agora envolvendo os modelos sem restrição de identificabilidade. Da mesma forma, a figura 4.34 é semelhante à figura 4.33 mas agora envolvendo os modelos sem restrição. 
Tabela 4.16: Estimativa do Fator de Bayes (pelo método de Newton e Raftery), do Pseudo-Fator de Bayes, da Medida L e da soma das normas dos resíduos sob cada um dos modelos (utilizando os dados da tabela 4.1).

\begin{tabular}{|c|c|c|c|}
\hline medidas & $\begin{array}{l}\text { modelo com } \\
\text { mudanca }\end{array}$ & $\begin{array}{l}\text { modelo sem } \\
\text { mudanca }\end{array}$ & \\
\hline $\begin{aligned} \operatorname{det}(L 2) & \delta=0.0 \\
\delta & =0.1 \\
\delta & =0.5 \\
\delta & =0.9 \\
\delta & =1.0\end{aligned}$ & $\begin{array}{l}4033.32 \\
4647.45 \\
7509.92 \\
11021.90 \\
12001.40\end{array}$ & $\begin{array}{l}8219.96 \\
9717.95 \\
16943.70 \\
26143.50 \\
28751.90\end{array}$ & \\
\hline $\begin{aligned} \operatorname{tr}\left(L_{2}\right) & \delta=0.0 \\
\delta & =0.1 \\
\delta & =0.5 \\
\delta & =0.9 \\
\delta & =1.0\end{aligned}$ & $\begin{array}{l}262.79 \\
286.54 \\
381.54 \\
476.54 \\
500.29\end{array}$ & $\begin{array}{l}555.08 \\
608.86 \\
824.02 \\
1039.17 \\
1092.96\end{array}$ & \\
\hline $\begin{array}{l}\text { Soma das normas } \\
\text { dos residuos }\end{array}$ & 92.38 & 109.90 & \\
\hline$\prod_{i=1}^{n} C P O_{i}^{M_{1}}$ & $3.04 \times 10^{-27}$ & $3.18 \times 10^{-34}$ & $\begin{array}{l}\text { Pseudo-Fator de } \\
\text { Baves }=9.6 \times 10^{6}\end{array}$ \\
\hline 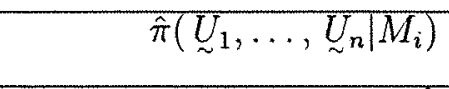 & $1.68 \times 10^{-40}$ & $3.56 \times 10^{-55}$ & $\begin{array}{l}\text { Fator de Bayes } \\
=4.7 \times 10^{14}\end{array}$ \\
\hline$\hat{k}$ & 20.93 & & \\
\hline
\end{tabular}

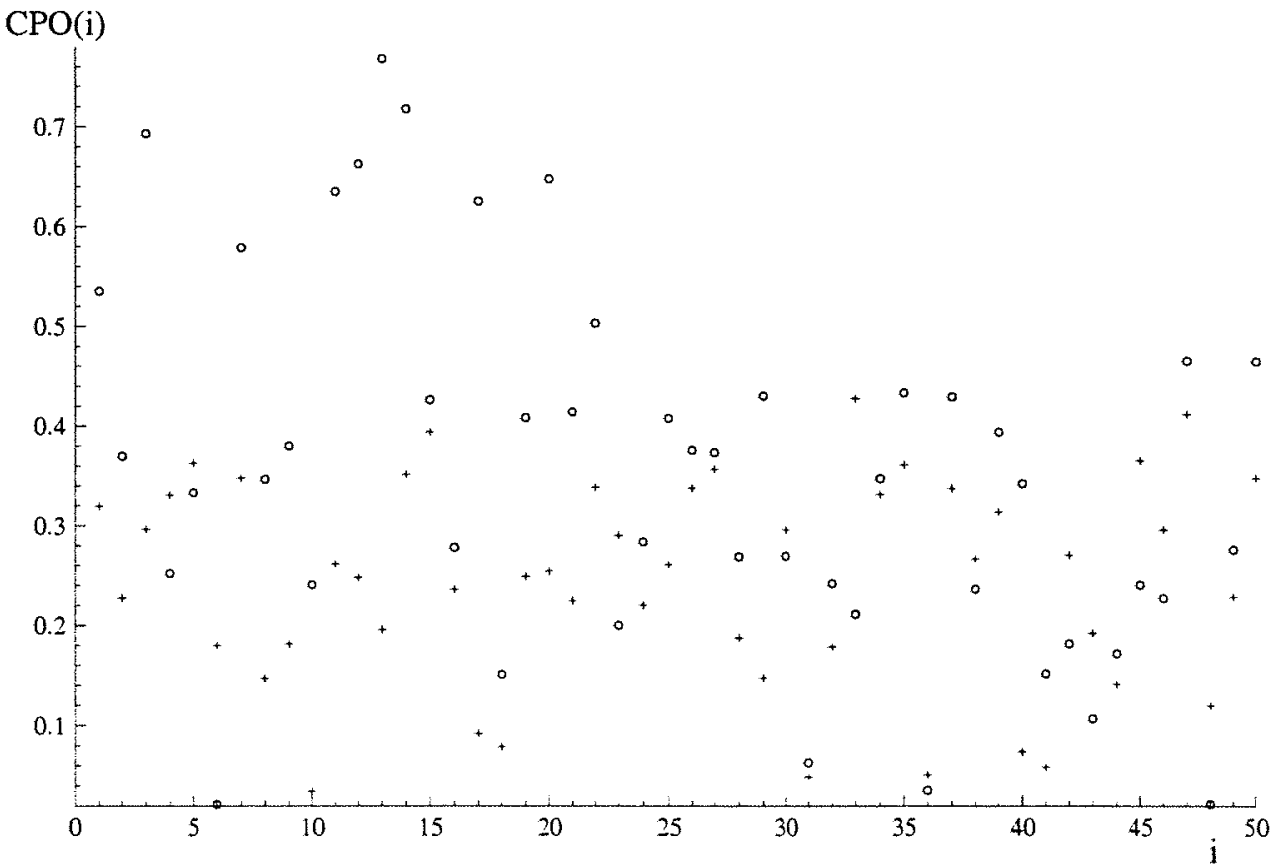

Figura 4.34: Valores da densidade condicional preditjva $(\mathrm{CPO}(\mathrm{i}))$ versus $i$, sob o modelo com mudança (o) e sob o modelo sem mudança( + ). 


\section{COMENTÁRIOS:}

Analisando as tabelas 4.15 e 4.16 e os gráficos 4.33 e 4.34, observamos que há uma forte evidência a favor do modelo que permite mudança em todos os parâmetros (do que o modelo sem nenhuma mudança) tanto no caso com restrição como no caso sem restrição de identificabilidade, ou seja existe evidência de que a hipótese nula (de que não há mudança) deve ser rejeitada nos dois casos.

Isso já era esperado pelo fato de que os dados da tabela 4.1 foram simulados apartir de um modelo onde todos os parâmetros mudam após a vigésima observação da seqüência de dados e a priori envolvida é muito pouco informativa.

É importante observar que os valores do Fator de Bayes e Pseudo-Fator de Bayes do exemplo (tabelas 4.15 e 4.16) nos pareceram extremamente grandes, além disso eles oscilam muito dependendo do algoritmo utilizado como também do ponto inicial escolhido. Nesse caso uma solução alternativa para o cálculo do Fator de Bayes, que deve ser tentada, é o método de Chib (1995) descrito na seção 4.4.3.

\subsubsection{Resumo dos resultados}

Na seção 4.7 basicamente estimamos os parâmetros dos modelos com e sem restrição de identificabilidade e que permitem mudança em todos os parâmetros após o ponto $k$. Ilustramos também a aplicação de três métodos para detectar mudança nesses modelos.

As observações a seguir referem-se apenas aos particulares exemplos apresentados nas subseções 4.7 .1 a 4.7 .5 .

(a)- Nos particulares exemplos tratados o algoritmo M.Collap. Gibbs teve um melhor desempemho que o algoritmo de Gibbs simples (principalmente em termos da função de autocorrelação e da matriz de correlações cruzadas).

Os dispositivos de diagnóstico de convergência apresentados nessa seção não 
sugerem que haja falhas na convergência dos algoritmos em nenhum dos exemplos. (b)- Nos modelos com e sem restrição de identificabilidade, os intervalos de credibilidade de $95 \%$ contiveram os verdadeiros valores dos parâmetros mas os comprimentos desses intervalos foram em geral ligeiramente menores no modelo com restrição. Entretanto, levando em conta que a qualidade do ajuste aparentemente não diferiu e que na prática dificilmente o valor de $\lambda$ é conhecido então seria bastante razoável escolher o modelo sem restrição de identificabilidade (quando os dados são os da tabela 4.1 e as priores são as descritas nas seções 4.7 .1 e 4.7.2).

Obs: As estimativas dos parâmetros nos modelos das seções 4.7.1 e 4.7.3 foram bastante semelhantes para o particular conjunto de dados.

(c)- No modelo que permite $k$ assumir o valor $n$ (com a particular distr. à priori dada na seção 4.7.3), as estimativas dos parâmetros da distribuição à posteriori, bem como a distribuição de freqüências à posteriori do ponto de mudança $k$, aparentemente não são sensiveis a variação do valor de c (onde c é a prob.à priori de não haver mudança), quando a amostra de dados é a da tabela 4.1.

(d)- O método 1, usado para detetar mudança, quando aplicado ao particular conjunto de dados da tabela 4.1 (e particular priori vaga da seção 4.7.3), detectou corretamente que a hipotese nula (de que nao há mudança) deve ser rejeitada e ao mesmo tempo forneceu as estimativas dos parâmetros do modelo.

O método 2 para detetar mudança, aparentemente não é muito preciso para detetar qual parâmetro está mudando, entretanto no particular exemplo envolvendo os dados da tabela 4.1 (e particular distr. à priori da seção 4.7.1), o método conseguiu detetar corretamente que todos os parâmetros mudam (tanto no modelo identificável como no modelo não identificável). Ele também forneceu a decisão correta no caso particular de um conjunto de dados gerados a partir de um modelo identificável sem 
nenhuma mudança (com a mesma distribuição à priori).

No método 3 (que usa os critérios de seleção de modelos do Fator de Bayes, Peudo-Fator de Bayes, Medida L e a soma dos módulos dos resíduos) vimos que todos esses critérios concordaram entre si (em rejeitar a hipótese $H_{0}$ de que não há mudança) no particular exemplo apresentado, independentemente do modelo ser ou não identificável.

É importante enfatizar que não estamos concluindo que os critérios de comparação aqui descritos são sempre adequados para detetar mudança e muito menos comparando os diversos critérios entre si, mas apenas exibindo os resultados para um particular conjunto de dados simulados. Um estudo mais completo deve ser conduzido futuramente com essa finalidade.

\section{Observações gerais:}

(1) As médias aritméticas e as médias R-Blackwelizadas ficaram sempre muito próximas independentemente do modelo estudado.

(2) A medida L baseada no traço aparentemente não serviu para diferenciar os modelos. 


\section{Capítulo 5}

\section{Uma análise do modelo linear estrutural $t$-Student com erros nas variáveis com um ponto de mudança desconhecido}

Neste capítulo faremos um estudo semelhante ao do capítulo 4, porém agora para os modelos estruturais $t$-independente e $t$-dependente com um ponto de mudança desconhecido, considerando a mesma restrição de identificabilidade utilizada no capítulo 4.

5.1 O modelo $t$ com erros independentes, com particular priori de componentes independentes

5.1.1 O modelo que permite mudança em todos os parâmetros após o ponto de mudança $k$

O modelo $t$-independente formulado como mistura de escala da distribuição Normal é definido a seguir:

$$
\left\{\begin{array}{l}
Y_{i}= \begin{cases}\alpha_{1}+\beta_{1} x_{i}+e_{i}, & i=1, \ldots, k, \text { onde } 1 \leq k \leq n-1, \\
\alpha_{2}+\beta_{2} x_{i}+e_{i}, & i=k+1, \ldots, n,\end{cases} \\
X_{i}=x_{i}+u_{i}, \quad i=1, \ldots, n
\end{array}\right.
$$


onde $Y_{i}$ e $X_{i}$ são as variáveis aleatórias observáveis, $x_{i}$ é variável aleatória latente e $e_{i}$ e $u_{i}$ são os erros aleatórios.

Seja $\underset{\sim}{Z}(1)=\left(\begin{array}{c}{\underset{\sim}{Z}}_{1} \\ \vdots \\ {\underset{\sim}{Z}}_{k}\end{array}\right)$ e $\underset{\sim}{Z}{ }^{(2)}=\left(\begin{array}{c}Z_{k+1} \\ \vdots \\ \underset{\sim}{Z}\end{array}\right)$, onde $\underset{\sim}{Z}{ }_{i}=\left(\begin{array}{c}Y_{i} \\ X_{i} \\ x_{i}\end{array}\right)$.

$\mathrm{O}$ modelo que vamos considerar tem a seguinte suposição:

$$
Z_{i} \mid \underset{\sim}{\stackrel{\text { indep }}{\sim}} \begin{cases}N_{3}\left(\tilde{\sim}_{1}, \sum_{1} w_{i}\right), & i=1, \ldots, k, \\ N_{3}\left(\underset{\sim}{\mu}, \sum_{2} w_{i}\right), & i=k+1, \ldots, n,\end{cases}
$$

onde

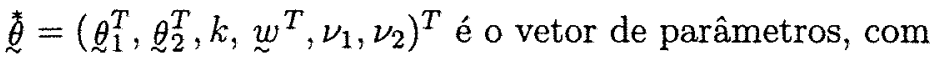

$$
\begin{aligned}
& \underline{\theta}_{i}=\left(\alpha_{i}, \beta_{i}, \mu_{i}, \sigma_{x(i)}^{2}, \sigma_{i}^{2}\right)^{T}, i=1,2, \underset{\sim}{w}=\left(w_{1}, \ldots, w_{n}\right)^{T},\left({\underset{\sim}{1}}_{1}^{T}, \nu_{1}\right) \neq\left(\underline{\theta}_{2}^{T}, \nu_{2}\right) \\
& \mu_{1}=\left(\begin{array}{c}
\alpha_{1}+\beta_{1} \mu_{1} \\
\mu_{1} \\
\mu_{1}
\end{array}\right), \quad \sum_{1}=\left(\begin{array}{lll}
\beta_{1} \sigma_{x(1)}^{2}+\lambda \sigma_{1}^{2} & \beta_{1} \sigma_{x(1)}^{2} & \beta_{1} \sigma_{x(1)}^{2} \\
\beta_{1} \sigma_{x(1)}^{2} & \sigma_{x(1)}^{2}+\sigma_{1}^{2} & \sigma_{x(1)}^{2} \\
\beta_{1} \sigma_{x(1)}^{2} & \sigma_{x(1)}^{2} & \sigma_{x(1)}^{2}
\end{array}\right)
\end{aligned}
$$

$\mathrm{e}$

$$
\mu_{2}=\left(\begin{array}{c}
\alpha_{2}+\beta_{2} \mu_{2} \\
\mu_{2} \\
\mu_{2}
\end{array}\right), \quad \Sigma_{2}=\left(\begin{array}{lll}
\beta_{2} \sigma_{x(2)}^{2}+\lambda \sigma_{2}^{2} & \beta_{2} \sigma_{x(2)}^{2} & \beta_{2} \sigma_{x(2)}^{2} \\
\beta_{2} \sigma_{x(2)}^{2} & \sigma_{x(2)}^{2}+\sigma_{2}^{2} & \sigma_{x(2)}^{2} \\
\beta_{2} \sigma_{x(2)}^{2} & \sigma_{x(2)}^{2} & \sigma_{x(2)}^{2}
\end{array}\right)
$$

$\nu_{1}=$ número de graus de liberdade antes da mudança

$\nu_{2}=$ número de graus de liberdade após a mudança $\left(\nu_{i}>2, i=1,2\right)$,

$\lambda$ é a razão das variâncias dos erros (conhecida) e $\sum_{i}>0, i=1,2$.

A distribuição à priori é dada por

$$
\pi(\stackrel{*}{\underset{\sim}{*}}) \propto \pi\left(k,{\underset{\sim}{1}}_{1}, \theta_{2}, \nu_{1}, \nu_{2}, \underset{\sim}{w}\right)=p(k) \pi\left({\underset{\sim}{1}}_{1}\right) \pi\left({\underset{\sim}{2}}_{2}\right) \pi\left(\underset{\sim}{w} \mid \nu_{1}, \nu_{2}\right) \pi\left(\nu_{1}\right) \pi\left(\nu_{2}\right),
$$

onde

$$
\begin{aligned}
& w_{i} \mid \nu_{1}, \nu_{2} \stackrel{\text { indep }}{\sim} \begin{cases}I G\left(\frac{\nu_{1}}{2}, \frac{\nu_{1}}{2}\right), & i=1, \ldots, k, \\
I G\left(\frac{\nu_{2}}{2}, \frac{\nu_{2}}{2}\right), & i=k+1, \ldots, n,\end{cases} \\
& p(k)= \begin{cases}P[K=k]=\frac{1}{\# \mathcal{K}}, & k \in \mathcal{K}, \\
0, & \text { caso contrário. }\end{cases} \\
& \pi\left(\theta_{i}\right)=\pi\left(\alpha_{i}\right) \pi\left(\beta_{i}\right) \pi\left(\mu_{i}\right) \pi\left(\sigma_{x(i)}^{2}\right) \pi\left(\sigma_{i}^{2}\right), i=1,2,
\end{aligned}
$$


onde

$$
\begin{aligned}
& \pi\left(\alpha_{i}\right) \sim N\left(a_{i}, \sigma_{\alpha_{i}}^{2}\right), \quad \pi\left(\beta_{i}\right) \sim N\left(b_{i}, \sigma_{\beta_{i}}^{2}\right), \quad \pi\left(\mu_{i}\right) \sim N\left(m_{i}, \sigma_{\mu_{i}}^{2}\right), \\
& \pi\left(\sigma_{x(i)}^{2}\right) \sim I G\left(c_{i}, d_{i}\right), \quad \pi\left(\sigma_{i}^{2}\right) \sim I G\left(f_{i}, g_{i}\right), i=1,2, \\
& \text { e } \quad \pi\left(\nu_{i}\right)=p\left(\mathcal{V}_{i}=\nu_{i}\right)= \begin{cases}\frac{1}{n_{0_{i}}}, & c_{i}-n_{0_{i}}+1 \leq \nu_{i} \leq c_{i}, \\
0, & \text { caso contrário, }\end{cases}
\end{aligned}
$$

onde $i=1,2, n_{0_{i}}=$ número de elementos do domínio da variável aleatória discreta $\mathcal{V}_{i}$ cuja probabilidade é positiva e $c_{i}$ é uma constante inteira (podemos tomar $c_{i} \geq 3$ para assegurar a existência dos momentos de ordem 1 e 2).

observação: $\mathrm{O}$ caso em que os números de graus de liberdade $\nu_{1}$ e $\nu_{2}$ são conhecidos é um caso particular de (5.1.4) quando $n_{0_{i}}=1$

\section{Distribuição à posteriori}

A posteriori pode ser escrita como

$$
p\left(\stackrel{*}{\stackrel{*}{*}} \mid D_{\mathrm{obs}}\right)=\int_{\mathfrak{X}} p\left(\underset{\sim}{x}, \stackrel{\stackrel{*}{\theta}}{\sim} \mid D_{\mathrm{obs}}\right) \mathrm{d} \underset{\sim}{x},
$$

onde $\mathscr{X}=\left\{\left(x_{1}, \ldots, x_{n}\right)^{T}: x_{i} \in \mathbb{R}\right\}$ e $p\left(\underset{\sim}{x}, \underset{\sim}{\stackrel{*}{*}} \mid D_{\text {obs }}\right) \propto L(\underset{\sim}{\stackrel{*}{\theta}} \mid D) \pi(\underset{\sim}{\stackrel{*}{\theta}})$, onde $L(\underset{\sim}{\stackrel{*}{*}} \mid D)$ é a verossimilhança baseada nos dados completos $D=\{\underset{\sim}{Z}: i=1,2, \ldots, n\}$, dada por

$$
\begin{aligned}
& L(\underset{\sim}{\stackrel{*}{*} \mid} \mid D)=L\left(k, \theta_{1}, \theta_{2}, \underset{\sim}{w} \mid D\right) \propto \frac{1}{\left(\prod_{i=1}^{k} w_{i}^{3 / 2}\right)\left(\sigma_{1}^{2}\right)^{k}\left(\sigma_{x(1)}^{2}\right)^{\frac{k}{2}}} \\
& . \exp \left\{-\frac{1}{2}\left[\sum_{i=1}^{k} \frac{\left(Y_{i}-\alpha_{1}-\beta_{1} x_{i}\right)^{2}}{w_{i} \lambda \sigma_{1}^{2}}+\sum_{i=1}^{k} \frac{\left(X_{i}-x_{i}\right)^{2}}{w_{i} \sigma_{1}^{2}}+\sum_{i=1}^{k} \frac{\left(x_{i}-\mu_{1}\right)^{2}}{w_{i} \sigma_{x(1)}^{2}}\right]\right\} \\
& \cdot \frac{1}{\left(\prod_{i=k+1}^{n} w_{i}^{3 / 2}\right)\left(\sigma_{2}^{2}\right)^{n-k}\left(\sigma_{x(2)}^{2}\right)^{\frac{n-k}{2}}} \exp \left\{-\frac{1}{2}\left[\sum_{i=k+1}^{n} \frac{\left(Y_{i}-\alpha_{2}-\beta_{2} x_{i}\right)^{2}}{w_{i} \lambda \sigma_{2}^{2}}\right.\right. \\
& \left.\left.+\sum_{i=k+1}^{n} \frac{\left(X_{i}-x_{i}\right)^{2}}{w_{i} \sigma_{2}^{2}}+\sum_{i=k+1}^{n} \frac{\left(x_{i}-\mu_{2}\right)^{2}}{w_{i} \sigma_{x(2)}^{2}}\right]\right\}
\end{aligned}
$$

$\propto L_{1}\left(k,{\underset{\theta}{1}}_{1}, \nu_{1} \underset{\sim}{w}{ }_{1} \mid D_{1}\right) L_{2}\left(k,{\underset{\sim}{\theta}}_{2}, \nu_{2}, \underset{\sim}{w} 2 \mid D_{2}\right)$,

onde ${\underset{\sim}{w}}_{1}=\left(w_{1}, \ldots, w_{k}\right)^{T}$ e $\underset{\sim}{w_{2}}=\left(w_{k+1}, \ldots, w_{n}\right)^{T}$ e onde o primeiro fator é a verossimilhança baseada nos dados completos $\left(D_{1}\right)$ até a mudança e o segundo fator a verossimilhança baseada nos dados completos $\left(D_{2}\right)$ após a mudança. 


\subsubsection{Implementação do algoritmo de Gibbs no modelo que permite mudança em todos os parâmetros}

Usando a distribuição $p\left(\underset{\sim}{x}, \underset{e}{\stackrel{*}{e} \mid} \mid D_{\text {obs }}\right)$ acima, obtemos as condicionais completas de $x_{1}, \ldots, x_{n}, \alpha_{1}, \alpha_{2}, \beta_{1}, \beta_{2}, \mu_{1}, \mu_{2}, \sigma_{x(1)}^{2}, \sigma_{x(2)}^{2}, \sigma_{1}^{2}, \sigma_{2}^{2}, \nu_{1}, \nu_{2}$ e $k$, que apresentamos a seguir.

$$
\begin{aligned}
& x_{i} \mid x_{(-i)}, \alpha_{1}, \alpha_{2}, \beta_{1}, \beta_{2}, \mu_{1}, \mu_{2}, \sigma_{x(1)}^{2}, \sigma_{x(2)}^{2}, \sigma_{1}^{2}, \sigma_{2}^{2}, \underset{\sim}{w}, \nu_{1}, \nu_{2}, k, D_{\mathrm{obs}} \\
& \sim N\left(\frac{\sigma_{x(1)}^{2}\left[\lambda X_{i}+\beta_{1}\left(Y_{i}-\alpha_{1}\right)+\lambda \sigma_{1}^{2} \mu_{1}\right]}{\sigma_{x(1)}^{2}\left[\lambda+\beta_{1}^{2}\right]+\lambda \sigma_{1}^{2}} ; \frac{\lambda \sigma_{1}^{2} \sigma_{x(1)}^{2} w_{i}}{\sigma_{x(1)}^{2}\left[\lambda+\beta_{1}^{2}\right]+\lambda \sigma_{1}^{2}}\right), \\
& \quad i=1, \ldots, k \\
& N\left(\frac{\sigma_{x(2)}^{2}\left[\lambda X_{i}+\beta_{2}\left(Y_{i}-\alpha_{2}\right)+\lambda \sigma_{2}^{2} \mu_{2}\right]}{\sigma_{x(2)}^{2}\left[\lambda+\beta_{2}^{2}\right]+\lambda \sigma_{2}^{2}} ; \frac{\lambda \sigma_{2}^{2} \sigma_{x(2)}^{2} w_{i}}{\sigma_{x(2)}^{2}\left[\lambda+\beta_{2}^{2}\right]+\lambda \sigma_{2}^{2}}\right), \\
& \quad i=k+1, \ldots, n .
\end{aligned}
$$

$\alpha_{1} \mid \underset{\sim}{x}, \alpha_{2}, \beta_{1}, \beta_{2}, \mu_{1}, \mu_{2}, \sigma_{x(1)}^{2}, \sigma_{x(2)}^{2}, \sigma_{1}^{2}, \sigma_{2}^{2}, \underset{\sim}{w}, \nu_{1}, \nu_{2}, k, D_{\mathrm{obs}}$ tem distribuição

$$
N\left(\frac{\sigma_{\alpha_{1}}^{2} \sum_{i=1}^{k} \frac{\left(Y_{i}-\beta_{1} x_{2}\right)}{w_{i}}+\lambda \sigma_{1}^{2} a_{1}}{\left.\sigma_{\alpha_{1}}^{2} \sum_{i=1}^{k} \frac{1}{w_{i}}+\lambda \sigma_{1}^{2}\right)} ; \frac{\lambda \sigma_{1}^{2} \sigma_{\alpha_{1}}^{2}}{\sigma_{\alpha_{1}}^{2} \sum_{i=1}^{k} \frac{1}{w_{i}}+\lambda \sigma_{1}^{2}}\right),
$$

$\alpha_{2} \mid \underset{\sim}{x}, \alpha_{1}, \beta_{1}, \beta_{2}, \mu_{1}, \mu_{2}, \sigma_{x(1)}^{2}, \sigma_{x(2)}^{2}, \sigma_{1}^{2}, \sigma_{2}^{2}, \underset{\sim}{w}, \nu_{1}, \nu_{2}, k, D_{\text {obs }}$ tem distribuição

$$
N\left(\frac{\sigma_{\alpha_{2}}^{2} \sum_{i=k+1}^{n} \frac{\left(Y_{i}-\beta_{2} x_{i}\right)}{w_{i}}+\lambda \sigma_{2}^{2} a_{2}}{\sigma_{\alpha_{2}}^{2} \sum_{i=k+1}^{n} \frac{1}{w_{i}}+\lambda \sigma_{2}^{2}} ; \frac{\lambda \sigma_{2}^{2} \sigma_{\alpha_{2}}^{2}}{\sigma_{\alpha_{2}}^{2} \sum_{i=k+1}^{n} \frac{1}{w_{i}}+\lambda \sigma_{2}^{2}}\right),
$$

$\beta_{1} \mid \underset{\sim}{x}, \alpha_{1}, \alpha_{2}, \beta_{2}, \mu_{1}, \mu_{2}, \sigma_{x(1)}^{2}, \sigma_{x(2)}^{2}, \sigma_{1}^{2}, \sigma_{2}^{2}, k, \nu_{1}, \nu_{2}, D_{\text {obs }}$ tem distribuição

$$
\begin{array}{r}
N\left(\frac{\sigma_{\beta_{1}}^{2} \sum_{i=1}^{k} \frac{\left(Y_{i}-\alpha_{1}\right) x_{i}}{w_{i}}+\lambda b_{1} \sigma_{1}^{2}}{\sigma_{\beta_{1}}^{2} \sum_{i=1}^{k} \frac{x_{i}^{2}}{w_{i}}+\lambda \sigma_{1}^{2}} ; \frac{\lambda \sigma_{1}^{2} \sigma_{\beta_{1}}^{2}}{\sigma_{\beta_{1}}^{2} \sum_{i=1}^{k} \frac{x_{1}^{2}}{w_{i}}+\lambda \sigma_{1}^{2}}\right), \\
\beta_{2} \mid \underset{\sim}{x}, \alpha_{1}, \alpha_{2}, \beta_{1}, \mu_{1}, \mu_{2}, \sigma_{x(1)}^{2}, \sigma_{x(2)}^{2}, \sigma_{1}^{2}, \sigma_{2}^{2}, \underset{\sim}{w}, \nu_{1}, \nu_{2}, k, D_{\text {obs }} \\
\sim N\left(\frac{\sigma_{\beta_{2}}^{2} \sum_{i=k+1}^{n} \frac{\left(Y_{i}-\alpha_{2}\right) x_{1}}{w_{i}}+\lambda b_{2} \sigma_{2}^{2}}{\sigma_{\beta_{2}}^{2} \sum_{i=k+1}^{n} \frac{x_{1}^{2}}{w_{i}}+\lambda \sigma_{2}^{2}} ; \frac{\lambda \sigma_{2}^{2} \sigma_{3_{2}}^{2}}{\sigma_{\beta_{2}}^{2} \sum_{i=k+1}^{n} \frac{x_{i}^{2}}{w_{i}}+\lambda \sigma_{2}^{2}}\right), \\
\mu_{1} \mid \underset{\sim}{x}, \alpha_{1}, \alpha_{2}, \beta_{1}, \beta_{2}, \mu_{2}, \sigma_{x(1)}^{2}, \sigma_{x(2)}^{2}, \sigma_{1}^{2}, \sigma_{2}^{2}, \underset{\sim}{w}, \nu_{1}, \nu_{2}, k, D_{\text {obs }}
\end{array}
$$


$\sim N\left(\frac{\sigma_{\mu_{1}}^{2} \sum_{i=1}^{k} \frac{x_{i}}{w_{i}}+\sigma_{x(1)}^{2} m_{1}}{\sigma_{\mu_{1}}^{2} \sum_{i=1}^{k} \frac{1}{w_{i}}+\sigma_{x(1)}^{2}} ; \frac{\sigma_{\mu_{1}}^{2} \sigma_{x(1)}^{2}}{\sigma_{\mu_{1}}^{2} \sum_{i=1}^{k} \frac{1}{w_{i}}+\sigma_{x(1)}^{2}}\right)$

$\mu_{2} \mid \underset{\sim}{x}, \alpha_{1}, \alpha_{2}, \beta_{1}, \beta_{2}, \mu_{1}, \sigma_{x(1)}^{2}, \sigma_{x(2)}^{2}, \sigma_{1}^{2}, \sigma_{2}^{2}, \underset{\sim}{w}, \nu_{1}, \nu_{2}, k, D_{\mathrm{obs}}$

$\sim N\left(\frac{\sigma_{\mu_{2}}^{2} \sum_{i=k+1}^{n} \frac{x_{i}}{w_{i}}+\sigma_{x(2)}^{2} m_{2}}{\sigma_{\mu_{2}}^{2} \sum_{i=k+1}^{n} \frac{1}{w_{i}}+\sigma_{x(2)}^{2}} ; \frac{\sigma_{\mu_{2}}^{2} \sigma_{x(2)}^{2}}{\sigma_{\mu_{2}}^{2} \sum_{i=k+1}^{n} \frac{1}{w_{i}}+\sigma_{x(2)}^{2}}\right)$,

$\sigma_{x(1)}^{2} \mid \underset{\sim}{x}, \alpha_{1}, \alpha_{2}, \beta_{1}, \beta_{2}, \mu_{1}, \mu_{2}, \sigma_{x(2)}^{2}, \sigma_{1}^{2}, \sigma_{2}^{2}, \nu_{1}, \nu_{2}, k, \underset{\sim}{w}, D_{\mathrm{obs}}$

$\sim I G\left(\frac{k}{2}+c_{1} ; \frac{1}{2} \sum_{i=1}^{k} \frac{\left(x_{i}-\mu_{1}\right)^{2}}{w_{i}}+d_{1}\right)$

$\sigma_{x(2)}^{2} \mid \underset{\sim}{x}, \alpha_{1}, \alpha_{2}, \beta_{1}, \beta_{2}, \mu_{1}, \mu_{2}, \sigma_{x(1)}^{2}, \sigma_{1}^{2}, \sigma_{2}^{2}, \nu_{1}, \nu_{2}, k, \underset{\sim}{w}, D_{\mathrm{obs}}$

$\sim I G\left(\frac{n-k}{2}+c_{2} ; \frac{1}{2} \sum_{i=k+1}^{n} \frac{\left(x_{i}-\mu_{2}\right)^{2}}{w_{i}}+d_{2}\right)$,

$\sigma_{1}^{2} \mid \underset{\sim}{x}, \alpha_{1}, \alpha_{2}, \beta_{1}, \beta_{2}, \mu_{1}, \mu_{2}, \sigma_{x(1)}^{2}, \sigma_{x(2)}^{2}, \sigma_{2}^{2}, \nu_{1}, \nu_{2}, k, \underset{\sim}{w}, D_{\mathrm{obs}}$

$\sim I G\left(k+f_{1} ; \frac{1}{2 \lambda}\left(\sum_{i=1}^{k} \frac{\left(Y_{i}-\alpha_{1}-\beta_{1} x_{i}\right)^{2}}{w_{i}}+\sum_{i=1}^{k} \frac{\left(X_{i}-x_{i}\right)^{2}}{w_{i}}\right)+g_{1}\right)$,

$\sigma_{2}^{2} \mid \underset{\sim}{x}, \alpha_{1}, \alpha_{2}, \beta_{1}, \beta_{2}, \mu_{1}, \mu_{2}, \sigma_{x(1)}^{2}, \sigma_{x(2)}^{2}, \sigma_{1}^{2}, \nu_{1}, \nu_{2}, k, \underset{\sim}{w}, D_{\text {obs }}$

$\sim I G\left(n-k+f_{2} ; \frac{1}{2 \lambda}\left(\sum_{i=k+1}^{n} \frac{\left(Y_{i}-\alpha_{2}-\beta_{2} x_{i}\right)^{2}}{w_{i}}+\sum_{i=k+1}^{n} \frac{\left(X_{i}-x_{i}\right)^{2}}{w_{i}}\right)+g_{2}\right)$,

$w_{i} \mid \underset{\sim}{x}, \alpha_{1}, \alpha_{2}, \beta_{1}, \beta_{2}, \mu_{1}, \mu_{2}, \sigma_{x(1)}^{2}, \sigma_{x(2)}^{2}, \sigma_{1}^{2}, \sigma_{2}^{2}, \nu_{1}, \nu_{2}, w_{(-i)}, k, D_{\text {obs }}$

$\sim I G\left(\frac{3+\nu_{1}}{2} ; \frac{1}{2}\left[\left(\frac{\left(Y_{i}-\alpha_{1}-\beta_{1} x_{i}\right)^{2}+\lambda\left(X_{i}-x_{i}\right)^{2}}{\lambda \sigma_{1}^{2}}+\frac{\left(x_{i}-\mu_{1}\right)^{2}}{\sigma_{x(1)}^{2}}\right)+\nu_{1}\right]\right)$, $i=1, \ldots, k$,

$\sim I G\left(\frac{3+\nu_{2}}{2}: \frac{1}{2}\left[\left(\frac{\left(Y_{i}-\alpha_{2}-\beta_{2} x_{i}\right)^{2}+\lambda\left(X_{i}-x_{i}\right)^{2}}{\lambda \sigma_{2}^{2}}+\frac{\left(x_{i}-\mu_{2}\right)^{2}}{\sigma_{x(2)}^{2}}\right)+\nu_{2}\right]\right)$, 


$$
\begin{aligned}
& P\left[K=k \mid \underset{\sim}{x}, \alpha_{1}, \alpha_{2}, \beta_{1}, \beta_{2}, \mu_{1}, \mu_{2}, \sigma_{x(1)}^{2}, \sigma_{x(2)}^{2}, \sigma_{1}^{2}, \sigma_{2}^{2}, \nu_{1}, \nu_{2}, \underset{\sim}{w}, D_{\mathrm{obs}}\right] \\
& =\frac{L\left(k, \theta_{1}, \underline{\theta}_{2}, \underset{\sim}{w}, \nu_{1}, \nu_{2} \mid D\right)}{\sum_{k \in \mathcal{K}} L\left(k,{\underset{\theta}{1}}_{1},{\underset{\sim}{2}}_{2}, \underset{\sim}{w}, \nu_{1}, \nu_{2} \mid D\right)},
\end{aligned}
$$

$k \in \mathcal{K}$, onde $L\left(k, \underset{\theta_{1}}{\underbrace{}_{\sim}} \underset{2}{\underset{\sim}{w}} \mid D\right)$ é dada em (5.1.5).

$$
p\left(\mathcal{V}_{i}=\nu_{i} \mid \underset{\sim}{x}, \alpha_{1}, \alpha_{2}, \ldots, \nu_{1}, \nu_{2}, \underset{\sim}{w}, D_{\mathrm{obs}}\right)=\frac{L_{i}\left(k,{\underset{\sim}{i}}_{i}, \underset{\sim}{w_{i}}, \nu_{i} \mid D_{i}\right)}{\sum_{\nu_{i}=c_{i}-n_{0_{i}}+1}^{c_{i}} L_{i}\left(k,{\underset{\theta}{i}}_{i}, \underset{\sim}{w}, \nu_{i} \mid D_{i}\right)}, \quad i=1,2
$$

onde $L_{i}\left(k,{\underset{\sim}{i}}_{i}, \nu_{i}, \underset{\sim}{w_{i}} \mid D_{i}\right)$ é dada em (5.1.5) e $n_{0_{i}}$ e $c_{i}$ são definidos em (5.1.4).

Observação 1: No caso em que $\nu_{1}$ e $\nu_{2}$ são conhecidos devemos desconsiderar as distribuições em (5.1.21).

Observação2: Fazendo $w_{i}=1, i=1, \ldots, n$ e desprezando as distribuições em (5.1.21), obtemos as condicionais completas do modelo Normal dadas na Seção 4.2.2.

\subsubsection{Implementação do algoritmo de Gibbs no modelo com mudança em um subconjunto qualquer de parâmetros}

Nessa subseção o objetivo é implementar o algoritmo de Gibbs para amostrar da distribuição à posteriori de um modelo que permite mudança em um subconjunto qualquer não vazio de parâmetros estritamente contidos em $\left\{\alpha, \beta, \mu, \sigma_{x}^{2}, \sigma^{2}\right\}$. Para esse propósito, utilizamos as distribuições condicionais completas à posteriori do modelo com mudança em todos os parâmetros (que são dadas pelas fórmulas (5.1.6) a (5.1.21), fazendo-se as devidas restrições; utilizamos também as distribuições condicionais completas a seguir (fazendo-se também as devidas restrições):

- $\left[\alpha \mid \beta_{1}, \beta_{2}, \mu_{1}, \mu_{2}, \sigma_{x(1)}^{2}, \sigma_{x(2)}^{2}, \sigma_{1}^{2}, \sigma_{2}^{2}, \underset{\sim}{w}, \nu_{1}, \nu_{2}, \underset{\sim}{x}, D_{\mathrm{obs}}\right]$ 


$$
\begin{gathered}
\sim N\left(\frac{\sigma_{2}^{2} \sigma_{\alpha}^{2} \sum_{i=1}^{k} \frac{\left(Y_{i}-\beta_{1} x_{i}\right)}{w_{i}}+\sigma_{1}^{2} \sigma_{\alpha}^{2} \sum_{i=k+1}^{n} \frac{\left(Y_{i}-\beta_{2} x_{i}\right)}{w_{i}}+\lambda \sigma_{1}^{2} \sigma_{2}^{2} a}{\sigma_{2}^{2} \sigma_{\alpha}^{2} \sum_{i=1}^{k} \frac{1}{w_{i}}+\sigma_{1}^{2} \sigma_{\alpha}^{2} \sum_{i=k+1}^{n} \frac{1}{w_{i}}+\lambda \sigma_{1}^{2} \sigma_{2}^{2}}\right. \\
\left.\frac{\lambda \sigma_{1}^{2} \sigma_{2}^{2} \sigma_{\alpha}^{2}}{\sigma_{2}^{2} \sigma_{\alpha}^{2} \sum_{i=1}^{k} \frac{1}{w_{i}}+\sigma_{1}^{2} \sigma_{\alpha}^{2} \sum_{i=k+1}^{n} \frac{1}{w_{i}}+\lambda \sigma_{1}^{2} \sigma_{2}^{2}}\right)
\end{gathered}
$$

- $\left[\beta \mid \alpha_{1}, \alpha_{2}, \mu_{1}, \mu_{2}, \sigma_{x(1)}^{2}, \sigma_{x(2)}^{2}, \sigma_{1}^{2}, \sigma_{2}^{2}, k, \underset{\sim}{w}, \nu_{1}, \nu_{2}, \underset{\sim}{x}, D_{\text {obs }}\right]$,

$$
\begin{gathered}
\sim N\left(\frac{\sigma_{2}^{2} \sigma_{\beta}^{2} \sum_{i=1}^{k} \frac{\left(Y_{i}-\alpha_{1}\right) x_{i}}{w_{i}}+\sigma_{1}^{2} \sigma_{\beta}^{2} \sum_{i=k+1}^{n} \frac{\left(Y_{i}-\alpha_{2}\right) x_{i}}{w_{i}}+\lambda \sigma_{1}^{2} \sigma_{2}^{2} b}{\sigma_{2}^{2} \sigma_{\beta}^{2} \sum_{i=1}^{k} \frac{x_{i}^{2}}{w_{i}}+\sigma_{1}^{2} \sigma_{\beta}^{2} \sum_{i=k+1}^{n} \frac{x_{i}^{2}}{w_{i}}+\lambda \sigma_{1}^{2} \sigma_{2}^{2}} ;\right. \\
\left.\frac{\lambda \sigma_{1}^{2} \sigma_{2}^{2} \sigma_{\beta}^{2}}{\sigma_{2}^{2} \sigma_{\beta}^{2} \sum_{i=1}^{k} \frac{x_{i}^{2}}{w_{i}}+\sigma_{1}^{2} \sigma_{\beta}^{2} \sum_{i=k+1}^{n} \frac{x_{i}^{2}}{w_{i}}+\lambda \sigma_{1}^{2} \sigma_{2}^{2}}\right)
\end{gathered}
$$

- $\left[\mu \mid \alpha_{1}, \alpha_{2}, \beta_{1}, \beta_{2}, \sigma_{x(1)}^{2}, \sigma_{x(2)}^{2}, \sigma_{1}^{2}, \sigma_{2}^{2}, k, \nu_{1}, \nu_{2}, \underset{\sim}{w}, D_{\mathrm{obs}}\right]$,

$$
\begin{gathered}
\sim N\left(\frac{\sigma_{x(2)}^{2} \sigma_{\mu}^{2} \sum_{i=1}^{k} \frac{x_{i}}{w_{i}}+\sigma_{x(1)}^{2} \sigma_{\mu}^{2} \sum_{i=k+1}^{n} \frac{x_{i}}{w_{i}}+\sigma_{x(1)}^{2} \sigma_{x(2)}^{2} m}{\sigma_{x(2)}^{2} \sigma_{\mu}^{2} \sum_{i=1}^{k} \frac{1}{w_{i}}+\sigma_{x(1)}^{2} \sigma_{\mu}^{2} \sum_{i=k+1}^{n} \frac{1}{w_{i}}+\sigma_{x(1)}^{2} \sigma_{x(2)}^{2}} ;\right. \\
\left.\frac{\sigma_{x(1)}^{2} \sigma_{x(2)}^{2} \sigma_{\mu}^{2}}{\sigma_{x(2)}^{2} \sigma_{\mu}^{2} \sum_{i=1}^{k} \frac{1}{w_{i}}+\sigma_{x(1)}^{2} \sigma_{\mu}^{2} \sum_{i=k+1}^{n} \frac{1}{w_{i}}+\sigma_{x(1)}^{2} \sigma_{x(2)}^{2}}\right)
\end{gathered}
$$

- $\left[\sigma_{x}^{2} \mid \alpha_{1}, \alpha_{2}, \beta_{1}, \beta_{2}, \mu_{1}, \mu_{2}, \sigma_{1}^{2}, \sigma_{2}^{2}, k, \underset{\sim}{w}, \nu_{1}, \nu_{2}, \underset{\sim}{x}, D_{\text {obs }}\right]$

$$
\sim I G\left(\frac{n}{2}+c ; \frac{1}{2}\left(\sum_{i=1}^{k} \frac{\left(x_{i}-\mu_{1}\right)^{2}}{w_{i}}+\sum_{i=k+1}^{n} \frac{\left(x_{i}-\mu_{2}\right)^{2}}{w_{i}}\right)+d\right),
$$

- $\left[\sigma^{2} \mid \alpha_{1}, \alpha_{2}, \beta_{1}, \beta_{2}, \mu_{1}, \mu_{2}, \sigma_{x(1)}^{2}, \sigma_{x(2)}^{2}, k, \underset{\sim}{w}, \nu_{1}, \nu_{2}, \underset{\sim}{x}, D_{\text {obs }}\right]$

$$
\begin{aligned}
\sim I G[n & +f ; \frac{1}{2}\left(\sum_{i=1}^{k} \frac{\left(Y_{i}-\alpha_{1}-\beta_{1} x_{i}\right)^{2}}{\lambda w_{i}}+\sum_{i=k+1}^{n} \frac{\left(Y_{i}-\alpha_{2}-\beta_{2} x_{i}\right)^{2}}{\lambda w_{i}}\right. \\
& \left.\left.+\sum_{i=1}^{k} \frac{\left(X_{i}-x_{i}\right)^{2}}{w_{i}}+\sum_{i=k+1}^{n} \frac{\left(X_{i}-x_{i}\right)^{2}}{w_{i}}\right)+g\right]
\end{aligned}
$$

Assim, por exemplo, para implementar o algoritmo de Gibbs no modelo que permite mudança apenas em $\left\{\alpha, \beta, \sigma^{2}\right\}$, as distribuições condicionais completas necessárias são as distribuições de (5.1.6) a (5.1.11), (5.1.24) a (5.1.25) e (5.1.16) a (5.1.21), impondo-se as restriçōes $\mu_{1}=\mu_{2}$ e $\sigma_{x(1)}^{2}=\sigma_{r(2)}^{2}$ nas distribuições que envolverem os parâmetros $\mu_{i}$ e $\sigma_{x(i)}^{2}, i=1,2$, como também no valor inicial, da mesma forma que na Seção 4.3. 


\subsection{O modelo $t$-dependente com particular priori de componentes independentes}

\subsubsection{O modelo que permite mudança em todos os parâme- tros após o ponto $k$}

O modelo $t$-dependente formulado como mistura de escala de normais é dado por:

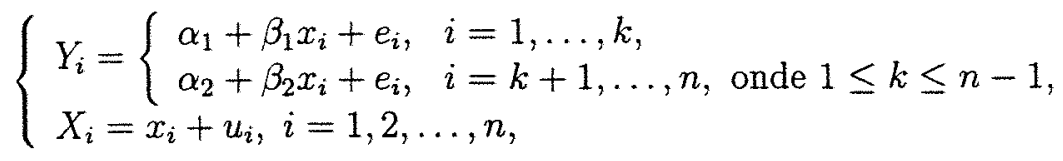

onde $X_{i}$ e $Y_{i}$ são v.a. observáveis, $x_{i}$ é v.a. latente e $\left(e_{i}, u_{i}\right)$ os erros aleatórios.

Sejam $\underset{\sim}{Z^{(1)}}=\left(\begin{array}{c}\underset{\sim}{Z} \\ \vdots \\ \underset{\sim}{Z}\end{array}\right),{\underset{\sim}{Z}}^{(2)}=\left(\begin{array}{c}\underset{\sim}{Z_{k+1}} \\ \vdots \\ \underset{\sim}{Z}\end{array}\right)$, onde $\underset{\sim}{\underset{Z}{Z}}=\left(\begin{array}{c}Y_{i} \\ X_{i} \\ x_{i}\end{array}\right)$.

O modelo assume que $Z^{(1)}$ e ${\underset{\sim}{Z}}^{(2)}$ são independentes com

$$
\underset{\sim}{Z^{(1)}} \mid w_{1}, \theta_{1}, \nu_{1} \sim N_{3 k}\left(\underset{\sim}{\mathbb{1}}{ }_{k \times 1} \otimes \underset{\sim}{\mu} ; I_{k \times k} \otimes \sum_{\sim} 1 w_{1}\right)
$$

e

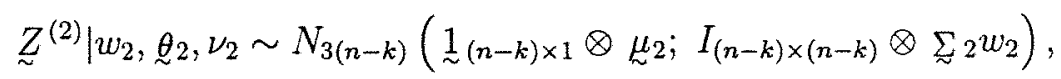

onde

$$
\begin{aligned}
\mu_{1} & =\left(\begin{array}{c}
\alpha_{1}+\beta_{1} \mu_{1} \\
\mu_{1} \\
\mu_{1}
\end{array}\right), \quad \mu_{2}=\left(\begin{array}{c}
\alpha_{2}+\beta_{2} \mu_{2} \\
\mu_{2} \\
\mu_{2}
\end{array}\right), \\
\Sigma_{1} & =\left(\begin{array}{ccc}
\beta_{1} \sigma_{x(1)}^{2}+\lambda \sigma_{1}^{2} & \beta_{1} \sigma_{x(1)}^{2} & \beta_{1} \sigma_{x(1)}^{2} \\
\beta_{1} \sigma_{x(1)}^{2} & \sigma_{x(1)}^{2}+\sigma_{1}^{2} & \sigma_{x(1)}^{2} \\
\beta_{1} \sigma_{x(1)}^{2} & \sigma_{x(1)}^{2} & \sigma_{x(1)}^{2}
\end{array}\right) \\
\mathrm{e} \quad \sum_{2}= & \left(\begin{array}{ccc}
\beta_{2} \sigma_{x(2)}^{2}+\lambda \sigma_{2}^{2} & \beta_{2} \sigma_{x(2)}^{2} & \beta_{2} \sigma_{x(2)}^{2} \\
\beta_{2} \sigma_{x(2)}^{2} & \sigma_{x(2)}^{2}+\sigma_{2}^{2} & \sigma_{x(2)}^{2} \\
\beta_{2} \sigma_{x(2)}^{2} & \sigma_{x(2)}^{2} & \sigma_{x(2)}^{2}
\end{array}\right),
\end{aligned}
$$

onde $\lambda=$ razão das variâncias dos erros (conhecida), $\nu_{1}=$ número de graus de liberdade antes e $\nu_{2}=$ número de graus de liberdade após a mudança, além disso $\sum_{i}>0, i=1,2, \underset{\theta}{*}=\left({\underset{\sim}{1}}_{1}^{T}, \underset{2}{\theta_{2}^{T}}, k, w_{1}, w_{2}, \nu_{1}, \nu_{2}\right)^{T}$ é o vetor de parâmetros do modelo, onde $\underline{\theta}_{i}=\left(\alpha_{i}, \beta_{i}, \mu_{i}, \sigma_{x(i)}^{2}, \sigma_{i}^{2}\right)^{T}$, 
A distribuição à priori escolhida para $\underset{\sim}{\theta}$ é da forma

$$
\pi\left(\underset{\stackrel{*}{g}}{)}=p(k) \prod_{i=1}^{2} \pi\left({\underset{\theta}{i}}_{i}\right) \pi\left(w_{i} \mid \nu_{i}\right) \pi\left(\nu_{i}\right)\right.
$$

onde $\pi\left(w_{i} \mid \nu_{i}\right) \sim I G\left(\frac{\nu_{i}}{2}, \frac{\nu_{i}}{2}\right), i=1,2$, e $\pi\left(\theta_{i}\right), p(k)$ e $\pi\left(\nu_{i}\right)$ coincidem com as distribuições em (5.1.4)

\section{- Função de verossimilhança}

A função de verossimilhança baseada nos dados "completos" $D=\left\{\underset{\sim}{Z} \underset{i}{ }=\left(Y_{i}, X_{i}\right.\right.$, $\left.\left.x_{i}\right)^{\top}, i=1, \ldots, n\right\}$ é a seguinte:

$$
\begin{aligned}
& L(\underset{\sim}{*} \mid D) \propto\left|\sum_{1} w_{1}\right|^{-\frac{k}{2}}\left|\sum_{2} w_{2}\right|^{-\frac{(n-k)}{2}} \exp \left\{-\frac{1}{2}\left[\left(Z^{(1)}-\underset{\sim}{1} k \times 1 \otimes \mu_{1}\right)^{\top}\right.\right.
\end{aligned}
$$

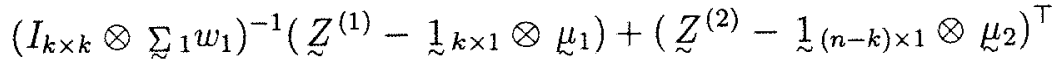

$$
\begin{aligned}
& \left.\left..\left(I_{(n-k) \times(n-k)} \otimes \sum_{\sim} w_{2}\right)^{-1}\left(Z_{\sim}^{(2)}-\underset{\sim}{1}(n-k) \times 1 \otimes \sim_{\sim}\right)\right]\right\} \\
& \propto\left|\sum_{i} w_{1}\right|^{-\frac{k}{2}}\left|\sum_{2} w_{2}\right|^{-\frac{(n-k)}{2}} \exp \left\{-\frac{1}{2}\left[\sum_{i=1}^{k}\left(\underset{\sim}{Z_{i}}-\mu_{1}\right)^{\top}\left(\sum_{1} w_{1}\right)^{-1}\left(\underset{\sim}{Z_{i}}-\mu_{1}\right)\right.\right. \\
& \left.\left.+\sum_{i=k+1}^{n}\left(\underset{\sim}{Z_{i}}-\mu_{2}\right)\left(\sum_{2} w_{2}\right)^{-1}\left(\underset{\sim}{Z_{i}}-\mu_{2}\right)\right]\right\} \\
& \propto L_{1}\left(\underset{\theta_{1}}{\theta_{1}}, w_{1}, \nu_{1} \mid D_{1}\right) L_{2}\left(\underset{\sim}{\theta_{2}}, w_{2}, \nu_{2} \mid D_{2}\right)=L_{1}\left(\underset{\sim}{*}\left|D_{1}\right| D_{1}\right) L_{2}\left(\underset{\sim}{*}\left|D_{2}\right| D_{2}\right),
\end{aligned}
$$

onde $D_{1}=\{\underset{\sim}{Z} i, i=1, \ldots, k\}=\underset{\sim}{\underset{\sim}{(1)}}, D_{2}=\{\underset{\sim}{Z} i, i=k+1, \ldots, n\}=\underset{\sim}{Z^{(2)}}$,

$$
\begin{aligned}
L_{1}\left(\stackrel{\sim}{\theta}_{1} \mid D_{1}\right)= & L_{1}\left(\theta_{1}, w_{1}, \nu_{1} \mid D_{1}\right) \\
\propto & \left|\Sigma_{1} w_{1}\right|^{-\frac{k}{2}} \exp \left\{-\frac{1}{2} \sum_{i=1}^{k}\left(Z_{i}-\mu_{1}\right)^{\top}\left(\sum_{i} w_{1}\right)^{-1}\left(Z_{\sim}-\mu_{1}\right)\right\} \\
= & w_{1}^{-\frac{3 k}{2}}\left(\sigma_{x(1)}^{2}\right)^{-\frac{k}{2}}\left(\sigma_{1}^{2}\right)^{-k} \exp \left\{-\frac{1}{2}\left[\frac{\sum_{i=1}^{k}\left(Y_{i}-\alpha_{1}-\beta_{1} x_{i}\right)^{2}}{\lambda \sigma_{1}^{2} w_{1}}\right.\right. \\
& \left.\left.+\frac{\sum_{i=1}^{k}\left(X_{i}-x_{i}\right)^{2}}{\sigma_{1}^{2} w_{1}}+\frac{\sum_{i=1}^{k}\left(x_{i}-\mu_{1}\right)^{2}}{\sigma_{I(1)}^{2} w_{1}}\right]\right\}
\end{aligned}
$$

é a verossimilhança para os dados completos, $D_{1}$, e

$$
L_{2}\left(\stackrel{*}{*}_{2} \mid D_{2}\right)=L_{2}\left(\underset{\theta_{2}}{\theta_{2}} w_{2}, \nu_{2} \mid D_{2}\right), \text { onde }
$$




$$
\begin{aligned}
L_{2}\left(\theta_{2}, w_{2}, \nu_{2} \mid D_{2}\right) \propto & w_{2}^{-\frac{3(n-k)}{2}}\left(\sigma_{x(2)}^{2}\right)^{-\frac{(n-k)}{2}}\left(\sigma_{2}^{2}\right)^{(n-k)} \exp \left\{-\frac{1}{2}\left[\sum_{i=k+1}^{n} \frac{\left(Y_{i}-\alpha_{2}-\beta_{2} x_{i}\right)^{2}}{\lambda \sigma_{2}^{2} w_{2}}\right.\right. \\
& \left.\left.+\sum_{i=k+1}^{n} \frac{\left(X_{i}-x_{i}\right)^{2}}{\sigma_{1}^{2} w_{2}}+\sum_{i=k+1}^{n} \frac{\left(x_{i}-\mu_{2}\right)^{2}}{\sigma_{x(2)}^{2} w_{2}}\right]\right\}
\end{aligned}
$$

é a verossimilhaną para o conjunto de dados completos, $D_{2}$, após a mudança.

\section{- Distribuição à posteriori}

Seja $\mathscr{X}=\left\{\underset{\sim}{x}=\left(x_{1}, \ldots, x_{n}\right)^{T}: x_{i} \in \mathbb{R}\right\}$.

A distribuição à posteriori

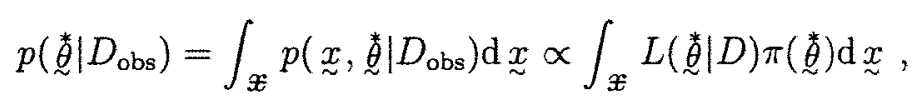

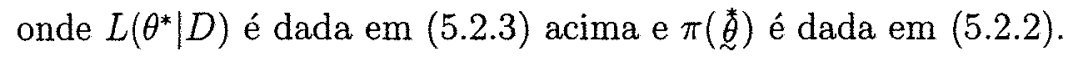

\subsubsection{Implementação do algoritmo de Gibbs no modelo com mudança em um subconjunto qualquer de parâmetros}

Para implementar o algoritmo de Gibbs no modelo com mudança em todos os parâmetros, necessitamos das distribuições condicionais completas à posteriori de $x_{i}$ (para $i=1, \ldots, n$ ), e de $\alpha_{1}, \alpha_{2}, \beta_{1}, \beta_{2}, \mu_{1}, \mu_{2}, \sigma_{x(1)}^{2}, \sigma_{x(2)}^{2}, \sigma_{1}^{2}, \sigma_{2}^{2}, \nu_{1}, \nu_{2}$ e $k$, que coincidem com as do modelo $t$-independente da Seção 5.1 .2 quando substituímos $w_{i}$ por $w_{i}=\left\{\begin{array}{ll}w_{1}, & \text { se } 1 \leq i \leq k, \\ w_{2}, & \text { se } k+1 \leq i \leq n .\end{array}\right.$ As distribuições condicionais completas à posteriori de $w_{1}$ e $w_{2}$, nesse caso, são dadas, respectivamente, por (5.2.5) e (5.2.6) a seguir.

$$
\begin{aligned}
& I G\left(\frac{3 k+\nu_{1}}{2} ; \frac{1}{2}\left[\frac{\sum_{i=1}^{k}\left(Y_{i}-\alpha_{1}-\beta_{1} x_{i}\right)^{2}}{\lambda \sigma_{1}^{2}}+\frac{\sum_{i=1}^{k}\left(X_{i}-x_{i}\right)^{2}}{\sigma_{1}^{2}}\right.\right. \\
& \left.\left.\quad+\frac{\sum_{i=1}^{k}\left(x_{i}-\mu_{1}\right)^{2}}{\sigma_{x(1)}^{2}}+\nu_{1}\right]\right)
\end{aligned}
$$

$\mathrm{e}$

$$
I G\left(\frac{3(n-k)+\nu_{2}}{2} ; \frac{1}{2}\left\{\frac{\sum_{i=1}^{k}\left(Y_{i}-\alpha_{2}-\beta_{2} x_{i}\right)^{2}}{\lambda \sigma_{2}^{2}}+\frac{\sum_{i=1}^{k}\left(X_{i}-x_{i}\right)^{2}}{\sigma_{2}^{2}}\right.\right.
$$




$$
\left.\left.+\frac{\sum_{i=1}^{k}\left(x_{i}-\mu_{2}\right)^{2}}{\sigma_{x(2)}^{2}}+\nu_{2}\right\}\right)
$$

No caso do modelo geral que permite mudança apenas em algum subconjunto de parâmetros estritamente contido em $\left\{\alpha, \beta, \mu, \sigma_{x}^{2}, \sigma^{2}\right\}$, precisamos ainda conhecer as distribuições condicionais completas de $\alpha$ do modelo com mudança em $\left\{\beta, \mu, \sigma_{x}^{2}, \sigma^{2}\right\}$, de $\beta$ do modelo com mudança em $\left\{\alpha, \mu, \sigma_{x}^{2}, \sigma^{2}\right\}$, de $\mu$ do modelo com mudança em $\left\{\alpha, \beta, \sigma_{x}^{2}, \sigma^{2}\right\}$, de $\sigma_{x}^{2}$ do modelo com mudança em $\left\{\alpha, \beta, \mu, \sigma^{2}\right\}$ e de $\sigma^{2}$ do modelo com mudança em $\left\{\alpha, \beta, \mu, \sigma_{x}^{2}\right\}$. Essas distribuições coincidem com as da Seção 5.1.3

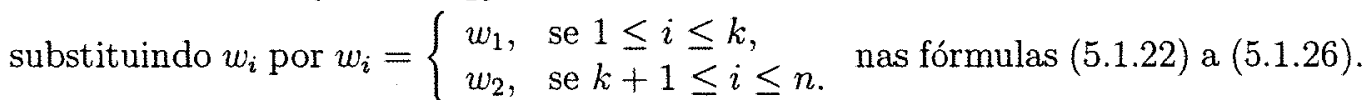

Com estas distribuições, e fazendo as devidas restrições, implementamos o algoritmo de Gibbs para o modelo que permite mudança em qualquer subconjunto de parâmetros (como fizemos na Seção 4.3 e na Seção 5.1.3).

\subsection{Detecção de mudança e estimação do modelo}

Para detectar mudança nos parâmetros do modelo $t$-independente e $t$-dependente, utilizamos as mesmas técnicas da seção 4.4 adaptadas a esses modelos.

\subsection{Condições para a existência da posteriori no modelo $t$-dependente sob diferentes escolhas de prioris impróprias}

Nessa seção apresentamos teoremas que estabelecem condições para a existência da posteriori sob o modelo $t$-dependente com graus de liberdade conhecidos e prioris impróprias análogas as usadas na seção 3.5 . 


\subsubsection{Condições no caso do modelo que permite mudança em todos os parâmetros}

Nesta subseção apresentamos alguns teoremas que garantem a existência da posteriori no modelo $t$-dependente que permite mudança em todos os parâmetros e tem priori imprópria do tipo:

\section{Distribuição à priori}

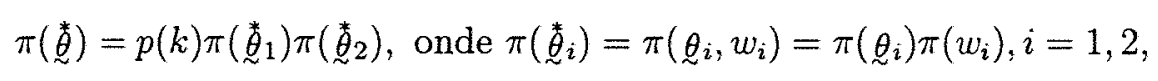

onde $w_{i} \sim I G\left(\frac{\nu_{i}}{2}, \frac{\nu_{i}}{2}\right), i=1,2$, para $n \geq 3, p(k)=P[K=k]$ é uma distribuição discreta definida num conjunto $\mathcal{K}$ finito e $\pi\left(\underset{i}{\theta_{i}}\right) \propto \pi\left(\sigma_{x(i)}^{2}\right) \pi\left(\sigma_{i}^{2}\right), i=1,2$, onde $\underline{\theta}_{i}=\left(\alpha_{i}, \beta_{i}, \mu_{i}, \sigma_{x(i)}^{2}, \sigma_{i}^{2}\right)^{T}$.

\section{Distribuição à posteriori}

No modelo com mudança em todos os parâmetros e priori $\pi(\underset{\sim}{\stackrel{*}{*}})=p(k) \pi\left({\underset{\sim}{\theta}}_{1}\right) \pi\left(\stackrel{\sim}{*}_{2}\right)$, a distribuição à posteriori $p\left(\underset{d}{\stackrel{*}{*} \mid} D_{\mathrm{obs}}\right)$ é proporcional a

$$
\int_{x=\left\{\underset{\sim}{x}=\left(x_{1}, \ldots, x_{n}\right): x_{i} \in \mathbb{R}\right\}} L(\stackrel{*}{\stackrel{*}{\theta}} \mid D) \pi(\underset{\sim}{\stackrel{*}{*}}) \mathrm{d} \underset{\sim}{x}, \text { onde } L(\underset{\sim}{\stackrel{*}{\theta}} \mid D) \text { é dado em }(5.2 .3)
$$

e portanto

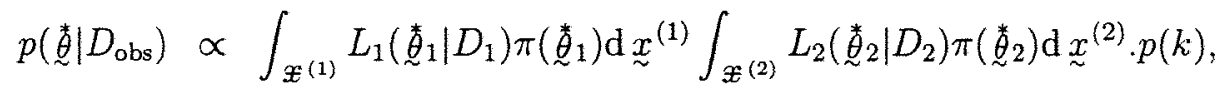

$$
\begin{aligned}
& \propto p_{1}\left(\stackrel{*}{*} 1 \mid D_{\text {obsi }}{ }^{(k)}\right) p_{2}\left(\stackrel{*}{*}_{2}^{*} \mid D_{\text {obs } 2}{ }^{(n-k)}\right) p(k),
\end{aligned}
$$

onde $p_{1}\left({\underset{\sim}{*}}_{1}^{*} \mid D_{\text {obs }}{ }_{1}^{(k)}\right)$ é a posteriori baseada nos dados até a mudança e $p_{2}\left(\stackrel{\sim}{\theta}_{2}^{*} \mid D_{\text {obs2 }}{ }^{(n-k)}\right)$ é a posteriori baseada nos dados após a mudança.

\section{Observação:}

Para mostrar que $p\left(\stackrel{*}{\theta} \mid D_{\text {obs }}\right)$ é própria, devemos mostrar que

$$
\sum_{k \in \mathcal{K}} p(k) \int_{W_{1}} \int_{\Theta_{1}} p\left({ }_{\ddot{\theta}} \mid D_{\text {obsi }}{ }^{(k)}\right) \mathrm{d} \underline{\theta}_{1} \mathrm{~d} w_{1} \int_{W_{2}} \int_{\Theta_{2}} p_{2}\left(\underline{\theta}_{2} \mid D_{\text {obs } 2}{ }^{(n-k)}\right) \mathrm{d} \underline{\theta}_{2} \mathrm{~d} w_{2}<\infty
$$


onde $W_{i}=\mathbb{R}_{+}, i=1,2, \Theta_{i}=\left\{\underline{\theta}_{i}=\left(\alpha_{i}, \beta_{i}, \mu_{i}, \sigma_{x(i)}^{2}, \sigma_{i}^{2}\right)^{T}: \alpha_{i}, \beta_{i}, \mu_{i} \in \mathbb{R}\right.$ e $\sigma_{x(i)}^{2}$, $\left.\sigma_{i}^{2} \in \mathbb{R}_{+}\right\}, i=1,2$, e $\mathcal{K}$ é um conjunto finito.

Como $\mathcal{K}$ é finito, então para mostrar a existência da posteriori $p\left(\underset{\Theta}{\theta} \mid D_{\mathrm{obs}}\right)$, basta mostrar que $p_{1}\left({\underset{\sim}{\theta}}_{1}^{*} \mid D_{\text {obs }}{ }^{(k)}\right)$ e $p\left({\underset{\sim}{*}}_{2}^{*} \mid D_{\text {obs2 }}^{(n-k)}\right)$ são próprias para um $k$ fixado.

\section{Teoremas}

Teorema 5.4.1 No modelo t-dependente com graus de liberdade conhecidos, que permite mudança em todos os parâmetros e tem distribuição à priori dada por

$$
\pi(\underset{\sim}{\stackrel{*}{\theta}})=p(k) \prod_{i=1}^{2} \pi\left({\underset{\sim}{\theta}}_{i}^{*}\right)=p(k) \prod_{i=1}^{2} \pi\left(\theta_{i}\right) \pi\left(w_{i}\right),
$$

onde $\pi\left(w_{i}\right) \sim I G\left(\frac{\nu_{i}}{2}, \frac{\nu_{i}}{2}\right), \nu_{i} \geq 3, i=1,2, \pi\left(\theta_{i}\right) \propto \pi\left(\sigma_{x(i)}^{2}\right) \pi\left(\sigma_{i}^{2}\right)$ (onde $\pi\left(\sigma_{x(i)}^{2}\right) \sim$ $\left.I G\left(c_{i}, d_{i}\right), \pi\left(\sigma_{i}^{2}\right) \sim I G\left(f_{i}, g_{i}\right), i=1,2\right)$ e $p(k)$ é uma distribuição discreta num conjunto finito $\mathcal{K}$. Então uma condição suficiente para que a distribuição à posteriori $p\left(\stackrel{*}{\theta} \mid D_{\mathrm{obs}}\right)$ seja própria é que $k \geq 3,(n-k) \geq 3,0<c_{1}<k+\frac{\left(\nu_{1}-3\right)}{2}$ e $0<c_{2}<$ $(n-k)+\frac{\left(\nu_{2}-3\right)}{2}$. Outra condição suficiente é que $k \geq 3, n-k \geq 3,0<f_{1}<\frac{k+\nu_{1}-1}{2}$ $e 0<f_{2}<\frac{n-k+\nu_{2}-1}{2}$.

Demonstração: O Teorema 3.5.2 garante que para $k$ fixado a integral

$$
\int_{W_{1}} \int_{\Theta_{1}} p_{1}\left({\underset{\sim}{1}}_{1}, w_{1} \mid D_{\mathrm{obs}}^{(k)}\right) \mathrm{d} \underset{\sim}{\theta_{1}} \mathrm{~d} w_{1}
$$

converge se $k \geq 3$ e $0<c_{1}<k+\frac{\left(\nu_{1}-3\right)}{2}$ (ou então $k \geq 3$ e $0<f_{1}<\frac{k+\nu_{2}-1}{3}$ ) e a integral

$$
\int_{W_{2}} \int_{\Theta_{2}} p_{2}\left(\theta_{2}, w_{2} \mid D_{\text {obs }}^{(n-k)}\right) \mathrm{d} \theta_{2} \mathrm{~d} w_{2}
$$

converge se $n-k \geq 3$ e $0<c_{2}<(n-k)+\frac{\left(\nu_{2}-3\right)}{2}$ (ou seja $n-k \geq 3$ e $0<f_{2}<$ $\frac{n-k+\nu_{2}-1}{3}$ ) e daí segue a conclusão.

Teorema 5.4.2 No modelo do Teorema 5.4.1 se a distribuição $\pi\left(\sigma_{x(i)}^{2}\right)$ for substituída por $\pi\left(\sigma_{x(i)}^{2}\right) \propto\left(\sigma_{x(i)}^{2}\right)^{-r_{1}}, r_{i}>0, i=1,2$, então uma condição suficiente 
para a existência da correspondente posteriori do modelo com mudança é que $k \geq 2$, $n-k \geq 2,0<r_{i}<\frac{1}{2}$ e $f_{i}<\frac{\nu_{i}}{2}+1-r_{i}, i=1,2$.

Demonstração: Como $p_{1}\left(\theta_{i}, w_{1} \mid D_{\text {obs1 }}^{(k)}\right)$ e $p_{2}\left(\theta_{2}, w_{2} \mid D_{\text {obs2 }}^{(n-k)}\right)$ estão sob as mesmas condições do Teorema 3.5.3 (que envolve o modelo $t$ sem mudança), então para cada $k$ fixado a integral

$$
\int_{W_{1}} \int_{\Theta_{1}} p_{1}\left(\theta_{1}, w_{1} \mid D_{\text {obs }}^{(k)}\right) \mathrm{d} \theta_{1} \mathrm{~d} w_{1}
$$

é finita se $k \geq 2,0<r_{1}<\frac{1}{2}$ e $0<f_{1}<\frac{\nu_{1}}{2}+1-r_{1}$ e a integral

$$
\int_{w_{2}} \int_{\Theta_{2}} p_{2}\left(\theta_{2}, w_{2} \mid D_{\mathrm{obs}_{2}}^{(k)}\right) \mathrm{d} \theta_{2} \mathrm{~d} w_{2}
$$

é finita se $n-k \geq 2,0<r_{2}<\frac{1}{2}$ e $0<f_{2}<\frac{\nu_{2}}{2}+1-r_{2}$ e daí segue a conclusão.

Teorema 5.4.3 No modelo do Teorema 5.4.1, se a distribuição de $\pi\left(\sigma_{i}^{2}\right)$ for substituída por $\pi\left(\sigma_{i}^{2}\right) \propto\left(\sigma_{i}^{2}\right)^{-s_{i}}, i=1,2$, e se os vetores de dados $\underset{\sim}{X^{(i)}}, \underset{\sim}{Y^{(i)}}$ sáo linearmente independentes para $i=1,2$ e tais que as componentes de $X^{(i)}$ (bem como as de $\left.\stackrel{\sim}{Y}^{(i)}\right)$ não são todas iguais, então a distribuição à posteriori do modelo com mudança correspondente é própria se $k \geq 3, n-k \geq 3, k+\nu_{1}>2 s_{1}-1$ e $n-k+\nu_{2}>2 s_{2}-1$.

Demonstração: Analogamente, a demonstração segue usando a relação em (5.4.1) e o Teorema 3.5.4.

Teorema 5.4.4 No modelo do Teorema 5.4.1 se as distribuições de $\pi\left(\sigma_{x(i)}^{2}\right)$ e $\pi\left(\sigma_{i}^{2}\right)$ forem substituídas respectivamente por $\pi\left(\sigma_{x(i)}^{2}\right) \propto\left(\sigma_{x(i)}^{2}\right)^{-r_{i}}$ e $\pi\left(\sigma_{i}^{2}\right) \propto\left(\sigma_{i}^{2}\right)^{-s_{i}}$ e os vetores de dados $\underset{\sim}{X^{(i)}}$ e $\underset{\sim}{Y}(i)$ são linearmente independentes para $i=1,2$ e as componentes de $\underset{\sim}{X^{(i)}}$ (bem como as de $\underset{\sim}{Y^{(i)}}$ ) não são todas iguais, então a condição necessária e suficiente para a existência da correspondente posteriori é que $k+r_{1}+$ $s_{1}>3,(n-k)+r_{2}+s_{2}>3,0<r_{i}<\frac{1}{2} e 0<s_{i}<\frac{\nu_{i}}{2}+2-r_{i}, i=1,2$. 
Demonstração: Usando a relação em (5.4.1) e o Teorema 3.5.5, a demonstração segue.

Observação: Nos teoremas 4.6.1 a 4.6.4, se considerarmos que os números de graus de liberdade não são conhecidos, mas são variáveis aleatórias discretas definidas em conjuntos finitos, as demonstrações seriam basicamente as mesmas.

\subsubsection{Condições no caso do modelo com mudança apenas em $\sigma_{x}^{2}$}

Teorema 5.4.5 No modelo estrutural $t$-dependente que permite mudança apenas em $\sigma_{x}^{2}$, com priori $\pi(\underset{\Theta}{\stackrel{*}{\theta}}) \propto p(k) \pi(w) \pi\left(\sigma_{i}^{2}\right) \prod_{i=1}^{2}\left(\pi\left(\sigma_{x(i)}^{2}\right)\right)$, onde $p(k)=P[K=k]$, $k \in \mathcal{K}$ (onde $\mathcal{K}$ é finito); $\pi\left(\sigma_{x(i)}^{2}\right) \sim I G\left(c_{i}, d_{i}\right), i=1,2, \pi\left(\sigma^{2}\right) \sim I G(f, g)$ e $\pi(w) \sim$ $I G\left(\frac{\nu}{2}, \frac{\nu}{2}\right)$, então uma condição suficiente para que a posteriori seja própria é que $n \geq 3$.

Demonstração: O objetivo é mostrar que $\sum_{k \in \mathcal{K}} \int_{W} \int_{\Theta} p\left(k, \underline{\theta}, w \mid D_{\text {obs }}\right) \mathrm{d} \theta \underset{\theta}{\mathrm{d} w}<\infty$, ou seja, $\sum_{k \in \mathcal{K}} \int_{W} \int_{\mathscr{X}} \int_{\Theta} p\left(k, w, \underset{\sim}{x}, \underset{\theta}{\theta} \mid D_{\text {obs }}\right) \mathrm{d} \underset{\sim}{\theta} \mathrm{d} \underset{\sim}{x} \mathrm{~d} w<\infty$,

onde $\Theta=\left\{\underset{\sim}{\theta}=\left(\alpha, \beta, \mu, \sigma_{x(1)}^{2}, \sigma_{x(2)}^{2}, \sigma^{2}\right)^{T} \in \mathbb{R}^{3} \times \mathbb{R}_{+}^{3}\right\}, W=\left\{w \in \mathbb{R}^{+}\right\}, \mathscr{X}=\{\underset{\sim}{x}=$ $\left.\left(x_{1}, \ldots, x_{n}\right)^{T}: x_{i} \in \mathbb{R}\right\}$ e $\mathcal{K}$ é um conjunto finito, onde

$$
\begin{aligned}
& \quad p\left(k, w, \underset{\sim}{x} \underset{\sim}{\theta} \mid D_{\text {obs }}\right) \propto L(k, w, \underset{\sim}{\theta} \mid D) \pi(k, w, \underset{\sim}{\theta}), \text { que é proporcional a } \\
& w^{-\left(\frac{3 n+\nu}{2}-\frac{1}{2}\right)}\left(\sigma^{2}\right)^{-(n+f+1)}\left(\sigma_{x(1)}^{2}\right)^{-\left(\frac{k}{2}+c_{1}+1\right)}\left(\sigma_{x(2)}^{2}\right)^{-\left(\frac{n-k}{2}+c_{2}+1\right)} \exp \left\{-\frac{1}{2}\left[\frac{\nu}{w}+\frac{2 d_{1}}{\sigma_{x(1)}^{2}}\right.\right. \\
& +\frac{2 d_{2}}{\sigma^{2}}+\frac{2 g}{\sigma^{2}}+\frac{\sum_{i=1}^{n}\left(Y-\alpha-3 x_{i}\right)^{2}}{\lambda \sigma^{2} w}+\frac{\sum_{i=1}^{n}\left(X_{i}-x_{i}\right)^{2}}{\sigma^{2} w}+\frac{\sum_{i=1}^{k}\left(x_{i}-\mu\right)^{2}}{\sigma_{x(1)}^{2} w} \\
& \left.\left.+\frac{\sum_{i=k+1}^{n}\left(x_{i}-\mu\right)^{2}}{\sigma_{x(1)}^{2} w}\right]\right\} .
\end{aligned}
$$

Seja $f\left(k, w, \underset{\sim}{x}, \underset{\sim}{\theta}, D_{\text {obs }}\right)$ a função que coincide com (5.4.2) quando substituímos a 
última parcela, $\frac{\sum_{i=k+1}^{n}\left(x_{i}-\mu\right)^{2}}{\sigma_{x(1)}^{2} w}$ por zero. Então

$$
\int_{W} \int_{\mathfrak{X}} \int_{\Theta} p\left(k, w, \underset{\sim}{x}, \underset{\sim}{\theta} \mid D_{\mathrm{obs}}\right) \mathrm{d} \underset{\sim}{\theta} \mathrm{d} \underset{\sim}{x} \mathrm{~d} w \leq \int_{W} \int_{\mathfrak{X}} \int_{\Theta} f\left(k, w, \underset{\sim}{x}, \underset{\sim}{\theta}, D_{\mathrm{obs}}\right) \mathrm{d} \underset{\underset{\sim}{\theta}}{\mathrm{d} \underset{\sim}{x} \mathrm{~d} w},
$$

onde

$$
\begin{aligned}
& \int_{x} \int_{\Theta} f\left(k, w, \underset{\sim}{x}, \underset{\sim}{\theta}, D_{\mathrm{obs}}\right) \mathrm{d} \underset{\sim}{\theta} \mathrm{d} \underset{\sim}{x} \propto \\
& \int_{x} \frac{w^{-\left(\frac{3 n+\nu-1}{2}\right)} \exp \left\{-\frac{\nu}{2 w}\right\}}{S_{x x}^{\frac{1}{2}}\left[2 d+\frac{S_{x_{1} x_{1}}}{w}\right]^{\frac{k+2 c_{1}-1}{2}}\left[2 g+\frac{\sum_{i=3}^{n}\left(X_{i}-x_{i}\right)^{2}}{w}+\frac{S_{x x} S_{Y Y}-S_{x Y}^{2}}{\lambda S_{x x} w}\right]^{n+f-1}} \mathrm{~d} \underset{\sim}{\sim} \\
& \leq \frac{1}{(2 d)^{\frac{k+2 c_{1}-1}{2}}} \int_{\mathscr{X}} \frac{w^{-\left(\frac{3 n+\nu-1}{2}\right)} \exp \left\{-\frac{\nu}{2 w}\right\}}{S_{x x}^{\frac{1}{2}}\left[2 g+\frac{\sum_{1=1}^{n}\left(X_{i}-x_{i}\right)^{2}}{w}+\frac{S_{x x} S_{Y Y}-S_{x Y}^{2}}{\lambda S_{x x} w}\right]^{n+f-1}} \mathrm{~d} \underset{\sim}{x} .
\end{aligned}
$$

A integral $\int_{X} \frac{w^{-\left(\frac{3 n+\nu-1}{2}\right)} \exp \left\{-\frac{\nu}{2 w}\right\}}{S_{x x}^{\frac{1}{2}}\left[2 g+\frac{\sum_{i=1}^{n}\left(X_{i}-x_{i}\right)^{2}}{w}+\frac{S_{x x} S_{Y Y}-S_{x Y}^{2}}{\lambda S_{x x} w}\right]^{n+f-1}} \mathrm{~d} \underset{\sim}{x}$ pode ser analisada facilmente aplicando-se a mesma transformação de variáveis usada na seção 2.8 (que a cada $(\underset{\sim}{x}, \underset{\sim}{X}, \underset{\sim}{Y})$ associa $(\underset{\sim}{z} \underset{\sim}{b}, c)=H(\underset{\sim}{x}, \underset{\sim}{X}, \underset{\sim}{Y})$, onde $H$ é a matriz de Helmert $)$. De fato, após a transformação, essa integral fica igual a

$$
\begin{aligned}
& \left.\int_{Z} \frac{w^{-\left(\frac{3 n+\nu-1}{2}\right)} \exp \left\{-\frac{\nu}{2 w}\right\}}{\left\|z_{x}\right\|\left[2 g+\frac{\| z_{x}-b}{w} x \|^{2}\right.}+\frac{\left(\sqrt{n} \bar{X}-z_{n}\right)^{2}}{w}+\frac{\|\mathcal{C} Y\|^{2} \operatorname{sen}^{2} \theta}{\lambda w}\right]^{n+f-1} \mathrm{~d} z \\
& \propto \int_{Z_{x}} \frac{w^{-\left(\frac{3 n+\nu-1}{2}\right)} \exp \left\{-\frac{\nu}{2 w}\right\}}{\left\|Z_{\sim}\right\|\left[2 g+\frac{\left\|z_{x}-b_{X}\right\|^{2}}{w}+\frac{\left\|C_{Y}\right\|^{2} \operatorname{sen}^{2} \theta}{\lambda w}\right]^{n+f-\frac{3}{2}}} \mathrm{~d} z \\
& \propto \int_{Z_{x}} \frac{\exp \left\{-\frac{\nu}{2 w}\right\}}{w^{\frac{n+\nu}{2}-f+1}\left\|{\underset{\sim}{z} x}_{x}\right\|\left[2 g w+\left\|{\underset{\sim}{z} x}_{x}-{\underset{\sim}{X}}_{X}\right\|^{2}+\frac{\left\|\mathcal{C}_{Y}\right\|^{2} \operatorname{sen}^{2} \theta}{\lambda}\right]^{n+f-\frac{3}{2}}} \mathrm{~d}{\underset{\sim}{x}}_{x} .
\end{aligned}
$$

Portanto, a integral da posteriori $\int_{W} \int_{X} \int_{\Theta} p\left(k, w, \underset{\sim}{x}, \underset{\sim}{\theta} \mid D_{\text {obs }}\right) \mathrm{d} \underset{\sim}{\theta} \mathrm{d} \underset{\sim}{x} \mathrm{~d} w$ é majorada por uma integral proporcional a

$$
\int_{W} \int_{Z_{x}} \frac{\exp \left\{\frac{-\nu / 2}{w}\right\}}{w^{\frac{n+\nu}{2}-f+1}\left\|\underset{\sim}{z_{x}}\right\|\left[2 g w+\| \underset{\sim}{\left.z_{x}-\underset{\sim}{b} X \|^{2}+\frac{\left\|\mathcal{C}_{Y}\right\|^{2} \operatorname{sen}^{2} \theta}{\lambda}\right]^{n+f-\frac{3}{2}}} \mathrm{~d}{\underset{\sim}{x}}_{x} \mathrm{~d} w\right.}
$$

que é muito semelhante à integral que aparece na demonstração do Teorema 3.5.3.

A regiāo de integração $W \times Z_{x}$ é particionada em $W \times R_{1}$ e $W \times R_{1}^{c}$, onde $R_{1}=\left\{z_{x} \in \mathbb{R}^{n-1}:\left\|z_{x}\right\|<\varepsilon\right\}$. 


\section{AVALIAÇÃO DA INTEGRAL NA REGIÃO $W \times R_{1}$}

Nessa região a integral pode ser majorada por

$$
\int_{W} \int_{\left\|Z_{x}\right\|<\varepsilon} \frac{\exp \left\{\frac{-\nu / 2}{w}\right\}}{w^{\frac{n+\nu}{2}-f+1}\left\|z_{x}\right\|[2 g w]^{n+f-\frac{3}{2}}} \mathrm{~d} z_{\sim} \mathrm{d} w
$$

que é proporcional a

$$
\int_{0}^{\infty} \frac{\exp \left\{\frac{-\nu / 2}{w}\right\}}{w^{\frac{3 n+\nu-1}{2}}} \mathrm{~d} w \int_{\left\|z_{x}\right\|<\varepsilon} \frac{1}{\left\|z_{\sim}\right\|} \mathrm{d} z_{\sim}
$$

que é finita se e somente se $\frac{3 n+\nu-1}{2}>1$ e $1<n-1$, isto é, $n \geq 3$.

\section{AVALIAÇÃO DA INTEGRAL NA REGIÃO $W \times R_{2}$}

Nessa região a integral pode ser majorada por

$$
\int_{W} \int_{\left\|Z_{x}\right\|<\varepsilon} \frac{\exp \left\{\frac{-\nu / 2}{w}\right\}}{w^{\frac{n+\nu}{2}-f+1}\|\underset{\sim}{z}\| \|\left[\left\|\underset{\sim}{z_{x}}-\underset{\sim}{b} X\right\|^{2}+\frac{\left\|\mathcal{L}_{Y}\right\|^{2} \operatorname{sen}^{2} \theta}{\lambda}\right]^{n+f-\frac{3}{2}}} \mathrm{~d}{\underset{\sim}{z}}_{x} \mathrm{~d} w
$$

que é proporcional ao produto

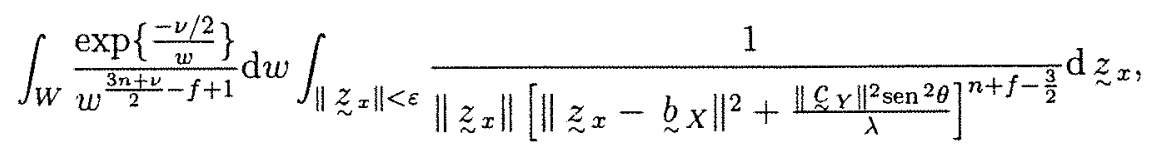

que é finita se e somente se $\frac{3 n+\nu-1}{2}>1$ e $1+2\left(n+f-\frac{3}{2}\right)>n-1$, isto é, $3 n+\nu>3$ e $n+2 f>1$.

Analisando simultaneamente essas regiões, concluímos que uma condição suficiente para que a posteriori seja própria é que $n \geq 3$.

Teorema 5.4.6 No modelo do Teorema 5.4 .5 se trocarmos a distribuição de $\pi\left(\sigma^{2}\right) \propto$ $I G(f, g)$ por $\pi\left(\sigma^{2}\right) \propto\left(\sigma^{2}\right)^{-s}, s>0$, e se $\underset{\sim}{X}$ e $\underset{\sim}{Y}$ forem linearmente independentes onde as componentes de $\underset{\sim}{X}$ e as de $\underset{\sim}{Y}$ não são todas iguais, então uma condição suficiente para a existência da posteriori é que $n \geq 3$. 
Demonstração: Substituindo $f$ por $s-1$ e $g$ por zero na demonstração do teorema anterior, temos que a integral da posteriori é majorada por uma integral proporcional a

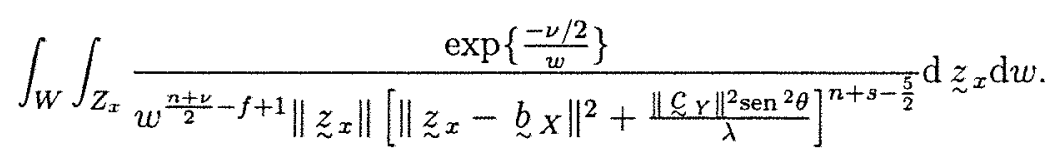

Essa integral é semelhante àquela que aparece na demonstração do Teorema 3.5.5. Para analisá-la particionamos a região de integração $W \times Z_{x}$ da mesma forma que no Teorema 3.5 .5 e concluímos que ela é finita se $\frac{n+\nu-2 f+2}{2}>1,1<n-1$ e $1+2\left(n+s-\frac{5}{2}\right)>n-1$, ou seja, $n \geq 3$ e $f<\frac{n+\nu}{2}$.

\subsection{Distribuição preditiva e avaliação do ajuste}

Podemos obter a distribuição preditiva e avaliar o ajuste do modelo $t$-independente com um ponto de mudança, de forma análoga ao modelo Normal com ponto de mudança da seção 4.5. Além disso, as seções 3.1 .3 e 3.1.4 (que tratam do modelo $t$-independente sem ponto de mudança) são também úteis para ajudar na dedução das fórmulas correspondentes. 


\section{Capítulo 6}

\section{Considerações Finais}

\subsubsection{Resultados do estudo sobre ponto de mudança}

Nesse trabalho fizemos uma análise bayesiana do modelo de regressão simples com erros de medida e que permite um único ponto de mudança numa seqüência de $n$ observações, sendo que essa "mudança" pode ocorrer em qualquer um dos $2^{5}$ ou $2^{6}$ subconjuntos de parâmetros, dependendo se o modelo é identificável ou não identificável respectivamente, isto é, manteve-se a forma da distribuição das observações (normal ou $t$-Student) e permitiu-se mudança apenas nos parâmetros dessas distribuições.

Foram estudados basicamente dois modelos. O primeiro, em que o ponto de mudança $k$ pode variar entre 1 e $n-1$ e o segundo em que o ponto de mudança pode variar entre 1 e $n$. No primeiro modelo o caso em que não há mudança ocorre apenas quando $\underset{\sim}{\theta_{1}}={\underset{\sim}{2}}_{2}$, caso contrário pelo menos um parâmetro muda. No segundo modelo não há mudança quando $k=n$ (ou $\underset{\sim}{\theta_{1}}=\underset{\sim}{\theta_{2}}$ ).

Nesse trabalho muitos resultados foram obtidos porém há também várias limitações e vários pontos que precisam ser ampliados ou mesmo completados.

1- Resultados no modelo Normal:

(a)- Algoritmos:

Para obter amostras das distribuições posterioris foram implementados apenas os 
algoritmos de Gibbs e M. Collapsed Gibbs (muito embora o algoritmo de MetropolisHastings em Gibbs, visto no capítulo 2, também possa ser implementado com base nos dados observados). Observou-se que o algoritmo M.Collapsed Gibbs, baseado no modelo que permite mudança em todos os parâmetros com particular priori própria "pouco informativa" apresentou um melhor desempenho que o algoritmo de Gibbs simples para o particular conjunto de dados simulados utilizado, independentemente se o modelo é ou não é identificável. Além disso aparentemente não foram observadas falhas na convergência desses algoritmos quando os critérios de diagnostico descritos no Apêndice foram empregados.

\section{(b)- Estimação e métodos para detectar mudança}

Para detetar se existe mudança no modelo identificável (ou não identificável), foram derivados três métodos:

(a) O método 1 que usa a distribuição à posteriori do ponto de mudança $k$ do modelo que permite mudança num subconjunto qualquer de parâmetros, onde $1 \leq k \leq n$. Este método detecta mudança e estima os parâmetros do modelo simultaneamente. (b) $\mathrm{O}$ método 2 que examina as distribuições marginais à posteriori das diferenças (ou quocientes) entre os parâmetros "antes" e "depois da mudança" no modelo que permite mudança em todos os parâmetros e $1 \leq k \leq n-1$.

(c) O método 3 que consiste em usar os critérios de seleção de modelos do Fator de Bayes, Pseudo-Fator de Bayes, Medida L e soma dos resíduos em valor absoluto (descritos na literatura), para comparar o modelo sem mudança com o modelo com mudança em qualquer subconjunto de parâmetros e $1 \leq k \leq n-1$, usando o mesmo conjunto de dados.

Utilizando um particular conjunto de dados simulados (dados gerados a partir do modelo com mudança em todos os parâmetros após o vigésimo ponto de uma seqüência de 50 observações) e escolhendo distribuições à priori "muito pouco 
informativas" vimos que os métodos 1 e 3 , implementados sob o modelo que permite mudança em todos os parâmetros, detectaram corretamente a existência de mudança independentemente do modelo ser ou não identificável. Entretanto é importante comentar que a medida $L$ baseada no traço da matriz $\left.L_{2} \underset{\sim}{Y}, \delta\right)$ não serviu para diferenciar entre os modelos com e sem mudança nesse exemplo; além disso, embora seja esperado que os valores das estimativas do Fator de Bayes sejam altos, as estimativas obtidas pelo método de Newton e Raftery (94) nos parecem muito grandes. Nesse caso, o método de Chib (95), descrito no capítulo 4, deveria ser implementado e as estimativas comparadas.

O método 2 não foi suficientemente preciso para detetar qual (quais) parmetro(s) está(ão) mudando em alguns dos exemplos rodados, principalmente no caso não identificável.

No modelo do método 1 , onde $1 \leq k \leq n$, foi feita uma análise descritiva da sensibilidade das estimativas a variações no valor do hiperparâmetro $c$ (onde $c$ é a probabilidade à priori de não haver mudança) com base em um particular conjunto de dados, e usando priori "muito pouco informativa". Essa análise sugeriu que as estimativas dos parâmetros da distr. à posteriori, bem como a distribuição marginal à posteriori de $k$, não são muito sensíveis à mudança no valor desse hiperparâmetro. (c)- Ajuste

Para avaliar a qualidade do ajuste derivamos as fórmulas da densidade preditiva de validação cruzada (CPO) e dos resíduos bayesianos de validação cruzada para o modelo com mudança onde $1 \leq k \leq n-1$. Nesse estudo, entretanto, a análise de resíduos está bastante limitada e deve ser mais explorada futuramente.

\section{(d)- Identificabilidade}

Para o particular conjunto de dados simulados da seção 4.7, os modelos com e sem restrição de identificabilidade com as correspondentes prioris "pouco informativas" 
não diferiram muito com relação a qualidade do ajuste, além disso, embora os comprimentos dos intervalos de credibilidade no modelo identificável são ligeiramente menores, os intervalos de $95 \%$ de credibilidade contiveram os valores verdadeiros dos parâmetros em ambos os modelos. Assim, levando em conta também o fato de que na prática dificilmente o valor de $\lambda=\sigma^{e} / \sigma_{u}^{2}$ é conhecido, adotar o modelo não identificável seria uma boa opção nesse particular exemplo.

Seria interessante rodar mais exemplos com dados simulados e ver se este padrão tende a se manter.

\section{(e)- Melhor modelo}

Com a metodologia apresentada na seção 4.3 é possivel amostrar facilmente de cada uma das $2^{5}$ (ou $2^{6}$, no caso não identificável) posterioris associadas aos modelos com mudança em todos os possíveis subconjuntos de parâmetros. Desse modo podemos escolher o "melhor modelo", usando por exemplo alguns dos critérios de seleção de modelos que compõe o método 3 acima.

\section{(f)- Distribuição preditiva}

Para gerar uma observação futura no modelo que permite mudança em todos os parâmetros basta simplesmente seguir os passos descritos na seção 4.5.1. O caso de mudança em apenas um subconjunto de parâmetros é totalmente análogo.

\section{(g)- Teoremas}

As condições para a existência da posteriori para certas prioris impróprias foram estudadas apenas para o modelo identificável. O estabelecimento das condições no caso do modelo não identificavel é totalmente análogo (Na seção 2.8 este estudo é feito para o modelo sem mudança).

\section{(h)- Exemplos}

Os exemplos apresentados nesse trabalho basearam-se apenas em dados simulados. Um exemplo com dados reais, como por exemplo os dados de Chang e Huang 
(97), complementaria esse estudo.

\section{2- Resultados no modelo t-Student:}

Embora os resultados teóricos (semelhantes aos resultados do modelo normal), sobre os modelos $t$-dependente e $t$-independente, com número de graus de liberdade conhecidos ou desconhecidos, estejam descritos no capítulo 5, falta ainda complementar esse estudo com exemplos numéricos. Os programas computacionais a serem usados no caso $t$ são basicamente os mesmos do caso normal, bastando fazer as devidas adaptações.

As condições para a existência da posteriori no modelo $t$, que foram desenvolvidas apenas para o caso $t$-dependente, podem ser estendidas (de forma análoga) para o caso $t$-independente tanto com pontos de mudança como sem pontos de mudaça.

\subsubsection{Perspectivas para trabalhos futuros}

Nesse trabalho analisamos apenas o modelo com erros aditivos normais e $t$-Student. Esse estudo pode ser facilmente estendido para o caso em que os erros têm distribuição elíptica e também para o caso de regressão linear múltipla tanto com erros aditivos como multiplicativos.

A seguir listamos mais alguns tópicos que acreditamos ser de interesse para ampliar e dar continuidade aos resultados obtidos.

- Investigação de métodos bayesianos para analisar a influência local no modelo com erros nas variáveis com ou sem pontos de mudança.

- Análise bayesiana do modelo linear com erros de medida e pontos de mudança onde os erros tem distribuição assimétrica.

- Análise bayesiana do modelo linear de calibração comparativa com erros nas variáveis e pontos de mudança no caso em que os erros são normais e t-Student. 
- Análise bayesiana do modelo linear generalizado com erros nas variáveis com ponto de mudança.

- Análise bayesiana de modelos não lineares com erros nas variáveis com e sem pontos de mudança.

- Estudo de um método global para detectar simultaneamente a presença de múltiplos pontos de mudança no modelo com erros nas variáveis (baseando-se no modelo PPM, "Partition Product Model", introduzido por Hartigan(1990) e Barry e Hartigan(1992)).

- Uso de procedimentos seqüenciais para detectar mudança em modelos estruturais com erros nas variáveis com mudança de regime markoviano. 


\section{Apêndice}

\section{Seção 2.4 Métodos Computacionais Descritos na Literatura}

\subsubsection{Classificação geral}

$\mathrm{Na}$ literatura existem vários procedimentos para aproximar quantidades de interesse na estatística bayesiana. Eles podem ser classificados basicamente em três grandes grupos: (1) os procedimentos de aproximação analíticos, (2) os procedimentos de simulação estáticos (ou "não iterativos") e (3) os procedimentos de simulação dinâmicos ("iterativos").

Os procedimentos analíticos nos permitem obter aproximações analíticas para a distribuição conjunta e para as distribuiçōes marginais à posteriori, como também para o valor esperado de qualquer função de $\underset{\sim}{\theta}$ à posteriori (incluindo os momentos por exemplo). O método mais simples usa um resultado assintótico para aproximar a distribuição à posteriori por uma distribuição Normal em torno da moda da posteriori ("Teorema do Limite Central Bayesiano"). A moda freqüentemente é obtida por meio de algoritmos de otimização numérica. Como Exemplo desses algorítmos temos o método de Newton, o método "BFGS" (Broyden, Fletcher, Goldfarb, Shano, que é um método de Quase-Newton) e o algoritmo EM. No caso da distribuição ser multimodal, pode-se aproximar a posteriori localmente, em cada uma das modas, pelas Normais (ou t's) correspondentes e tomar a distribuição da mistura dessas Normais (ou $t$ 's) como aproximação global da distribuição à posteriori. (Gelman 
et al., 1997, páginas 234 e 275). Outro procedimento, também baseado na teoria assintótica, é o método de Laplace (Tanner, 1993), conhecido principalmente por fornecer aproximações analíticas mais precisas (de segunda ordem) para o valor esperado de funções de $\underset{\sim}{\theta}$ à posteriori (como, por exemplo, os momentos, a própria função geradora de momentos e a densidade preditiva à posteriori), ele pode ser usado também para obter aproximações analíticas de segunda ordem para as distribuições marginais à posteriori (Carlin e Louis, 2000).

O principal problema com os métodos de aproximação pela Normal e de Laplace é que o número de parâmetros do modelo precisa ser pequeno para que esses métodos possam fornecer aproximações razoáveis (assim eles podem ser usados, por exemplo, para estudar subconjuntos de parâmetros à posteriori ou distribuições condicionais à posteriori). Esses métodos requerem ainda que o tamanho da amostra seja "grande" (e não sabemos quão grande é "grande"), requerem também que certas condições de regularidade estejam satisfeitas, além disso dependem também da parametrização (o método de Laplace, por exemplo, requer ainda que a posteriori seja unimodal).

Outro procedimento analítico alternativo para o cálculo de integrais na estatística bayesiana é utilizar integração numérica. Os métodos mais conhecidos de integração numérica são os da "Quadratura Gaussiana", o "método de Romberg" (Lange, 1999) e o método "Quadratura de Gauss-Hermite" (Smith, 1987). Esses métodos, entretanto, são usados apenas quando o número de parâmetros do problema é pequeno.

Outra maneira de obter aproximações para as quantidades de interesse na inferência estatística bayesiana é usar simulação. Existem dois tipos de técnicas de simulação: as estáticas (ou "não iterativas") e as dinâmicas (ou "iterativas").

Nas técnicas estáticas geramos "amostras aleatórias" diretamente da distribuição à posteriori ou indiretamente por meio de uma distribuição conhecida que serve como intermediária e portanto a qualidade das aproximações pode ser melhorada sempre 
que aumentarmos o número de elementos gerados.

Os métodos de simulação direta mais conhecidos são: o método da "grade de pontos" e o método da "aproximação trapezoidal" (Gelman et al., 2000). No método da "grade de pontos" a idéia é aproximar uma distribuição à posteriori contínua por uma discreta, calculando-se o valor da posteriori (ou de seu núcleo) nos pontos da grade e depois gerando uma amostra a partir da distribuição discreta correspondente.

Quando os valores da posteriori calculados nos pontos da grade são ligados por segmentos de reta no caso unidimensional, seções de um plano no caso bidimensional, etc. e uma amostra é gerada a partir dessa aproximação, obtemos o chamado "método trapezoidal". O problema com essas técnicas é que se o espaço paramétrico não for bem pequeno elas são inviáveis para um estudo conjunto dos parâmetros.

Nos métodos de simulação indiretos, as amostras não são geradas diretamente da posteriori, mas sim de uma distribuição $g(\theta)$ conhecida (a função $g(\underset{\theta}{\theta})$ é também chamada de "função importance sampling").

$\mathrm{Na}$ literatura os métodos mais conhecidos de simulação indireta são: o "método de rejeição" e o método "weighted bootstrap", que é bastante semelhante ao "sampling-importance-resampling" (Tanner, 1993; Geweke, 1989; Smith and Gelfand, 1992).

Quando o objetivo é apenas obter aproximações para o valor esperado de funções de $\underset{\sim}{\theta}$ à posteriori (e além disso não sabemos gerar amostras diretamente da posteriori mas sim de uma distribuição $g(\theta)$ conhecida), então podemos usar um método denominado "importance sampling" (Geweke, 1989). Esse procedimento, para essa finalidade é mais eficiente do que gerar uma amostra (usando qualquer um dos métodos mencionados acima) e depois obter as estimativas das quantidades desejadas (por exemplo, por meio do método simples de Monte Carlo para o cálculo de integrais). 
Os três métodos acima apresentam limitações importantes nas aplicações, pois eles são eficientes apenas quando a função $g(\underset{\theta}{\theta})$ (da qual geramos as amostras) for aproximadamente uniformemente proporcional ao núcleo da posteriori para todo $\underset{\sim}{\theta}$ do domínio da posteriori. O problema de achar uma distribuição $g$ com essas características, e que ao mesmo tempo seja simples de ser amostrada, pode ser uma tarefa praticamente impossível se a dimensão do espaço de parâmetros for grande.

Como pudemos ver, todos os métodos de aproximação analítica como os de simulação não iterativa têm limitações nas aplicações. Gelman et al. (1997) sugerem o uso combinado desses procedimentos para se obter melhores aproximações. Um exemplo (descrito na página 312 de seu livro) considera primeiramente uma aproximação da distribuição à posteriori por uma mistura de Normais (ou t's) a partir dessa distribuição aproximada, gera-se uma amostra de valores de $\underset{\sim}{\theta}$ e a seguir utiliza-se o "algoritmo de rejeição" ou o algoritmo "weighted bootstrap" para "filtrar" essa amostra. Note que aqui a distribuição da mistura está fazendo o mesmo papel da distribuição "importance sampling" $g(\underset{\sim}{\theta})$ descrita anteriormente, outro método semelhante é combinar o "método trapezoidal" com o algoritmo de rejeição (esse método é descrito na página 305 de Gelman et al., 1997).

Segundo Gelman et al. (1997), mesmo utilizando essas combinações (de métodos), ainda assim as simulações obtidas a partir dessas aproximações podem não ser adequadas, dependendo do modelo que se está estudando. Em tais casos eles sugerem que a amostra gerada por esses métodos seja utilizada para se obter "bons" valores iniciais, que por sua vez são usados em procedimentos iterativos (ou dinâmicos) de simulação, como os métodos MCMC ("Markov Chain Monte Carlo").

Um método de simulação MCMC tem por objetivo (poder) gerar uma cadeia de Markov cuja distribuição estacionária coincida com a distribuição alvo $\pi(\theta)$ da qual queremos amostrar (por exemplo, a posteriori). Usando as observaçōes geradas por 
essa cadeia podemos explorar aspectos de interesse sobre $\pi(\underset{\sim}{\theta})$ como, por exemplo, os momentos, os quantis e as densidades marginais, como também podemos gerar amostra da distribuição preditiva à posteriori (com a qual podemos explorar certos aspectos dessa distribuição).

Existe na literatura uma quantidade muito grande de algoritmos do tipo MCMC (ver os livros de Chen, 2000, e Robert e Casella, 1999). Os algoritmos mais populares são o "amostrador de Gibbs" (Geman e Geman, 1984; Gelfand e Smith, 1990) e o algoritmo de Metropolis-Hastings (Metropolis et al., 1993; Hastings, 1970).

Neste trabalho analisamos o modelo com erros nas variáveis Normal e $t$-Student, com e sem mudança nos parâmetros, usando métodos $\mathrm{MCMC}$ como técnica principal (embora algumas das técnicas mencionadas anteriormente também sejam utilizadas para a obtenção de bons valores iniciais) . A razão dessa escolha (métodos MCMC) é que o número de "parâmetros" do modelo pode ser bem grande, especialmente quando se trabalha com a posteriori baseada nos dados completos, que envolve $n+p$ "parâmetros" onde $n$ é o tamanho da amostra. ( O valor de p no caso por exemplo do modelo de regressão linear simples com restrição de identificabilidade e com ponto de mudança é onze).

Embora possamos trabalhar com a "posteriori baseada nos dados observados", que no caso da regressão simples com um ponto de mudança e restrição de identificabilidade envolve apenas 11 parâmetros (o que é relativamente pequeno), a ídéia nesse trabalho é propor um método geral que possa também vir a ser usado em em regressão múltipla.

Os métodos $\mathrm{MCMC}$ são muito poderosos, não tendo restrições quanto ao número de parâmetros envolvidos e ao mesmo tempo são relativamente simples de serem implementados. 


\subsubsection{Métodos MCMC: idéias básicas}

Quando uma distribuição $\pi(\underset{\sim}{\theta}), \underset{\sim}{\theta} \in E \subset \mathbb{R}^{p}$ (por exemplo, a posteriori) é difícil de ser amostrada (ou porque $p$ é muito grande, ou porque a própria distribuição é intratável) então uma solução para o problema seria obtida se pudéssemos construir uma cadeia de Markov homogênea e aperiódica com "espaço de estados" $E$ e distribuição estacionária igual a $\pi(\underset{\sim}{\theta})$, e daí então amostrar dessa cadeia. Isso porque se uma cadeia de Markov é irredutível e aperiódica a distribuição estacionária (ou distribuição invariante) é única e coincide com a distribuição limite (ou distribuição de equilibrio), portanto, para $n$ "suficientemente grande" a observação gerada na $n$-ésima iteração (da cadeia) "essencialmente" é uma observação da distribuição de $\pi(\underset{\sim}{\theta})$ (assim como todas as observações geradas a partir da $(n+1)$-ésima iteração).

Para implementar a idéia acima temos na literatura os algoritmos denominados MCMC ("Markov-Chain-Monte Carlo"). Num algoritmo MCMC constrói-se um núcleo de transição de tal modo que a cadeia de Markov gerada por ele tenha distribuição estacionária igual a $\pi(\underset{\sim}{\theta})$. Entretanto, para que a distribuição limite dessa cadeia exista e coincida com $\pi(\theta)$ (onde $\pi(\underset{\sigma}{\theta})$ é a única distribuição estacionária da cadeia), é necessário que ela seja irredutível e aperiódica. Na literatura muitos autores têm estabelecido condições para a convergência de vários desses algoritmos MCMC. Por exemplo, Roberts e Smith (1994) estabelecem condições suficientes relativamente simples sobre $\pi(\underset{\theta}{\theta})$ de modo que as cadeias induzidas pelos algoritmos de Gibbs e Metropolis-Hastings (que tem $\pi(\underset{\sim}{)}$ como distribuição estacionária) sejam irredutíveis e aperiódicas (e portanto convirjam em distribuição para uma variável com distribuição $\pi(\theta)$ ). Outra referência é Roberts e Tweedie (1996) que mostra a ergodicidade geométrica de uma classe bem ampla de algoritmos do tipo MetropolisHastings. O problema é que mesmo quando condições teóricas estão "satisfeitas" pode não ocorrer convergência dessas cadeias. Por exemplo, uma cadeia pode ser tec- 
nicamente irredutível mas na prática se comportar como se fosse redutível quando a probabilidade de transição de um determinado estado para outro é próxima de zero. Isso ocorre, por exemplo, quando a densidade $\pi(\underset{\sim}{\theta})$ tem várias modas (máximos locais) e a massa da densidade entre essas modas (embora positiva) é bem pequena (nesse caso se o ponto inicial da cadeia pertence à região em torno de uma das modas e essa região tem uma massa substancial da densidade, então a cadeia tenderá a não sair dessa região.

Nesse trabalho não vamos explorar resultados teóricos sobre condições de convergência (como os de Roberts e Tweedie, 1996; Meyn e Tweedie, 1993; Roberts e Smith, 1994), mas vamos simplesmente fazer uma análise exploratória (a partir dos dados simulados) para verificar se houve falha na convergência desses algoritmos, utilizando diagnósticos de convergência. Na literatura existem muitas técnicas de diagnóstico com essa finalidade (ver Best, Cowler e Venes, 1996, Cowles e Carlson, 1996, e Mangersen et al., 1999). É muito importante ressaltar entretanto que nenhuma dessas técnicas fornece uma "prova" de convergência (a começar pelo simples fato de que apenas um número finito de observações é analisado). Na subseção 2.4.6 apresentamos algumas das técnicas de diagnóstico mais conhecidas na literatura.

Os algoritmos MCMC mais conhecidos são os algoritmos de Gibbs e o MetropolisHastings. Entretanto, na literatura existe uma grande quantidade de métodos, incluindo variações dos algoritmos de Gibbs e Metropolis-Hastings, como também combinações desses algorimos, como os métodos híbridos (ver Chen, 2000, capítulo 2. e Robert e Casella, 1999). Entretanto, nessa seção descrevemos apenas os métodos básicos de Gibbs e Metropolis-Hastings. Nas subseções 2.4 .3 e 2.4.4 descrevemos duas variaçōes do método de Gibbs, destinadas a acelerar a convergência do algoritmo original. o "grouped Gibbs" e o "collapsed Gibbs", e na subseção 2.4.5 descrevemos o algorimto hibrido de Metropolis-Hastings em Gibbs. 


\section{$\mathrm{O}$ algoritmo de Metrópolis-Hastings}

Seja $\pi(\underset{\sim}{\theta}), \underline{\theta} \in A \subset \mathbb{R}^{p}$ a distribuição alvo da qual desejamos amostrar (por exemplo, a posteriori $\left.p\left(\underset{\sim}{\theta} \mid D_{\text {obs }}\right)\right)$.

No algoritmo de Metropolis-Hastings, primeiramente escolhe-se arbitrariamente uma densidade (ou núcleo) de transição (auxiliar) em uma etapa $q\left(\underset{\sim}{\theta_{b}} \mid{\underset{\sim}{a}}_{a}\right), \forall{\underset{\sim}{a}}_{a}, \underset{\sim}{\theta_{b}}$, de uma cadeia com espaço de estados igual ao domínio de $\pi(\underset{\sim}{\theta})$ (o conjunto $A$ ). Esta densidade deve ser tal que na $(i+1)$-ésima iteração do algoritmo possamos gerar $\underset{\sim}{*}$ a partir de $q\left(\cdot \mid{\underset{\sim}{\theta}}^{(i)}=\underset{\sim}{\theta}\right), \forall \underset{\sim}{\forall} \in A$. Além disso, quanto mais próxima $q\left(\underset{\sim}{\stackrel{*}{\theta}} \mid{ }_{\sim}^{(i)}=\underset{\sim}{\theta}\right)$ estiver de $\pi(\underset{\theta}{\theta})$, mais eficiente será o algoritmo, ou seja, $q(\cdot \underbrace{(i)}_{\theta^{(i)}}$ faz um papel mais ou menos semelhante à função "importance sampling" citada na subseção 2.4.1.

Assim, em cada iteração do algoritmo de Metropolis-Hastings amostramos $\underset{\sim}{\stackrel{*}{d}}$ de $q\left(\cdot \mid \theta^{(i)}\right)$ e tomamos

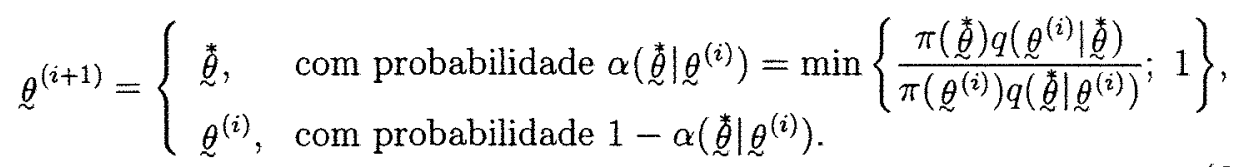

$\mathrm{Na}$ prática, o algoritmo de M-H (Metropolis-Hastings) pode ser implementado da seguinte maneira:

Passo 0: Escolher um ponto inicial ${\underset{\theta}{(0)}}^{(0)}=\left(\theta_{1}^{(0)}, \theta_{2}^{(0)}, \ldots, \theta_{p}^{(0)}\right)^{T}$ e fazer $i=0$.

Passo 1: Gerar um ponto $\underset{\ddot{\theta}}{\stackrel{*}{*}}=\left(\stackrel{*}{\theta}_{1}, \ldots, \stackrel{*}{\theta}_{p}\right)^{T}$ da densidade de transição auxiliar $q(\cdot \mid \underset{\sim}{\underbrace{(i)}})$ e gerar $U$ de uma $U(0,1)$.

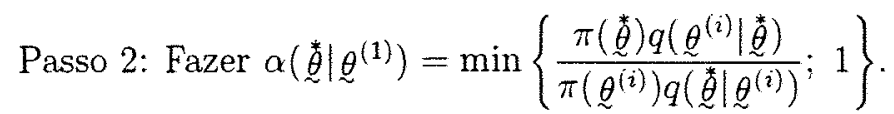

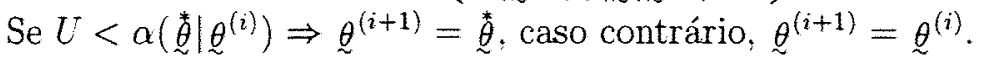

Passo 3: Fazer $i=i+1$ e voltar ao passo 1 .

\section{Observações}




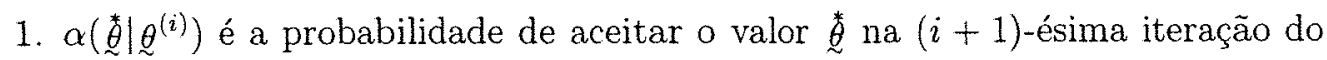
algoritmo de $\mathrm{M}-\mathrm{H}$.

2. É bem simples ver que o algoritmo de $\mathrm{M}-\mathrm{H}$ acima define uma cadeia de Markov com núcleo de transição (ou probabilidade de transição no caso discreto) do estado $\underset{\sim}{\theta_{a}}$ na $i$-ésima iteração para o estado ${\underset{\sim}{b}}_{b}$ na $(i+1)$-ésima iteração dada por

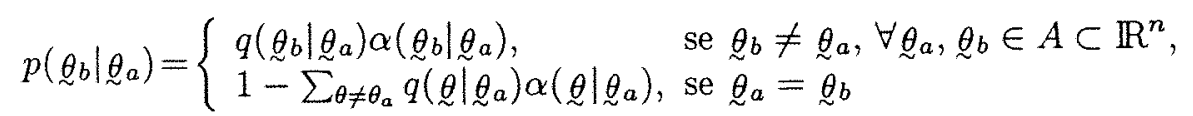

3. Usando o núcleo de transição $p\left(\underset{b}{\theta_{b}} \mid \theta_{a}\right)$ acima, é simples também verificar que $\pi(\underset{\theta}{\theta})$ satisfaz a condição de reversibilidade $\pi\left(\theta_{a}\right) p\left(\underset{\sim}{\theta_{b}} \mid \underline{\sim}_{a}\right)=\pi\left(\theta_{b}\right) p\left({\underset{\sim}{a}}_{a} \mid \underline{\sim}_{b}\right)$, $\left.\forall \underset{\sim}{\theta_{a}}, \underset{\sim}{\theta_{b}}\right)$, o que implica que $\pi(\underset{\sim}{\theta})$ é uma distribuição estacionária da cadeia definida por esse núcleo (ou seja, o algoritmo proposto induz uma CM com distribuição estacionária igual a $\pi(\underset{\theta}{\theta})$ ).

4. Não é necessário conhecer a constante de normalização da distribuição alvo $\pi(\theta)$, já que ela se cancela na expressão entre chaves dada em (6.0.1).

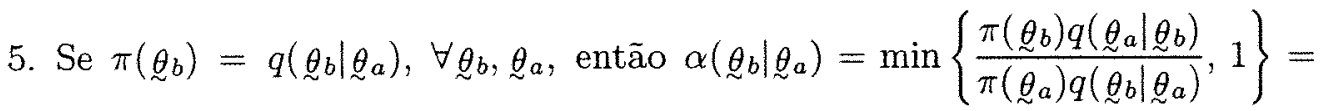
1. Nesse caso, o valor $\underset{\sim}{*}$ gerado na $(i+1)$-ésima iteração pela densidade de transição auxiliar $q\left(\cdot \mid{\underset{\theta}{\theta}}^{(i)}\right)$ é aceito com probabilidade $\alpha\left(\underset{\sim}{*} \mid \underset{\sim}{\theta^{(i)}}\right)=1$ (isso quer dizer que se a densidade auxiliar coincide com a densidade alvo aceitamos o candidato $\underset{\sim}{*}$ com probabilidade 1$)$.

6. No caso particular em que $q\left(\underset{b}{\theta_{b}} \mid{\underset{\sim}{a}}_{a}\right)=q\left({\underset{\sim}{a}}_{a} \mid \theta_{b}\right), \forall \underline{\sim}_{a},{\underset{\theta}{b}}_{b}$, caímos no algoritmo de Metropolis (Metropolis et al., 1953) e nesse caso $\alpha\left(\theta_{b} \mid{\underset{a}{a}}_{a}\right)=\min \left\{\frac{\pi\left(\theta_{b}\right)}{\pi\left(\underline{\sigma}_{a}\right)}, 1\right\}$ (e portanto $\alpha\left(\underline{\theta}^{\theta} \mid \underline{\theta}^{(i)}\right)=\min \left\{\frac{\pi(\stackrel{*}{\theta})}{\pi\left(\underline{\theta}^{(i)}\right)}, 1\right\}$ ).

7. No caso particular em que $q\left({\underset{\sim}{b}}_{b} \mid \theta_{a}\right)$ não é função de ${\underset{\sim}{a}}_{a}, \forall \theta_{a}, \theta_{b},\left(q\left(\underline{\sim}_{b} \mid \theta_{a}\right)=\right.$ 
$\left.h\left(\theta_{b}\right)\right)$, caímos no algoritmo denominado "Independence Chain MetropolisHastings" (Tierney, 1994) e nesse caso

$$
\alpha\left(\underline{\theta}_{b} \mid \underline{\theta}_{a}\right)=\min \left\{\frac{\pi\left(\underline{\theta}_{b}\right)}{\pi\left(\underline{\theta}_{a}\right)} \frac{h\left(\underline{\theta}_{a}\right)}{h\left(\underline{\theta}_{b}\right)}, 1\right\}
$$

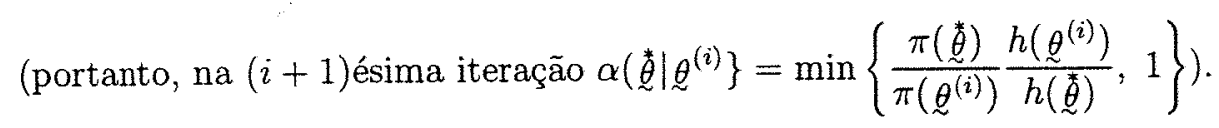

8. No caso particular em que $\pi(\underset{\sim}{\theta})$ pode ser escrita como $\pi(\underset{\sim}{\theta}) \propto \psi(\underset{\sim}{\theta}) . h(\underset{\sim}{\theta})$, onde $h(\underset{\sim}{\theta})$ é uma densidade que pode ser facilmente amostrada e $\psi(\underset{\sim}{\theta})$ é uma função uniformemente limitada, então tomando-se a função de transição $q\left(\theta_{b} \mid \theta_{a}\right)=$ $h\left({\underset{\sim}{\theta}}_{b}\right), \forall \underbrace{}_{a}, \underline{\sim}_{b}$, então

$$
\alpha\left(\underline{\theta}_{b} \mid \underline{\theta}_{a}\right)=\min \left\{\frac{\psi\left(\theta_{b}\right) h\left(\theta_{b}\right)}{\psi\left(\underline{\sigma}_{a}\right) h\left(\underline{\theta}_{a}\right)} \frac{h\left(\theta_{a}\right)}{h\left(\underline{\sim}_{b}\right)} ; 1\right\}=\min \left\{\frac{\psi\left(\theta_{b}\right)}{\psi\left(\underline{\sim}_{a}\right)}, 1\right\}
$$

(e portanto na $(i+1)$-ésima iteração $\alpha\left(\underset{\sim}{\theta} \mid \theta^{(i)}\right)=\min \left\{\frac{\psi(\underset{\theta}{\theta})}{\psi\left(\underset{\theta^{(i)}}{ }\right)}, 1\right\}$ (ver Chib e Greenberg, 1995)).

9. Outras escolhas para a função de transição auxiliar $q$ são descritas em Chib e Greenberg (1995).

10. Quando o espaço (amostral) é contínuo e $\pi \underset{\sim}{(\theta)}$ é unimodal com caudas não muito pesadas, uma escolha conveniente de $q\left(\cdot \mid \theta^{(i)}\right)$ é a distribuição Normal (pela simetria e pela simplicidade para gerar amostras).

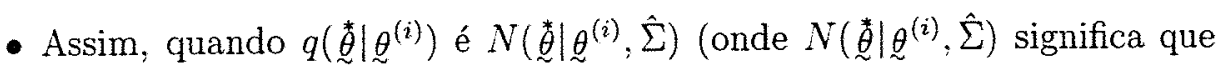
$\left.\stackrel{*}{\theta} \sim N\left(\stackrel{\theta}{\theta}^{(i)}, \hat{\Sigma}\right)\right)$, temos que

$$
q\left(\stackrel{\vec{\theta}}{\underset{\theta}{\theta^{(i)}}}{ }^{(i)}=\frac{1}{\sqrt{2 \pi}}|\hat{\Sigma}| \exp \left\{-\frac{1}{2}\left(\underset{\theta}{\theta}-\underline{\sim}^{(i)}\right)^{\top} \hat{\Sigma}\left(\stackrel{*}{\underline{\theta}}-\underline{\sim}^{(i)}\right)\right\}\right.
$$

e portanto

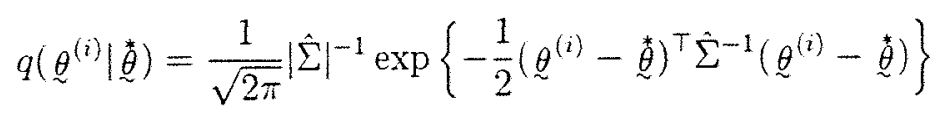




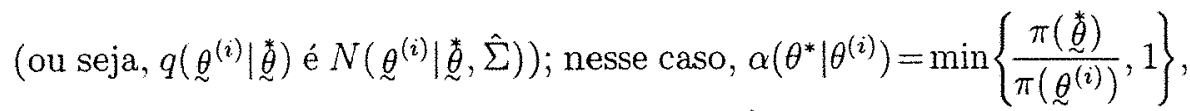
onde $\underset{\sim}{*}$ é gerado de $q\left(\cdot \mid \mathscr{\theta}^{(i)}\right)$ que é $N\left({\underset{\sim}{\theta}}^{(i)}, \hat{\Sigma}\right)$ e temos o algoritmo de Metropolis.

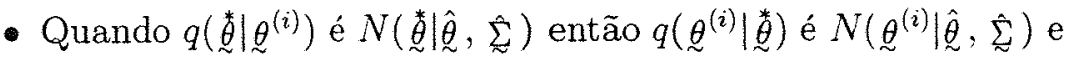

$$
\frac{q\left(\stackrel{*}{\theta} \mid \underline{\theta}^{(i)}\right)}{q\left(\underline{\theta}^{(i)} \mid \underset{\sim}{*}\right)}=\frac{\exp \left\{-\frac{1}{2}(\underset{\theta}{*}-\hat{\theta})^{\top} \hat{\sim}^{-1}(\stackrel{*}{\theta}-\hat{\theta})\right\}}{\exp \left\{-\frac{1}{2}\left(\underline{\theta}^{(i)}-\hat{\theta}\right)^{\top} \hat{\Sigma}^{-1}\left(\underline{\sim}^{(i)}-\hat{\theta}\right)\right\}},
$$

onde $\underset{\theta}{*}$ é gerado de $N(\underset{\theta}{\hat{\theta}}, \underset{\sim}{\hat{\Sigma}})$. Nesse caso temos o algoritmo "Independence Metropolis-Hastings".

Uma possível escolha para $\underset{\sim}{\hat{\theta}}$ é a moda de $\pi(\underset{\sim}{\theta})$ (que pode ser obtida por algum método de otimização como o BFGS (Gill et al., 1981) ou o algoritmo EM). Uma possível escolha para $\underset{\sim}{\hat{\Sigma}}$ é $-\left[\frac{\partial^{2} \log \pi(\underline{\theta})}{\partial \underset{\sim}{\theta} \partial_{\sim}^{\top}{ }^{\top}}\right]^{-1}$ calculada no ponto $\hat{\theta}$.

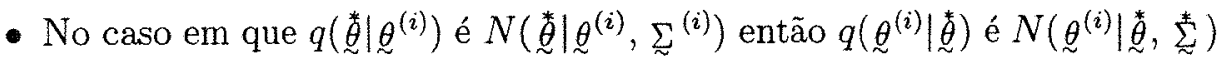
$\mathrm{e}$

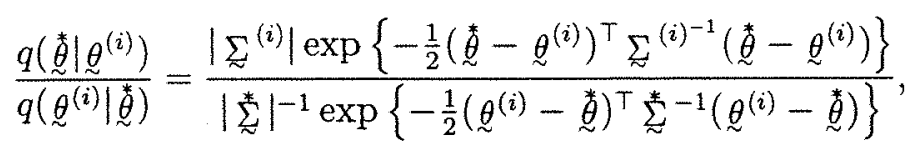

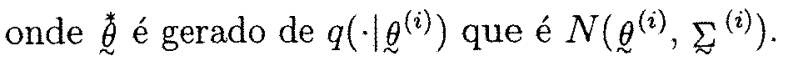

Uma possível escolha para $\sum_{\sim}^{(i)}$ e $\sum_{\sim}^{*}$ é calcular $\left[-\frac{\partial^{2} \log \pi(\theta)}{\partial \theta^{\partial} \theta}\right]^{-1}$ nos ponto $\stackrel{\theta}{\sim}^{(i)}$ e $\stackrel{*}{\stackrel{*}{*}}$, respectivamente.

Quando as caudas de $\pi(\underset{\sim}{\theta})$ forem "pesadas" outra alternativa conveniente para $q\left(\cdot \mid \underline{\theta}^{(i)}\right)$ é a distribuição $t$-Student.

11. Para a eficiência do algoritmo de $\mathrm{M}-\mathrm{H}$ é importante escolher bem os parâmetros de dispersão da distribuição de transição auxiliar $q\left(\cdot \mid \underline{\theta}^{(i)}\right)$. No caso do algoritmo de Metropolis, Roberts et al. (1996) mostraram que se $\pi(\underset{\theta}{\theta})$ e $q(\cdot \mid \underbrace{(i)})$ são normais univariadas com parâmetros de dispersão $\Sigma$ e $\hat{\Sigma}$, respectivamente, então, $\hat{\Sigma}=(2,4)^{2} \Sigma$ é a escolha "ótima". ou seja. que minimiza a 
auto-correlação de primeira ordem da cadeia, e que conseqüentemente leva a uma taxa de aceitação de aproximadamente $44 \%$ (no caso multivariado os autores mostraram que essa escolha leva a uma taxa de aceitação de aproximadamente $23 \%$ para $p>5$ ). Baseados nesse resultado, Gelman et al. (1997) sugerem que no caso não Normal iniciemos o processo usando $\hat{\Sigma}=$

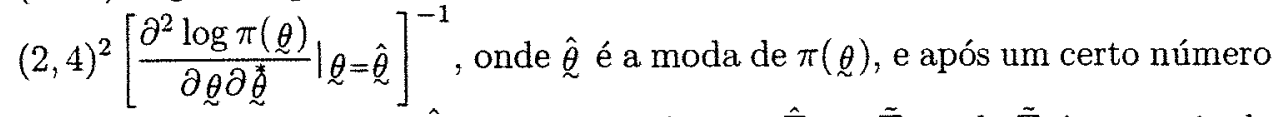
de iterações atualizemos $\hat{\Sigma}$ da seguinte forma: $\hat{\Sigma}=c \tilde{\Sigma}$, onde $\tilde{\Sigma}$ é a matriz de covariância à posteriori obtida a partir dos dados simulados e $c$ é uma constante a ser ajustada de tal modo que a taxa de aceitação da cadeia $\alpha(\underset{\sim}{\stackrel{*}{*} \mid \underbrace{(i)})}$ seja de aproximadamente $44 \%$ no caso unidimensional e $23 \%$ no caso multidimensional.

12. Nos casos mais complexos, escolher a densidade auxiliar de transição como uma mistura de normais (ou $t$ 's) pode ser uma solução (por exemplo, se $\pi(\underset{\sim}{\theta})$ é multimodal).

13. Para melhorar a estabilidade do algoritmo é melhor trabalhar com a escala

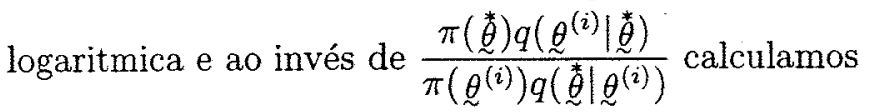

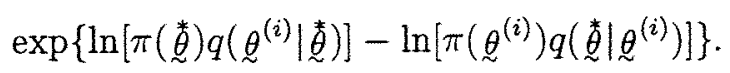

\section{$\mathrm{O}$ algoritmo original de Gibbs}

Seja $\pi(\underset{\theta}{\theta}), \underset{\theta}{\theta} \in A \subset \mathbb{R}^{p}$; a distribuição de interesse (por exemplo, a posteriori) e suponhamos que amostrar dessa distribuição é extremamente complicado, mas que as distribuições condicionais completas $\pi\left(\theta_{i} \mid \theta_{(-i)}\right), i=1, \ldots, p$ (ou $\pi\left(\theta_{i} \mid \theta_{(-i)}, D_{\text {obs }}\right)$, no caso em que $\pi(\underset{\theta}{\theta})$ é uma distribuição à posteriori), são distribuições totalmente 
conhecidas e podem ser "facilmente" amostradas por algum método, então, nesse caso, o algoritmo de Gibbs é uma alternativa. A idéia de gerar dados das distribuiçōes condicionais completas foi discutida pela primeira vez por Geman e Ge$\operatorname{man}(1984)$.

O Algorimo de Gibbs na sua forma mais simples é descrito a seguir.

Passo 0: Escolha um valor inicial $\theta^{(0)}=\left(\theta_{1}^{(0)}, \ldots, \theta_{p}^{(0)}\right)^{T}$ e faça $i=0$ (onde $i$ é o contador das iterações);

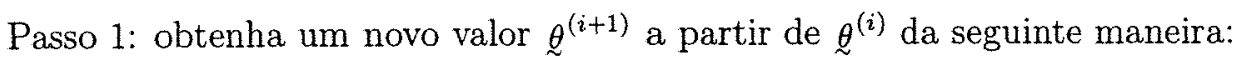

- gere $\theta_{1}^{(i+1)}$ de $\pi\left(\theta_{1} \mid \theta_{2}^{(i)}, \theta_{3}^{(i)}, \theta_{4}^{(i)}, \ldots, \theta_{p}^{(i)}\right)$,

- gere $\theta_{2}^{(i+1)}$ de $\pi\left(\theta_{2} \mid \theta_{1}^{(i+1)}, \theta_{3}^{(i)}, \theta_{4}^{(i)}, \ldots, \theta_{p}^{(i)}\right)$,

- gere $\theta_{3}^{(i+1)}$ de $\pi\left(\theta_{3} \mid \theta_{1}^{(i+1)}, \theta_{2}^{(i+1)}, \theta_{4}^{(i)}, \ldots, \theta_{p}^{(i)}\right)$,

- gere $\theta_{p}^{(i+1)}$ de $\pi\left(\theta_{p} \mid \theta_{1}^{(i+1)}, \theta_{2}^{(i+1)}, \theta_{3}^{(i+1)}, \ldots, \theta_{p-1}^{(i+1)}\right)$,

Passo 2: faça $i=i+1$ e volte ao passo 1 (até a convergência).

\section{Observações}

1. O passo 1 constitui um ciclo ou uma iteração do algoritmo de Gibbs.

2. As componentes $\theta_{i}$ podem ser escalares ou vetores.

3. $\pi\left(\theta_{i} \mid \theta_{(-i)}\right)$ pode ser uma distribuição desconhecida mas deve ser facilmente amostrada (por exemplo, pelo algoritmo de rejeição adaptativo (Gilks, 1995), pelo algoritmo de $\mathrm{M}-\mathrm{H}$, ou por qualquer outro método).

4. O algoritmo original de Gibbs descrito acima define uma CM com núcleo de transiçāo (ou probabilidade de transição) do estado $\underline{\theta}^{(i)}$ para o estado $\underline{\theta}^{(i+1)}$ 
dado por

$$
p\left(\theta^{(i+1)} \mid \underline{\theta}^{(i)}\right)=\prod_{j=1}^{p} \pi\left(\theta_{j}^{(i+1)} \mid \theta_{k<i}^{(i+1)}, \theta_{k>i}^{(i)}\right) .
$$

No caso particular de espaço de estados discreto e $p=2$, escolhida uma ordem para o movimento, por exemplo atualizar primeiro a primeira componente $\theta_{1}$, depois a segunda componente $\theta_{2}$, é fácil ver que

$$
\begin{aligned}
& p\left[\underline{\theta}^{(i+1)}=(c, d)^{T} \mid \underline{\sim}^{(i)}=(a, b)^{T}\right]=p[(a, b) \rightarrow(c, b) \rightarrow(c, d)] \\
& =p[(c, d) \mid(c, b)] \cdot p[(c, b) \mid(a, b)]=p\left(\theta_{2}^{(i+1)}=d \mid \theta_{1}^{(i+1)}=c\right) \cdot p\left(\theta_{1}^{(i+1)}=c \mid \theta_{2}^{(i)}=b\right) .
\end{aligned}
$$

5. Embora o núcleo de transição $p(\cdot \mid)$ acima geralmente não satisfaz a condição de reversibilidade, ele define uma cadeia que tem $\pi \underset{\sim}{\theta})$ como uma distribuição estacionária. Sem perda de generalidade, mostramos esse fato apenas para o caso $p=2$ :

Demonstração:

$$
\begin{aligned}
& \int_{\Theta_{i}} p\left(\underline{\sim}^{(i+1)} \mid \underline{\theta}^{(i)}\right) \pi\left(\underline{\theta}^{(i)}\right) \mathrm{d} \underline{\sim}^{(i)} \\
& =\int_{\Theta_{1}} \int_{\Theta_{2}} \pi\left(\theta_{2}^{(i+1)} \mid \theta_{1}^{(i+1)}\right) \pi\left(\theta_{1}^{(i+1)} \mid \theta_{2}^{(i)}\right) \pi\left(\theta_{1}^{(i)}, \theta_{2}^{(i)}\right) \mathrm{d} \underset{\underset{1}{\theta}}{(i)} \mathrm{d} \underset{\sim}{\theta_{2}^{(i)}} \\
& =\pi\left(\theta_{2}^{(i+1)} \mid \theta_{1}^{(i+1)}\right) \int_{\Theta_{1}} \pi\left(\theta_{1}^{(i+1)} \mid \theta_{2}^{(i)}\right) \pi\left(\theta_{2}^{(i)}\right) \mathrm{d} \theta_{2}^{(i)} \\
& =\pi\left(\theta_{2}^{(i+1)} \mid \theta_{1}^{(i+1)}\right) \int_{\Theta_{1}} \pi\left(\theta_{1}^{(i+1)}, \theta_{2}^{(i)}\right) \mathrm{d} \theta_{2}^{(i)}=\pi\left(\theta_{2}^{(i+1)} \mid \theta_{1}^{(i+1)}\right) \pi\left(\theta_{1}^{(i+1)}\right) \\
& =\pi\left(\theta_{1}^{(i+1)}, \theta_{2}^{(i+1)}\right)=\pi\left(\stackrel{(}{(i+1)}^{(i+1)}\right. \text {. }
\end{aligned}
$$

6. Existe na literatura, vários algoritmos que são variações do algoritmo de Gibbs (ver Chen, 2000, Carlin e Louis, 2000, e Robert e Casella, 1999).

\subsubsection{O algoritmo de Gibbs em Blocos ("Grouped Gibbs)}

Quando o algoritmo original de Gibbs converge lentamente devido as fortes correlações entre os parâmetros do modelo ou devido as autocorrelações entre as it- 
eraçōes do algoritmo para cada parâmetro, pode-se tomar certas medidas para melhorar o desempenho desse algoritmo como, por exemplo, reparametrizar o modelo ou usar variáveis auxiliares, como também utilizar variações do algoritmo original como, por exemplo, os métodos "grouped Gibbs" (Liu, 1994, e Roberts e Sahu, 1997), "collapsed Gibbs" (Liu, 1994) e "covariance-adjusted MCMC" (Liu et al., 1994), dentre outros.

No algoritmo original de Gibbs, amostramos cada componente $\theta_{i}$ (do vetor de parâmetros $\underset{\sim}{\theta}$ ), de sua respectiva distribuição condicional completa. No "Gibbs em blocos" particionamos as componentes de $\theta$ em grupos (de um ou mais componentes); e os grupos que tiverem mais de uma componente são amostrados conjuntamente (de suas respectivas distribuições condicionais completas conjuntas). Segundo Chen (2000), o procedimento de amostrar em blocos traz benefícios e segundo Gemermann (1996), quando amostramos os parâmetros conjuntamente deixa-se de fazer a cadeia percorrer ao longo dos eixos para fezê-la percorrer trajetórias ditadas pela distribuição condicional completa do bloco. Segundo Roberts e Sahu (1997), nem sempre o uso de blocos traz benefícios e eles mostram alguns exemplos em que o uso desse método é ruim. A seguir ilustramos o método por meio de um exemplo.

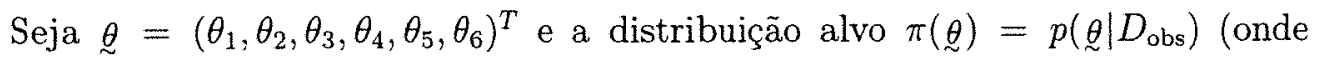
$p\left(\underset{\theta}{\theta} \mid D_{\text {obs }}\right)$ é a posteriori de $\left.\underset{\sim}{\theta}\right)$ e suponha que sabemos amostrar $\left(\theta_{1}, \theta_{2}, \theta_{3}\right)$ conjuntamente de $\pi\left(\theta_{1}, \theta_{2}, \theta_{3} \mid \theta_{4}, \theta_{5}, \theta_{6}, D_{\text {obs }}\right)$ como também $\left(\theta_{4}, \theta_{5}\right)$ de $\pi\left(\theta_{4}, \theta_{5} \mid \theta_{1}, \theta_{2}, \theta_{3}, \theta_{6}\right.$, $\left.D_{\text {obs }}\right)$ e $\theta_{6}$ de $\pi\left(\theta_{6} \mid \theta_{1}, \theta_{2}, \theta_{3}, \theta_{4}, \theta_{5}, D_{\text {obs }}\right)$, então teríamos o seguinte algoritmo:

Passo 0: escolha ${\underset{\sim}{(0)}}^{(0)}=\left(\theta_{1}^{(0)}, \theta_{2}^{(0)}, \theta_{3}^{(0)}, \theta_{4}^{(0)}, \theta_{5}^{(0)}, \theta_{6}^{(0)}\right)^{T}$ e faça $i=0$.

Passo 1: gere $\left(\theta_{1}^{(i+1)}, \theta_{2}^{(i+1)}, \theta_{3}^{(i+1)}\right)$ de $\pi\left(\theta_{1}, \theta_{2}, \theta_{3} \mid \theta_{4}^{(i)}, \theta_{5}^{(i)}, \theta_{6}^{(i)}, D_{\text {obs }}\right)$.

Passo 2: gere $\left(\theta_{4}^{(i+1)}, \theta_{5}^{(i+1)}\right)$ de $\pi\left(\theta_{4}, \theta_{5} \mid \theta_{1}^{(i+1)}, \theta_{2}^{(i+1)}, \theta_{3}^{(i+1)}, \theta_{6}^{(i)}, D_{\text {obs }}\right)$.

Passo 3: gere $\theta_{6}^{(i+1)}$ de $\pi\left(\theta_{6} \mid \theta_{1}^{(i+1)}, \theta_{2}^{(i+1)}, \theta_{3}^{(i+1)}, \theta_{4}^{(i+1)}, \theta_{5}^{(i+1)}, \theta_{6}^{(i+1)}, D_{\text {obs }}\right)$. 
Passo 4: faça $i=i+1$ e volte ao passo 1 , até a convergência.

Observação: É fácil ver que a CM induzida pelo algoritmo de Gibbs em blocos tem $\pi(\underset{\sim}{\theta})$ como distribuição invariante. A demonstração para o caso particular em que $\underset{\theta}{\theta}=\left(\theta_{1}, \theta_{2}, \theta_{3}\right)^{T}$, onde primeiramente $\left(\theta_{1}, \theta_{2}\right)$ é amostrado de $\pi\left(\theta_{1}, \theta_{2} \mid \theta_{3}, D_{\text {obs }}\right)$ e depois $\theta_{3}$ é amostrado de $\pi\left(\theta_{3} \mid \theta_{1}, \theta_{2}, D_{\text {obs }}\right)$ é dado abaixo:

demonstração;

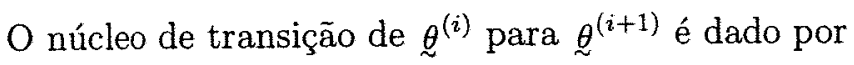

$$
p\left(\stackrel{(}{\theta}^{(i+1)} \mid{\underset{\sim}{(i)}}^{(i)}\right)=\pi\left(\theta_{1}^{(i+1)}, \theta_{2}^{(i+1)} \mid \theta_{3}^{(i)}, D_{\mathrm{obs}}\right) \pi\left(\theta_{3}^{(i+1)} \mid \theta_{1}^{(i+1)}, \theta_{2}^{(i+1)}, D_{\mathrm{obs}}\right) .
$$

Nesse caso,

$$
\begin{aligned}
& \int_{\Theta_{1}} \int_{\Theta_{2}} \int_{\Theta_{3}} p\left(\underline{\theta}^{(i+1)} \mid \underline{\sim}^{(i)}\right) \pi\left(\underline{\sim}^{(i)} \mid D_{\text {obs }}\right) \mathrm{d} \theta^{(i)} \\
& =\int_{\Theta_{1}} \int_{\theta_{2}} \int_{\Theta_{3}} \pi\left(\theta_{1}^{(i+1)}, \theta_{2}^{(i+1)} \mid \theta_{3}^{(i)}, D_{\text {obs }}\right) \pi\left(\theta_{3}^{(i+1)} \mid \theta_{1}^{(i+1)}, \theta_{2}^{(i+1)}, D_{\text {obs }}\right) \text {. } \\
& . \pi\left(\theta_{1}^{(i)}, \theta_{2}^{(i)}, \theta_{3}^{(i)} \mid D_{\mathrm{obs}}\right) \mathrm{d} \theta_{1}^{(i)} \mathrm{d} \theta_{2}^{(i)} \mathrm{d} \theta_{3}^{(i)} \\
& =\pi\left(\theta_{3}^{(i+1)} \mid \theta_{1}^{(i+1)}, \theta_{2}^{(i+1)}, D_{\mathrm{obs}}\right) \int_{\Theta_{3}} \pi\left(\theta_{1}^{(i+1)}, \theta_{2}^{(i+1)} \mid \theta_{3}^{(i)}, D_{\mathrm{obs}}\right) \pi\left(\theta_{3}^{(i)} \mid D_{\mathrm{obs}}\right) \mathrm{d} \theta_{3} \\
& =\pi\left(\theta_{3}^{(i+1)} \mid \theta_{1}^{(i+1)}, \theta_{2}^{(i+1)}, D_{\text {obs }}\right) \int_{\Theta_{3}} \pi\left(\theta_{1}^{(i+1)}, \theta_{2}^{(i+1)}, \theta_{3}^{(i)} \mid D_{\text {obs }}\right) \mathrm{d} \theta_{3} \\
& =\pi\left(\theta_{3}^{(i+1)} \mid \theta_{1}^{(i+1)}, \theta_{2}^{(i+1)}, D_{\text {obs }}\right) \pi\left(\theta_{1}^{(i+1)}, \theta_{2}^{(i+1)} \mid D_{\text {obs }}\right)=\pi\left(\theta_{1}^{(i+1)}, \theta_{2}^{(i+1)}, \theta_{3}^{(i+1)} \mid D_{\text {obs }}\right) \\
& =\pi\left(\underline{\theta}^{(i+1)} \mid D_{\mathrm{obs}}\right) \text {. }
\end{aligned}
$$

\subsubsection{O algoritmo "Collapsed Gibbs"}

O algoritmo "collapsed Gibbs" foi proposto por Liu (1994) com o objetivo de acelerar a convergência e o "mixing" do amostrador de Gibbs. Este método é uma variação do algoritmo original de Gibbs e para ilustrar como ele funciona usamos o seguinte exemplo:

Seja $\underset{\theta}{\theta}=\left(\theta_{1}, \theta_{2}, \theta_{3}\right)^{T}$ e a distribuição alvo $\pi(\underset{\sim}{\theta})=\pi\left(\underset{\sim}{\theta} \mid D_{\text {obs }}\right)$ (a posteriori de $\underset{\sim}{\theta}$ ). 
Se, por exemplo, pudermos eliminar por integração a componente $\theta_{3}$ de $\pi\left(\theta_{1}, \theta_{2}, \theta_{3} \mid D_{\text {obs }}\right)$ (ou seja, obter a marginal à posteriori $\left.\pi\left(\theta_{1}, \theta_{2} \mid D_{\text {obs }}\right)\right)$, então os passos do algoritmo são os seguintes:

Passo 0: faça $i=0$, escolha $\underline{\theta}^{(0)}=\left(\theta_{1}^{(0)}, \theta_{2}^{(0)}\right)^{T}$;

Passo 1: $\operatorname{gerar}\left(\theta_{1}^{(i+1)}, \theta_{2}^{(i+1)}\right)$ de $\pi\left(\theta_{1}, \theta_{2} \mid D_{\text {obs }}\right)$;

Passo 2: gerar $\theta_{3}^{(i+1)}$ de $\pi\left(\theta_{3} \mid \theta_{1}^{(i+1)}, \theta_{2}^{(i+1)}, D_{\text {obs }}\right)$;

Passo 3: fazer $i=i+1$ e voltar para o passo 1 .

No caso em que não sabemos amostrar diretamente $\left(\theta_{1}, \theta_{2}\right)$ simultaneamente, podemos usar uma versão desse algoritmo denominada "modified collapsed Gibbs sampler" e, nesse caso, no passo 1 podemos usar um mini-Gibbs para amostrar $\left(\theta_{1}^{(i+1)}, \theta_{2}^{(i+1)}\right)$ de $\pi\left(\theta_{1}, \theta_{2} \mid D_{\text {obs }}\right)$, Liu, 1994, menciona que nesse caso a cadeia induzida pelo mini-Gibbs deve convergir antes de prosseguir para o passo 2 e o algoritmo seria o seguinte, em cada iteração:

Passo 1: gerar $\left(\theta_{1}, \theta_{2}\right)$ de $\pi\left(\theta_{1}, \theta_{2} \mid D_{\text {obs }}\right)$ da seguinte maneira:

- gerar $\theta_{2}$ de $\pi\left(\theta_{2} \mid \theta_{1}, D_{\text {obs }}\right)$,

- gerar $\theta_{2}$ de $\pi\left(\theta_{1} \mid \theta_{2}, D_{\text {obs }}\right)$;

Passo 2: gerar $\theta_{3}$ de $\pi\left(\theta_{3} \mid \theta_{1}, \theta_{2}, D_{\text {obs }}\right)$.

Mais precisamente, teríamos os seguintes passos:

Passo 0: faça $i=0$, faça $j=0$ e escolha $\theta_{1 j}^{(0)}$.

Passo 1: gere $\left(\theta_{1, j+1}^{(i+1)}, \theta_{2, j+1}^{(i+1)}\right)$ da seguinte maneira:

- gere $\theta_{2, j+1}^{(i+1)}$ de $\pi\left(\theta_{2} \mid \theta_{1, j}^{(i)}, D_{\text {obs }}\right)$.

- gere $\theta_{i, j+1}^{(i+1)}$ de $\pi\left(\theta_{1} \mid \theta_{2, j+1}^{(i+1)}, D_{\text {obs }}\right)$; 
Passo 2: faça $j=j+1$, se $j<N$ volte ao passo 1 (onde $N$ é fixado e suficientemente grande);

Passo 3: gere $\theta_{3}^{(i+1)}$ de $\pi\left(\theta_{3} \mid \theta_{1, N}^{(i+1)}, \theta_{2, N}^{(i+1)}\right)$;

Passo 5: fazer $i=i+1$ e volte ao passo 1 , até a convergência.

\section{Observações}

1. O algoritmo "collapsed Gibbs" induz uma CM onde $\pi \underset{\sim}{(\theta)}$ é uma distribuição invariante (ou estacionária). É muito simples demonstrar isso no exemplo acima

\section{demonstração}

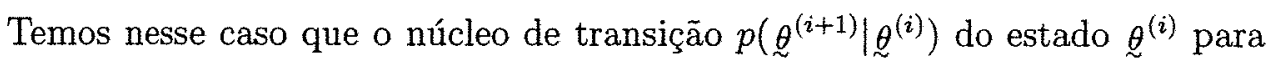

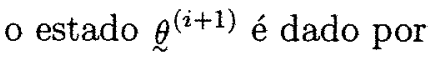

$$
p\left(\stackrel{\theta}{(i+1)}^{\left(i+\underline{\theta}^{(i)}\right.}\right)=\pi\left(\theta_{1}^{(i+1)}, \theta_{2}^{(i+1)} \mid D_{\mathrm{obs}}\right) \pi\left(\theta_{3}^{(i+1)} \mid \theta_{1}^{(i+1)}, \theta_{2}^{(i+1)}, D_{\mathrm{obs}}\right)
$$

e portanto

$$
\begin{aligned}
& \int_{\Theta_{1}} \int_{\Theta_{2}} \int_{\Theta_{3}} p\left(\underline{\theta}^{(i+1)} \mid \underline{\theta}^{(i)}\right) \pi\left(\underline{\theta}^{(i)} \mid D_{\mathrm{obs}}\right) \mathrm{d} \underline{\theta}^{(i)} \\
& =\int_{\Theta_{1}} \int_{\Theta_{2}} \int_{\Theta_{3}} \pi\left(\theta_{1}^{(i+1)} \theta_{2}^{(i+1)} \mid D_{\mathrm{obs}}\right) \pi\left(\theta_{3}^{(i+1)} \mid \theta_{1}^{(i+1)}, \theta_{2}^{(i+1)}, D_{\mathrm{obs}}\right) . \\
& =\pi\left(\theta_{1}^{(i)}, \theta_{2}^{(i)}, \theta_{3}^{(i)} \mid D_{\mathrm{obs}}\right) \mathrm{d} \theta_{1}^{(i)} \mathrm{d} \theta_{2}^{(i)} \mathrm{d} \theta_{3}^{(i)} \\
& =\pi\left(\theta_{1}^{(i+1)} \theta_{2}^{(i+1)} \mid D_{\mathrm{obs}}\right) \pi\left(\theta_{3}^{(i+1)} \mid \theta_{1}^{(i+1)}, \theta_{2}^{(i+1)}, D_{\text {obs }}\right) \\
& =\pi\left(\theta_{1}^{(i+1)}, \theta_{2}^{(i+1)}, \theta_{3}^{(i+1)} \mid D_{\text {obs }}\right)=\pi\left(\underline{\theta}^{(i+1)} \mid D_{\text {obs }}\right)
\end{aligned}
$$

2. O algoritmo modificado também induz uma CM com uma distribuição esta-

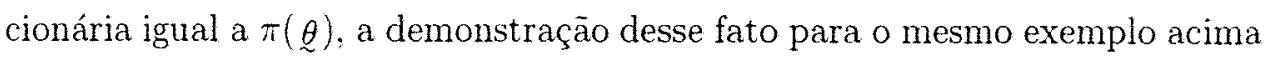
segue abaixo. 
Demonstração: Seja

$p\left(\stackrel{(}{(}^{(i+1)} \mid \underline{\sim}^{(i)}\right)=\pi\left(\theta_{1}^{(i+1)} \mid \theta_{2}^{(i)}, D_{\text {obs }}\right) \pi\left(\theta_{2}^{(i+1)} \mid \theta_{1}^{(i+1)}, D_{\text {obs }}\right) \pi\left(\theta_{3}^{(i+1)} \mid \theta_{1}^{(i+1)}, \theta_{2}^{(i+1)}, D_{\text {obs }}\right)$

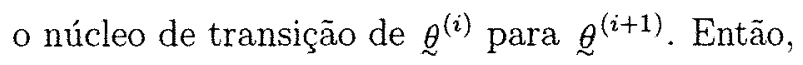

$$
\begin{aligned}
& \int_{\Theta_{1}} \int_{\Theta_{2}} \int_{\Theta_{3}} p\left(\dot{\theta}^{(i+1)} \mid \theta^{(i)}\right) \pi\left(\dot{\theta}^{(i)} \mid D_{\mathrm{obs}}\right) \mathrm{d} \dot{\sim}^{(i)} \\
& =\int_{\Theta_{1}} \int_{\Theta_{2}} \int_{\Theta_{3}} p\left(\underline{\theta}^{(i+1)} \mid \underline{\theta}^{(i)}\right) \pi\left(\underline{\theta}^{(i)} \mid D_{\text {obs }}\right) \mathrm{d} \theta_{1}^{(i)} \mathrm{d} \theta_{2}^{(i)} \mathrm{d} \theta_{3}^{(i)} \\
& =\int_{\Theta_{1}} \int_{\Theta_{2}} \int_{\Theta_{3}} \pi\left(\theta_{1}^{(i+1)} \mid \theta_{2}^{(i)}, D_{\mathrm{obs}}\right) \pi\left(\theta_{2}^{(i+1)} \mid \theta_{1}^{(i+1)}, D_{\mathrm{obs}}\right) \pi\left(\theta_{3}^{(i+1)} \mid \theta_{1}^{(i+1)} \theta_{2}^{(i+1)}, D_{\mathrm{obs}}\right) \text {. } \\
& . \pi\left(\theta_{1}^{(i)}, \theta_{2}^{(i)}, \theta_{3}^{(i)} \mid D_{\text {obs }}\right) \mathrm{d} \theta_{1}^{(i)} \mathrm{d} \theta_{2}^{(i)} \mathrm{d} \theta_{3}^{(i)} \\
& =\int_{\Theta_{1}} \int_{\Theta_{2}} \pi\left(\theta_{1}^{(i+1)} \mid \theta_{2}^{(i)}, D_{\mathrm{obs}}\right) \pi\left(\theta_{2}^{(i+1)} \mid \theta_{1}^{(i+1)}, D_{\mathrm{obs}}\right) \pi\left(\theta_{3}^{(i+1)} \mid \theta_{1}^{(i+1)}, \theta_{2}^{(i+1)}, D_{\mathrm{obs}}\right) \text {. } \\
& . \pi\left(\theta_{1}^{(i)}, \theta_{2}^{(i)} \mid D_{\mathrm{obs}}\right) \mathrm{d} \theta_{1}^{(i)} \mathrm{d} \theta_{2}^{(i)} \\
& =\int_{\Theta_{2}} \pi\left(\theta_{1}^{(i+1)} \mid \theta_{2}^{(i)}, D_{\mathrm{obs}}\right) \pi\left(\theta_{2}^{(i+1)} \mid \theta_{1}^{(i+1)}, D_{\mathrm{obs}}\right) \pi\left(\theta_{3}^{(i+1)} \mid \theta_{1}^{(i+1)}, \theta_{2}^{(i+1)}, D_{\mathrm{obs}}\right) \pi\left(\theta_{2}^{(i)} \mid D_{\mathrm{obs}}\right) \mathrm{d} \theta_{2}^{(i)} \\
& =\pi\left(\theta_{3}^{(i+1)} \mid \theta_{1}^{(i+1)}, \theta_{2}^{(i+1)}, D_{\mathrm{obs}}\right) \pi\left(\theta_{2}^{(i+1)} \mid \theta_{1}^{(i+1)}, D_{\mathrm{obs}}\right) \int_{\theta_{2}} \pi\left(\theta_{1}^{(i+1)} \mid \theta_{2}^{(i)}, D_{\mathrm{obs}}\right) \pi\left(\theta_{2}^{(i)} \mid D_{\mathrm{obs}}\right) \mathrm{d} \theta_{2}^{(i)} \\
& =\pi\left(\theta_{3}^{(i+1)} \mid \theta_{1}^{(i+1)}, \theta_{2}^{(i+1)}, D_{\mathrm{obs}}\right) \pi\left(\theta_{2}^{(i+1)} \mid \theta_{1}^{(i+1)}, D_{\mathrm{obs}}\right) \int_{\Theta_{2}} \pi\left(\theta_{1}^{(i+1)}, \theta_{2}^{(i)} \mid D_{\mathrm{obs}}\right) \mathrm{d} \theta_{2}^{(i)} \\
& =\pi\left(\theta_{3}^{(i+1)} \mid \theta_{1}^{(i+1)}, \theta_{2}^{(i+1)}, D_{\text {obs }}\right) \pi\left(\theta_{2}^{(i+1)} \mid \theta_{1}^{(i+1)}, D_{\text {obs }}\right) \pi\left(\theta_{1}^{(i+1)} \mid D_{\text {obs }}\right) \\
& =\pi\left(\theta_{1}^{(i+1)}, \theta_{2}^{(i+1)}, \theta_{3}^{(i+1)} \mid D_{\text {obs }}\right)=\pi(\underbrace{(i+1)} \mid D_{\text {obs }})
\end{aligned}
$$

3. Segundo Liu (1994), uma vantagem de eliminar componentes por integração ("collapsed down components") é que em geral as auto-correlações entre as iterações são reduzidas. No exemplo acima vemos que $\theta_{3}^{(i+1)}$ depende de $\theta_{1}^{(i+1)}$ e $\theta_{2}^{(i+1)}$, mas $\theta_{1}^{(i+1)}$ e $\theta_{2}^{(i+1)}$ não dependem de $\theta_{3}^{(i)}$.

4. Segundo Chen (2000), e de acordo com Liu (1994), o collapsed Gibbs "funciona melhor" que o "grouped Gibbs", enquanto que o "grouped" é melhor que o Gibbs original. Também espera-se que o "collapsed Gibbs" seja melhor do que o "modified collapsed Gibbs" e que este por sua vez seja melhor do que o Gibbs original. Porém, entre o "grouped" e o "modified collapsed Gibbs", é 
difícil dizer qual é o melhor e vai depender das correlações dentro dos grupos (ou blocos) (se elas forem "altas" espera-se que o "grouped" funcione melhor).

\subsubsection{O algoritmo Híbrido de Metropolis-Hastings em Gibbs}

Seja $\pi(\underset{\sim}{\theta})$ a distribuição da qual queremos amostrar e suponhamos que nem todas as distribuições condicionais completas, $\pi\left(\theta_{i} \mid \theta_{(-i)}\right)$, possam ser amostradas facilmente (por exemplo, quando apenas os seus núcleos são conhecidos). Em princípio podemos utilizar qualquer um dos métodos de simulação estática descritos na seção 2.4.1 para amostrar dessas distribuições mais complicadas (de serem amostradas) e assim completar o ciclo do amostrador de Gibbs. O problema entretanto é que esses métodos são numericamente intensivos e, ao serem re-utilizados em cada uma das iterações do algoritmo principal de Gibbs, leva a um "custo" computacional muito grande no final (sem contar o fato que em cada iteração do algoritmo de Gibbs amostramos um grande número de observações para salvar apenas uma observação, e também há o problema do "re-envelopamento" em cada iteração quando usamos o método de rejeição ou o método "weighted bootstrap").

Uma solução elegante para amostrar das condicionais completas complicadas (sendo ou não log-côncavas), especialmente quando o número dessas condicionais é relativamente grande, é utilizar o algoritmo de Metropolis-Hastings (M-H). Nesse caso, geramos sub-cadeias de M-H dentro do ciclo amostrador de Gibbs. Por exemplo, se $\pi\left(\theta_{j} \mid \theta_{(-j)}\right)$ é difícil de ser amostrada, então na $(i+1)$-ésima iteração do algoritmo principal de Gibbs geramos $\theta_{j}^{(i+1)}$ de $\pi\left(\theta_{j} \mid \theta_{1}^{(i)}, \ldots, \theta_{j-1}^{(i)}, \theta_{j+1}^{(i-1)}, \ldots, \theta_{p}^{(i-1)}\right)$

por meio do algoritm de M-H (onde $\theta_{j}^{(i+1)}$ é o resultado da última iteração de $\mathrm{M}-\mathrm{H}$ ). Esse procedimento é conhecido como "Metropolis-Hastings em Gibbs", ou também como "passos de Metropolis-Hastings" ou "Localized Metropolis-Hastings".

\section{Observações}


1. Esse método híbrido é tal que a cadeia de Markov induzida por ele tem $\pi(\theta)$ (a distribuição alvo) como uma distribuição estacionária ou invariante (e portanto para haver convergência da cadeia para essa distribuição é necessário que a cadeia seja irredutível e aperiódica).

2. O número de iterações $T$ do sub-algoritmo de M-H é arbitrário. Entretanto, a escolha de um valor muito grande para $T$ (digamos 10.000) é puro desperdício, especialmente nas primeiras iterações do algoritmo principal de Gibbs, pois estaríamos ainda longe da distribuição estacionária. Na prática é muito comum usar $T=1$, entretanto, não existe uma recomendação de um valor de $T$ ideal. Intuitivamente, concordamos que nas primeiras iterações do algoritmo principal ("burn in period"), $T$ deveria ser pequeno, mas à medida que o número de iterações aumenta, $T$ deveria também aumentar.

A seguir, por meio de um exemplo, apresentamos os passos do algoritmo de Metropolis-Hastings em Gibbs na sua forma mais simples (isto é, no caso em que o número de iterações, $T$, dos sub-algoritmos de $\mathrm{M}-\mathrm{H}$ para cada iteração do algoritmo principal de Gibbs é $T=1$ ).

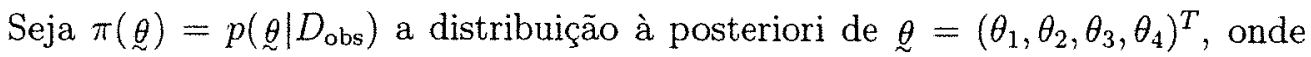
$\pi\left(\theta_{1} \mid \theta_{2}, \theta_{3}, \theta_{4}, D_{\text {obs }}\right), \pi\left(\theta_{2} \mid \theta_{1}, \theta_{3}, \theta_{4}, D_{\text {obs }}\right)$ e $\pi\left(\theta_{4} \mid \theta_{1}, \theta_{2}, \theta_{3}, D_{\text {obs }}\right)$ podem ser amostradas facilmente, mas $\pi\left(\theta_{3} \mid \theta_{1}, \theta_{2}, \theta_{4}, D_{\text {obs }}\right)$ é difícil de ser amostrada. Então temos os seguintes passos:

Passo 0: Escolher um vetor inicial ${\underset{\theta}{(0)}}^{(0)}=\left(\theta_{1}^{(0)}, \theta_{2}^{(0)}, \theta_{3}^{(0)}, \theta_{4}^{(0)}\right)^{T}$ e fazer $i=0$;

Passo 1: gerar $\left.\stackrel{\theta}{(i+1)}^{\left(i+\theta_{1}^{(i+1)}\right.}, \theta_{2}^{(i+1)}, \theta_{3}^{(i+1)}, \theta_{4}^{(i+1)}\right)$ da seguinte forma:

- $\theta_{1}^{(i+1)} \sim \pi\left(\theta_{1} \mid \theta_{2}^{(i)}, \theta_{3}^{(i)}, \theta_{4}^{(i)}, D_{\text {obs }}\right)$.

- gerar $\theta_{2}^{(i+1)} \sim \pi\left(\theta_{2} \mid \theta_{1}^{(i+1)}, \theta_{3}^{(i)}, \theta_{4}^{(i)}, D_{\text {obs }}\right)$,

- $\operatorname{gerar} \theta_{3}^{(i+1)} \sim \pi\left(\theta_{3} \mid \theta_{1}^{(i+1)}, \theta_{2}^{(i+1)}, \theta_{4}^{(i)}, D_{\mathrm{obs}}\right)$ da seguinte forma: 
sub-passo 1: gere o candidato $\theta_{3}^{*(i+1)}$ de $q\left(\cdot \mid \theta_{1}^{(i+1)}, \theta_{2}^{(i+1)}, \theta_{3}^{(i)}, \theta_{4}^{(i)}\right)$ e gere $U \sim$ $N(0,1)$,

sub-passo 2: faça $\alpha\left(\theta_{3}^{*(i+1)} \mid \theta_{3}^{(i)}\right)=$

$$
\min \left\{\frac{\pi\left(\theta_{3}^{*(i+1)} \mid \theta_{1}^{(i+1)}, \theta_{2}^{(i+1)}, \theta_{4}^{(i)}, D_{\text {obs }}\right) \cdot q\left(\theta_{3}^{(i)} \mid \theta_{1}^{(i+1)}, \theta_{2}^{(i+1)}, \theta_{3}^{*(i+1)}, \theta_{4}^{(i)}\right)}{\pi\left(\theta_{3}^{(i)} \mid \theta_{1}^{(i+1)}, \theta_{2}^{(i+1)}, \theta_{4}^{(i)}, D_{\text {obs }}\right) \cdot q\left(\theta_{3}^{*(i+1)} \mid \theta_{1}^{(i+1)}, \theta_{2}^{(i+1)}, \theta_{3}^{(i)}, \theta_{4}^{(i)}\right)} ; 1\right\}
$$

sub-passo 3: se $U<\alpha\left(\theta_{3}^{*(i+1)} \mid \theta_{3}^{(i)}\right)$ então faça $\theta_{3}^{(i+1)}=\theta_{3}^{*(i+1)}$, caso contrário faça $\theta_{3}^{(i+1)}=\theta_{3}^{(i)}$.

- $\operatorname{gerar} \theta_{4}^{(i+1)} \sim \pi\left(\theta_{4} \mid \theta_{1}^{(i+1)}, \theta_{2}^{(i+1)}, \theta_{3}^{(i+1)}, D_{\text {obs }}\right)$

Passo 2: faça $i=i+1$ e volte ao passo 1 .

\section{Observações}

1. Se $q\left(\theta_{3}^{*(i+1)} \mid \theta_{1}^{(i+1)}, \theta_{2}^{(i+1)}, \theta_{3}^{(i)}, \theta_{4}^{(i)}\right)=q\left(\theta_{3}^{(i)} \mid \theta_{1}^{(i+1)}, \theta_{2}^{(i+1)}, \theta_{3}^{*(i+1)}, \theta_{4}^{(i)}\right)$, temos o algoritmo de Metropolis.

2. Se $q\left(\theta_{3}^{*(i+1)} \mid \theta_{1}^{(i+1)}, \theta_{2}^{(i+1)}, \theta_{3}^{(i)}, \theta_{4}^{(i)}\right)$ não depende de $\theta_{1}^{(i+1)}, \theta_{2}^{(i+1)}, \theta_{3}^{(i)}$ e $\theta_{4}^{(i)}$, temos o algoritmo "Independence M-H".

3. Se $\pi\left(\theta_{3} \mid \theta_{1}, \theta_{2}, \theta_{4}\right)$ puder ser escrita como o produto $\psi\left(\theta_{3} \mid \theta_{1}, \theta_{2}, \theta_{4}\right) \cdot h\left(\theta_{3} \mid \theta_{1}, \theta_{2}\right.$, $\left.\theta_{4}\right)$, então

$$
\alpha\left(\theta_{3}^{*(i+1)} \mid \theta_{3}^{(i)}\right)=\min \left\{\frac{\psi\left(\theta_{3}^{*(i+1)} \mid \theta_{1}^{(i+1)}, \theta_{2}^{(i+1)}, \theta_{4}^{(i)}\right)}{\psi\left(\theta_{3}^{(i)} \mid \theta_{1}^{(i+1)}, \theta_{2}^{(i+1)}, \theta_{4}^{(i)}\right)} ; 1\right\} .
$$

\subsubsection{Diagnósticos de Convergência}

Conceitualmente dizemos que um algoritmo $\mathrm{MCMC}$ converge no tempo $T_{0}$ quando os dados gerados após $T_{0}$ podem ser considerados "com segurança" como uma amostra da distribuição alvo e portanto os dados simulados antes de $T_{0}$ (no período préconvergência, ou "burn-in period") não devem fazer parte nas inferências. 
Essa definição, embora não seja muito precisa (pois o termo "com segurança" não está bem definido) serve como um guia. Na prática, gostaríamos de saber não somente se o algoritmo converge, mas o valor de $T_{0}$ e também o número de iteraçōes que devem ser consideradas após esse tempo $T_{0}$ para que a amostra seja representativa.

Na literatura existem vários métodos para diagnosticar possíveis falhas na convergência dos algoritmos MCMC e nessa subseção apresentamos alguns deles. Entretanto, é importante ressaltar que não existe método que possa "provar" convergência (pelo simples fato de que são utilizadas apenas um número finito de observações simuladas). Por esse motivo, vários autores recomendam que seja utilizada uma combinação de várias técnicas de diagnóstico para analisar um mesmo problema. Desse modo espera-se ter um "certo grau de confiança" na análise da convergência. Entretanto, é importante ter em mente que mesmo utilizando um grande número de técnicas de diagnóstico, não podemos afirmar com certeza que os dados simulados são representativos da distribuição que queremos amostrar.

A seguir consideramos alguns aspectos ligados aos problemas de simulação iterativa.

\section{NÚMERO DE CADEIAS GERADAS}

Um dos tópicos de grande debate é se é melhor analisar uma única cadeia MCMC ou então múltiplas cadeias partindo-se de pontos iniciais distintos no espaço paramétrico.

Uma única cadeia pode aparentemente exibir um comportamento aceitável. Entretanto, ela pode estar "confinada" a apenas uma certa região do espaço paramétrico, por exemplo em torno de um máximo local (e nesse caso a amostra final não seria representativa da distribuição alvo, já que a cadeia não "varreu" ou explorou 
todo o domínio dessa distribuição).

Para não correr esse risco, podemos considerar o uso de múltiplas cadeias partindo-se de pontos iniciais bem dispersos no espaço paramétrico. Esse procedimento, embora tenha um melhor poder exploratório, depende totalmente da escolha dos valores iniciais (ou seja, da habilidade em escolher esses pontos "bem dispersos"). Outro problema é que o número de simulações descartadas (referente ao período "burn-in") é multiplicado pelo número de cadeias, o que pode ser um grande desperdício.

\section{ESCOLHA DOS VALORES INICIAIS}

Para a escolha dos valores iniciais, Carlin e Louis (2000) recomendam fazer uma grade de pontos na região do espaço paramétrico (centrado por exemplo na média da priori e cobrindo mais ou menos três desvios-padrão a partir dessa média) e depois tomar sistematicamente todo ponto da grade como um valor inicial. Gelman et al. (1997) recomendam um procedimento aleatório para a escolha dos valores iniciais partindo do pressuposto de que a distribuição alvo é multimodal. O procedimento é o seguinte:

- Achar as modas da posteriori (por exemplo pelo algoritmo EM ou algum outro procedimento de otimização numérica);

- Aproximar a posteriori por uma mistura de $\mathrm{k}$ Normais multivariadas $N\left(\theta \mid \theta_{\theta_{i}}, \widehat{V_{\theta_{i}}}\right)$, $i=1, \ldots, k$, onde $k$ é o número de modas encontradas no passo anterior, $\hat{\theta}_{i}$ é a i-ésima moda e $\widehat{V_{\theta_{2}}}=-\left[\left.\frac{\partial \log p\left(\theta \mid D_{o b s}\right)}{\partial \underline{\theta}_{\partial} \partial \underline{\theta}^{\prime}}\right|_{\theta=\theta_{i}}\right]^{-1}$. Ou então por uma mistura de $t^{\prime}$ 's multivariadas com quatro graus de liberdade, $t_{\nu}\left(\mu_{i}, \Sigma_{i}\right)$, onde $\nu=4, \mu_{i}=\underline{\theta}_{i}$ e $\Sigma_{i}=\widehat{V_{\underline{\theta}}}$

Os pesos $w_{i}$ de cada distribuição são dados por $w_{i}=q\left(\underset{\theta_{i}}{ } \mid D_{o b s}\right) \cdot\left|\widehat{V_{\underline{\theta}}}\right|^{\frac{1}{2}}$, onde 
$q\left(\hat{\theta}_{i} \mid D_{o b s}\right)$ é a posteriori não normalizada calculada no ponto ${\underset{\sim}{\hat{\theta}}}_{i}$.

- Amostrar 1000 valores da distribuição de mistura e depois fazer uma reamostragem ponderada ("weighted bootstrap") para "filtrar" uma amostra por exemplo de dez pontos, que serão usados como pontos iniciais do processo iterativo (ver página 312 de Gelman et al., 1997).

\section{MONITORAMENTO DA CONVERGÊNCIA}

Os diagnósticos podem ser qualitativos ou quantitativos. Os qualitativos utilizam dispositivos gráficos para descrever os dados simulados e os quantitativos fornecem medidas resumo desses dados. Essas técnicas podem ser univariadas ou multivariadas. As univariadas descrevem cada parâmetro individualmente e as multivariadas, a posteriori conjunta. A maioria das técnicas de diagnóstico exploram a idéia de viés (ou "distância" entre a quantidade de interesse numa particular iteração e seu verdadeiro valor) outras exploram a idéia de variância (ou precisão das estimativas).

\section{(a) MÉTODOS QUANTITATIVOS}

Um dos diagnósticos mais conhecidos é a medida de Gelman e Rubin (1992), que descrevemos a seguir.

\section{Medida de Gelman e Rubin (1992)}

A idéia desse método é utilizar $m$ cadeias paralelas $(m \geq 2)$ partindo de pontos iniciais bem dispersos dentro do espaço paramétrico, e para cada quantidade de interesse $\psi$, comparar a variabilidade "entre" as cadeias com a variabilidade "dentro" das cadeias, calculadas com base nas últimas $n=T-T_{0}$ iterações (onde $T$ é o número total de iterações). 
Segundo Gelman et al. (1997), essa medida foi originalmente calculada como

$$
\sqrt{\hat{R}}=\sqrt{\frac{n-1}{n}+\frac{B}{n W}} \text {, onde } W=\frac{1}{m} \sum_{j=1}^{n} S_{j}^{2} \text { e } B=n \frac{1}{m-1} \sum_{j=1}^{m}\left(\bar{\psi}_{\cdot j}-\overline{\bar{\psi}}\right)^{2},
$$

onde $S_{j}^{2}=\frac{1}{n-1} \sum_{i=1}^{n}\left(\psi_{i j}-\bar{\psi}_{\cdot j}\right)^{2}, \bar{\psi}_{\cdot j}=\frac{1}{n} \sum_{i=1}^{n} \psi_{i j}, \mathrm{e} \overline{\bar{\psi}}=\frac{1}{m} \sum_{i=1}^{m} \bar{\psi}_{c d o t j}=\frac{1}{m n} \sum_{i=1}^{m} \sum_{j=1}^{n} \psi_{i j}$, ou seja, $W$ é a média das $m$ variâncias dentro das cadeias e $\frac{B}{n}$ é a variância entre as médias das $m$ cadeias paralelas $(\sqrt{\hat{R}}$ é conhecido como "Potential Scale Reduction Factors(PSRF)").

Interpretação: No caso em que há convergência, espera-se que a variabilidade entre as cadeias seja pequena quando comparada com a variabilidade total (e portanto pequena quando comparada com a variabilidade dentro) e nesse caso $\hat{R}$ deve estar próximo de 1 (ou então que o quantil 0.975 da distribuição amostral é $\leq 1.2$ ).

Limitações do método:

(a) O sucesso desse método depende totalmente da habilidade do usuário em encontrar valores iniciais bem espalhados no domínio da distribuição à posteriori (sem o conhecimento da mesma);

(b) É uma técnica univariada.

Observação 1: O programa BOA ("Bayesian Output Analysis")(Smith, 2003) calcula a medida acima (PSRF) fazendo a correção proposta por Brooks e Gelman (1998), dada pela seguinte fórmula

$$
\sqrt{\left(\frac{n+1}{n}+\frac{B}{n W} \frac{m+1}{m}\right) \frac{d f+3}{d f+1}}
$$

onde df é o número de graus de liberdade de uma densidade $t$ que aproxima a distribuição à posteriori. (Esta medida é também conhecida como "Corrected Scale Reduction Factor (CSRF)"). O programa calcula também a mediana e o quantil 0.975 da distribuição amostral para CSRF. 
O programa BOA calcula também uma medida denominada "Multivariate Potential Scale Reduction Factor (MPSRF)", proposta por Brooks e Gelman(1998), que é uma extensão multivariada da medida PSRF.

Observação 2 Os diagnósticos de Raftery e Lewis (1992), Geweke (1992) e Heidelberg e Welch (1983) são implementados pelo programa BOA.

\section{(b) MÉTODOS GRÁFICOS}

Os diagnósticos mais comuns consistem em contruir, para cada parâmetro, os seguintes gráficos:

- Gráfico da série temporal (seqüência simulada).

- Gráfico da densidade (ou do núcleo da densidade) estimada.

No caso em que a densidade condicional completa da componente $\theta_{i}$ é conhecida, a densidade estimada de $\theta_{i}$ à posteriori é dada por

$$
\begin{aligned}
& \hat{\pi}\left(\theta_{i} \mid \theta_{1}, \ldots, \theta_{i=1}, \theta_{i+1}, \ldots, \theta_{p}, D_{\text {obs }}\right) \\
& =\frac{1}{m\left(T-T_{0}\right)} \sum_{i=1}^{m} \sum_{g=T_{0}+1}^{T} \pi\left(\theta_{i} \mid \theta_{1}^{(g)}, \ldots, \theta_{i-1}^{(g)}, \theta_{i+1}^{(g)}, \ldots, \theta_{p}^{(g)}, D_{\mathrm{obs}}\right),
\end{aligned}
$$

onde $m$ é o número de cadeias paralelas e $T$ é o número total de iterações de cada cadeia.

- Gráfico da função de autocorrelação amostral.

Observação: O programa BOA (Smith, 2003) fornece também outras opções como

- Graáfico das médias móveis ("running mean").

- Gráfico da medida de diagnóstico de Gelman e Rubin. 
- Gráfico da medida de Brooks e Gelman (que é uma extensão multivariada da medida de Gelman e Rubin).

- Gráfico da medida de diagnóstico de Geweke.

\section{GUIA PRÁTICO}

Uma possível estratégia para diagnosticar falha na convergência dos algorítmos:

(1) Gerar $m$ cadeias paralelas e simultaneamente uma cadeia mais longa (digamos com 50000 iterações), onde $3 \leq m \leq 10$.

(2) Para cada componente do vetor de parâmetros, fazer os seguintes gráficos:

(a) Série temporal usando cadeia mais longa.

(b) Autocorrelação usando a cadeia mais longa.

(c) Fazer o gráfico das médias móveis para cada parâmetro

(1) usando a cadeia mais longa ou

(2) usando as $m$ cadeias paralelas

(i) plotando as $m$ cadeias num mesmo sistema de coordendas ou

(ii) plotando todos os parâmetros num mesmo sistema de coordenadas para cada cadeia.

(d) Fazer um gráfico com as $m+1$ densidades estimadas (ou núcleos das densidades estimadas) num mesmo sistema de coordenadas.

(e) Fazer o gráfico com a medida de Gelman e Rubin (que requer $m \geq 2$ cadeias). Aumentar o número de simulações (iterações do algoritmo) caso o gráfico não se estabilize em torno de 1 (ou o quantil $0,975 \leq 1,2$ ).

(f) Fazer o gráfico de Geweke (implementado no programa BOA). 
(3) Fazer o gráfico com a medida multivariada de Brooks e Gelman (que utiliza as $m$ cadeias e está implementado no programa BOA).

(4) Avaliar as correlações cruzadas entre os parâmetros.

Observação sobre o período pré-convergência:

Os autores Gelman et al. (1997) recomendam descartar sempre a primeira metade de cada seqüência, outros autores, apenas $1 \%$. Uma outra alternativa é utilizar por exemplo os dispositivos $2 \mathrm{a}$ a $2 \mathrm{f}$ para auxiliar nesta questão. 


\section{Seção 2.5 .1}

A seguir apresentamos os cálculos para a obtenção das distribuições condicionais completas da posteriori $p\left(\alpha, \beta, \mu, \sigma_{x}^{2}, \sigma^{2} \mid D_{\text {obs }}\right)$ dada em (2.3.3).

- Fórmula (2.5.1)

$$
\begin{aligned}
& \pi\left(\alpha \mid \beta, \mu, \sigma_{x}^{2}, \sigma^{2}, D_{\text {obs }}\right) \propto \exp \left\{-\frac{1}{2|\Sigma|}\left[-2 \beta \sigma_{x}^{2} \sum_{i=1}^{n}\left(Y_{i}-\alpha-\beta \mu\right)\left(X_{i}-\mu\right)\right.\right. \\
& \left.\left.\quad+\left(\sigma_{x}+\sigma^{2}\right) \sum_{i=1}^{n}\left(Y_{i}-\alpha-\beta \mu\right)^{2}\right]\right\} \exp \left\{-\frac{1}{2} \frac{(\alpha-a)^{2}}{\sigma_{\alpha}^{2}}\right\} \\
& \propto \exp \left\{-\frac{1}{2}\left[\frac{2 \beta \sigma_{x}^{2} \alpha \sum_{i=1}^{n}\left(X_{i}-\mu\right)+\left(\sigma_{x}^{2}+\sigma^{2}\right)\left(n \alpha^{2}-2 \alpha \sum_{i=1}^{n}\left(Y_{i}-\beta \mu\right)\right)}{|\Sigma|}\right.\right. \\
& \left.\left.\quad+\frac{\alpha^{2}-2 \alpha a}{\sigma_{\alpha}^{2}}\right]\right\} \\
& \propto \exp \left\{-\frac{1}{2}\left[\frac{2 \alpha n \beta \sigma_{x}^{2}(\bar{X}-\mu)+n \alpha^{2}\left(\sigma_{x}^{2}+\sigma^{2}\right)-2 \alpha n\left(\sigma_{x}^{2}+\sigma^{2}\right)(\bar{Y}-\beta \mu)}{|\Sigma|}\right.\right. \\
& \left.\left.\quad+\frac{\alpha^{2}-2 \alpha a}{\sigma_{\alpha}^{2}}\right]\right\} \\
& \propto \exp \left\{-\frac{1}{2}\left[\alpha^{2}\left(\frac{n\left(\sigma_{x}^{2}+\sigma^{2}\right)}{|\Sigma|}+\frac{1}{\sigma_{\alpha}^{2}}\right)\right.\right. \\
& \left.\left.\quad-2 \alpha\left(\frac{-n \beta \sigma_{x}^{2}(\bar{X}-\mu)+n\left(\sigma_{x}^{2}+\sigma^{2}\right)(\bar{Y}-\beta \mu)}{|\Sigma|}+\frac{a}{\sigma_{\alpha}^{2}}\right)\right]\right\} .
\end{aligned}
$$

Fazendo

$$
\begin{aligned}
A & =\frac{n\left(\sigma_{x}^{2}+\sigma^{2}\right)}{|\Sigma|}+\frac{1}{\sigma_{\alpha}^{2}} \mathrm{e} \\
B & =\frac{-n \beta \sigma_{x}^{2}(\bar{X}-\mu)+n\left(\sigma_{x}^{2}+\sigma^{2}\right)(\bar{Y}-\beta \mu)}{|\Sigma|}+\frac{a}{\sigma_{\alpha}^{2}} \\
& =\frac{n \sigma_{x}^{2}(\bar{Y}-\beta \bar{X})+n \sigma^{2}(Y-\beta \mu)}{|\Sigma|}+\frac{a}{\sigma_{\alpha}^{2}},
\end{aligned}
$$

a expressão acima é proporcional a

$$
\begin{aligned}
\exp \left\{-\frac{1}{2}\left[\alpha^{2} A-2 \alpha B\right]\right\} & \propto \exp \left\{-\frac{A}{2}\left[\alpha^{2}-2 \alpha \frac{B}{A}\right]\right\} \\
& \propto \exp \left\{-\frac{1}{2 \cdot \frac{1}{A}}\left[\alpha-\frac{B}{A}\right]^{2}\right\}
\end{aligned}
$$


ou seja, é uma $N\left(\frac{B}{A}, \frac{1}{A}\right)$ e segue a fórmula (2.5.1).

\section{- Fórmula (2.5.3)}

$$
\begin{aligned}
\pi & \left.\mu \mid \alpha, \beta, \sigma_{x}^{2}, \sigma^{2}, D_{\text {obs }}\right) \propto \exp \left\{-\frac{1}{2}\left[\left(\left(\beta^{2} \sigma_{x}^{2}+\lambda \sigma^{2}\right)\left(n \mu^{2}-2 n \bar{X} \mu\right)\right.\right.\right. \\
& -2 \beta \sigma_{x}^{2}\left(\sum_{i=1}^{n}\left(Y_{i}-\alpha\right)\left(X_{i}-\mu\right)-\beta \mu \sum_{i=1}^{n}\left(X_{i}-\mu\right)\right)+\left(\sigma_{x}^{2}+\sigma^{2}\right)\left(n \mu^{2} \beta^{2}\right. \\
& \left.\left.\left.\left.-2 \mu \beta \sum_{i=1}^{n}\left(Y_{i}-\alpha\right)\right)\right) /|\Sigma|+\frac{(\mu-m)^{2}}{\sigma_{\mu}^{2}}\right]\right\} \\
\propto & \exp \left\{-\frac{1}{2}\left[\left(\left(\beta^{2} \sigma_{x}^{2}+\lambda \sigma^{2}\right) n \mu^{2}-2 n \bar{X}\left(\beta^{2} \sigma_{x}^{2}+\lambda \sigma^{2}\right) \mu+2 \beta \sigma_{x}^{2} n(\bar{Y}-\alpha) \mu\right.\right.\right. \\
& \left.+2 \beta^{2} \sigma_{x}^{2} n(\bar{X}-\mu) \mu+\left(\sigma_{x}^{2}+\sigma^{2}\right) n \beta^{2} \mu^{2}-2 n\left(\sigma_{x}^{2}+\sigma^{2}\right) \beta(\bar{Y}-\alpha) \mu\right) / \\
& \left.\left./|\Sigma|+\frac{\left.\mu^{2}-2 m \mu\right]}{\sigma_{\mu}^{2}}\right]\right\} \\
\propto & \exp \left\{-\frac{1}{2}\left[\left(\frac{n\left(\beta^{2} \sigma_{x}^{2}+\lambda \sigma^{2}\right)-2 n \beta^{2} \sigma_{x}^{2}+n \beta^{2}\left(\sigma_{x}^{2}+\sigma^{2}\right)}{|\Sigma|}+\frac{1}{\sigma_{\mu}^{2}}\right) \mu^{2}\right.\right. \\
& -2 n \mu\left(\left(\bar{X}\left(\beta^{2} \sigma_{x}^{2}+\lambda \sigma^{2}\right)-\beta \sigma_{x}^{2}(\bar{Y}-\alpha)-\beta^{2} \sigma_{x}^{2} \bar{X}+\left(\sigma_{x}^{2}+\sigma^{2}\right)(\bar{Y}-\alpha) \beta\right) /\right. \\
& \left.\left.\left./|\Sigma|+\frac{m}{\sigma_{\mu}^{2}}\right)\right]\right\} \\
& / \Sigma(\Sigma) \mid \sigma_{\mu}^{2} \\
\propto & \exp \left\{-\frac{1}{2}\left[\left(\frac{n\left(\lambda \sigma^{2}+\beta^{2} \sigma^{2}\right) \sigma_{\mu}^{2}+|\Sigma|}{|\Sigma| \sigma_{\mu}^{2}}\right) \mu^{2}\right.\right. \\
& \left.\left.-2 n \mu\left(\sigma_{\mu}^{2}\left(\bar{X} \lambda \sigma^{2}-\beta \sigma_{x}^{2}(\bar{Y}-\alpha)+\beta\left(\sigma_{x}^{2}+\sigma^{2}\right)(\bar{Y}-\alpha)\right)+m|\Sigma|\right)\right]\right\} \\
\propto & \exp \left\{\left[\left(\frac{n \sigma_{\alpha}^{2} \sigma^{2}\left(\lambda+\beta^{2}\right)+|\Sigma|}{|\Sigma| \sigma_{\mu}^{2}}\right) \mu^{2}-2 \mu\left(\frac{n \sigma_{\mu}^{2}\left(\lambda \bar{X} \sigma^{2}+\beta \sigma^{2}(\bar{Y}-\alpha)\right)+m|\Sigma|}{|\Sigma|}\right)\right] .\right.
\end{aligned}
$$

Portanto,

$$
\mu \mid \alpha, \beta, \sigma_{x}, \sigma, D_{\mathrm{obs}} \sim N\left(\frac{n \sigma_{\mu}^{2}\left(\lambda \bar{X} \sigma^{2}+3 \sigma^{2}(\bar{Y}-\alpha)\right)+m|\Sigma|}{n \sigma_{\mu}^{2} \sigma^{2}\left(\lambda+\beta^{2}\right)+|\Sigma|} ; \frac{\sigma_{\mu}^{2}|\Sigma|}{n \sigma_{\mu}^{2} \sigma^{2}\left(\lambda+\beta^{2}\right)+|\Sigma|}\right)
$$

- Fórmula (2.5.5)

$$
\pi\left(\mu \mid \beta, \sigma_{x}^{2}, \sigma^{2}, D_{\text {obs }}\right) \propto \pi\left(\mu, \beta, \sigma_{x}^{2}, \sigma^{2} \mid D_{\text {obs }}\right)=\int_{\mathbb{R}} \pi\left(\alpha, \mu, \beta, \sigma_{x}^{2}, \sigma^{2} \mid D_{\text {obs }}\right) \mathrm{d} \alpha
$$


(onde o integrando é olhado como função de $\mu$ e $\alpha$ ), onde

$$
\begin{aligned}
& \int_{\mathbb{R}} \pi\left(\alpha, \beta, \mu, \sigma_{x}^{2}, \sigma^{2} \mid D_{\mathrm{obs}}\right) \mathrm{d} \alpha \propto \int_{\mathbb{R}} \exp \left\{-\frac{1}{2}\left[\left(\left(\beta^{2} \sigma_{x}^{2}+\lambda \sigma^{2}\right) \sum_{i=1}^{n}\left(X_{i}-\mu\right)^{2}\right.\right.\right. \\
& \left.-2 \beta \sigma_{x}^{2} \sum_{i=1}^{n}\left(Y_{i}-\alpha-\beta \mu\right)\left(X_{i}-\mu\right)+\left(\sigma_{x}^{2}+\sigma^{2}\right) \sum_{i=1}^{n}\left(Y_{i}-\alpha-\beta \mu\right)^{2}\right) /|\Sigma| \\
& \left.\left.+\frac{(\alpha-a)^{2}}{\sigma_{\alpha}^{2}}+\frac{(\mu-m)^{2}}{\sigma_{\mu}^{2}}\right]\right\} \mathrm{d} \alpha \text {, onde }|\Sigma| \text { é dada em (2.3.3), } \\
& \propto \exp \left\{-\frac{1}{2}\left[\left(\left(\beta^{2} \sigma_{x}^{2}+\lambda \sigma^{2}\right)\left(n \mu^{2}-2 n \bar{X} \mu\right)-2 \beta \sigma_{x}^{2} \sum_{i=1}^{n}\left(Y_{i}-\beta \mu\right)\left(X_{i}-\mu\right)\right.\right.\right. \\
& \left.\left.\left.+\left(\sigma_{x}^{2}+\sigma^{2}\right) \sum_{i=1}^{n}\left(Y_{i}-\beta \mu\right)^{2}\right) /|\Sigma|+\frac{\left.(\mu-m)^{2}\right)}{\sigma_{\mu}^{2}}\right]\right\} \text {. } \\
& \int_{\mathbb{R}} \exp \left\{-\frac{1}{2}\left[\left(2 \beta \sigma_{x}^{2} \alpha \sum_{i=1}^{n}\left(X_{i}-\mu\right)+\left(\sigma_{x}^{2}+\sigma^{2}\right)\left(n \alpha^{2}-2 \alpha \sum_{i=1}^{n}\left(Y_{i}-\beta \mu\right)\right)\right) /\right.\right. \\
& \left.\left./|\Sigma|+\frac{(\alpha-a)^{2}}{\sigma_{\alpha}^{2}}\right]\right\} \mathrm{d} \alpha \\
& \propto \exp \left\{-\frac{1}{2}\left[\left(\left(\beta^{2} \sigma_{x}^{2}+\lambda \sigma^{2}\right)\left(n \mu^{2}-2 n \bar{X} \mu\right)-2 \beta \sigma_{x}^{2}\left(-n \mu \bar{Y}-n \beta \mu \bar{X}+n \beta \mu^{2}\right)\right.\right.\right. \\
& \left.+\left(\sigma_{x}^{2}+\sigma^{2}\right)\left(n \beta^{2} \mu^{2}-2 \beta \mu n \bar{Y}\right)\right) / \\
& \left.\left./|\Sigma|+\frac{\mu^{2}-2 m \mu}{\sigma_{\mu}^{2}}\right]\right\} \cdot \int_{\mathbb{R}} \exp \left\{-\frac{1}{2}\left[\left(2 n \beta \sigma_{x}^{2}(\bar{X}-\mu) \alpha+n\left(\sigma_{x}^{2}+\sigma^{2}\right) \alpha^{2}\right.\right.\right. \\
& \left.\left.\left.-2 n \alpha(\bar{Y}-\beta \mu)\left(\sigma_{x}^{2}+\sigma^{2}\right)\right) /|\Sigma|+\frac{\alpha^{2}-2 a \alpha}{\sigma_{\alpha}^{2}}\right]\right\} \\
& \propto \exp \left\{-\frac{1}{2}\left[\mu^{2}\left(\left(n\left(\beta^{2} \sigma_{x}^{2}+\lambda \sigma^{2}\right)-2 n \beta^{2} \sigma_{x}^{2}+\left(\sigma_{x}^{2}+\sigma^{2}\right) n \beta^{2}\right) /|\Sigma|+\frac{1}{\sigma_{\mu}^{2}}\right)\right.\right. \\
& \left.\left.-2 \mu\left(\left(n \bar{X}\left(\beta^{2} \sigma_{x}^{2}+\lambda \sigma^{2}\right)-n \bar{Y} \beta \sigma_{x}^{2}-n \beta^{2} \sigma_{x}^{2} \bar{X}+\beta n \bar{Y}\left(\sigma_{x}^{2}+\sigma^{2}\right)\right) /|\Sigma|+\frac{m}{\sigma_{\mu}^{2}}\right)\right]\right\} \\
& \int_{\mathbb{R}} \exp \left\{-\frac{1}{2}\left[\alpha^{2}\left(\frac{n\left(\sigma_{x}^{2}+\sigma^{2}\right)}{|\Sigma|}+\frac{1}{\sigma_{\alpha}^{2}}\right)\right.\right. \\
& \left.\left.-2 \alpha\left(\frac{-n \beta \sigma_{x}^{2}(\bar{X}-\mu)+n(\bar{Y}-\beta \mu)\left(\sigma_{x}^{2}+\sigma^{2}\right)}{|\Sigma|}+\frac{a}{\sigma_{a}^{2}}\right)\right]\right\} \mathrm{d} \alpha \\
& =\exp \left\{-\frac{1}{2} \mu^{2}\left(\frac{n \lambda \sigma^{2}+n \sigma^{2} \beta^{2}}{|\Sigma|}+\frac{1}{\sigma_{\mu}^{2}}\right)-2 \mu\left(\frac{n \bar{X} \lambda \sigma^{2}+n \beta \bar{Y} \sigma^{2}}{|\Sigma|}+\frac{n}{\sigma_{\mu}^{2}}\right)\right. \\
& \int_{\mathbb{R}} \exp \left\{-\frac{1}{2}\left[\left(\frac{n \sigma_{\alpha}^{2}\left(\sigma_{x}+\sigma^{2}\right)+|\Sigma|}{\sigma_{\alpha}^{2}|\Sigma|}\right) a^{2}\right.\right.
\end{aligned}
$$




$$
\left.\left.-2 \alpha\left[\left(\frac{-n \beta \sigma_{x}^{2} \bar{X}+n \bar{Y}\left(\sigma_{x}^{2}+\sigma^{2}\right)-n \beta \sigma^{2} \mu}{|\Sigma|}\right)+\frac{a}{\sigma_{\alpha}^{2}}\right]\right]\right\} \mathrm{d} \alpha .
$$

Chamando

$$
\begin{aligned}
& A=\frac{n \lambda \sigma^{2}+n \sigma^{2} \beta^{2}}{|\Sigma|}+\frac{1}{\sigma_{\mu}^{2}} \\
& B=\frac{n \bar{X} \lambda \sigma^{2}+n \beta \bar{Y} \sigma^{2}}{|\Sigma|}+\frac{m}{\sigma_{\mu}^{2}}=\frac{n \sigma^{2}(\lambda \bar{X}+\beta \bar{Y})}{|\Sigma|}+\frac{m}{\sigma_{\mu}^{2}} \\
& C=\frac{n \sigma_{a}^{2}\left(\sigma_{x}^{2}+\sigma^{2}\right)+|\Sigma|}{\sigma_{\alpha}^{2}|\Sigma|} \mathrm{e} \\
& \left.D=\frac{-n \beta \sigma_{x}^{2} \bar{X}+n \bar{Y}\left(\sigma_{x}^{2}+\sigma^{2}\right)-n \beta \sigma^{2} \mu}{|\Sigma|}+\frac{a}{\sigma_{\alpha}^{2}} \quad \text { (que é função de } \mu\right)
\end{aligned}
$$

temos que a expressão acima é igual a

$$
\begin{aligned}
& \exp \left\{-\frac{1}{2}\left[\mu^{2} A-2 \mu B\right]\right\} \int_{\mathbb{R}} \exp \left\{-\frac{1}{2}\left[C \alpha^{2}-2 \alpha D\right]\right\} \mathrm{d} \alpha \\
& =\exp \left\{-\frac{1}{2}\left[A \mu^{2}-2 B \mu\right]\right\} \int_{\mathbb{R}} \exp \left\{-\frac{C}{2}\left[\alpha^{2}-2 \alpha \frac{D}{C}+\left(\frac{D}{C}\right)^{2}-\left(\frac{D}{C}\right)^{2}\right]\right\} \mathrm{d} \alpha \\
& =\exp \left\{-\frac{1}{2}\left[A \mu^{2}-2 B \mu\right]\right\} \exp \left\{\frac{D^{2}}{2 C}\right\} \int_{\mathbb{R}} \exp \left\{-\frac{1}{2 \cdot \frac{1}{C}}\left(\alpha-\frac{D}{C}\right)^{2}\right\} \mathrm{d} \alpha \\
& \propto \exp \left\{-\frac{1}{2}\left(A \mu^{2}-2 B \mu\right)\right\} \exp \left\{\frac{D^{2}}{2 C}\right\}
\end{aligned}
$$

onde

$$
D=\frac{\sigma_{\alpha}^{2}\left(-n \beta \sigma_{x}^{2} \bar{X}+n \bar{Y}\left(\sigma_{x}^{2}+\sigma^{2}\right)\right)+a|\Sigma|}{|\Sigma| \sigma_{\alpha}^{2}}-\frac{n \beta \sigma^{2} \sigma_{\alpha}^{2}}{|\Sigma| \sigma_{\alpha}^{2}} \mu .
$$

Chamando

$$
E=\frac{\sigma_{\alpha}^{2}\left(-n \beta \sigma_{x}^{2} \bar{X}+n \bar{Y}\left(\sigma_{x}^{2}+\sigma^{2}\right)\right)+a|\Sigma|}{|\Sigma| \sigma_{\alpha}^{2}} \text { e } F=\frac{n \beta \sigma^{2}}{|\Sigma|}
$$

então temos que a expressão acima é proporcional a

$$
\begin{aligned}
& \exp \left\{-\frac{1}{2}\left[A \mu^{2}-2 B \mu\right]\right\} \exp \left\{\frac{1}{2 C}[E-F \mu]^{2}\right\} \\
& =\exp \left\{-\frac{1}{2}\left[A \mu^{2}-2 B \mu-\frac{(E-F \mu)^{2}}{C}\right]\right\} \\
& \times \exp \left\{-\frac{1}{2}\left[A \mu^{2}-2 B \mu-\frac{F^{2} \mu^{2}}{C}+\frac{2 F E}{C} \mu\right]\right\} \\
& =\exp \left\{-\frac{1}{2}\left[\left(A-\frac{F^{2}}{C}\right) \mu^{2}-2 \mu\left(B-\frac{F E}{C}\right)\right]\right\} .
\end{aligned}
$$


Chamando

$$
G=A-\frac{F^{2}}{C} \quad \text { e } \quad H=B-\frac{F E}{C}
$$

temos que

$$
\pi\left(\mu \mid \beta, \sigma_{x}^{2}, \sigma^{2}, D_{\mathrm{obs}}\right) \propto \exp \left\{-\frac{1}{2}\left[G \mu^{2}-2 H \mu\right]\right\} \propto \exp \left\{-\frac{1}{2 \frac{1}{G}}\left(\mu-\frac{H}{G}\right)^{2}\right\},
$$

ou seja, é uma $N\left(\frac{H}{G}, \frac{1}{G}\right)$, onde

$$
\begin{aligned}
G & =\frac{n \sigma^{2}\left(\lambda+\beta^{2}\right)}{|\Sigma|}+\frac{1}{\sigma_{\mu}^{2}}-\frac{n^{2} \beta^{2}\left(\sigma^{2}\right)^{2} \sigma_{\alpha}^{2}}{|\Sigma|\left(n \sigma_{\alpha}^{2}\left(\sigma_{x}^{2}+\sigma^{2}\right)+|\Sigma|\right)} \\
H & =\frac{n \sigma^{2}(\lambda \bar{X}+\beta \bar{Y})}{|\Sigma|}+\frac{m}{\sigma_{\mu}^{2}}-\frac{n \beta \sigma^{2}\left\{n \sigma_{\alpha}^{2}\left(\bar{Y}\left(\sigma_{x}^{2}+\sigma^{2}\right)-\beta \sigma_{x}^{2} \bar{X}\right)+a|\Sigma|\right\}}{|\Sigma|\left[n \sigma_{\alpha}^{2}\left(\sigma_{x}^{2}+\sigma^{2}\right)+|\Sigma|\right]} \\
|\Sigma| & =\beta^{2} \sigma^{2} \sigma_{x}^{2}+\lambda \sigma^{2} \sigma_{x}^{2}+\lambda\left(\sigma^{2}\right)^{2} .
\end{aligned}
$$

- Fórmula (2.5.6)

$$
\begin{aligned}
\pi & \left(\beta \mid \alpha, \mu, \sigma_{x}^{2}, \sigma^{2}, D_{\text {obs }}\right) \propto|\Sigma|^{-\frac{n}{2}} \exp \left\{-\frac{1}{2}\left[\left(\left(\beta^{2} \sigma_{x}^{2}+\lambda \sigma^{2}\right) \sum_{i=1}^{n}\left(X_{i}-\mu\right)^{2}\right.\right.\right. \\
& \left.-2 \beta \sigma_{x}^{2} \sum_{i=1}^{n}\left(Y_{i}-\alpha-\beta \mu\right)\left(X_{i}-\mu\right)+\left(\sigma_{x}^{2}+\sigma^{2}\right) \sum_{i=1}^{n}\left(Y_{i}-\alpha-\beta \mu\right)^{2}\right) / \\
& \left.\left./|\Sigma|+\frac{(\beta-b)^{2}}{\sigma_{\beta}^{2}}\right]\right\} \\
\propto & |\Sigma|^{-\frac{n}{2}} \exp \left\{-\frac{1}{2}\left[\left(\beta^{2} \sigma_{x}^{2} \sum_{i=1}^{n}\left(X_{i}-\mu\right)^{2}-2 \beta \sigma_{x}^{2}\left(\sum_{i=1}^{n}\left(Y_{i}-\alpha\right)\left(X_{i}-\mu\right)\right.\right.\right.\right. \\
& \left.\left.\left.\left.-\beta \mu \sum_{i=1}^{n}\left(X_{i}-\mu\right)\right)+\left(\sigma_{x}^{2}+\sigma^{2}\right)\left(n \beta^{2} \mu^{2}-2 \beta \mu \sum_{i=1}^{n}\left(Y_{i}-\alpha\right)\right)\right) /|\Sigma|+\frac{\beta^{2}-2 \beta b}{\sigma_{\beta}^{2}}\right]\right\} \\
\propto & |\Sigma|^{-\frac{n}{2}} \exp \left\{-\frac{1}{2}\left[\left(\beta^{2} \sigma_{x}^{2} \sum_{i=1}^{n}\left(X_{i}-\mu\right)^{2}-2 \beta \sigma_{x}^{2} \sum_{i=1}^{n}\left(Y_{i}-\alpha\right)\left(X_{i}-\mu\right)\right.\right.\right. \\
& \left.+2 n \beta^{2} \sigma_{x}^{2} \mu(\bar{X}-\mu)+n \beta^{2} \mu^{2}\left(\sigma_{x}^{2}+\sigma^{2}\right)-2 n 3 \mu(\bar{Y}-\alpha)\left(\sigma_{x}^{2}+\sigma^{2}\right)\right) / \\
& \left.\left./|\Sigma|+\frac{\left.\beta^{2}-23 b\right]}{\sigma_{\beta}^{2}}\right]\right\} \\
\times & |\Sigma|^{-\frac{n}{2}} \exp \left\{-\frac{1}{2}\left[\beta^{2}\left(\frac{\sigma_{x}^{2} \sum_{i=1}^{n}\left(X_{i}-\mu\right)^{2}+2 n \sigma_{x}^{2} \mu(\bar{X}-\mu)+n \mu^{2}\left(\sigma_{x}^{2}+\sigma^{2}\right)}{|\Sigma|}+\frac{1}{\sigma_{3}^{2}}\right)\right.\right.
\end{aligned}
$$




$$
\begin{aligned}
& \left.\left.-2 \beta\left(\frac{\sigma_{x}^{2} \sum_{i=1}^{n}\left(Y_{i}-\alpha\right)\left(X_{i}-\mu\right)+n \mu(\bar{Y}-\alpha)\left(\sigma_{x}^{2}+\sigma^{2}\right)}{|\Sigma|}+\frac{b}{\sigma_{\beta}^{2}}\right)\right]\right\} \\
& \propto|\Sigma|^{-\frac{n}{2}} \exp \left\{-\frac{1}{2|\Sigma| \sigma_{\beta}^{2}}\left[\beta ^ { 2 } \left\{\sigma _ { \beta } ^ { 2 } \left[\sigma_{x}^{2} \sum_{i=1}^{n}\left(X_{i}-\mu\right)^{2}+2 n \sigma_{x}^{2} \mu(\bar{X}-\mu)\right.\right.\right.\right. \\
& \left.\left.+n \mu^{2}\left(\sigma_{x}^{2}+\sigma^{2}\right)\right]+|\Sigma|\right\}-2 \beta\left\{\sigma _ { \beta } ^ { 2 } \left[\sigma_{x}^{2}\left(\sum_{i=1}^{n} X_{i} Y_{i}-n \alpha \bar{X}\right)\right.\right. \\
& \left.\left.\left.\left.+n \mu \sigma^{2}(\bar{Y}-\alpha)\right]+b|\Sigma|\right\}\right]\right\}
\end{aligned}
$$

- Fórmula (2.5.7)

$$
\begin{aligned}
& \pi\left(\sigma_{x}^{2} \mid \alpha, \beta, \mu, \sigma^{2}, D_{\text {obs }}\right) \propto|\Sigma|^{-\frac{n}{2}}\left(\sigma_{x}^{2}\right)^{-(c+1)} \exp \left\{-\frac{1}{2}\left[\left(\beta^{2} \sigma_{x}^{2} \sum_{i=1}^{n}\left(X_{i}-\mu\right)^{2}\right.\right.\right. \\
& \left.\left.\left.-2 \beta \sigma_{x}^{2} \sum_{i=1}^{n}\left(Y_{i}-\alpha-\beta \mu\right)\left(X_{i}-\mu\right)+\sigma_{x}^{2} \sum_{i=1}^{n}\left(Y_{i}-\alpha-\beta \mu\right)^{2}\right) /|\Sigma|+\frac{2 d}{\sigma_{x}^{2}}\right]\right\} \\
& \propto|\Sigma|^{-\frac{n}{2}}\left(\sigma_{x}^{2}\right)^{-(c+1)} \exp \left\{-\frac{1}{2}\left[\sigma_{x}^{2} \frac{\sum_{i=1}^{n}\left(Y_{i}-\alpha-\beta X_{i}\right)^{2}}{|\Sigma|}+\frac{2 d}{\sigma_{x}^{2}}\right]\right.
\end{aligned}
$$

- Fórmula (2.5.8)

$$
\begin{aligned}
& \pi\left(\sigma^{2} \mid \alpha, \beta, \mu, \sigma_{x}^{2}, D_{\text {obs }}\right) \propto \\
& |\Sigma|^{-\frac{n}{2}}\left(\sigma^{2}\right)^{-(f+1)} \exp \left\{-\frac{1}{2}\left[\frac{\lambda \sigma^{2} \sum_{i=1}^{n}(X-\mu)^{2}+\sigma^{2} \sum_{i=1}^{n}\left(Y_{i}-\alpha-\beta \mu\right)^{2}}{|\Sigma|}+\frac{2 g}{\sigma^{2}}\right]\right\} \\
& =|\Sigma|^{-\frac{n}{2}}\left(\sigma^{2}\right)^{-(f+1)} \exp \left\{-\frac{1}{2}\left[\frac{\sigma^{2}}{|\Sigma|}\left(\sum_{i=1}^{n}\left(Y_{i}-\alpha-\beta \mu\right)^{2}+\lambda \sum_{i=1}^{n}\left(X_{i}-\mu\right)^{2}\right)+\frac{2 g}{\sigma^{2}}\right]\right\}
\end{aligned}
$$




\section{Seção 2.8}

- Derivação da fórmula (2.8.2)

$$
\begin{aligned}
& \int_{-\infty}^{\infty} p\left(\underset{\sim}{\theta}, \underset{\sim}{x} \mid D_{\text {obs }}\right) \mathrm{d} \alpha \propto \sigma_{x}^{2^{-\left(\frac{n}{2}+c+1\right)}}\left(\sigma^{2}\right)^{-(n+f+1)} \exp \left\{-\frac{1}{2}\left[\frac{2 d}{\sigma_{x}^{2}}+\frac{2 g}{\sigma^{2}}\right.\right. \\
& \left.\left.+\frac{\sum_{i=1}^{n}\left(X_{i}-x_{i}\right)^{2}}{\sigma^{2}}+\frac{\sum^{i}\left(x_{i}-\mu\right)^{2}}{\sigma_{x}^{2}}\right]\right\} \int_{-\infty}^{\infty} \exp \left(-\frac{1}{2 \lambda \sigma^{2}} \sum_{i=1}^{n}\left(Y_{i}-\alpha-\beta x_{i}\right)^{2}\right) \mathrm{d} \alpha
\end{aligned}
$$

onde

$$
\begin{aligned}
& \int_{-\infty}^{\infty} \exp \left(-\frac{1}{2 \lambda \sigma^{2}} \sum_{i=1}^{n}\left(Y_{i}-\alpha-\beta x_{i}\right)^{2}\right) \mathrm{d} \alpha \\
& =\int_{-\infty}^{\infty} \exp \left\{-\frac{1}{2 \lambda \sigma^{2}}\left[\sum_{i=1}^{n}\left(Y_{i}-\beta x_{i}\right)^{2}+n \alpha^{2}-2 \alpha \sum_{i=1}^{n}\left(Y_{i}-\beta x_{i}\right)\right]\right\} \mathrm{d} \alpha \\
& =\int_{-\infty}^{\infty} \exp \left\{-\frac{1}{2 \lambda \sigma^{2}}\left[n \alpha^{2}-2 n \alpha(\bar{Y}-\beta \bar{x})+\sum_{i=1}^{n}\left(Y_{i}-\beta x_{i}\right)^{2}\right]\right\} \mathrm{d} \alpha \\
& =\int_{-\infty}^{\infty} \exp \left(-\frac{1}{2 \lambda \sigma^{2}} \sum_{i=1}^{n}\left(Y_{i}-\beta x_{i}\right)^{2}\right) \exp \left\{-\frac{n}{2 \lambda \sigma^{2}}\left[\alpha^{2}-2 \alpha(\bar{Y}-\beta \bar{x})\right]\right\} \mathrm{d} \alpha \\
& =\exp \left\{-\frac{1}{2 \lambda \sigma^{2}}\left[\sum_{i=1}^{n}\left(Y_{i}-\beta x_{i}\right)^{2}-n(\bar{Y}-\beta \bar{x})^{2}\right]\right\} \int_{-\infty}^{\infty} \exp \left\{-\frac{n}{2 \lambda \sigma^{2}}[\alpha-(\bar{Y}-\beta \bar{x})]^{2}\right\} \mathrm{d} \alpha \\
& \propto \exp \left\{-\frac{1}{2 \lambda \sigma^{2}}\left[\sum_{i=1}^{n}\left(Y_{i}-\beta x_{i}\right)^{2}-n(\bar{Y}-\beta \bar{x})^{2}\right]\right\}\left(\sigma^{2}\right)^{\frac{2}{2}}
\end{aligned}
$$

Portanto,

$$
\begin{aligned}
& \int_{-\infty}^{\infty} p\left(\underset{\sim}{\theta}, \underset{\sim}{x} \mid D_{\mathrm{obs}}\right) \mathrm{d} \alpha \propto \sigma_{x}^{2^{-\left(\frac{n}{2}+c+1\right)}\left(\sigma^{2}\right)^{-\left(n+f+\frac{1}{2}\right)} \exp \left\{-\frac{1}{2}\left[\frac{2 d}{\sigma_{x}^{2}}+\frac{2 g}{\sigma^{2}}\right.\right.} \\
& \left.\left.+\frac{\sum_{i=1}^{n}\left(X_{i}-x_{i}\right)^{2}}{\sigma^{2}}+\frac{\sum_{i=1}^{n}\left(x_{i}-\mu\right)^{2}}{\sigma_{x}^{2}}+\frac{\sum\left(Y_{i}-\beta x_{i}\right)^{2}-n(\bar{Y}-\beta \bar{x})^{2}}{\lambda \sigma^{2}}\right]\right\} .
\end{aligned}
$$

- Derivação da fórmula (2.8.3)

$$
\begin{aligned}
& \int_{-\infty}^{\infty} \int_{-\infty}^{\infty} p\left(\underset{\sim}{x} \underset{\sim}{x} \mid D_{\text {obs }}\right) \mathrm{d} \alpha \mathrm{d} \mu \propto \sigma_{x}^{2^{-\left(\frac{n}{2}+c+\frac{3}{2}\right)}}\left(\sigma^{2}\right)^{-(n+f+1)} \exp \left\{-\frac{1}{2}\left[\frac{2 d}{\sigma_{x}^{2}}+\frac{2 g}{\sigma^{2}}\right.\right. \\
& \left.\left.+\frac{\sum_{i=1}^{n}\left(X_{i}-x_{i}\right)^{2}}{\sigma^{2}}+\frac{\sum_{i=1}^{n}\left(Y_{i}-\beta x_{i}\right)^{2}-n(\bar{Y}-\beta \bar{x})^{2}}{\lambda \sigma^{2}}\right]\right\} \int_{-\infty}^{\infty} \exp \left\{-\frac{1}{2 \sigma_{x}^{2}} \sum_{i=1}^{n}\left(x_{i}-\mu\right)^{2}\right\} \mathrm{d} \mu,
\end{aligned}
$$

onde

$$
\int_{-\infty}^{\infty} \exp \left[-\frac{1}{2 \sigma_{x}^{2}} \sum_{i=1}^{n}\left(x_{i}-\mu\right)^{2}\right] \mathrm{d} \mu
$$




$$
\begin{aligned}
& =\int_{-\infty}^{\infty} \exp \left\{-\frac{1}{2 \sigma_{x}^{2}}\left(\sum x_{i}^{2}-2 n \bar{x} \mu+n \mu^{2}\right)\right\} \mathrm{d} \mu \\
& =\int_{-\infty}^{\infty} \exp \left\{-\frac{n}{2 \sigma_{x}}\left(\mu^{2}-2 \mu \bar{x}\right)\right\} \exp \left(-\frac{\sum_{i=1}^{n} x_{i}^{2}}{2 \sigma_{x}^{2}}\right) \mathrm{d} \mu \\
& =\exp \left(-\frac{\sum_{i=1}^{n} x_{i}^{2}}{2 \sigma_{x}^{2}}\right) \exp \left(\frac{n \bar{x}^{2}}{2 \sigma_{x}^{2}}\right) \int_{-\infty}^{\infty} \exp \left\{-\frac{n}{2 \sigma_{x}^{2}}\left(\mu^{2}-2 \mu \bar{x}+\bar{x}^{2}\right)\right\} \mathrm{d} \mu \\
& \propto \exp \left\{-\frac{1}{2 \sigma_{x}^{2}}\left(\sum_{i=1}^{n} x_{i}^{2}-n \bar{x}^{2}\right)\right\} \int_{-\infty}^{\infty} \exp \left\{-\frac{1}{2 \frac{\sigma_{x}^{2}}{n}}(\mu-\bar{x})^{2}\right\} \mathrm{d} \mu \\
& \propto\left(\sigma_{x}^{2}\right)^{\frac{1}{2}} \exp \left\{-\frac{1}{2 \sigma_{x}^{2}} \sum_{i=1}^{n}\left(x_{i}-\bar{x}\right)^{2}\right\} .
\end{aligned}
$$

Portanto,

$$
\begin{aligned}
& \int_{-\infty}^{\infty} \int_{-\infty}^{\infty} p\left(\underset{\sim}{\theta}, \underset{\sim}{x} \mid D_{\text {obs }}\right) \mathrm{d} \alpha \mathrm{d} \mu \propto\left(\sigma_{x}^{2}\right)^{-\left(\frac{n}{2}+c+\frac{1}{2}\right)}\left(\sigma^{2}\right)^{-\left(n+f+\frac{1}{2}\right)} \exp \left\{-\frac{1}{2}\left[\frac{2 d}{\sigma_{x}^{2}}+\frac{2 g}{\sigma^{2}}\right.\right. \\
& \left.\left.+\frac{\sum_{i=1}^{n}\left(X_{i}-x_{i}\right)^{2}}{\sigma^{2}}+\frac{\sum_{i=1}^{n}\left(Y_{i}-\beta x_{i}\right)^{2}-n(\bar{Y}-\beta \bar{x})^{2}}{\lambda \sigma^{2}}+\frac{\sum_{i=1}^{n}\left(x_{i}-\bar{x}\right)^{2}}{\sigma_{x}^{2}}\right]\right\}
\end{aligned}
$$

- Derivação da fórmula (2.8.4)

$\int_{-\infty}^{\infty} \int_{-\infty}^{\infty} \int_{-\infty}^{\infty} p\left(\underset{\sim}{\theta}, \underset{\sim}{x} \mid D_{\text {obs }}\right) \mathrm{d} \alpha \mathrm{d} \mu \mathrm{d} \beta$

$\propto\left(\sigma_{x}^{2}\right)^{-\left(\frac{n}{2}+c+1\right)}\left(\sigma^{2}\right)^{-\left(n+f+\frac{1}{2}\right)} \exp \left\{-\frac{1}{2}\left[\frac{2 d}{\sigma_{x}^{2}}+\frac{2 g}{\sigma^{2}}+\frac{\sum_{i=1}^{n}\left(X_{i}-x_{i}\right)^{2}}{\sigma^{2}}+\frac{\sum_{i=1}^{n}\left(x_{i}-\bar{x}\right)^{2}}{\sigma_{x}^{2}}\right]\right\}$

$\int_{-\infty}^{\infty} \exp \left\{-\frac{1}{2 \lambda \sigma^{2}}\left(\sum_{i=1}^{n}\left(Y_{i}-\beta x_{i}\right)^{2}-n(\bar{Y}-\beta \bar{x})^{2}\right)\right\} \mathrm{d} \beta$

onde

$$
\begin{aligned}
& \int_{-\infty}^{\infty} \exp \left\{-\frac{1}{2 \lambda \sigma^{2}}\left(\sum_{i=1}^{n}\left(Y_{i}-\beta x_{i}\right)^{2}-n(\bar{Y}-\beta \bar{x})^{2}\right)\right\} \mathrm{d} \beta \\
& \propto \int_{-\infty}^{\infty} \exp \left\{-\frac{1}{2 \lambda \sigma^{2}}\left[\sum_{i=1}^{n} Y_{i}^{2}-2 \beta \sum_{i=1}^{n} Y_{i} x_{i}+\beta^{2} \sum_{i=1}^{n} x_{i}^{2}-n \bar{Y}^{2}+2 n \bar{Y} \beta \bar{x}-n \beta^{2} \bar{x}^{2}\right]\right\} \mathrm{d} \beta
\end{aligned}
$$

Sejam $S_{Y Y}=\sum_{i=1}^{n} Y_{i}^{2}-n \bar{Y}^{2}, S_{x x}=\sum_{i=1}^{n} x_{i}^{2}-n \bar{x}^{2}, S_{Y x}=\sum_{i=1}^{n} Y_{i} x_{i}-n \bar{x} \bar{Y}$.

Portanto, a última integral é igual a

$$
\int_{-\infty}^{\infty} \exp \left\{-\frac{1}{2 \lambda \sigma^{2}}\left[S_{Y Y}-2 \beta S_{Y^{\prime} x}+\beta^{2} S_{x x}\right]\right\} \mathrm{d} \beta
$$




$$
\begin{aligned}
& =\exp \left\{-\frac{S_{Y Y}}{2 \lambda \sigma^{2}}\right\} \int_{-\infty}^{\infty} \exp \left\{-\frac{1}{2 \lambda \sigma^{2}}\left(\beta^{2} S_{x x}-2 \beta S_{Y x}\right)\right\} \\
& =\exp \left\{-\frac{S_{Y Y}}{2 \lambda \sigma^{2}}\right\} \int_{-\infty}^{\infty}\left\{-\frac{S_{x x}}{2 \lambda \sigma^{2}}\left[\beta^{2}-2 \beta \frac{S_{Y x}}{S_{x x}}\right]\right\} \mathrm{d} \beta \\
& =\exp \left\{-\frac{S_{Y Y}}{2 \lambda \sigma^{2}}\right\} \exp \left\{\frac{S_{Y x}^{2}}{2 \lambda \sigma^{2} S_{x x}}\right\} \int_{-\infty}^{\infty} \exp \left\{-\frac{1}{2 \lambda \frac{\sigma^{2}}{S_{x x}}}\left(\beta-\frac{S_{x Y}}{S_{x x}}\right)^{2}\right\} \mathrm{d} \beta \\
& \propto \exp \left\{-\frac{1}{2 \lambda \sigma^{2}}\left(S_{Y Y}-\frac{S_{x Y}^{2}}{S_{x x}}\right)\right\} \sqrt{\frac{\lambda \sigma^{2}}{S_{x x}}} \\
& \propto \frac{\left(\sigma^{2}\right)^{\frac{1}{2}}}{\left(S_{x x}\right)^{\frac{1}{2}}} \exp \left\{-\frac{1}{2 \lambda \sigma^{2}} \frac{\left[S_{Y Y} S_{x x}-S_{x Y}^{2}\right]}{S_{x x}}\right\}
\end{aligned}
$$

e, portanto,

$$
\begin{aligned}
& \int_{-\infty}^{\infty} \int_{-\infty}^{\infty} \int_{-\infty}^{\infty} p\left(\underset{\sim}{\theta}, \underset{\sim}{x} \mid D_{\mathrm{obs}}\right) \mathrm{d} \alpha \mathrm{d} \mu \mathrm{d} \beta \propto\left(\sigma_{x}^{2}\right)^{-\left(\frac{n}{2}+c+\frac{1}{2}\right)} \frac{\left(\sigma^{2}\right)^{-(n+f)}}{\left(S_{x x}\right)^{\frac{1}{2}}} \exp \left\{-\frac{1}{2}\left[\frac{2 d}{\sigma_{x}^{2}}\right.\right. \\
& \left.\left.+\frac{2 g}{\sigma^{2}}+\frac{\sum_{i=1}^{n}\left(X_{i}-x_{i}\right)^{2}}{\sigma^{2}}+\frac{S_{x x}}{\sigma_{x}^{2}}+\frac{S_{Y Y} S_{x x}-S_{x Y}^{2}}{\lambda \sigma^{2} S_{x x}}\right]\right\}
\end{aligned}
$$

- Derivação da fórmula (2.8.5)

$$
\begin{aligned}
& \int_{-\infty}^{\infty} \int_{-\infty}^{\infty} \int_{-\infty}^{\infty} \int_{-\infty}^{\infty} p\left(\underset{\sim}{\theta}, \underset{\sim}{x} \mid D_{\text {obs }}\right) \mathrm{d} \alpha \mathrm{d} \mu \mathrm{d} \beta \mathrm{d} \sigma_{x}^{2} \propto \frac{\left(\sigma^{2}\right)^{-(n+f)}}{\left(S_{x x}\right)^{\frac{1}{2}}} \exp \left\{-\frac{1}{2}\left[\frac{2 g}{\sigma^{2}}\right.\right. \\
& \left.\left.+\frac{\sum_{i=1}^{n}\left(X_{i}-x_{i}\right)^{2}}{\sigma^{2}}+\frac{\left(S_{Y Y} S_{x x}-S_{x Y}^{2}\right)}{\lambda \sigma^{2} S_{x x}}\right]\right\} \int_{0}^{\infty}\left(\sigma_{x}^{2}\right)^{-\left(\frac{n}{2}+c+\frac{1}{2}\right)} \exp \left\{-\frac{1}{2}\left(\frac{2 d}{\sigma_{x}^{2}}+\frac{S_{x x}}{\sigma_{x}^{2}}\right)\right\} \mathrm{d} \sigma_{x}^{2},
\end{aligned}
$$

onde

$$
\begin{aligned}
& \int_{0}^{\infty}\left(\sigma_{x}^{2}\right)^{-\left(\frac{n+2 c-1}{2}+1\right)} \exp \left\{-\frac{1}{2 \sigma_{x}^{2}}\left(2 d+S_{x x}\right)\right\} \mathrm{d} \sigma_{x}^{2} \\
& =\int_{0}^{\infty}\left(s_{x}^{2}\right)^{-\left(\frac{n+2 c-1}{2}+1\right)} \exp \left\{\frac{-\frac{1}{2}\left[2 d+S_{x x}\right]}{\sigma_{x}^{2}}\right\} \mathrm{d} \sigma_{x}^{2},
\end{aligned}
$$

onde o integrando é o núcleo de uma Gama Invertida com parâmetros $\frac{n+2 c-1}{2} \mathrm{e}$ $\frac{2 d+S_{x x}}{2}$ e portanto a última integral é igual a

$$
\frac{\Gamma\left(\frac{n+2 c-1}{2}\right)}{\frac{1}{2}\left(2 d+S_{x x}\right)^{\frac{n+2 c-1}{2}}} \propto \frac{1}{\left(2 d+S_{x x}\right)^{\frac{n+2 c-1}{2}}} .
$$

Portanto.

$$
\int_{-\infty}^{\infty} \int_{-\infty}^{\infty} \int_{-\infty}^{\infty} \int_{-\infty}^{\infty} p\left(\theta, \underset{\sim}{x} \mid D_{\mathrm{obs}}\right) \mathrm{d} \alpha \mathrm{d} \mu \mathrm{d} \sigma_{x}^{2} \propto \frac{\left(\sigma^{2}\right)^{-(n+f)}}{\left(S_{x x}\right)^{\frac{1}{2}}\left(2 d+S_{x x}\right)^{\frac{n+2 c-1}{2}}} \exp \left\{-\frac{1}{2}\left[\frac{2 g}{\sigma^{2}}\right.\right.
$$




$$
\left.\left.+\frac{\sum_{i=1}^{n}\left(X_{i}-x_{i}\right)^{2}}{\sigma^{2}}+\frac{\left(S_{Y Y} S_{x x}-S_{x Y}^{2}\right)}{\lambda \sigma^{2} S_{x x}}\right]\right\}
$$

- Derivação da fórmula (2.8.6)

$$
\begin{aligned}
& \int_{\Theta} p\left(\underset{\sim}{\theta}, \underset{\sim}{x} \mid D_{\mathrm{obs}}\right) \mathrm{d} \underset{\sim}{\theta} \propto \frac{1}{\left(S_{x x}\right)^{\frac{1}{2}\left(2 d+S_{x x}\right)^{\frac{n+2 c-1}{2}}} .} \\
& \int_{0}^{\infty}\left(\sigma^{2}\right)^{-(n+f)} \exp \left\{\frac{-\frac{1}{2}\left[\sum_{i=1}^{n}\left(X_{i}-x_{i}\right)^{2}+\frac{S_{Y Y} S_{x x}-S_{x Y}^{2}}{\lambda S_{x x}}+2 g\right]}{\sigma^{2}}\right\} \mathrm{d} \sigma_{x}^{2},
\end{aligned}
$$

onde

$$
\begin{aligned}
& \int_{0}^{\infty}\left(\sigma^{2}\right)^{-(n+f)} \exp \left\{\frac{-\frac{1}{2}\left[\sum_{i=1}^{n}\left(X_{i}-x_{i}\right)^{2}+\frac{S_{Y Y} S_{x x}-S_{x Y}^{2}}{\lambda S_{x x}}+2 g\right]}{\sigma^{2}}\right\} \mathrm{d} \sigma_{x}^{2} \\
& =\int_{0}^{\infty}\left(\sigma^{2}\right)^{-(n+f-1+1)} \exp \left\{\frac{-\frac{1}{2}\left[\sum_{i=1}^{n}\left(X_{i}-x_{i}\right)^{2}+\frac{S_{Y Y} S_{x x}-S_{x Y}^{2}}{\lambda S_{x x}}+2 g\right]}{\sigma^{2}}\right\} \mathrm{d} \sigma_{x}^{2} \\
& =\frac{\Gamma(n+f-1)}{\frac{1}{2}\left[\sum_{i=1}^{n}\left(X_{i}-x_{i}\right)^{2}+\frac{S_{Y Y} S_{x x}-S_{x Y}^{2}}{\lambda S_{x x}}+2 g\right]^{n+f-1}} \\
& \propto \frac{1}{\left[\sum_{i=1}^{n}\left(X_{i}-x_{i}\right)^{2}+\frac{S_{Y Y} S_{x x}-S_{x Y}^{2}}{\lambda S_{x x}}+2 g\right]^{n+f-1}} \\
& =\frac{1}{\left[\sum_{i=1}^{n}\left(X_{i}-x_{i}\right)^{2}+\frac{S_{Y Y} S_{x x}-S_{x Y}^{2}}{\lambda S_{x x}}+2 g\right]^{n+f-1} .}
\end{aligned}
$$

Portanto,

$$
\int_{\Theta} p\left(\underset{\sim}{\theta}, \underset{\sim}{x} \mid D_{\mathrm{obs}}\right) \mathrm{d} \theta \underset{\sim}{\left(S_{x x}\right)^{\frac{1}{2}}\left(2 d+S_{x x}\right)^{\frac{n+2 c-1}{2}}\left(\sum_{i=1}^{n}\left(X_{i}-x_{i}\right)^{2}+\frac{S_{Y Y} S_{x x}-S_{x Y}^{2}}{\lambda S_{x x}}+2 g\right)^{n+f-1}} .
$$




\section{Seção 4.4.3}

\section{(a) O critério do Fator de Bayes}

Suponha que estamos interessados em comparar dois modelos: o modelo $M_{1}$ (com vetor de parâmetros ${\underset{\sim}{M_{1}}}_{1}$ e priori $\pi\left(\underset{\sim}{\theta_{M}}{ }_{1}\right)$ ) e o modelo $M_{2}$ (com vetor de parâmetros $\underset{\sim}{\theta} M_{2}$ e priori $\left.\pi\left(\underset{\sim}{\left(M_{2}\right.}\right)\right)$ para o mesmo conjunto de dados $\underset{\sim}{Y}$.

Então o Fator de Bayes (BF) para o modelo 1 versus o modelo 2 é dado por

$$
\mathrm{BF}_{12} \frac{\pi\left(M_{1} \mid \underset{\sim}{Y}\right) / \pi\left(M_{2} \mid \underset{\sim}{Y}\right)}{\pi\left(M_{1}\right) / \pi\left(M_{2}\right)}=\frac{\pi\left(\underset{\sim}{Y} \mid M_{1}\right)}{\pi\left(\underset{\sim}{Y} \mid M_{2}\right)}
$$

onde $\pi\left(\underset{\sim}{Y} \mid M_{i}\right)=\int_{\Theta_{M_{i}}} \pi\left(\underset{\sim}{Y} \mid \underset{M_{i}}{\mid}\right) \pi\left(\theta_{M_{i}}\right) \mathrm{d} \theta_{M_{i}}, i=1,2$, isto é, $\pi\left(\underset{\sim}{Y} \mid M_{i}\right)$ é a distribuição marginal de $\underset{\sim}{Y}$ sob o modelo $M_{i}$ (ou distribuição priori preditiva sob o modelo $\left.M_{i}\right)$ e portanto $\pi\left(\underset{\sim}{Y} \mid M_{i}\right)$ é essencialmente a constante de proporcionalidade (ou de normalização) da posteriori sob o modelo $M_{i}$, isto é, $\pi\left(\underset{\sim}{(\underset{\sim}{Y}}, M_{i}\right)$.

Interpretação: Valores altos de $\mathrm{BF}_{12}$ favorecem o modelo 1.

Observação: Se $\pi\left(\theta_{M_{1}}\right)$ ou $\pi\left(\theta_{M_{2}}\right)$ for imprópria, o fator de Bayes não está bem definido pois envolve uma constante arbitrária (e portanto não é interpretável). Além desse problema, essa medida tem sido criticada pois fere princípios bayesianos.

Jeffreys (1961) e Kass e Raftery (1995) sugerem as seguintes tabelas de referência para o Fator de Bayes $\left(\mathrm{BF}_{12}\right)$ :

\begin{tabular}{l|l|l}
\hline \hline & $\mathrm{BF}_{12}$ & Evidência a favor do modelo $M_{1}$ \\
\hline \hline & $1 \leq \mathrm{BF} \leq 3.2$ & Fraca \\
Jeffrey & $3.2<\mathrm{BF} \leq 10$ & Moderada \\
& $10<\mathrm{BF} \leq 100$ & Forte \\
& $\mathrm{BF}>100$ & Decisiva \\
\hline \hline & $1 \leq \mathrm{BF} \leq 3$ & Fraca \\
Kass e & $3<\mathrm{BF} \leq 20$ & Moderada \\
Raftery & $20<\mathrm{BF} \leq 150$ & Forte \\
& $\mathrm{BF}>150$ & Muito forte \\
\hline \hline
\end{tabular}


$\mathrm{Na}$ literatura existem vários métodos para estimar essa razão de constantes de proporcionalidade (ver Chen et al., 2000). Um método é estimar a verossimilhança marginal de cada modelo $\hat{p}\left(\underset{\sim}{Y} \mid M_{i}\right)$ e tomar o quociente.

Newton e Raftery (1994) propõe um método direto de estimar $p\left(\underset{\sim}{Y} \mid M_{i}\right)$ :

$$
\hat{p}\left(\underset{\sim}{Y} \mid M_{i}\right)=\left[\frac{1}{G} \sum_{g=1}^{G} \frac{1}{f\left(\underset{\sim}{Y} \mid \underset{\sim}{\theta_{M_{i}}^{(g)}}\right)}\right]^{-1}
$$

onde $\left({\underset{\sim}{(}}^{(1)}, \ldots,{\underset{\theta}{(G)}}^{(G)}\right)$ é uma amostra da posteriori $p\left(\underset{\sim}{\theta \mid} \underset{\sim}{Y}, M_{i}\right)$ e $f\left(\underset{\sim}{Y} \mid \underset{{ }^{\prime}}{M_{i}}\right)$ é a função densidade das observações. Esse método, embora altamente eficiente, pode ficar instável, já que o denominador pode ficar muito próximo de zero para algumas parcelas dessa soma.

Um método relativamente fácil de ser implementado e também seguro é o método proposto por Chib (1995) que se baseia também na verossimilhança marginal dos modelos sendo comparados (isto é, se o objetivo é comparar dois modelos $M_{1}$ e $M_{2}$, então o fator de Bayes $\mathrm{BF}_{1,2}$ é estimado por $\left.\widehat{\mathrm{BF}}_{1,2}=\frac{\hat{p}\left(\underset{\sim}{Y} \mid M_{1}\right)}{\hat{p}\left(\underset{\sim}{Y} \mid M_{2}\right)}\right)$. Esse método é usado somente no caso em que todas as distribuições condicionais completas são conhecidas (isto é, no caso em que o algoritmo de Gibbs possa ser utilizado).

\section{Descrição do método de Chib}

Pela regra de Bayes, temos que a posteriori $p(\underset{\sim}{\theta} \mid \underset{\sim}{Y})=\frac{p(\underset{\sim}{Y} \mid \underset{\sim}{\mid}) p(\theta)}{p(\underset{\sim}{Y})}$, e portanto $p(\underset{\sim}{Y})=\frac{p(\underset{\sim}{Y} \mid \underline{\theta}) p(\underline{\theta})}{p(\underset{\sim}{\theta} \mid \underset{\sim}{Y})}$. Essa igualdade vale para todo $\theta$, e em particular para um $\underset{\sim}{\theta}=\underset{\sim}{\theta^{\prime}}$ qualquer (nesse método é usual tomar $\underset{\sim}{\theta^{\prime}}$ como a moda ou a média da posteriori para garantir estabilidade numérica). Portanto, $p(\underset{\sim}{Y})=\frac{p\left(\underset{\sim}{Y} \mid \underline{\Theta}^{\prime}\right) p\left(\underline{\theta}^{\prime}\right)}{p\left(\underline{\theta}^{\prime} \mid \underset{\sim}{Y}\right)}$, onde $p\left(\underline{\theta}^{\prime} \mid Y\right)$ deve ser estimado, já que freqüentemente conhecemos apenas o núcleo da posteriori $p(\underset{\sim}{\theta} \mid \underset{\sim}{Y})$.

A seguir descrevemos o método para o caso particular em que $\underset{\sim}{\theta}$ é composto de

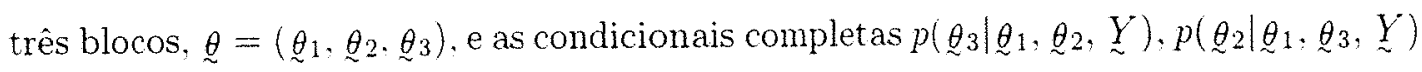


e $p\left(\theta_{1} \mid \theta_{2}, \theta_{3}, \underset{\sim}{Y}\right)$ são conhecidas explicitamente. Nesse caso, $p\left(\theta^{\prime} \mid \underset{\sim}{Y}\right)=$

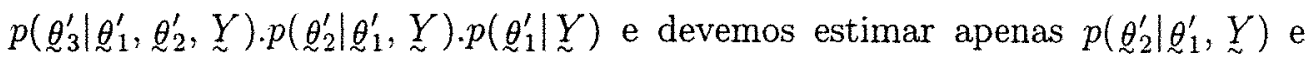
$p\left(\theta_{1}^{\prime} \mid \underset{\sim}{Y}\right)$, já que $p\left(\theta^{\prime} \mid \theta_{1}^{\prime}, \theta_{2}^{\prime}, \underset{\sim}{Y}\right)$ é conhecido.

Para estimar $p\left(\theta_{1}^{\prime} \mid Y \sim \sim\right.$ ) podemos usar o estimador de Monte Carlo $\hat{p}\left(\theta_{\sim}^{\prime} \mid Y\right)=$

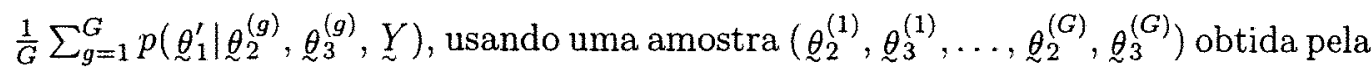
algoritmo de Gibbs quando amostramos da posteriori $p\left(\theta_{1}, \theta_{2}, \theta_{3} \mid \underset{\sim}{Y}\right)$, onde $\theta_{1}=\theta_{\sim}^{\prime}$.

Para estimar $p\left(\Theta_{2}^{\prime} \mid \theta_{1}^{\prime}, \underset{\sim}{Y}\right)$, devemos notar primeiramente que

$$
p\left(\theta_{2}^{\prime} \mid \theta_{1}^{\prime}, \underset{\sim}{Y}\right)=\int_{\Theta_{1}} p\left(\theta_{2}^{\prime}, \theta_{3} \mid \theta_{1}^{\prime}, \underset{\sim}{Y}\right) \mathrm{d} \theta_{3}=\int_{\Theta_{3}} p\left(\theta_{2}^{\prime} \mid \theta_{1}^{\prime}, \theta_{3}, \underset{\sim}{Y}\right) p\left(\theta_{3} \mid \theta_{1}^{\prime}, \underset{\sim}{Y}\right) \mathrm{d} \theta_{3},
$$

e portanto se pudermos gerar $\left(\theta_{3}^{(1)}, \ldots,{\underset{\sim}{3}}_{3}^{(G)}\right)$ de $p\left(\theta_{3} \mid \theta_{1}^{\prime}, \underset{\sim}{Y}\right)$, um estimador de Monte Carlo de $p\left(\theta_{2}^{\prime} \mid \theta_{1}^{\prime}, \underset{\sim}{Y}\right)$ é dado por

$$
\hat{p}\left(\theta_{2}^{\prime} \mid \theta_{1}^{\prime}, \underset{\sim}{Y}\right)=\frac{1}{G} \sum_{g=1}^{G} p\left(\underline{\theta}_{2}^{\prime} \mid \theta_{1}^{\prime}, \Theta_{3}^{(g)}, \underset{\sim}{Y}\right) .
$$

O problema então é como gerar essa amostra.

A seguir descrevemos o algoritmo de Gibbs para amostrar de $p\left(\theta_{3} \mid \theta_{1}^{\prime}, \underset{\sim}{Y}\right)$.

Passo 1: dar um valor inicial $\theta_{3}=\theta_{3}^{(0)}$ e fazer $i=0$,

Passo 2: gerar $\theta_{2}^{(i+1)}$ de $p\left(\theta_{2} \mid \theta_{1}^{\prime}, \theta_{3}^{(i)}, \underset{\sim}{Y}\right)=p\left(\theta_{2} \mid \theta_{1}={\underset{\theta}{1}}_{1}^{\prime}, \theta_{3}=\theta_{3}^{\prime}, \underset{\sim}{Y}\right)$,

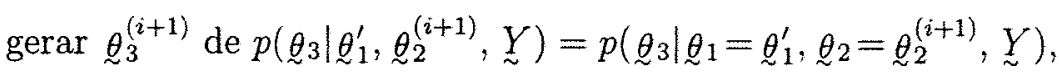

Passo 3: fazer $i=i+1$ e voltar ao passo 2 , até a convergência.

Finalmente, tendo obtido $\hat{p}\left(\underline{\theta}_{1}^{\prime} \mid \underset{\sim}{Y}\right)$ e $\hat{p}(\underbrace{\prime}_{2} \mid \theta_{1}^{\prime}, \underset{\sim}{Y})$, temos que

$$
\hat{p}(Y)=\frac{p\left(\underline{Y} \mid \underline{\theta}^{\prime}\right) p\left(\underline{\theta}^{\prime}\right)}{\hat{p}\left(\underline{\theta}^{\prime} \mid Y\right)},
$$

ou seja, $\log \hat{p}(\underset{\sim}{Y})=\log p\left(\underset{\sim}{Y} \mid \underline{\theta}^{\prime}\right)+\log p\left(\underline{\theta}^{\prime}\right)-\log \hat{p}\left(\underline{\theta}^{\prime} \mid \underset{\sim}{Y}\right)$, onde

$$
\log \hat{p}\left(\underline{\theta}^{\prime} \mid Y\right)=\log p\left(\theta_{3}^{\prime} \mid \theta_{1}^{\prime} \theta_{2}^{\prime}, \underset{\sim}{Y}\right)+\log \hat{p}\left(\theta_{2}^{\prime} \mid \theta_{1}^{\prime}, Y\right)+\log \hat{p}\left(\theta_{1}^{\prime} \mid Y\right)
$$




\section{(b) O critério do Pseudo Fator de Bayes - formulação geral}

Vimos que o Fator de Bayes não está definido quando a priori é imprópria. Uma medida alternativa robusta nesse caso é o Pseudo Fator de Bayes, definido por Geisser e Eddy (1979).

O Pseudo Fator de Bayes (PBF) para dois modelos competitivos $M_{1}$ e $M_{2}$ é definido por

$$
\operatorname{PBF}_{12}=\frac{\prod_{i=1}^{n} \pi_{1}\left(Y_{i} \mid Y_{(-i)}\right)}{\prod_{i=1}^{n} \pi_{2}\left(Y_{i} \mid Y_{(-i)}\right)}=\frac{\prod_{i=1}^{n} \mathrm{CPO}_{i}^{\left(M_{1}\right)}}{\prod_{i=1}^{n} \mathrm{CPO}_{i}^{\left(M_{2}\right)}}
$$

onde $\mathrm{CPO}_{i}^{\left(M_{l}\right)}=\pi_{l}\left(Y_{i} \mid Y_{(-i)}\right)$, que é a densidade de validação cruzada no modelo $M_{l}$, $l=1,2$.

Interpretação: Um valor alto de PBF na fórmula acima favorece o modelo $M_{1}$.

\section{(c) O critério "BIC" ("Bayesian Information Criterion")}

Esta é uma medida proposta por Schwarz (1978) que pode ser usada para comparar modelos e que não depende de conhecimento à priori, mas apenas da verossimilhança.

O "BIC" do modelo $M_{i}$ é definido por

$$
\mathrm{BIC}_{M_{i}}=-2 \log \left(\sup _{M_{i}} f(\underset{\sim}{Y} \mid \underset{\sim}{\mid \theta})\right)+p_{i} \log n=-2 \log \left(f_{M_{i}}\left(\underset{\sim}{Y} \mid \hat{\theta}_{i}\right)\right)+p_{i} \log n
$$

onde $\underline{\sim}_{i}$ é o ponto de máximo de $f_{M_{i}}\left(\underset{\sim}{Y} \mid \underset{\sim}{\theta_{i}}\right)$, ou seja, $\hat{\sim}_{i}$ é o estimador de máxima verossimilhança de ${\underset{\sim}{i}}_{i}$ e $p_{i} \log n$ é a penalidade (onde $n$ é o tamanho da amostra e $p_{i}$ é a dimensão do espaço paramétrico do modelo $M_{i}$ ).

Interpretação: O modelo com o menor BIC é o "melhor".

Schwarz (1978) mostrou que para $n$ grande e sob certas condições, $\Delta \mathrm{BIC} \approx$ $-2 \log B F$, ou seja, $B F \approx \exp \left\{-\frac{\Delta \mathrm{BIC}}{2}\right\}$, onde $\mathrm{BF}$ é o Fator de Bayes e $\triangle \mathrm{BIC}=$ $\mathrm{BIC}_{M_{1}}-\mathrm{BIC}_{M_{2}}$. Ver também Gelfand e Dey (1994). 


\section{Referências bibliográficas}

Arellano-Valle, R. B. (1994). Distribuições elípticas: propriedades, inferência e aplicações a modelos de regressão. 262p. Tese (Doutorado). Instituto de Matemática e Estatística. Universidade de São Paulo.

Berger, J. O. (1980). Statistical Decision Theory - Foundations, Concepts and Methods. New York, Heidelberg, Berlin: Springer-Verlag.

Berger, J. O. (2000). Bayesian analysis: A look at today and thoughts of tomorrow. Journal of the American Statistical Association, 95(452).

Bernardo, J. M. (1979). Reference posterior distributions for Bayesian inference (with discussion). Journal of The Royal Statistical Society, B, 41, 113-147.

Best, N. G.; Cowles, M. K. and Vines, K. (1995). CODA: Convergence diagnosis and output analysis software for Gibbs sampling output, version 0.30. Technical Report, Medical Research Council Biostatistics Unit, Institute of Public Health, Cambridge University.

Box, G. E. P. and Tiao, G. (1973). Bayesian Inference in Statistical Analysis. Londo: Addison-Wesley.

Brooks, S. and Gelman, A. (1998). General Methods for Monitoring Convergence of Iterative Simulations. Journal of Computation and Graphical Statistics, $7(4), 434-455$.

Brodsky, B. E.; Darkhovsky, B. S. (1993). Nonparametric Methods in Change-Point Problems. Kluwer Academic Publishers.

Broemeling, L. D.; Chin Choy, J. H. (1981). Detecting structural change in linear models. Communications in Statistics, 4, 33-47.

Carlin, B. P.; Gelfand. A.; Smith, A. F. (1992). Hierarchical bayesian analysis of changepoint problems. Applied Statistics, 41(2), 389-405. 
Carlin, B. P. and Louis, T. A. (2000). Bayes and Empirical Bayes Methods for Data Analysis. Champman \& Hall/CRC.

Carroll, R. J.; Ruppert, D. and Stefanski, L. A. (1995). Measurement Error in Nonlinear Models. Chapman \& Hall.

Casella, G. and George, E. L. (1992). Explaining the Gibbs sampler. The American Statistician, 46, 167-174.

Caussinus, H. e Lyazrhi, F. (1997). Choosing a linear model with a random number of change-points and ouliers.

Chang, M. P.; Huang, W. T. (1997). Inferences for the linear errors-in-variables with changepoint models. J. Amer. Statist. Association, 92(437), 171-178.

Chong, T-L.T. (1999). Estimation of and testing for structural break in the errorsin-variables model. Journal of Applied Statistical Science, 9(2), 147-158.

Chen, J. e Gupta, A. K. (1997). Testing and locating variance change-points with applications to stock prices. Journal of the American Statistical Association, 92, 739-747.

Chen, M. H.; Shao, Q. M. and Ibrahim, J. G. (2000). Monte Carlo Methods in Bayesian Computation. New York: Springer-Verlag.

Chib, S. and Greenberg, E. (1995). Understanding the Metropolis-Hastings algorithm. The American Statistician, 49, 327-335.

Chin Choy, J. H. e Broemeling, L. D. (1980). Some bayesian inference for a changing linear model. Technometrics, 22(1), 71-78.

Chung, Y. e Dey, D. K. (1996). Bayes factor for change point problem with conjugate prior. Journal of the Korean Statistical Society, 25(4).

Cowles, M. K. and Carlin, B. P. (1996). Markov chain Monte Carlo convergence diagnostics: A comparative review. J. Amer. Statist. Association, 91, 883904.

Dey, D. K. (1997). Bayesian approach to change point problems. Commun. Statist. - Theory Meth., 26(8), 2035-2047.

Dey, D. K.: Maiti, T. (2002). An Appraisal from Bayesian Perspective. Technical Report. 
Ferreira, P. E. (1975). A bayesian analysis of a switching regression model: known number of regimes. Joumal of the American Statistical Association, 70, 370374 .

Florens, J. P.; Mouchart, M. e Richards, J. F. (1974). Bayesian inference in errorsin-variables models. Journal of Multivariate Analysis, 4, 419-456.

Fuller, W. A. (1980). Measurement Error Models. New York: Erickson, T.

Gamermann, D. (1996). Simulação Estocástica via Cadeias de Markov. $12^{\circ}$ SINAPE-Caxambu.

Gbur, E. E. e Dahm, P. F. (1985). Estimation of the linear-linear segmented regression model in the presence of measurement error. Communication in Statistics, A, 14(4), 809-826.

Geisser, S. (1982). Predictive inference in duscussion of G. E. Box. Journal of the Royal Statistical Society, Series A, 143, 416-417.

Geisser, S. and Eddy, W. (1979). A predictive approach to model selection. Journal of the American Statistical Association, 74, 153-160.

Gelfand, A. E. and Dey, D. K. (1994). Bayesian model choice: asymptotics and exact calculations. J. R. Statist. Soc., B, 56, 501-514.

Gelfand, A. E.; Dey, D.K. and Chang, H. (1992). Model determination using predictive distributions with implementation via sampling-based methods (with discussion). In Bayesian Statistics, 4, J. M. Bernardo, J. O. Berger, A. P. Dawid and A. F. M. Smith, eds., Oxford: Oxford University Press, 147-167.

Gelfand, A. E., and Sahu, S. K. (1999). Identifiability improper priors, and Gibbs sampling for generalized linear models. Journal of the American Statistical Association, 94, 247-253.

Gelfand, A. E. and Smith, A. F. M. (1990). Sampling based approaches to calculating marginal densities. Journal of the American Statistical Association, 85, $398-409$.

Gelman, A.; Carlin, J.: Stern, H., and Rubin, D. B. (1997). Bayesian Data Analysis. London: Chapman and Hall/CRC. 
Gelman, A. and Rubin, D.B. (1992). Inference from iterative simulation using multiple sequences. Statistical Science, 7, 457-511.

Geman, S. and Geman, D. (1984). Stochastic relaxation, Gibbs distributions and the Bayesian restoration of images. IEEE Transactions on Pattern Analysis and Machine Intelligence, 6, 721-741.

Geweke, J. (1989). Bayesian inference in econometric models using Monte Carlo integration. Econometrica, 57, 1317-1339.

Geweke, J. (1993). Bayesian treatment of the independent Student- $t$ linear model. Journal of Applied Econometrics, 8, 519-540.

Gilks, W. R.; Best, N., and Tan, K. K. C. (1995). Adaptative rejection Metropolis sampling within Gibbs sampling. Applied Statistics, 44, 455-472.

Gill, P. E.; Murray, W; Wrigert, M. H. (1981). Practical Optimization. Academic Press. London, New York, Toronto, Sydney and S. Francisco.

Grimshaw, S. D. (1992). Estimation of the linear-Plateau segmented regression model in the presence of measurement error. Communication in Statistics, A, 21(8), 2399-2413.

Hastings, W. K. (1970). Monte Carlo sampling methods using Markov chains and their applications. Biometrika, 57, 97-109.

Hinkley, D. V. (1970). Inference about the change-point in a sequence of random variables. Biometrika, 57, 1-16.

Holbert, D. (1982). A bayesian analysis of a switching linear model. Journal of Econometrics, 19, 77-87.

Ibrahim, J. G., Chen, M. H., Sinha (2001). Criterion based methods for bayesian model assessment. Statistica Sinica, 11, 419-443.

Jeffrey, H. (1961). Theory of Probability. 3.ed. Oxford: Cniversity Press.

Johnson, R. A.; Wichern, D. W. (1998). Applied Multivariate Statistical Analysis. Prentice Hall, W. J. 07458.

Kass, R. E. and Raftery, A. E. (1995). Bayes factors. J. Amer. Statist. Assoc., 90. 773-795. 
Kass, R. E. and Wasserman, L. (1996). The selection of prior distribuitions by formal rules. Journal of The American Statistical Association, 91, 1343-1370.

Kim, D. (1991). A bayesian significance test of the stationarity of regression parameters. Biometrika, $78(3), 667-75$.

Land, M. e Broemeling, L. (1983). Bayesian forecasting with changing linear models. Communications in Statistics, A, 12(12), 1421-1430.

Lange, K. (1999). Numerical Analysis for Statisticians. New York: SpringerVerlag, Inc.

Leiva, R. A. (1994). Bayesian prediction of the number of changepoints in the piecewise linear model. Journal of the Italian Statistical Society, 3(2), 271289 .

Liu, J. S. (1994). The collapsed Gibbs sampler in Bayesian computations with applications to a gene regulation problem. Journal of the American Statistical Association, 89, 958-966.

Liu, J. S., Wong, W. H., and Kong, A. (1995). Covariance structure of the Gibbs sampler with applications to the comparisons of estimators and augmentations. Biometrika, 81, 27-40.

Lyazrhi, F. (1997). Bayesian criteria for discriminating among regression models with one possible change point. Joumal of Statistical Planning and Inference, 59, 317-353.

Mengersen, K. L.; Robert, C. P. and Guihennec-Jouyavx, C. (1999). MCMC convergence diagnostics: a review (with discussion). In Bayesian Statistics 6, J. M. Bernardo, J. O. Berger, A. P. Dawid, and A. F. M. Smith, eds., Oxford; Oxford University Press, pp. 415-440.

Meyn, S. P. and Tweedie, R. I. (1993). Markov Chains and Stochastic Stability. London: Springer-Verlag.

Metropolis, N.; Rosenbluth, A. W.; Rosenbluth, M. W.; Teller, A. H. and Teller, E. (1953). Equations of state calculations by fast computing machines. J. Chem. Physics, 21, 1087-1092.

Moen. D. H.; Salazar, D. e Broemeling, L. D. Structural changes in multivariate regression models. Communications in Statistics, A, 14, 1757-1768. 
Newton, M. A. and Raftery, A. E. (1994). Aproximate Bayesian inference by the weighted likelihood bootstrap (with discussion). J. Roy. Statist. Soc., Ser. $B, \mathbf{5 6}, 1-48$.

Quandt, R. E. (1958). The estimation of the parameters of a linear regression system obeys a two separate regimes. Journal of the American Statistical Association, 53, 873-880.

Quandt, R. E. (1960). Tests of the hypothesis that a linear regression system obeys two separate regimes. Journal of the American Statistical Association, 55, 324-330.

Robert, C. P. and Casella, G. (1999). Monte Carlo Statistical Methods. New York: Springer-Verlag.

Roberts, G. O. and Sahu, S. K. (1997). Updating schemes, correlation structure, blocking and parametrization for the Gibbs sampler. Joumal of the Royal Statistical Society, Series B, 59, 291-317.

Roberts, G. O. and Smith, A. F. M. (1993). Simple conditions for the convergence of the Gibbs sampler and Metropolis-Hastings algorithm. Stochastic Processes and their Applications, 49, 207-216.

Roberts, G. O. and Tweedie, R. I. (1996). Geometric convergence and central limit theorems for multidimensional Hastings and Metropolis algorithms. Biometrika, 83, 95-110.

Schwarz, G. (1978). Estimating the dimension of a model. Ann. Statist., 6, 461464.

Searle, S. R. (1971). Linear Models. New York, London, Sydney, Toronto: John Wiley \& Sons, Inc.

Smith, A. F. M. (1975). A bayesian approach to inference about change point in sequence of random variables. Biometrika, $62,407-416$.

Smith, A. F. M. and Gelfand, A. E. (1992). Bayesian statistics without tears. A sampling-resampling perspective. The American Statistician. 46, 84-88.

Smith, A. F. M.; Skeene, A. A.; Shaw, J. E. H., and Naylor (1987). Progress with numerical and graphical methods for practical Bayesian statistics. The Statistician. 36, 75-82. 
Smith, B. J. (2003). Bayesian Output Analysis Program (BOA) Version 1.0 User's Manual. Disponível em http://www.public-health.uiowa.edu.

Spiegelhalter, D. J.; Best, N. G.; Carlin, B. P. e Van der Linde, A. (2002). Bayesian measures of model complexity and fit (with discussion). Journal of the Royal Statistical Society, Series B, 64, 583-639.

Stefanski, L. A. (2000). Measurement error models. Journal of The American Statistical Association, 95(452), 1353-1358.

Stephens, D. A. and Dellaportas, P. (1992). Bayesian analysis of generalized linear models with covariate measurement error. Bayesian Statistics, 4, 813-820. Oxford University Press.

Tanner, M. A. (1993). Tools for Statistical Inference: Methods for the Exploration of Posterior Distributions and Likelihood Functions. 2.ed. New York: Springer-Verlag.

Teeter, R. (1979). Effects of measurement error in piecewise regression models. American Statistical Association, 1979. Proceedings of the Social Statistics Section, 422-426.

Teeter, R. (1985). The application of linear pricewise regression to basal body temperature data. Biom. J., 27(7), 750-773.

Tierney, L. (1994). Markov chains for exploring posterior distributions (with discussion). The Annals of Statistics, 22, 1701-1762.

Wang, C. H. e Lee, C. B. (1993). Bayesian analysis for a change in the intercept of simple linear regression. Communication in Statistics, A, 22(11), 3031-3057.

Worsley, K. J. (1983). The power of likelihood ratio and cumulative sum tests for a change in a binomial probability. Biometrika, 70, 455-465.

Zacks, S. (1983). Survey of classical and bayesian approaches to the change-point problem: fixed sample and sequential procedures of testing and estimation. Recent Advances in Statistics, Academic Press, 245-269. 